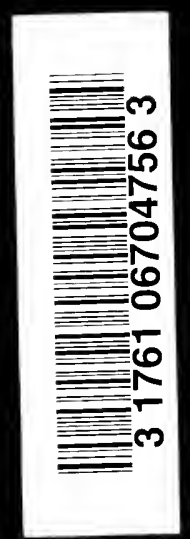




$$
\mathrm{meq}^{13^{10^{\circ}}}
$$


Digitized by the Internet Archive in 2007 with funding frọm Microsoft Corporation 

.

. 





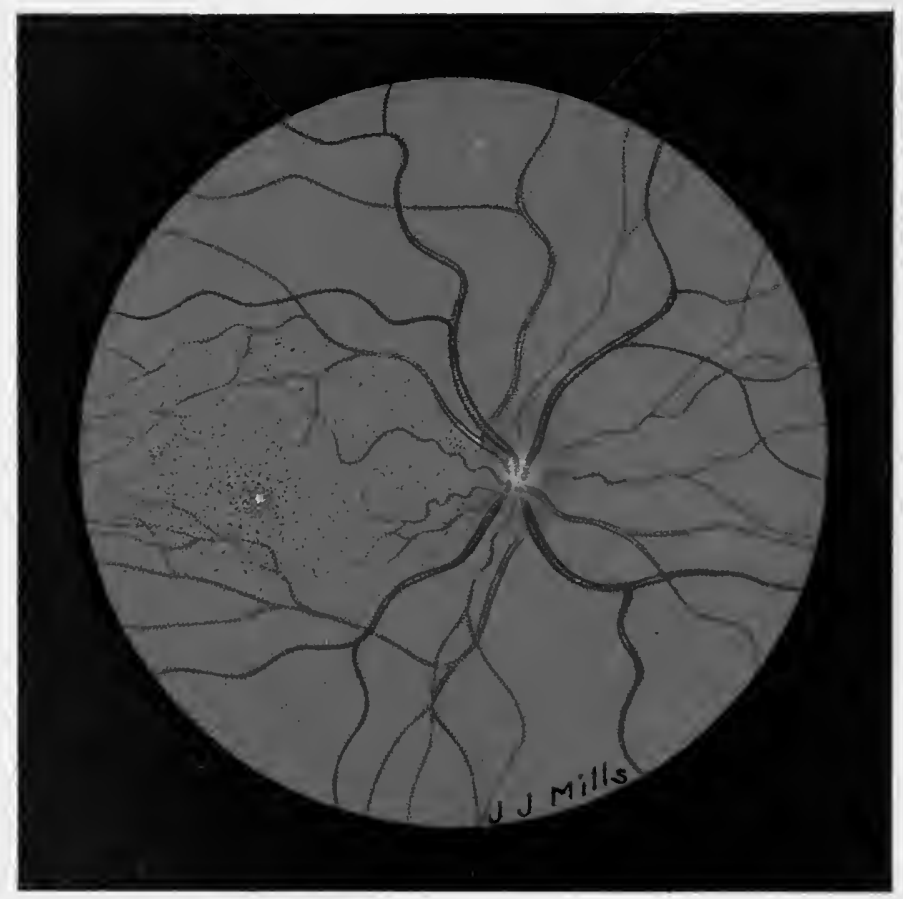

Miliary Choroido-Retinitis.

(Sef PAge 344.) 


\section{PREVALENTT DISEASES}

\section{OF THE EYE}

A REFERENCE HANDBOOK, ESPECIALLY ADAPTED TO THE NEEDS OF THE GENERAL PRACTITIONER AND THE MEDICAL STUDENT

BY

\section{SAMUEL THEOBALD, M.D.}

Clinical Professor of Ophthalmology and Otology in the Johns Hopkins University ; Ophthaimic and Aural Surgeon to the Johns Hopkins Hospital, and to the Baltimore Eye, Ear, and Throat Charity Hospital ; Consulting Ophthalmic and Aural Surgeon to the Home for Incurables, and to the South Baltimore Eye, Ear, and Throat Cirarity Hospital.

\section{WITH 219 ILLUSTRATIONS} AND IO COLORED PLATES

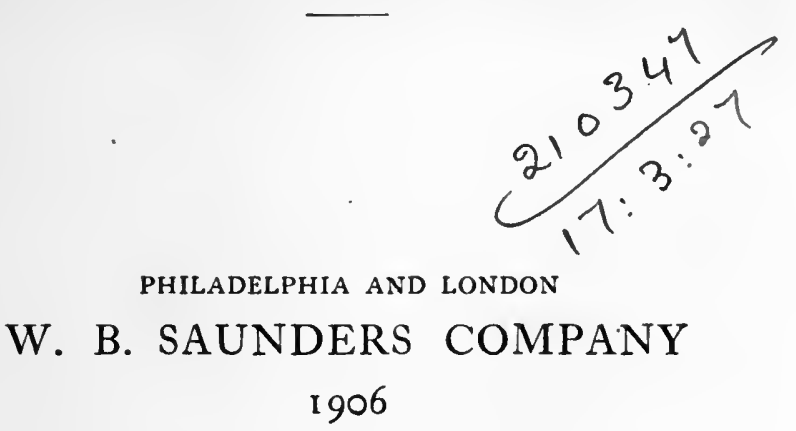


Copyright, 1906, by W. B. Saunders Company

$$
\text { PRESS OF }
$$

w. B. saunoers company

PHILADELPHIA 


\section{PREFACE.}

Although most treatises upon diseases of the eye have been written ostensibly for the general practitioner and the medical student, they have been, with few exceptions, adapted in reality not to their needs, but to the requirements of the specialist in this department of medicine. A treatise upon the eye really intended to meet the needs of the general practitioner, it has seemed to the author, should take into account the fact that the great majority of physicians are not skilled in the use of the ophthalmoscope, and are not likely ever to be; that they possess neither the experience nor the necessary paraphernalia to make trustworthy tests of refractive errors, of muscular anomalies, or of the visual fields; and that they are not qualified to perform, and have no desire to undertake, the more delicate eye operations. It should recognize, further, that because of these limitations the general practitioner is hopelessly handicapped as regards the diagnosis, and consequently the treatment, of many important eye affections; but, on the other hand, that there are other important affections of the eye which he is competent, or should be competent, to diagnosticate correctly, and to treat successfully.

It should, therefore, devote especial attention to this latter class of maladies, and, particularly, should help him to discriminate between these cases and those 
which are without his province, so that, on the one hand, he may not send trivial affections, which he is himself competent to treat, to the distant specialist, or, on the other hand, lose precious time, and thus permit sight to become irreparably damaged, through attempting to cope with maladies which urgently demand the skill of the trained ophthalmologist.

Again, such a work should take into account that the general practitioner is most in need of definite information as to the more frequently encountered diseases of the eye, and, while these should be dwelt upon, that it is a work of supererogation, so far as he is concerned, to give space to the description of rare affections which, in all probability, he will never encounter. It should also make clear to him the sharp line to be drawn between those diseases of the eye in which delay in the employment of remedial measures is permissible, or even desirable, and those in the treatment of which time is an allimportant factor.

In offering suggestions as to treatment, it should be concise, unambiguous, and as specific as practicable, not giving a long list of remedies, and leaving the inexperienced reader to choose from among them, but recommending definitely what the author-if he is qualified to write such a book-should know, from his own observation, is best adapted to the condition under consideration. The simpler operations, which the general practitioner may perform, it should describe in detail; the more difficult ones, which he is not warranted in undertaking, need not be so described, though the indications for them should be clearly set forth.

For such a book-not a complete treatise upon diseases of the eye, but a concise description of the commoner ocular maladies-designed solely to meet the 
needs of the physician engaged in general practice, the author is convinced there is a real want; and this want, with what measure of success it remains to be seen, he has endeavored to supply.

Having in mind the well-worn proverb, "a little knowledge is a dangerous thing," he has not thought it desirable to encourage the general practitioner to rely upon the ophthalmoscope as a means of diagnosis. Indeed, he has not even given a description of it, or explained its use. Furthermore, he has constantly assumed that the knowledge which it affords is not at the reader's command. For the same reason, he has not given a detailed account of the several methods of measuring the refractive and muscular anomalies of the eyes, of charting the visual fields, etc. On the other hand, especial pains have been taken to describe fully such aids to diagnosis (the examination by oblique illumination, for example) as can but prove helpful, and such therapeutic measures, operative and non-operative, as the general practitioner may employ with advantage. The hope is indulged that the introductory chapters upon "Diagnosis" and upon "Treatment" will prove of practical value.

For greatly appreciated advice and many helpful suggestions in connection with the preparation and publication of this work, the author takes pleasure in acknowledging his indebtedness to Dr. Wm. H. Welch and to Dr. Henry M. Hurd. He is also indebted to Dr. Albert H. Buck, editor of the "Reference Handbook of the Medical Sciences," for permission kindly accorded him to make free use, in writing Chapters V and VII, of the articles upon "Affections of the Conjunctiva" and upon "Iritis," which it was the author's privilege to contribute to that standard work. 
PREFACE.

To Dr. A. Maitland Ramsay he is under obligation for permission to use several admirable illustrations from his "Atlas of External Diseases of the Eye."

304 West Monument Street,

BaLtimore, JUly, igo6. 


\section{SYNOPSIS OF CONTENTS.}

\section{CHAPTER I.}

General Observations upon the Diagnosis of Diseases of the

Eye. Description of the Methods of Examining the Eye Available to the General Practitioner ...............

The difficulties in diagnosticating diseases of the eye with which the general practitioner has to contend, I7. Some of the errors into which he is apt to fall, I 7. His skill in the use of the ophthalmoscope rarely such as to make it a trustworthy means of diagnosis, 19. Other methods of examining the eye, 19. Oblique illumination, 19. Value of the information which it affords, 2 r. Facility in its employment easily acquired, 21. The assistance afforded by a transitory mydriatic, $2 \mathrm{I}$. Eversion of the eyelids, 22. Determination of intra-ocular tension, 23. The reactions of the pupil, 24. Variations in the size and reactions of the pupil in health and disease, 25. Determination of visual acuteness, 27. Simple method of measuring the visual field, 29. The patient's description of his symptoms often misleading, $3 \mathrm{I}$. Pertinent questions which he should be asked, $3 \mathbf{r}$. Significance of his replies, $3 \mathrm{r}$. Inspection of the eye by daylight, 34. The condition of the lids, lacrimal apparatus, conjunctiva, cornea, anterior chamber, iris, the appearance and behavior of the pupil, the tension of the globe, and the movements of the eyes, to be noted, 34 . Loss of sensibility of the cornea-how determined, 35. Diagnostic significance of the different types of vascular injection of the eye, and of the character and amount of the discharge present, 35. Examination of the anterior structures of the eye by oblique illumination, with and without the application of a mydriatic, 36 .

\section{CHAPTER II.}

\section{General Observations upon the Treatment of Diseases of} THE EYE

A correct diagnosis having been reached the selection of the proper remedy to meet the condition usually not difficult, 38 . Importance of constitutional remedies in the treatment of diseases of the eye, 38 . The local and constitutional remedies especially useful in the treatment of eye diseases, 39. Indications for, and contraindications to, their use, and the methods of employing 
them, 39, 40. Importance of an early diagnosis, and the prompt employment of therapeutic measures, in many affections of the eye, 4o. Transference of infectious material through the careless use of eye-droppers, $4 \mathrm{I}$. Cautions necessary in the employment of poisonous collyria, 43. Collyria as commonly prepared by the apothecary often contaminated, 43. Methods of applying colly ria and ointments to the eye, 44. Mydriatics, 46. Atropin, the most valuable local remedy in the treatment of diseases of the eye, capable of doing much harm when injudiciously employed, 46 . Possibility of its inducing glaucoma in persons beyond middle life, 47. Follicular conjunctivitis caused by its long-continued use, 48 . Idiosyncrasies met with in individuals with reference to atropin and other mydriatics, 48 . Myotics, 48 . Indications for their employment, 48 . Their mode of action in glaucoma, 48. Eserin the most valuable, 49. How it should be employed, 49. Contraindications to its use; 5०. Astringent and antiseptic agents, 50. Useful especially in conjunctival affections, 5०. Value of silver nitrate, protargol, and argyrol in the severer types of conjunctivitis, $5 \mathrm{I}$. The application of carbolic acid and tincture of iodin to corneal ulcers, $5 \mathrm{r}$. Cocain useful only as an anesthetic, 5 $\mathrm{I}$. Harmful when otherwise employed, 52. Causes desquamation of the corneal epithelium, and favors bacterial invasion, 52. Dionin a valuable analgesic and lymphagogue, 52 . Opium a valuable local remedy in ocular inflammations, 53. Method of employing it, 53. Ointments, their preparation and uses, 53. Castor-oil in burns of the eye and abrasions of the cornea, 54. Light not the reprehensible thing it was formerly supposed to be, 54. . The application of heavy bandages to the eyes, designed to exclude it, and confinement in very dark rooms detrimental rather than helpful, 54 . Inflamed and painful eyes, nevertheless, should be protected from undue exposure to light, 54. Method of examining and making applications to the eyes of children, 57. Constitutional remedies useful in the treatment of diseases of the eye, 57. The value, and indications for the employment, of mercury, potassium iodid, quinin, iron, strychnin, arsenic, sodium pyrophosphate, pilocarpin, etc., in diseases of the eye, 57-6r. Efficacy of the antitoxin treatment in diphtheritic conjunctivitis, $6 \mathrm{I}$.

\section{CHAPTER III.}

\section{Diseases of the Eyelids and Orbit............... $63^{-117}$}

\section{Diseases of the Eyelids.}

Blepharitis marginalis, 63 . Commonly a chronic condition, 63 . In children, eczematous in character, and usually due to malnutrition and disturbed digestion; in adults, to accommodative 
strain, 64. Treatrient, $6_{5}$ Hordeolum, $6_{7}$. Etiology and treatment, 69. Eczema, 7r. Constitutional and local causes, $7 \mathrm{I}$. Gencral and local treatment, 71. Chalazion, 73. Etiology, 74 . Often multiple, 74. Chronic blepharitis a predisposing cause, 74. Treatment, 74. Non-operative treatment seldom of avail, 74. Description of operation found most effectual and easiest of performance, 75. Milium, 77. Hydrocystoma, 78 . Warts, 78 . Malignant growths, 78 . Tarsitis, 79. Commonly of syphilitic origin, 79. Entropion, 80. Spasmodic entropion, 81. May result from spasm of the orbicularis, commonly dependent upon photophobia, or from senile relaxation of the lid-structures, $8 \mathrm{I}$. Faulty construction of the tarsal cartilage or of the arrangement of the orbicularis a predisposing cause, 81. Organic entropion, 8I. Produced by contraction of scar-tissue in or beneath the palpebral conjunctiva, 81 . Usually consequent upon trachoma, $8 \mathrm{r}$. Treatment of spasmodic and organic entropion, $8 \mathrm{r}$. Operative procedures indicated, 82 . Correction of entropion of the lower lid by the production of an eschar with caustic potash, 82 . Ectropion, 88. Like entropion, may be caused by spasm of the orbicularis, by contraction of scar tissue, or by senile relaxation of the lid-structures, 88. Spasmodic ectropion, 88. Etiology, 88. Organic ectropion, 89. May result from any lesion which leads to destruction of the external integument of the lid or neighboring parts, 89. Incomplete ectropion of the lower lid a not infrequent complication of disease of the lacrimal apparatus, 89 . Non-operative and operative treatment of the different varieties of ectropion, 91. Snellen's operation for ectropion, 92. Value of Wolfe and Thiersch grafts in operations for the correction of ectropion, 97. Ptosis, 98. Occurs as a congenital and as an acquired fault, 98. Acquired ptosis, 98. Commonly due to paralysis of the third nerve, 98 . Generally unilateral and of syphilitic origin, 98. Congenital ptosis, 98. Usually bilateral and caused by faulty innervation, or imperfect development or absence, of the levator muscle, 98 . Treatment, 99. Only operative treatment of avail in congenital ptosis, 99. In acquired ptosis operative measures indicated only after other means have proved ineffectual, 99. Gruening's modification of Bowman's operation for ptosis, I02. Panas' operation, 104. Paralysis of the facial nerve, 105. Symptoms and consequences, 105. Etiology. ro6. Treatment, 106.

\section{Diseases of the Orbit.}

Cellulitis of the orbit, 108. Acute and chronic forms of the affection, 108. Symptoms, 108. Etiology, 108. Fatal consequences from cerebral implication, Io9. Treatment, 109. Periostitis, caries, and necrosis of the orbital walls, II 1 . Causes and conse- 
quences, III. Treatment, II2. Value of hydrochloric acid, PAGE. 113. Tumors of the orbit, II3. Mode of origin, II3. Varieties, II4. Consequences, II4. Diagnosis, Ir5. Treatment, I 6 .

\section{CHAPTER IV.}

Diseases of the Lacrimal Apparatus................. ir8-i5o

\section{Diseases of the Lacrimal Gland.}

Of infrequent occurrence, because of the protected position of the gland and its multiple system of ducts, ir8. Dacryoadenitis, I19. Occurs as an acute and as a chronic affection, I I8. Possible relationship to mumps, I I9. Acute dacryoadenitis, 1 19. Chronic dacryoadenitis, I 19. Treatment, 120. Fistula of the lacrimal gland, г20. Dacryops, I2 . Dacryoliths, I22. Dislocation of the lacrimal gland, I22. Met with as a spontaneous condition and as a consequence of trauma, r22. Hypertrophy of the lacrimal gland, I23. Atrophy of the lacrimal gland, 124. Tumors of the lacrimal gland, 125 . Are rare, and not infrequently of traumatic origin, I25. Varieties, I25. Treatment, I25.

\section{Diseases of the Drainage Apparatus.}

Of common occurrence, 125. Intimate pathological relationship between the drainage apparatus and the nasal passages, 125. Atresia of the lacrimal puncta, I26. Occurs as a congenital and as an acquired condition, I26. Treatment, 127. Malposition of the puncta, 128 . Eversion of the puncta, I28. Inversion of the puncta, I28. Etiology and treatment, I28. Division of the canaliculus the efficient remedy, I 28. Description of the operation, 128. Atresia of the canaliculi, 130. Dacryoliths, г31. Polypi, г3r. Dacryocystitis, I31. Etiology, г31. Primary inflammation of the lacrimal sac, $\mathrm{I} 3 \mathrm{I}$. Blennorrhea of the lacrimal sac, I32. Symptoms, I32. Mucocele, I32. Course of the disease, 132. Acute dacryocystitis, 133. Lacrimal fistula an occasional consequence, $\mathrm{r} 34$. Dacryocystitis rarely dependent upon ocular disease, I35. Treatment, I36. Prelacrimal abscess, I37. Stricture of the nasal duct, I37. Its etiology, 137. Symptoms and consequences, 138 . Treatment, I40. The efficacy of thorough dilatation by means of probes large enough to restore the normal caliber of the duct, I 40. Author's series of lacrimal probes, I4r. Electrolysis, I49. Removal of the lacrimal gland, I49. Excision of the lacrimal sac and its destruction with the actual cautery, 149. Dacryocystitis and occlusion of the nasal duct in the new-born, 150. 
Diseases of the Conjunctiva...................... $5^{1-201}$

Diseases of the conjunctiva of common occurrence, I5I. Importance of a correct diagnosis in dealing with them, $15 \mathrm{I}$. Signs and symptoms of inflammation of the conjunctiva, $15 \mathrm{r}$. Severe remedies, which cause pain and increase photophobia and lacri-

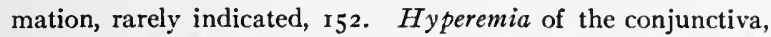
152. Conjunctivitis, 154. Catarrhal conjunctivitis, 154. Purulent or gonorrheal conjunctivitis, 158 . Ophthalmia neonatorum, 165. Croupous conjunctivitis, 168 . Diphtheritic conjunctivitis, 169. Follicular conjunctivitis, 173. Trachomatous conjunctivitis, I 74. Serious consequences to which it gives rise, 174. Vernal conjunctivitis, I 83. Bulbar and palpebral types, 183. Phlyctenular conjunctivitis, 186 . Of constitutional origin, 186. Toxic conjunctivitis, 192. Argyria conjunctive, 193. Subconjunctival hemorrhage, 193. Pinguecula, 194. Pterygium, 195. Author's view of its etiology, 196. Pseudo-pterygium, 197. Operative treatment of pterygium, 198 .

\section{CHAPTER VI.}

\section{Diseases of the Cornea And Sclera................ 202-245}

\section{Diseases of the Cornea.}

Keratitis, 202. Divisible into suppurative and non-suppurative keratitis, 202. Suppurative keratitis, 204. Etiology, 204. Tractable and intractable types, 204. Phlyctenular keratitis, 205. Abscess and ulcer of the cornea, 207. Pathology, 207. Etiology, 210. Often of traumatic origin, 21 r. Treatment, local and constitutional, 2 I I. Employment of carbolic acid, 2 I 3. Use of thermo-cautery, 214. Keratomalacia, 215. Neuropathic keratitis, 2 16. Herpes zoster ophthalmicus, 218. Post-malaria! keratitis, 220. Dendritic keratitis, 220. Herpes cornea febrilis, 220. Non-suppurative keratitis, 233. Interstitial keratitis, 223. Always dependent upon inherited syphilis, 223. Obstinacy its chief characteristic, 225. Though ultimate prognosis good, 226. Treatment, 227. Pannitic keratitis, 23 I. Canthotomy in treatment of pannitic keratitis, 233. Opacities of the cornea, 235. Arcus senilis, 235. Nebulx, Maculo, Leucomata, 236. Leucoma adherens, 236 . Staphyloma of the cornea, 239. Etiology, 238. May be partial or complete, 238 . Conical cornea, 241. Attended by a high degree of myopia, 24I.

\section{Diseases of the Sclera.}

The tough and non-vascular sclera not often the seat of disease, 242. Scleritis, 242. Acute, diffuse scleritis, 242. Commonly of rheumatic origin, 242. Chronic scleritis, 243. Complicated by involvement of the cornea, 213. Episcleritis, 244. 


\section{Diseases of the Iris.}

Iritis, 246. Diagnostic signs and symptoms, 246. Etiology, 248. Consequences of neglected iritis, 250. Varieties of iritis, 250. Plastic, serous, and purulent iritis, 250. Rheumatic iritis, 251. Gonorrheal iritis, 251. "Trophic" nerve iritis, 252. Serous iritis, 252. Plastic iritis, 253. Syphilitic iritis, 254. Iritis condylomatosa, 255. Iritis gummosa, 255. Varieties of "trophic" nerve, or neuropathic, iritis, 255. Sympathetic iritis (sympathetic ophthalmitis), 256. Etiology, 256. Sympathetic irritation, 257. Iritis due to herpes zoster ophthalmicus, 259. Spongy iritis, 259. Chronic iritis, 260. Treatment of the different varieties of iritis, 260.

\section{Diseases of the Ciliary Body.}

Cyclitis, 268. Plastic cyclitis, 268. Purulent cyclitis, 268. Serous cyclitis, 268 . Treatment of the several varieties of cyclitis, 269. Tumors of the ciliary body, 269. Often of malignant type, 269. An early diagnosis of the utmost importance, 269. Can be made only with the aid of the ophthalmoscope, 269.

\section{CHAPTER VIII.}

\section{Glaucoma}

Importance of an early diagnosis, 275. Consequences of failure to recognize glaucoma in its incipiency, 275. Hardening of the eyeball the essential feature of glaucoma, 275. Impairment of vision in glaucoma, 275. Primary glaucoma, 276. Secondary glaucoma, 276. Primary glaucoma extremely rare in persons under thirty years of age, 276 . Secondary glaucoma may manifest itself at any period of life, 276. Varieties of primary glaucoma, 276. Buphthalmos, 276. Symptoms, subjective and objective, of inflammatory glaucoma, 277. Prodromal symptoms, 279. Factors which have to do with precipitating the acute exacerbations of glaucoma, 280. Consequences of unchecked inflammatory glaucoma, 28r. Inexcusable mistakes made in diagnosticating glaucoma, 28I. Glaucoma to be susspected whenever a painful inflammation of the eye, attended by impairment of sight, is encountered in an individual who has reached middle life, 281. Etiology of inflammatory glaucoma, 28I. Accommodative strain an important factor in the causation of glaucoma, 285. Influence of mydriatics in precipitating an attack of glaucoma, when a predisposition to the disease exists, 285. Contraction of the visual field in glaucoma, 286 . Treatment of inflammatory glaucoma, 287 . Iridectomy the sov- 
ereign remedy, 287 . The value of myotics in glaucoma, 288 . Nature of their astion, 288. Manner of their employment, 288. Dionin useful in conjunction with eserin, 289. Other non-operative measures useful in glaucoma, 289. Importance of correcting refractive and muscular anomalies in the incipient stage of glaucoma, 290. Simple glaucoma, 29г. Symptoms and clinical history, 291. The ophthalmoscope and perimeter necessary to its diagnosis, 291. Treatment, 292. Iridectomy not the effective remedy it is in the inflammatory type of the disease, 292. Sympathectomy of doubtful value, 293. The systematic use of eserin, 293. The constitutional measures indicated, 293. Secondary glaucoma, 293. Etiology, 293. Symptoms and consequences, 294. Treatment, 294.

\section{CHAPTER IX.}

Diseases of the Crystalline Lens and Vitreous Humor. . . . 295-337

\section{Diseases of the Crystalline Lens.}

Anatomy and histology of the lens, 295. Growth of the lens, 295. Changes which it undergoes with advancing age, 296. Significance of these changes, 297. With reference to presbyopia, 297. With reference to the development of cataract, 297. Nutrition of the lens, 297. Cataract, 297. Origin of the name, 297. Mistaken views of the Greeks and Romans as to the real nature of cataract, 297. Though encountered most frequently in old age, occurs at all periods of life, and may be of congenital origin, 298. Different classifications of cataract, 298. Senile or hard cataract, 298. Juvenile or soft cataract, 298. General cataract, 298. Partial cataracts, 298. Varieties of partial cataract, 298. Zonular cataract, 299. Anterior polar cataract, 299. Posterior polar cataract, 299. Congenital cataract, 299. Idiopathic cataract, 299. Complicated cataract, 299. Traumatic cataract, 299. Immature, mature, and hypermature cataract, 299. Secondary or capsular cataract, 299. General cataract, 299. Features common to the several varieties of general cataract, 299. Progressive impairment of vision in general cataract, 299. Characteristics of soft and of hard cataract, $30 \mathrm{r}$. "Second sight" a premonitory symptom of senile cataract, 302 . Etiology of general cataract, 302. The development of cataract a degenerative, not an inflammatory, process, 302 . Faulty nutrition of the lens the immediate cause of cataract, 302 . This may be the result of a constitutional disorder, of senile decay, or of pathological changes in the eye itself, 302. Life-long accommodative strain a not unimportant factor in the causation of senile cataract, 303. Etiology of traumatic cataract, 303. Diagnosis of cataract, 304 . Assistance afforded by oblique illumination and the instillation of a mydri- 
atic, 304. Determination of the maturity of cataract, 307 . Treatment of cataract, 310 . Improvement in vision in incipient cataract from the use of a mydriatic, 3 ro. Discission, the procedure applicable to soft or juvenile cataract, 3I4. Extraction, applicable to hard or senile cataract, 315. Simple and combined extraction, $3{ }^{1} 5$. The success which attends extraction of cataract at the present day, 3 I6. Conditions which militate against the success of the operation, 316 . Linear extraction, 319. Suction extraction, 319. Partial Cataract, 320. Anterior polar cataract, 320. Pyramidal cataract, 321. Posterior polar cataract, 322. Zonular cataract, 324. Treatment of the several varieties of partial cataract, 325. Capsular cataract, 326. Etiology and treatment of capsular cataract, 326, 327 . Dislocation of the crystalline lens, 327 . May be complete or incomplete, 327 . May be forward, into the anterior chamber, or backward, into the vitreous chamber, 327. Effect upon vision, 329. Treatment, $33 \mathrm{I}$.

\section{Diseases of the Vitreous Humor.}

Pathological changes in the vitreous humor usually due to traumatism or to pre-existent disease of the uveal coat or retina, 333 . Purulent panophthalmitis, 333. Treatment, 334. Fluidity of the vitreous humor, 334 . Opacities of the vitreous humor, 334 . Musce volitantes, 335. Hemorrhage into the vitreous humor, 336. Etiology, 336. Treatment, 337 .

CHAPTER X.

Diseases of the Choroid Coat, Retina, and Optic Nerve....338-387 General description of the symptoms indicative of disease of the choroid, retina, and optic nerve, which, when taken into account, enable the general practitioner, even without the aid of the ophthalmoscope, to reach an approximately accurate diagnosis, $33^{8}$.

\section{Diseases of the Choroid Coat.}

Choroiditis, 340. May be plastic, purulent, or serous in type, 340. Purulent choroiditis, synonymous with purulent panophthalmitis, described in Chapter IX, 340. Serous choroiditis, synonymous with uveitis, considered in Chapter VII, 340. Plastic choroiditis, 340. Frequently dependent upon syphilis, 340. Syphilitic choroiditis, 340 . Commonly involves the retina, 340 . Runs a tedious course, $34 \mathrm{I}$. Both eyes usually affected, $34 \mathrm{I}$. If neglected may lead to loss of sight and to the development of cataract, 342. Choroiditis of high myopia, 342. Traumatic choroiditis, 342. Miliary choroido-retinitis, 343. A not uncommon consequence of accommodative strain, 343. Probably a factor, 
of no mean importance, in the causation of glaucoma and of senile cataract, 344 . Treatment of the several varieties of choroiditis. 345 . Tumors of the choroid, 348 .

\section{Diseases of the Retina.}

Retinitis, 348. Divisible into primary and secondary retinitis, 348. Also into serous and parenchymatous retinitis, 348 . Retinitis unattended by photophobia or pain, 348 . Impairment of vision the chief subjective symptom, 348 . Causes of primary and of secondary retinitis, 349. Retinitis albuminurica, 349. Occurs in all forms of disease of the kidney accompanied by albuminuria, and not infrequently as an early symptom, 349. Also in the albuminuria of pregnancy and of scarlatina, 349. Nearly always bilateral, 349. Uremic amblyopia, 35I. Treatment to be directed to the nephritis and to the general condition of the patient, $35^{\mathrm{I}}$. Diabetic retinitis, $35^{\mathrm{I}}$. Is always bilateral, $35^{\mathrm{I}}$. Not infrequently accompanied by cataract, and occasionally by iritis or glaucoma, 351. Prognosis unfavorable, 352. Treatment, 352. Leucocythemic retinitis, 352. Retinitis of pernicious anemia, 352. Syphilitic retinitis, 353. Energetic antisyphilitic treatment demanded, 353. Retinitis from exposure of the eyes to intense light, 353 . Usually most marked in the macular region, 353. Treatment, 354. Retinitis pigmentosa, 354. Begins in early childhood, 354. May be congenital, 354 . Often associated with congenital anomalies of the eye or of other organs, 354. Met with in the offspring of consanguineous marriages, 354. Night-blindness an early symptom, 355. Gradual contraction of visual field, with ultimate loss of central vision, 355. Treatment of little avail, 356. Embolism of the central artery of the retina, 357 . Characterized by sudden loss of sight of the affected eye, 357. Striking ophthalmoscopic picture, exhibiting the "cherry-red spot at the macula," 357 . Treatment, $35^{8}$. Thrombosis of the central artery of the retina, 359. Probably the cause of the sudden loss of sight occasionally observed in acute anemia, 359. Symptoms and ophthalmoscopic picture similar to those observed in embolism, 359 . Thrombosis of the central retinal vein, 359 . Usually met with in elderly persons with organic heart disease or angiosclerosis, 359 . May be caused by the extension of facial erysipelas to the orbit, 359. The ophthalmoscope shows enormous distention and great tortuosity of the retinal veins, with numerous hemorrhages scattered over the entire fundus, 360 . Treatment of little avail, 360. Detachment of the retina, 360. Its etiology, 36r. Farfetched theories advanced to explain its occurrence unsatisfying, 36r. Oftenest met with in high myopia, 361 . May result from trauma, $36 \mathrm{r}$. Occurs in conjunction with intraocular growths, 362. Symptoms, 362. Annoying disturbance of vision to 
which it gives rise, $3^{6}{ }_{3}$. Prognosis most unfavorable, $36_{3}$. Operative and non-operative treatment, $36_{4}$. Glioma of the retina, 364 . One of the most malignant of pathological newgrowths, 364 . Possibility of diagnosticating it by daylight inspection, with the assistance of a mydriatic, 365 . Symptoms and course of the disease, 365 . Enuclcation of the eye at the earliest moment possible indicated, 366 . But recurrence of the growth, espccially in the brain, to be feared, $3^{67}$. A disease of childhood, $3^{6} 5$.

\section{Diseases of the Optic Nerve.}

Optic neuritis, 367 . Divisible into retrobulbar neuritis and intraocular neuritis, 367 . The latter again divisible into papillitis, or choked disc, and descending optic neuritis, 367 . Etiology of descending optic neuritis and of choked disc, 368 . . Choked disc (papillitis), 37o. Vision at first may be but slightly impaired, though pronounced fundus changes are present, 37 . Usually dependent upon intracranial tumor, 37r. Present in from eighty to ninety per cent. of intracranial new-growths, $37 \mathrm{I}$. Descending optic neuritis, 372. Fundus changes less marked than in choked disc, 372. Oftenest due to basilar meningitis attended by inflammation of the contiguous brain substance, 372. The prognosis in both choked disc and descending optic neuritis largely dependent upon the nature of the lesion which has led to their development, 372. Treatment, 373. Retrobulbar optic neuritis (orbital neuritis), 374. Occurs under two forms-Acute retrobulbar neuritis and chronic retrobulbar neuritis, or toxic amblyopia, 374, 375. In each the orbital portion of the nerve is first involved, and especially the papillo-macular fibers, 375. Acute retrobulbar neuritis, 374. Etiology, 374. Symptoms and course of the disease, 374,375 . Treatment, 375 . Chronic retrobulbar neuritis (toxic amblyopia), 375. Etiology, 375. Symptoms, 375. Ophthalmoscopic changes observed, 377. Prognosis and treatment, 377. Quinin blindness, 377. Atrophy of the optic nerve, 378 . Primary (simple) atrophy, 378. Nearly always bilateral, 379. Etiology, 379. Early symptoms, 379. Ophthalmoscopic signs, 379. Treatment usually of little avail, $38 \mathrm{r}$. Consecutive atrophy of the optic nerve (inflammatory atrophy), $38 \mathrm{r}$. Causes, $38 \mathrm{r}$. Subjective and objective symptoms, $3^{81}$. Diagnosis to be established only by the ophthalmoscope, $3^{8 \mathrm{r}}$. Usually distinguishable from simple atrophy, $38 \mathrm{r}$. Prognosis not so unfavorable as in simple atrophy, $3^{82}$. Treatment, 382 . Hemianopsia, $3^{8} 3$. Varieties, $3^{8} 3$. Binasal and horizontal hemianopsia, uncommon, 384. Homonymous lateral hemianopsia and bitemporal hemianopsia more frequently encountered, 385 . Explanation of the occurrence of the different varieties of hemian- 
opsia, 385. Homonymous lateral hemianopsia, 385 . Causes, PAGE. 385 . Early and later symptoms, 385 . Central vision seldom lost, 385. Transient hemianopsia, 386. Bitemporal hemianopsia, 386 . Treatment, except in cases of syphilitic origin, usually without avail, 387 .

\section{CHAPTER XI.}

ANomalies of Refraction and Accommodation............. $388-437$

\section{Anomalies of Refraction.}

Prevalent misconceptions in regard to the measurement and correction of the refractive and muscular anomalies of the eyes, 388. No branch of ophthalmic practice more imperatively demands especial skill and training, 388. The harm which comes from the hap-hazard methods of the tyro who, after a few weeks' instruction in an "optical college," announces himself as an "ophthalmic optician," 388 . Importance of the rôle errors of refraction play in the causation of ocular maladies, 389. Also in the production of maladies other than those of the eye, though, as to these, there has been much exaggeration, 389 . Eye-strain a common cause of headache, neurasthenia, vertigo, disturbance of mental concentration, etc., 390. Observations upon some of the prevalent misconceptions regarding refractive errors, and concerning the indication for "glasses" and their influence upon sight, 391. Errors of refraction rarely "outgrown," and not to be gotten rid of by "rest of the eyes," massage, the application of drugs, etc., 391. Glasses not to be regarded as a dernier ressort, 391. Glasses do not "weaken the sight," as is popularly supposed, 39I. No foundation for the belief that if glasses are worn in childhood a time may come when it will be impossible to obtain them of the required strength, 391. Glasses given not merely to sharpen vision, but to relieve eye-strain, 39r. Often demanded when there is normal acuteness of sight, 392. Exert a marked influence in checking the progress of myopia, 392. But it is not to be expected that they will "cure" the refractive fault for which they are prescribed, and only exceptionally does it happen that they can be put aside, 392. Not all eyes needing glasses make a direct appeal for their aid, 392: Not infrequently, when the remote consequences of eyestrain are pronounced, there may be no complaint of the eyes themselves, 392. Emmetropia, 393. Ametropia, 394. A general term denoting a departure from the normal in the optical construction of the eye, 394. Hvpermetropia (far-sightednes.), 395. The most prevalent form of ametropia, and nearly always of congenital origin, 395. Owing to an abnormal flatness of the eyeball, or a lack of refractive power in its lens system, the hyper- 
metropic eye is incapable of focusing upon the retina parallel rays of light, without an effort of accommodation, 395. Facultative and non-facultative hypermetropia, 395. Axial hypermetropia, 397. Curvature hypermetropia, 397. The ill consequences of hypermetropia, 398 . The most important factor in the causation of convergent squint, 398 . Asthenopia, headache, blepharitis marginalis, etc., common consequences of the eye strain to which it gives rise, 398 . The ill effects of the higher grades of hypermetropia manifest themselves in early childhood, 398. The lower grades may cause no inconvenience until the presbyopic agc is approached, 399. The whole treatment of hypermetropia comprised in the careful adjustment of glasses, 399. Whether these must be worn constantly, or only in near vision, will depend upon the degree of the refractive fault, the power of accommodation, and the relative strength of external and internal recti muscles, 399. With properly adjusted glasses the hypermetropic eye is relieved of all strain, and is capable of doing the work which the normal eye does without assistance, 400 . Influence which the correction of hypermetropia by glasses exerts upon the development of convergent squint, 402. A squint, as yet not fully established, can always be corrected by properly adjusted glasses, 402. A fully established squint, only exceptionally, 402 . More often than not hypermetropia is complicated by the coexistence of astigmatism, 403. For this reason, and because the judicious correction of hypermetropia presupposes a clear comprehension of the muscular anomalies of the eye, it is manifest that the treatment of this very prevalent refractive fault should be undertaken only by the physician who has had especial training and experience, 403. Myopia (short-sightedness), 403. Much less common than hypermetropia, and nearly always an acquired fault, 403. Owing either to the antero-posterior axis of the eye being too long, or to an excess of power in its lens system, rays of light are brought to a focus before reaching the retina, 403. Axial myopia, 404. Curvature myopia, 404. . Axial myopia, the more common type, usually due to the development of a posterior staphyloma, 404. Conical cornea a striking example of curvature myopia, 405. Etiology of axial myopia, 405. Disturbed relation of accommodation and convergence in myopia, 407. A cause of asthenopia, and may lead to the development of divergent squint, 408. Myopia occasionally a result of acute systemic disease, 408. Origin of the name "myopia," 409. The ophthalmoscope affords the readiest means of diagnosticating myopia, 409 . Treatment, 409 . Whether the defect shall increase to the danger-point, or shall be arrested before the deeper structures of the eye have suffered irreparable damage, hinges upon the skill exercised in its cor- 
rection by glasses, 410 . Importance of correcting associated astigmatism, and of taking into account the muscle-balance in both far and near vision, $4 \mathrm{ro}$. The widespread belief that in myopia glasses are called for only in distant vision, seldom well founded, 4II. In distant vision they are a convenience; in near vision, a therapeutic agent of great value, $4 \mathrm{II}$. Operative procedures in myopia, $4^{\mathrm{I}} 3$. When there is pronounced insufficiency of the internal recti muscles a guarded tenotomy of one or both of the opponent muscles may be indicated, 4r3. Removal of the crystalline lens in high myopia a procedure attended by considerable risk, and of doubtful utility, 4I3. Astigmatism, 4I3. Definition of the term, 4I3. Regularastigmatism, 4I3. Commonly due to asymmetry of the cornea, less often to asymmetry or obliquity of the lens, $4 \mathrm{I} 3$. Usually a congenital and often an inherited fault, $4^{\mathrm{I}} 3$. Disturbance of vision in astigmatism, $4^{\mathrm{I}} 4$. Latent astigmatism, 4I4. Explanation of the asthenopia commonly associated with astigmatism, 4I4. Improvement in vision secured by nipping the lids, $4 \mathrm{I} 4$. Varieties of regular astigmatism, 415. Simple astigmatism, 415. Compound astigmatism, 4I5. Mixed astigmatism, 4I 5. Astigmatism "according to the rule" and "against the rule," 4I5. Importance of correcting even the lowest degrees of astigmatism "against the rulc," $4 \mathrm{I} 6$. The apparent increase of astigmatism usually due to the total defect becoming manifest, as a result of correcting glasses, 4 I 6 . Acquired astigmatism commonly the result of traumatism or of ulcerative keratitis, $4^{1} 7$. Astigmatism capable of giving rise to pronounced asthenopic symptoms, not incompatible with normal acuteness of vision, $4^{\mathrm{I}} 7$. Simple method of detecting the presence of astigmatism marked in degree, 4I 7. Astigmatism a prevalent fault, and often a chief factor in the causation not only of many ocular maladies but of many obscure disturbances of the nervous system, 4r8. Treatment of astigmatism, 4I9. Its correction by cylindrical glasses, 419. These commonly bring vision up to the normal standard, and, more important still, by eliminating the previously existing accommodative strain, do away with the asthenopia, headache, etc., 420. Methods of detecting and measuring astigmatism, 421. Anisometropia, 424. A not uncommon cause of asthenopia, 424. Exceptionally it proves a blessing in disguise, 424 . Indications for its correction by glasses, 424 .

\section{Anomalies of Accommodation.}

Theory of the accommodation of the eye as propounded by Helmholtz, 426. Different ways in which the ability of the eye to change its focus may be impaired, 427. Presbyopia (oldsightedness), 427. Due to loss of elasticity of the lens, 427. Commonly manifests itself about the forty-fifth year, and 
interferes with the sharp-seeing of near objects, 428 . Its oncoming influenced by the existence of refractive errors, 428 . Cannot be cured or its development postponed by massage, the use of "eye-cups," etc., 429. Glasses the only remedy, 429. Fallaciousness of the common belief that the needed glasses can be "fitted" by anyone who "carries a stock" of spectacles, 429. As presbyopia is a progressive condition, the glasses prescribed must be increased in strength from time to time, 429. Bifocal lenses in presbyopia associated with refractive faults, 430. Paralysis of the ciliary muscle, 430 . Diphtheria and syphilis the commonest causes, $43 \mathrm{I}$. Sudden impairment of near vision the most prominent symptom, 431. Prognosis usually favorable, 431. Treatment, 432. Spasm of the ciliary muscle, 432 . Commonly the result of uncorrected refractive errors, 432 . Treatment, 433. Subnormal accommodative power, 433. A not infrequent cause of asthenopia in young persons, 433 . Due to congenital lack of elasticity of the crystalline lens or to insufficiency of the ciliary muscle, 433. Rules for its detection and measurement, 435 . Its treatment by convex glasses and by spheroprismatic lenses, 436,437 . Method of determining the required lenses, $43^{6}$. When associated with faults of refraction, different glasses may be required for far and near vision, even in quite young subjects, 436,437 .

\section{CHAPTER XII.}

Muscular Anomalies of the Eyes.................. $43^{8-479}$

Manifest and latent muscular anomalies, 438 . May be of paralytic or of congenital origin, or a consequence of refractive errors, 438. The manifest muscular anomalies - the actual squints-not provocative of eve-strain, 439. The latent muscular anomalies - the several varieties of heterophoria-on the other hand, commonly give rise to marked asthenopic symptoms, 439 .

\section{Manifest Muscular Anomalies.}

Paralytic squint, 440. The nervous supply of the extrinsic ocular muscles, 440. Etiology of paralytic squint, 44I. Paralysis of the external rectus muscle, 442 . Symptoms, diagnosis, and treatment, 443. Paralysis of the superior oblique muscle, 444. Paralysis of the oculomotorius, 444. Oftenest dependent upon acquired syphilis, 444. Varieties, 445. Frequently attended by ptosis, 445. Ophthalmoplegia totalis, 445. Ophthalmoplegia externa, 445. Ophthalmoplegia interna, 445 . Usually caused by diphtheria, 445. Treatment of the different varieties of paralysis of the oculomotorius, 446 . Operative procedures not to be resorted to hastily, 446. Conjugate ocular paralyses, 
446. Nystagmus, congenital and acquired, 447. Concomitant squint, 448. How different from paralytic squint, 448. May be constant, periodic, or alternating, 448. Etiology of concomitant squint, 448. Amblyopia of the squinting eye, 449. Not an example of "amblyopia exanopsia," 449. Commonly a consequence, not a cause, of the squint, 449. Explanation of its development, 449. Significance of its regional character, $45^{\circ}$. Tests for the detection of squint, 452. Convergent concomitant squint, 453. Develops in early childhood, 453. The sufficiency of Donders' explanation of why many hypermetropes do not squint, 453. Hypermetropia the most important factor in the causation of convergent squint, 453. The coexistence of insufficiency of the external recti muscles or of subnormal accommodative power greatly increases the likelihood of its occurrence, 454. Explanation of the occurrence of convergent squint in high myopia, 454. Treatment, operative and non-operative, of concomitant convergent squint, 455. Early correction of the squint desirable, 455 . Established convergent squint seldom corrected without operation, 455. Importance of determining the refractive condition and visual acuteness in all cases of squint, 455. Periodic convergent squint always capable of correction by glasses alone, 456 . Re-establishment of binocular vision the ideal result aimed at in the treatment of squint, 456 . Factors which sometimes render this difficult of accomplishment, 456. Operative treatment of convergent . squint, 457. "Tenotomy" preferable to "advancement," 457. Arlt's method of performing tenotomy the simplest and best, 457. Description of his operation and enumeration of the instruments required in its performance, 457 . The unsightly sinking of the caruncle in awkwardly executed tenotomies upon the internal rectus, 459. How obviated, 459. Essential difference between the modern operation of tenotomy and the clumsy procedures in vogue fifty years ago, 462 . Divergent concomitant squint, $46_{3}$. Myopia, congenital or acquired insufficiency of the internal recti muscles, and marked difference in the visual acuteness of the eyes the most potent factors in its production, ${ }_{463}$. Usually develops in adult life, ${ }_{464}$. May be present only in near vision, 464 . Regional amblyopia, as observed in convergent concomitant squint, rarely present, 464. Treatment, 464. Exceptional conditions which render its correction, without operation, possible, 464 . Its correction in high myopia often inadvisable, 465 . Not so easily corrected by tenotomy as convergent squint, 465 . Tenotomy must often be supplemented by advancement, 465 . Free tenotomy of both external recti the best procedure in some instances, 465 . Vertical concomitant squint, 466 . 


\section{Latent Muscular Anomalies.}

PAGE.

Under this head are included all the varieties of heterophoria, 466. Less frequently encountered than refractive errors, but as capable of producing the manifold symptoms which we have learned to attribute to eye-strain, 466 . When associated with ametropia, may greatly aggravate the ill consequences of the refractive fault, 467 . Exceptionally, may have the contrary effect, 467. Apparent and actual muscular anomalies, 467 . How they may be distinguished, 467 . Influence of refractive errors upon muscle-imbalance, 467 . Brief mention of the tests employed for the detection and measurement of heterophoria, 469. Importance of determining the muscle-balance for near, as well as for distant, vision, 469. Schild's pin-hole light, 470 . Heterophoria to be corrected by glasses or by operative procedure, 470. Exophoria, $47 \mathrm{I}$. Frequently only apparent, and dependent upon myopia, 472 . Treatment by glasses, tenotomy, and, exceptionally, by advancement, 472. Esophoria, 473. Hypermetropia and hypermetropic astigmatism important factors in its causation, 473. In every case their existence should be suspected, and carefully searched for, 473. Not always dependent, however, upon these faults, 474. Marked examples met with in emmetropia, 474. Here there is actual insufficiency of the external recti muscles, 474. The tests for esophoria should be applied in near, as well as in distant, vision, 474. Treatment, 474. Exact determination of the refractive condition of the eyes the first step, 474. If a marked degree of hypermetropia is present, it is best to correct this by glasses and postpone operative procedure until the effect is observed, 475 . When relief is not secured in this way, prisms may be combined with the glasses required for the correction of the refractive fault or a tenotomy performed, 475. When no error of refraction exists the choice lies between prisms or a tenotomy, and hinges upon the degree of the muscle-fault, 475 . Conservatism to be commended in operating for latent muscular anomalies, 476 . But, when the indications are clear, the fullest confidence may be felt that a well executed tenotomy will result in marked benefit, 476 . By "tenotomy" is meant a real division of the tendon, 476 . No excuse for "graduated" or partial tenotomies, 476 . A "guarded tenotomy" not infrequently indicated, 477. Actual squint often corrected by less free tenotomizing than is sometimes demanded in latent muscular faults, 477. Hyperphoria, 477. Definition, 477. Etiology, 477. Capable of producing all of the distressing symptoms arising from eye-strain, 477,478 . Should be sought for in every case of asthenopia, 478 . Exceptionally present only in near, or only in distant, vision. Should therefore be sought for in both, 478 . Maddox's multiple rod the best test for 
hyperphoria, 478 . In the test for near vision, should be used in

PAGE. conjunction with the pin-hole light of Schild, 478 . Treatment, 478. The lower degrees should be corrected by prisms, 478 . Operation indicated only when the defect is pronounced, 478 .

\section{CHAPTER XIII.}

INJURIES of the Eye and its APPENDAGES . . . . . . . . . . . . $480-504$

Injuries of the eyelids, 480 . May result in malposition of the lid margins and lacrimal puncta, also in anchyloblepharon or symblepharon, 480 . Burns of the lids from caustic agents, molten metal, etc., $48 \mathrm{I}$. Treatment, 482 . Value of Thiersch grafts, 483 . Injuries of the bulbar conjunctiva, 483 . Danger of symblepharon resulting, 483 . Superficial injuries of the cornea, 485 . Usually not of serious moment unless infection occurs, 485. Method of applying carbolic acid under such circumstances, 486. Other remedial measures, 486. Superficial lodgment of foreign bodies in the eye, 486 . One of the commonest accidents to which the eye is subject, 486 . Nearly always found adherent to the cornea or upon the under surface of the upper lid, 487 . Occasionally find their way into the canaliculus, 488. Fragments of grass seed hulls sometimes remain attached to the cornea for months, 488 . Their true character easily overlooked, 488 . Little reliance to be placed upon the convictions of the patient as to the presence or non-presence of a foreign body, 489. Best methods of removing foreign bodies from the eye, 490 . Cocain required only when they are adherent to the cornea, 490 . Contusions of the eye, $49 \mathrm{r}$. Though of common occurrence, seldom of serious moment because of the protection afforded by the bony orbital margin and the elastic cushion of fat upon which the eyeball rests, 49I. Rupture of the eyeball, dislocation of the lens, traumatic cataract, laceration of the iris, and detachment of the retina among the serious consequences apt to result from severe contusions, 49r. Commoner causes of such injuries, 493. Treatment demanded in slight and in severe contusions, 493. Penetrating wounds of the eye, 494. Always of serious concern, are made more so because of the danger of infection, 494. Wounds of the cornea, iris and lens, 494. Of the sclera and ciliary body, 494. Of the sclera, choroid, and retina, 495 . Consequences of such injuries, 495. Treatment, 495. Antiseptic precautions of the first importance, 495. Wounds of the eye complicated by the lodgment of foreign bodies within the ball, 497. Of still more serious moment, 497. Much depends, however, upon the nature of the foreign body and upon its location, 497. Least dangerous when sterile and when incapable of undergoing chemical change, 497. May find lodgment in the iris, the lens, the vitreous body, the deeper tunics of the eye, or, 
more rarely, may fall into the anterior chamber, or pass through the eye and reach the depths of the orbit, 498. Treatment, 499. Great assistance afforded by skiagraphy and the electro-magnet, 499. Nowadays, eyes often saved, and with useful vision, which formerly were enucleated without hesitation, 499. Pain reaction test, 500 . Removal of non-mugnetic foreign bodies, 502. Difficulties which attend the removal of foreign bodies from the anterior chamber, 502. Gunshot wounds, 503 . Wounds caused by penetrating foreign bodies may be of such a character as to demand immediate enucleation of the eye, 504 . This is especially the case when their nature is such as to render the development of sympathetic ophthalmitis not improbable, 504 .

APPENDIX

Formulæ, for the niost part in general use, of proved efficacy in the treatment of diseases of the eye, $5 \circ 5$.

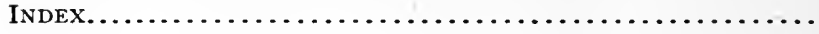




\section{PREVALENT DISEASES OF THE EYE.}

CHAPTER I.

GENERAL OBSERVATIONS UPON THE DIAGNOSIS OF DISEASES OF THE EYE. DESCRIPTION OF THE METHODS OF EXAMINING THE EYE AVAILABLE TO THE GENERAL PRACTITIONER.

The weak point of the general practitioner in dealing with eye diseases is, unquestionably, in reaching a correct diagnosis; and this is not surprising in view of the fact that in most instances he works without the help of the ophthalmoscope, the trial case, and the various contrivances for testing the central and peripheral visual acuteness, the muscle balance, etc., aids which the specialist in diseases of the eye always has at command, and without which even he would often be at fault. But, it must be confessed, the general practitioner's errors of diagnosis are not always limited to the class of cases in which these aids are essential.

For example, he not infrequently fails to draw a distinction between the several varieties of conjunctivitis, and, in consequence, is led into errors in the therapeutic measures which he employs. Again, he mistakes a corneal inflammation or an iritis, with its attendant conjunctival injection, for a simple conjunctivitis, with still more serious consequences; or, in order to avoid this mistake, he goes to the other 
extreme, and causes his patient much unnecessary inconvenience by prescribing atropin when only a mild astringent is called for. In other instances, he mistakes the photophobia, lacrimation and hyperemia of the conjunctiva due to the presence of a foreign body upon the cornea or beneath the upper lid for a commencing ocular inflammation; and treats it accordingly, without success. A chronic conjunctivitis or blepharitis, dependent upon an error of refraction or a muscular anomaly or secondary to disease of the lacrimal apparatus, is dealt with without reference to its primary cause and, therefore, to no effect.

Still more disastrous in its results is the failure of the general practitioner to recognize promptly inflammatory glaucoma. Not infrequently this disease is mistaken for iritis or keratitis, and atropin, the remedy distinctly contraindicated, is prescribed, or, in the quiet periods between the inflammatory outbreaks, owing to the apparent or actual loss of transparency of the crystalline lens, for cataract, which usually leads to the patient's being advised to defer any operative procedure until the supposed cataract is "ripe." Under such circumstances, when the case ultimately comes under the observation of the ophthalmic surgeon the eye too often is absolutely blind, and nothing that he can do is of avail, at least so far as the restoration of sight is concerned. To mistake an acute glaucomatous attack for severe facial neuralgia, as sometimes happens, though a most unfortunate mistake, is less reprehensible; for in neuralgia of the fifth nerve the eye upon the affected side is not infrequently injected, sensitive to the touch and photophobic, and the pain experienced in the two conditions is of much the same character. 
It is with the view of helping the general practitioner to avoid such mistakes as have been enumerated and others of similar character, and, further, to enable him to recognize the probable existence of other troubles, such as refractive and muscular anomalies and diseases of the deeper structures of the eye-as to which he can hardly be expected to make a definite and exact diagnosis, and which, therefore, if he is conscientious and discreet, he will not be inclined to treat-that this chapter is written.

Doubtless, it would be an excellent thing if every physician were an expert ophthalmoscopist; but, however desirable, there are not many who would contend that, for the present at least, this is practicable. As a matter of fact, the number of general practitioners at the present day who are, or who are ever likely to be, sufficiently versed in the use of the ophthalmoscope to make it of any real diagnostic value to them is, relatively, so small that it may be treated as a negligible quantity. Such being the case, it is assumed throughout this work-which, as has been stated, is designed solely for physicians engaged in general practice-that the reader is not skilled in the use of the ophthalmoscope; and this has materially modified the treatment of several important subjects.

\section{METHODS OF EXAMINING THE EYE.}

Oblique Illumination.-Although skill in the employment of the ophthalmoscope, as has just been said, is not likely to be acquired by very many general practitioners, there is a most valuable method of examining the eye which every physician can easily master, and which he will find of the greatest possible assistance as an aid to diagnosis. I refer to what is known as 
oblique illumination of the eye. A room which can be made, at least, moderately dark, a brightly burning candle, a lamp (a "student's lamp" is the best) or a steadily burning gas-light, and a biconvex lens of from two to two and a half inches diameter and of two and a half inches focal length, are all the paraphernalia necessary, and to employ these to good purpose but

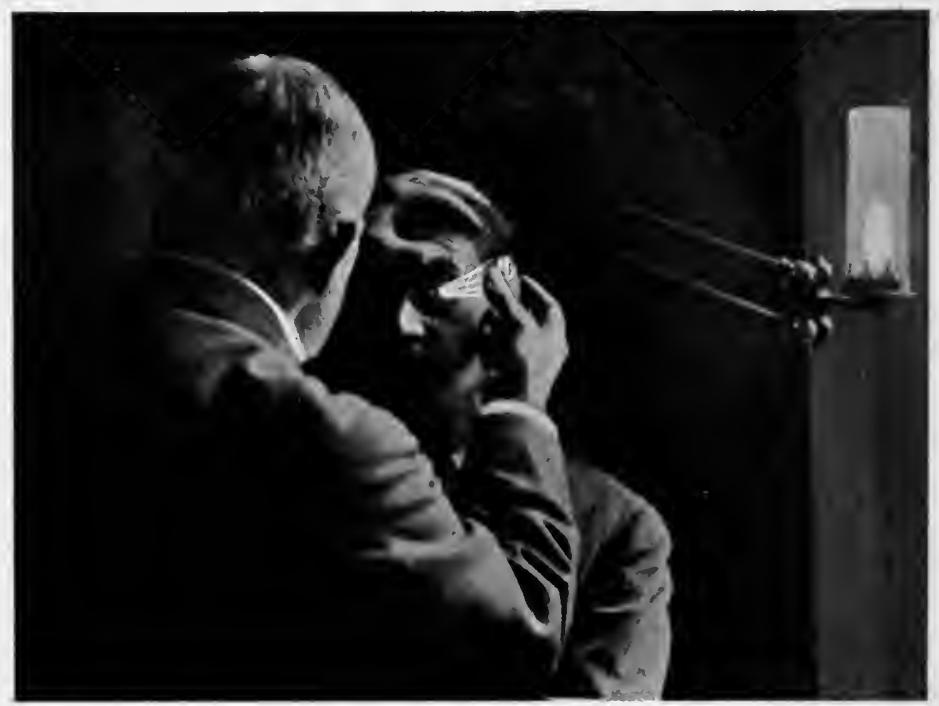

Fig. I.-Examination of the eye by oblique illumination.

little practice (and for this the normal eye will suffice) is required.

"Oblique illumination" means, simply, the focusing, by means of the lens just described, of a beam of artificial light upon the anterior structures of the eye. The examination is most conveniently made, as is shown in the accompanying illustration (Fig. I), by having the light to the left and slightly in front of the patient, approximately on a level with his eyes, and at a dis- 
tance of about eighteen inches from his face. The lens should be held between the thumb and forefinger of the examiner, with one of its convex surfaces towards the light, the other towards the eye to be examined, and at a distance of about three inches from the latter. The concentration of the light upon the eye can be regulated more accurately if the examiner steadies his hand by resting the ring-finger upon the patient's cheek.

The great help which this method of inspection affords in the investigation of abnormal conditions in the anterior structures of the eye-that is to say, the cornea, anterior chamber, iris and crystalline lenscan scarcely be realized by those who have not employed it. In searching for foreign bodies, whether lodged upon or in the cornea, the iris, the lens or within the anterior chamber; in ascertaining the presence and character of opacities in the cornea or anterior portion of the lens; in examining corneal abscesses and ulcers; in investigating the condition of the iris and the pupil, determining whether suspected iritis exists, whether posterior synechiæ have formed or hypopyon is present -in all these conditions, and in others likely to be met with from time to time, its value is hardly to be estimated. While, therefore, I do not deem it worth while to advise physicians in general to endeavor to become ophthalmoscopists, I do urgently recommend that every practitioner likely to be called upon to give an opinion regarding an injured or diseased eye should provide himself with the means, and acquire the easily gained skill, necessary to the successful employment of oblique illumination.

As a further important aid to accuracy in diagnosis, every physician should have at hand a mydriatic which 
is evanescent in its action, such as homatropin hydrobromate in one per cent. solution or euphthalmin hydrochlorate in five per cent. solution; for, as will be seen later, by its use he will often gain valuable and much-needed information. He should, moreover, acquire facility in everting the upper lid; in determining the tension of the eye; in observing the pupillary reaction, and in testing, roughly at least, central and peripheral vision.

Eversion of the Eyelids.-To facilitate eversion of

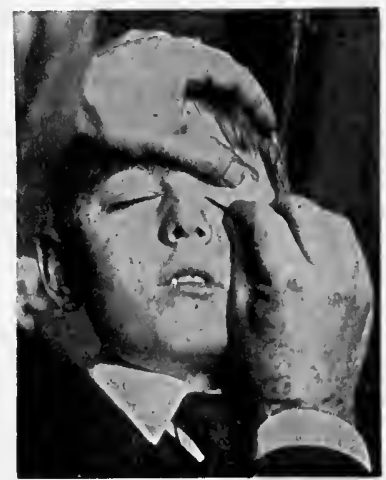

Fig. 2.-Eversion of the upper lid (Hansell and Sweet).

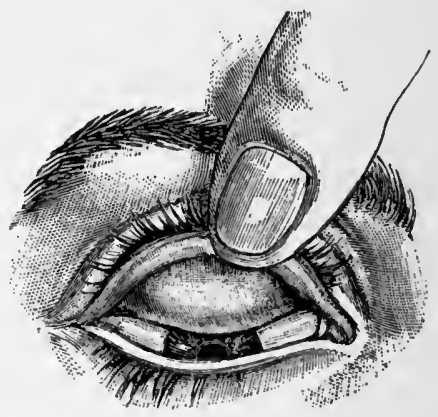

Fig. 3.-Method of holding the everted lid (Lawson).

the upper lid the patient should look strongly downward, without closing the eye. If the examiner now seizes the lashes and draws the lid away from the ball while at the same moment he depresses the upper edge of the tarsal cartilage either with a finger of his other hand or a slender penholder or pencil, the eversion is, in most instances, easily accomplished (Figs. 2 and 3 ). The inner surface of the lower lid may, of course, be inspected with more ease. Its eversion is effected by having the patient look upward, and by drawing the 
margin of the lid downward and pressing it against the lower border of the orbit with the finger-tip.

Tension of the Eye.-The intraocular tension or hardness of the eyeball, which varies greatly in different affections of the eye, is determined by having the patient look downward and making gentle pressure upon the eyeball, through the upper lid, with the tip

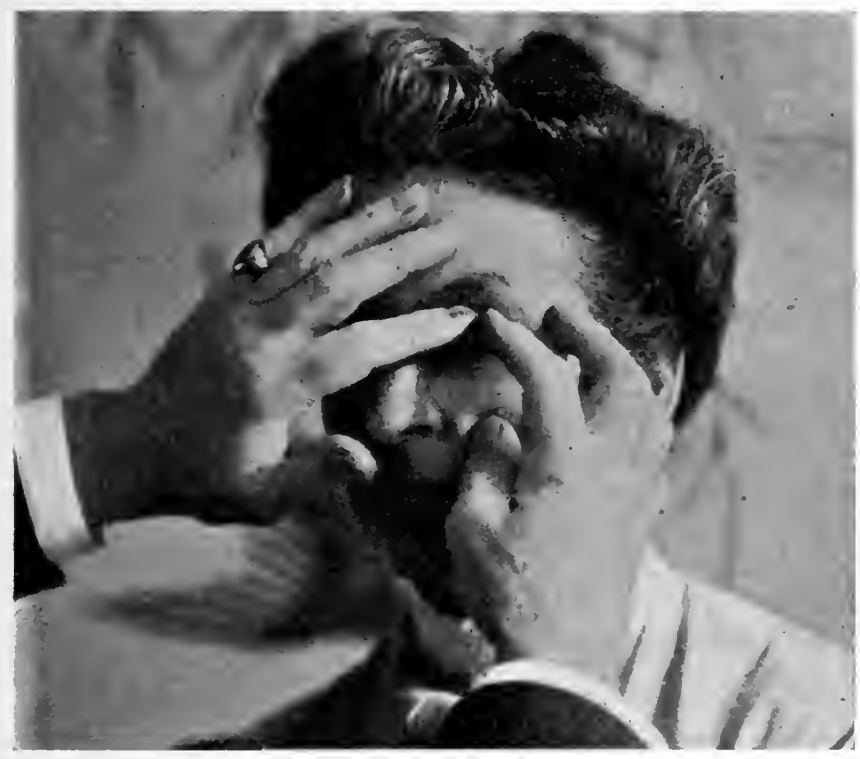

Fig. 4.-Position of hands in determining intraocular tension.

of the forefinger or second finger of each hand (Fig. 4). The pressure should alternate, not too quickly, between the two fingers, and should be made well back of the corneal border. By allowing the ring or middle finger of each hand to rest upon the temple or brow of the patient, so as to support the weight of the hand, the test is rendered more delicate. In recording the result of such an examination it is usual to employ a 
capital $\mathrm{T}$ as an abbreviation for "tension," $+\mathrm{T}$ indicating increased tension, or an abnormally hard eyeball, $-\mathrm{T}$, reduced tension, and $\mathrm{Tn}$, normal tension. The numerals $\mathrm{I}, 2,3$ following $+\mathrm{T}$ or $-\mathrm{T}$ are used to indicate varying degrees of increased or reduced tension.

Pupillary Reaction.--The patient should be seated facing the bright light of a window (not, however, the direct rays of the sun) and the pupillary reaction of each eye should be tested separately, the light, meantime, being carefully excluded from the other eye, which should be closed and covered by the patient's hand. The patient having been directed to look into the distance, the hand of the examiner should be held in front of, and quite close to, the eye under observation, (so close to it that the patient will not attempt to look at the hand-to "accommodate" for it-as this, of itself, would cause the pupil to contract) and then, after the eye has been shaded in this way for a few moments, the hand should be removed quickly, and the behavior of the pupil noted.

Under normal conditions the pupil, which will have dilated considerably under the shadow of the hand, contracts sharply and promptly when the light is allowed to fall upon the eye. This is the direct reflex action of the pupil, caused by contraction of the sphincter muscle of the iris. As is well known, it is due to the stimulation of the retina by the greater amount of light falling upon it, which leads, in turn, to stimulation of the sphincter pupillæ center in the oculomotor nucleus. The necessity for carefully excluding the light from the eye not under observation while testing the pupillary reaction of the other eye arises from the fact that, normally, both pupils contract when the retina of either eye is stimulated by light. This is 
known as the indirect or consensual reflex action of the pupil, and is a consequence of the semi-decussation of the optic nerves in the chiasm. A contraction of the pupils also occurs in accommodation (focusing) of the eyes for near objects and in the convergence of the visual axes which usually accompanies an effort of accommodation. This is called the associated action of the pupils.

Before describing the more important alterations in pupillary reaction due to pathological conditions, it will be well to mention that both the size and degree of reaction to light of the pupils vary greatly in persons who are entirely free from disease. In the first place, in youth the pupils are larger and respond more energetically to light than they do in advanced life. Again, the size of the pupils is influenced by the state of refraction of the eye, being, as a rule, smaller in hypermetropic, and larger in myopic than in normally constructed, or emmetropic, eyes. Frequently, however, the size and activity of the pupils vary markedly without assignable cause, just as the diameter of the cornea or iris varies in eyes that are entirely normal. In health the pupils of the two eyes are usually of the same size, though slight differences are not very uncommon, and are not significant. A marked difference in the refraction of the two eyes may cause a difference in the size of the pupils, the pupil being smaller in the eye in which a greater accommodative effort is required. In shape the pupils vary less than in size, being, in health, practically round.

There are many pathological conditions which influence the size, shape and reactions of the pupils. The iris itself may be the seat of disease; for example, iritis may be present, and this commonly causes con- 
traction and immobility of the pupil and not infrequently distortion, owing to the formation of adhesions between the iris and the lens capsule. On the other hand, when the tension of the eye becomes abnormally increased, as in glaucoma, the pupil is more or less widely dilated, is often oval in shape, and responds but slightly, if at all, to light. Inflammation of the cornea, the presence of a foreign body in the eye and, in fact, almost any condition accompanied by photophobia, lacrimation and ciliary irritation, if we except glaucoma, is attended by contraction of the pupil. Again, a moderately dilated and immovable pupil may be caused by paralysis of the sphincter muscle of the iris, which is commonly attended by paralysis of accommodation, and may, or may not, be accompanied by paralysis of the other ocular muscles supplied by the third nerve. A widely dilated and immovable pupil, if glaucoma be excluded, points strongly to the influence of a mydriatic. Marked and persistent contraction of the pupil may be due to eserin or some other myotic, to opium poisoning, to disease of the brain and its meninges, causing irritation of the sphincter pupillæ center, to spinal lesions, causing paralysis of the pupildilating center, or to paralysis of the cervical sympathetic. A dilated pupil which responds sluggishly or not at all to light, and is accompanied by impairment of vision for distant as well as near objects, glaucoma and adhesion of the iris to the lens capsule being excluded, indicates loss or impairment of function in the retina or optic nerve-tracts.

It should be remarked, however, that in rare instances, when a lesion exists high up in the optic tract, beyond the point where the fibers which pass to the pupillary center are given off, the pupil may react to 
light though absolute blindness be present. The opposite condition, in which the pupil fails to react to light though good vision exists, is frequently met with in the early stages of tabes dorsalis. When, under such circumstances, the reaction of the pupil to accommodation and convergence is retained we have what is known as the Argyll-Robertson symptom, due to a lesion involving the fibers, just mentioned, which pass from the optic tract to the center for pupillary movements. Usually the reflex immobility of the pupil met with in tabes dorsalis is associated with myosis, but exceptionally the pupil may be of normal size or even abnormally dilated.

Determination of Acuteness of Vision.- $\mathcal{T}_{0}$ determine the visual acuteness for distant objects it is customary to employ capital letters of different sizes, which are printed upon a sheet of paper or upon cardboard. The test-letters suggested by Snellen (which are to be had of most opticians) are those commonly employed. The largest of Snellen's letters, designated by the Roman numeral CC, is of such size that it should be recognized by an eye having normal sight at two hundred feet. The letters next smaller should be distinguished at one hundred feet, and finally there are letters recognizable at only fifteen feet, which is as small as is usually required for testing distant vision. If practicable, the letters, with a good light falling upon them, should be placed twenty feet from the patient; but whatever the distance it should be known, if the test is to be of even approximate accuracy. The sight of each eye should be determined separately, the other eye being "excluded" by holding a card or other opaque object before it. If at twenty feet the letters in the row marked $\mathrm{XX}$, or if at fifteen feet those 
marked $\mathrm{XV}$, can be distinguished, vision (abbreviated " $V$ ") is practically normal, and is recorded thus: $\mathrm{V}=\frac{20}{\mathrm{xx}}$ or $\frac{15}{\mathrm{xV}}$, as the case may be. If, however, at twenty feet only the letters which should be recognized at, let us say, fifty feet are distinguished, such a subnormal acuity of sight would be noted as $\mathrm{V}=\frac{20}{\mathrm{~L}}$. Sometimes it will happen that not even the largest letter can be made out at the usual testing distance, and then it will be necessary to lessen the distance, until finally, at six feet, perhaps, it can be distinguished, when the record would be $\mathrm{V}=\frac{6}{\mathrm{cc}}$.

When the impairment of vision is still more marked, the patient may be placed with his back to the light, and his ability to count fingers ascertained. In making this test the examiner holds up one hand, with the fingers separated, and slowly approaches the patient until he can state correctly the number of fingers extended. If three feet should be the greatest distance at which he can do this, the record would be: "Fingers (or Fingers counted) at 3 feet." If he is unable at any distance to tell how many fingers are held up, we next try whether he can distinguish the movements of the hand by reflected light (his back being still to the source of illumination, usually a window). When even this is impossible, he may still have "light perception"; that is, he may be able, when facing the light, to tell when the hand is held in front of the eye and when it is removed.

For testing near vision-ability to see at the usual reading or sewing distance - the test-types of Jaeger are commonly used. These consist simply of sentences printed in letters of different sizes, varying from No. I, "diamond" type, to No. 24, in which the letters are nearly an inch and three-fourths in height. The smallest type which the patient is able to read is as- 
certained, and the result is noted as J. (or Jaeger) No. I, No. 6, No. 24, as the case may be. In this test, as in the test for distant vision, the eyes should be examined separately, and the patient should be seated with his back to the light.

For testing accurately eccentric vision-determining the "field of vision"- - perimeter (Fig. 5) is required; but any considerable defect or contraction of the visual field may be detected in the following manner: Let the patient, with his back to the light, sit facing the examiner, at a distance of about two feet, and, having closed one of his eyes, have him with the eye under examination look at the open eye of the examiner (who also should close one eye, the right if he is testing the patient's right eye and vice versa). Watching the patient to see that he does not change the direction of his gaze, the examiner now moves his hand from different parts of the periphery toward the center of the visual field, and in a plane about midway between his own and the patient's face. Using his own field of vision as a standard of comparison, and requiring the patient to tell the number of fingers he extends, and whether they are held still or moved, the examiner is able, in a few moments, to determine whether the eccentric vision of the patient is defective, whether, in any direction, his field of vision falls appreciably short of what it should be. By this method of examination such conditions as hemianopsia, the contraction of the field of vision characteristic of glaucoma, and that which occurs in detachment of the retina, in retinitis pigmentosa, etc., may be readily detected.

In examining the eyes with a view to reaching a correct diagnosis it is of the first importance that the examination should be conducted in a systematic 


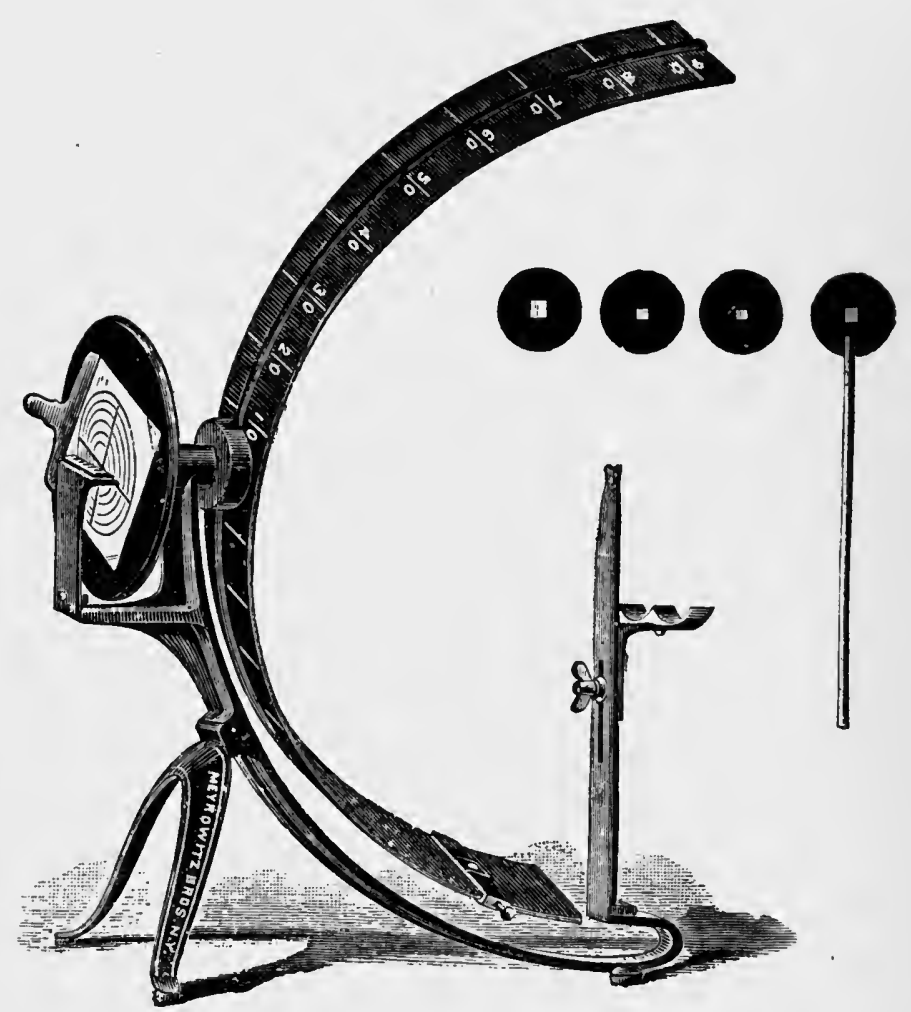

Fig. 5. - Standard registering perimeter. The examination may be made with the carrier which moves along the semicircle, or the test-object may be carried along this by means of dark discs attached to a long handle, each disc containing in its center the test-object. The patient's chin is placed in the curved chin-rest; the notched end of the upright bar is brought in contact with the face, directly beneath the eye to be examined, which attentively fixes the center of the semicircle. The other eye should be covered, preferably with a neatly-adjusted bandage. The record-chart is inserted at the back of the instrument, and by means of an ivory vernier the examiner is enabled to mark exactly with a pencil the point on the chart corresponding to the position on the semicircle at which the patient sees the test-object. The various marks are then joined by a continuous. line, and a map of the field is obtained. 
manner. Although the patient's account of his malady is, more often than not, indefinite and unsatisfactory and not infrequently misleading, it should be elicited at the outset; and if there is any reason to suspect that the eye affection is dependent upon a constitutional cause this too should be carefully inquired into. How long have the eye symptoms lasted is a most important question, which, if intelligently answered, will probably afford the examiner more assistance than any other one inquiry. Other important questions are, as to the existence of pain, photophobia, lacrimation, discharge (gumming of the lashes during sleep), and impairment of sight. In inquiring as to the last mentioned symptom it is essential that we should learn whether vision is defective for distant objects only, or for near objects only, or whether both are seen indistinctly, also whether diplopia exists.

The answers to these questions should give, at least, a clue as to what to look for when the next step in the examination - the careful inspection of the eye by daylight-is begun. The existence of pain, at all pronounced, for example, would suggest the probability of inflammation of the cornea, iris, or ciliary body being present, or, perhaps, glaucoma, or a foreign body or other traumatic lesion. Marked photophobia and lacrimation, usually coëxistent with pain, have a like significance. Considerable muco-purulent or purulent discharge is indicative of inflammation of the conjunctiva, which, except in the more severe types, such as gonorrheal conjunctivitis, is not usually attended by pain, but rather by a sensation of irritation, as though "sand were in the eyes." Again, if the symptoms (impaired vision, irritability of the eyes, etc.) have lasted but a few days, one may put aside errors of 
refraction and muscular anomalies, and search for a distinctly acute affection. A history of declining vision, without pain or external signs of inflammation, especially in a person beyond middle life, would point to cataract or, this being excluded, to disease of the retina or optic nerve. The existence of syphilis, nephritis, diabetes, alcoholism, or arteriosclerosis is. especially significant in this connection. Photophobia, it should be remarked, is not, as might be supposed, a usual symptom of retinitis or neuritis.

Epiphora of long standing suggests stricture of the lacrimal duct or canaliculi, or malposition or occlusion of the lacrimal puncta, and should lead to a careful examination of these structures. Poor vision for near objects, as in reading, with good distant vision, indicates failure or loss of the accommodative power of the eye, and in middle life usually means presbyopia; in childhood or early life, especially if occurring suddenly, paralysis of the ciliary muscles, as from diphtheria. A sudden onset of photophobia, lacrimation, and conjunctival injection, especially when limited to one eye, suggests the presence of a foreign body, which should be carefully looked for, even though the patient is not aware of its entrance.

Long-continued discomfort in the eyes (asthenopia), usually more marked after near work, and which may or may not be accompanied by imperfect sight; headaches, frontal or occipital, precipitated by reading, sewing or gazing fixedly at distant objects; chronic inflammation of the lid margins (blepharitis marginalis), without other signs of constitutional disorder, and persistent hyperemia of the conjunctiva, indicate refractive errors, and less frequently muscular anomalies, and call for carefully adjusted glasses. If, under such 
circumstances, there is good near vision and poor vision for distance, near-sightedness (myopia) probably exists; if both near and far vision are poor, hypermetropia of high grade or astigmatism. Ability to read ordinary print at the usual reading distance, it should be remarked, does not exclude myopia, but only myopia of high grade. Inability to obtain satisfactory glasses at the usual presbyopic age (about forty-five) suggests difference in the refraction of the eyes, astigmatism or a muscular error. Diplopia of sudden onset, often but not always attended by an evident squint, is commonly due to paralysis of one or more of the extrinsic eye muscles. Squint of long standing (rarely attended by diplopia), though it may have had its origin in an old paralysis, usually indicates the existence of a refractive error of high grade.

Although it seems almost incredible, it occasionally happens that the sight of one eye is lost, and monocular blindness exists for weeks or months, without the individual being conscious of the fact. When, under such circumstances, the discovery is finally made, the account usually given by the patient is that the loss of sight has only just occurred. The knowledge that such a thing is possible may prevent much perplexity in reaching a correct diagnosis. It is well, moreover, to bear in mind that, in general, the statements of patients as to the amount of sight they possess, particularly if the impairment of vision is limited to one eye, are often misleading. Influenced by especial considerations, they may claim to have better vision than they really possess; but commonly they go to the other extreme, and describe the loss of sight as being more complete than is actually the case. The patients themselves are often surprised to find their own convictions so much at 
fault; but occasionally it is evident that they are wilfully attempting to deceive the examiner.

Inspection of the Eye by Daylight.-Guided by the information gained by the questions which have been enumerated, the examiner should next proceed to a careful inspection of the lids and the superficial structures of the eye. A single glance may, in some. instances, suffice to confirm beyond doubt the tentative diagnosis already made, perhaps, as a result of these questions; but, at all events, the examination will seldom fail to afford distinctly helpful information.

As a matter of routine, it will be well to glance at the lids, to see whether they are swollen, whether their position and movements are normal, their margins free from inflammation, and whether the eyelashes occupy their proper position and are neither deficient in number nor matted together by discharge. If there is a history of epiphora the position and perviousness of the lacrimal puncta should be observed, and pressure should be made upon the lacrimal sac; for if there is occlusion of the nasal duct there will almost surely be an accumulation of tears and mucus in the sac, and the pressure exerted by the finger will cause regurgitation through the lacrimal puncta. If for any reason it is desirable to inspect the inner surface of the lids - to search, for example, for a foreign body or to observe the condition of the palpebral conjunctivathey should be everted in the manner already described.

Next an examination of the eye itself should be made, and this should include a determination of the tension (T) of the ball, a careful inspection of the conjunctiva, with reference to injection and the presence of discharge, of the smoothness of the surface and the transparency of the cornea, of the color and appearance 
of the iris, of the size, shape, blackness, and reaction of the pupil, of the depth of the anterior chamber, and of the clearness of the aqueous humor. The movements of the eyes, binocular as well as monocular, should also be observed, especially if diplopia is complained of or strabismus is suspected. This may be done conveniently by means of a pencil, held fifteen inches from the eyes and moved in various directions, or a lighted candle at the distance of as many feet. The sensibility of the cornea, when glaucoma or paralysis of the ciliary nerves is suspected, should be determined by touching its surface lightly with a spill of tissue paper or absorbent cotton.

The presence of marked opacity of the crystalline lens, especially if the opacity involves the anterior cortical layers, may usually be detected by simple, daylight inspection; but if it is limited to the deeper cortical layers, to the nucleus, or to the periphery of the lens, or if the cataract, though fully formed, be amber-colored, it may easily escape detection. On the other hand, it should be remarked that, owing to the greater amount of light reflected by the crystalline lens in persons advanced in life, a mistaken impression that a cataract is present is often gained by mere daylight inspection, which is dispelled by a glance into the eye with the ophthalmoscope.

When vascular injection of the eye is present much information may be gained by carefully noting the character of the hyperemia. A diffuse injection of the conjunctiva, least pronounced near the corneal limbus, of a brick-red color, the injected vessels being large, tortuous, and movable, is indicative of an inflammation limited to the conjunctiva. On the other hand, a zone of pericorneal injection, pinkish in color, composed of 
fine, subconjunctival vessels, usually radiating from the corneal border, is of more serious import, and points to keratitis, iritis, cyclitis, inflammatory glaucoma or, perhaps, to the presence of a foreign body upon the cornea or beneath the upper lid.

The character and amount of the discharge from the eye are also of diagnostic value. In the milder conjunctival inflammations the discharge is mucoid or muco-purulent in character and slight in amount, manifesting itself chiefly by gumming the lashes together during sleep; in the severer types of conjunctivitis, more especially in gonorrheal ophthalmia, it is very profuse and distinctly purulent, and a usual accompaniment is marked edema of the lids; in keratitis, iritis, and inflammatory glaucoma the discharge, due to excessive lacrimation, is watery, with but a slight admixture of mucus. It is a good rule in all inflammatory affections of the eyes, especially when met with in persons over forty years of age, to test the intraocular tension, or, in other words, to be on the lookout for glaucoma; for, as has been said already, no more unfortunate mistake can be made than to overlook the existence of this disease.

Inspection of the Eye by Oblique Illumination.Having completed the examination of the eye by daylight, if the diagnosis is still in doubt or further information bearing upon the treatment of the case is needed, the inspection of the eye by oblique illumination, with or without the aid of a mydriatic, should next be undertaken. In the manner already described, by concentrating the light first upon one and then upon another of the superficial structures of the eye, the cornea, anterior chamber, aqueous humor, iris, and lens should be carefully scrutinized. In examining the cornea 
one should look for disturbance of the epithelium, loss of substance, diminished transparency, superficial or deep, the deposition of exudates upon its inner surface, and the possible presence of a foreign body. In inspecting the anterior chamber one should consider its depth, the clearness of the aqueous humor, and the existence of hypopyon. The iris should be examined for alterations in color (as compared with the iris of the opposite eye), for swelling of its tissue, for inflammatory exudates, changes in position, and for the existence of anterior or posterior synechiæ. We may also examine by this method the size and shape of the pupil, its reaction to light and its clearness.

If we suspect iritis and the presence of posterior synechiæ, but owing to the small size of the pupil are left in doubt, or if we wish to examine the lens for commencing opacity, a mydriatic will afford the greatest possible assistance. A delay of, perhaps, fifteen minutes will be necessitated; but in the former case, if our suspicions are well founded, we shall obtain an irregularly dilated pupil, showing plainly the synechiæ, and so putting the question of iritis beyond doubt; and in the latter, nearly the whole anterior surface of the lens will be exposed to view, and opacities will be readily detected, which before could not be seen. As has been pointed out, a mydriatic transient in its effect, such as homatropin or euphthalmin, is best adapted for this purpose. 


\section{CHAPTER II.}

\section{GENERAL OBSERVATIONS UPON THE TREATMENT OF DISEASES OF THE EYE.}

In the management of eye diseases, a correct diagnosis having been reached, the selection of the proper remedy to meet the condition is not usually a difficult matter, even for those who have not paid especial attention to this branch of medicine. This is so because, in the first place, the indications are usually definite and clear and, in the next place, the list of available remedies is not a long one.

In writing for the general practitioner there is, perhaps, less reason than there would be in addressing the specialist in ophthalmology to emphasize the fact that eye diseases should not be treated simply as local maladies, and that in their management constitutional remedies are at times even more important than local ones. There are, of course, diseases of the eye which are purely local affections, and which demand only local treatment; but there are many others which are but local manifestations of a constitutional disorder, and in which general measures, aimed at this disorder, are an essential part of their successful treatment.

To enumerate all the drugs and therapeutic agents apt to be needed in treating the commoner diseases of the eye is not a difficult task, and the list need not be a long one. It would naturally be divided into local remedies and constitutional remedies. Such a list, fairly comprehensive, would be as follows: 


\section{LOCAL REMEDIES.}

Zinc sulphate,

Boracic acid,

Mercury bichlorid,

Sodium chlorid,

Silver nitrate,

Protargol, or

Argyrol,

Copper sulphate,

Chlorin water,

Carbolic acid,

Salicylic acid,

Yellow oxid of mercury,

Alum,

Zinc oxid,

Caustic potash,

Mercurial ointment,

Atropin sulphate,

Eserin sulphate,
Pilocarpin hydrochlorate,

Holocain hydrochlorate,

Hyoscyamin hydrobromate,

Homatropin hydrobromate, or

Euphthalmin hydrobromate,

Cocain hydrochlorate,

Adrenalin,

Dionin,

Tincture of iodin,

Extract of opium,

Extract of belladonna,

Castor oil,

Veratrin oleate,

Jequirity,

Cold (ice-cloths),

Heat (dry or moist),

Galvano-cautery,

Local bloodletting (leeching).

\section{CONSTITUTIONAL REMEDIES.}

Mercury biniodid,

Mercury protoiodid,

Mercury bichlorid,

Calomel,

Mercurial ointment,

Potassium iodid,

Quinin sulphate,

Strychnin sulphate,

Extract or tincture of nux vomica,

Iron phosphate,

Iron iodid,

Iron carbonate,

Opium,

Morphin sulphate,
Pilocarpin hydrochlorate,

Arsenic (Fowler's solution),

Lithium,

Colchicum,

Cod-liver oil,

Sodium pyrophosphate,

Trional,

Phenacetin,

Diphtheria antitoxin,

An energetic purgative (such as the "compound calomel powder," mentioned in the appendix).

Speaking broadly, it may be said, as to local remedies, that astringents and antiseptics are indicated when there is a considerable discharge from the eye of mucus 
or pus, such discharge being indicative of inflammation of the conjunctiva. On the other hand, the existence of pain, photophobia, and lacrimation, symptoms characteristic of keratitis, iritis, etc., commonly contraindicates their employment and calls for soothing remedies, especially atropin (unless there is $+T$ of the eye) and opium. As the different varieties of inflammation of the conjunctiva, if we except phlyctenular and diphtheritic conjunctivitis, are essentially local affections, it is evident that the field for astringents and antiseptics is mainly in local maladies. Atropin, on the other hand, while useful in certain purely local affections, as, for example, corneal ulcer due to direct infection, is more especially indicated in inflammations of the eye which have a constitutional origin, such as iritis, cyclitis, and phlyctenular, herpetic, and interstitial keratitis.

In no department of medicine are an early diagnosis and a prompt employment of remedial measures more important than in ophthalmic practice. Especially is this true in the treatment of purulent (gonorrheal) conjunctivitis, of diphtheritic conjunctivitis, of iritis and of inflammatory glaucoma. In the first named affection, and the observation applies as well to diphtheritic conjunctivitis, a delay of two or three days may mean loss of sight through necrosis of the cornea; in iritis it may mean a permanently damaged eye through the formation of unbreakable posterior synechix, while in the severer types of acute glaucoma a still briefer delay, of twenty-four or forty-eight hours, may result in absolute blindness from irreparable damage to the optic nerve and retina.

Local Remedies.-Topical remedies for the eye are commonly in the shape of collyria-solutions in 
water of various drugs for instillation into the conjunctival sac; lotions, to be applied on linen or gauze pads to the closed lids; ointments, for application to the eye itself or to the lids; powders, to be dusted into the eye, and crystals or crayons (copper sulphate, alum, silver nitrate) for direct application to the eye, usually to the palpebral conjunctiva or to the lids. Strong antiseptic agents, such as pure carbolic acid and tincture of iodin, are applied accurately and in small quantities to threatening corneal ulcers, and the actual cautery-the galvanocautery being best adapted to the purpose-is similarly employed. Heat and cold, the former in the shape of hot fomentations or dry heat (Japanese stove), the latter in the form of ice-cloths or small ice-bags, are also used. Ice-cloths, which are especially useful in gonorrheal ophthalmia, should be kept lying upon a block of ice, from which they must be transferred at brief intervals to the lids of the affected eye. Moist heat may be conveniently applied by means of a soft, bird's-nestshaped sponge, which should be kept at the desired temperature by being repeatedly dipped in hot water.

For the application of collyria an eye-dropper is essential, and one with a bent nozzle is more convenient than a straight one (Fig. 6 and Fig. 7). As it is possible to transfer infectious material from one eye to another by means of eye-droppers, certain precautions should be observed in their use. The danger of doing this is slight if the dropper is not allowed to come in contact with the lids or eye, which, of course, need not, and should not, happen. It is best, however, in the management of distinctly contagious diseases - such as gonorrheal conjunctivitis-that a special dropper should be set apart for each case, and this should not be used 
for another patient unless it has been sterilized by boiling. It is important also that a dropper which

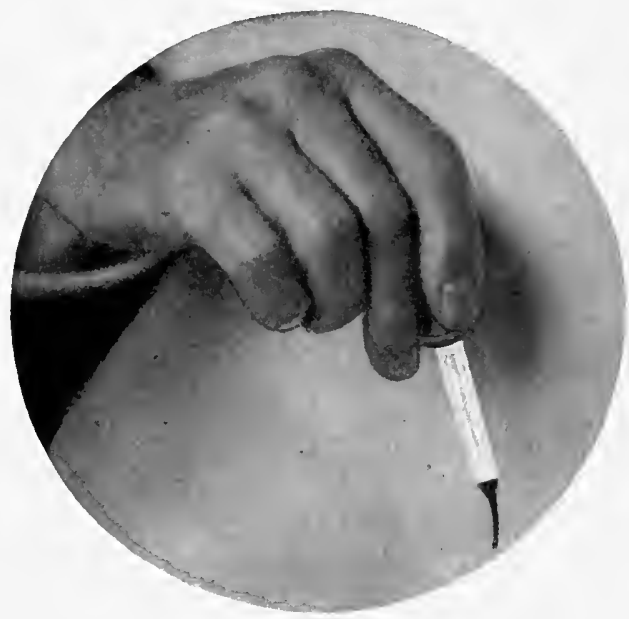

Fig. 6. -The right way to hold an eye-dropper.

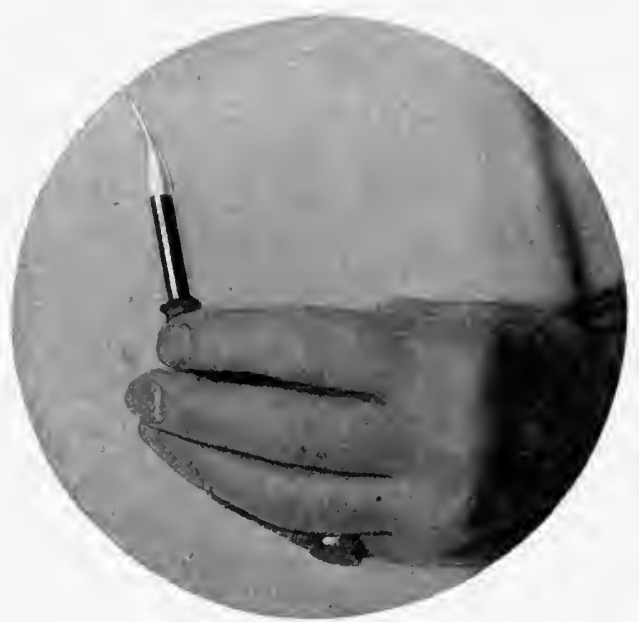

Fig. 7.-'The wrong way to hold an eye-dropper.

has been used for a mydriatic-atropin, for exampleshould not be used for the instillation of other collyria, 
as neglect of this precaution may result in a dilatation of the pupil and a blurring. of vision that will cause the patient much needless inconvenience.

As a rule, to apply more than one or two drops to the eye, as is often done, is unnecessary, since hardly that quantity is retained in the conjunctival sac, and in the case of poisonous solutions-atropin, hyoscyamin and the like-it is especially to be avoided, as general intoxication may be induced in this way. It is not uncommon for individuals to "taste" such medicines as those just mentioned shortly after their application to the eye, indicating that they have passed through the lacrimal passages and have reached the nose and pharynx. It is in this way that they impress the system, and for this reason it is better that poisonous collyria should be instilled near the outer, rather than near the inner, canthus. If it is desirable that the solution used should exert its full effect upon the cornea, as in keratitis or iritis, the patient's head should be thrown back and he should be directed to look downward; the upper lid being then drawn up, it is easy to cause the drop to fall directly upon the exposed cornea. Under other circumstances it is more convenient to apply the drop between the lower lid and the eyeball, the patient looking upward and the lid being drawn slightly away from the ball (Fig. 8).

Apothecaries have a reprehensible habit, in preparing solutions for the eye, of using a mortar and pestle to mix the ingredients indicated. This is wholly unnecessary, and nearly always results in contaminating the solution, as is shown in a few days by the appearance in it of a fungous growth. It is a practice which is without excuse, and which ought unquestionably to be abandoned. Equally deserving of condemnation, and 
for much the same reason, is the habit common among physicians of prescribing rose-water, instead of distilled water, as a solvent for drugs intended for application to the eye, because it has, or is supposed to have, a pleasant odor. There is a very general popular belief that collyria in order to be efficacious must be

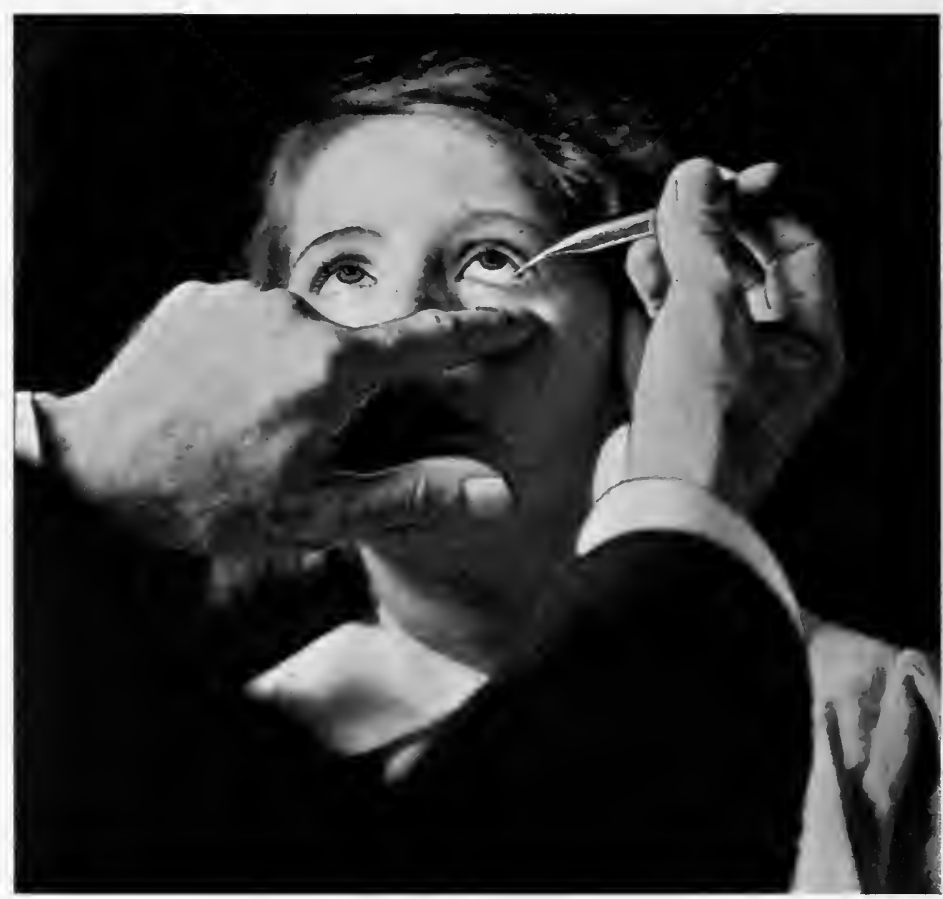

Fig. 8.-Convenient method of dropping solutions into the eye.

"strong," must cause decided smarting when applied to the eye. It is scarcely necessary to say that this belief is without warrant.

Such agents as protargol, strong solutions of silver nitrate, etc., which are commonly applied to the everted lids, can be applied most conveniently by means 
of a mop made by wrapping a little absorbent cotton around the sharp end of a wooden toothpick. Ointments may be applied to the inner surface of the upper or lower lid, the eye being directed downward in one

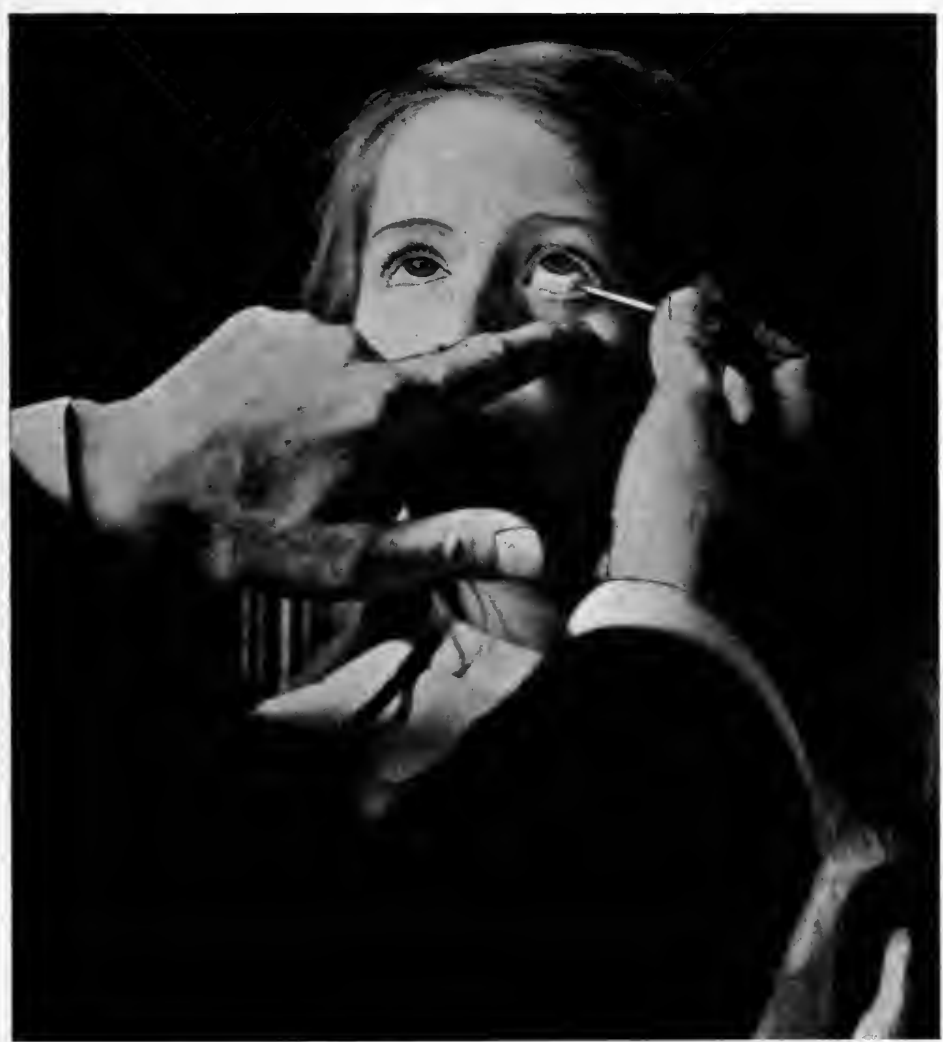

Fig. 9.-Applying ointment to the eye with a wooden toothpick.

case and upward in the other, with the broad, flat end of a toothpick (Fig. 9). If intended for application to the lids only, they may be rubbed on with the finger-tip. In preparing ointments for application to the eye vaselin or some similar preparation should be used 
as a base, since it is advantageous that they should melt at the temperature of the body and so become diffused throughout the conjunctival sac; but when intended for application to the lids this is a disadvantage, and a firmer base, such as "vaselin cerate"* or cold-cream, should be used.

Of all local remedies employed in the treatment of eye diseases, atropin is, perhaps, the most valuable. It is commonly prescribed in aqueous solution, the sulphate being used because it is freely soluble in water. Its value depends not only upon its efficacy in relieving pain and photophobia and favorably influencing inflammation of various structures of the eye, but upon its action as a mydriatic, its mechanical effect in enlarging the pupil, which, as is well known, is of inestimable value in the treatment of iritis. It is prescribed in solutions varying in strength from one-eighth of a grain to eight grains to the ounce, according to the effect desired. In iritis, and in other non-glaucomatous inflammations attended by pain, lacrimation and photophobia, a solution of the strength of four grains to the ounce (exceptionally, an eight-grain solution) is commonly employed. In inflammations in which these symptoms are not so marked, as, for example, the usual type of phlyctenular kerato-conjunctivitis, a strength of one grain to the ounce generally suffices. The very weak solutions, the quarter-grain- or eighthgrain-to-the-ounce solutions, are used chiefly for their mydriatic effect, the former being applied once in three or four days to produce a continuous but not very

* Composed of yellow wax, one part; vaselin, four parts. An ointment suggested by the author some years since. It is of suitable consistence, and will keep for a very long time without becoming rancid. 
marked mydriasis - to improve the vision, for example, in incipient cataract when the opacity is confined to the central portion of the lens; the latter, as a substitute for the more evanescent mydriatics, when a transient dilatation of the pupil is needed to facilitate inspection of the lens or deeper eye structures.

It is well to remember that the mydriasis produced by a strong solution of atropin-a four-grain solutionwill frequently not disappear entirely for fifteen or sixteen days. It is inexcusable, therefore, to use such a solution when only a transient effect upon the pupil is desired. Euphthalmin is the most evanescent mydriatic that we possess, its effect lasting scarcely twenty-four hours; but it is a very expensive drug, and must be used in quite strong solutions-four to five per cent.- to insure the desired result. Homatropin is effective in much weaker solutions-two to four grains to the ounce-and, though its action upon the pupil is somewhat more prolonged, it is a very satisfactory agent to employ when only a brief mydriasis is desired.

Although, as has just been said, atropin is so valuable an agent, its use is attended with certain unfavorable possibilities. In persons advanced in life it sometimes precipitates an attack of glaucoma. Probably, this happens only when there exists a predisposition to this disease; but, at all events, it has led to caution in its use in old persons and an indisposition to prescribe it under such circumstances unless the need for it is clear. It is, doubtless, by dilating the pupil and crowding the iris into the periphery of the anterior chamber, and thus clogging the lymph-passages in this region, that this unfortunate result is brought about. In penetrating wounds near the border of the cornea, 
and in ulcers in this region which have perforated, or are about to perforate, into the anterior chamber, its employment is contraindicated, since through its paralyzing effect upon the sphincter pupilla it favors the occurrence of hernia of the iris.

Again, the prolonged use of atropin may cause a conjunctivitis of follicular type, which is usually accompanied by annoying itching, and in persons peculiarly susceptible to the action of belladonna even a single application of an atropin solution to the eye may produce a conjunctivitis of this character, accompanied at times by marked edema and redness of the lids and face. Occasionally, too, when used in old persons, especially when both eyes are closed, as after operations for cataract, it causes delirium, which under such circumstances may have serious consequences. Any one of the commonly employed mydriatics, it should be said, may produce the same unpleasant consequences as atropin, and the contraindications to their use are the same. Homatropin is the least poisonous, and, therefore, is the least likely to cause delirium. A peculiar susceptibility to atropin, such as has been described, does not necessarily imply a like susceptibility to all the other mydriatics; so that when it is encountered some other member of the group-such as hyoscyamin, scopolamin, or duboisin-may, not infrequently, be substituted with good effect.

As mydriatics tend to induce glaucoma by dilating the pupil and obstructing the filtration angle at the periphery of the anterior chamber, myotics (eserin and pilocarpin) tend to reduce increased intraocular tension, to control glaucoma, by contracting the pupil (which under such circumstances is almost always abnormally dilated), drawing the iris toward the center 
of the anterior chamber and freeing the lymph-spaces at its periphery. Such being the case, it is evident that we have in the behavior of the pupil a guide to the strength of the myotic solution required in the treatment of glaucoma. The stronger solutions of eserin, a much more energetic myotic than pilocarpin, through the vigorous contraction of the sphincter pupillæ and the ciliary muscle which they induce, often cause pain in the eye, and when their use is long continued may even excite iritis. It is desirable, therefore, that they should not be used except when clearly demanded. In a word, the weakest solution of eserin which will cause the pupil to contract, and will maintain it in a state of contraction (not excessive), is what one should aim to employ in endeavoring to control increased intraocular tension.

In an acute attack of inflammatory glaucoma it often happens that even the strongest solution of eserin that we are in the habit of using (four grains to the ounce) will fail to overcome the dilatation of the pupil; but, on the other hand, in the intervals between the attacks, when it is used as a prophylactic, and in glaucoma simplex a strength of a quarter of a grain, or even an eighth of a grain, to the ounce usually suffices to accomplish the desired result. In inflammatory glaucoma iridectomy, of course, is the sovereign remedy, and resort to it should not be unnecessarily delayed; but, if during an acute exacerbation eserin is to be used, a strong solution (four grains to the ounce) should be prescribed at once, without waiting to ascertain whether a weaker solution will cause the pupil to contract. When, however, the conditions are not urgent, one should determine by trial the weakest solution of eserin that will maintain the pupil in a state of 
moderate contraction when applied twice or, at most, three times a day, and direct this for future use.

The prolonged use of a collyrium of eserin, which is commonly prescribed in the form of the sulphate, occasionally excites a follicular conjunctivitis very similar to that sometimes induced by atropin. When this happens, or when for any reason eserin is not well borne, the hydrochlorate of pilocarpin should be substituted, and since its myotic action, as has been said, is much less powerful than that of eserin, it must be prescribed in considerably stronger solutions-in solutions of from one to eight grains to the ounce. The myotics, eserin especially, are distinctly contraindicated in iritis, and if used through misapprehension of the true condition are sure to do harm, increasing the pain and aggravating the inflammation.

Astringents and antiseptic agents, as has been pointed out, are especially indicated in the treatment of inflammations of the conjunctiva. In the milder types of conjunctivitis, such as the catarrhal and follicular varieties, they should be used in comparatively weak solutions. Zinc sulphate, on the whole, is the most useful astringent, and boracic acid and bichlorid of mercury are the most useful antiseptics, in these conditions. The collyrium which I have found especially efficacious in catarrhal conjunctivitis is one containing half a grain of sulphate of zinc and ten to twelve grains of boracic acid to the ounce. Dropped into the eye freely, three times a day, it will seldom fail to cure an acute attack in a very few days. Bichlorid of mercury, which is useful more particularly in follicular conjunctivitis, in vernal catarrh, and in blennorrhea of the lacrimal passages, should be prescribed in solutions varying in strength from $I: 12,000$ 
to I : 8000. The addition of sodium chlorid to the solution, in the proportion of three grains to the ounce (about the strength of normal salt solution), seems to add to its efficacy, and, moreover, renders it more acceptable to the eye. In asthenopia, in hyperemia of the conjunctiva, in mild cases of catarrhal conjunctivitis, and, again, when one is uncertain as to the diagnosis, and wishes to prescribe something that will do a measure of good, at least, and will surely do no harm, boracic acid (IO to I 2 grains to the ounce) is especially to be commended. In addition to its slight antiseptic and astringent action, it is decidedly soothing and grateful to irritable eyes.

In the severer types of conjunctivitis, in purulent (gonorrheal) and trachomatous conjunctivitis, more energetic treatment is demanded, much stronger astringent and antiseptic solutions are called for, and in the first-named affection, especially, very much more assiduous attention. Silver nitrate in 2 per cent. solution or, preferably, protargol or argyrol in 20 to 40 per cent. solution must here be employed.

In applying tincture of iodin or pure carbolic acid to corneal ulcers, the application may be conveniently made by means of a sharply pointed wooden toothpick, about the tip of which a very little absorbent cotton - a few fibers only-has been wound. To prevent the cornea being acted upon more extensively than is desired the improvised applicator should carry only a very small quantity of the fluid.

Cocain may be used with good effect to do away with the pain caused by the application to the eye of such severe remedies as silver nitrate, copper sulphate, carbolic acid, iodin, etc.; but as a remedial agent per $s e$, apart from its use as a local anesthetic, it should 
never, in my judgment, be employed. For not only is the relief which it affords-from pain, photophobia, etc.--very evanescent, but it markedly disturbs the nutrition of the cornea, causes, not infrequently, desquamation of the corneal epithelium, and brings about a condition peculiarly favorable to the invasion of pathogenic bacteria. From time to time cases of inflammation of the superficial structures of the eye have come under my observation in which cocain had been used, and in which its ill effects were plainly manifest:

Dionin, one of the more recent additions to the pharmacopœia of the ophthalmologist, owes its value to its action as an analgesic and a lymphagogue. It is useful in iritis, not only because of its a nalgesic effect, but because it increases the mydriatic action of the atropin, in association with which it is employed. In inflammatory glaucoma it tends to reduce the intraocular tension, as well as to relieve pain. It is beneficial also in keratitis, more especially in parenchymatous keratitis, and through its action as a lymphagogue it promotes the absorption of recent corneal opacities and the remnants of cortical lens substance left after operations for cataract. It is used commonly in five per cent. solution, and in this strength may be applied to the eye twice to four times daily. The immediate effect of its application is to cause considerable irritation, not infrequently decided pain, and pronounced edema and congestion of the conjunctiva. The analgesia which supervenes in fifteen or twenty minutes lasts for some hours. A tolerance to dionin is established usually after it has been used a few days, and after this its further employment is of doubtful value. 
The use of opium as a local application in eye diseases, formerly much in vogue, does not receive at the present day the attention it deserves. I am not inclined to employ it as a collyrium-for application to the eye itself; but, as a lotion to be applied by means of a gauze or linen pad to the closed lids, I have found it a most useful remedy in all painful inflammations of the eye, and especially in those of traumatic origin. It should be prescribed in the strength of from ten to fifteen grains of the extract of opium to four ounces of distilled water, to which it is often advantageous to add from forty to sixty grains of boracic acid. When its action is favorable, it can hardly be applied too persistently, though it is well to remember that in susceptible individuals opium used in this way occasionally produces its characteristic constitutional effect. As a rule, it should be applied at the temperature of the atmosphere, but occasionally it affords greater relief when used as hot as can be conveniently borne. A gauze pad of suitable thickness, wet with a saturated solution of boracic acid or with the lotion of opium and boracic acid just mentioned, and covered with a piece of rubber protective or oiled silk or muslin, makes an excellent "poultice," and one which is much more cleanly and convenient of application than the poultices of flaxseed-meal, etc., which are commonly employed.

Ointments are applied to the lids to prevent their becoming. stuck together by the drying of discharge upon the eyelashes, as in the different types of conjunctivitis, and to cure inflammation of the lids or their margins (blepharitis marginalis). For the purpose first named a simple, bland ointment, such as coldcream, to which boracic acid may be added (five grains to the dram), is best adapted. For blepharitis 
marginalis nothing is so generally efficacious as the so-called "yellow salve" (yellow oxid of mercury, 2 grains; cold-cream or "vaselin cerate," I dram). An ointment of salicylic acid of the same strength is also useful in this condition, as well as in eczema involving the lids or neighboring parts.

In burns of the eye, whether from hot substances or caustic agents, and in abrasions of the cornea, castor oil is a useful application, affording, as it does, a protective covering to the inflamed surfaces. A still more soothing application under such circumstances is a solution of atropin (the alkaloid, not the sulphate) in castor oil (four to eight grains to the ounce).

Light, in so far as its influence upon eyes which are the seat of pathological changes is concerned, is not the reprehensible thing it was once supposed to be, and the confinement of patients with ocular inflammations in quite dark rooms, as well as the closure of the eyes by thick bandages designed to exclude light, very generally practised in former days, is now regarded not only as uncalled for but as actually harmful in many conditions. The misadventures, especially those occurring after important operations upon the eye, formerly attributed to premature or undue exposure of the eyes to light, are now known to be caused-in the great majority of instances, at all events-by bacterial infection, against which, at the present day, we guard with greater assiduity than we display in trying to avoid the supposed ill effects of light.

In saying this, however, I do not wish to be understood as holding that inflamed and painful eyes should not be protected from undue exposure to light; for, as a rule, it is desirable, unquestionably, that they should be. An eye in which there exists inflammation 
of the iris, of the ciliary body, or the cornea, or which is the seat of an attack of acute glaucoma, will certainly be made more photophobic and painful and, perhaps, actually worse, so far as the inflammatory condition is concerned, by undue exposure to light, and this should, without doubt, be avoided; but this does not mean that the patient must be shut up in an absolutely dark room, or that his eyes must be

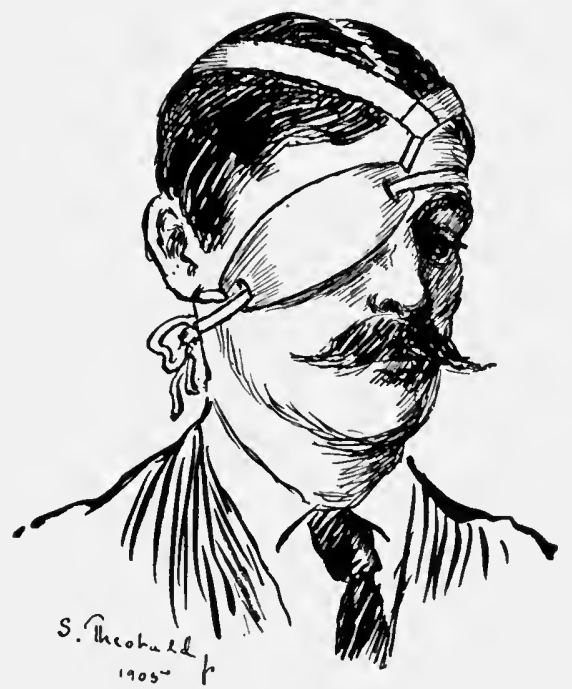

Fig. I0.-Author's bandage, as applied to one eye.

confined by, and subjected to the poultice-like action of, a roller bandage of many thicknesses. It means simply that he should avoid very light rooms, and that he should wear smoke-tinted (but not too darkly tinted) spectacles (coquilles), which may be supplemented by a monocular or a binocular eye-shade or a piece of black court-plaster attached to the glass which covers the affected eye, if photophobia be a marked symptom of the attack. 
Bandages are seldom necessary except after certain operations, or after serious injuries of the eye, or for the purpose of holding in position pads used for the application of lotions to the lids; and for these purposes a light, easily applied and easily removed bandage, such as that contrived some years ago by the author* (Figs. IO and I I), is greatly to be preferred to the clumsy

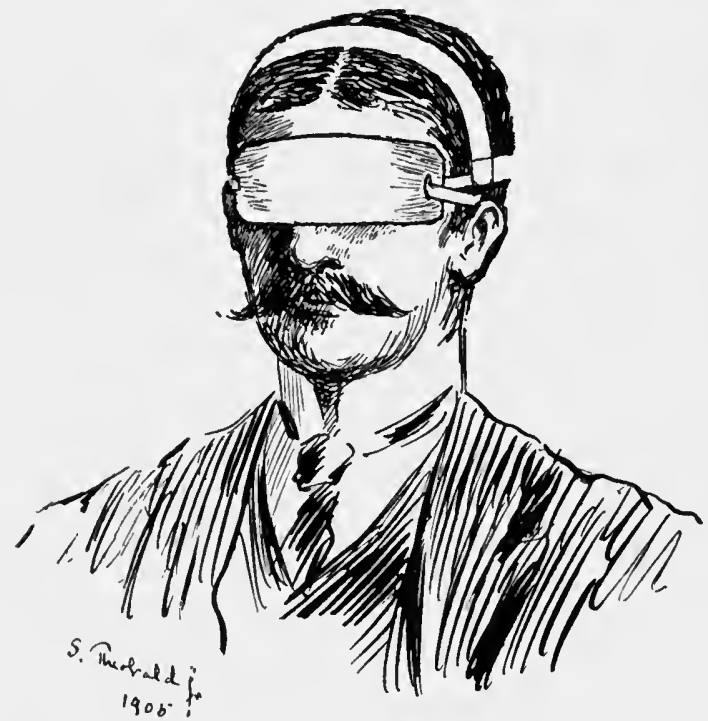

Fig. II.-Author's bandage, as applied to both eyes.

* This bandage, which can be used for one or for both eyes, as shown in the illustrations, consists of a head-piece, to which the tapes are attached, and an eye-piece with a buttonhole at each end, through which the tapes are passed. The head-piece consists of two straight strips of cotton cloth, of good quality, twelve inches long and one inch and three-quarters wide, the ends of which are sewed together so that the strips shall form a right angle. The eye-piece, made of the same material cut bias, when intended for one eye, is of oval shape, six inches and a half long by two inches and three-quarters wide at its widest part; when intended for closing both eyes, it is rectangular, and should be seven inches and a half in length by two inches and three-quarters in width. The size of the head-piece may be varied, to fit small or large heads, by cutting the strips a half-inch shorter or longer. 
roller bandage, which, however, many ophthalmic surgeons employ even at the present day.

It is hardly necessary to say that inflamed and painful eyes should be given complete rest from such work as reading, writing, sewing, and the like; but it may be well to point out that this is as true when only one eye is affected as it is when both are involved, since it is almost as trying to the inflamed eye to have its fellow taxed as it is to be taxed itself.

It is often extremely difficult to examine, or to make applications to, the eyes of unruly children. When this difficulty is met with, it may be easily overcome in the following manner: Let the child be placed across the lap of an attendant or nurse, who is instructed to hold its hands firmly. Then let the physician, seating himself in a convenient position for the purpose, and having thrown a towel over his lap, take the child's head between his knees. In this way he is enabled to hold it very securely, while, both his hands being free, it is not difficult for him to separate the lids in order to inspect the eye, or to make such applications as may be needed.

Constitutional Remedies.-As is indicated by the briefness of the list given in the early part of this chapter, the number of constitutional remedies required in the treatment of diseases of the eye is small.

Since syphilis plays a very important rôle in the etiology of eye diseases, the antisyphilitic drugs-mercury and potassium iodid-are among the most useful remedial agents employed in ophthalmic practice; but their value, it should be said, is not limited to diseases of syphilitic origin only. On the contrary, they are of great value in all ocular inflammations in which there is a tendency to plastic exudation and proliferation 
of connective tissue. This is especially true of inflammations of the uveal coat (iris, ciliary body, and choroid), of the retina and optic nerve, and of the motor nerves which supply the extrinsic eye muscles. On the other hand, a tendency to purulent infiltration, to ulceration and necrosis, especially of the cornea, is a distinct contraindication to their use.

When it is important to bring the system promptly under the influence of mercury, as in syphilitic iritis or irido-choroiditis of severe type, we can not do better than to administer calomel in small, frequently repeated doses, - half a grain every hour or every two hours,-guarded, if need be, by small doses of opium. Inunctions of mercurial ointment may supplement the calomel, if thought desirable. When the purpose of the inunctions is to impress the system, they should be made, in the usual way, to the inside of the thighs and arms. With less effect upon the system, a decided impression may be made upon the eyes by applying mercurial ointment several times daily to the forehead and temples. In iritis and cyclitis the addition of extract of belladonna to the mercurial ointment, in the proportion of one dram to the ounce, renders this procedure still more efficacious.

When less urgency is demanded, one of the best ways of administering mercury in diseases of the eye is in the form of the biniodid, which may be given in solution (with the addition of a few grains of potassium iodid) or in tablet-triturates, and in doses varying from a sixteenth to a thirty-second of a grain, three times a day. When the exhibition of iron as well as of mercury is indicated, as is frequently the case in ocular affections dependent upon inherited syphilis, the syrup of the iodid of iron may be added, in such 
proportion as desired, to the biniodid solution, or, if the iodids are not well borne, the bichlorid of mercury in solution, with the addition of the tincture of chlorid of iron, may be given instead.

Potassium iodid, in order to secure the best results in the ocular maladies in which it is indicated, must often be given in liberal doses. This is especially true of the diseases of the eye occurring in the tertiary stage of syphilis, of the disturbances of sight, whether strictly visual or motor, arising from intracranial affections, of the so-called neuropathic inflammations of the eye, and of that obscure and frequently intractable disease, serous iritis, or, as it is more correctly denominated, general uveitis. When it is to be administered in increasing doses, it is convenient to prescribe it in saturated solution, so that each drop shall represent a grain of the iodid. It is frequently given, with good effect, in combination with the biniodid or bichlorid of mercury.

The salicylates, sodium and lithium, are remedies of much value, not only in rheumatic affections of the eye (which comprise a not insignificant group), but also in traumatic and post-operative inflammations, and even in iritis and iridocyclitis of syphilitic origin. The lithium salt is supposed to have a somewhat less disturbing effect upon the stomach. The dose of each is the same-ten to twenty grains-every three hours. When they-and this is true also of potassium iodidare not well borne by the stomach, two teaspoonfuls of Fairchild's essence of pepsin given with each dose will often obviate completely this difficulty.

In suppuration, ulceration and necrosis of the cornea-the conditions which, as has been said, especially contraindicate the administration of mercury 
and to a less degree that of potassium iodid-quinin is of undoubted value, and should be given in such doses as to produce cinchonism. It is also extremely useful, especially in combination with iron and strychnin or nux vomica, in those affections of the eye which are dependent upon an impaired state of the general health, such as phlyctenular conjunctivitis or keratitis, blepharitis marginalis, eczema of the lids, etc. A favorite combination with me in such cases is the elixir of the phosphates of iron, quinin, and strychnin, preference being given to the elixir prepared by Wyeth and Bro., since it contains, besides a sixtieth of a grain of strychnin, two grains of iron and one grain of quinin to the dram-more than twice as much of the two last-named ingredients as do many of the preparations which are called by the same name. The syrup of the iodid of iron, which is more often administered in these affections, is useful when they are associated with a distinctly strumous diathesis, manifested by enlarged lymphatic glands, etc.; ' but, except under such circumstances, the iron, quinin, and strychnin combination just mentioned has afforded me decidedly better results, and I have no hesitation in strongly commending it.

Strychnin, in gradually increasing doses, given by the mouth and not, as some (absurdly, I think) recommend, by hypodermic injection, and usually in connection with potassium iodid, is valuable in amblyopic affections, and in paralyses of the ocular muscles of not too long standing. Opium and morphin are chiefly useful for the relief of pain, in such diseases as iritis, cyclitis, and inflammatory glaucoma. Sulphonal and trional I have found especially useful in giving quiet sleep and relief from nervousness after important 
operations upon the eye, and, as a matter of routine, I prescribe one or the other of these drugs, to be given several hours before bedtime, after cataract extractions, iridectomies, etc.

There can be no question as to the value of an energetic cathartic, particularly one containing a liberal proportion of calomel, in many inflammatory affections of the eye, and especially in iritis, in acute glaucoma, and in phlyctenular kerato-conjunctivitis, accompanied, as it so often is, by eczema of the lids and face and by nasal catarrh. In the last-named condition the good which it accomplishes so promptly seems, in great measure, to be due to its action in ridding the alimentary canal of bacteria and their toxins, or, as the older writers used to express it, in "Cleaning out the prima via."

Pilocarpin, which, like strychnin, may be given by the mouth with good effect and without inconvenience, and which, therefore, should not be administered hypodermically, is useful at times in retinitis, in choroiditis, and in detachment of the retina. Colchicum and lithium (lithia water or the citrate or carbonate of lithium tablets) are indicated in gouty inflammations of the eye (iritis, retinitis, scleritis, and chronic conjunctivitis), and arsenic, generally in the form of Fowler's solution, in the different varieties of herpes.

The diphtheria antitoxin, as reported by trustworthy observers, has proved so eminently efficacious in controlling diphtheritic conjunctivitis-a disease hitherto regarded as one of the most dangerous to which the eye is liable-as to have completely overshadowed all local measures. It should be administered as in diphtheria affecting the fauces. 
In acute suppurative processes involving the lids, the lacrimal sac, or the orbit, sodium pyrophosphate, in liberal doses, is of undoubted value. For an adult the dose is twenty grains every two hours; for a child, from ten to fifteen grains every two or three hours. It should be prescribed in solution, and, as it is not very soluble, as much as half an ounce of water should be allowed for each twenty grains of the salt. 
CHAPTER III.

\section{DISEASES OF THE EYELIDS AND ORBIT.}

DISEASES OF THE EYELIDS.

Diseases of the eyelids are of common occurrence and, as a rule, may be dealt with satisfactorily by the general practitioner. Usually, they require local treatment only, but this is not always the case.

Blepharitis Marginalis.-Inflammation of the lid-

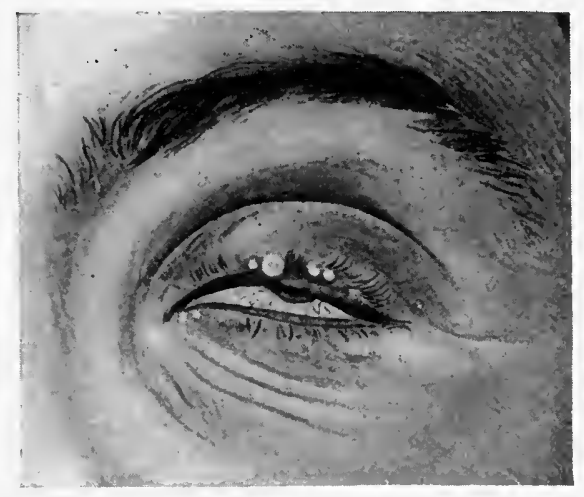

Fig. I 2.-Blepharitis marginalis (Haab).

margin, or blepharitis marginalis (Fig. I2), a condition not infrequently met with in both children and adults, is characterized by redness of the edges of the lids, the formation of crusts upon them, and, in severe and protracted cases, by more or less complete loss of the eyelashes. Because the inflammation is not limited to the surface of the lid, but involves as well the hairfollicles and accompanying sebaceous glands, it is sometimes called blepharo-adenitis. In severe cases ulcera- 
tion occurs about the orifices of the follicles (Fig. I3); it is usually superficial, however, and the loss of tissue is slight. It is commonly a chronic condition, and, unless its etiology is understood and the treatment regulated with reference thereto, it is apt to be an intractable one. In children, it is usually due to malnutrition and a consequent depraved state of the system, and under such circumstances it is often accompanied by eczema of the face or ears or by phlyctenular conjunctivitis. In adults, it arises exceptionally, espe-

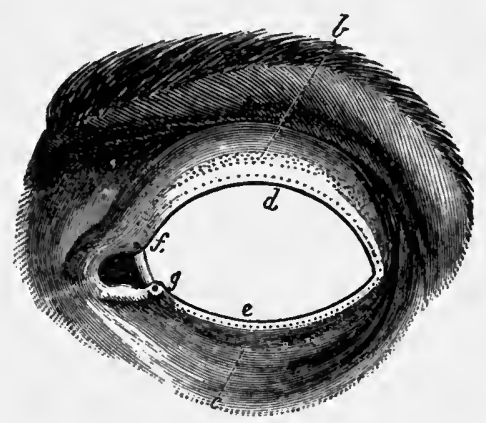

Fig. 13.-The palpebral aperture; the lid margin somewhat everted, so as to show the openings of the ducts of the meibomian glands, $d, e$, the follicles of the cilia (the lashes having been removed), $b, c$, and the lacrimal puncta, $f, g$ (Nunneley).

cially in strumous subjects, from a like cause; but much more frequently it is produced by accommodative strain, that is to say, is dependent upon an error of refraction, such, for example, as hypermetropia or astigmatism. The severe cases, attended by ulceration, destruction of the hair-follicles, and permanent loss of the eyelashes, are seldom met with except in the strumous variety of the disease. Those cases which have their origin in accommodative strain, though prone to chronicity, are not of this severe type. 
Exceptionally, blepharitis marginalis is dependent upon lacrimal disease-when it is apt to be unilateralor upon chronic rhinitis. It occurs also in connection with acne rosacea, but then the inflammation usually is not confined to the lid-margin.

Treatment.-The treatment of blepharitis marginalis, when regard is had to the underlying cause which has given rise to it, generally yields most gratifying results; on the other hand, if this is not taken into account, the outcome is likely to be far from satisfactory. Speaking broadly, it may be said that when a case of chronic blepharitis marginalis is encountered in an adult or in a child old enough to attend school, without other signs of constitutional disorder, the presumption is warranted that an error of refraction, or possibly an anomaly of the ocular muscles, exists, and it may be added that the cure of the lid affection will necessarily involve the adjustment of suitable glasses. On the other hand, when the disease is met with in young children, especially in association with facial eczema or phlyctenular conjunctivitis, remedial measures having reference to the disordered state of the system are of the first importance, and this is true also of those cases which occur in strumous adults.

The most useful local remedy in all varieties of blepharitis is the yellow oxid of mercury. It should be used in the form of an ointment, of the strength of two grains to the dram (hydrarg. ox. flav., gr. viij; ung. aquæ rosæ vel "vaselin cerat.," כ̋ss). A single application in twenty-four hours usually suffices, the best time for this being just before going to bed. Before each application the margins of the lids should be carefully freed of all crusts by persistent bathing with warm water, a bit of soft sponge or rag being 
used to facilitate the detachment of the scabs, and all loose eyelashes should be removed by gentle traction with the thumb and finger. The efficacy of the treatment depends, in no small degree, upon the thoroughness with which this preliminary cleansing is done. In the rare instances in which the "yellow oxid" ointment does not act favorably, one may employ instead, and in the same manner, an ointment of salicylic acid (gr. $\mathrm{j}-\mathrm{ij}$ to $3 \mathrm{j}$ ).

In obstinate cases, and especially in those of severe type, attended by ulceration, much benefit results from touching lightly the margins of the lids (previously freed of crusts) with a pointed crayon of silver nitrate. In doing this care should be exercised to prevent the silver salt from coming in contact with the conjunctival surface of the lid, otherwise considerable irritation of the eye will result.

When the blepharitis is dependent upon a disordered state of the system, and especially when it is accompanied by eczema of the face, phlyctenular conjunctivitis or otorrhea, as a step preliminary to the tonic treatment which is indicated, the bowels should be moved freely by one or more doses of calomel, scammony, and rhubarb, an excellent purgative combination, which will, hereafter, be spoken of as "compound calomel powder," the formula for the same being given under this name in the "Appendix."

The good effects of this "unloading of the primæ viæ," as the older writers used to express it, are, as a rule, promptly manifested, and it will sometimes happen that the case is well on the way to recovery before other treatment (apart from the yellow oxid ointment, which should be prescribed when the purgative is ordered) is begun. The most useful tonic in these 
cases is a combination of the phosphates of iron, quinin, and strychnin.* Exceptionally, in distinctly strumous subjects, exhibiting enlarged lymphatic glands, etc., the syrup of the iodid of iron or one of the codliver oil emulsions may answer a better purpose.

From what has been said regarding the frequent dependence of blepharitis upon errors of refraction, it is obviously the duty of the physician when he meets with an intractable case of this affection, to direct the patient, without unnecessary delay, to a competent specialist, in order that the glasses, which it is probable are urgently demanded, may be prescribed.

Hordeolum (Stye).-Styes are of such common occurrence that every physician is familiar with their appearance. Very considerable diffuse edema of the lid commonly marks the incipient stage of a stye. Presently, at some point near the lid-margin a more defined swelling, attended by redness and tenderness, makes its appearance. Within a day or two suppuration takes place at this point, the overlying tissue softens, and there occurs a slight discharge of thickish pus. The pain, perhaps quite severe, which has been experienced up to this time now quickly subsides, and here the trouble may end. However, so fortunate an outcome as this is rather exceptional, for styes are, so to speak, gregarious, and when one has made its appearance others are apt to follow. The explanation of this is not far to seek, when we consider their etiology. Almost invariably styes, which are simply furuncles occurring in the lids, have their starting-point in some one of the numerous glands with which the eyelids

* The elixir of the phosphates of iron, quinin and strychnin prepared by Wyeth and Bro. and a syrup of about the same strength made by Sharp and Dohme are especially to be commended. 
are so plentifully supplied (Fig. I4). The conditions being such as to favor its development, the Staphylococ-

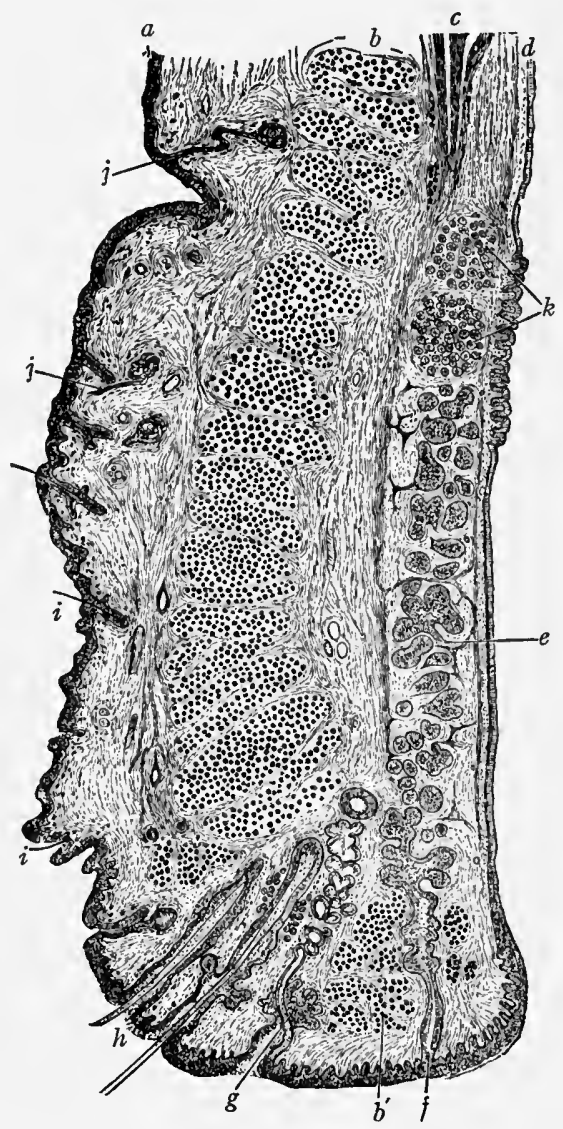

Fig. 14.-Vertical section through the upper eyelid (Waldeyer): $a$, Skin; $b$, cut fibers of the orbicularis; $b^{\prime}$, ciliary bundle of orbicularis; $c$, muscle (involuntary) of Müller; $d$, conjunctiva; $e$, tarsal plate in which are embedded the meibomian glands $(f) ; g$, sebaceous glands near cilia (h); $i$, small hairs of integument; $j$, sweat-glands; $k$, posterior tarsal glands.

cus aureus, or some other pyogenic organism, invades one of the meibomian, or one of the sebaceous, glands or the follicle of an eyelash, and the inflammatory pro- 
cess is started. Suppuration once established, the infection of other glands is almost sure to occur, and so it happens that a sequence of styes is the rule rather than the exception.

The existence of blepharitis marginalis is the most common predisposing cause of styes. Accommodative strain is another predisposing cause; for, even when it does not excite an actual blepharitis, it is apt to induce a hyperemic condition of the lids which favors furunculosis. A "run-down" state of the system also may be a cause of styes, as it may be of furuncles in other regions. Habitual constipation is still another predisposing cause.

Treatment.-Prophylactic: Whether blepharitis be present or not, the history of repeated attacks of styes should suggest the probable existence of accommodative strain. Therefore, in all such cases a careful test of the refraction should be made. If blepharitis exists, the yellow oxid ointment should be prescribed and should be used systematically. This ointment is also useful, since it lessens the danger of secondary infections, in preventing the recurrence of styes. For overcoming habitual constipation aloin, in doses of a tenth to a fifth of a grain, at bedtime, is especially efficacious.

Abortive: If a stye is seen in its incipiency, it is usually possible to prevent its development. One way of doing this is to apply a strong solution of sulphate of zinc (gr. $x x x$ to $z \mathrm{j}$ ) to the external surface of the eyelid, over the sensitive region where it is evident the stye is about to form. To be effectual the applications must be frequently repeated-at intervals of half an hour throughout the day. The solution may be applied with the tip of the finger, care being exercised 
to prevent its flowing into the eye, as this would cause considerable irritation. A few fibers of absorbent cotton placed over the region of the stye will adhere to the surface of the lid after having been once wet, and, by holding a greater quantity of the solution in contact with it, will make the application more efficacious.

Another method of aborting a stye is to introduce a minute quantity of pure carbolic acid into the infected follicle. A careful inspection of the margin of the lid will frequently show which follicle is involved, for the orifice of the infected follicle will be either slightly swollen and congested or there will be a little discharge oozing from it. When this has been determined, a wooden toothpick, made quite slender and sharppointed, should be dipped into carbolic acid and insinuated, as far as practicable, into the follicle. One should, of course, be careful to prevent the acid coming in contact with the eye; but this is not likely to happen if the toothpick is slender, and the precaution is taken to shake off any excess of the acid from its tip. This procedure is usually effectual, if resort to it is not too long delayed.

When it is evident that abortive treatment is not likely to be successful, the suppurative process should be hastened by the application of poultices or hot fomentations, and as soon as pus has formed it should be evacuated by an incision made, with a keen-edged and sharp-pointed knife, parallel to the border of the lid. For this purpose the old-fashioned, triangular cataract knife or a Graefe cataract knife is well adapted. As soon as the pain and discharge have abated the poultices should be discontinued, and the ointment of yellow oxid of mercury (gr. ij to "vaselin cerate" $\mathbf{3} \mathbf{j}$ ) should be used instead. This will not only tend to 
dissipate the remaining inflammation and induration; but, as has already been said, will, perhaps, prevent the development of other styes. When the system needs building up the tonics likely to prove most useful are the tincture of chlorid of iron, the elixir of phosphates of iron, quinin, and strychnin (as recommended in blepharitis), and the well-known combination of quinin, carbonate of iron, and nux vomica, which may be given conveniently in capsules or in pills. When a purgative is indicated, which is not infrequently the case, the "compound calomel powder" will be found to answer an excellent purpose.

Eczema.-Eczema of the eyelids is commonly associated with eczema upon other parts of the face; it is also a not infrequent accompaniment of phlyctenular ophthalmia (Fig. I 5). Epiphora, due to malposition of the lacrimal puncta or to stricture of the nasal duct, often causes an eczema of the lower lid and occasionally of the cheek, the skin being irritated by the constant overflow of tears and mucus. Eczema limited to the inner canthus, and less often to the outer canthus, is not uncommon. When occurring in the former position, it is usually dependent upon inflammation of the lacrimal passages or upon chronic rhinitis. In children eczema, like phlyctenular conjunctivitis, is often due to faulty digestion consequent upon improper food and unsanitary surroundings; in adults it may be dependent upon a gouty diathesis.

Treatment.-The ointments of yellow oxid of mercury and of salicylic acid, as recommended in blepharitis marginalis, are the most useful local remedies. Another useful application is an ointment of oxid of zinc and boracic acid (zinci oxid., gr. ij; acid. boracic., gr. iv; ung. aquæ rosæ, $\bar{j} \mathrm{j}$ ). If the eczema is caused 
by epiphora, this must be remedied. If the overflow of tears is due to malposition (usually eversion) of the puncta, the lower canaliculus must be slit; if dependent upon occlusion of the lacrimal duct, this must be overcome by the systematic use of probes, as described in the succeeding chapter.

The condition of the general health should also be

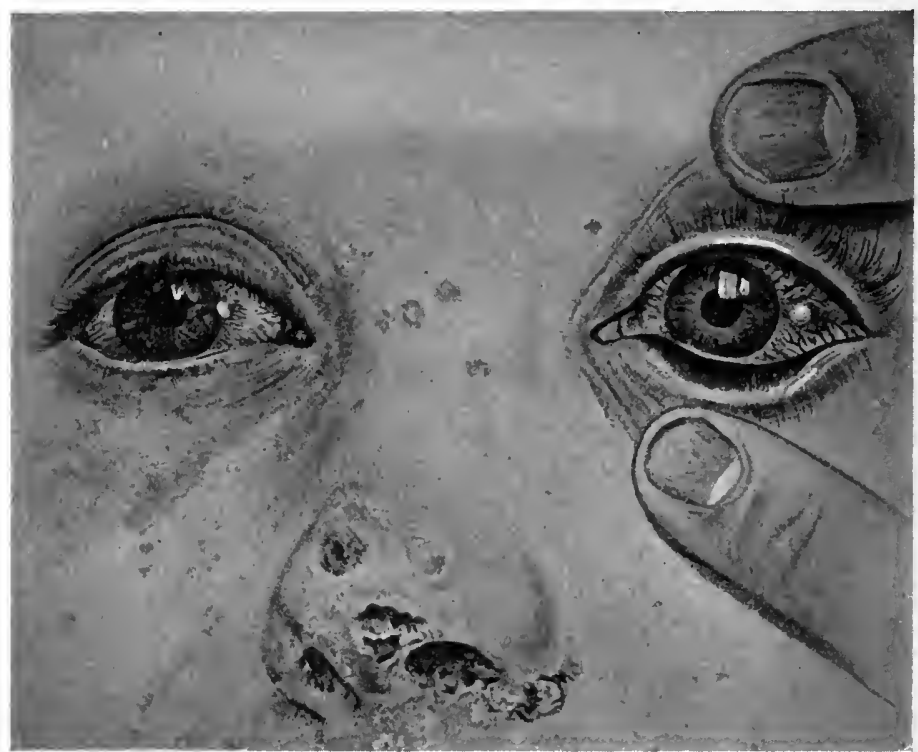

Fig. 15.--Eczema of the lids and face, with associated phlyctenular conjunctivitis (Haab).

looked to-the bowels should be opened, the diet regulated and suitable tonics administered. When gout is present, the natural lithia waters will be found beneficial. Especially when the eczema is limited to. the margin of the lids, and has existed for a considerable time, accommodative strain should be suspected, and the refractive condition of the eyes should be looked into. 

PLATE 1.

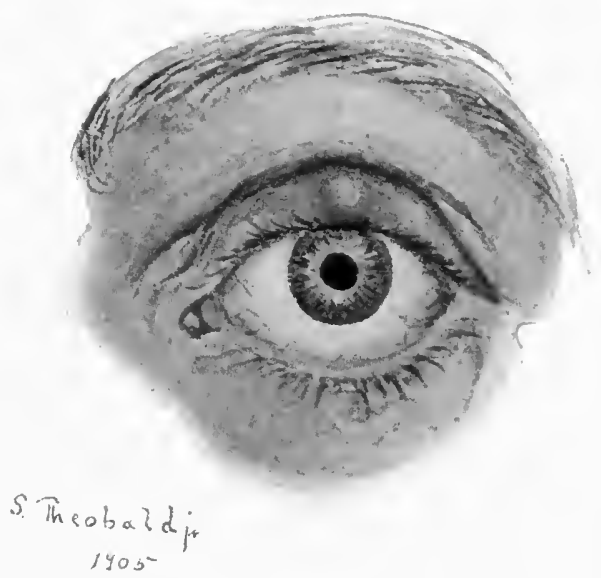

Chalazion of the Upper Lid. 
Chalazion ( $\mathcal{T}$ arsal $C$ yst).-An acute inflammation of a meibomian gland, ending in suppuration, constitutes, as has been said, one variety of stye. A chronic inflammation of one of these glands, or an inflammation which, though acute at the outset, does not go on to suppuration, frequently leads to the development of a chalazion or tarsal cyst. A chalazion, after slowly increasing in size for some weeks, or even for several months, until it becomes, perhaps, larger than a large

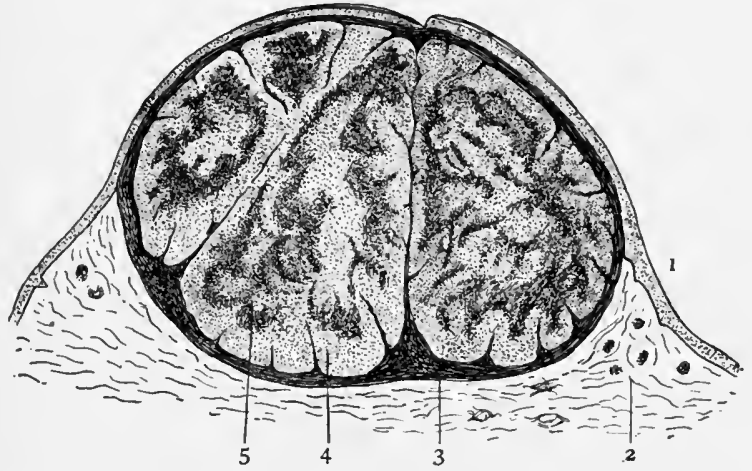

Fig. I6.-Vertical section of chalazion (meibomian cyst); $\times$ то, glycerin: I, Stratified epithelium continued over the surface; 2, connective-tissue outside tumor; 3 , capsule of fibrous tissue from which septa pass inward, dividing the cyst into lobules; 4 , epithelial cells inside capsule; 5. fatty material occupying center of lobules, the outer layers being more opaque (Pollock).

split pea, and forms upon the outer surface of the lid a conspicuous little tumor, often oval in shape, with its long axis vertical (in correspondence with the direction of the follicle) (see Plate I), not infrequently opens upon the conjunctival aspect of the lid-a rather lame effort of nature to bring the process to an end. Before this occurs the contents of the cyst, previously gelatinous, and consisting of the retained and altered secretions of the gland, usually become purulent (Fig. 
r6). After the perforation takes place a slight discharge from the cyst may continue for an indefinite period, and very often granulations sprout up from the edges of the fistula-like opening through which the discharge escapes. In rare instances the anterior wall of the cyst breaks down, and its contents are discharged through the dermal surface of the lid.

Before the stage of perforation is reached, the chalazion, unless it has become large enough to increase appreciably the weight of the lid or to interfere with its movements, gives rise to but little inconvenience, apart from its unsightliness; but, after the perforation occurs, and especially if granulations have formed, it causes more discomfort, partly from the discharge spreading over the cornea and partly from the mechanical irritation produced by the presence of the granulations. Chalazia form rather more frequently in the upper than in the lower lid, are prone to recurrence, though not in the same follicle, are often multiple, several occurring simultaneously in the lids of one or both eyes, and are most common in early adult life. The existence of chronic blepharitis marginalis is a distinctly predisposing cause, and so also, though less directly, is accommodative strain. As may be inferred from what has been said regarding their etiology, they are often encountered in persons who are subject to styes.

Treatment.-It is sometimes possible to dissipate a chalazion, which is small and has only recently formed, by the application of the ointment of the yellow oxid of mercury or the ordinary mercurial ointment; but, as a rule, the only effectual method of treatment is operative The suggestion, often met with in text-books upon the eye, that chalazia should 
be "dissected out" is ridiculous and should not be followed, because, in the first place, owing to the thinness of the cyst walls it is an almost impossible procedure and, in the next place, it involves a very unnecessary traumatism of the eyelid. Except in rare instances, when the cyst is very superficial and shows

Fig. I7.-Chalazion knife and sharp curet (about two-thirds actual size).

a disposition to break through the skin, they should be attacked from the inner surface of the lid. When the lid is everted, a circumscribed purplish area is observed. This marks the location of the cyst, which will be found directly beneath it, and indicates the point at which the incision should be made.

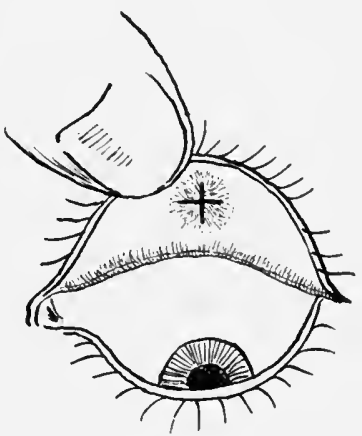

Fig. I8.-Operation for chalazion. Crucial incision into sac through conjunctival aspect of lid.

The operation which I have found effectual, and which is very easy of performance, is as follows: The eye having been anesthetized by several applications of cocain and adrenalin solution ( $\mathrm{I}$ : I OOO), the lid is everted and held securely in this position, either with or without the aid of a lid-clamp, as may be preferred. With a 
knife such as is shown in the illustration (Fig. 17) a crucial incision (the cuts being about $4 \mathrm{~mm}$. in length) is made directly into the cyst (Fig. I8). The contents of the cyst are then removed with a sharp spoon (also shown in Fig. I7), and in doing this its walls are thoroughly scraped (Fig. I9). The small, bulbous tip of a silver probe, which has been previously coated with silver nitrate by being heated in a flame and brought in contact with a crystal or crayon of lunar

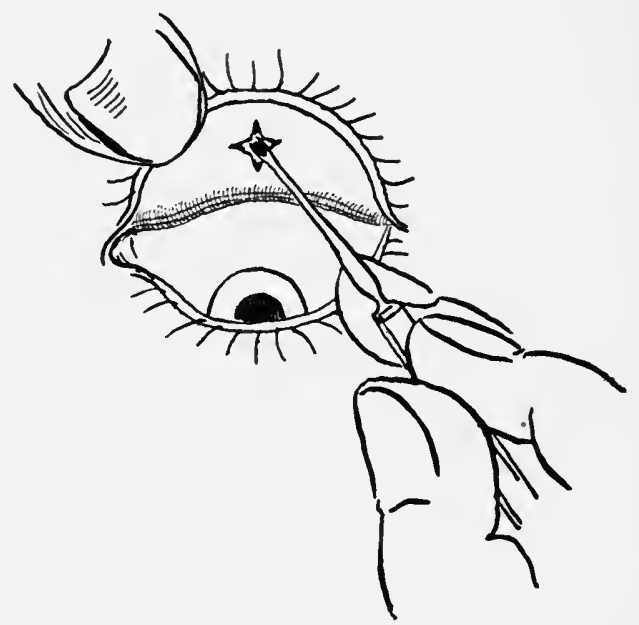

Fig. 19.--Removing contents of sac and curetting its walls with sharp spoon.

caustic, is now introduced into the cyst, and moved about so as to cauterize it thoroughly (Fig. 20). This completes the operation, and the lid, after having been washed clean with sterile water or a solution of boracic acid, is allowed to resume its normal position. No afterdressing is required, but it is a good plan to apply a poultice of flaxseed-meal to the lids the succeeding night. Considerable inflammatory reaction follows the operation; but this subsides quickly, leaving some 
induration which disappears in the course of a few weeks. The curetting and cauterization are necessary to prevent a re-formation of the cyst. The smaller the chalazion, the more difficult, as a rule, is the operation. When the chalazion is in the lower lid, the operation is also rather more difficult. In operating through the external surface of the lid the same procedure is followed.

Milium is the name given to a variety of sebaceous

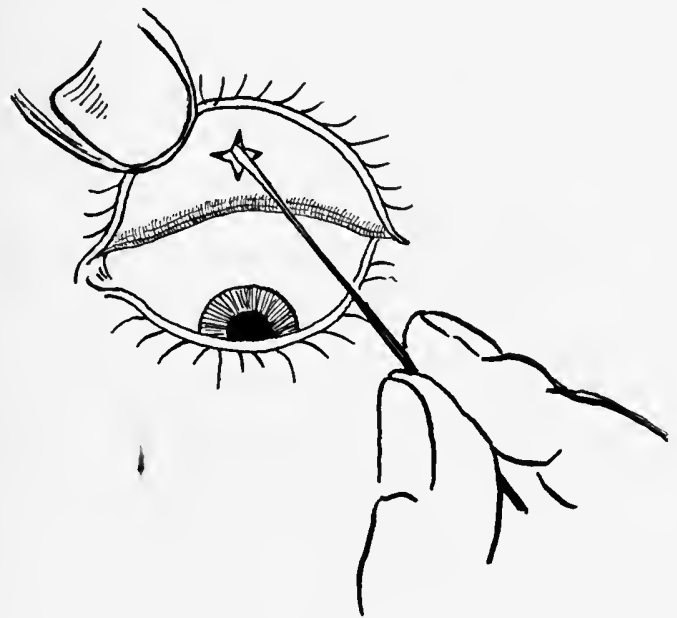

Fig. 20.-Cauterizing walls of sac with silver nitrate fused upon bulbous tip of silver probe.

cyst, about the size of a millet-seed (hence the term), which occurs in the eyelid. It is white in color, roundish, and slightly prominent. It causes no especial inconvenience, but is rather unsightly.

Treatment.-It should be incised, the sebaceous contents removed with a small curet, and the cyst wall peeled out with slender, toothless forceps. It exhibits but little tendency to recur, when dealt with in this way. 
Hydrocystoma.-Small, translucent cysts, containing a clear, watery fluid, are occasionally observed in the lid. They result from occlusion of the duct of a sweat-gland, and may be gotten rid of by simple incision or, more surely, by excising, with slender curved scissors, the outer half of the cyst wall.

Warts, usually of small size, are not uncommon upon the lids. They are found generally upon the

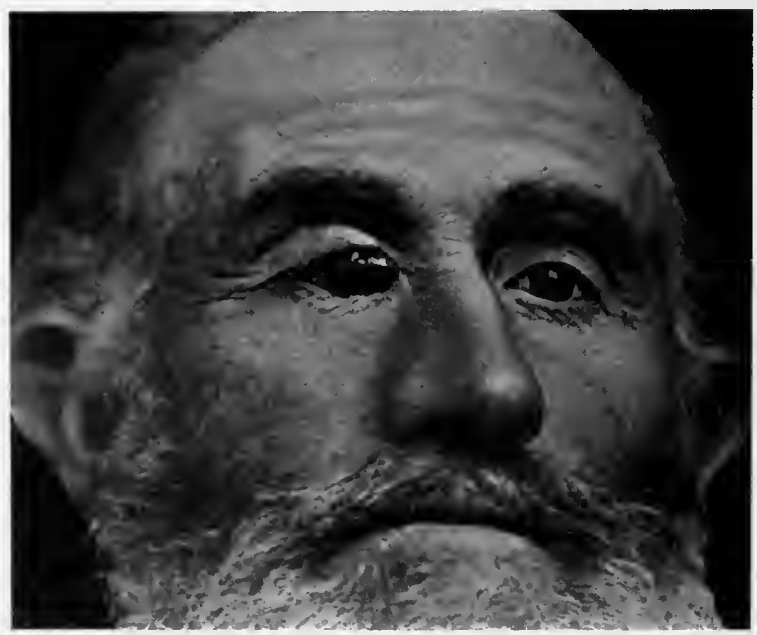

Fig. 21.-Epithelioma of the eyelid (Ramsay).

lid-margin, about the roots of the eyelashes. They are unsightly, but otherwise cause no inconvenience. They should be snipped off with curved scissors, and, to lessen the possibility of a recurrence, the base should be cauterized with a pointed crayon of silver nitrate.

Malignant tumors of the eyelid are rare, if we except epithelioma, which is prone to occur here, as it does in other regions of the body where the skin and mucous membrane join (Fig. 2I). They should be 
dealt with promptly and radically, as they tend to invade the orbit (Figs. 23 and 24), causing not only loss of sight but loss of life from secondary involvement of the brain. They afford an especially favorable

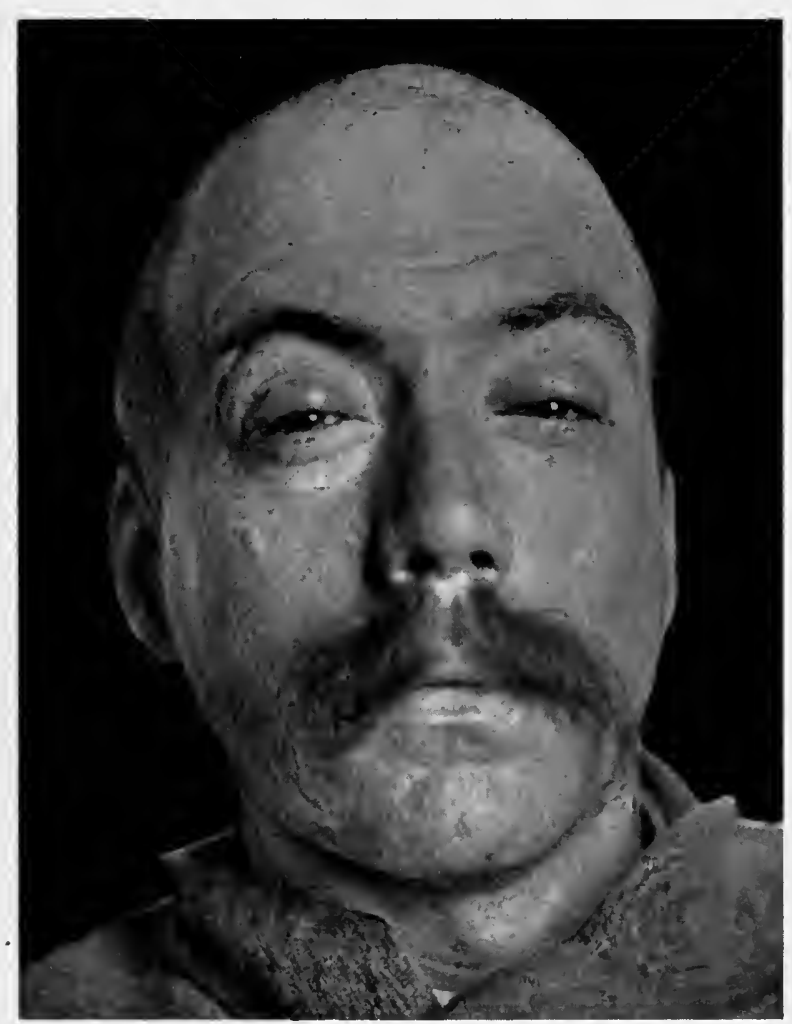

Fig. 22.-Syphilitic tarsitis (de Schweinitz).

field for the Röntgen-ray treatment, provided this is employed before extension to the orbit has occurred. Tarsitis, or inflammation of the tarsal cartilage, characterized by hyperemia and nodular swelling of the lid, is commonly dependent upon acquired syphilis, occurring in the tertiary stage of the disease (Fig. 22). 
It tends to run a chronic course, and is frequently accompanied by palpebral conjunctivitis.

Constitutional as well as local treatment is called for. The biniodid of mercury, in doses of $\frac{1}{24}$ to $\frac{1}{16}$ of a grain, three times a day, and the yellow oxid ointment, applied to the lid morning and night, are the remedies indicated.

Entropion, inversion of the eyelid, a very annoying

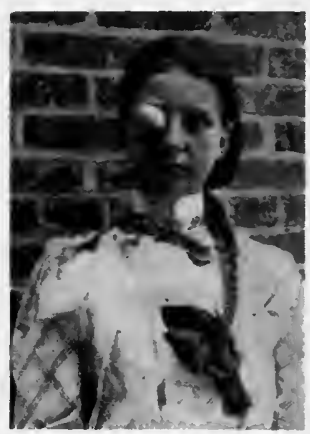

Fig. 23.-Sarcoma of the lid and orbit (Friedenwald).

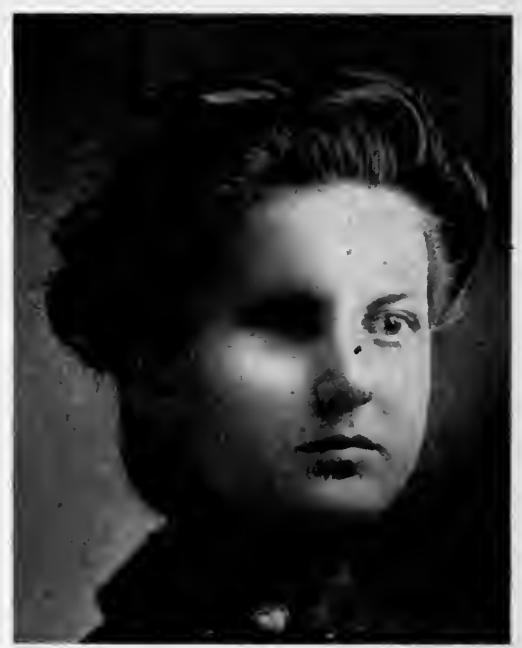

Fig. 24.-Dr. Friedenwald's case of sarcoma of the lid and orbit three months after operation.*

condition because the eyelashes come into contact with the cornea, causing much irritation and not infrequently superficial keratitis, occurs under two forms, one variety being known as spasmodic, the other as organic or cicatricial entropion.

* The description of this case will be found in the "Trans. Am. Ophthalmological Soc." for I 900. There was no local recurrence of the disease; but Dr. Friedenwald informs me the patient died fifteen months after the date of the operation, with symptoms indicative of metastatic involvement of the right lung. 
Spasmodic entropion, as its name implies, results usually from undue contraction of the orbicularis muscle, commonly dependent upon photophobia. It occurs also as a senile condition, arising from relaxation of the lid-structures, and occasionally develops as a result of bandaging the eyes after operations, such as cataract extraction. A predisposing cause-some fault, perhaps, in the form or firmness of the tarsal cartilage or in the arrangement of the orbicularis muscle-it would seem probable, is always present.

Organic entropion is produced by the contraction of scar-tissue in or beneath the palpebral conjunctiva,

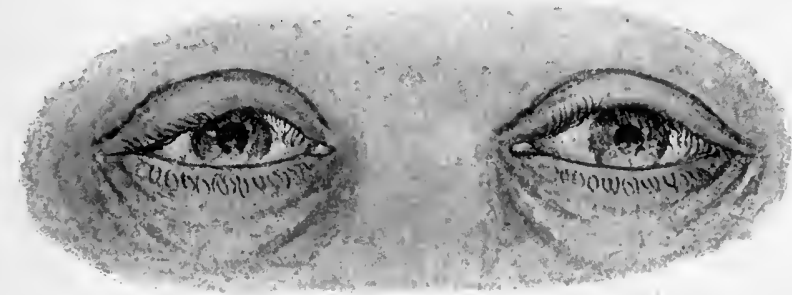

Fig. 25.-Incomplete entropion of the upper lids with consequent trichiasis (de Schweinitz).

and almost invariably is dependent upon chronic trachoma; exceptionally it is of traumatic origin. Both the upper and lower lids are liable to be involved. When, however, the fault is consequent upon trachoma, it is usually the upper lid that is most in-turned, and causes the greatest amount of trouble (Fig. 25). On the other hand, the lower lid is almost always affected in senile entropion, and, indeed, in the several varieties of spasmodic entropion it is commonly the lower lid that is misplaced.

The treatment of entropion, which has for its object the replacement of the lid in its normal position, differs 
materially in the different types of the affection; the procedure to be adopted depends also upon whether the upper or the lower lid is involved. In spasmodic entropion, including the senile type, if the fault has existed only a short time, it is often possible to effect a cure by putting the lid in its normal position, and keeping it there for some days. When only the upper lid is involved, this may be accomplished by the careful application of a pressure bandage. A more effectual method of preventing the in-turning-applicable, especially, to the lower lid-is the painting of several coats of contractile collodion upon the loose skin of the lid. The application must be repeated of ten enough to keep the lid constantly in proper position-usually once in two or three days. The collodion will "hold" very

- much better if, before applying it, the skin is sponged with alcohol or ether.

When these expedients fail to remedy the defect, resort must be had to one of the many operations which have been devised for the cure of entropion.

The procedure best adapted, in my judgment, to the correction of inversion of the lower lid-whether due to trachoma or of spasmodic origin-is one that, in recent years, at all events, has not received the consideration which, I think, it deserves. I refer to the production of a linear eschar near the margin of the lid by the application of caustic potash. My experience with this operation - if it deserves to be called an operation-has been most satisfactory, and has induced me, heretofore, to point out its merits.* The aim is to produce an eschar, 4 or $5 \mathrm{~mm}$. wide, parallel with the

* In a paper published in the "Transactions of the American Ophthalmological Society" for the year 1898 , and in the "American Journal of Ophthalmology," October, 1898. 
lid-margin and extending nearly the whole length of the tarsus, the contraction resulting from which shall hold the lid in its normal position.

In order that the caustic may be applied with the requisite degree of exactness, one end of the crayon employed must be carefully sharpened. This is easily accomplished by rubbing it upon wet blotting-paper. The very considerable pain caused by the action of the caustic may be materially lessened by soaking the lid for ten or fifteen minutes with a ten per cent. solution of cocain, applied by means of a pledget of absorbent cotton. As the destruction of tissue tends to spread considerably beyond the point where the caustic is applied, and as it is desirable that this should not approach nearer the lid-margin than $1 \frac{1}{2}$ or $2 \mathrm{~mm}$., the line of application of the crayon should be about. 4 or $5 \mathrm{~mm}$. from the ciliary border. Along this line, the lid being held upon the stretch and pulled away from the eyeball, the point of the crayon should be drawn, back and forth, a number of times, until the epidermis is destroyed and the tissues beneath assume a brownish appearance (Fig. 26). The lid being still held so that it shall not become inverted, the action of the caustic is allowed to extend as far as may seem desirable. When this point is reached, its further action is arrested by the application of vinegar and water, equal parts, or acetic acid diluted with water to about an equivalent strength. Within a few minutes the eschar begins to contract, and the lid may then be released without fear of its turning in, as already, in most instances, the tendency to entropion has been overcome.

It is seldom necessary to repeat the application of the caustic; but this can be readily done, if the effect of the first application has proved insufficient. Within 
a few weeks all traces of the eschar have disappeared, and usually it is not possible to detect that any operation has been performed.

In entropion of the upper. lid the procedure just described is not effectual; for the cartilage of the upper lid, which is broader and thicker than that of the lower lid (Fig. 27), usually plays a more essential part in the production of the deformity, so that not much can be expected of any operation which accomplishes little

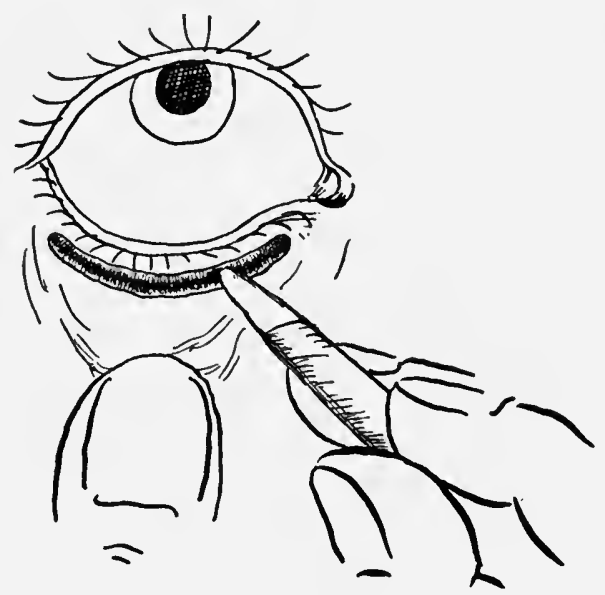

Fig. 26.-The correction of entropion of the lower lid by the production of an eschar with caustic potash.

else than the removal of a portion of the external integument. However, in spasmodic entropion of the upper lid, which, as has been said, is a condition seldom encountered, if the skin of the lid is redundant, it is sometimes possible to correct the fault by the simple excision of a semilunar piece of the integument, which is done in the following manner: A lidspatula is placed beneath the lid, to support it, and two incisions are made through the skin, one parallel 
with and about $2 \mathrm{~mm}$. from the line of the lashes, extending nearly the whole length of the tarsus; the other curvilinear, and reaching from one end to the other of the first incision. The degree of upward curve of the second incision will depend upon the amount of integument it is thought desirable to excise. The skin lying between the two incisions and a portion

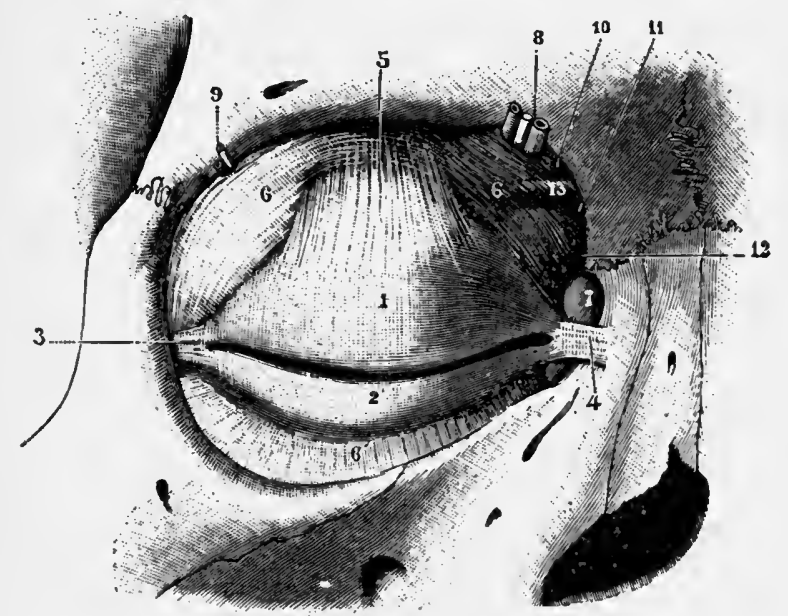

Fig. 27.-Dissection of the tarsal plates and their ligaments (Testut): I, 2, Upper and lower tarsus; 3, 4, external and internal tarsal ligaments; 5 , expanded tendon of levator palpebræ; 6, 6', septum orbitale; 7 , lacrimal sac; 8 , supraorbital vessels and nerve; 9 , lacrimal artery and nerve; Io, II, openings for supratrochlear and infratrochlear nerves; I2, opening for the angular vein; 13, tendon of superior oblique muscle.

of the orbicularis muscle beneath it are next removed, with the knife or scissors as may be preferred, and the operation is completed by bringing together the edges of the wound with three or four fine silk sutures. After three days union will have taken place, and the stitches may be removed.

In the so-called organic entropion of the upper lidthat form which is commonly induced by trachoma- 
a more radical procedure than the foregoing is demanded.

The operation which I have most frequently employed in this condition, and usually with satisfactory results, is that which was suggested some years ago by Dr. John Green, of St. Louis. It is open to the objection, however, that it necessarily involves a very considerable traumatism of the tarsal cartilage and of the palpebral conjunctiva, and it has been largely supplanted by the procedure originally proposed by Anagnostakis, but which commonly goes by the name of Dr. Hotz, of Chicago, who, without knowledge of what had been done in this direction by Anagnostakis, re-contrived and perfected the operation, and brought its merits to the notice of the ophthalmic surgeons of the present day.

Dr. Hotz's description of the operation, made very easy of comprehension by the accompanying excellent illustrations (Fig. 28), for which I am indebted to him, is as follows:

"While an assistant fixes the skin at the supraorbital margin the operator, seizing the center of the lid-border with fingers or forceps, draws the lid downward to put its skin well on a stretch, and makes a transverse incision through the skin and orbicularis muscle from a point 2 or $3 \mathrm{~mm}$. above the punctum lachrymale to a point 2 or $3 \mathrm{~mm}$. above the external canthus. This incision (Fig. 28, A) divides the lid-skin in a line parallel to and a little below the upper border of the tarsal cartilage, and is therefore from 4 to $8 \mathrm{~mm}$. distant from the free border in the center of the lid. The skin and muscular layer are now dissected from the incision down to the roots of the eyelashes, and, while an assistant is holding the edges of the wound well separated, the operator seizes with forceps and excises with curved scissors the muscular fibers running transversely across the upper border of the tarsus. Next the sutures are inserted. Three sutures are usually sufficient-one in the center of the wound and one at each side of the central suture. The curved needle, armed with black silk No. 3, is first passed through the wound-border of the lid-skin ( $A, a)$; then it is thrust through the upper horder of the tarsus and returned through the tarso-orbital fascia just above this 
border; and finally it is carried through the upper wound-border (b). When the sutures are tied the skin is drawn upward and fixed to the upper tarsal border (Fig. 28, B), and this slight traction is sufficient to turn the inverted lid-border and eyelashes to their normal position; and, as the skin becomes firmly united with the tarsal border, the tension thus produced upon the lid-border is permanently secured."

Under aseptic dressings the wound commonly heals without suppuration, and the stitches may be removed on the third or fourth day.

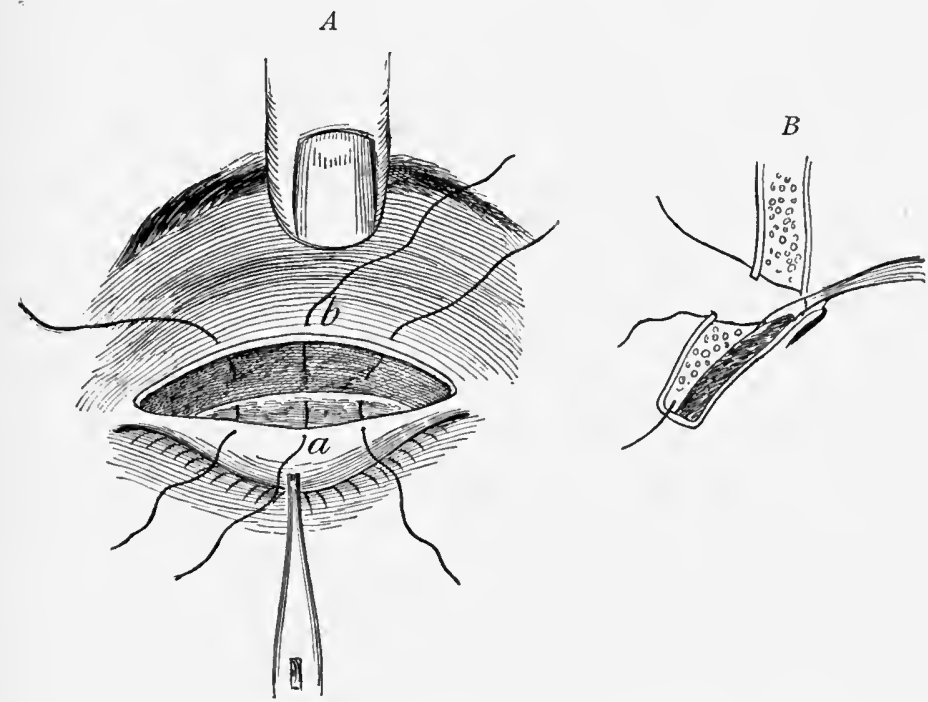

Fig. 28.- $A$ and $B$, Anagnostakis-Hotz operation for entropion.

In the worst forms of entropion Dr. Hotz combines with the procedure just described a "reconstruction of the lid-margin." A deep incision is made in the free border of the lid, extending nearly its whole length and just behind the line of the lashes, great care being exercised that no lashes are left in the posterior lip of the divided lid-margin. The tension of the external integument of the lid produced by its attachment to the upper margin of the tarsal cartilage causes this 
incision to gape considerably, and this gap is filled by a Thiersch graft-a narrow, wedge-shaped strip of skin, of suitable length, which may be obtained conveniently from the posterior surface of the auricle or from the integument which covers the mastoid process. Sutures are not necessary to retain the graft in position; but it is desirable to bandage both eyes-for twentyfour or forty-eight hours- until it has become adherent.

Ectropion.-Like entropion, eversion of the eyelid, or ectropion, may be produced by spasm of the orbicularis muscle, by contraction of scar tissue, or by senile relaxation and loss of tone of the lid-structures. It may occur also in consequence of paralysis of the orbicularis muscle. Both lids, the upper and the lower, are liable to be affected, the less pronounced forms being found, as a rule, when the displacement is in the lower lid; for the degree of eversion, it should be remarked, varies greatly-from a slight drooping of the lower lid, just sufficient to cause eversion of the punctum and consequent epiphora, to complete turning out of the conjunctival surface of the lid, giving rise to a revolting deformity. The slighter forms, affecting the lower lid, are those which result from senile changes, from paralysis of the orbicularis, and from eczema of the lid and cheek. The more pronounced types are due to spasm of the orbicularis or to cicatricial contraction.

Spasmodic ectropion develops usually during the course of an acute conjunctivitis or kerato-conjunctivitis, attended by congestion and edema of the palpebral conjunctiva. As a rule, these conditions are accompanied by photophobia and blepharospasm. When an attempt is made to examine such eyes, or to make applications to them, it is not uncommon for the lids, 
the upper lid especially, to become everted. If this happens under the observation of the surgeon, the eversion, of course, is corrected at once and without difficulty. If, however, it occurs under other circumstances, and if, so occurring, the displacement of the lid is allowed to remain for several days, the correction of the fault is no longer an easy matter. In fact, a condition comparable to paraphimosis has been brought about, and the everted conjunctiva is now congested and greatly swollen, as a consequence of the strangulation produced by the action of the distorted orbicularis -a typical spasmodic ectropion has, indeed, already become established. Now, if the lid is returned to its normal position, it refuses to stay there. The faulty position has become, as it were, the "natural" position, and so it will ever remain, unless proper measures are taken to remedy the defect. Briefly told, this is the usual history of the development of spasmodic ectropion.

Cicatricial or organic ectropion is commonly of traumatic origin, though it may result from any lesion which leads to destruction of the external integument of the lid or of the neighboring parts. Burns, whether from hot substances or caustic agents, lacerated wounds, malignant growths, lupus, and caries of the bones forming the border of the orbit, are some of the conditions apt to produce it. The distortion and displacement of the lid occurring in this form of ectropion are often excessive (Fig. 29); nevertheless, the repulsive appearance characteristic of spasmodic ectropion is seldom present.

The incomplete eversion of the lower lid caused by eczema of the lid and cheek is a not unusual complication of disease of the lacrimal apparatus - of any con- 
dition, in fact, whether displacement or occlusion of the punctum, or stricture of the canaliculus or lacrimal duct, which may give rise to epiphora, the overflow of tears and mucus being the exciting and continuing

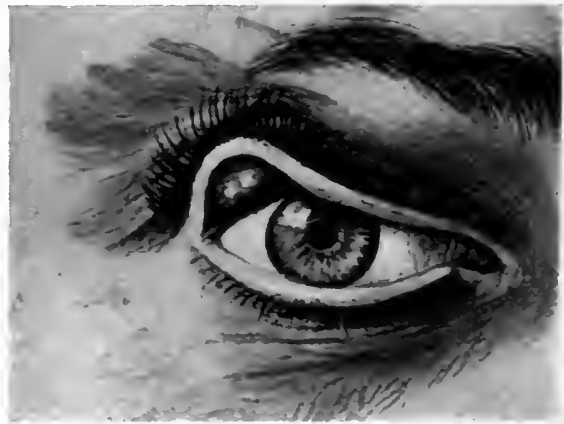

Fig. 29.-Cicatricial ectropion following burn by molten lead (Haab).

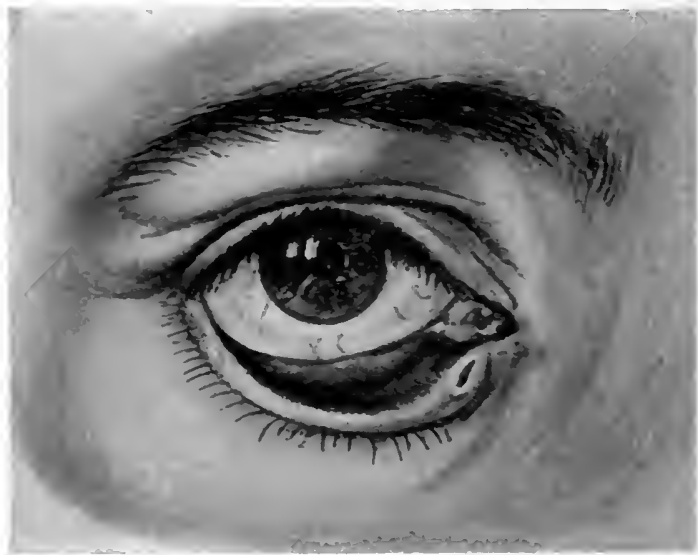

Fig. 30.-Ectropion of the lower lid due to facial paralysis (Haab).

cause of the eczema, which, through the resulting skin contraction, drags the lid from its normal position. In this way, too, senile ectropion and the ectropion of facial paralysis (Fig. 30) are of ten greatly aggravated; for the malposition of the lower punctum present in 
both of these conditions usually gives rise to epiphora, and, sooner or later, a dermatitis of the lid and cheek develops.

A more marked type of ectropion of the lower lid, attended by considerable elongation of the lid-margin, is met with in certain cases of chronic inflammation of the lids and conjunctiva (Fig. 3I). Its occurrence is

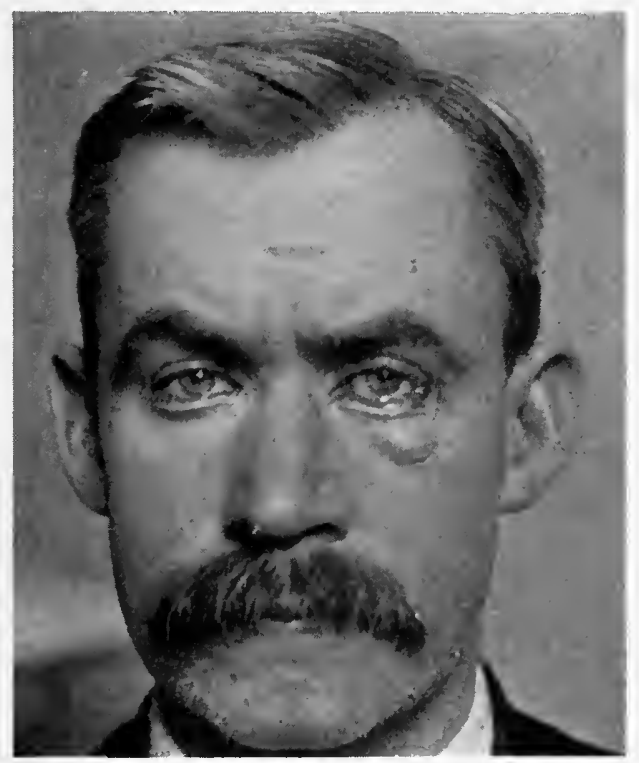

Fig. 31.-Ectropion of the lower lids (de Schweinitz and Randall).

due measurably to the hypertrophied condition of the palpebral conjunctiva; but it seems probable that other factors, such as an ill-shaped tarsus or a badly disposed orbicularis muscle, have to do with its development (Fig. 32).

Treatment.-The slighter degrees of ectropion of the lower lid, such as occur in facial paralysis or from senile atrophy or eczema of the cheek, are chiefly 
annoying because of the epiphora to which they give rise. They require, therefore, no treatment beyond the slitting of the lower canaliculus. This simple procedure not only does away with the epiphora, but, by preventing the excoriation of the skin by the overflowing tears, not infrequently materially lessens the malposition of the lid.

In spasmodic ectropion, of not too long duration, it is often possible to effect a cure by replacing the everted

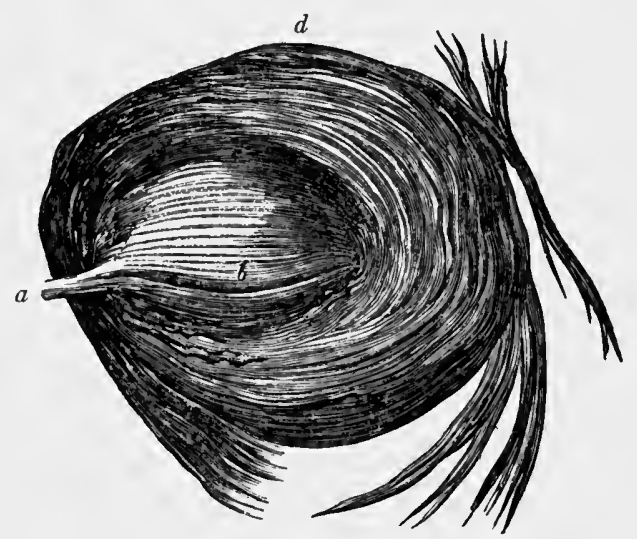

Fig. 32.-The orbicularis muscle and the internal palpebral ligament (Nunneley). The palpebral ligament is seen at $a ; b$ and $d$ indicate the palpebral and orbital portions of the orbicularis muscle.

lid and applying a compress bandage. After this has been worn for two or three days the tendency of the lid to become everted will have been overcome. When this plan fails, the procedure suggested by Snellen should be ressorted to, and will usually be found efficacious, unless the condition has lasted so long that the lid-margin has become considerably elongated. The steps of Snellen's operation, which is applicable to either lid, are as follows: A curved needle is attached to each end of a silk thread, and both needles are 
passed, from within outward, through the whole thickness of the lid. The points of entrance should be 5 or $6 \mathrm{~mm}$. apart, and at such a distance from the border of the lid that the needles shall pass through the convex margin of the tarsus. The points of exit should be about a centimeter apart and two centimeters from the lid-margin. Sufficient traction is now made upon the thread to cause the lid to assume its proper position, and the two ends are then tied rather tightly over a piece of small rubber tubing or a roll of adhesive plaster, which prevents the thread cutting into the skin. To effect the desired result two, or possibly three, threads may sometimes be required. A light bandage should be worn for several days, and on the fourth day the stitches should be removed.

To facilitate the replacement of the lid, excision of a portion of the congested and swollen palpebral conjunctiva is recommended; but the end in view, it would seem, might be attained as well through the markedly astringent action of adrenalin.

When ectropion is attended by considerable elongation of the lid-border, a shortening of the lid becomes an essential part of any operation undertaken for the correction of the deformity. This was accomplished at one time by excising a wedge-shaped piece from the center of the lid, but a better procedure, and the one now usually employed, is to remove the piece at the outer canthus, as suggested by von Ammon. The amount of shortening required will, of course, determine the size of the wedge to be removed. The edges of the incision (Fig. 33, A) should be brought together accurately by three or four silk sutures, or by a harelip pin, as shown in Fig. 33, B.

The form of ectropion most difficult to correct is 
that which is produced by the contraction of scartissue, and innumerable ingenious operations have been contrived for this purpose. In cicatricial ectropion the lid, besides being everted, usually is elongated and dragged away from its proper position. It may be also so immovably fixed in this new position that closure of the eye is quite impossible.

The first step in every operation for the correction of this variety of ectropion is the freeing of the lid from its attachments, so that it may be brought into normal relation with the eye and with the fellow-lid. The completion of this step usually leaves, in the

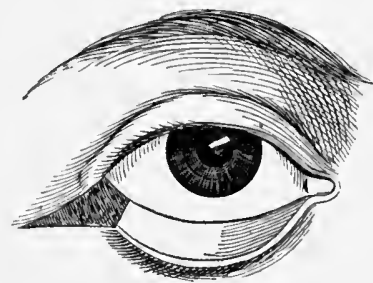

A

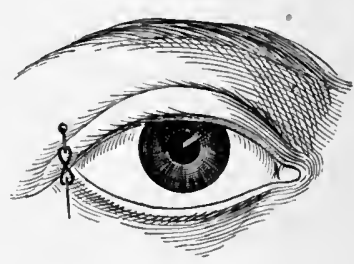

B

Fig. 33.-Von Ammon's operation for shortening the lid.

neighborhood of the orbital margin, a more or less extensive area denuded of integument, and the filling of this gap with skin, which is necessary to prevent a re-displacement of the lid, constitutes the second and final step of the operation. Formerly this was accomplished by some form of plastic operation, usually by the transplantation of flaps of skin from the neighboring parts-the forehead, the temple, or the cheek. There were, however, many difficulties and disadvantages connected with such operative procedures, and of late they have been in large measure abandoned, and have been supplanted by the modern methods of skin-grafting devised by Wolfe and Thiersch. 
When the ectropion involves the lower lid its replacement, which usually includes a shortening of the lid-border, is commonly effected by the operation proposed by Arlt. As represented in Fig. 34, A, two converging incisions, $a b, b d$, are made through the whole thickness of the skin, or through the scar-tissue which has taken its place. The included integument is then dissected up until the lid is free, and can be restored to its normal position. Next, the lid-border is shortened at the outer canthus, in the manner already

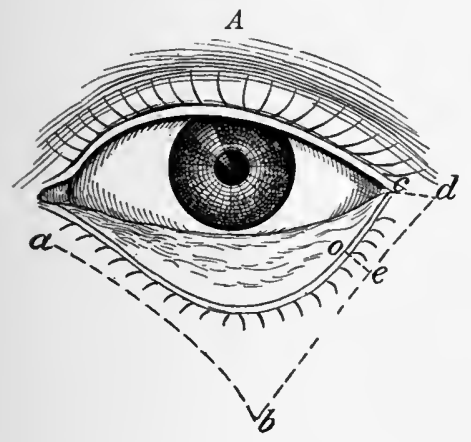

$B$

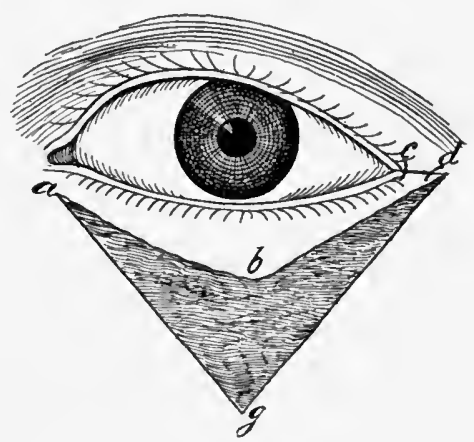

Fig. 34. $-A$, Arlt's operation for cicatricial ectropion of lower lid; $B$, final stage.

described. An effort is then made, by undermining the margins of the incision, a $g d$ (Fig. 34, B), to close the $\mathrm{V}$-shaped gap, the edges being brought together by harelip pins. If this cannot be done without undue traction, or if after it is accomplished a gap is left above which cannot be closed except by such tension upon the flap, $a b d$, as might result ultimately in a recurrence of the ectropion, resort should be had to skin-grafts with which to fill in any existing gaps.

Hotz, who has given special attention to operations upon the lids, recommends that for this purpose grafts 
obtained by the Wolfe method should be employed. The grafts, which include the whole thickness of the skin, but which should be freed carefully from any underlying fat, may be obtained most advantageously from the inside of the arm or forearm. They should be shaped to fit accurately the space into which they are to be inserted, and as outlined upon the skin of the arm should be about one-third larger than this space, to allow for the usual shrinkage. No sutures are required to maintain them in position; but, after they have been adjusted carefully, they should be covered with several layers of silver-foil. Over this a pad of sterile gauze and absorbent cotton, of sufficient thickness to afford equable support, is placed, and is kept in position by a light bandage or by several strips of rubber adhesive plaster. Owing to the overflow of the secretions from the eye, it is usually necessary to change the dressings on the third or fourth day; otherwise, it would be advantageous to allow them to remain for ten or twelve days.

The use of silver-foil as a surgical dressing, first suggested by Dr. Wm. S. Halsted, has been employed extensively for some years in the Johns Hopkins Hospital. All fresh wounds, both closed and open, are invariably dressed with it, and as a covering for the moist bloodclot and for skin grafts it has been found especially valuable. Dr. Halsted advises that it should be "laid on without stint, at least three or four layers thick, and should be protected from the outer (gauze) dressing by two or three layers of the foil-paper."

To maintain the replaced lid in its proper position and to guard against a recurrence of the ectropion, it is usual, before inserting the grafts, to unite the margins of the upper and lower lids by three sutures, 
which, if deemed necessary, may be left in position for several weeks.

In dealing with ectropion of the upper lid, owing measurably to the presence of the eyebrow, the V-shaped incision of Arlt does not yield satisfactory results. The method of procedure to be adopted for the correction of this defect will vary necessarily with the conditions to be met; but, in general, it will consist of a freeing of the retracted and everted upper lid, so that it can be brought into proper relation with the eye and with the fellow-lid (to which it should be attached by sutures, as in the previously described operation), and the filling of the resulting gap by Wolfe or Thiersch grafts. The Thiersch grafts afford a thinner and more flexible tissue, and for this reason are preferable if they are to form a part of the lid proper (Hotz); but, owing to the marked shrinkage which they undergo, a recurrence of the ectropion is liable to occur if they are depended upon to fill in gaps of considerable size. A combination of the two methods, therefore, may be employed advantageously when this condition has to be met-Wolfe grafts being used to fill large gaps upon the temple or above the brow; Thiersch grafts, to build up the lid itself.

The Thiersch grafts, which consist only of the epidermis and the very superficial layers of the dermis, carefully shaved off with a razor or other keen-edged blade, may be employed to excellent purpose to prevent the development of ectropion, since they can be "planted" upon granulating surfaces as well as upon fresh wounds. For this reason, too, it is possible to apply supplementary grafts, should any of those applied at a previous operation fail to "take."

Sepsis should be carefully guarded against in skin- 
grafting. The surface from which the graft is to be taken, as well as that to which it is to be transferred, should be rendered as nearly as possible aseptic, and all dressings employed, including, of course, the silverleaf, should be sterile. For this purpose sublimate solutions are used more freely in the surgical service of the Johns Hopkins Hospital than was at one time thought advisable.

Ptosis, a drooping of the upper lid with inability to elevate it, occurs as a congenital and as an acquired condition. The fault is usually in the levator muscle, though exceptionally the defect may be due to increased weight of the lid from inflammatory hypertrophy or other cause. Acquired ptosis is commonly a result of paralysis of the third nerve, a twig from which supplies the levator muscle, and is very often dependent upon syphilis. When this is the case, the other ocular muscles supplied by the third nerve are usually involved, and, besides drooping of the lid, we have divergent squint, inability to turn the eye inward, upward, or downward, mydriasis, and loss of accommodative power. Exceptionally only that branch of the third nerve which supplies the levator muscle is affected. Such a condition may be caused by central disease, or it may occur as a result of traumatism or from pathological processes in the orbit.

Congenital ptosis, which is commonly bilateralacquired ptosis being more often monolateral-is not infrequently an inherited condition. It is caused by faulty innervation, or by imperfect development or actual absence, of the elevator muscle of the lid. The subjects of this defect acquire the habit of employing the occipitofrontalis muscle to lift the lids, so that the eyebrows are unduly elevated. They are inclined 
also to throw the head backward, since this makes it easier for them to see straight forward, as the upper lid, under such circumstances, need not be elevated as much as would otherwise be necessary. They present, therefore, an odd and characteristic appearance, which is well shown in the accompanying illustration (Fig. 35). The appearance exhibited in acquired monolateral ptosis, if it is complete-for varying degrees of both congenital and acquired ptosis are met

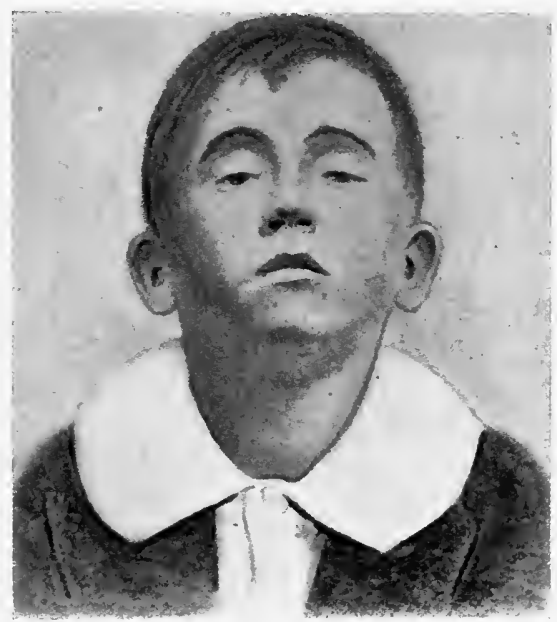

Fig. 35,-Congenital ptosis (T. C. Evans).

with-is exactly that of a person who, keeping one eye open, succeeds in closing the other without apparent muscular effort (Fig. 36).

Treatment.-In congenital ptosis only operative treatment is of avail; on the other hand, in acquired ptosis operative measures should be resorted to only when all other means have been tried, and have proved ineffectual. As a rule, if the condition is not of long standing, the prognosis in acquired ptosis is favorable. 
This is true, especially, of the many cases which are of luetic origin. In these cases, as well as in those dependent upon rheumatism, potassium iodid, in liberal doses, is perhaps the most valuable remedy we possess. The biniodid of mercury is another valuable remedy, and with either of these strychnin may be advantageously combined. Counter-irritation, by

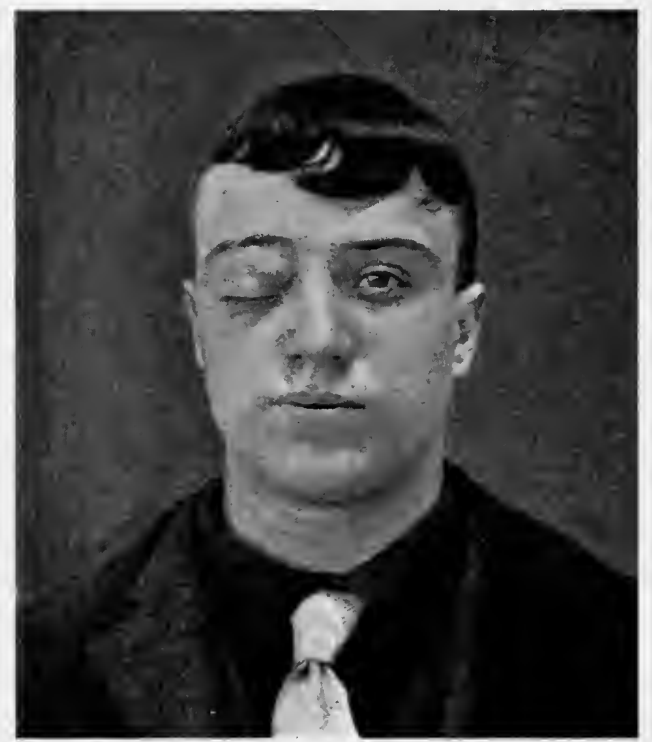

Fig. 36.-Acquired ptosis with cystic tumor of orbit (de Schweinitz and Randall).

means of blisters applied to the forehead or temples, at times seems to exert a good effect. I have not much confidence in the efficacy of galvanism in these cases; but some authorities, probably with larger experience in its use, hold it in higher esteem. When constitutional treatment has been given a thorough trial without effect, and only then, resort should be had to one of the operative measures presently to be described. 
For the correction, more especially, of congenital ptosis, innumerable operative procedures, and numberless modifications of previously suggested operations, have been proposed. When so many plans are suggested to meet a given condition the inference is warranted that no one of them is entirely satisfactory, and this is certainly true of the many operations devised for the correction of ptosis.

All of these procedures, with one or two unimportant exceptions, aim either to shorten the eyelid, so that it shall not interfere so greatly with vision, or to render more effective the vicarious action of the occipitofrontalis muscle in its endeavor to take the place of the incapacitated levator palpebræ.

The objection to those operations which aim at shortening the lid is that in ptosis, as a rule, the lid is not elongated. Indeed, in congenital ptosis, perhaps in consequence of the long-continued action of the occipitofrontalis, it not infrequently appears to be preternaturally short. If under such circumstances it is shortened still more, sufficiently to raise it above the level of the pupil, there is great danger of producing the condition known as lagophthalmos-inability to close the eye or, at all events, to keep it closed without actual effort-a condition which, especially through lack of protection of the cornea during sleep, may lead to serious consequences. If, however, there is actual elongation of the lid, and sometimes when it is only of the usual length, as is more apt to be the case in acquired ptosis, much relief may be afforded by an operation which will shorten it to the degree required.

The shortcoming in those procedures which undertake to render the action of the occipitofrontalis more effective is that, notwithstanding our best efforts in this 
direction, the part of levator of the lid is played by this muscle in rather lame fashion.

Of the various operations proposed for shortening the lid, the most rational is the procedure first suggested by Bowman, in 1859 -the removal of a portion of the tarsal cartilage.* The credit of originating this operation is commonly given to Gillet de Grandmont; but the procedure which he described in $1891,+$ though differing in minor details, is essentially that which Bowman employed thirty-two years before. Quite recently this operation has been somewhat modified and improved upon by Gruening, who speaks enthusiastically of the excellent results which it has afforded him. His modification consists chiefly in the method of introducing the sutures which are employed to bring together the edges of the divided cartilage.

Like de Grandmont, Gruening makes an incision, corresponding in length with the tarsus, through the external integument of the lid, parallel with and about $3 \mathrm{~mm}$. from its free border, and, after dissecting back the skin and orbicularis muscle until the whole tarsal cartilage is exposed, removes a semilunar piece of the cartilage and the subjacent conjunctiva by two incisions, one straight and parallel with the lid-margin, the other curvilinear, with its convexity upward, and joining the extremities of the first incision. The breadth of the piece of cartilage to be removed is determined by the effect desired. If a shortening of the lid amounting to 6 $\mathrm{mm}$. is desired, the excised piece should have a breadth in the center of $6 \mathrm{~mm}$. and at each extremity of $2 \mathrm{~mm}$. The upper border of the cartilage Gruening, following

* " Royal London Ophthalmic Hospital Reports," Vol. I, p. 34.

† “Journ. de Méd. de Paris,” ı89ı, p. 296. 
the example of Heisrath,* leaves intact, so that the cartilage is actually cut in two, a crescentic piece being left above, a long strip, with nearly parallel sides, below (Fig. 37).

In de Grandmont's operation the deep parts of the wound are united by three catgut sutures, which are left to undergo absorption, the skin being closed over them. Gruening, on the other hand, brings the lips of the cartilage wound into apposition by means of three double-armed silk threads. The needles at-

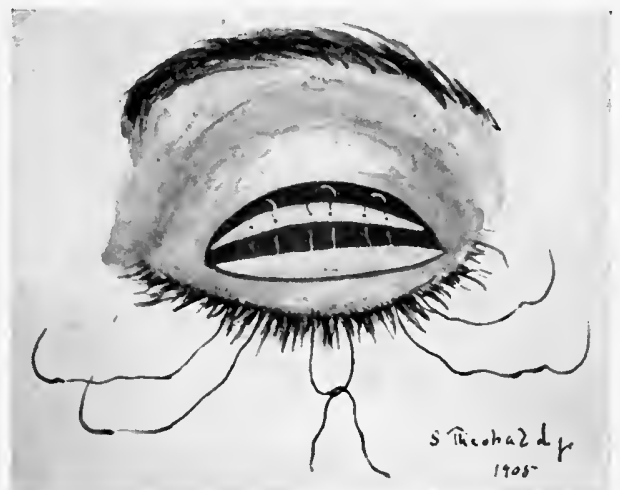

Fig. 37.-Gruening's modification of the Bowman-deGrandmont operation for ptosis.

tached to each of these are passed first through the lower edge of the upper, crescentic portion of the divided cartilage, then downward through the lid structures, as shown in the illustration, and, finally, are brought out upon the free border of the lid, behind the lashes, where each pair of threads is tied, after having been drawn tightly enough to close the cartilage wound. The skin wound may be closed by several superficial sutures, though this is hardly necessary.

* "Berliner klin. Wochenschrift," I89I, p.58. 
The deep sutures are removed on the fifth day. With proper antiseptic precaution these sutures are not likely to excite undue inflammatory reaction, and, on the whole, are to be preferred to the buried stitches of de Grandmont.

Of the operations designed to render more effective the action of the occipitofrontalis in lifting the lid, that contrived by Panas affords the best results. By reference to the illustrations (Figs. $3^{8}$ and 39) the steps of this operation are made easily comprehensible. A horizontal incision, $2 \mathrm{~cm}$. long, is made through the
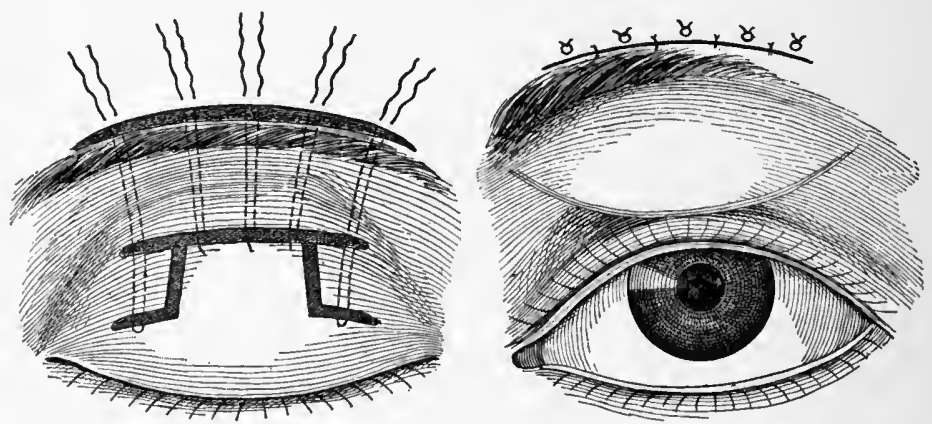

Fig. 38.-Panas's operation for ptosis. Fig. 39.-Panas's operation concluded.

skin and orbicularis muscle a short distance below the upper margin of the orbit. From near each extremity of this incision two vertical incisions are carried downward to a point 2 or $3 \mathrm{~mm}$. below the upper margin of the tarsal cartilage, where each incision is continued horizontally, as shown in the illustration. The flap of skin and muscle thus formed is then dissected up from the underlying fascia and cartilage. Another incision, $3 \mathrm{~cm}$. long, is next made through the skin and muscle just above, and following the curve of, the eyebrow, and the bridge of skin between this incision and 
the lower incision is undermined. By means of three sutures introduced near its upper margin, and passed beneath this bridge of skin, the flap is now drawn up under the bridge, and attached to the upper edge of the incision above the brow. To prevent the traction which results from causing eversion of the lid-margin, two lateral sutures, as shown in the illustration, are passed through the tarso-orbital fascia and conjunctiva, without including the skin, and are carried up subcutaneously and attached, like the previously mentioned sutures, to the lip of the upper incision.

The effect of the operation, in lifting the lid, will depend largely upon the width of the undermined bridge of skin, or, in other words, upon the distance between the two horizontal incisions. If this is too great, the effect will be excessive; if not great enough, it will be insufficient. It is a matter of importance, therefore, taking into account the degree of ptosis and the redundancy or scantiness of the integument of the lid, to determine how considerable this distance shall be. The operation being completed, the wounds should be covered with silver-foil, and over this a pad of sterilized gauze should be applied; and on the fourth or fifth day the stitches should be removed.

Paralysis of the facial nerve, a condition nearly always unilateral, affects the eye through loss of power of the orbicularis muscle (Fig. 40) The lids do not close properly, and the lower lid tends to sag, so that the lacrimal punctum fails to maintain its normal position with reference to the eyeball. In consequence of this, epiphora is commonly present. Partly owing to the epiphora, and still more because the lids do not afford the usual protection to the eye, conjunctivitis, and less frequently keratitis, may develop. When the paralysis 
is complete, the eye cannot be closed, and when an effort is made to close it, the eyeball is turned strongly upward in a manner which is quite characteristic.

The lesion causing the palsy is usually peripheral, and may involve the nerve during its course through the temporal bone or after its emergence from the stylomastoid foramen. Disease of the middle ear is probably the commonest cause of facial paralysis. It

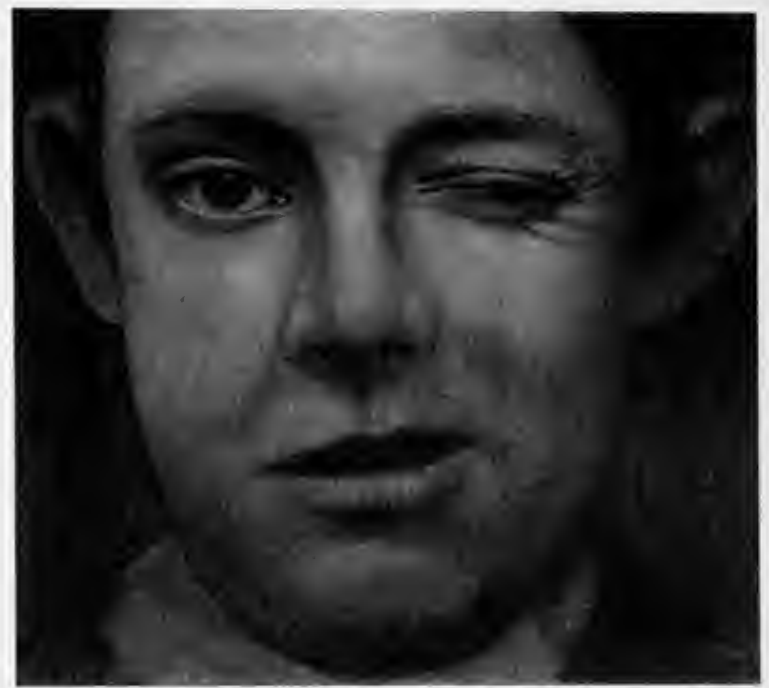

Fig. 40.- Right-sided facial paralysis, showing inability to close the lids from involvement of the orbicularis palpebrarum (Ramsay).

may also follow surgical operations or other traumatisms involving the tympanum. It is sometimes dependent upon syphilis, and it may be brought on by exposure to draughts or to cold. It is also met with in connection with hemiplegia, and, according to the location of the intracranial lesion, may be on the same side as the hemiplegia or on the opposite side.

Treatment.-This will depend, of course, upon the 
cause of the attack. In recent cases the prognosis usually is favorable. As a matter of routine, it is well always to examine the ear, unless it is manifest that the lesion is located elsewhere. Potassium iodid in liberal doses is indicated, and its usefulness is by no means limited to the cases of syphilitic origin. Strychnin also is useful, and some authorities place reliance in electricity. Small blisters, applied in front of the auricle, seem at times to be of benefit. When disease of the middle ear is present, its treatment is of the first importance.

If keratitis develops, measures should be taken to insure proper protection of the cornea by the lids. This is especially important during sleep, as more prolonged exposure of the eye is then apt to occur. This may be accomplished by the application over the lids of a light bandage, or a gauze compress, held in place by two or three strips of rubber adhesive plaster.

The most useful application for the keratitis is a collyrium of holocain and boracic acid (holocain hydrochlorate, gr. j; acid. boracic., gr. x; aquæ. destil., $\tilde{5} \mathbf{j}$ ), which may be dropped into the eye three to five times a day. When only conjunctivitis is present, a ten- to fifteen-grain solution of boracic acid, or a sublimate solution ( $1: 8000$ to $\mathrm{I}: \mathrm{I} 2,000$ ), should be prescribed.

\section{DISEASES OF THE ORBIT.}

Diseases of the orbit hardly deserve to be regarded as among the more prevalent affections of the eye. However, it will not be out of place to treat briefly of cellulitis of the orbit, of periostitis and caries of the orbital walls, and of the orbital tumors which are most frequently encountered. 
Cellulitis of the orbit, leading usually to abscess, or phlegmon (Fig. 4I), occurs as an acute affection and, less frequently, as a subacute or chronic process. It may arise from a variety of causes, such as local injuries of various sorts, "cold," the extension of inflammation from the integument of the face, as in facial erysipelas, or from the sinuses accessory to the orbit, periostitis of the orbital walls, panophthalmitis, and meningitis. It occurs also as a post-typhoidal and a post-scarlatinal affection, and as a manifestation of a general pyemia. The acute form of the disease is characterized by

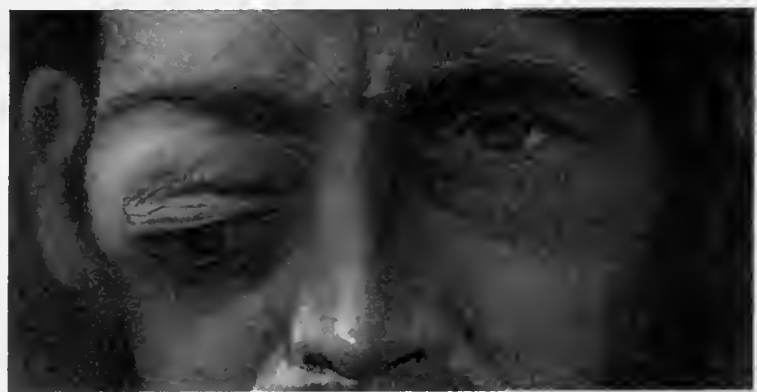

Fig. 4I.-Orbital abscess (Ramsay).

headache, severe pain in the orbit, increased by pressure upon the eye or by an attempt to rotate it, protrusion of the eyeball, with limitation of its movements, marked injection and chemosis of the conjunctiva, and redness and brawny swelling of the lids. It is commonly attended also by evidences of constitutional disturbance, such as elevation of temperature, loss of appetite, sleeplessness, etc. In the chronic type of the affection all the symptoms are less pronounced; they are also less typical, and in consequence a correct diagnosis is not so easily made.

The prognosis in the milder cases, which may ter- 
minate without suppuration, is favorable. But in the more severe cases, especially in those which are consequent upon facial erysipelas, the prognosis is grave; for not only is there danger of loss of sight from involvement of the optic nerve, or from necrosis of the cornea or the supervention of panophthalmitis, but a fatal result may ensue, through extension of the suppurative process to the brain or through the occurrence of a general pyemia.

Because of the great swelling of the lids and the conjunctival chemosis, acute cellulitis of the orbit may be mistaken for gonorrheal conjunctivitis or for panophthalmitis. However, it may be distinguished from the former affection by the existence of exophthalmos, by limitation of the movements of the eye, and by the absence of copious purulent discharge, and from the latter, by the fact that the cornea is clear-not opaque and necrotic, as it usually is in panophthalmitis.

Treatment.-This should be both local and constitutional. In the acute form of the disease the local treatment should consist in the constant application of a warm anodyne fomentation-the lotion of opium and boracic acid, applied by means of a gauze pad covered with rubber protective, as previously described (ext. opii., gr. x-xv; acid. boracic., gr. xl; aq. destil., そ̋jv) - and an early incision, to afford efficient drainage.

The incision, which it may be necessary to extend deeply into the orbit, should be made from the conjunctival cul-de-sac or through the lid, as may seem to be indicated. A straight, narrow bistoury should be used, and the blade should be entered flatwise with reference to the eyeball, to avoid the risk of wounding it. It is well, too, to bear in mind that if the incision is made in the upper nasal, or in the lower nasal, angle 
of the orbit, there is danger that one or the other of the oblique muscles may be divided. In severe cases, especially those of erysipelatous origin, it may be necessary to make several incisions. The introduction of an iodoform-gauze drain is indicated when the suppuration is deeply seated or when the external wound shows a disposition to close. Systematic syringing of the pus cavity with a warm antiseptic solution (a saturated solution of boracic acid, a one per cent. solution of carbolic acid, or a I : 8000 to I $: 4000$ sublimate solution) should be practised, and, if the discharge is considerable, should be repeated two or three times a day.

Should panophthalmitis develop during an attack of orbital cellulitis, enucleation of the eye, in my opinion, should be performed without unnecessary delay. Resort to this measure, under such circumstances, is commonly condemned, upon the ground that the danger of cerebral infection is increased through the consequent opening of the lymph-channels of the optic nerve; but, it seems to me, any added risk from this source is more than offset by the greatly improved drainage of the orbit afforded by the removal of the eye, besides which the operation may be counted upon to relieve the patient of much suffering and, in all probability, to considerably curtail the duration of the orbital inflammation. The possible dependence of the cellulitis upon periostitis or caries of the orbital walls should not be lost sight of.

The constitutional treatment of the acute form of the disease should consist in the administration of an energetic mercurial purgative, to be followed by liberal doses (gr. xx every two hours) of sodium pyrophosphate. Iron and quinin may also be called for. In the chronic form, tonics and alteratives are indicated. 


\section{Periostitis, Caries, and Necrosis of the Orbital} Walls.-Periostitis of the walls of the orbit, leading frequently to caries and not rarely to necrosis, is met with as a chronic and as an acute affection. Its most common causes are syphilis, inherited and acquired, rheumatism, scrofula, local injuries, and disease of the accessory cavities.

The symptoms of acute periostitis resemble those of orbital cellulitis, to which, indeed, it not infrequently gives rise. Deep-seated pain, increased by pressure upon the eyeball, edema of the lids, conjunctival hyperemia, limitation of the movements of the eye, attended, perhaps, by diplopia, and more or less evident exophthalmos, are the usual symptoms when the disease occurs in the deeper parts of the orbit. When it is situated nearer the orbital margin, localized tenderness, manifested upon pressure on or beneath the border of the orbit, is a characteristic symptom, and, under such circumstances, it is often possible to detect the thickening of the periosteum.

Suppuration is more apt to occur when the periostitis is of tuberculous or of traumatic origin, or when it is secondary to disease of the neighboring cavities. It is least apt to happen, and the affection is more prone to be chronic, when it is dependent upon rheumatism or syphilis.

When the inflammation is pronounced, and involves the apex of the orbit, loss of sight may occur through compression of the optic nerve. Again, if caries and necrosis supervene, especially when the roof of the orbit is involved, intracranial complications may result. If suppuration takes place in the cellular tissue of the orbit, any of the untoward consequences which have just been described in treating of this condition may 
ensue. Furthermore, when exit has been given to the pus the discharge is apt to persist, and a fistula to become established, which will not close until the disease of the bone has been overcome. One of the unpleasant consequences to which this is apt to give rise is a permanent distortion of the lid, usually a more or less pronounced ectropion (Fig. 42).

Treatment.-This will depend, of course, upon the character of the attack, its cause, and upon the extent

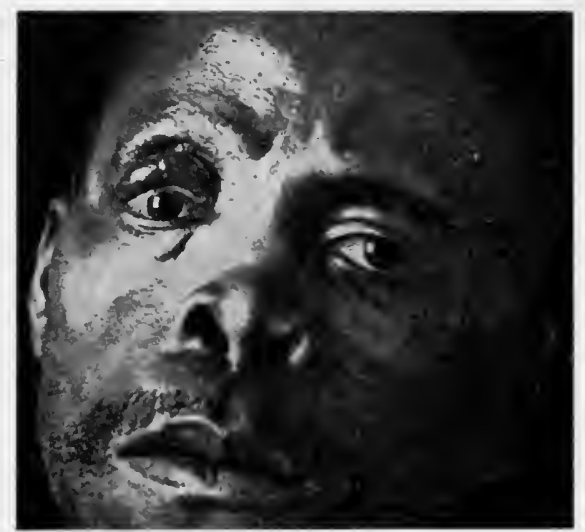

Fig. 42.-Ectropion of upper lid from caries of orbital roof (author's case).

to which the bone has become involved. The salicylates and potassium iodid are indicated when the affection is of rheumatic origin, and the latter, in combination, perhaps, with mercury, when it is dependent upon constitutional syphilis; iodid of iron, cod-liver oil, and the hypophosphites when it is tuberculous.

An early incision is called for when suppuration supervenes, to be followed by systematic syringing with an antiseptic solution; and, as caries is usually present under such circumstances, treatment directed to this 
condition should be instituted when the acute symptoms have subsided. Careful curetting may become necessary; but before this is resorted to, or as supplementary to it, an effort should be made to remove any dead bone which may be present, and to bring about a healthier action in the periosteum and in the bone itself, by the use of hydrochloric acid.

If the carious bone is so situated as to be readily reached, the application of the acid may be made most effectually by means of a probe armed with a bit of absorbent cotton. The acid (c. p.) should be diluted at first with three or four parts of water; but, if well received, the strength of the solution may be gradually increased to equal parts of each. I have had but little experience in treating caries of the orbit in this way; but I have obtained very satisfactory results in caries of the walls of the auditory canal from the employment of hydrochloric acid in this manner. If it is impracticable to make the application in this direct way, the sinus leading to the carious bone may be syringed with a much weaker solution of the acid-a two to four per cent. solution to begin with, which may be gradually increased to twenty or twenty-five per cent. if well borne.

Tumors of the Orbit.- Tumors of many kinds, malignant as well as benign, are met with in the orbit. They may have their starting-point in the orbital cellular tissue, in the lacrimal gland, in the optic nerve (Fig. 43), in the bony walls which surround the orbit; they may invade the orbit from one of the neighboring cavities, or they may begin as intraocular growths.

Benign tumors developing in the orbit may cause serious consequences through interference with vision. In the case of malignant tumors the prognosis is most 
unfavorable, not only as to sight, but as to life, since they are prone to recur even after most thorough removal, and, sooner or later, are apt to involve the brain by extension along the optic nerve or through the roof of the orbit.

Among the benign tumors occurring in the orbit

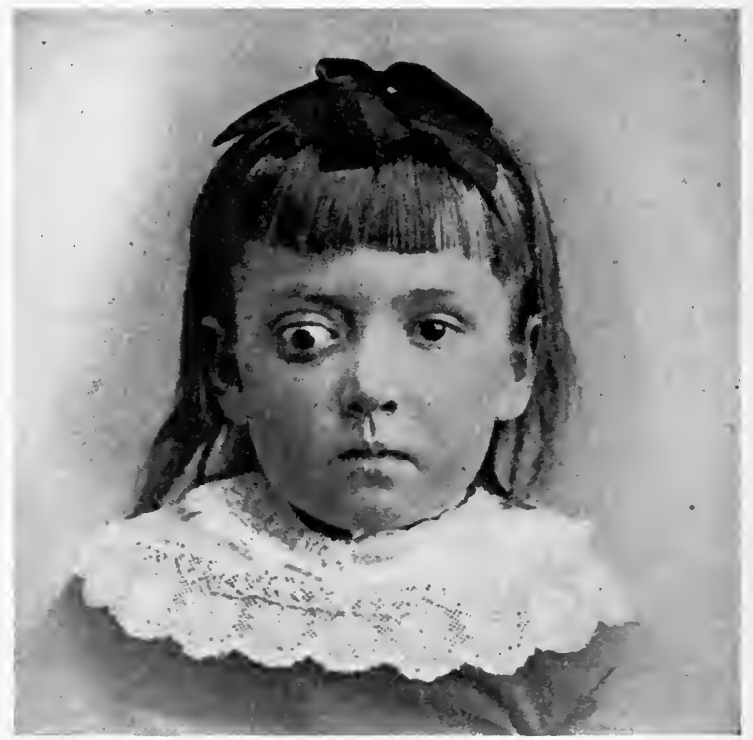

Fig. 43--Exophthalmos from fibroma of the optic nerve. The morbid growth in this case extended into the optic foramen, at which point chlorid-of-zinc paste was applied after removal of the eyeball and growth, without exenteration. Ten years later there had been no recurrence (Buller).

may be mentioned lipoma, fibroma, dermoid, sebaceous and hydatid cysts, angioma, osteoma, and gumma. The malignant growths include the several varieties of sarcoma (Fig. 44), epithelioma, usually through extension from the lids or eyeball, and carcinoma, commonly having its starting-point in the lacrimal gland. Exophthalmos, lateral displacement and limitation of 
the movements of the eyeball, diplopia, impairment of vision through involvement or compression of the optic nerve, and in the end desiccation and necrosis of the cornea from lack of protection, are commonly observed when the growth has attained a considerable size.

In endeavoring to determine the character of the growth, palpation is at times of much assistance. One

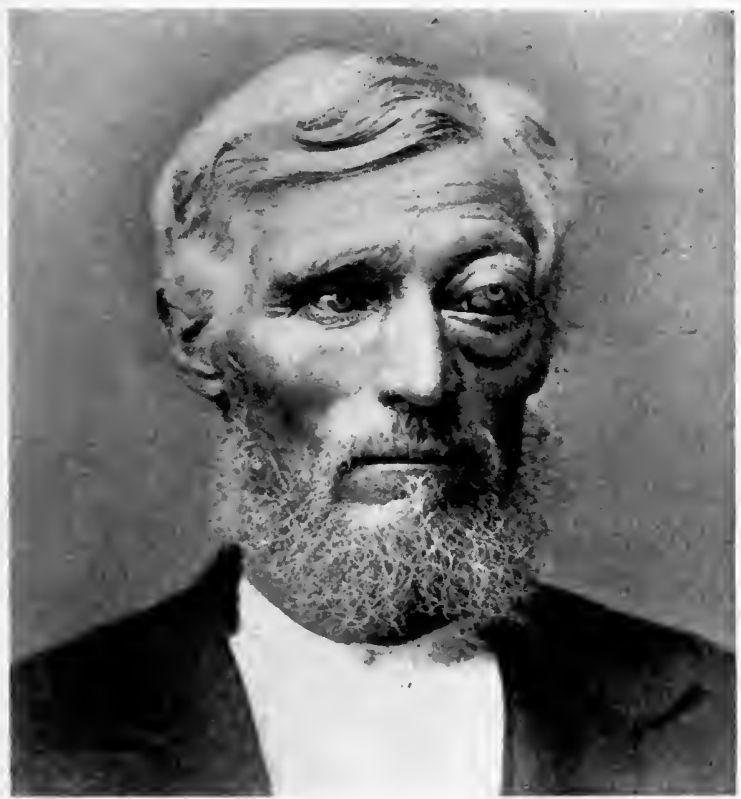

Fig. 44.-Sarcoma of the orbit originating in the tissues of the apex (Buller).

should also take into account the history of the case, the rapidity with which the tumor has developed, the presence or absence of pain, the age and general condition of the patient, and the state of the nose and of the other cavities accessory to the orbit. The possibility that the growth may be of syphilitic origin should not be lost sight of; for when this is the case not 
only is the treatment different, but the prognosis is decidedly more favorable.

Treatment.-The presence of an orbital tumor having been definitely determined, its removal is commonly indicated. There are exceptions, however, to this rule. For example, if the tumor is benign, is causing no inconvenience, is not increasing in size, and is not readily accessible, it is permissible to postpone operative interference as long as these conditions maintain. Again, when it is evident that a malignant growth starting in the orbit has already invaded the neighboring cavities, or it is clear that such a growth has involved the orbit only secondarily, having had its starting-point in one of these cavities, since no operative procedure, however radical, is likely to be of avail, either in relieving suffering or in prolonging the life of the patient, there is little justification for undertaking it.

In dealing with benign tumors it is usually practicable, and one should always endeavor, to accomplish their removal without sacrificing the eyeball or in any way impairing the sight. In the case of malignant growths, however, the preservation of sight becomes a matter of secondary importance, and not only the eyeball but all the contents of the orbit (exenteration of the orbit), and in some instances the lids and portions of the orbital walls, must be removed (Fig. 45). Cosmetically, the effect of such an operation is, at first, rather shocking; but it is surprising how well nature copes with a condition so unpromising. In time the orbit becomes greatly lessened in depth, and its walls become lined with skin and scar-tissue, so that the unsightliness in large measure disappears. (In illustration of this, compare Figs. 45 and 24.)

Whenever there is ground for suspecting that an 
orbital growth may be of syphilitic origin, mercury and potassium iodid should be given a thorough trial before resort is had to operation.

The removal of intraorbital growths, it should be remarked, ought to be undertaken only by those

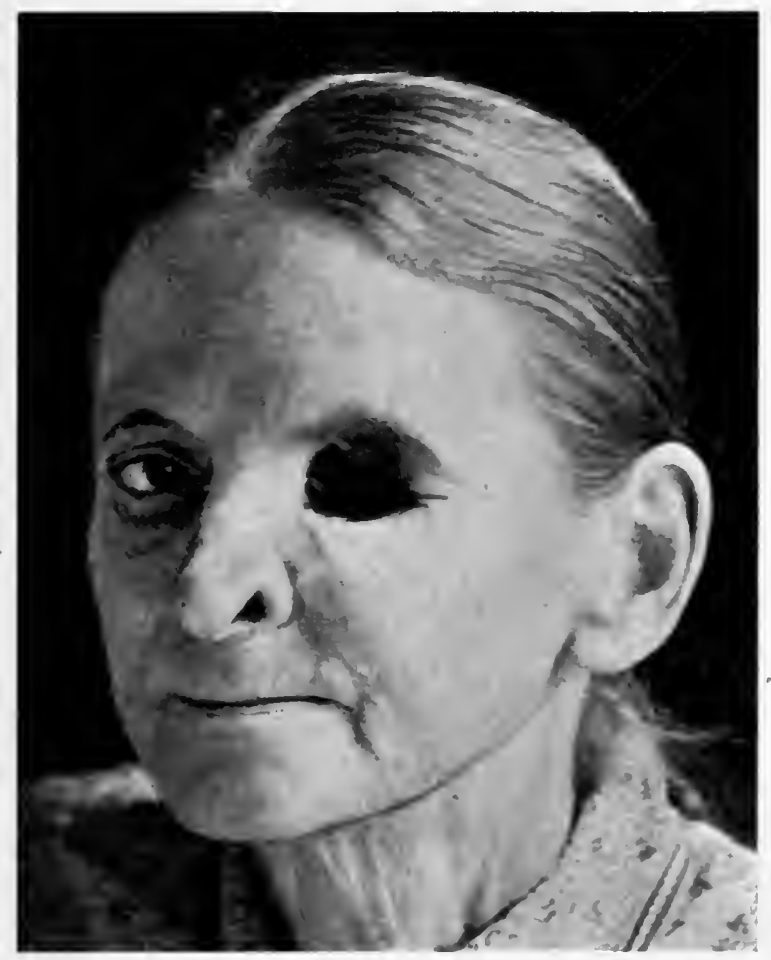

Fig. 45.-Exenteration of the orbit, with removal of the major part of the lids, for epithelioma of the lids and orbit (author's case; photograph taken two weeks after operation).

who, in addition to surgical skill, possess thorough familiarity with the anatomy of the parts; for, even when they seem to be superficial, their extirpation may entail invasion of the deeper parts of the orbit, and under such circumstances irreparable damage to sight may result from awkward manipulation. 


\section{CHAPTER IV.}

\section{DISEASES OF THE LACRIMAL APPARATUS.}

In treating of diseases of the lacrimal apparatus it is usual to consider, first, those affections which have to do with the lacrimal gland and its ducts, and, second, those which pertain to the drainage apparatus, including in this term the puncta, the canaliculi, the lacrimal sac, and the nasal, or lacrimal, duct. As, however,

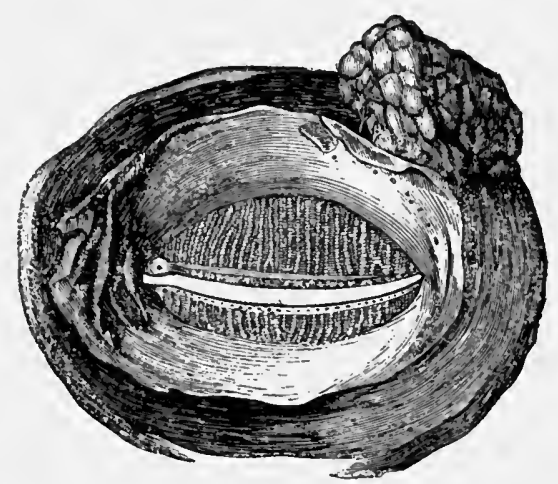

Fig. 46.-The lacrimal gland, the mouths of its ducts showing; the meibomian glands, seen upon the under surface of the lids, and the lacrimal puncta (Nunneley).

diseases of the lacrimal gland, owing to the protected position of the gland and its system of multiple ducts (Fig. 46), are of infrequent occurrence, they will be treated of very briefly; while, on the other hand, affections of the drainage apparatus, since they are common, and, as a rule, may be successfully treated by the general practitioner, will be considered at greater length. 


\section{DISEASES OF THE LACRIMAL GLAND.}

Dacryoadenitis, or inflammation of the lacrimal gland, occurs as an acute and as a chronic affection. Both varieties are rare, though it seems probable that acute inflammation of the gland is sometimes mistaken for cellulitis of the orbit, from which it is not always easy to distinguish it. It occurs more frequently in children than in adults and oftener in females than in males. It has been known to assume an epidemic character, and Galezowski once met with an unusual number of cases during an epidemic of mumps. Other causes to which it has been ascribed are traumatism, "cold," rheumatism, gout, tuberculosis, syphilis, gonorrhea, and the extension of inflammation from the conjunctiva and cornea. It is usually unilateral, but not infrequently both glands are involved.

Acute dacryoadenitis gives rise to severe pain, which may be accompanied by fever, sleeplessness, and delirium. The lids, the upper lid especially, are greatly swollen, and there is marked chemosis of the conjunctiva, the general appearance of the eye being not unlike that which characterizes purulent conjunctivitis (S. C. Ayres). Through the enlargement of the gland the eyeball may be displaced and its movements restricted. Palpation of the gland, because of its exquisite sensitiveness and the swelling of the lid, is difficult, and eversion of the lid, to permit of its inspection, is impracticable. Suppuration may supervene within a few days, the pus making its way through the integument of the lid or into the conjunctival culde-sac, or the inflammation may subside without the formation of pus.

In chronic dacryoadenitis the enlargement of the 
gland may be detected by palpation, and in some instances by simple inspection, and upon everting the lid the swollen gland may be brought into view as a red, tongue-shaped, nodular mass (Hirschberg). Though the gland is usually sensitive to pressure, there is an absence of the pain, edema of the lids, and conjunctival chemosis which characterize the acute variety of the disease. Marked displacement of the eyeball, usually downward and inward, may occur, and this is commonly accompanied by diplopia.

Treatment.-The treatment of acute dacryoadenitis, if the case is seen in its incipiency, should consist in the local abstraction of blood by leeches, the application of ice-cloths or, if more acceptable to the patient, of a lotion of lead acetate and opium (plumbi acetatis, gr. xv; ext. opii, gr. x-xv; aq. destil., §iv), and the administration of an energetic mercurial purgative, to be followed by liberal doses (twenty grains every two hours) of sodium pyrophosphate. Should these measures fail to cut short the attack, warm fomentations, containing opium or belladonna, should be employed, and as soon as the presence of pus can be detected it should be evacuated by an incision through the integument of the lid or through the conjunctival culde-sac, as may seem to be indicated.

In chronic inflammation of the gland benefit may be expected from the application of mercurial or compound iodin ointment, combined with the administration of potassium iodid or biniodid of mercury. Should the gland become so enlarged as to endanger the integrity of the eyeball, its extirpation may be necessary.

Fistula of the lacrimal gland may occur as a sequel of dacryoadenitis or may be of traumatic origin. 
In rare instances it has been observed as a congenital defect. The fistulous orifice is usually situated in the upper lid, and much annoyance results from the tears which constantly flow from it.

Treatment.- It is not easy to bring about a healing of the fistula, and if this is accomplished it is at the risk of precipitating a recurrence of the dacryoadenitis. The operative procedure which has proved most effectual is that suggested by Bowman. Its purpose is to convert the annoying, external cutaneous fistula into one opening into the conjunctival sac, and, therefore, causing little or no inconvenience. A needle, attached to a silk thread, is passed a short distance into the fistula, and is then made to transfix the lid, being brought out upon its conjunctival surface. A second needle, upon the other end of the thread, is next passed through the lid, close to the orifice of the fistula. The two ends are then tied tightly, and the thread is left to cut its way out. To promote its closure, the edges of the external orifice of the fistula are freshened.

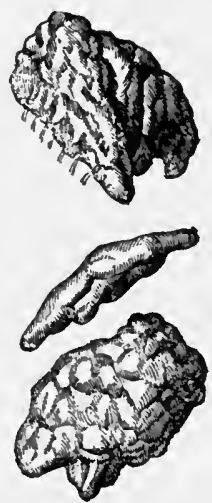

Fig. 47.-Three views of the lacrimal gland, the efferent ducts shown in one of them (Nunneley).

Dacryops, or cyst of the lacrimal gland, results from occlusion of one or more of the efferent ducts of the gland (Fig. 47). It has also been met with as a congenital condition. Upon eversion of the upper lid the cyst may be observed as a translucent, at times lobulated, swelling. Marked distention of the cyst may occur from crying.

Treatment.-This consists in the establishment of a permanent opening between the cyst and the con- 
junctival sac. It may be accomplished by excising a portion of the cyst-wall and preventing closure of the wound by the repeated introduction of a probe, or, as suggested by von Graefe, by passing a silk thread through the wall of the cyst, tying it in a loop, and leaving it to cut its way out.

Dacryoliths, chalky concretions, occasionally form in the lacrimal gland. They are liable to cause me-

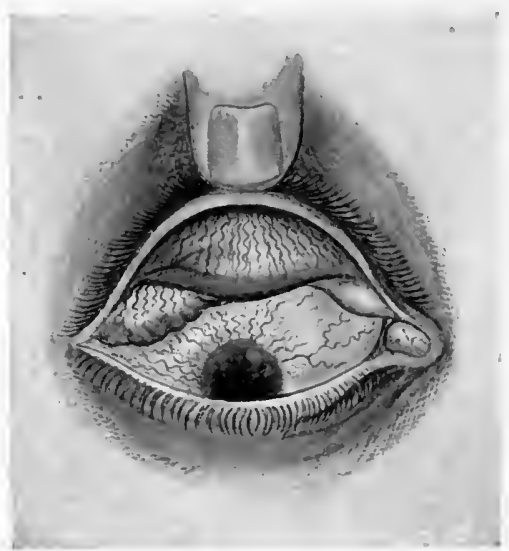

Fig. 48.- Enlargement and prolapse of the palpebral portion of the lacrimal gland in an eye with kerato-iritis (de Schweinitz).

chanical irritation, and, if this happens, they should be removed through a conjunctival incision.

Dislocation of the lacrimal gland, sometimes described as hernia or prolapse of the gland (Fig. 48), has been met with as a spontaneous condition, and as a consequence of injury involving the neighboring parts.

Treatment.-If possible, the gland should be restored to its normal position, and a compress bandage should be applied, and worn continuously for some time to prevent a redislocation. If this is impracticable, removal of the gland may become necessary. 
Hypertrophy of the lacrimal gland occurs more frequently in children than in adults, and has been known to be of congenital origin. The gland may become so greatly enlarged as to force the eyeball from the orbit, and destroy sight through stretching and compression of the optic nerve. Cases have been observed, however, in which there was marked displacement of the eye, with great elongation of the optic

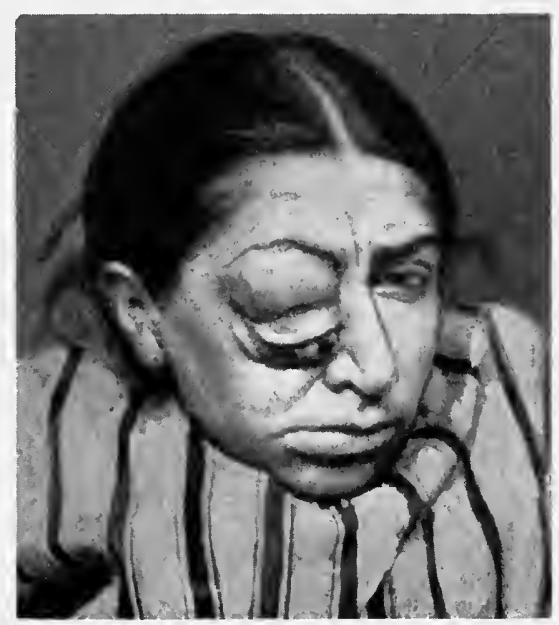

Fig. 49.-Hypertrophy of the lacrimal gland.

nerve and external ocular muscles, and yet fairly good vision and ability to rotate the eye were retained.

The accompanying illustration (Fig. 49) represents a remarkable case of this character, which occurred in the practice of the late Prof. Christopher Johnston, of Baltimore. The hypertrophied gland, which was about the size of a hen's egg, and contained numerous dacryoliths, was removed by Dr. Johnston through an incision made parallel with the orbital margin. The eye subsequently resumed nearly its normal position, 
and retained vision equal, at least, to counting fingers.

Treatment.-If the enlargement of the gland is not so great as to interfere with vision, an effort should be made, by the application of mercurial or compound iodin ointment and the administration of potassium iodid, to arrest the hypertrophic process. The possibility that the condition may be of syphilitic origin should be borne in mind. If, however, the gland is so greatly enlarged as to endanger the integrity of the eye, it should be removed without unnecessary delay.

Removal of the lacrimal gland may be accomplished by either of two procedures: The gland may be exposed by an incision through the integument of the upper lid parallel with the orbital margin, drawn out with a tenaculum, and separated from its attachments with a knife or scissors. The objection to this method is that it involves a more or less complete division of the tendon of the levator palpebræ superioris muscle, which may result in the production of ptosis.

The other, and probably better, plan, suggested by Velpeau, is to divide the external canthus, evert the upper lid, and cut down upon the gland from the superior conjunctival cul-de-sac. This method does not endanger the integrity of the levator muscle, and leaves a less conspicuous scar than the first-described procedure.

Atrophy of the lacrimal gland has been observed as one of the late consequences of trachomatous conjunctivitis, in the condition known as xerophthalmia. Arlt has described a case of this character in which the efferent ducts of the gland were obliterated, and the gland itself was reduced to one-third its normal size. Paralysis of the trigeminus may result in abol- 
ishment of the functional activity of the lacrimal gland.

Tumors of the lacrimal gland are rare, and, not infrequently, are of traumatic origin. They are usually of slow growth, and occur oftenest in advanced life. As they increase in size they interfere with the movements of the eyeball, giving rise to diplopia. Later they produce exophthalmos, and, eventually, may not only destroy sight by pressure upon the optic nerve, but may cause death by extension to the brain.

The following varieties of tumors, believed to have had their origin in the lacrimal gland, have been observed: adenoma, myxoma, myxosarcoma, lymphosarcoma, spindle-cell sarcoma, epithelioma, cylindroma, chloroma, and carcinoma.

Treatment.-Early and complete removal of the growth, including, of course, the gland itself, is indicated. Whether this can be accomplished without sacrificing the eye, will depend up on the size of the tumor and the extent to which it has invaded the deeper parts of the orbit.

\section{DISEASES OF THE DRAINAGE APPARATUS.}

The lacrimal drainage apparatus (Fig. 50), as has been said, is frequently the seat of pathological changes. This is due not only to the fact that the mechanism by which the tears are carried from the conjunctival sac to the nose is complex, and a disarrangement of any one of its parts is apt to disturb the normal action of the whole, but to the further fact that this apparatus, while an appendage of the eye, is, pathologically considered, a part, rather, of the nasal passages, and so prone to participate in the many maladies to which these passages are liable. 
Whatever may be the nature of the pathological changes which affect the drainage apparatus, and wherever they may be located, a common symptom characterizes them all: the tears are no longer carried, as they should be, from the conjunctival sac to the nose, and in consequence they overflow the lids, giving rise to the annoying condition known as epiphora. This condition is not only, in itself, very annoying, but it leads to chronic

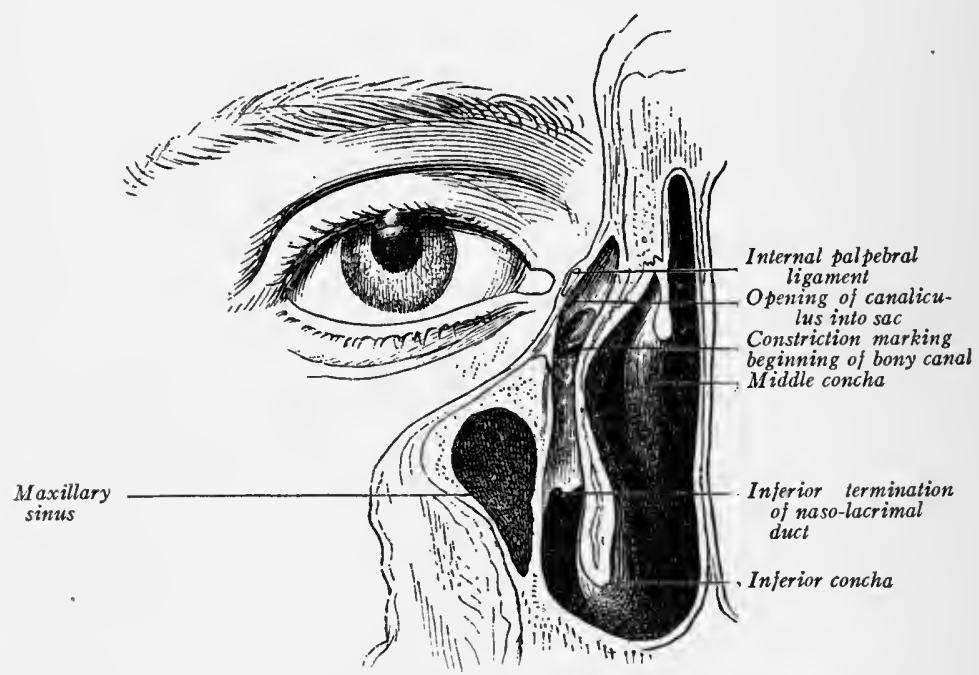

Fig. 50.-Section showing the course and relations of the lacrimal sac and nasal duct (Merkel).

conjunctivitis, to blepharitis, and, not infrequently, to eczema of the lids and cheek.

Atresia of the lacrimal puncta is met with as a congenital and as an acquired anomaly. Congenital atresia of the puncta, of which not many authentic cases have been reported, may affect one or both eyes, and may be attended by absence of the corresponding canaliculi. I have encountered one case of this char- 
acter, in which, however, but one punctum, with its canaliculus, was absent.

As an acquired condition, complete obliteration of the puncta occurs usually as a result of destruction of neighboring parts, such as may happen from burns of the lids by molten metal or lime. It has been known also to follow the cicatrization of a smallpox pustule and of a chancre of the lid. A superficial occlusion, usually of the lower punctum, which is easily overcome, and which is due chiefly to desiccation of the parts, is often observed in blepharitis marginalis complicated by ectropion.

Treatment.-The occlusion, whether congenital or acquired, may commonly be overcome without much difficulty, provided the canaliculus is not absent or

Fig. 51.-Straight, sharp-pointed probe.

has not been obliterated. A slight depression usually marks the site of the occluded punctum, and at this point an opening may be drilled into the canaliculus with a straight, rather sharp-pointed probe, such as is represented in Fig. 5I. After this has been accomplished it is best, as a rule, to slit the canaliculus; though it may be possible to prevent a recurrence of the occlusion by the occasional introduction of a No. 2 or No. 3 probe. If the canaliculus as well as the punctum is obliterated, an opening must be made, starting from where the punctum should be, and following the usual direction of the canaliculus, into the lacrimal sac. For this purpose a sharp-pointed knife is required. We shall scarcely succeed, even by persistent probing, in keeping open a considerable part of this artificial canaliculus; but, aided by the action of the tears, we 
may, at least, be able to establish a permanent opening into the lacrimal sac-as I succeeded in doing in the case of congenital absence of the punctum and canaliculus to which reference has been made-and so get rid of the epiphora and the great discomfort to which it gives rise.

Malpositions of the Puncta.-Normally the puncta lie in contact with the eyeball, and this position is essential to the proper performance of their office. Malpositions of the upper puncta are not common, but misplacements of the lower puncta are more frequently met with, and usually give rise to greater annoyance.

Eversion of the puncta is present in most cases of ectropion; it occurs also in inflammatory thickening of the lid-margin, in senile relaxation of the tissues of the lid, and in facial paralysis. Inversion of the puncta, which is less frequently encountered, is usually a result of entropion. Another faulty position of the puncta results from the eye being exceptionally small or being deeply set. Under such circumstances a triangular space is present, in the neighborhood of the inner canthus, between the lids and the eye, and in consequence the puncta and the eyeball are not in apposition.

Treatment.-The efficient remedy for all malpositions of the puncta is division of the corresponding canaliculus. This not only relieves the epiphora, but leads to the rapid disappearance of the conjunctivitis and the blepharitis which are its usual accompaniments.

Division of the lower canaliculus (the upper canaliculus, in my experience, seldom needs to be divided) is accomplished most conveniently with the straight, probe-pointed canaliculus knife (Fig. 52), a modification of the beak-pointed knife of Weber (Fig. 53). Hav- 
ing previously dilated the punctum and canaliculus by the passage of a small probe, the operator, standing behind the patient, and putting the lid upon the stretch, introduces vertically into the punctum the probed tip of the canaliculus knife. Then, changing the direction of the knife, he passes it horizontally along the canaliculus until its progress is arrested by the inner wall

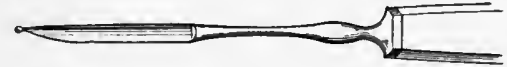

Fig. 52.-Straight, probe-pointed canaliculus-knife.

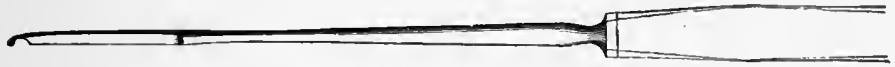

Fig. 53.-Weber's beak-pointed canaliculus-knife.

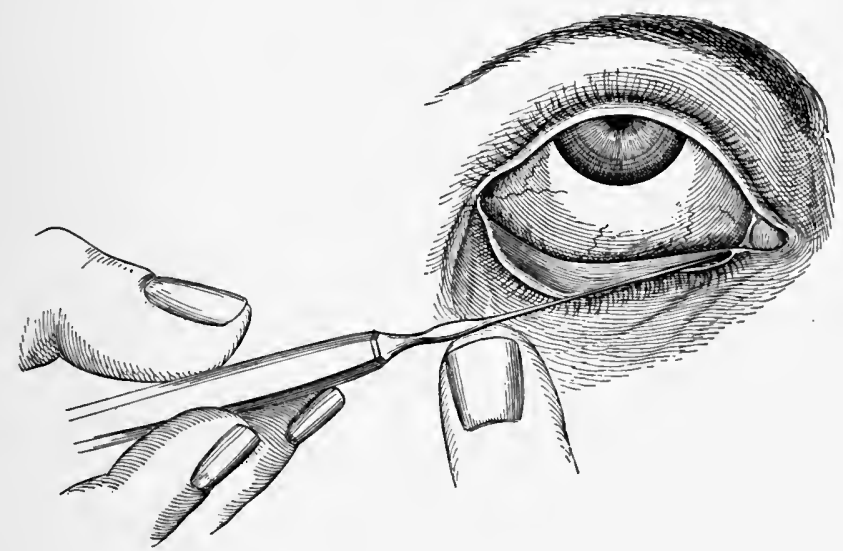

Fig. 54.-Introduction of canaliculus-knife.

of the lacrimal sac (Fig. 54). This point having been reached, and the edge of the knife being directed upward and slightly backward, the lid still being kept well upon the stretch, the canaliculus is divided by simply elevating the handle of the knife. If the operation is done as a step preliminary to the probing of the lacrimal duct, the canaliculus should be divided well up to its 
juncture with the sac; but if done for some other purpose, such as eversion of the punctum, it may not be necessary to carry the division quite to this point.

A few instillations of a four per cent. solution of cocain renders the operation of division of the canaliculus almost painless. As in all operations upon the eye, the instruments used should be sterilized by a brief immersion in boiling water; after which, to facilitate their introduction, it is well to dip the blade of the knife and the probes employed into sterilized vaselin.

The edges of the divided canaliculus, for several days, usually show a disposition to grow together, and to prevent this they must be separated, once in fortyeight hours, by the passage of a greased probe, until this disposition is overcome.

Division of the upper canaliculus, which, as has been said, is seldom called for, is accomplished by essentially the same procedure, except that the operator should stand or sit in front of the patient.

Atresia of the canaliculi may occur as a congenital defect, in association with absence of the puncta, as has already been mentioned. It may occur also as a consequence of traumatism or of an ulcerative process involving the region of the inner canthus. Circumscribed strictures of the canaliculi are of frequent occurrence, especially in connection with stenosis of the lacrimal duct. They are usually located near the point of juncture of the canaliculus with the lacrimal sac.

Treatment.-When the canaliculi are completely. obliterated, their restoration by operative procedure is impracticable. It may be possible, however, to make an opening directly into the lacrimal sac, and by repeated probings to render it permanently patulous. The circumscribed strictures may be overcome by the 
passage of a small lacrimal probe or with the straight probe represented in Fig. 5I. If, however, they show a disposition to recur, the canaliculus should be slit.

Dacryoliths, small concretions composed of lime and of a fungous growth (leptothrix), occasionally form in the canaliculi. Their presence is indicated by a circumscribed swelling. As they cause more or less irritation, and give rise to epiphora, they should be removed without delay. To effect their removal, slitting of the canaliculus may be required.

Polypi sometimes form in the canaliculi, and may project through the puncta. Their removal may necessitate division of the canaliculus.

Small foreign bodies, eyelashes especially, at times find their way into the canaliculi, where they may remain for a long time, causing considerable annoyance.

Treatment.-If they project through the puncta, as they usually do, they may be seized with forceps and easily withdrawn; otherwise, division of the canaliculus may be required to effect their removal.

Dacryocystitis, or inflammation of the lacrimal sac, occurs as an acute and as a chronic affection. The former is often spoken of as abscess of the lacrimal sac; the latter is usually denominated blennorrhea of the sac.

In the great majority of cases dacryocystitis is secondary to, and dependent upon, stricture of the nasal duct. Primary inflammation of the lacrimal sac, that is to say, inflammation occurring independently of stenosis of the nasal duct, though comparatively a rare affection, is occasionally encountered. It is met with most frequently in the new-born, usually in the form of a mild blennorrhea; it is said to occur also in strumous children, and it may be brought on by 
external violence or through the entrance into the sac of an irritant fluid.

Blennorrhea of the Lacrimal Sac.-Inflammation of the lacrimal sac, secondary to disease of the nasal duct, usually begins as a chronic affection. It is unattended by pain, and manifests itself chiefly through the accumulation of tears and mucus in the sac, their regurgitation through the puncta, and the existence

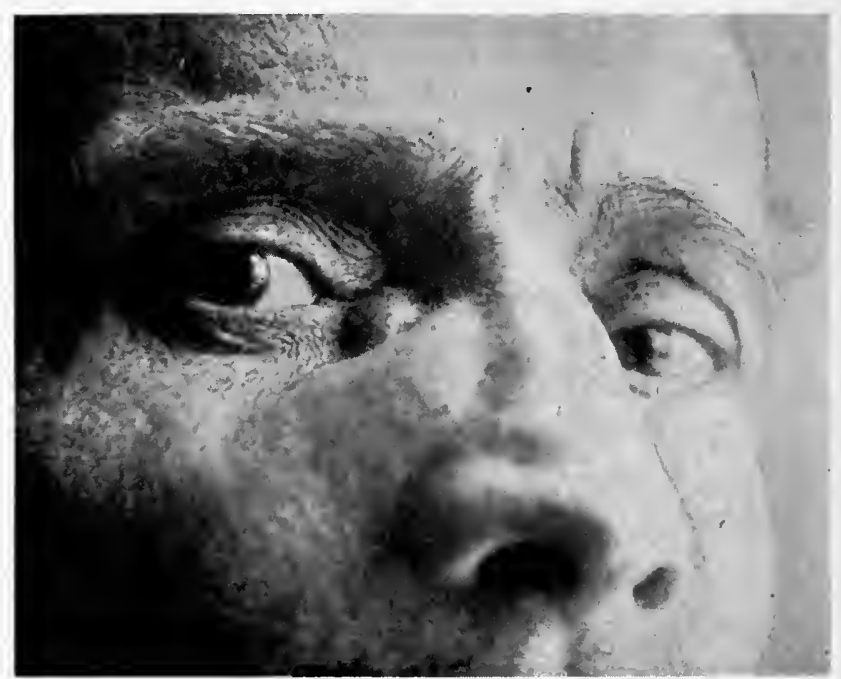

Fig. 55--Mucocele; fracture of superior maxilla; exostoses of nasal bones (de Schweinitz).

of epiphora. Frequently, there is a perceptible distention of the sac (mucocele) (Fig. 55), which, under slight pressure with the tip of the finger, disappears, the contents of the sac regurgitating through the puncta and flowing over the front of the eye, or, exceptionally, if the stenosis of the duct is incomplete, escaping into the nose.

This state of chronic catarrhal inflammation some- 
times continues indefinitely, without undergoing appreciable change; but, on the other hand, through the influence of "cold," a slight traumatism, the entrance into the sac of a pyogenic organism of unusual virulence, or, as seems to happen not infrequently, through the sudden occlusion of the canaliculi at their point of juncture with the sac, the inflammation is liable at any moment to undergo a sudden and acute aggravation. Severe pain, accompanied by marked edema and redness of the lids, and often of the whole side of the face, comes on; thick, creamy pus forms in the sac, and decided evidences of constitutional disturbance, such as fever, loss of appetite, sleeplessness, etc., may manifest themselves. Indeed, the local appearance and the general disturbance of the system are such as not infrequently to lead to a mistaken diagnosis of facial erysipelas.

These are the symptoms which characterize acute dacryocystitis, or abscess of the lacrimal sac (Fig. 56), and which, in many cases of stricture of the nasal duct, recur from time to time as long as the occlusion of the duct is permitted to remain.

After several days of intense suffering the integument over the sac assumes a yellowish appearance, becomes thinned, and, if left to itself, usually gives way at a point just below the internal palpebral ligament, permitting the contents of the sac to escape, and affording immediate and almost complete relief from suffering. Exceptionally, the inflammation subsides without perforation of the sac, and the pus escapes ultimately through the canaliculi and puncta.

After the contents of the acutely inflamed lacrimal sac have been evacuated, either spontaneously or by an incision, the inflammation rapidly subsides, and 
within ten days or two weeks the opening through which the discharge has occurred usually closes, and the sac resumes its previous condition of chronic blennorrhea. It may happen, however, that the cicatrization of this opening is prevented by the discharge through it of tears and muco-pus, and thus there is established the troublesome condition known as lac-

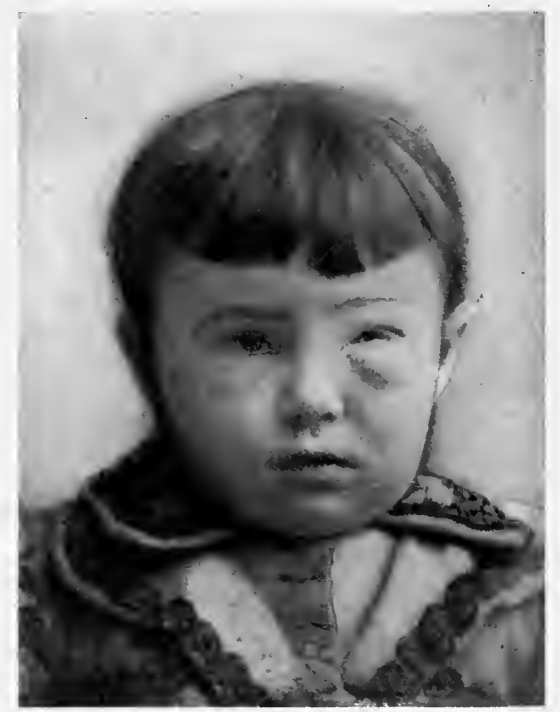

Fig. 56.-Acute dacryocystitis (author's case).

rimal fistula - a condition which may persist for an indefinite period (Fig. 57).

It is worthy of remark that during an acute attack of dacryocystitis it is seldom possible to empty the distended sac by pressure, as can usually be done in the intervals between such attacks. From this it would appear likely that when the sac is unduly distended a valve-like closure of the canaliculi at their point of juncture with the sac takes place; and it seems not 
improbable that such an occurrence as this, which would necessarily interfere with the previously existing drainage of the sac, is often a potent factor in the causation of the acute outbreaks.

Although inflammation of the lacrimal sac, through the regurgitation of muco-pus which commonly attends it, not infrequently gives rise to inflammation of the conjunctiva and cornea, the reverse-the secondary involvement of the lacrimal sac in an inflammation having its starting-point in the superficial ocular tunics

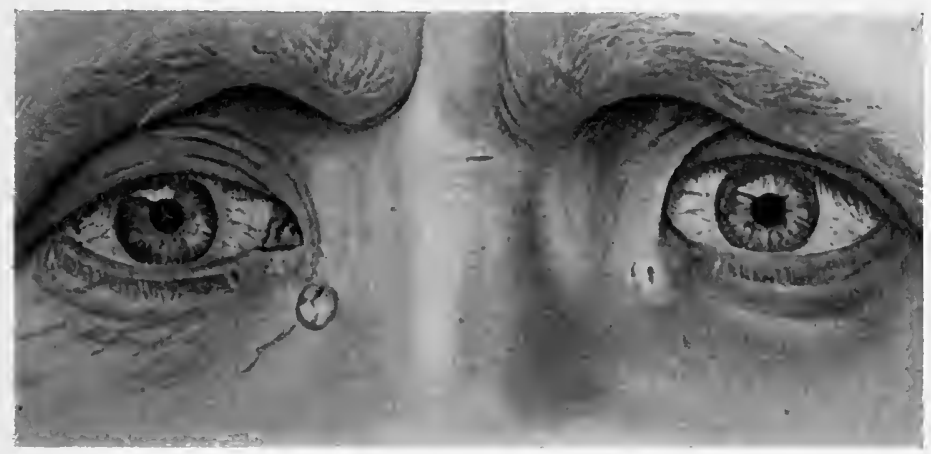

Fig. 57.-Lacrimal fistulæ-a tear-drop escaping from the fistula on the right side (Haab).

-is an occurrence of extreme rarity. The truth of this statement is strikingly illustrated in gonorrheal conjunctivitis; for, though the gonococci doubtless find their way in great numbers into the lacrimal sac, dacryocystitis as a complication of gonorrheal ophthalmia is, so far as I can learn, practically unknown.

On the other hand, as has already been intimated, there is the closest pathological sympathy between the drainage apparatus of the eye and the nasal passages, and without doubt, in the majority of instances, dacryocystitis is traceable, directly or indirectly, to nasal disease. 
Treatment.-From what has been said regarding the etiology of dacryocystitis-that it is almost invariably dependent upon stricture of the nasal duct-it follows that a description of the treatment of this affection, or, at least, of the chronic variety of it, is practically a description of the treatment of stenosis of the nasal duct, of which we shall speak presently. It will be in place, however, to consider here the treatment of acute dacryocystitis.

It does not often happen that we can cut short an attack of acute inflammation of the lacrimal sac; but, if the case is seen in its incipiency, an effort should be made to accomplish this result. The application of a lotion of boracic acid and opium (ext. opii, gr. $\mathrm{x}-\mathrm{xv}$; acid. boracic., gr. xl; aqux destil., ziv), and the administration of an energetic cathartic ("compound calomel powder," gr. x), followed by liberal doses of sodium pyrophosphate (gr. xx, every two or three hours), are the measures which are most likely to prove effectual.

If these measures fail to subdue the inflammation, the pad of absorbent gauze, wet with the lotion of opium and boracic acid, should be covered with a piece of oiled silk or muslin, or rubber protective, to give it a poultice-like action (a convenient and cleanly substitute for a poultice), and, as soon as it is evident that pus has formed, and is endeavoring to make its way to the surface, a free incision should be made into the distended sac, usually at a point beneath the internal palpebral ligament. As such an incision leaves no perceptible scar, provided it is made in the direction in which the skin tends to wrinkle-that is, from above and toward the nose downward and outward-it is much better to give the pus free exit in this way than 
to attempt to drain the sac by simply slitting the canaliculus. After this has been done, and until the discharge has lessened markedly, the application of the gauze pads should be continued as before. Until all the evidences of acute inflammation of the sac have disappeared no attempt-it should be said with emphasis-ought to be made to deal with the stenosis of the nasal duct by the introduction of probes or otherwise; for a fresh outbreak of dacryocystitis is likely to be the result of a disregard of this precaution.

In chronic inflammation of the lacrimal sac, if for any reason treatment of the strictured nasal duct is not practicable, a considerable measure of relief may be obtained from slitting the lower canaliculus, and prescribing a collyrium of mercury bichlorid (I : I 2,000 to I : 8000) or of argyrol (5 per cent.) or protargol ( 2 per cent.), to be dropped into the inner corner of the eye two or three times a day, explicit instructions being given that, before each instillation of the "drops," the sac shall be emptied of its contents by pressure with the finger-tip.

It is well to bear in mind that abscesses occasionally occur in the neighborhood of the lacrimal sac (prelacrimal abscess) which, from their appearance only, cannot well be distinguished from dacryocystitis. However, the history of the case, showing the absence of pre-existent lacrimal disease, will usually make the diagnosis plain.

Stricture of the Nasal Duct.--In order to comprehend the etiology of this affection, one needs but to call to mind the anatomical and histological peculiarities of the membranous lining of the duct; to remember that it is, at once, a mucous membrane and a periosteal membrane; that it contains a dense plexus of veins, 
resembling those of the turbinate bodies; and that here and there it is thrown into valve-like folds, which encroach considerably upon the lumen of the canal. In the presence of such conditions it is evident that even a trivial inflammation occurring here is liable to cause, at least, a transient occlusion of the duct; and, further, it is manifest that, though the inflammation may begin as a simple catarrhal process, involving only the mucous membrane, it is apt, if it be prolonged or become more intense, to extend to the underlying fibrous tissue, and so develop into an actual periostitis. Thus it happens that the transient occlusion of the duct caused by edema of the mucous membrane and engorgement of the underlying venous plexus, which under favorable circumstances passes away without leaving any permanent ill effects, may give place to a persistent stenosis, dependent upon periosteal and osteal thickening.

Again, if we would understand how it is that the conditions which favor such permanent occlusion of the nasal duct occur as often as they do, we have but to recall the fact, to which reference has already been made, that this canal, although an appendage of the eye, is in reality a part rather of the nasal cavity into which it opens, and that its relation to this cavity, prone as it is to inflammatory affections, is as close pathologically as it is anatomically.

Watering of the eyes, as is well known, is a usual symptom of acute rhinitis, and it is probable that in pronounced cases of this affection the mucous membrane lining the lacrimal drainage apparatus commonly participates, to a greater or less degree, in the catarrhal process. With the subsidence of the rhinitis the lacrimal symptoms usually disappear, and the parts return to their 
normal condition. Exceptionally, however, because of the greater intensity of the inflammation, the occurrence of a second or third attack before the first has been recovered from, a congenital narrowness of the duct, or a peculiar susceptibility of the lacrimal passages to disease (a susceptibility which, not infrequently, is inherited), the inflammation of the lining membrane of the duct does not subside with the nasal affection, but assumes the more serious character which has just been described.

In this way, and, perhaps, still more frequently from the extension of chronic inflammatory affections of the nose to the lacrimal passages, stricture of the nasal duct, which, as has been said, is the usual forerunner of dacryocystitis, commonly arises.

The nasal affections of inherited and of acquired syphilis, it should be remarked, are especially liable to involve the lacrimal apparatus. Gummata have been met with in the lacrimal sac, as well as in the duct. Tuberculosis of the nose, through extension to the lacrimal passages, has been known to cause occlusion of the nasal duct. The exanthematous fevers also may lead to this condition, as a consequence of the inflammation of the nasal mucous membrane which attends them; and, it may be added, not only inflammation of the lacrimal sac, as has been indicated, but stricture of the duct is occasionally brought about by a blow upon the bridge of the nose or in the region of the inner canthus.

As to the location of the strictures, although their most common situation is at the upper extremity of the duct, there is no part of the canal in which they are not frequently encountered. Multiple strictures, at least in cases of long standing, are the rule. As they 
are the outcome of periosteal inflammation, the strictures are almost always, in part at least, of bony structure. In form they may be circumscribed and annular (a thin bony septum being occasionally met with) or ill defined and of wide extent, involving a considerable part of the length of the canal. A stricture located at the lower extremity of the duct, it is well to bear in mind, is more easily overlooked; and it may happen that a mistake of this character will render of no avail a course of treatment which otherwise would prove successful.

Treatment.-Of the many ingenious surgical procedures for dealing with occlusion of the nasal duct which have been suggested from time to time, there are but very few which have for us, at the present day, an interest that is other than historical.

The invaluable suggestion of Bowman (1857) that the canaliculus should be slit as a preliminary step to the treatment of stenosis of the nasal duct may be said to mark the beginning of a new era in the surgery of the lacrimal apparatus. The great merit of this procedure is that it renders possible the use of lacrimal probes sufficiently large to completely obliterate the strictures, and restore the normal caliber of the canal. It was not, however, until some years after Bowman's operation was generally adopted that the opportunity which it affords in this respect was fully appreciated and taken advantage of; but, when this finally came about, the treatment of lacrimal strictures ceased to be what it previously had been-an opprobrium of ophthalmic surgery.

Dr. E. Williams, of Cincinnati, and Dr. H. D. Noyes, of New York, were the first to realize the inadequate size of the probes employed by Bowman and his followers, and to urge the necessity of using probes 
of considerably larger caliber. A brief experience in the treatment of diseases of the lacrimal apparatus led me to a similar conclusion, and induced me (in 1877 ) to undertake the measurement of a large number of nasal ducts (in the dried skull and in the cadaver) with a view to determining the usual size of the duct, and how large a lacrimal probe it would be practicable, and probably advantageous, to employ.*

As a result of these measurements (Fig. 58), I devised the series of probes - sixteen in number, the smallest

- Bowman's largest, No. 6, probe; diameter $=1.50 \mathrm{~mm}$.

Author's largest, No. 16, probe; diameter $=4 \mathrm{~mm}$.

Average size of 10 adult nasal ducts, cadaver; diameter $=$ $4.47+\mathrm{mm}$.

Largest of ro adult nasal ducts, cadaver; diameter $=5.25 \mathrm{~mm}$.

Largest of 70 bony nasal ducts; diameter $=7 \mathrm{~mm}$.

Fig. 58.-Diameters of probes, and of nasal ducts as determined by author.

size, No. I, having a diameter of 0.25 of a millimetre, and each number increasing in size by 0.25 of a millimetre, so that the largest, No. 16 , has a diameter of 4 mm.--which I have used since with great satisfaction, and which are now quite generally employed. The accompanying illustration (Fig. 59) represents the actual size of No. 15 and No. I6, the largest probes of the series, and shows the correct shape of the tips and the curve which experience has shown to be most convenient.

* "The Use of Large Probes in the Treatment of Strictures of the Nasal Duct," "Trans. of the Medical and Chirurgical Faculty of Maryland," I877, p. 154 . 
Although other ways of treating strictures of the nasal duct are advocated, such as dividing them with a suitably shaped knife, ${ }^{*}$ inserting styles, etc., their

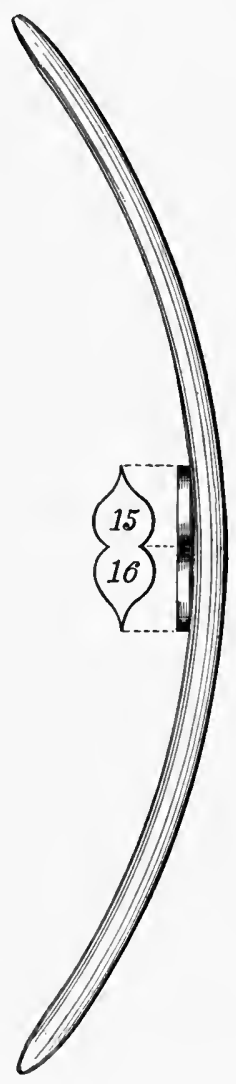

Fig. 59.-Author's lacrimal probe.

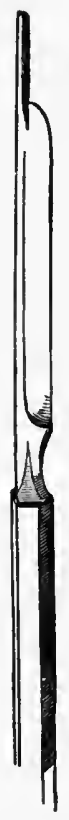

Fig. 6o.-Dr. N. R. Smith's knife for dividing strictures of the nảsal duct (Norris and Oliver).

* This plan of treatment was first practised (in 1 846 ) by my grandfather, the late Prof. Nathan R. Smith, of Baltimore, who devised a knife of peculiar pattern for the purpose (Fig. 60). Many years afterward it was revived, improved upon, and brought more prominently into notice by Stilling, of Cassel. (See the author's article upon "Diseases of the Lacrymal Apparatus" in Norris and Oliver's "System of Diseases of the Eye," Vol. III.) 
thorough dilatation by means of probes sufficiently large for the purpose is the method which, in my opinion, yields by far the best results, and which I unhesitatingly commend to others.

Unquestionably there are cases of stenosis of the duct, especially such as are associated with and dependent upon severe chronic nasal disease, that are not amenable to any plan of treatment; but such cases are rare, and, apart from these, the dilatation treatment with large probes, if systematically carried out, commonly yields most gratifying results, a complete and permanent cure, even in cases of long standing, being its usual outcome.

Briefly described, the method of dealing with strictures of the nasal duct which long experience has led me to place such confidence in is as follows:

Cocain (4 per cent. solution) or holocain (I per cent. solution) having been instilled several times into the inner corner of the eye, and, alternating with these instillations, several applications of adrenalin solution ( I : I000) having been made, a No. 2 probe, or a No. I followed by a No. 2, is passed through the lower canaliculus into the sac, for the double purpose of slightly dilating the punctum and canaliculus and of discovering, and, if possible, overcoming, any stricture which may exist at this point. If a stricture too firm to be overcome by either of these probes is encountered (the usual site of such strictures being at the juncture of the canaliculus with the lacrimal sac) the straight, sharppointed probe (Fig. 5I) is substituted, and with a drilllike motion the obstruction is penetrated. This preliminary probing I regard as important, as it insures the complete division of the canaliculus which is to follow. In the manner already described (p. 128) and with 
the straight, probe-pointed knife (Fig. 52) the canaliculus (always the lower canaliculus) is now divided well up to its juncture with the sac. The next step is the passage of the probe through the occluded nasal duct, as large a probe being used as can be readily introduced into the sac through the divided canaliculus. This is usually a No. 5, exceptionally a No. 6, probe. In passing the probe I prefer, as in dividing the canaliculus, to stand behind the patient, using the right hand

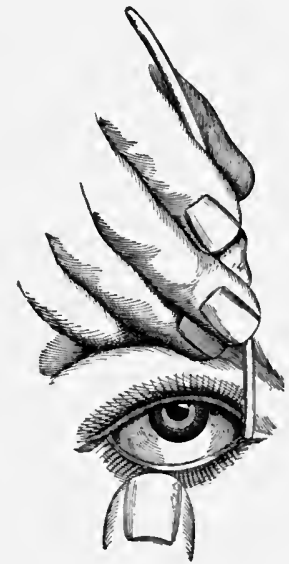

Fig. 6r.-Introduction of lacrimal probe. for the right eye and the left hand for the left eye, because the patient's head can be more easily steadied in this position, and because, moreover, it is more convenient for the operator.

The probe selected, which previously has been sterilized by brief boiling, and anointed with sterile vaselin, is passed in the usual way, first, horizontally, along the divided canaliculus, the lid being kept upon the stretch with the thumb of the opposite hand, until its point has entered the sac and come in contact with its inner wall; then it is turned into a vertical position, and passed slowly through the duct until it reaches the floor of the nose (Fig. 6I). Provided the probe has entered fairly into the lacrimal sac, any reasonable amount of force that may be required to pass it through the occluded duct is considered permissible, care, of course, being exercised that it does not take a wrong direction. The probe is withdrawn after having been allowed to remain in the duct for from ten to twenty minutes. 
If, as sometimes happens, neither a No. 5 nor a No. 4 probe can be made to enter the sac directly after the division of the canaliculus - an indication that there is an undivided constriction at the juncture of the canaliculus and the sac, or that the point of the probe has caught in the freshly cut tissues-an attempt is made to dilate the constriction by means of the "supplementary probe" (Fig. 62), which was devised to meet this particular condition.*

If this attempt also fails, if even the slender tip of this probe cannot be made to enter the sac, an interval of forty-eight hours is allowed to elapse without further effort; when the difficulty previously encountered will

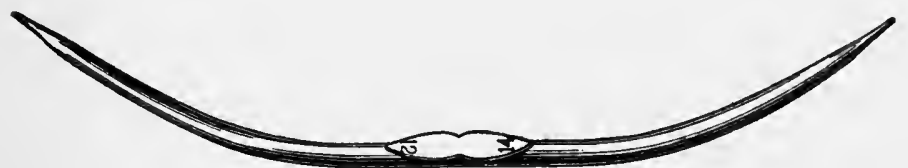

Fig. 62.-Author's supplementary lacrimal probe (about two-thirds actual size).

often be found in great measure to have disappeared. Should this not prove to be the case, an opening is drilled through the constriction with the straight, sharp probe (Fig. 5I), or a No. 5 probe is passed along the divided canaliculus to the point of resistance, the lid being kept well upon the stretch, and is then turned vertically and forced through the obstruction-a procedure, however, which it is desirable to avoid, as it may result in making a false passage from the canaliculus directly into the duct. Exceptionally, it is found necessary to divide the constriction with a sharp-pointed knife, for which purpose a narrow-bladed Sichel cataract knife has been found especially convenient.

* "Trans. American Ophthalmological Society," 1901, p. 398. 
During the early stages of the treatment the probing is repeated every other day, usually a size larger probe being passed each time. Occasionally, if the probe last introduced was passed very easily, a size is skipped, while, on the other hand, the same probe is passed more than once, if found to be tighter than usual.

The size of the largest probe which it is desirable to use varies, of course, in different cases, but it is seldom best to stop short of No. 14; for it is to be borne in mind that the end in view is not simply the making of a small opening through the strictures, as was formerly done with such unsatisfactory results, but their complete obliteration, and the restoration of the normal caliber of the duct, since it is only in this way that frequent relapses are to be avoided and permanently good results assured. In about twothirds of the cases, including those occurring in children as well as those in adults, the No. 16 probe is used.

In passing the larger probes considerable force is sometimes employed. Experience has shown not only that this is permissible, but that, instead of being harmful as many maintain it must be, the effect upon the carious walls of the duct is distinctly beneficial, the result being not unlike that produced by the curetting of diseased bone in other parts of the body (Fig. 63).

When as large a probe has been introduced as is deemed advisable, the interval between the probings is gradually increased, first to three or four days, then to a week, a fortnight, and finally to a month or two months; and when several of these longer intervals have elapsed, without any tendency to recontraction having manifested itself, the case is dismissed with full assurance that a permanent cure has been effected. 
Including these longer intervals, the treatment frequently extends over a period of eight or ten months; but the active treatment, involving the frequent probings, is comprised within as many weeks.

The improvement occurs much more rapidly in some cases than in others, so that the length of time during which it is necessary to use the probes varies considerably. It is a safe rule not to discontinue the treatment as long as there is any dacryocystitis or any roughness of the walls of the duct noticeable upon passing the

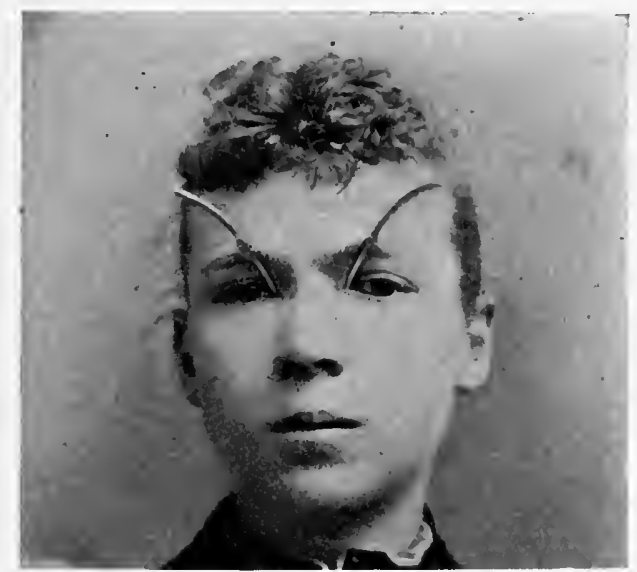

Fig. 63.-Position of lacrimal probes introduced through lower canaliculus.

probe. However, as it sometimes happens that the inflammation is kept up by the too frequent use of the probe, it is well, in obstinate cases, to try the effect of lengthening the interval between the probings.

When an attempt is made to pass a probe after the lapse of six or eight weeks, it is sometimes found difficult or impossible, owing to a contraction having occurred at the juncture of the canaliculus and the sac, to introduce the size which was previously employed. When this happens, the constriction is dilated by means 
of the "supplementary probe" (Fig. 62), after which the probe previously used may commonly be passed without difficulty.

No attempt is made to inject with a syringe antiseptic or other solutions into the lacrimal sac. Instead, a collyrium is prescribed, which the patient is instructed to drop into the inner corner of the eye three times a day, after having previously pressed out the contents of the sac with the finger-tip. The collyrium which has been found most useful for this purpose is a solution of bichlorid of mercury (I : I2,000 to I : 8000) with the addition of sodium chlorid ( $\mathrm{I}$ per cent.). A solution of alum and boracic acid (alum., gr. j-ij; acid. boracic., gr. x; aquæ destil., $\mathbf{3}$ j) has also given good results, and so has a weak solution ( 2 per cent.) of protargol.

The presence of a lacrimal fistula, even when accompanied by caries of the underlying bone, has not seemed to call for special treatment, beyond the snipping off, or cauterization with silver nitrate, of exuberant granulations, if they happen to be present; for it has been found that, as soon as thorough drainage has been established by the passage of the large probes, the fistula heals, and the carious bone, taking on a healthier action, becomes re-covered with periosteum.

The frequent dependence of disease of the lacrimal apparatus upon nasal catarrh is kept in mind, and whenever it seems to be indicated treatment is directed to the nose. A solution of bichlorid of mercury, sodium chlorid and glycerin (hydrarg. bichlorid., gr. ss; sodii chlorid., gr. xv; glycerin., §̋ss; aquæ destil., §̋ivss) applied to the nose three times a day, by means of a hand atomizer, has been found especially useful under such circumstances. Constitutional treatment is occa- 
sionally called for, more especially when the lacrimal affection is dependent upon inherited or acquired syphilis or upon struma. Ammonium chlorid, in tengrain doses, has been found beneficial, more particularly when nasal catarrh is present.

When patients from a distance are urable to remain under treatment as long as is thought desirable, it has been found practicable, after the duct has been thoroughly dilated, to teach them to introduce the probes themselves. In this way relapses, which might have occurred from a too early discontinuance of the treatment, have been avoided. The probe represented in Fig. 64 (usually made to correspond in size with No.

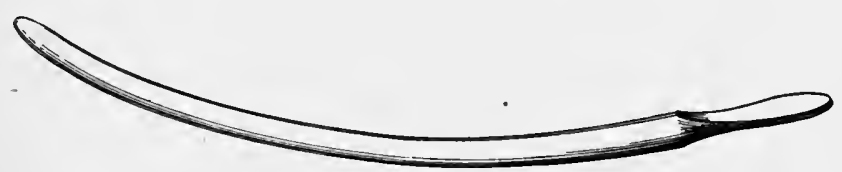

Fig. 64.-Author's lacrimal probe for use by patients (actual size).

I3) was devised for this purpose, and has proved very useful.

Electrolysis has been tried to a limited extent, in connection with the large probes, to hasten the obliteration of the strictures, but its effect was inappreciable.

In intractable cases of stenosis of the nasal duct, accompanied by persistent dacryocystitis, which have failed to yield to less radical measures, removal of the lacrimal gland, and also excision of the lacrimal sac or its destruction by means of caustics or by means of the galvanocautery or thermocautery, are practised by some surgeons, and, it is claimed, with excellent results. I have had no experience with these procedures, and have seldom encountered cases in which they seemed to be demanded. As to the use of styles, 
which some authorities still commend, I abandoned them, after a thorough test of their merits, many years ago, because the good which they accomplished proved, in almost every instance, to be but temporary.

In the transient occlusion of the nasal duct which commonly accompanies the dacryocystitis of the newborn, previously referred to, operative treatment is seldom called for, since the blennorrhea of the sac and the epiphora usually disappear as a result of the use of one of the collyria which have been spoken of (bichlorid of mercury, alum and boracic acid, or protargol). Should this, after persistent trial, not prove to be the case, however, the canaliculus should be divided, and the duct probed. The outcome of this treatment is usually very satisfactory, and it is seldom necessary to repeat the probing oftener than five or six times. In a case of this character which was operated upon when the child was fifteen months old, a No. I 2 probe was passed without difficulty, and a complete cure was soon effected. 


\section{CHAPTER V. \\ DISEASES OF THE CONJUNCTIVA.}

There are no diseases of the eye with which it is more important that the general practitioner should be familiar than those which have to do with the conjunctiva. This is true not only because they are of very common occurrence, and it must needs happen that he will often be called upon to treat them; but because it is frequently impracticable for him to refer such cases to the specialist, and because, moreover, there is, in most instances, little reason why he should not himself be able to deal with them successfully.

As has already been intimated, the usual shortcoming of the general practitioner in dealing with diseases of the conjunctiva is in the direction of faulty diagnosis; for the indications for treatment are commonly clear when once a correct diagnosis has been reached. It is of the first importance, therefore, that he should be able not only to distinguish conjunctival inflammation from inflammation of the deeper structures of the eye, but to recognize the several varieties of conjunctivitis, since the treatment which they call for is essentially different.

The medical practitioner unfamiliar with diseases of the eye will be saved, at least, a goodly number of the diagnostic errors into which he is apt to fall, if he will bear in mind that, speaking broadly, inflammation of the conjunctiva is not accompanied by severe pain or pronounced photophobia; that it is attended 
by more or less abundant secretion of mucus or mucopus; that it does not appreciably impair sight, except through the presence of this discharge, or through implication of the cornea, as in purulent or trachomatous conjunctivitis; that the vascular injection which attends it is made up largely of coarse, superficial, movable vessels, which run irregularly in all directions; that the injection is brick-red in color, and that the redness is not confined to, or especially marked in the neighborhood of, the corneal border; and if he will remember, on the other hand, that the presence of severe pain, accompanied usually by photophobia and lacrimation, and by more or less pronounced obscuration of vision, and attended by pericorneal injection, pinkish in color, and composed of fine, immovable, subconjunctival vessels, points unmistakably either to corneal inflammation or to inflammation of the deeper structures of the eye, such as iritis, cyclitis, glaucoma, etc.

Again, in the matter of ocular therapeutics, he will have made a long stride in the right direction, if he will further bear in mind that in the treatment of conjunctival inflammations, as well as of other inflammations of the eye, severe remedies-applications which cause pain and which increase photophobia and lacrimation-are, almost without exception, contraindicated.

In the account about to be given of the more prevalent affections of the conjunctiva the difficulties with which the general practitioner has to contend in the matter of differential diagnosis will be kept in mind, and every effort will be made to afford him assistance in this direction. The suggestions regarding treatment will also be made as definite and concise as practicable.

Hyperemia of the Conjunctiva.-Hyperemia of 
the conjunctiva, when pronounced, is usually accompanied by some discomfort of the eyes-a sensation of burning or itching-and by undue lacrimation. It occurs as a transient and also as a chronic condition.

Transient or acute conjunctival hyperemia may arise from a variety of causes, and, as a rule, is a matter of but little moment. The presence of a foreign body upon the cornea or conjunctiva, exposure of the eyes to a strong wind, to undue heat or light or to irritant gases, prolonged use of the eyes, especially with imperfect illumination, crying, etc., are some of the causes which may give rise to it. It also marks the onset of most superficial inflammations of the eye, and is a frequent accompaniment of acute rhinitis and of facial neuralgia.

Chronic hyperemia of the conjunctiva, a condition of greater significance, is more frequently due to eyestrain, the result of errors of refraction or anomalies of the ocular muscles, than to any other one.cause. It may be dependent also upon chronic rhinitis, inflammation of the lacrimal passages, trichiasis, alcoholism, and gout.

In the treatment of this condition the most important consideration is the removal of the cause. In chronic hyperemia the refraction and the muscular balance of the eyes should be examined, and glasses should be prescribed if found to be indicated. Nasal or lacrimal disease, if present, should be treated, and measures should be taken to combat any disorder of the system, such as a gouty diathesis, which might be a factor in the causation of the local affection. As supplementary measures, a collyrium of boracic acid (acid. boracic., gr. $\mathrm{x}$; aq. destil.,, $\mathbf{j}$ ) or of boracic acid and sulphate of zinc (zinci sulphat., gr. $\frac{1}{4}$; acid. boracic., gr. x; aq. 
destil., $\tilde{5}$ j), to be dropped into the eyes three times a day, and, especially in acute cases, the application to the lids of cold water or ice-cloths, are useful.

\section{CONJUNCTIVITIS.}

Whether regarded from a clinical or a pathological point of view, all of the commonly recognized types of conjunctivitis may very properly, and with practical advantage, be classified as follows: First, catarrbal or simple conjunctivitis; second, purulent or gonorrbeal conjunctivitis; third, croupous or membranous conjunctivitis; fourth, diphtheritic conjunctivitis; fifth, follicular conjunctivitis; sixth, tracbomatous or granular conjunctivitis; seventh, vernal conjunctivitis or spring catarrb; and, eighth, phlyctenular or scrofulous conjunctivitis.

As a rule, it is not difficult to differentiate these several varieties, which, though they possess certain features in common, exhibit other well-marked and distinctive characteristics; but, occasionally, the most experienced observer may find himself at fault in this respect, being unable to determine, simply from inspection of the eye, to which class a particular case should be assigned. However, the history of the case, and especially its behavior under treatment, will usually dispel any doubt upon this point. With the exception of phlyctenular conjunctivitis and of diphtheritic conjunctivitis, all of these different types of conjunctival inflammation are essentially local disorders. As regards their etiology, much has yet to be learned, though considerable progress has been made in this direction within the past few years.

Catarrhal or Simple Conjunctivitis.-This variety 
of inflammation of the conjunctiva, which is of very common occurrence, is met with as an acute, and, less frequently, as a.chronic, affection.

In a well-marked attack of acute catarrbal conjunctivitis the patient complains of a sensation as though sand were in the eyes, exhibits some little dread of light, and, upon being questioned, commonly states that his eyes smart when he attempts to read or write, that after sleep the lashes are stuck together by discharge, and that during the day some discharge collects about the inner cornea of the eyes and upon the lid-margins, and requires to be wiped away from time to time. Upon inspection, the lids will be found to be somewhat edematous, and the bulbar conjunctiva to be markedly injected and of a nearly uniform brickred color, the injected blood-vessels being superficial, coarse, tortuous, and movable. The palpebral conjunctiva also will be congested and somewhat swollen, and strings of mucus will be discovered upon the inner surface of the lids and in the retrotarsal folds. The cornea and iris present a normal appearance, and the pupil responds to light as in health (Plate III, Fig. I).

In the milder cases, which are the more common, the lids are not edematous, and the bulbar conjunctiva is but slightly, if at all, injected, the evidences of inflammation being limited to the palpebral conjunctiva and to the retrotarsal folds, the diagnosis, under such circumstances, being based mainly upon the appearance of the inner surface of the lids, the sudden onset of the attack, and the gumming of the eyelashes during sleep. Another feature of acute catarrhal conjunctivitis, which is of decided diagnostic value, is that it is essentially a binocular affection. One eye, it is true, 
is often attacked before the other, but within twentyfour hours both eyes are sure to be affected.

A variety of phlyctenular conjunctivitis-the catarrhal type-very closely resembles acute catarrhal conjunctivitis; but as this affection is usually monocular, and is commonly accompanied by blepharitis, eczema of the lids or face, or other signs of constitutional disorder, it is not difficult, as a rule, to recognize its true character. The character of the conjunctival injection, the absence of changes in the cornea or iris, the normal size and reaction of the pupil, and the nonexistence of pain suffice to differentiate the disease under consideration from inflammation of the cornea or iris and from inflammatory glaucoma. As the symptoms produced by the presence of a foreign body upon the cornea or beneath the upper lid very closely resemble those of a commencing conjunctivitis, one should be careful to avoid falling into a diagnostic error of this character.

Acute catarrhal conjunctivitis, which occasionally assumes an epidemic character, and under such circumstances is popularly known as "pink eye," is unquestionably, though not markedly, contagious. As to its etiology, there can be no. doubt that in most instances it is of bacterial origin. "Cold" probably is at times a factor in its causation; but doubtless acts chiefly by rendering the conditions more favorable for the development of the invading bacteria. The Weeks bacillus and the pneumococcus are the microorganisms which are most frequently concerned in its production.

Under favorable hygienic conditions the disease is usually self-limited, and disappears within ten days or a fortnight; but occasionally, when neglected, it 
runs a protracted course, lasting, perhaps, for many weeks.

Treatment.-The treatment of this variety of conjunctivitis, which has proved so efficacious that I seldom have occasion to employ other measures, consists in the use of a collyrium of zinc sulphate and boracic acid (zinci sulphat., gr. ss; acid. boracic., gr. $\mathrm{x}$; aq. destil., $\tilde{5} \mathrm{j}$ ), which is dropped into the eyes three times a day, and the application of a bland ointment to the lids at bed-time. For this latter purpose "coldcream," to which boracic acid may be added in the proportion of five grains to the dram, is well adapted. When the eyes are more than usually irritable and uncomfortable, relief is afforded by the application to the lids of pads of absorbent gauze wet with a lotion of opium and boracic acid (ext. opii., gr. x; acid. boracic., gr. xl; aq. destil., ऊiv). In the event of this treatment not proving promptly efficacious, a weak solution of argyrol (5 per cent.) or protargol (2 per cent.) may be substituted for the zinc and boracic acid, being dropped into the eyes three times a day.

Chronic catarbal conjunctivitis, which, as has been said, is less common than the acute type, owes its chronicity, as a rule, to some extraneous cause. Among the conditions that may give rise to it may be mentioned accommodative strain, disease of the lacrimal apparatus-especially when attended by blennorrhea of the lacrimal sac-partial ectropion, chronic rhinitis, unfavorable hygienic surroundings, as in crowded reformatories, etc., and habitual exposure of the eyes to irritating gases or to heat and dust. The conjunctival injection and discharge may be, but usually are not, pronounced.

Treatment.-The collyrium of zinc sulphate and 
boracic acid, as recommended in the acute form of the disease, is the most efficacious local remedy; but of greater importance is it to discover, and, if possible, to eliminate, the condition upon which the affection depends. Any refractive or muscular anomaly found to be present should be corrected by the careful adjustment of glasses; disease of the lacrimal apparatus or of the nose, if it exists, should receive attention; the canaliculus should be slit, if there is eversion of the punctum; and the patient's surroundings and his occupation, if they are such as to favor the continuance of the disease, should, if possible, be changed for the better. Good food and tonics-iron, quinin, and strychnin, especially-by building up the system will often accomplish much.

Purulent or Gonorrheal Conjunctivitis.-This severe type of conjunctival inflammation is met with in the new-born-the so-called ophthalmia neonatorum - as a result of infection of the eyes during the passage of the child's head through the vagina, and in later life in consequence of accidental inoculation of the eyes with gonorrheal discharge from a specific urethritis or vaginitis, or from a previously infected eye. The purulent conjunctivitis of the infant and the gonorrheal ophthalmia of the adult are, therefore, essentially one and the same disease, each being due to inoculation of the conjunctiva with the gonococcus. (See Plate II, Fig. I.)

In purulent conjunctivitis the inflammation is much more intense than in catarrhal conjunctivitis. The discharge, which is thick and creamy and distinctly purulent, is very profuse, so that it overflows the lids, and runs down upon the cheek. The lids are greatly swollen, often of a dusky red color, and so tense that 
PLATE II.
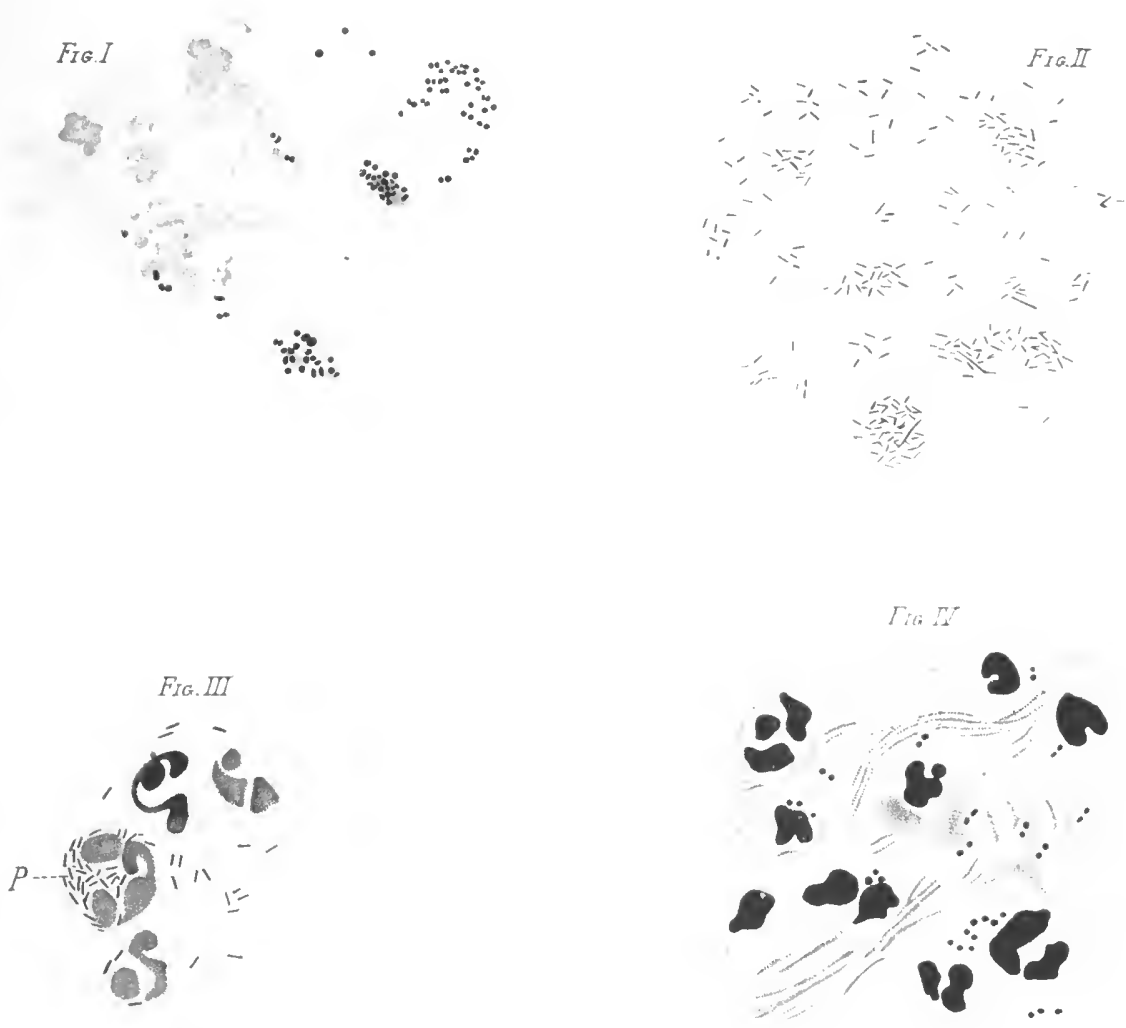

Fig. I.-Discharge from Right Ere in a Case of Purulent conjunctivitis; Gonococci Numerous in Cells (Stephenson).

Fig. II.-Bacillus of Weeks in Pure Culture (from a PhotoGRAPH) (WEEKS).

Fig. III.-Conjunctival Secretion from Acute Contagious Conjunctivitis; Polynuclear Leukocytes with the Bacillus of Wheks; $P$, Phagocyte Containing Bacillus of Weeks; Imales. $\frac{1}{1}$, OC. iii (MORAx).

Fig. IV.-Secretion from a Case of Conjunctivitis, Showing PNEUMoCoCCI; ImMers, $\frac{1}{12}$, OC. iii (MORAX). 

it is usually impossible to evert them (Plate III, Fig. 2). The bulbar conjunctiva is intensely injected, and so chemotic that it overlaps the cornea, and may even hide it completely from view.

As a rule, in ophthalmia neonatorum the disease runs a less malignant course, and the prognosis is less grave, than in the adult. This, it would seem, is largely because of the fact that the discharge which infects the eyes of infants is seldom due to a recently acquired gonorrhea, whereas in adults the inoculation is more apt to occur during the height of the urethritis or vaginitis, when the infectious power of the discharge is greatest. Apart from this, however, it would appear that the eyes of the new-born are capable of withstanding gonorrheal infection better than are those of adults. The interval between the inoculation of the eye and the appearance of the first signs of the disease-which at the outset are much like those of a well-marked attack of acute catarrhal conjunctivitis-is somewhat greater in the infantile than it is in the adult form of the affection, the incubation period in the latter being from twelve to forty-eight hours, whereas in the former it is usually from forty-eight to seventy-two hours. In ophthalmia neonatorum (Fig. 65) both eyes are usually affected, because each is almost sure to be inoculated with the vaginal discharge; in adults, on the other hand, the disease is commonly monocular, though there is always great danger that the second eye may become affected through the transference of discharge from the one first involved.

The disease runs a tedious course, and, even when promptly and carefully treated, is seldom cured under four to six weeks. Severe pain, marked photophobia, and lacrimation characterize the height of the attack. 
The great danger to be feared, in both forms of the disease, is necrosis of the cornea (Fig. 66). This probably results, not infrequently, from a secondary infection; but it is due, primarily, to the nutrition of the cornea being seriously interfered with

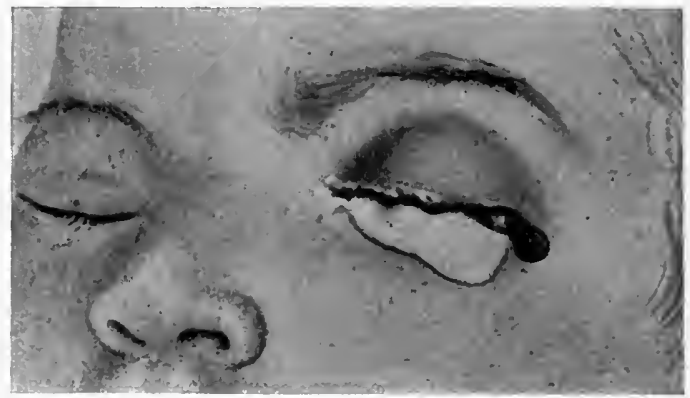

Fig. 65-Purulent (gonor-heal) conjunctivitis in the new-born (Haab).

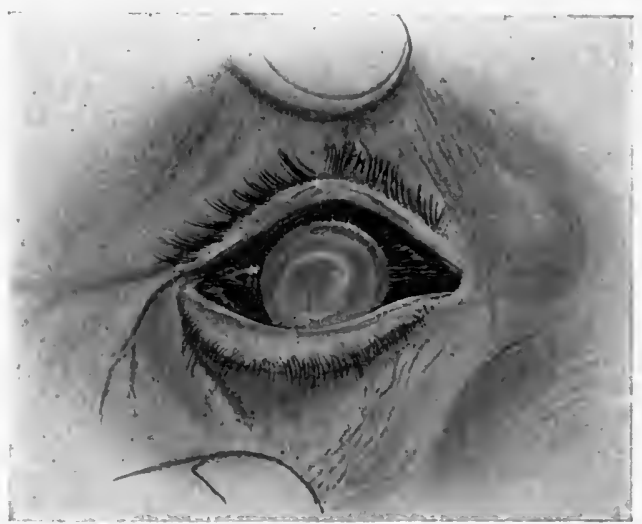

Fig. 66.- Extensive necrosis of the cornea (Ramsay).

through the intensity of the conjunctival inflammation. Complete destruction of the cornea, which is by no means uncommon, necessarily involves loss of useful vision; but, if the destruction is not complete, a considerable amount of sight may be regained, either 
PLATE III.

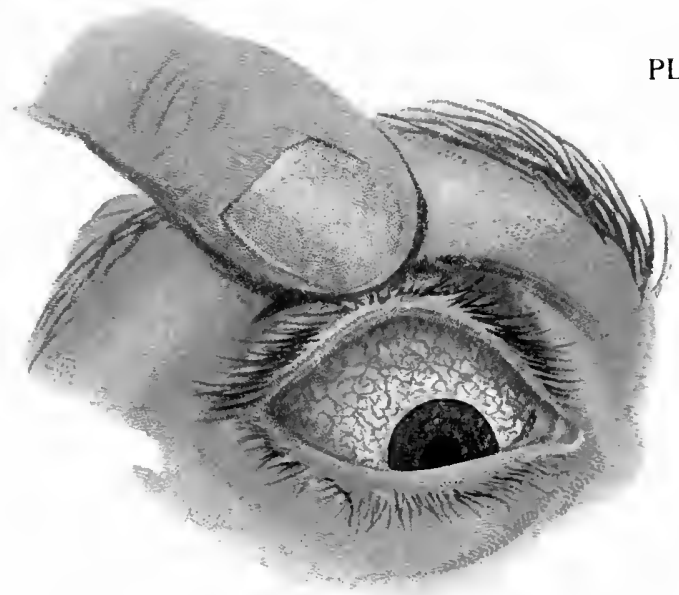

Fig. i.-Acute Catarkhal Conjunctivitis.

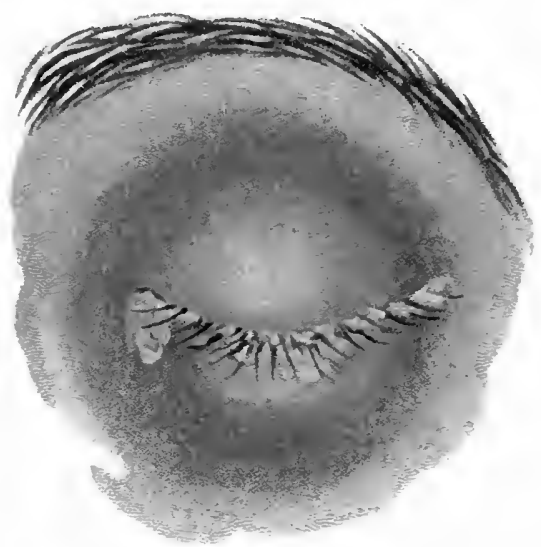

Fig. 2.-Purulent Conjunctivitis (Gonorrieal).

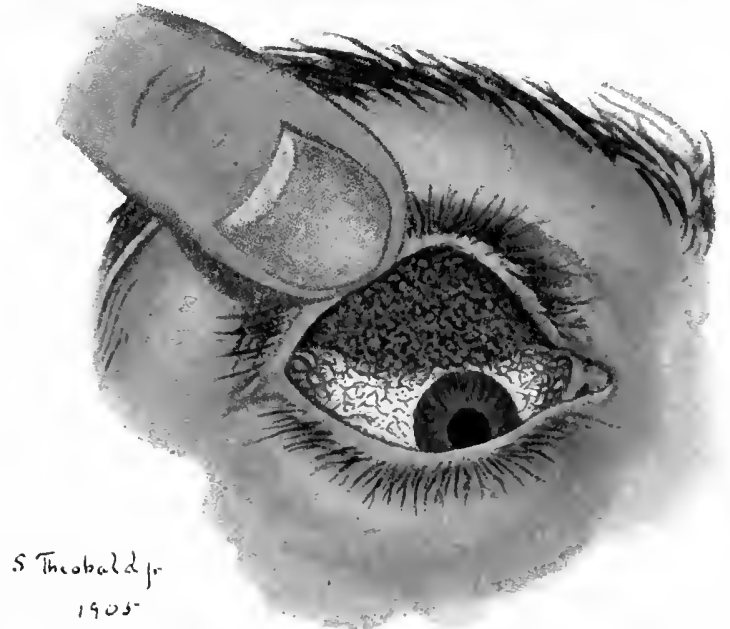

Fig. 3--Acute Trachomatous Conjunctivitis (Papillary Variety). 

with or without the help of an iridectomy. When the discharge, instead of being thick and creamy, is thin and watery, and is accompanied by a membranous exudation upon the surface of the palpebral or bulbar conjunctiva and by a plastic infiltration into the subconjunctival cellular tissue, the prognosis is distinctly unfavorable, and the danger of corneal complications very much greater.

Although more tractable than the adult form of the disease, ophthalmia neonatorum, because its treatment is so often neglected, is the most fruitful source of incurable blindness. According to the statistics gathered by Magnus, twenty-four per cent. of the inmates of the institutions for the blind in the different countries of Europe owe their loss of sight to this one disease-a showing wholly inexcusable, as it is largely the result of unpardonable neglect and ignorance on the part of those having the care of the new-born.

One of the not very unusual consequences of gonorrheal conjunctivitis is anterior staphyloma. (See Plate VI, Fig. I.) In the less severe cases, or in those in which treatment has been begun promptly, necrosis of the cornea, if it occurs, is more apt to be circumscribed. Under such circumstances an opacity of the cornea is left, and to this a knuckle of the iris is frequently adherent (anterior synechia). (See Fig. Ioo.) The degree of impairment of vision in such cases will depend upon the density and the location of the opacity, whether it is central or peripheral, and upon the situation of the displaced pupil.

Treatment.-As a preliminary step to the employment of remedial measures, if there be any doubt as to the diagnosis, the discharge from the eye should be subjected to microscopic examination, in order that the 
presence or absence of the gonococcus may be determined. Its presence in the discharge definitely establishes the diagnosis (Fig. 67). Failure to find it at one examination, however, is not conclusive. Therefore, if the general symptoms point to gonorrheal infection repeated microscopic examinations should be made, and, meantime, it is safest to treat the case as though its specific character had been established. The fact that the disease is acquired at times in an entirely

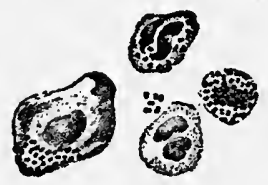

$a$

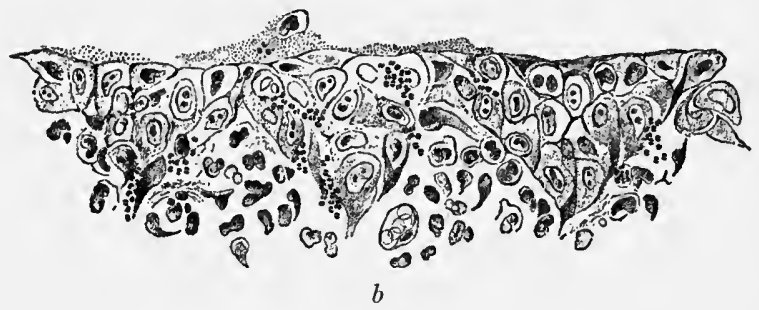

Fig. 67.- $a$, Gonococci free and in the cells (Bumm); $b$, gonococci in the conjunctival tissues (Bumm).

innocent manner, and by persons who have not themselves had gonorrhea of the genito-urinary tract, should not be lost sight of.

To secure the best results, it is essential that the treatment should be begun promptly, and that it should be carried out intelligently and assiduously; for there is no other disease of the eyes, it may be remarked, which demands such unremitting attention.

Until the disease is well under control, that is to say, 
until there has been a decided abatement of the conjunctival inflammation, of the edema of the lids, and of the discharge, the eye should be bathed as often as every hour or, if practicable, every half hour, day and night, with a saturated solution (gr. xviij to $\tilde{j}$ ) of boracic acid, pledgets of absorbent cotton, wet with this solution, being used to douche the eye, and to remove the discharge from the lids and from the conjunctival sac. While this should be done as thoroughly as practicable, it should be done with a gentle hand, and the greatest care should be exercised not to abrade the corneal epithelium, since this accident materially increases the danger of corneal ulceration. - In addition to this careful cleansing of the eye, in the adult form of the disease ice-cloths, if found to afford relief, as is usually the case, should be applied constantly to the lids. The only contraindication to their use is the existence of a thin, watery discharge, accompanied by a membranous exudation upon the surface of the conjunctiva. In such cases the likelihood of corneal necrosis-always great, as has been pointed out-may be increased by the depressing effect of cold; therefore, until the character of the discharge changes, until it becomes purulent, it may be desirable even to employ hot fomentations instead of the cold compresses.

The "ice-cloths," consisting of pads of absorbent gauze made cold by lying upon a block of ice, which is kept close at hand, must be changed frequently to afford the best results. An intelligent and not too awkward patient may be trusted to carry out this feature of the treatment himself. In ophthalmia neonatorum the application of cold is not, in my judgment, called for. In the first place, it is impracticable to apply it 
effectively, and, in the next place, as abundant experience proves, the withholding of it does not influence unfavorably the progress of the disease.

The other measure of importance in the treatment of purulent ophthalmia consists in the careful application of a sufficiently strong solution of argyrol or protargol. Formerly, in common with most ophthalmic surgeons, I employed in this disease silver nitrate, usually in two per cent. solution; but my experience with these newer agents has been so eminently satisfactory that $I$ now feel warranted in using one or the other, as a matter of routine, instead of the silver nitrate. The advantages of argyrol and protargol are that they are more penetrating in their action, that they seem to control the inflammation more effectually, and that even the strongest solutions are far less irritating than are the comparatively weak solutions of the older salt. To be effective, however, it is essential that they should be used in strong solution. A forty per cent. solution (forty parts to sixty parts of water) is about a saturated solution of protargol, and this is the strength in which I am in the habit of using it, one application being made to the eye daily, in addition to which a weaker solution (ten to twenty per cent.) is applied twice each day. Argyrol, being more soluble, can be used in stronger (fifty per cent.) solution, and, being less irritating, can be applied more freely-as often as three times a day.

When practicable, the application should be made to the everted lids, by means of a cotton mop; but when, as usually happens, it is impracticable to evert the lids the solution should be applied, as thoroughly as possible, to the palpebral and bulbar conjunctiva with a mop or an eye-dropper, as may be found more effective. An- 
other useful measure is the instillation, once or twice a day, of a sterile solution of atropin-a four-grain-tothe-ounce solution in the case of adults, a one-grain solution in infants. This, besides affording the patient a measure of relief, favorably influences any corneal complication which may be present.

In ophthalmia neonatorum both eyes, as has been stated, are usually infected at birth, and when this is not the case subsequent infection of the second eye is almost sure to occur, in spite of any efforts which may be made to prevent it. In the adult form of the disease, on the other hand, while the danger of involvement of the fellow-eye is always great, it is, as a rule, possible with proper precautions to avoid this. There are several ways in which infection of the sound eye may occur. The greatest danger is that the patient may transfer the discharge from one eye to the other with his fingers. There is also the possibility that in bathing the affected eye some of the pus may find its way into the other eye; and, again, if, through lack of attention, the discharge is allowed to accumulate, it may flow across the bridge of the nose, and thus reach the sound eye.

To lessen the risk of the second eye becoming infected, it is the practice with many surgeons to seal up this eye hermetically by means of what is known as Buller's shield (Fig. 68). This consists of a watch-glass secured between two suitably shaped pieces of rubber adhesive plaster, in each of which a circular opening, smaller than the watch-glass, has been cut. This is fastened over the eye in such manner as to permit free movements of the lids, being applied with exactness to the brow, side of the nose, and lower margin of the orbit. To render it more secure, reinforcing strips of plaster 
should be used, and, as infection is most apt to occur at the nasal edge of the shield, a coating of collodion should be applied here. The employment of the watchglass (the suggestion of Dr. Buller) enables the patient to use the eye, and the surgeon to inspect it, and to watch for signs of commencing inflammation.

An objection to the use of the shield is that one seldom knows, when a case of monocular purulent conjunctivitis comes under observation, whether infection of the other eye may not have occurred already.

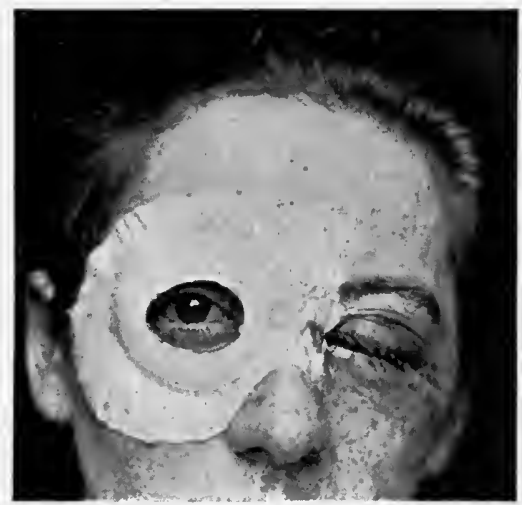

Fig. 68.-Buller's shield (Hansell and Sweet).

If this has happened, it is of the utmost importance, in order that no time should be lost in the employment of energetic therapeutic measures, ${ }^{*}$ that we should be

* The advice given in most text-books upon diseases of the eye, that in gonorrheal conjunctivitis the use of silver nitrate should not be begun too early, should not be commenced, in fact, until the discharge has assumed a distinctly purulent character, is, in my opinion, wholly bad. The sooner the silver salt (whether the nitrate or protargol or argyrol) is begun, the greater is the probability that the disease will be kept under control, and that the eye will be saved. That I have never lost the second eye, when it became involved while the case was under my observation, I attribute to the fact that the nature of the affection was recognized at the very outset, and that energetic treatment (the use of a silver salt) was begun without a moment's delay. 
able to detect the very first signs of beginning conjunctivitis, and, unquestionably, this cannot be done as well when the shield is in position. Moreover, the confining effect of the shield (although, with the view of obviating this, its temporal edge is usually left free for ventilation) tends of itself to excite a conjunctivitis of catarrhal type, which must necessarily befog the situation.

In addition to warning the patient as to the risk of infecting his sound eye, it is the duty of the physician to impress upon those, nurses or others, who may have charge of a case of gonorrheal conjunctivitis, how important it is that they should exercise the greatest care to guard against infection not only of the patient's other eye but of their own eyes and of the eyes of others about them. He should instruct them not to touch the patient's sound eye with infected fingers; to be careful, in bathing and making applications to the affected eye, not to permit any discharge or any possibly infected fluid to find its way into the other eye; to allow no infected dressings or solutions to stand about where harm might come from them, and after each handling of the patient's eye to wash their hands, immediately and thoroughly, with soap and water.

In adults, internal remedies are at times indicatedmorphin to relieve severe pain, trional to control less severe pain and to induce sleep, quinin in liberal doses when necrosis of the cornea threatens, or in combination with iron when the vitality of the patient is reduced.

Much benefit has resulted from the propbylactic measures proposed by Credé for the prevention of ophthalmia neonatorum, and they should be resorted to in every case in which the mother is known to have 
a specific vaginitis, or in which there is a suspicion that such is the case. These measures consist in the syringing of the vagina for some days previous to and during labor with a three per cent. solution of carbolic acid, and in a single careful application of a two per cent. solution of silver nitrate to the infant's eyes directly after birth or, at least, as soon as they have been thoroughly cleansed. Protargol has recently been substituted for the silver nitrate with excellent results. It should be used in ten per cent. solution, in which strength it is far less irritating than the two per cent. silver nitrate solution recommended by Credé.

Croupous or Membranous Conjunctivitis. - It is with some hesitation that this variety of conjunctival inflammation is described as a distinct disease, since there are excellent reasons for regarding it rather as a type of inflammation prone to occur, under favoring conditions, in several different kinds of conjunctivitis. Reference has been made to the fact that in certain unfavorable cases of purulent conjunctivitis, attended by a thin, watery discharge, a membranous exudation tends to form upon the palpebral, and at times upon the bulbar, conjunctiva; and it may be added that a similar disposition occasionally manifests itself in catarrhal conjunctivitis. A typical form of membranous conjunctivitis is that which is induced by the application of the jequirity bean in the treatment of trachoma. Rarely, a chronic form of croupous conjunctivitis is met with, in which the membrane forms and re-forms for weeks and even months.

In true diphtheria of the conjunctiva the membrane frequently forms upon the bulbar as well as upon the palpebral conjunctiva; but in the milder affection under consideration the exudate, which does not invade 
the subconjunctival tissue as in true diphtheria, and can, as a rule, be easily detached, is usually confined to the conjunctiva of the lids. In croupous conjunctivitis the danger of corneal implication is slight; but there is commonly more pronounced ciliary irritation and more decided edema of the lids than is found in catarrhal conjunctivitis. The discharge is scant and watery. Usually after a few days the membranewhich consists of a meshwork of coagulated fibrin, pus corpuscles, and epithelial cells-ceases to be formed, and the case assumes the features of a severe catarrhal, or, perhaps, of a purulent, conjunctivitis.

The condition of the system seems to have much to do in determining this type of conjunctival inflammation. Unhealthy, ill-nourished children-the subjects of inherited syphilis, for example-are especially prone to it. It may be induced also by the injudicious employment of too severe remedies in catarrhal and in purulent conjunctivitis, as, for example, unduly strong solutions of silver nitrate.

Treatment.-This should be constitutional as well as local. Iron and quinin internally, and mild applications to the eyes, are indicated. Boracic acid (gr. $\mathrm{x}-\mathrm{xv}$ to $\mathbf{z} \mathrm{j}$ ) is useful, as is also a $\mathbf{I}: 8000$ solution of corrosive sublimate. Atropin (gr. $\mathrm{j}$ to $\mathbf{5} \mathrm{j}$ ) may be employed when there is marked ciliary irritation. After the formation of the membrane has ceased, and the discharge, previously watery, has become mucopurulent, astringents (zinc. sulphat., gr. ss; acid. boracic., gr. x; aq. destil., $\tilde{z} \mathrm{j}$, or protargol in two to four per cent. solution) are called for, but should be used with caution.

Diphtheritic Conjunctivitis. - True diphtheria of the conjunctiva, characterized by the presence of 
the Klebs-Löffler bacillus-to which condition the term "diphtheritic conjunctivitis" should be restricted -is not among the commoner diseases of the eye; still, as it is an affection which may be encountered at any time by the general practitioner, a brief account of it seems to be called for.

Like faucial diphtheria, diphtheritic conjunctivitis, one of the most dangerous diseases to which the eye is subject, occurs more frequently in children than in adults. The onset of the disease is sudden, and its

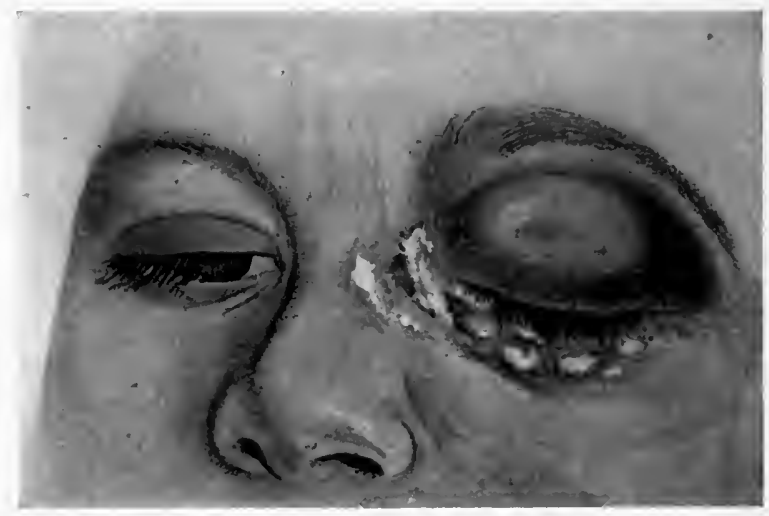

Fig. 69.-Diphtheritic conjunctivitis in a child (Haab).

development rapid. Severe pain is experienced, and the lids are not only red and greatly swollen, but, owing to the character of the infiltration, are tense and brawny (Fig. 69). The discharge at first is thin, ichorous, and scanty, but at a later stage frequently becomes purulent. The membrane, which usually forms upon the ocular, as well as upon the palpebral, conjunctiva, is thick and coherent, grayish in color, and extends so deeply into the subconjunctival tissue that it is not possible to detach it (Fig. 70). Destruction of the cornea, in consequence of the rapid and extensive 
infiltration of the bulbar conjunctiva interfering with its nourishment, is the result which is most to be dreaded. Deep sloughs, involving not only the conjunctiva but the subconjunctival cellular tissue, may occur, and the contraction resulting from this destruction of tissue is apt to produce entropion with its attendant ill consequences. The constitutional symptoms which are present in faucial diphtheria-fever, general depression, etc.-manifest themselves. Usually both eyes are involved, though this is not always the case.

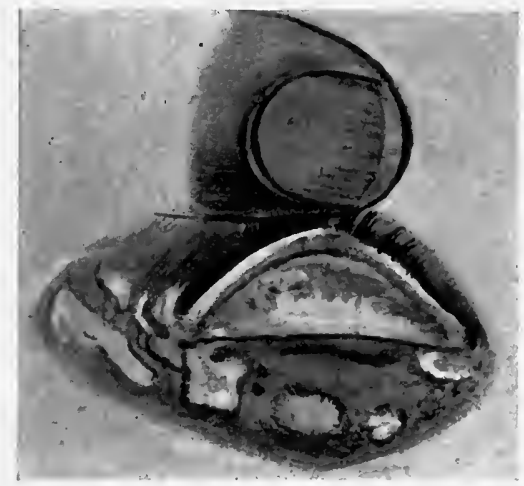

Fig. 70.-Diphtheritic conjunctivitis in a child (Haab).

The infection of the eye may be direct and primary, or it may be secondary to diphtheria of the throat and nose, occurring as a result of accidental inoculation or of extension through the lacrimal passages. Occasionally the disease manifests itself after operative procedures upon the eye. Atypical examples of diphtheritic conjunctivitis have been observed, in which the brawny infiltration of the lids and the conjunctival false membrane are absent, and yet rapid destruction of the cornea occurs, and the Klebs-Löffler bacillus is found to be present (Fig. 7I). 
Treatment.-The most important therapeutic measure, as in the faucial type of the disease, is the injection of the diphtheria antitoxin. The results of this plan of treatment have been most gratifying, and, as Jackson expresses it, "its importance overshadows that of all local remedies." Quinin, iron, stimulants, and a nutritious diet are also indicated. The local treatment consists in the application of warm fomentations, the lotion of opium and boracic acid (ext. opii, gr. x; acid.

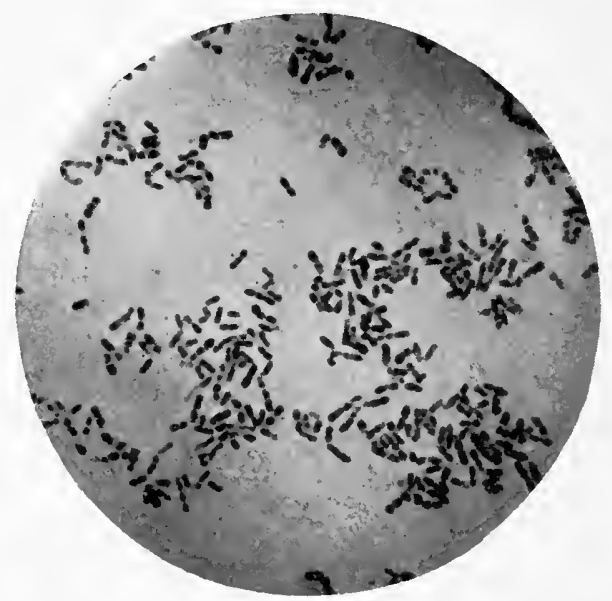

Fig. 71--Bacillus diphtheriæ, from a culture upon blood-serum; $X$ I000 (Fränkel and Pfeiffer).

boracic., gr. xl; aq. destil., ziv) being one of the best, and, for direct application to the eye, mild antiseptic rather than caustic or astringent solutions. Among these boracic acid, in saturated solution, and bichlorid of mercury ( 1 : 8000) are, perhaps, the most useful. A two per cent. solution of potassium permanganate is also recommended.

When the disease is limited to one eye, the same precautions should be taken as in purulent conjunc- 
tivitis to prevent inoculation of the fellow-eve. Those in attendance should also be warned of the danger of inoculating their own ocular or faucial mucous membrane. During the declining stage of the disease, when the membrane has been thrown off, and the discharge has become purulent, protargol in ten per cent., or silver nitrate in one per cent., solution may be employed with advantage.

Follicular Conjunctivitis.-The seat of this affection is chiefly in the palpebral conjunctiva and in the retrotarsal folds, though more or less pronounced hyperemia of the bulbar conjunctiva is often observed. Its characteristic feature is the presence of enlarged follicles-hypertrophied lymphoid tissue-in the superior and inferior retrotarsal folds. In well-marked cases it bears a close resemblance to the follicular type of trachomatous conjunctivitis; but the hypertrophied papillæ observed in this disease are not present, the enlarged follicles are more definitely limited to the retrotarsal folds, and it does not lead to pannus or deformity of the lids. It is at times, however, an obstinate affection, and not infrequently shows but little disposition to respond to treatment. It is an interesting fact that the negro race, which is almost immune from trachomatous conjunctivitis, is especially prone to this variety of conjunctival inflammation. It is probably of microbic origin, but this has not been demonstrated. It is attended by a slight, mucous discharge, by a feeling as though dust were in the eyes, and sometimes by itching.

Treatment.-A collyrium of corrosive sublimate (I : 12,000 to I : 8000) with sodium chlorid (one per cent.) is the remedy which I have found most useful. Zinc sulphate and boracic acid, as recommended in 
catarrhal conjunctivitis, and protargol (in two to five per cent. solution) I have also employed with good effect.

Trachomatous or Granular Conjunctivitis (Granular Lids).-The distinctive characteristics of this variety of conjunctivitis, which expends its force chiefly upon that portion of the conjunctiva which lines the lids and constitutes the retrotarsal folds, are its chronicity, the marked structural changes which it causes not only in the conjunctiva but in the subconjunctival tissue as well, and the secondary changes, known as pannus, which it induces in the cornea.

Its pathology is as yet but imperfectly understood; but it is, without doubt, a contagious disease, the product of a specific organism. A small diplococcus, first described by Sattler, is held by some to be the organism which produces it, but this has not been conclusively shown.

Although one of the most intractable diseases of the eye with which we have to deal, and in its ultimate consequences as disastrous to sight as any, trachomatous conjunctivitis does not, like purulent conjunctivitis, threaten the eye with immediate destruction. The inflammation does not approach in intensity that which characterizes the latter disease; nevertheless, during the acute stage which supervenes upon inoculation, there is usually marked conjunctival injection, with considerable swelling of the lids, and pronounced photophobia, lacrimation, and blepharospasm. The discharge, which is not abundant, is mucoid or mucopurulent in character. The first evidences of corneal implication, a slight roughening of the external epithelial layer, may become manifest within a few weeks of the onset of the attack. 
With the subsidence of the more acute symptoms the chronic stage of the disease begins, and this, if left to itself, may last a lifetime, rendering existence miserable, and reducing the individual to a state of helplessness and dependence; for not only is the sight greatly impaired, as a result of the corneal changes, but the eyes (for both eyes are commonly involved, though exceptions to this rule are encountered) are persistently irritable

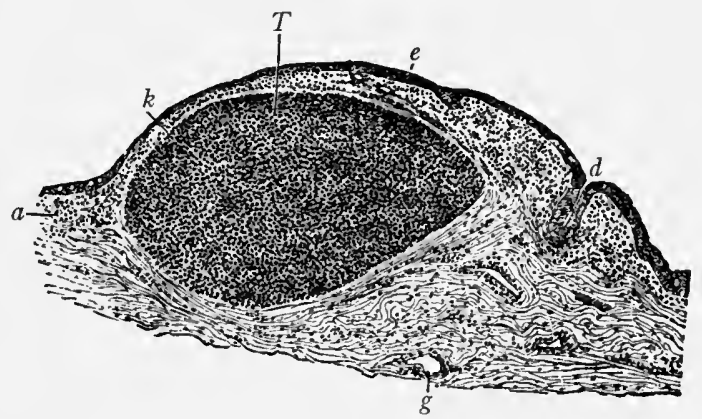

Fig. 72.-Section of a trachoma follicle from the retrotarsal fold. Magnified $24 \times I$ (Fuchs). The trachomatous granulation, $T$, pushes up the conjunctiva in the form of an elevation, and is inclosed by a layer of thickened connective tissue, the capsule, $k$. The conjunctiva is infiltrated with cells, both in its upper layers, $a$, and along the vessels, $g$; the epithelium, $e$, shows, above the place marked $a$, bright spots which correspond to the goblet cells; at $d$, it lines one of Henle's glands.

and photophobic, and lacrimation and blepharospasm are generally present.

It is usual to describe two varieties of the diseasea papillary and a follicular. In the former the distinctive feature is hypertrophy of the papilla of the tarsal conjunctiva (Plate III, Fig. 3); in the latter, the presence in the conjunctiva of the tarsus and in the retrotarsal folds of the so-called "trachoma granules" or "trachoma follicles." More of ten the affection is of a mixed type, both of these features being present. The 
trachoma follicles, which are made conspicuous by everting the lids and causing the retrotarsal folds to protrude, are translucent bodies, having a resemblance to boiled sago grains or frog's spawn. Recent investigation seems to show that they are, in fact, hypertrophied lymphoid and connective-tissue cells, enclosed in a fibrous envelope (Fig. 72). The hypertrophy of the papillæ of the tarsal conjunctiva, which is observable

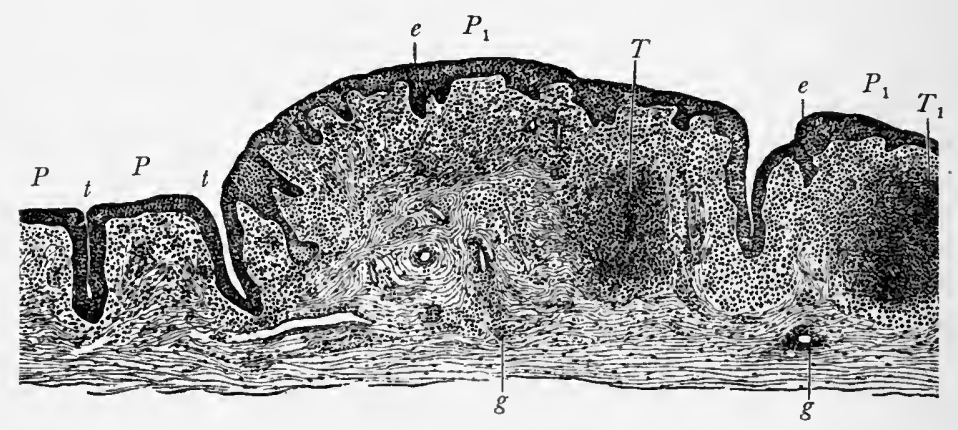

Fig. 73.-Cross-section through the trachomatous conjunctiva of the upper lid. Magnified $24 \times$ I (Fuchs). Both small papillæ, $P, P$, and large ones, $P_{1}, P_{1}$, are found. The former stand side by side like the pickets of a palisade; the depressions, $t, t$, lying between them and coated with epithelium, look like the tubules of glands. The large papillæ contain trachoma granules, $T, T_{1}$, which are not sharply limited and do not possess a capsule. The epithelium of the conjunctiva is in many places, $e, e$, thickened. The mucous coat is in a condition of cellular infiltration, which is especially marked in the vicinity of the blood-vessels, $g, g$.

chiefly upon the upper lid (Fig. 73), and the development of the trachoma follicles are accompanied by pronounced hyperplasia of the submucous connective tissue.

Ultimately there supervenes a stage of atrophy (Fig. 74 ), which in the worst cases results in the condition known as xerophthalmia, in which the conjunctivaitself so atrophied that the retrotarsal folds are obliter- 
ated, and free movements of the lids and eyeball curtailed-loses the character of a mucous membrane, and becomes dry and cuticular, while the cornea becomes so opaque that vision is reduced to little better than light perception. A more common consequence of this atrophic process and of the contraction which accompanies it, is the development of entropion (Fig. 75), that very annoying condition in which the lid-margins are inverted, and the eyelashes come in contact with, and

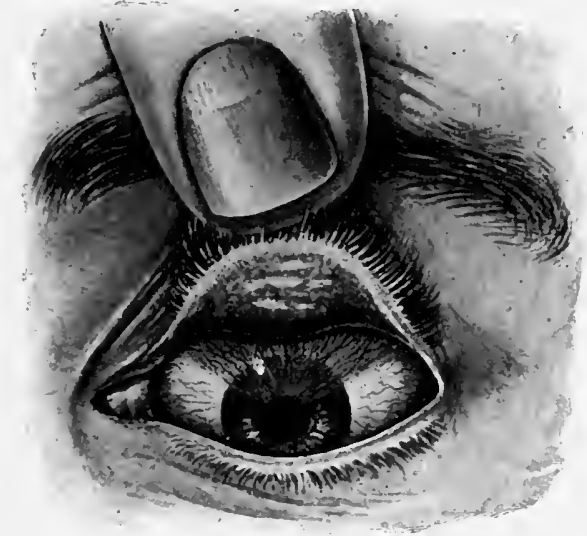

Fig. 74-Cicatricial stage of trachoma with pannus (Hansell and Sweet).

constantly irritate, the bulbar conjunctiva and the cornea. (See Chapter upon Diseases of the Eyelids and Orbit.)

The secondary changes which occur in the cornea, it seems probable, are largely due to the mechanical violence to which it is subjected through constant friction with the roughened inner surface of the lids. At first the cornea shows a mere loss of luster, a slight roughening of its surface; but soon it becomes more decidedly opaque; numerous blood-vessels develop 
upon it; its surface becomes uneven; and from time to time sluggish ulcers make their appearance-these

A

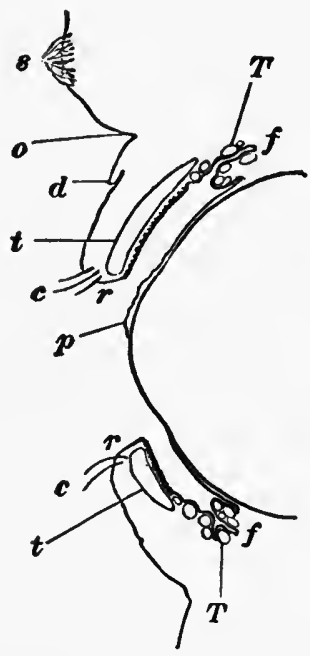

B

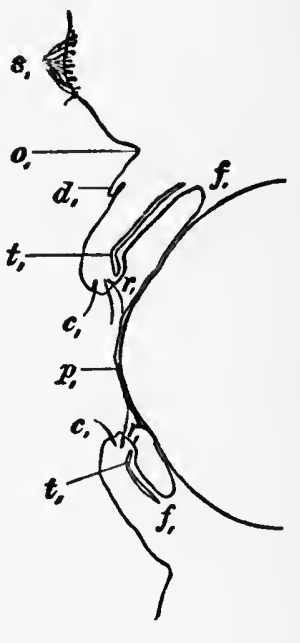

Fig. 75.-Schematic section through the lids and eyeball ( $A$, in recent, $B$, in old trachoma) (Fuchs). $A$ shows the way in which the two forms of hypertrophy of the conjunctiva are distributed among the separate divisions of the latter; $B$, the stage of sequelæ of trachoma. $s, s_{1}$, eyebrows; $o, o_{1}$, furrow between the brow and the lid (sulcus orbito-palpebralis); $d$, $d_{1}$, covering fold; $c$, cilia in their proper position; $c_{1}$, cilia turned toward the cornea; $r$, free border of the lid, with the borders of the upper and lower lids running parallel and the posterior margins of the lids acute; $r_{1}$, free border of the lid, looking backward, and with its posterior margin rounded; $t$, tarsus thickened by infiltration and covered with the velvety conjunctiva tarsi; $t_{1}$, tarsus thinned (atrophic), bent at an angle near its free extremity, and covered with smooth epithelium; $f$, fornix with numerous trachoma granulations, $T$, in the folds of the conjunctiva; $f_{1}$, fornix smooth, without folds (symblepharon posterius); $p$, thick pannus covering the upper half of the cornea; $p_{1}$, a shrunken pannus, extending over the whole cornea.

changes being more marked upon the upper half of the cornea, because here the lid friction is greatest.

Although, as has been stated, unquestionably a contagious disease, the contagium which gives rise 
to trachomatous conjunctivitis, fortunately, is nonvolatile, and so the disease can be communicated only by actual transference of the discharge from one eye to another. This commonly occurs through the medium of towels, handkerchiefs and the like, and it is for this reason that the affection so often flourishes in orphan asylums, reformatories, workhouses, etc.

Filth, unhygienic surroundings, uncleanly habits, and ill-nourishing food unquestionably favor the development and spread of this loathsome malady; but the view, not infrequently expressed, that trachoma may actually originate from such conditions, may develop without a pre-existent case to supply the contagium, is, it seems to me, absurd; as absurd, indeed, as to suppose that smallpox or cholera might originate in similar fashion. It is a fact of interest that certain races, the Jews and the Irish, for example, exhibit an especial predisposition to the disease, while, on the other hand, the negro is practically immune from it.

In the early stages of trachomatous conjunctivitis it is not always possible to reach a definite diagnosis; for there are less severe forms of conjunctival inflammation, more especially follicular conjunctivitis, in which hypertrophy of the palpebral lymphoid tissue and frog-spawn-like follicles are observed. However, the behavior of the case under treatment, and especially the occurrence of pannitic corneal changes, soon establish the true nature of the affection. In the chronic stage of the disease the distinctive features are pannus (Figs. 74 and 76 ), more or less evident entropion, and the presence of irregular, linear scars upon the conjunctival surface of the upper lid.

Treatment.-The treatment of this affection yields far from satisfactory results, and even in the most 
favorable cases must be long-continued to be effective. Moreover, relapses are of common occurrence, and, for this reason, it is well to give a guarded prognosis as to the future. Silver nitrate (in two to four per cent. solution) in the earlier stages, and copper sulphate (in the form of a crystal, applied to the everted lids) at a later stage, are the remedies which formerly were chiefly relied upon, and which are still extensively used. However, recent experience has led me to the

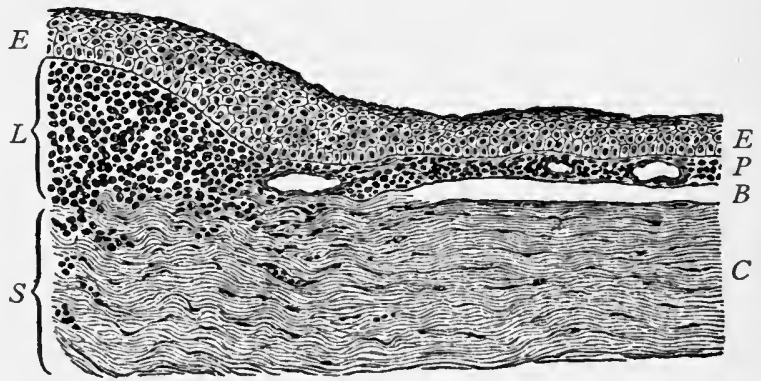

Fig. 76-- Cross-section through the margin of a cornea affected with pannus. Magnified I25 $X$ I (Fuchs). Beneath the epithelium, $E, E$, is the limbus, $L$, greatly thickened by cellular infiltration; from it the pannus, $P$, in which are perceived the cross-sections of several vessels, extends between the epithelium and Bowman's membrane, $B$, over the cornea, $C$. $S$, sclera.

conclusion that in trachomatous, as in purulent conjunctivitis, protargol may be employed with advantage as a substitute for the silver nitrate. I am also inclined to believe that it accomplishes as much in the chronic stage of the disease as any other remedy, and so I find myself using it instead of the copper sulphate, the application of which is so much more painful.

My practice, in the acute as well as in the chronic stage of the disease, is to apply to the everted lids, every other day, a forty per cent. solution of protargol, 
and to prescribe a weaker solution (ten to twenty per cent.) for application by the patient twice daily. If he can be taught to evert the lids, and make the application directly to their inner surfaces, this is distinctly advantageous; but, if this is not practicable, the solution is applied by means of an eye-dropper. When corneal ulcers, attended, as they usually are, by photophobia, are present, the protargol treatment is supplemented by the instillation, three times a day, of a two- to four-grain solution of atropin or a one- to twograin solution of holocain. The other remedial measure chiefly relied upon is the use of the roller forceps of Knapp (Fig. 77). This treatment has been found especially useful in the follicular type of the disease,
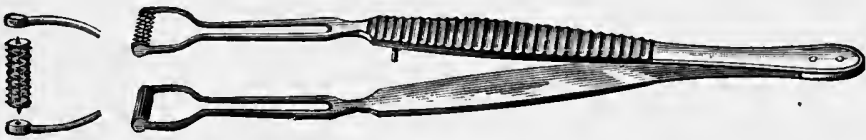

Fig. 77-Knapp's roller-forceps.

the forceps being employed, from time to time, to squeeze out any "trachoma follicles" which may be discovered in the tarsal conjunctiva or in the retrotarsal folds. To lessen the considerable pain which attends this procedure, the eye should be brought well under the influence of cocain. The lid is then everted, and the forceps are made to grasp the everted cartilage or the retrotarsal folds of the conjunctiva, and the follicles are expressed by gentle traction, combined with nottoo-firm compression of the blades (Fig. 78).

Should the condition of the eyes not improve under the use of protargol, silver nitrate in two per cent. solution, or the crystal of copper sulphate, to be applied not oftener than every other day, should be tried instead, or a crystal of alum, which the patient may apply to 
the everted lids two or three times a day, and which sometimes is very beneficial, may be prescribed. A five per cent. ointment of copper citrate, to be applied two or three times a day, is one of the newer remedies claimed to be useful in this condition. When entropion

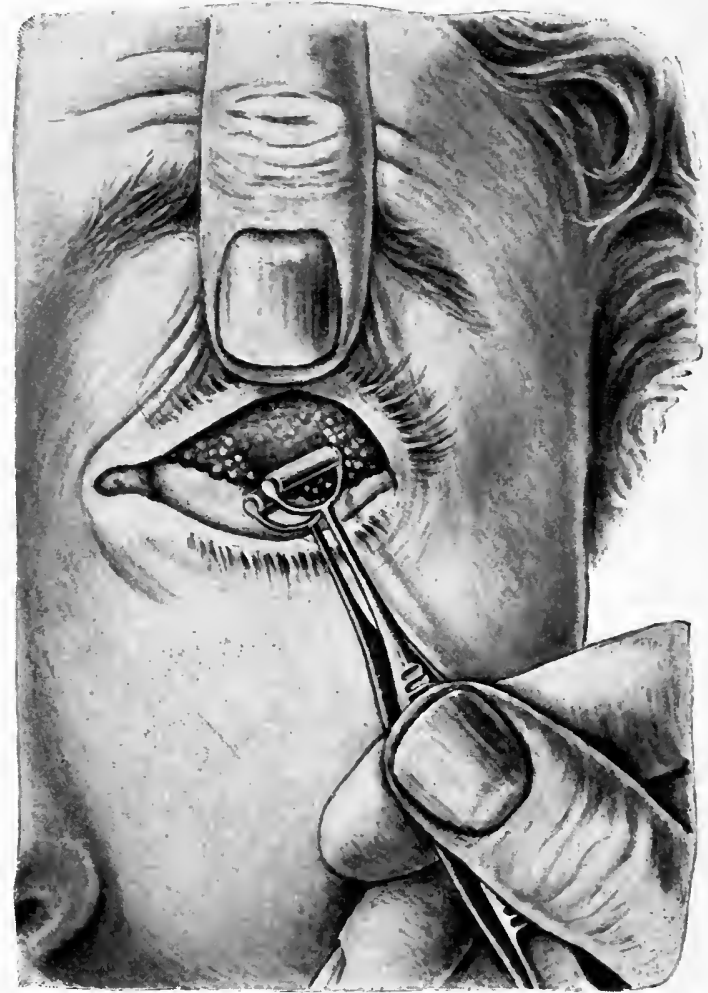

Fig. 78.-Expression of trachoma follicles with Knapp's roller forceps (Hansell and Sweet).

is present, removal of the inverted lashes affords temporary relief (Fig. 79). To secure permanent relief the lid-fault must be corrected by operative procedure (see Chapter III).

In cases which exhibit marked pannus, with a plenti- 
ful supply of blood-vessels upon the cornea, much may be accomplished by the jequirity treatment; but the risk of serious corneal complications attending the use of this remedy is so considerable, that one hardly feels warranted in recommending its employment by the general practitioner.

Vernal Conjunctivitis or Spring Catarrh.-This very obstinate form of conjunctival inflammation is met with chiefly in children. It usually attacks both eyes, and is probably mildly contagious. It derives its name from the fact that the disease remains comparatively dormant during the winter, and becomes more active and troublesome with the oncoming of warm weather. Though probably dependent upon

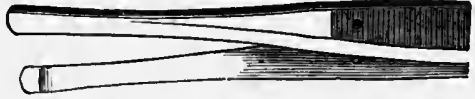

Fig. 79.-Epilation-forceps.

a specific germ, the efforts to discover this have so far proved fruitless.

Two types of the disease are met with. In one, the bulbar conjunctiva is the seat of the characteristic changes; in the other, they are found in the conjunctiva of the tarsus. Well-marked examples of the two types are rarely met with in the same individual; at least, this has been my experience. In the bulbar variety (Fig. 8o) there is observed a slightly elevated, nodular, gelatinous growth, of yellowish-brown color, upon the conjunctiva, close to the corneal border. In some instances this growth exhibits a tendency to encircle the cornea, as a rather narrow band; in others it tends to spread upon the conjunctiva, especially in the direction of the inner and outer canthi, and, to a less extent, 
upon the cornea. In rare cases the whole cornea is overrun, and the sight in consequence is greatly impaired. The nodular masses, which are stable and show no disposition to ulcerate, are composed of connective tissue and greatly thickened epithelium, the latter showing a tendency to extend into the underlying tissue in the form of solid epithelial plugs (Fuchs).

In the palpebral variety the papillæ of the tarsal conjunctiva, and to a less extent those of the re-

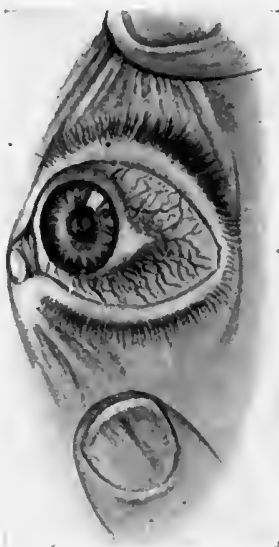

Fig. 8o.- Vernal conjunctivitis (bulbar type) (Haab).

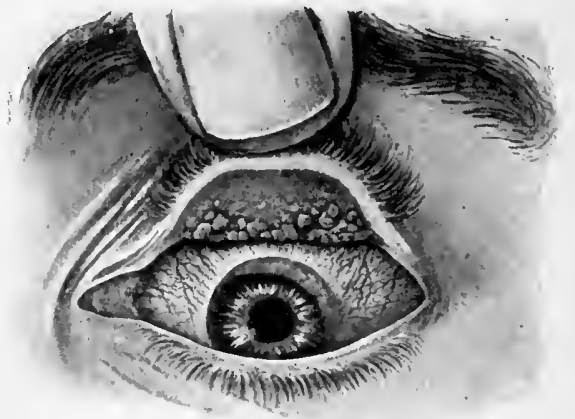

Fig. 8r.- Vernal conjunctivitis, showing typical palpebral as well as bulbar changes (Dr. Wm. Zentmayer's case) (Hansell and Sweet).

trotarsal folds, undergo a peculiar hypertrophy, and at the same time' become flattened (probably from the constant pressure to which they are subjected), so that the inner surface of the lid (it is the upper lid which commonly exhibits these changes) presents a strikingly tessellated appearance. The hypertrophied papillæ (admirably shown in the accompanying illustration, Fig. 8I) are as firm almost as cartilage, and their edges, which overhang in mushroom-like fashion, 
can be slightly elevated. According to Fuchs, they are composed of areolar connective tissue, with connectivetissue cells which have undergone a peculiar hyaline degeneration, and are covered by thickened epithelium, which gives to the conjunctival surface of the lid the bluish-white, skimmed-milk appearance that is a feature of the disease.

The most prominent symptom of vernal catarrh is persistent itching. Pain is not complained of, but rather a sensation of sand being in' the eyes. The discharge is slight. Not infrequently the disease lasts for years, and rarely is it of brief duration. It is not uncommon to meet with more than one case in members of the same family. For instance, the author has encountered marked examples of the palpebral variety in a father and his two sons, and also in sisters who were twins.

Treatment.-As a rule, the disease, especially when it is well marked, responds to treatment very unsatisfactorily. The remedy which has yielded me the best results is bichlorid of mercury. This is prescribed as a collyrium, in the strength of $\mathrm{I}: 8000$, with the addition of one per cent. sodium chlorid, and is dropped into the eyes three times a day. To be effective the remedy must be long continued, although in the milder cases improvement may manifest itself within two or three weeks; in the more severe cases it is a matter of months rather than weeks. Other remedies which may be tried are zinc sulphate and boracic acid, as recommended in catarrhal conjunctivitis; yellow oxid of mercury ointment (hydrarg. ox. flav., gr. j; vaselin, $5 \mathrm{j}$ ), to be applied to the eye once a day; dilute acetic acid (I part of the dilute acid to $25^{\circ}$ parts of water), to be dropped into the eye three times a day, as recommended by Van Millingen, and salicylic acid ointment, two 
to fifteen per cent., as suggested by Randolph. The internal administration of syrup of iodid of iron has seemed to me, at times, to be of benefit, and in the palpebral type of the disease I have thought that good resulted from the use of the roller forceps, employed as in trachoma.

Phlyctenular or Scrofulous Conjunctivitis.The essential feature of this form of conjunctivitis, which is known also as conjunctivitis lymphatica and as eczema conjunctiva, is its dependence upon a constitutional cause. Though not infrequently met with in adults, it is a disease to which children are especially disposed. It happens as often that both eyes are affected as that one only is involved.

In the typical form of the disease the conjunctival injection is not uniform, but is more marked in, or, perhaps, is confined to, the neighborhood of the phlyctenulæ (Plate IV, Fig. I). These are yellowish-red elevations, differing in size from that of a mustard-seed to a small split pea, and composed chiefly of lymphoid cells, which, in varying numbers,-usually two or three, -develop upon the ocular conjunctiva, more especially in the neighborhood of the corneal border, and are converted quickly into superficial ulcers through loss of their epithelial covering. Pain is not a symptom of the disease, but photophobia and lacrimation are frequently present. The discharge is mucoid, and is less abundant than in catarrhal conjunctivitis. The affection is not contagious.

As often as not the phlyctenulæ make their appearance upon the cornea as well as upon the conjunctiva, and then the affection is known as phlyctenular keratoconjunctivitis. Under such circumstances the photophobia is apt to be more pronounced-in some instances 
PLATE IV.

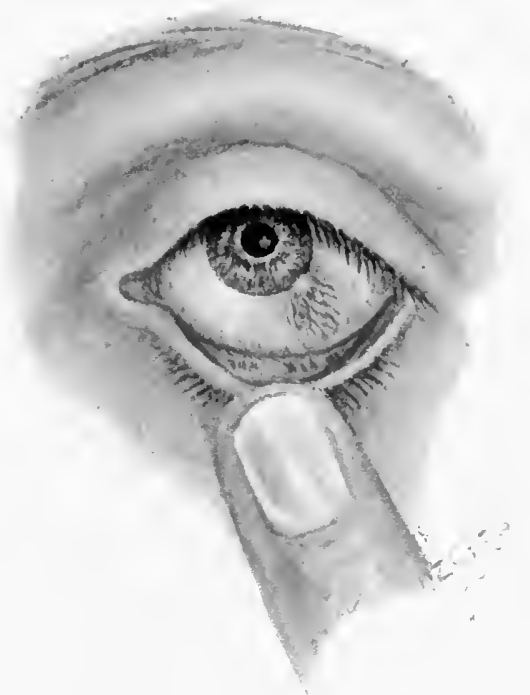

Fig. I.-Phlyctenular Conjunctivitis.

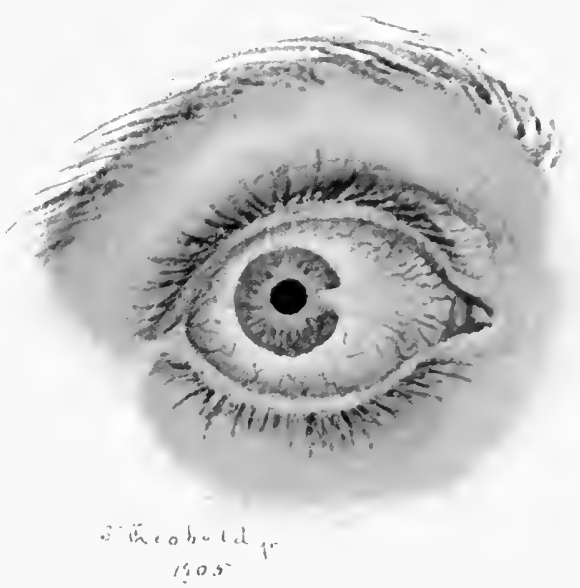

Fig. 2.-PTFrigium. 

intense-and to be attended by blepharospasm and profuse lacrimation. It is through involvement of the cornea that the disease, at times, permanently and seriously damages the eye. In consequence, usually, of neglect or of injudicious treatment, the corneal ulcers left by the breaking-down of the phlyctenulæ extend laterally and in depth until, perhaps, a perforation into the anterior chamber occurs or, at all events, until the cornea is seriously damaged. A leucoma, or dense opacity of the cornea, results, which, if central or nearly central, greatly impairs the sight. More than this, if the ulcer perforates into the anterior chamber, a permanent adhesion of the iris to the cornea at the point of perforation is almost sure to occur, and this is usually attended by distortion and displacement of the pupil (see Fig. IOO).

When phlyctenular conjunctivitis presents the typical appearance which has been described, it is not difficult of recognition. Occasionally, however, in what may be properly termed the catarrbal type of the disease, the conjunctival injection is diffuse, and the phlyctenula are absent or not distinguishable. Under such circumstances the eye presents almost the same appearance that it does in acute catarrhal conjunctivitis, with which it may be confounded, if only the condition of the conjunctiva is relied upon as a diagnostic guide. However, in true catarrhal conjunctivitis both eyes are almost invariably affected, and there are no evidences of constitutional derangement, whereas, in phlyctenular conjunctivitis; as has been pointed out, it often happens that only one eye is involved, and it is seldom the case that there are not present other evidences of constitutional disorder, such as blepharitis marginalis, eczema of the face, etc. As the treatment 
called for in catarrhal conjunctivitis (the use of an astringent collyrium) is sure to be harmful in phlyctenular conjunctivitis, it is most important that the two conditions should not be confounded. It may be added, that when the diagnosis is in doubt, especially in dealing with children, it is safer to treat the case as one of strumous character.

The name "scrofulous conjunctivitis," or "scrofulous ophthalmia," as applied to the affection under consideration is somewhat misleading; for, though distinctly scrofulous individuals show an especial predisposition to the disease, it is often met with in persons who can not properly be regarded as belonging to this category. This is particularly true of the cases which are so often encountered in children. Here the ocular affection, which is frequently accompanied by nasal catarrh, and by eczema of the lids, the upper lip, and the auricle, and not uncommonly by suppurative inflammation of the middle ear, seems to be largely dependent upon disorder of the digestive apparatus, brought about by improper feeding, by unhygienic surroundings, and by lack of pure air and sunlight. A furred tongue, "feverish" breath, loss of appetite, and constipation of the bowels are other symptoms which are often present.

That in these cases we have to do with a relatively mild form of septicemia, due in all probability to the entrance into the circulation of bacteria or their toxins from the alimentary canal, is a view which I have long held, and which I have advanced upon more than one occasion. This view seems to be supported not only by the clinical features of these cases, which are just such as might result from the presence in the blood of a relatively benign septic organism ("intestinal intoxi- 
cation" is the way it is expressed nowadays), but also by the character of the treatment which proves most effective. For of all remedial measures none produces so prompt and decided a change for the better-not only in the condition of the eye but in the patient's general condition - as the administration of a generous, old-fashioned, calomel purge, which gives the "prime vice" a thorough cleansing. In this connection it may be mentioned that careful bacterial investigations, made by Weeks and others, show that the Staphylococcus aureus is commonly present in the phlyctenulæ, as it is in the eczematous nodules which, as has been stated, are so often found in association with strumous conjunctivitis.

Treatment.-There are few diseases of the eye that respond to treatment so promptly and satisfactorily as does phlyctenular conjunctivitis; but, on the other hand, there are few in which injudicious treatment does more harm. Astringents-silver nitrate, zinc sulphate, etc.- - are distinctly contraindicated, and when used invariably make matters worse. It goes without saying, that the treatment of this affection, in view of its systemic origin, should be both constitutional and local.

The local treatment, which is so invariably effectual that it need scarcely ever be departed from, may be described in a few words. It consists in the application to the eye, three times a day, of a solution of atropin and boracic acid, and once a day (preferably in the morning, after the atropin has been instilled) of an ointment of yellow oxid of mercury and vaselin (hydrarg. ox. flav., gr. j; vaselin, 5j). The exact quantity of the ointment to be applied is a matter of little moment, but a bit the size of a match-head is 
sufficient. It should be carefully inserted between the upper or lower lid and the eyeball. This may be done conveniently with the broad end of a wooden toothpick (see Fig. 9), or, more safely, if the treatment is to be carried out by untrained hands, with a small camel's-hair brush. If blepharitis is present, or eczema of the lids, some of the same ointment (or, still better, an ointment of twice the strength mentioned, and having "vaselin cerate" rather than vaselin as a base, because of its higher melting-point) should be applied to the lids at bedtime.

The strength of the atropin solution prescribed should vary with the degree of photophobia, lacrimation, and blepharospasm present. When the inflammation is confined to the conjunctiva, and there are no phlyctenulæ upon the cornea, and when, therefore, as a rule, there is but little photophobia, a one-grainto-the-ounce solution of atropin, with ten grains of boracic acid, should be ordered. In most cases of kerato-conjunctivitis, especially those occurring in children, this same strength suffices; but the atropin should be increased to two, or even to four grains, to the ounce, if photophobia and lacrimation are pronounced.* Exceptionally, in mild cases, in which the conjunctiva only is involved, and in which the effect of the atropin upon the sight would cause considerable inconvenience, it is permissible to try a collyrium simply of boracic acid (ten to twelve grains to the ounce) in connection with the yellow oxid of mercury ointment; but my experience is that, almost

* Whenever atropin is prescribed, the effect it will have upon the pupil and upon the sight, and the fact that it is very poisonous, should always be impressed upon the patient or upon those in whose charge he is. 
without exception, the cases do better, and the affection yields much more promptly, when atropin is employed.

For the effectual application of the atropin solution an eye-dropper is indispensable, and even with its aid, owing to the spasm of the lids, this is not always easy of accomplishment. The patient, if a child, should be placed in a recumbent position, and will probably need to be held firmly while the drops are being instilled and the ointment inserted. Neither application, it may be observed, causes any pain.

As to constitutional treatment, this, of course, will be influenced largely by the age and the general condition of the patient. When there are present evidences -enlarged lymphatic glands, etc. - of a well-marked strumous diathesis, the syrup of the iodid of iron, and cod-liver oil combined with the hypophosphites are especially useful. In the cases which are less distinctly strumous, especially in those which have been described as being encountered so often in children, the remedy in which experience has led me to place the greatest confidence is a combination of the phosphates of iron, quinin, and strychnin, given in the form of an elixir or a syrup.* The preparations of "beef, wine, and iron," which have the advantage of being palatable, a matter of no little importance where children are concerned, I have also found useful.

* As has been mentioned before, the elixir of the phosphates of iron, quinin, and strychnin made by Wyeth \& Brother is especially to be commended, because it contains a much larger proportion of iron and quinin (two grains of the former and one grain of the latter to the dram) than do most of the preparations that are called by the same name. A syrup prepared by Sharp \& Dohme, which contains one grain, each, of the phosphates of iron and of quinin and one-sixtieth of a grain of phosphate of strychnin to the dram, is another preparation which I have used with excellent effect, especially in hospital practice. 
As a rule, it is well, especially in those cases, common in children, which exhibit besides the ocular inflammation other signs of constitutional disorder, such as blepharitis, eczema, nasal catarrh, otitis media, etc., to administer, at the outset of the treatment, an energetic mercurial purgative (calomel, scammony, āā gr. ij; rhubarb, gr. vj). The benefit resulting from this measure usually is strikingly manifest, and not infrequently the case is well on the road to recovery before the tonic treatment, to which it is the prelude, has had time to produce its effect.

The regulation of the diet, it should be added, especially in cases occurring in children, who should be allowed to have only simple, easily digested, and nourishing food, is a matter of prime importance. It is important also that patients suffering with phlyctenular conjunctivitis or kerato-conjunctivitis should be out of doors as much as practicable-should have plenty of fresh air and sunlight, and should not be shut up in darkened and ill-ventilated rooms, as too often happens.

The exanthematous fevers, measles especially, not infrequently give rise to inflammation of the conjunctiva, and writers often make of these cases a distinct variety of conjunctivitis, which they denominate "exanthematous." There seems to be no good reason for doing so, however, as they differ in no essential respect from the systemic conjunctivitis that has just been described, and, like it, present at times a distinctly phlyctenular character, with a tendency to corneal implication, and, at other times, a catarrhal type; moreover, the treatment which they require is exactly the same.

Toxic Conjunctivitis.-An inflammation of the conjunctiva of follicular type, usually accompanied by 
annoying itching, is occasionally excited by the longcontinued use of collyria containing atropin, hyoscyamin, eserin, and other drugs of similar character. Exceptionally, owing to a peculiar susceptibility on the part of the individual, a single application of an atropin collyrium will induce a marked conjunctivitis, which may be accompanied by decided redness and edema of the eyelids and even of the whole face. Sometimes this susceptibility is manifested to all of the commonly employed mydriatics, but usually this is not the case.

Treatment.- This consists in withholding the drug to which the susceptibility is shown, and in the use of a ten-grain-to-the-ounce solution of boracic acid, three or four times daily. This soon restores the eye to its normal condition.

Argyria Conjunctivæ.-From the long-continued application of silver nitrate to the eye a permanent stain of the lower tarsal and retrotarsal conjunctiva, and not infrequently of the bulbar conjunctiva as well, may result. The "white of the eye" assumes an olive tint, while the retrotarsal folds and the inner surface of the lids are stained a bluish-gray or slate color. A similar discoloration of the conjunctiva is said to occur in persons who, owing to the nature of their employment, are constantly exposed to the action of silver dust. Cases of argyria from protargol have been reported, and as this agent is more penetrating in its action, and can be used more freely than the silver nitrate, it is probable that this condition will be met with oftener in the future than it has been in the past. The stains are indelible. Different agents have been employed to remove them, but without success.

Subconjunctival Hemorrhage.-This condition 
(Fig. 82), which usually manifests itself suddenly, may occur spontaneously or may be of traumatic origin. Spells of coughing, vomiting, or sneezing may produce it, while at other times it occurs, it may be during sleep, without assignable cause. The existence of angiosclerosis predisposes to it, as it does to hemorrhages in other parts. The blood not infrequently encircles the cornea, and spreads over the whole anterior surface of the eyeball, giving to it

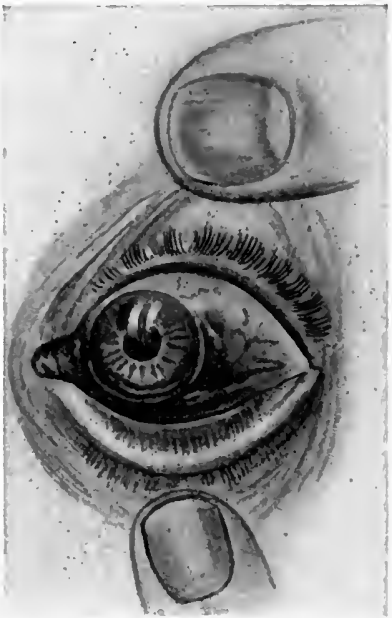

Fig. 82.-Subconjunctival hemorrhage (Haab). a bright-red appearance, which leads to the belief that something serious has happened. It is commonly mistaken for "inflammation"; but inspection of the eye shows that the redness is not due to injection of blood-vessels. It may give rise to a slight soreness of the eyeball; but beyond this it causes no inconvenience, apart from its unsightliness. During the process of absorption, which may occupy two or three weeks, the bright-red color of the freshly extravasated blood changes to a greenish-yellow.

Treatment is scarcely called for; but, if it is a matter of moment to hasten the restoration of the normal appearance of the eye, a compress bandage and the internal administration of potassium iodid may be of some avail. A lotion of opium and boracic acid is useful, when "soreness" of the eyeball is complained of.

Pinguecula.-An elevation, yellowish in color, and 
varying in size and shape, situatèd upon the conjunctiva in the interpalpebral space, usually in close proximity to the nasal, and less frequently to the temporal, margin of the cornea, was given the name pinguecula (from pinguis, "fat"), because formerly it was supposed to be due to the deposition of fat in the conjunctiva. It is seldom observed in young persons, and is oftenest met with in individuals who have passed middle life. The fact that it occurs upon the most exposed portion of the bulbar conjunctiva seems to indicate that it is, in some measure at least, due to the irritation caused by wind, dust, etc. Microscopic investigation has shown that the thickening of the conjunctiva is caused mainly by an increase in the number and size of its elastic fibers, and that the yellow color is due not to the deposition of fat, but to the presence of numerous concretions of a yellowish colloid substance (Fuchs).

Treatment.-Removal of the growth is sometimes indicated on account of its unsightliness, and in other instances because, when unusually prominent, it may cause mechanical irritation. It may be excised with slender curved scissors, care being taken to sacrifice as little as possible of the neighboring conjunctiva. The edges of the conjunctival wound should be slightly undermined, and brought together by one or two fine, black silk sutures, which should be removed after two or three days.

Pterygium is a circumscribed hypertrophy of the conjunctiva and subconjunctival tissue, of triangular shape, and more or less markedly vascular, which exhibits a tendency to encroach upon the cornea. The apex of the growth is always turned toward the center of the cornea; the base toward the equator of the eye- 
ball (Plate IV, Fig. 2). Its usual location is to the nasal side of the cornea, over the region of attachment of the tendon of the internal rectus muscle; exceptionally it is situated upon the outer side of the cornea. It develops very slowly, and months, or even years, may elapse without its extending far enough upon the cornea to impair vision. It is rarely met with in children, and it is more prevalent in tropical than in temperate countries.

The apex of a pterygium occasionally reaches, but very rarely passes beyond, the center of the cornea. I have, however, met with one case in which a pterygium of unusually large size, starting from the nasal side of the eye, grew entirely across the cornea to its external margin. The other eye of the same individual (a woman advanced in years) also exhibited a large pterygium, which already had passed beyond the center of the cornea. As long as the growth is confined to the conjunctiva and the periphery of the cornea it causes little or no inconvenience, apart from its unsightliness. When, however, it encroaches upon the pupillary area of the cornea it seriously impairs vision, not only by obstructing the passage of light into the eye, but because the curvature of the corneal surface about its apex is so altered as to produce a high grade of irregular astigmatism.

The question of the etiology of pterygium has been much discussed, and several theories have been advanced to account for its occurrence and for its growth. In order to a clear comprehension of the matter, it is essential that a sharp distinction should be drawn between true pterygium, which so far we have been considering, and pseudo-pterygium, since much of the confusion regarding the etiology of this affection has arisen from failure to do this. 
Pseudo-pterygium, which is as apt to develop at the upper or lower margin of the cornea as in any other direction (whereas true pterygium may be said never to occur in either of these positions), has its startingpoint in the presence of an ulcer or wound of the cornea near its margin, to which a knuckle of the swollen and overhanging conjunctiva becomes adherent. Occasionally, in this variety of pterygium, which is a not very uncommon consequence of gonorrheal conjunctivitis, and, as just indicated, of marginal wounds of the cornea, only the apex of the growth is adherent to the cornea, and it is possible to pass a probe under the body of the pterygium. It is manifest, therefore, that both clinically and etiologically this affection differs radically from true pterygium.

It has long been taught that the development of true pterygium, like that of pinguecula, is favored by conditions which bring about persistent hyperemia of the conjunctiva, as, for example, exposure of the eyes to the rays of a tropical sun, as in long sea voyages, or to the heat from furnaces or to the irritant action of dust and vapors, as in mills and other manufacturing establishments; and the evidence in favor of this view is much too strong to be put aside. Fuchs, indeed, holds that a pterygium is simply a pinguecula which has extended onto the cornea, and in doing so has drawn the conjunctiva after it. While it is undoubtedly true that pterygium not infrequently does originate in this manner, the view that it always does so is not, I believe, supported by clinical observation. Transitional types, which one observer might call pterygium, and another pinguecula, are often encountered.

Any theory which would satisfactorily explain the origin of pterygium must, it seems to me, account for 
the fact that in a large majority of instances the growth develops to the nasal side of the cornea, over the region of the internal rectus muscle. This the theory which I advanced some years ago-that insufficiency of the internal recti muscles (exophoria), by inducing hyperemia of the overlying conjunctiva, is an important factor in the causation of pterygium-does in an eminently satisfactory manner.* In the years that have elapsed since this view was advanced, the frequency with which I have found these two conditions-pterygium and exophoria-associated, has convinced me that it is not without substantial foundation.

Treatment.-In the treatment of pterygium little is to be expected except from operative interference. However, in its incipient stage its growth may be retarded, or possibly arrested, by the correction of any muscular anomaly or error of refraction which may be present; and it may be added that after operation the tendency to recurrence may be controlled in the same way. In this connection it should be observed that the discomfort (asthenopia) of which persons with pterygium often complain, is much more apt to be due to the existence of a muscular or refractive error than to the mere presence of the growth.

The propriety of resorting to operation depends upon several considerations. When the growth, though confined to the conjunctiva, is narrow and well-defined, it is advisable to operate; but if it is broad and illdefined, it is well not to interfere, unless it is known to be progressing, because, under such circumstances, there is no assurance that the condition and appear-

* See "Trans. Am. Ophthalmolog. Soc.," Vol. IV, p. 537; "Am. Journal Ophthalmology," Aug., I887; and "Reference Handbook of the Medical Sciences," Vol. VI, p. 58. 
ance of the eye will be better after operation than it was before. When, already, the growth has encroached upon the cornea, it is best not to postpone operation, for when this has happened it usually continues to advance, and as it does so produces changes in the corneal structure of such character as to leave a more or less pronounced permanent opacity, even when it has been removed with the greatest care.

Of the many operative procedures which have been proposed for the cure of pterygium, the operation of excision is the only one which I am disposed to commend. The end which all of these procedures seek to accomplish is the same-to minimize the loss of conjunctival tissue-and this, indeed, is of the first importance; but when excision is properly performed there need be but little sacrifice of tissue. The important point to bear in mind is that while the corneal portion of the growth should be carefully dissected up and completely removed, the conjunctival portion should be dealt with much less radically, should, indeed, be only in part removed. If this precaution is not observed, a large gap will be made in the conjunctiva, and after the completion of the healing process, which under such circumstances is apt to be slow and difficult, a conspicuous, vascular cicatrix will be left, which may interfere with the free movements of the eyeball, and which is as unsightly as was the pterygium itself.

The method of operating which I have employed for years, and which, as a rule, has yielded very satisfactory results, recurrence of the growth being decidedly exceptional, is as follows: With a pair of slender, toothed forceps (such as are used in operating for squint) the pterygium is seized near its apex, and, while considerable traction is made upon it, is carefully 
dissected from the cornea with a sharp and but slightly bent iridectomy knife, especial care being taken to detach its margins from the corneal limbus; its more loose attachments to the sclera are also divided for a distance of 3 or $4 \mathrm{~mm}$. from the border of the cornea. Then with a pair of slender scissors, curved on the flat, the whole of the corneal and a small part of the conjunctival portion of the growth is excised by two converging cuts. During this step of the operation but slight traction is exerted with the forceps; for, if the traction is considerable, and if at the same time the scissors are pressed against the sclera, a much larger part of the pterygium will be removed than is
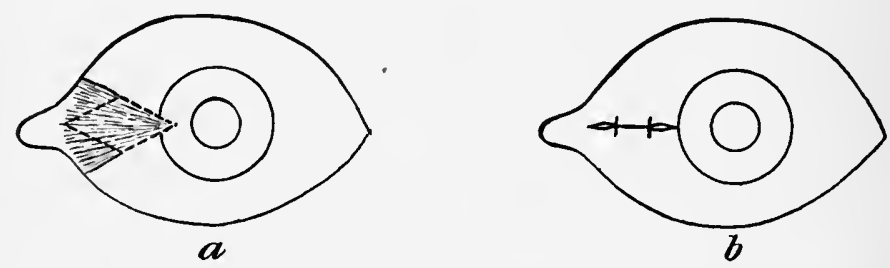

Fig. 83-Operation for the removal of pterygium. The dotted lines $(a)$ represent the portion of the growth which is excised; $b$ represents the conjunctival wound closed by two stitches.

desirable. The margins of the conjunctival wound are now slightly undermined with the scissors, so that they may be more readily brought together by two sutures of fine black silk, which are next introduced (Fig. 83). A light bandage is then applied, to be worn until the stitches are removed on the second or third day, when a collyrium of boracic acid (ten to twelve grains to the ounce) is prescribed, to be used until the inflammation consequent upon the operation has subsided. A speculum should, of course, be used during the operation, and the eye should be thoroughly under the 
influence of cocain. The instillation of a few drops of a $\mathrm{I}: 1000$ adrenalin solution is also of advantage, as it renders the operation nearly, if not quite, bloodless.

It is well to inform the patient that the improvement in the appearance of the eye will be slow, else he may suppose the operation has not been successful, and may mistake the localized vascularity, which remains for some time, for a return of the growth. 


\section{CHAPTER VI.}

\section{DISEASES OF THE CORNEA AND SCLERA.}

\section{DISEASES OF THE CORNEA. \\ KERATITIS.}

Inflammation of the cornea, keratitis, occurs as a primary and as a secondary condition. As a secondary condition it is oftenest observed in association with the severer forms of conjunctivitis. It may be acute or chronic in type, may involve one or both eyes, may be wholly dependent upon local causes or may be constitutional in its origin, and it is, not infrequently, a result of traumatism. It is commonly accompanied by pericorneal subconjunctival and conjunctival injection, a contracted pupil, and by more or less marked impairment of the transparency of the cornea and loss of its surface luster. In some forms of keratitis the development of blood-vessels upon or in the cornea is a striking feature. It is usually attended by pain, often very severe, and by photophobia and lacrimation. There may be also marked impairment of vision if the corneal opacity is dense and overlies the pupil.

The several varieties of keratitis may be grouped conveniently under two heads-suppurative keratitis and non-suppurative keratitis. To the former group, characterized by a tendency to tissue necrosis, belong phlyctenular keratitis, abscess and ulcer of the cornea, keratomalacia, and, as a rule, neuropatbic keratitis; to the latter, interstitial keratitis, pannus or pannitic 
keratitis, and certain comparatively rare forms of corneal inflammation-sclerosing keratitis and keratitis profunda-which need not engage our attention, the chief characteristic of this group being chronicity.

The dangers to be feared in keratitis are extensive necrosis of the corneal tissue, circumscribed necrosis, as in perforating ulcer, the formation of permanent opacities, which may seriously impair sight, and alterations in the shape of the cornea, which are scarcely less detrimental to vision (Fig. 84 ). The control of the in-

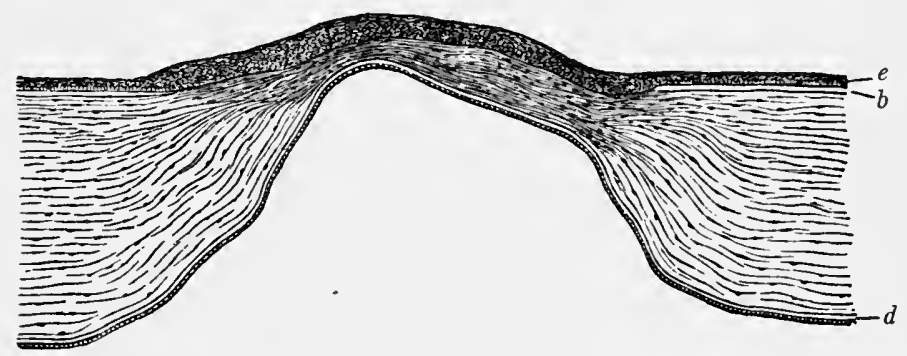

Fig. 84.-Keratectasia resulting from an ulcer. Magnified $25 \times$ I (Fuchs). The thinned and protruding cicatrix is distinguished by its denser texture from the adjacent normal cornea. The epithelium, $e$, over it is thickened, while Bowman's membrane, $b$, is wanting. On the other hand, Descemet's membrane, $d$, with its endothelium, is everywhere present-a proof that the ulcer has not perforated.

flammatory process as quickly as possible, so that the serious consequences just enumerated may not occur, and the relief of the attendant pain and photophobia, are the ends to which treatment should be directed.

The corneal epithelium (Fig. 85) when destroyed by accident or disease, it may be remarked, is quickly regenerated; but, on the other hand, Bowman's membrane is never regenerated, and the true corneal tissue (the substantia propria of the cornea) when lost is replaced by connective tissue, which seldom attains the transpar- 
ency of the normal cornea, though it is the fixed corneal cells which are mainly instrumental in its formation (Fig. 86).

SUPPURATIVE KERATITIS.

In the production of the different forms of suppurative keratitis bacteria play an important rôle.

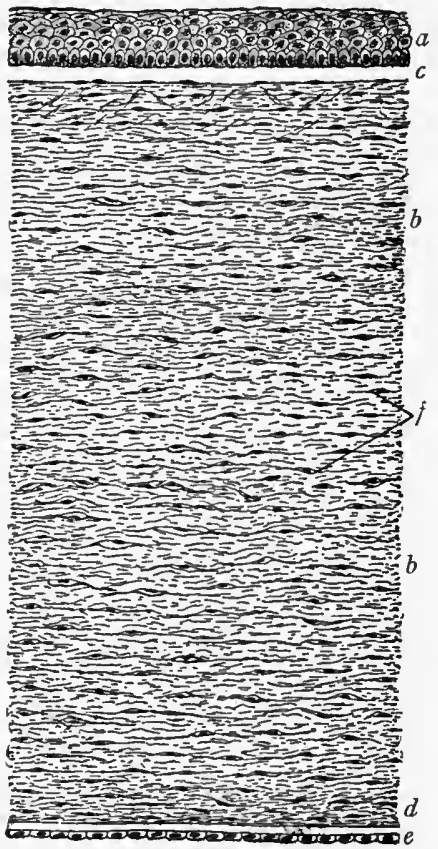

Fig. 85.-Section of cornea (Piersol): $a$, Anterior epithelium; $c$, anterior limiting (Bowman's) membrane; $b, b$, fibrous stroma of substantia propria, containing corneal corpuscles (f) lying within the corneal spaces; $d$, posterior imiting (Descemet's) membrane; $e$, endothelium lining anterior chamber.

Those oftenest concerned are the Staphylococcus aureus, the streptococcus and the pneumococcus. The infection usually is ectogenous, though in many instances undoubtedly it is entogenous. The tractability or intractability of this type of keratitis depends upon 
the micro-organism concerned in its production-the pneumococcus, for example, giving rise usually to a severe form of keratitis-and the bactericidal energy of the cells and the body juices which the invading bacteria must encounter. The worst cases, unquestionably, are those in which the more virulent bacteria play a part, and, from one cause or another, meet with exceptionally feeble resistance.

Phlyctenular Keratitis or Kerato-conjunctivi-

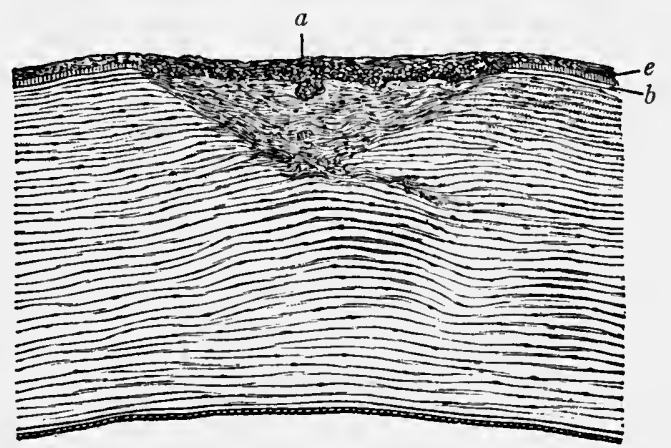

Fig. 86.-Cicatrix of the cornea (Saemisch). The epithelium, $e$, is everywhere present, but over the cicatrix it is irregular, and in places (at $a$ ) is thickened. Bowman's membrane, $b$, is wanting at the site of the cicatrix. The latter itself is distinguished from the tissue of the normal cornea by its denser and less regular texture.

tis.-In treating of phlyctenular conjunctivitis it was stated that, as often as not, the cornea is involved as well as the conjunctiva in this type of ophthalmia (Fig. 87). It was stated also that when this is the case the photophobia and lacrimation are commonly more pronounced, and, further, that it is through this involvement of the cornea that the sight is at times permanently impaired, the impairment of sight being caused either by the formation of a persistent corneal opacity or, in the case of an ulcer perforating into the anterior 
chamber, by the development of an anterior synechia and the consequent displacement or obliteration of the pupil (Fig. 100).

As to treatment, it was pointed out that when the involvement of the cornea is attended, as it often is, by pronounced photophobia and lacrimation, a considerably stronger solution of atropin (gr. $\mathbf{i j}$-iv to $\mathbf{z} \mathbf{j}$ ) than is commonly used when the inflammation is confined to the conjunctiva should be employed.

Since the two conditions, phlyctenular conjunctivitis

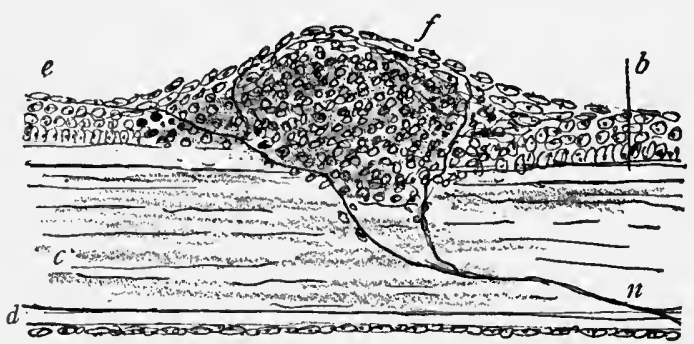

Fig. 87.--Section of corneal phlyctenule: $b$, Bowman's membrane; $c$, corneal substance; $d$, Descemet's membrane; $e$, epithelium; $f$, phlyctenule, consisting of a deposit of round-cells in the epithelial layer and along the course of the nerve; $n$, twig of nerve ending in epithelium (modified from Iwanoff).

and phlyctenular keratitis, are essentially the same not only in their etiology but, except in the particulars just pointed out, in their clinical history and their treatment as well, there remains but little to be added regarding the latter condition, except to say that when a threatening ulcer has taken the place of the superficial ulcer usually left by the breaking down of a phlyctenule, the therapeutic measures recommended in corneal ulcers having a different origin-the liberal administration of quinin being one of the most important-are indicated. 
Abscess and Ulcer of the Cornea.-In nonsuppurative keratitis the inflammatory infiltrate, which characterizes all types of corneal inflammation (Fig. 88), disappears more or less completely without the formation of pus and without necrosis of the corneal stroma. In suppurative keratitis, on the other hand, the infiltrate breaks down, pus is formed, and to a greater or less extent necrosis of the corneal tissue occurs. When this pus formation and this tissue necrosis take place in the superficial layers of the cornea, we have a corneal

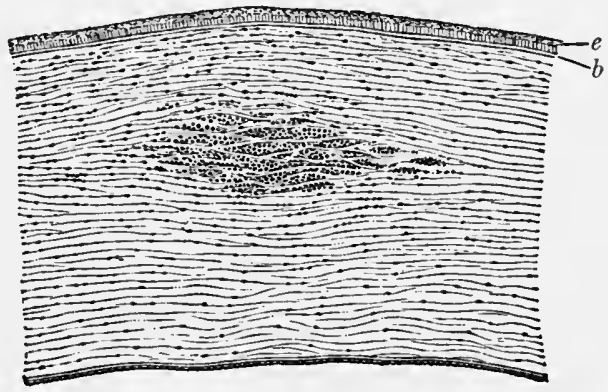

Fig. 88.- Infiltrate in the cornea; first stage of keratitis (Saemisch). The epithelium, $e$, and Bowman's membrane, $b$, over the infiltrate are preserved.

ulcer; when they occur in the deeper layers, and are shut in, in front and behind, by tissue which is relatively sound, an abscess of the cornea.

As a rule, a corneal abscess tends to become converted into an ulcer through breaking down of the overlying corneal substance. Exceptionally, the underlying tissue breaks down, and the abscess discharges into the anterior chamber; while in other instances the pus burrows extensively between the corneal layers, and shows little disposition to make toward either the anterior or the posterior surface of the cornea. 
As may be inferred from this description, abscess and ulcer of the cornea are alike in their etiology, and differ but little in the symptoms which attend them. Among their commoner causes may be mentioned traumatism, complicated by bacterial infection; purulent conjunctivitis; blennorrhea of the lacrimal sac; abnormal exposure of the cornea, such as may result from paralysis of the orbicularis muscle; a lowered state of the general system, as after typhoid fever or the exanthematous fevers; auto-infection, from the alimentary canal especially; senility; and disturbances in the nerve-supply of the cornea, having their origin usually, it seems probable, in the gasserian or in the ophthalmic ganglion.

A moment's reflection will show that the mode of action of these various causes is essentially the same; that they all-the traumatism, the neuro-paralysis, the lowered vitality of the system, etc.-pave the way for successful bacterial invasion of the corneal tissue.

The symptoms common to the two conditions are pain, often very severe, and referred not only to the eye but to the brow, temple, and side of the head, photophobia, lacrimation, and blepharospasm. Exceptionally, especially in the aged, an ulcer may develop in the cornea without attendant pain or photophobia. Inspection of the eye shows circumscribed opacity of the cornea, marked conjunctival injection, more pronounced in the neighborhood of the ulcer or abscess, a contracted pupil, frequently decided edema of the lids, slight muco-purulent discharge, and in the more unfavorable cases hypopyon-a collection of pus or of fibrin and round cells in the lower part of the anterior chamber (Plate V, Fig. I). In the case of an abscess, the opacity will be beneath the surface of the 


\section{PLATE V.}

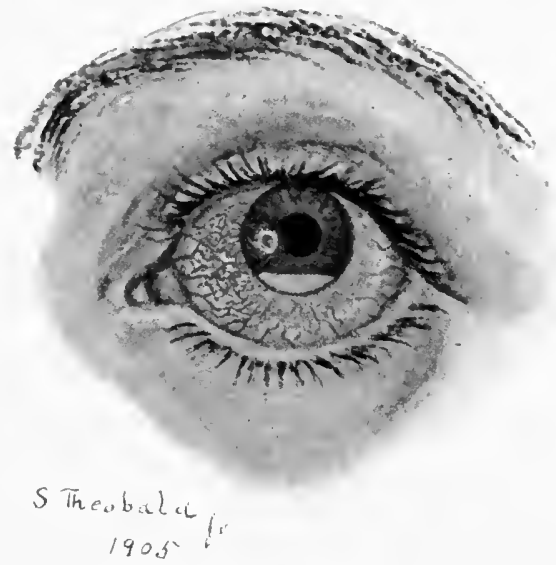

Fig. i.-Corneal Ulcer, with Hypopyon.

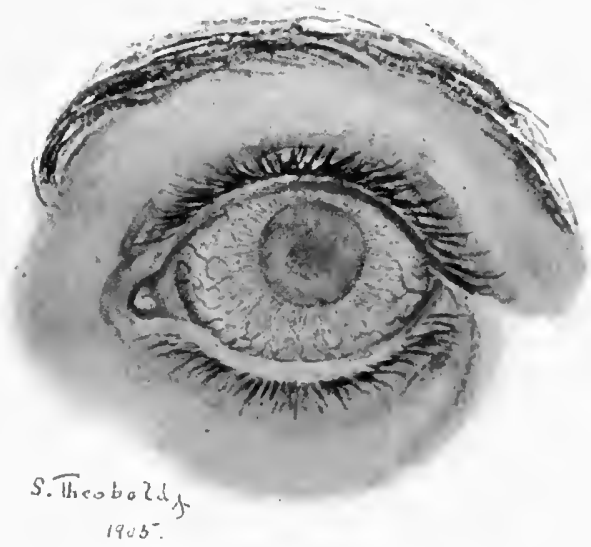

Fig. 2.-Interstitial Keratitis. 

cornea, which will be seen to be intact. If an ulcer be present, there will be a manifest loss of substance, an excavation, which may be shallow or deep, and vary greatly in extent.

Much may be learned as to the condition of a corneal ulcer by careful inspection, especially with the aid of oblique illumination. If it be in a progressive stage, its walls will be opaque and its edges ragged and somewhat undermined (Fig. 89); if in a regressive stage, if the re-

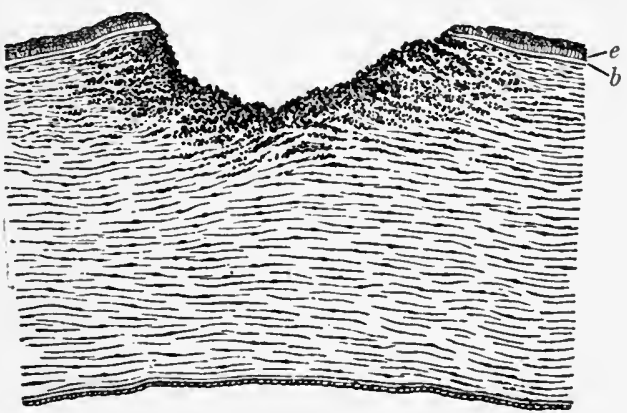

Fig. 89.-Corneal ulcer in progressive stage (Saemisch). The base of the ulcer is formed by an accumulation of pus cells, which also push their way some distance in between the lamellæ of the cornea that adjoin the ulcer. At the edges of the ulcer, which are somewhat raised, the epithelium, $e$, and Bowman's membrane, $b$, end as if cut short off.

parative process has been established, it will present a cleaner appearance, its walls will be nearly transparent, and its edges rounded off (Fig. 90), and, especially if it be near the corneal border, newly formed, superficial blood-vessels will, perhaps, be seen running to its margin from the neighboring conjunctiva.

There is a vast difference in the behavior and in the tractability of corneal ulcers. In some instances they are uncontrollable, and tend to go from bad to worse in spite of all that can be done to combat them; in 
others, they show a disposition to do well, and respond promptly and favorably to treatment. It is usual to denominate ulcers of the former type "infected" ulcers, and those of the latter, "simple" ulcers; but this nomenclature is open to objection, for even the mildest ulcers are "infected," in the sense that bacteria nearly always play a part in their production.

We have yet much to learn regarding the etiology and pathology of corneal ulcers; but enough already is known

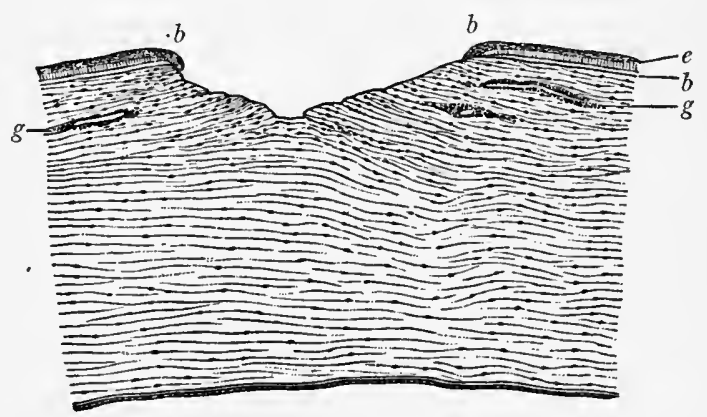

Fig. 90.-Corneal ulcer in regressive stage (Saemisch). The base of the ulcer is formed by the denuded lamellæ of the cornea; a slight increase in the number of cells between them can still be made out. At the edges, $b$, of the ulcer the epithelium, $e$, is beginning to grow out over the base. Newly formed blood-vessels $(g)$ lying in the upper layers of the cornea run to the ulcer.

to warrant the statement that their malignancy does not depend upon the mere presence of bacteria, but rather upon the nature of the bacteria and, scarcely to a less degree, upon the character of the opposition-feeble or energetic-which they encounter in their invasion of the corneal tissue. When, in consequence of lowered general vitality or of unusual local conditions, this resistance is exceptionally inefficient, even the less virulent bacteria, such as the Staphylococcus pyogenes aureus, may do irreparable damage; but usually when the infection is of 
this character the ulcer is benign and not difficult to control. On the other hand, when there is a streptococcus infection, or when the pneumococcus or the KlebsLöffler bacillus is present, the ulcer is apt to exhibit greater malignancy, and to tax our therapeutic powers.

Systematic writers upon diseases of the eye commonly speak not only of "simple" and "infected" ulcers, but they describe several kinds of "infected" ulcers, to which they give particular names, such as serpent or acute sloughing ulcer, marginal or ring ulcer, herpetic or dendritic ulcer, and hypopyon ulcer. It would serve no useful purpose to describe and consider each of these at length; but it may be stated briefly that the serpent ulcer, which shows a tendency to progress in a tortuous course upon the cornea, is often of traumatic origin, is usually attended by hypopyon, and is especially dangerous, causing loss of sight not infrequently through involvement of the deeper structures of the eye; that the ring ulcer, which tends to encircle the cornea close to its margin, often proves intractable, and in the worst cases may lead to blindness through complete necrosis of the cornea; and that the dendritic ulcer-named because of its fancied resemblance to a fern leaf, and also called mycotic ulcer, because in some instances it has been found to be infected with a fungous growth-is obstinate in character, owing to its herpetic origin is not infrequently attended by anesthesia of the cornea and by marked ciliary irritation, is met with at times as a sequel of malarial fever, and is prone to leave in its wake a much more persistent and conspicuous opacity than the slight loss of substance which commonly attends it would lead one to expect.

Treatment.-In the treatment of abscess and ulcer 
of the cornea constitutional measures are scarcely of less importance than local measures. By means of constitutional measures it is possible to strengthen the combative powers of the cornea, and, when the infection is entogenous, to rid the system, in large measure perhaps, of the bacteria and their toxins which are responsible for the local malady. By the use of local applications we are able to enfeeble or destroy the bacteria at their point of attack, and to put the eye in the most favorable condition to resist their destructive action.

Quinin, in liberal doses, is the constitutional remedy of greatest value, since, perhaps by increasing the phagocytic action of the cells or the bactericidal energy of the body juices, it unquestionably reinforces the resisting power of the cornea.* An energetic calomel purge is the agent which, by unloading the alimentary canal, accomplishes most toward ridding the system of bacteria and their poisonous products.

The most useful local remedies are atropin, holocain, dionin, occasionally eserin, and, as bactericides, boracic acid, yellow oxid of mercury, chlorin water, carbolic acid, curettage, and, under exceptional circumstances, the actual cautery. The opium and boracic acid lotion, which has been mentioned so often, is a valuable adjuvant. In benign ulcers, such, for example, as occur in phlyctenular keratitis, a one-grain solution of atropin, with the addition of boracic acid (gr. $x-x v$ to $\tilde{3} j$ ), applied three to four times a day, and supplemented by the daily application of yellow oxid of mercury ointment (gr.j to vaselin $\tilde{\jmath} \mathrm{j}$ ), is in most instances efficacious. Chlorin water, which should be of full strength and freshly

* Mercury, given so as to impress the system, produces exactly the opposite effect. 
prepared, is a valuable remedy in less benign ulcers, especially those of traumatic origin. Its application causes little or no discomfort, and it should be dropped into the eye freely every two or three hours, being used in conjunction with atropin or holocain.

The existence of pain, photophobia, and lacrimation is an indication for employing a strong solution of atropin (gr. iv to $3 \mathrm{j}$ ). Holocain (the hydrochlorate) is used in one- to two-grain solution, and with this also it is well to combine boracic acid. It should be applied once in three hours. It may be used instead of atropin, when the latter does not act favorably, or in conjunction with it. It relieves pain, and promotes the healing of the ulcer, partly, at least, by favoring the formation of new blood-vessels upon the cornea. Although an anesthetic, it does not, like cocain, dilate the pupil or disturb the corneal epithelium. Dionin, which, in conjunction with atropin or holocain, may be used in five per cent. solution, as often as three times a day, not only relieves pain, but furthers the process of repair and the absorption of inflammatory products through its stimulant action upon the lymph currents of the eye. The lotion of opium (ext. opii, gr. $x-x v$; acid. boracic., gr. $x l-l x$; aquæ destil., $\boldsymbol{3}$ iv), if acceptable to the eye, should be used freely, being applied more or less constantly to the closed lids upon a gauze or linen pad. Eserin is indicated, in my judgment, only when there is increased intraocular tension, and, because of its tendency to produce iritis, it should be used with caution and only in weak solution (gr. $\frac{1}{4}-\mathrm{j}$ to $5 \mathrm{j}$ ).

In ulcers that are foul and show a disposition to extend carbolic acid, in full strength, carefully applied directly to the ulcer, and usually preceded by cautious curetting, often accomplishes great good, changing the 
character of the ulcer, and inducing the process of repair. To facilitate the application the eye should be thoroughly cocainized, which renders the procedure painless. The application may be made conveniently by means of a finely pointed wooden toothpick, about the tip of which a few fibers of absorbent cotton have been wound. If much cotton is used, an excess of the acid will be taken up, and it will be almost impossible to prevent its spreading over healthy portions of the cornea. The acid should be applied to the ulcer thoroughly by a gentle rubbing movement, which is, in effect, a sort of curettage. If the ulcer is lined by infected and necrotic material, this should be removed with a small curet before the application of the acid. When, however, this condition is less pronounced, aided by the loosening action of the cocain, the cleaning of the ulcer may be effected satisfactorily by means of a toothpick, armed with a wisp of dry absorbent cotton. After the acid has been allowed to remain in contact with the ulcer for a few moments, the lids meantime being held apart, its further action should be arrested by flushing the cornea with sterile water, normal salt solution, or a saturated solution of boracic acid. Some smarting may be felt in the eye after the effect of the cocain has passed off, but usually this is not pronounced. The application may be repeated after twenty-four hours, should the ulcer still present a foul appearance.

The timely employment of carbolic acid in the careful manner just described will reduce to a minimum the cases in which resort to the actual cautery will be demanded. However, when the condition of the ulcer is not improved by the application of the acid, or when the ulcer is extending rapidly and perforation of the cornea is imminent, the actual cautery should be em- 
ployed. The galvanocautery, provided with a very delicate platinum tip, is best adapted for the purpose. So energetic an agent, however, should be employed only by those skilled in its use, since in untrained hands it is apt to do irreparable damage to the delicate structures of the eye.

Paracentesis of the anterior chamber for the purpose of removing the hypopyon, which, as has been said, is often present in the more malignant types of corneal ulcers, and incision of corneal abscesses with a view of giving vent to the insignificant amount of pus which they commonly contain, are procedures of doubtful value, which were formerly more in vogue than they are at present. The traumatism involved, more especially in the first-mentioned procedure, seems not infrequently to turn the scales, and to hasten the impending total necrosis of the cornea. However, an abscess which is extending laterally, and shows no disposition to reach the surface, should be opened by the removal of the overlying corneal tissue with a curet, and should then be treated as one would treat a malignant ulcerby the application of carbolic acid or the galvanocautery.

Keratomalacia.-As the name indicates, this is a softening or sloughing of the cornea. Though not one of the commoner diseases of the eye, it has seemed to me to demand, at least, brief consideration, since it is a malady which the general practitioner may be called upon at any time to recognize and treat. It is a condition rarely met with except in children, and occurs in them as a result usually of some exhausting disease, such as scarlet fever, measles, typhus fever, or inherited syphilis, or as a consequence of malnutrition from insufficient or improper food. It is attended by none 
of the usual symptoms of corneal inflammation, such as pain, photophobia, and lacrimation; indeed, diminished lacrimation is a feature of the disease. It is frequently preceded by night-blindness, which is simply another manifestation of the lowered general vitality, and by desiccation of the corneal and conjunctival epithelium. It commonly affects both eyes, and the necrotic process, which is clearly the result of unopposed bacterial invasion, may progress so rapidly as to cause complete destruction of the cornex within a few hours. (See Fig. 66.) It is accompanied by pericorneal, venous injection of a dusky red color.

Fortunately, the children attacked by this terrible malady seldom long survive the ocular involvement, but die from general exhaustion or from some intercurrent affection, such as pneumonia or bronchitis.

Treatment.-This is of little avail after the corneal necrosis has commenced. The indications are to inprove the general condition of the child by every means possible-by nourishing diet, stimulants, and tonics, quinin especially, and to apply soothing and antiseptic remedies to the eyes, atropin, in weak solution, or holocain, and chlorin water or a saturated solution of boracic acid. If the case is seen before the cornea has begun to break down, during the period of nightblindness and conjunctival desiccation, the energetic employment of the measures indicated may prevent the loss of sight.

Neuropathic Keratitis.-This variety of keratitis, that is to say, keratitis dependent primarily upon disorder of the nerve-supply of the cornea, is, in my opinion, of much more frequent occurrence than is commonly supposed. The more severe types of this affection, such as' arise from serious lesions of the fifth nerve or of the 
gasserian ganglion, it is true, are not common; but the milder forms, characterized by more or less pronounced anesthesia of the cornea, and having their origin, probably, in pathological changes in the ophthalmic ganglion, are often encountered. Doubtless in some cases of corneal inflammation originating in this manner bacteria play a part,- - an important part in those which are attended by suppuration and considerable loss of corneal substance,-but the prime factor in the production of this type of keratitis is the disturbance in the metabolism of the cornea due to the derangement of its nerve-supply. Probably the sympathetic fibers which pass from the ophthalmic ganglion to the eye have most to do with this disturbance; but, at all events, the sensory fibers, as well, usually are involved, as is shown by the corneal anesthesia.

Keratitis from Lesions of the Ophthalmic Division of the Fifth Nerve, its Nucleus, or the Gasserian Ganglion. -In serious lesions of the ophthalmic division of the fifth nerve or its nucleus, or of the gasserian ganglion, severe and rapidly destructive inflammation of the cornea at times occurs. Under such circumstances we first observe a lack of luster, quickly followed by desquamation, of the corneal epithelium. Then the anesthetic cornea, which, owing to the lessened activity of the lacrimal gland, is not bathed and moistened by the tears as it is under normal conditions, becomes centrally clouded and then, perhaps, rapidly necrotic.

The view, widely held, that this is a purely traumatic inflammation, due to injuries received in consequence of the want of sensibility of the cornea, always has seemed to me chimerical. A more reasonable explanation, as has already been suggested, is that owing to its deranged metabolism the cornea is in a state of 
unstable equilibrium, so to speak,-a state in which, with all other conditions favorable, its vitality is hardly maintained. Under such circumstances, with the corneal epithelium denuded, the conditions are ideally favorable for destructive bacterial action; so that even the less virulent micro-organisms that are not infrequently present in the conjunctival sac, and that usually are harmless, are now able to overcome the feeble resistance opposed to them, and to cause destruction of the corneal tissue.

Herpes Zoster Opbthalmicus.-Another severe type of neuropathic keratitis, which fortunately, like that just described, is not common, is that which is met with in herpes zoster ophthalmicus. Here, as is well known, the primary lesion is in the gasserian ganglion. Usually in this affection the ocular inflammation is not limited to the cornea, but involves the iris and at times the deeper portions of the uveal tract as well.

The keratitis of herpes zoster, which is almost always unilateral, is usually obstinate, deep-seated, and attended by severe pain and pronounced photophobia. At the outset vesicles, commonly in groups, make their appearance upon the cornea. These rupture early, and leave superficial ulcers, which may extend deeply into the corneal tissue. Anesthesia of the cornea, as well as of the lids and forehead, is present. The onesided eruption upon the upper lid, forehead, and scalp, and less frequently upon the side of the nose, is characteristic, and indicates the true nature of the affection (Fig. 9I). Perforation of the cornea rarely occurs, but indelible opacities are frequently left, which may seriously and permanently impair sight.

$T$ be milder forms of neuropatbic keratitis, under which head I would include herpes corneæ febrilis, post- 
malarial keratitis, and dendritic keratitis, are, as has been said, of frequent occurrence. They appear to arise from a variety of causes, and often occur without assignable reason. They are characterized by more or less pronounced anesthesia of the cornea; may be attended by the formation of vesicles, by superficial ulceration or simply by inflammatory infiltration; are inclined to be intractable; are almost invariably monocular, and are apt to leave persistent opacities, out of proportion to the loss of tissue which attends them (Fig. 92). Among

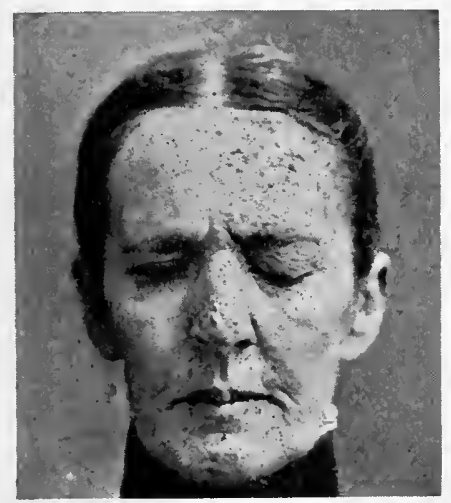

Fig. 91.-Herpes zoster ophthalmicus (Posey and Wright).

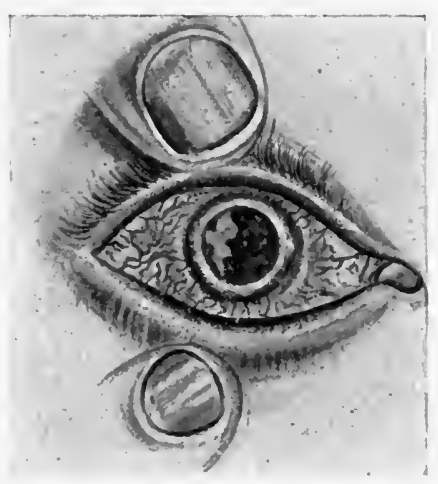

Fig. 92,-Herpetic (neuropathic) keratitis (Haab).

their known causes may be mentioned "cold," malarial fever, influenza, bronchitis, pneumonia, typhoid fever, reflex dental irritation, and probably rheumatism, gout, and syphilis.

I have long believed, though I have no definite evidence to offer in support of this belief, that the primary lesion in these types of keratitis is in the ophthalmic ganglion. That, in fact, we have here a condition closely related to herpes zoster ophthalmicus, the difference being that in one case, the primary lesion 
being in the gasserian ganglion, the resultant inflammatory changes and the attendant anesthesia manifest themselves not only in the eye but in other regionsthe lid, forehead, etc.-supplied by the fifth nerve; while in the other, in which the original fault is in the ophthalmic ganglion, the consequent inflammation and anesthesia, as might be expected, are limited to the eye itself. A significant fact, worthy of mention in this connection, is that malarial fever, which is one of the most definitely proved causes of the type of neuropathic keratitis we are considering, is also a recognized cause of herpes zoster. The unilateral character of both affections is also significant.

Post-malarial Keratitis.-One of the most typical examples of the milder variety of neuropathic keratitis is that which follows malarial fever. In this affection, which is unilateral, and is attended by marked ciliary irritation,-pain, photophobia, and lacrimation,--we have impairment of corneal sensibility and superficial inflammatory infiltration and ulceration, the ulcer showing but little tendency to spread, and often exhibiting a branched or arborescent form. Closely related to this, frequently not to be distinguished from it, and in many instances, probably, identical with it, is the so-called dendritic keratitis. The close resemblance of these two affections, for they are alike not only in appearance but in general behavior, is to be attributed, I believe, to the probable fact that, whatever the inducing cause, each has its origin in disease of the ophthalmic ganglion. The characteristic feature of berpes cornea febrilis, another nearly related affection, attended by impaired sensibility of the cornea and almost without exception unilateral, is the appearance at the outset of the attack of numerous small vesicles, 
which soon rupture, leaving superficial ulcers, that not infrequently present the same dendritic or arborescent form just spoken of.*

Treatment.-In the treatment of neuropathic keratitis, as may be inferred from what has been said as to its etiology, constitutional as well as local remedies are called for. The most useful local remedy, indicated in all of its forms, is atropin, with which it is well to combine boracic acid. Depending upon the amount of pain, photophobia, etc., present, the strength of the solution prescribed should vary from one to four grains to the ounce. Holocain, in one-grain-to-theounce solution, is also useful, and so is the lotion of opium and boracic acid. When, in the ulcerative forms, secondary bacterial infection is found to have occurred, chlorin water, employed as recommended in other intractable corneal ulcers, is valuable, and under such circumstances it may become necessary to resort to curettage and the application of pure carbolic acid.

In the severe type of neuropathic keratitis which results from lesion of the fifth nerve or its nucleus one of the most important measures is to keep the lids constantly closed by a light and evenly applied bandage, so as to afford the eye as complete protection as possible. In herpes zoster ophthalmicus the systematic use of atropin in strong solution (gr. iv to $5 \mathrm{j}$ ) is especially indicated, not only on account of the severe pain which commonly attends it, but because of the danger that iritis may develop at any time.

The most valuable constitutional remedy is quinin,

* Fuchs, than whom, on such a point, there is scarcely a higher authority, seems not disposed to make a distinction between herpes corneæ febrilis and keratitis dendritica. 
which should be given with sufficient freedom-three grains, four or five times a day-to produce moderate cinchonism. Its usefulness is by no means confined to the post-malarial type of neuropathic keratitis, but is manifested in all varieties of the affection. Strychnin, which may be given in association with the quinin, is useful also, and iron should be prescribed when the condition of the system seems to call for it. Arsenic, a remedy often recommended, I have found rather disappointing in its effect. Next to quinin, I am disposed to regard potassium iodid as the drug most apt to accomplish good. In prescribing it I have had in view its effect upon the hypothetical lesion of the ophthalmic ganglion-for it is especially in the milder forms of neuropathic keratitis that it has proved efficacious-rather than any direct influence which it might exert upon the keratitis itself. It should be given in five- to ten-grain doses, three times a day. Salicylic acid is another remedy which has been recommended, especially in herpes zoster ophthalmicus (Leber); but as to its value I can not speak from experience.

The possibility that an intractable keratitis of the type under consideration may be due to reflex dental irritation should not be lost sight of. Such cases, doubtless, are not common; but that they do occur I am convinced. "Dead" teeth, always, it would seem, on the side of the eye affected and usually in the upper jaw, are more apt to produce such consequences. If, therefore, such teeth, or others badly carious, are found to be present, and especially if they are painful or sensitive to pressure, they should be extracted without unnecessary delay. From this measure I have seen benefit result too often to leave any doubt in my mind as to the propriety of resorting to it. 
In this connection it may be not without interest to mention that I have met with two cases of monocular paralysis of accommodation, attended by mydriasis, which were clearly due to reflex dental irritation, and that from this same cause, as well as from the irritation attendant upon phimosis and the existence of adhesions between the prepuce and the glans penis, I have observed clonic spasm of the orbicular muscle of the lids.

\section{NON-SUPPURATIVE KERATITIS.}

\section{Interstitial Keratitis (Parenchymatous Kerati-} tis).- This interesting variety of corneal inflammation, the true nature of which was first recognized by that admirable clinical observer, Mr. Jonathan Hutchinson, of London, occurs only as a result of inherited syphilis. Other sorts of deep keratitis may resemble it in appearance; but they are not greatly like it in this respect, and are entirely unlike it in all other respects. In typical cases the appearance of the cornea-the socalled "ground-glass cornea"--is so characteristic that an error in diagnosis is scarcely possible. (Plate V, Fig. 2.) On the other hand, in less severe cases it is not always an easy matter to recognize its true character.

Although occasionally encountered in adults, this type of keratitis is essentially a disease of childhood, and commonly occurs between the ages of five and fifteen. Usually, but not always, it affects both eyes, generally manifesting itself first in one eye and subsequently, after days or weeks, attacking the other. It is seldom attended by pain, but is commonly accompanied by pronounced photophobia and lacrimation, and at times by almost uncontrollable blepharospasm. Depending upon the extent and density of 
the corneal opacity, vision may be but slightly affected, or may be reduced to mere light perception.

Interstitial keratitis is a disease of the substantia propria of the cornea, more especially of its deeper layers, and it is attended not only by dense inflammatory infiltration but by the formation of numerous fine blood-vessels, these vessels also being deeply seated, and not upon the surface of the cornea as we find them in pannus (Fig. 93). This feature, of new-vessel formation, varies greatly in different cases, being at times very pronounced and again scarcely perceptible or, it may be, entirely absent. When it is marked, it gives to the cornea a salmon-pink or even a crimson color. There is also injection of the conjunctival vessels, this injection being especially noticeable near the border of the cornea and particularly at points where the keratitis is pronounced.

The inflammatory infiltration may begin at the margin of the cornea or near its center. In the former case there is usually an ill-defined, deep-seated, nebulous opacity, which at a later stage may assume a salmonpink appearance from the formation of new blood-vessels, as has just been mentioned. Often there are several such areas of infiltration, and through their extension and confluence the whole cornea may become involved. When the inflammation begins centrally, the opacity may be of the same diffused and ill-defined character, or there may be many small, densely opaque, deeply-seated maculæ. The epithelium over the areas of infiltration loses its luster, and the surface of the cornea presents an appearance like that produced by breathing upon cold glass. The "ground-glass" effect is exhibited most strikingly in those cases in which the infiltration is dense and the formation of new vessels is 
slight. There is no tendency to tissue necrosis, and never any loss of corneal substance through ulceration or suppuration.

The most marked characteristic of the disease is its

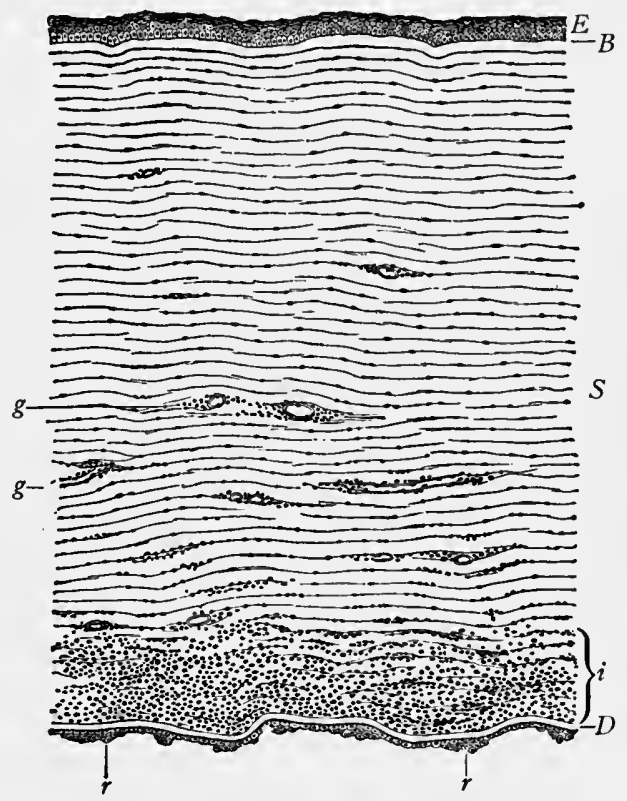

Fig. 93-Cross-section of cornea in interstitial keratitis (after a preparation of Dr. Nordenson) (Fuchs). The stroma, $S$, of the cornea shows an infiltration, which begins in the middle layer, and keeps on increasing more and more posteriorly, so that the deepest layers, $i$, have assumed the aspect of a granulating tissue. On account of the inequality in the degree of thickening of these layers, Descemet's membrane, $D$, is undulated; upon its endothelium there are deposited in places small accumulations of roundcells, $r$. In the middle and deep layers of the cornea we see the transverse and longitudinal section of newly formed blood-vessels, $g, g$, while the most anterior layers, and also Bowman's membrane, $B$, and the epithelium, $E$, are normal.

chronicity, the tedious course which it always runs-a course not of weeks, but of many months. Because of this, it is especially important that its true character should be recognized at the outset, in order that those 
who are interested in the welfare of the patient may know what is in store for them. Although the sight for a considerable time may be seriously impaired, reduced, it may be, to mere light perception, as has been stated, the ultimate prognosis is decidedly favorable. This is due to the remarkable manner in which the opacity of the cornea slowly, but in most instances almost completely, disappears. In no other variety of keratitis do we find so remarkable a change in this regard. However, in the bad cases, in which the corneal opacity renders inspection of the deeper structures of the eye and even of the iris impossible, it is well that the prognosis should be guarded, as under such circumstances iritis or choroido-retinitis may occur, without our being aware of it, and do serious damage to vision. In some instances, too, marked and permanent impairment of sight may result from persistence of the corneal opacity.

The occurrence of iritis is especially to be deplored because, owing to the condition of the cornea, atropin is very imperfectly absorbed, and it is often impossible to induce the pupil to dilate. Even when iritis is not present the pupil frequently remains contracted for weeks in spite of the daily application of a strong solution of atropin, and finally responds to its influence only when the keratitis has measurably subsided. The occurrence of pain, which, as has been remarked, is not a usual symptom of this type of corneal inflammation, points to the probable development of iritis.

Contrary to the opinion expressed by some excellent authorities, recurrent attacks of interstitial keratitis are by no means rare. Not very infrequently, after an intermission of months or even of years, I have seen the disease recur, although the inflammation is apt to be less severe than in the primary attack. 
As might be supposed, other evidences of the constitutional taint are often associated with the eye affection. The notched and pegged teeth-the Hutchinson teeth (Fig. 94) -and the physiognomy characteristic of inherited syphilis-the sunken nose-bridge, the prominent forehead, and the fissured mouth-angles-are those most frequently observed (Fig. 95), while periostitis of the long bones and pronounced labyrinthine deafness are

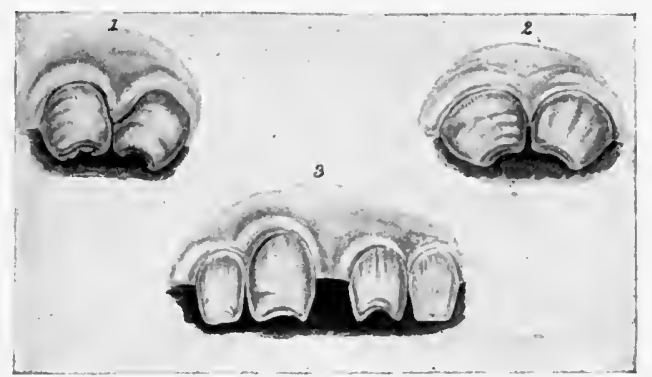

Fig. 94--Hutchinson's teeth (Hutchinson): I, The central upper incisors of a lad, aged fifteen years, the subject of inherited syphilis. The teeth are short, convergent, narrow from side to side at their edges, and show in each a vertical notch. 2, These teeth present similar characters. The notches, however, are less deep, while the narrowing from side to side is very marked. 3, The upper incisors of a girl aged seventeen years. There is a wide space between the central ones, and both these teeth, although of nearly normal length, are narrow, and show deep vertical notches. As is usual, the lateral incisors are more nearly normal in size and form. These teeth are not so typical as those shown in I and 2 .

not uncommon. It goes without saying, therefore, that in every case of supposed interstitial keratitis such signs of inherited lues should be searched for, and the family history should be inquired into.

Treatment.-Although it is not possible by any therapeutic measures to cut short an attack of interstitial keratitis, there can be no question as to the value of treatment, when instituted early and persisted in systematically. Besides lessening the discomfort of the patient 
- the photophobia, lacrimation, and blepharospasm-it unquestionably shortens the duration of the disease; not infrequently prevents the second eye from becoming involved; renders less probable the extension of the inflammation to the iris and deeper tunics of the eye, and greatly diminishes the likelihood of the sight being permanently impaired through the persistence of the cor-

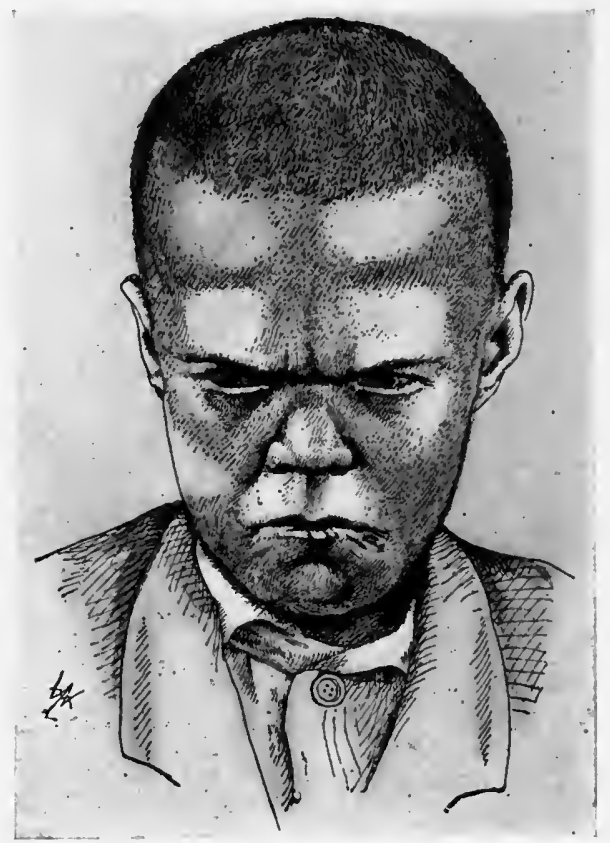

Fig. 95.-Physiognomy characteristic of inherited syphilis (de Schweinitz).

neal opacity. Furthermore, it improves the general health of the patient, and thus, perhaps, prevents the occurrence of other luetic lesions.

Locally, the treatment consists in the liberal use of atropin, the intermittent application of hot fomentations, and the wearing of smoke-tinted glasses. The application of mercurial ointment to the forehead and 
temples is another measure which at times is useful. Owing to the difficulty commonly experienced in dilating the pupil, and the importance, if possible, of accomplishing this, the atropin-which in this affection is well borne, even by young children-should be used in strong solution, usually four grains to the ounce. The hot fomentations are indicated especially during the earlier and more acute stage of the disease, and seem to afford the patient measurable relief. A convenient way of applying heat is by means of a soft, bird's-nest-shaped sponge, which should be dipped frequently into bot water, wrung out, and applied to the lids. This should be done, for fifteen minutes at a time, three or four times a day. During this stage, too, the atropin should be used as of ten as three, or even four, times in twenty-four hours. Later on, when the ciliary irritation and photophobia have subsided, and mydriasis has been established, it need not be used oftener than twice daily, and the hot fomentations may be discontinued. Bandaging the eyes is detrimental, and should not be practised. The yellow oxid of mercury ointment, so useful in phlyctenular kerato-conjunctivitis, contrary to what might be expected is of no value in this affection. It is, indeed, recommended by some authorities; but, in my judgment, it does more harm than good, for it irritates the eye and increases the discomfort of the patient. Dionin has been used, and it is claimed with good effect; but it seems hardly likely that it will prove of much value in so chronic an affection as interstitial keratitis.

Mercury, in easily borne doses, potassium iodid, and iron are the constitutional remedies to be relied upon, and with me it is a common practice to administer the three in combination. Inunctions of mercurial oint- 
ment are recommended, and doubtless are valuable, though it has been my habit to employ them only in very young children or when the digestive apparatus is disordered.

I have found it convenient to administer mercury in the form of the biniodid, giving it in doses of $\frac{1}{48}$ to $\frac{1}{24}$ of a grain, according to the age of the patient, and always prescribing it in solution with the addition of potassium iodid, either just enough of the latter to render the mercury soluble or in such quantity as to make the dose from one to five grains. The syrup of the iodid of iron often is added to this solution, or is given alone or in combination with potassium iodid. When the iodids are not well borne, or when they have been given for a considerable time, the bichlorid of mercury is substituted, and with this the tincture of chlorid of iron is frequently combined. Two favorite prescriptions with me are the following:

R. Hydrarg. biniodid................. i.

Potas. iodid. . . . . . . . . . . . gr. v.

Syr. ferri iodid. ............ J ss.

Aquæ ....................

R. Hydrarg. bichlorid................ gr. i.

Tinct. ferri chlorid. . . . . . . . . . 万 ss.

Aquæ ................

The dose of each is a teaspoonful, well diluted with water, three times a day, after meals.

As fresh air and sunlight are beneficial to the patient's general condition, and do the eyes no harm, he should not be housed or kept in the dark; but, in spite of his photophobia, should be encouraged to go into the open air and take plenty of out-of-door exercise. His diet should be regulated in accordance with common-sense 
rules, and condiments, such as pepper, mustard, and the like, especially should be interdicted. The near use of the eyes, as in reading, writing, and sewing, is impracticable during the active stage of the disease, and should be prohibited throughout the long period of convalescence.

The absorption of the corneal opacity is necessarily a slow process, and as this is true especially of that part of it which is situated in the center of the cornea and overlies the pupil, the improvement in vision is distressingly slow. Systematic massage of the eyes-rubbing the cornea gently through the lids with the finger-tipwhich can be done best by the patient himself, but should not be practised until all symptoms of irritation have subsided, probably hastens somewhat the clearing process.

Not infrequently after an attack of interstitial keratitis it will be found that the long-continued inflammation has produced a considerable amount of corneal astigmatism, and if this is not wholly irregular, as, unfortunately, too often is the case, glasses may be prescribed with great advantage.

Pannitic Keratitis or Pannus.-In treating of trachomatous conjunctivitis we have already spoken of this variety of corneal inflammation. As there stated, it is a usual accompaniment of "granular lids," and is largely the result of the mechanical irritation of the cornea by the roughened palpebral conjunctiva. Because of the greater friction exerted upon the cornea by the upper lid, pannus is always more pronounced upon, and is frequently confined to, the upper half of the cornea. Although in the beginning only the epithelium of the cornea is affected, a loss of luster and a slight roughening being observed, the super- 
ficial layers of the substantia propria in time are involved. This happens often in consequence of the formation of ulcers or abscesses, which usually are the result of secondary infections, the condition of the cornea, denuded of its epithelium, being such as to favor bacterial invasion.

The corneal opacity in this affection is less uniform and more superficial than in interstitial keratitis, and there is an unevenness of the surface of the cornea which is not present in the latter disease. A characteristic feature of pannitic keratitis is the formation of new

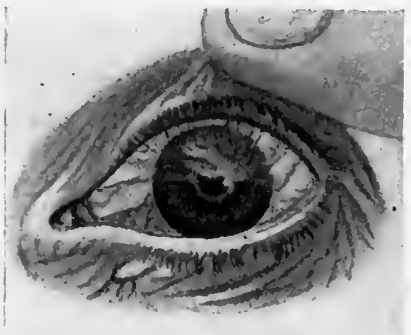

Fig. 96.-Trachoma with pannus (Haab). blood-vessels upon the cornea; but these vessels, which grow out from the neighboring conjunctiva, are coarse and superficial, and bear but little resemblance to those which are observed in the keratitis of inherited syphilis (Fig. 96).

In neglected cases the opacity of the cornea may be so great as to render the iris invisible, and to reduce sight to little better than light perception. While the diffuse opacity due to uncomplicated pannitic keratitis disappears in large measure with the subsidence of the inflammation of the conjunctiva upon which it depends, the opacity caused by intercurrent ulcers or abscesses, which is more dense in character, is apt to be indelible. Apart from this, however, ulcers or abscesses occurring under such circumstances, though they are frequently attended by severe pain and by an aggravation of the photophobia, lacrimation, and blepharospasm that are the usual accompaniments of trachomatous conjunctivitis, 
are not as apt to produce disastrous consequences, such as perforation into the anterior chamber, as are those which develop in a previously healthy cornea. This is because of the increased resisting power which the pannitic cornea derives from its abnormal vascularity.

The diagnosis of pannus usually is not difficult. The coarse and superficial character of the new bloodvessels, the unevenness of the corneal surface, the lack of uniformity in the opacity and the fact that it is commonly limited to the upper half of the cornea indicate its true nature, and should lead at once to an inspection of the tarsal conjunctiva of the upper lid, the appearance of which in trachoma is so typical as to place the diagnosis beyond doubt.

Treatment. - The treatment of pannus is the treatment of trachoma, and this has been fully considered already. It may be well, however, to say that the employment of atropin, and in strong solution, is especially indicated when intercurrent ulcers or abscesses occur, and that the lotion of opium, so often commended, is also very useful under the same circumstances; and, further, to mention the decided benefit that results in some obstinate cases of pannus, which do not respond to the usual remedies, from the performance of the simple operation of cantbotomy.

This operation, which can be done by any one who has a modicum of surgical skill, does good by lessening the tension of the upper lid, and so reducing the friction which it exerts upon the cornea. It consists in a lengthening of the palpebral aperture and a division of that part of the external canthal ligament which is attached to the tarsal cartilage of the upper lid. It is performed as follows: With a pair of straight scissors the external canthus, at one cut, is divided horizontally outward for 
a distance of 8 or $10 \mathrm{~mm}$. (Fig.97). The upper lid is then put upon the stretch, and the superior half of the external canthal ligament, which is thus made tense, is cut through with the scissors, the points of which are inserted vertically beneath the upper lip of the skin wound. The first incision will have divided both skin and conjunctiva, and the final step of the operation consists in attaching the edges of the conjunctival wound to those of the skin wound by several fine sutures, so that the cut edges of the skin shall not unite one with the other (Fig.98).

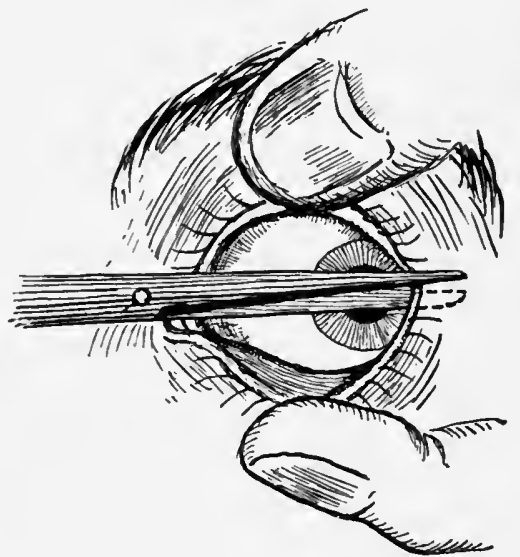

Fig. 97-Canthotomy. The incision (modified after Czermak).

For this purpose I employ fine black silk and half-curved needles, introducing four sutures, two to bring together the upper and two the lower lips of the wound. It is well to make the horizontal incision rather longer than would seem to be necessary, since during the healing process it is sure to shorten somewhat. The sutures may be removed after three or four days. Before, and for a time after, their removal the outer canthus should be anointed with borated vaselin or cold-cream, and any tendency to grow together which the edges of the 
skin wound may show should be overcome by gently stretching them apart. No deformity is left by the operation; indeed, when the palpebral aperture is preternaturally small, as is sometimes the case in old trachoma, a distinct improvement in the appearance of the eye results.

Opacities of the Cornea.-All varieties of keratitis, as has been stated, are attended by more or less pronounced impairment of the transparency of the

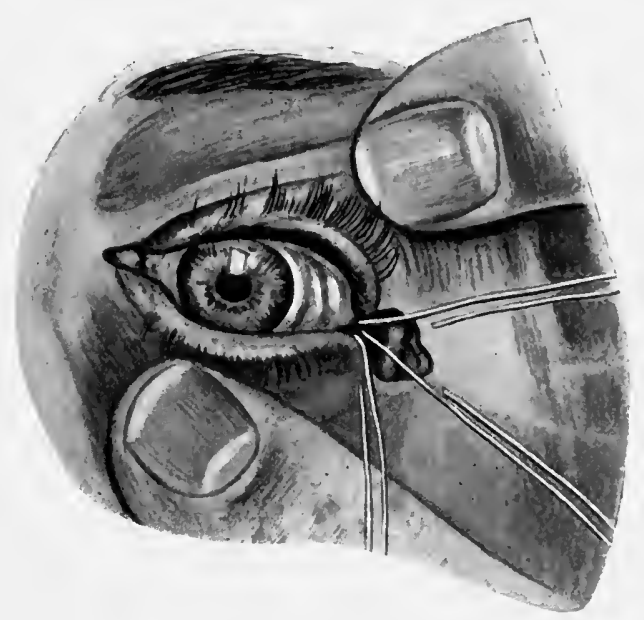

Fig. 98.-Canthotomy. The stitches ready to be tied (Haab).

cornea. Often this loss of transparency is evanescent, but not infrequently, especially when consequent upon suppurative keratitis, it is permanent. Permanent corneal opacities may be caused also by interstitial, and other forms of deep, keratitis, by wounds of the cornea, by severe iritis, and by protracted disease of the deeper tunics of the eye; while one common form of opacity, the well-known arcus senilis-due to colloid degeneration of the superficial layers of the cornea-occurs spontaneously, and, it may be remarked, is frequently 
observed, contrary to what its name would seem to indicate, in persons who are much too young to be designated as "senile." Apart from the trivial unsightliness which attends it, this last-mentioned variety of corneal opacity causes no inconvenience, and does not, as was once supposed, interfere in any degree with the success of operations, such as extraction of cataract, that involve section of the corneal limbus. Undoubtedly, the largest number of indelible opacities of the cornea are the result of ulcers, the density of the opacity being dependent in great measure upon the extent to

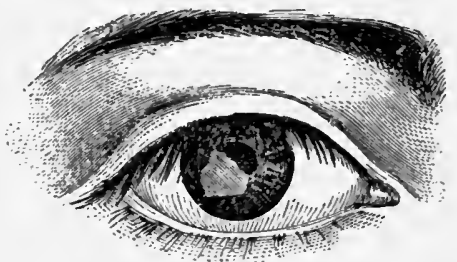

Fig. 99.-Leucoma resulting from a lime burn (Lawson).

which the substantia propria has been destroyed by the ulcerative process.

According to their density, corneal opacities are designated as nebula, macula, or leucomata (Fig. 99), and when, in consequence of an ulcer which has perforated into the anterior chamber, the iris is attached to the corneal scar, we have a leucoma adberens or a leucoma with anterior synechia (Fig. 100).

Marked corneal opacities are always disfiguring; but whether they cause impairment of sight or not depends less upon their density than upon their location, and upon whether they are attended by considerable distortion of the curvature of the cornea. Thus, a very slight nebulous opacity, scarcely perceptible by simple inspection, if situated in the center of the cornea, in the line of 
vision, may seriously impair the sight; whereas even a large and dense leucoma at the margin of the cornea may cause no visual disturbance whatever. The impairment of sight caused by such faint, central nebulæ, it is true, is due less to the slight opacity than to the marked irregular astigmatism which is frequently present under such circumstances, and which leads to the formation of distorted and imperfect retinal images. In the same way corneal scars, which are not in the direct line of vision, may give rise to great disturbance

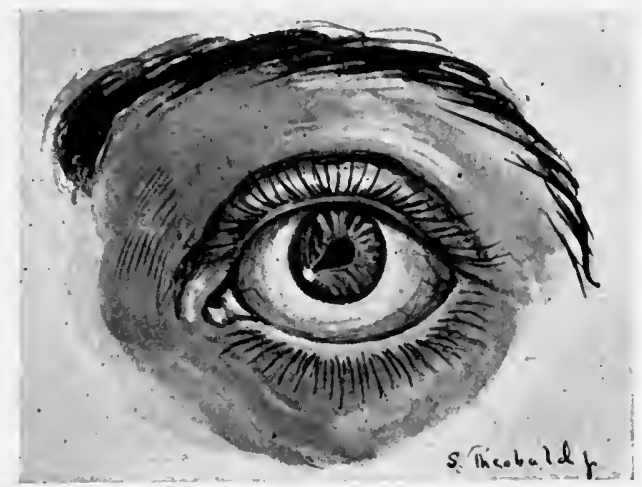

Fig. 100.-Leucoma with anterior synechia.

of sight if they are attended by alteration in the curvature of the cornea.

A superficial, milk-white opacity of the cornea results at times from the injudicious use of collyria containing lead acetate. This happens only when there is denudation of the cornea from ulceration or traumatism, and is caused by the deposit of an insoluble salt of lead upon the denuded surface. Because of its density, such an opacity, if central, produces marked disturbance of vision. To avoid the risk of such an accident, solutions of "sugar of lead" should never be applied 
to the eye when from any cause loss of corneal tissue has taken place.

There is little excuse for confounding corneal opacities with opacities situated in the crystalline lens, and yet, with those unfamiliar with diseases of the eye, this is an error of not very infrequent occurrence. If by simple daylight inspection the location of the opacity is not evident, it can be determined, beyond doubt, by the aid of oblique illumination, which, therefore, should always be employed under such circumstances.

Treatment.-Little can be done to promote the absorption of corneal opacities of long standing. Nature, as has been indicated, often accomplishes a great deal, especially when the opacity is the result of inflammatory infiltration unattended by necrosis of true corneal tissue. Massage,--rubbing the cornea through the upper lid with the finger-tip,-if employed perseveringly, may do some good, provided the opacity is not too dense and is not of too long standing. In connection with massage an ointment of yellow oxid of mercury ( $\mathrm{gr} . \mathrm{j}$ to vaselin 5j) may be used. Subconjunctival injections of salt solution (one per cent. to five per cent.) are not without value in suitable cases, that is to say, in opacities of not too long duration, and, under such circumstances, dionin (in five per cent. solution), which acts in much the same way as the salt injections do-by stimulating the lymph currents of the eye-is of undoubted value. The chances of improvement are better in children than in adults.

When the opacity overlies the pupil, but more especially when the sight of both eyes is impaired, considerable improvement in vision may be obtained by an iridectomy, the artificial pupil being placed behind a clear or relatively clear portion of the cornea. Con- 
spicuous leucomata are sometimes tattooed with India ink, in order to render them less unsightly.

Staphyloma of the Cornea (Anterior Staphyloma).-Occasionally as a result of a penetrating wound or of extensive and deep ulceration of the cornea, but usually in consequence of more or less complete corneal necrosis occurring during the course of a purulent (gonorrheal) conjunctivitis, an irregular protrusion of the anterior segment of the eyeball occurs. When this protrusion is confined to a limited area of the cornea, as is more apt to be the case when it arises from one of the two first-mentioned causes, it is known as partial stapbyloma; when it involves the whole cornea, or, as sometimes happens, the anterior portion of the sclera as well, it is known as total stapbyloma.

In total staphyloma the protrusion, which may be inconsiderable or so great that the lids cannot be closed over it, is irregularly globular in shape and occasionally somewhat lobulated, is bluish-white in color, and frequently exhibits upon its surface several coarse, tortuous veins (Plate VI, Fig. I). The protuberant tissue consists mainly of the iris reinforced by newly formed connective tissue, some remnants of the cornea, perhaps, being found incorporated with its base (Fig. IOI). The development of the staphyloma is due to the inability of this improvised tunic to resist the pressure exerted by the intraocular fluids. In partial staphyloma the walls of the ectasia are made up of corneal, iridic and newly formed scar tissue, and the protuberance is commonly near the margin of the cornea. When the whole cornea is involved in the staphylomatous process vision is necessarily reduced to mere perception of light; but when the defect is partial, good vision may be retained, though, owing to the distortion of 
the corneal curvature and the displacement of the pupil so often present, this is usually not the case.

Because of its poor resisting power, the staphylomatous tissue is not infrequently the seat of recurrent attacks of inflammation. This happens especially in those cases of total staphyloma in which the protuberance is so great as not to receive the protection of the lids. In such cases, too, accidental rupture of the thinned walls of the staphyloma is not uncommon. Infection

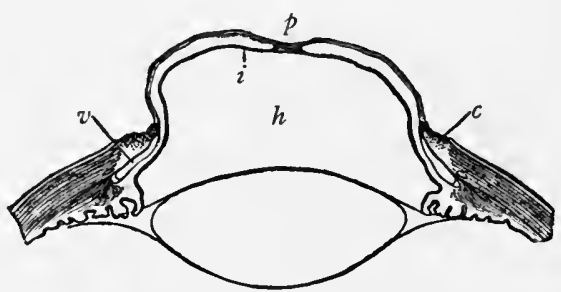

Fig. ror.-Cross-section of a total staphyloma, the result of extensive necrosis of the cornea (schematic) (Fuchs). Only the marginal portions, $c$, of the cornea are preserved, and these are still partially infiltrated. Between them bulges the iris, which is driven strongly forward and which consequently is thinned so that the pigment, $i$, upon its posterior surface shines through it and gives the prolapse a blackish hue. The pupil, $p$, is closed by a membrane. The space, $h$, between the iris and the lens is the enlarged posterior chamber. Of the anterior chamber only the shallow, slit-like, annular space, $v$, is left. This no longer communicates anywhere with the posterior chamber (seclusio pupillæ).

of the deeper tunics of the eye, leading to purulent panophthalmitis, is another accident which may occur at any time.

Treatment.-In its incipiency the development of a staphyloma - of a partial staphyloma more especiallymay be arrested, in some instances, by the continuous support of a not too tightly applied pressure bandage. At this stage, too, a partial abscission of the ectasia or a well-placed iridectomy may be very effective. In total staphyloma restoration of vision is impracticable, and 


\section{PLATE VI.}

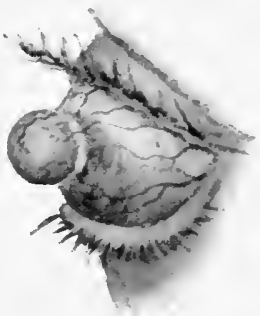

Fig. i.-Total Staphyloma (after Sichel).

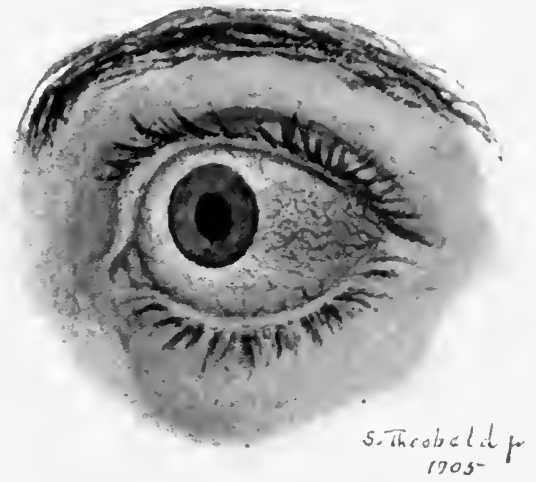

Fig. 2.-EPISCLERITIS.

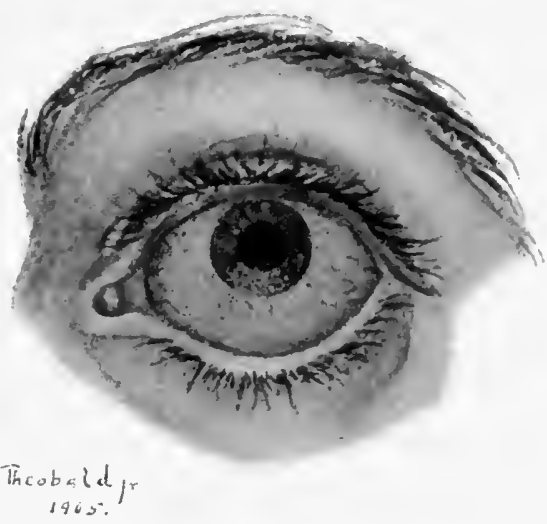

Fig. 3.-Serous Iritis (C'veitis). 

enucleation of the eye is commonly indicated, not for its cosmetic effect only, but because it eliminates all the possible complications mentioned as apt to occur. In children, too young to wear an artificial eye, abscission may be practised in accordance with the method recommended by Knapp, the wound being closed by bringing together, with four or five stitches, the previously undermined conjunctiva.

In partial staphyloma, if the vision is good and the ectasia shows no tendency to increase, surgical interference is usually contraindicated; but, on the other hand, if the vision is poor, and especially if the staphyloma is progressing, an iridectomy may do much good,

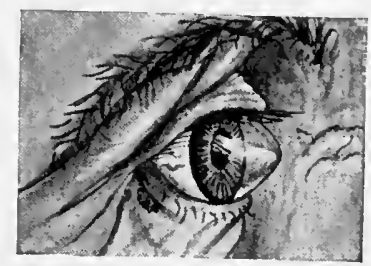

Fig. I02.-Conical cornea (de Schweinitz).

not only by its direct effect upon vision, but by reducing the intraocular tension, and so staying the increase of the staphyloma.

Conical Cornea (Keratoconus).-In this condition, which is comparatively rare and therefore calls for but brief mention, the cornea gradually assumes, usually without considerable loss of transparency, a distinctly conoidal shape (Fig. I02). This change in the form of the cornea gives rise to a high grade of myopia, attended by excessive symmetrical aberration of the eye, in consequence of which vision is greatly impaired.

Treatment.-In the lower grades of this anomaly 
carefully adjusted glasses are helpful. In the higher grades, especially if the condition is progressing, an effort should be made to reduce the protrusion by cautiously cauterizing the apex of the cone with the galvanocautery, care being taken not to perforate the cornea. This delicate procedure, it should be remarked, requires for its successful performance especial skill and experience.

Tumors of the cornea are exceedingly rare, and so do not demand our consideration.

Traumatic lesions of the cornea, which are of common occurrence, are treated of in Chapter XIII, devoted to injuries of the eye.

\section{DISEASES OF THE SCLERA.}

The tough and non-vascular sclera, or sclerotic coat of the eye, is not often the seat of diseased processes. In deep keratitis the neighboring sclera is at times involved, the condition being known as kerato-scleritis, and in syphilitic irido-cyclitis (especially in the negro race) the anterior portion of the sclera may become implicated, and a staphylomatous condition may result from the softening and thinning which it undergoes.

Scleritis (Sclerotitis; Sclero-conjunctivitis).Inflammation confined to, or having its starting-point in, the sclera is usually of rheumatic or gouty origin. Two varieties of scleritis are encountered-an acute, diffuse inflammation, which is aptly described by the name sclero-conjunctivitis, and a more persistent form, in which the inflammation is circumscribed.

Sclero-conjunctivitis.-In this type of scleritis the inflammation is often capricious and fleeting in character, attacking one eye to-day, and after twenty-four or forty-eight hours disappearing from this eye only to 
manifest itself in the other. It is characterized by marked, general injection of the conjunctival and subconjunctival vessels, and is usually attended by pronounced pain, photophobia, and lacrimation.

In chronic scleritis (Fig. I03) the injection and inflammation are confined to a limited area of the sclera, usually near the corneal margin, and the hyperemic vessels are finer and more deeply seated, in consequence of which the affected area presents a dusky red or purplish appearance. Pain and photophobia are less conspicuous in this variety, but involvement of the cornea or iris is more apt to occur.

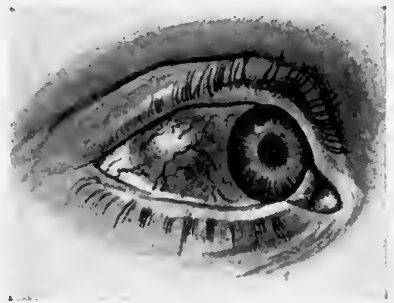

Fig. I03.-Scleritis (Haab).

Acute diffuse scleritis or sclero-conjunctivitis is to be distinguished from simple conjunctivitis by the pain and the greater photophobia and lacrimation which attend it, and by the fact that it is commonly monocular. Its association with other manifestations of a rheumatic or gouty diathesis affords additional evidence of its real character. Except by the course of events, it is not to be distinguished from the incipient stage of luetic or rheumatic iritis-the stage, often observed, in which, as yet, the iris is neither muddy nor swollen, and in which the pupil dilates fully and symmetrically to atropin.

Treatment.-In the acute form of the disease atropin 
in strong solution, and moist heat (hot water or, better still, the lotion of opium, applied hot) are indicated, and, internally, sodium salicylate in liberal doses, supplemented by a brisk purgative. In the circumscribed form, atropin and the lotion of opium are called for, while potassium iodid, sodium salicylate, lithium, and colchicum are the constitutional remedies apt to accomplish the greatest good.

Episcleritis.-This is an affection of not uncommon occurrence, and consists in a circumscribed inflammation, usually monocular, of the episcleral connective tissue, in which the superficial layers of the sclera are frequently involved (Plate VI, Fig. 2). The inflamed area, which is always adjacent to the corneal limbus, is somewhat elevated, and of a dark red or purplish color. Pain is not a usual symptom, but photophobia and an irritability of the eye, unfitting it for near work, are commonly present. This affection, which is rarely encountered in children, and is commoner in women than in men, does not respond satisfactorily to treatment, and, still worse, when seemingly cured, is prone to recur, it may be at the same spot or upon some other part of the pericorneal region.

Episcleritis at times bears a close resemblance to phlyctenular conjunctivitis, and it might be confounded, also, with the bulbar variety of vernal catarrh. Its course, however, is more protracted than the former disease and much less protracted than the latter. Moreover, there is no tendency to ulceration, as in phlyctenular conjunctivitis, and no itching, as in vernal catarrh.

It is often impossible to assign a cause for episcleritis; but there can be no doubt that in many instances it is dependent upon a rheumatic or gouty diathesis. 
Treatment.-This is much the same as in circumscribed scleritis. Atropin with boracic acid, the strength of the solution depending upon the ciliary irritation and photophobia present, or holocain with boracic acid (holocain muriatis, gr. i; acid. boracic., gr. $x$; aq. destil., $3 \mathrm{i}$, to be dropped into the eye every three or four hours), are the most useful local remedies, to which may be added the lotion of opium, if there is pain or marked ciliary irritation. The yellow oxid of mercury, so efficacious in phlyctenular conjunctivitis, is contraindicated. Potassium iodid in moderate doses, and frequently in combination with syrup of the iodid of iron, is the constitutional remedy I have found most beneficial. Other useful remedies are sodium salicylate, and lithium, preferably in the form of the natural mineral water. Regulation of the diet and of the bowels is also important. 


\section{CHAPTER VII.}

\section{DISEASES OF THE IRIS AND CILIARY BODY.}

\section{DISEASES OF THE IRIS.}

Iritis.-Iritis, or inflammation of the iris, is one of the common affections of the eye, and it is one with which it is especially important the general practitioner should be familiar. It arises from a variety of causes, may attack one or both eyes, and, though almost always amenable to treatment if recognized in its inception and judiciously managed, it is apt to impair the sight more or less seriously, and permanently damage the integrity of the eye if allowed to run its course unchecked, or if improperly or only tardily treated. It is essential, therefore, that its true character should be recognized at the outset, and that the requisite therapeutic measures should be resorted to without delay.

The diagnosis of inflammation of the iris is commonly not a difficult matter, and the indications for its treatment are usually plain. Nevertheless, it is frequently confounded with other forms of inflammation of the eye, and for this reason improperly treated. In consequence of this, or because of the ignorance or indifference of those whom it attacks, it is by no means an uncommon cause of blindness.

The existence of iritis is to be suspected whenever, without increase of intraocular tension or other evident cause, pain in and around the eye, usually worse at night, is complained of, and is accompanied by pericorneal subconjunctival injection and a contracted 
pupil. This concourse of symptoms does not necessarily indicate the presence of iritis, but it is distinctly suggestive, and should lead to a careful search for other evidences of its existence. A dull, lack-luster appearance of the iris, with appreciable change of color and more or less swelling of its tissue; immobility of the pupil, and perhaps loss of its circular form; diminished transparency of the aqueous humor, and frequently of the cornea as well, with consequent indistinctness of vision; adhesions between the margin of the pupil and the anterior capsule of the lens, which, however, are frequently not evident until a mydriatic has been used; and in severe cases a grayish opacity of the pupil from the deposition of an organized exudate upon the lens capsule, are the other changes which should be sought for, and which, if found, establish the diagnosis beyond question.

In examining a case of suspected iritis the use of "oblique illumination" is of great assistance, since it enables one to detect slight changes in the cornea and in the tissue of the iris, and in many cases to discover adhesions between the iris and lens, which can not be seen by simple inspection. If, however, any doubt remains as to the existence of iritis after this method of examination has been employed, a weak solution of atropin (gr. ss. $-\mathrm{i}$ to $\mathbf{3} \mathrm{i}$ ) or of homatropin (gr. iv to $\overline{\mathrm{z}}$ ) or euphthalmin (gr. viii to $\xi_{i}$ ) should be dropped into the eye, when, if iritis is present, the pupil will almost certainly dilate in an irregular manner, showing points of adhesion between its margin and the lens capsule.

The character of the vascular injection of the eyeball, while helpful, is not always an entirely trustworthy guide in the differential diagnosis of iritis. When, however, it is most marked around the corneal margin, is of a 
pinkish rather than a brick-red color, and the vessels involved are for the most part small, and radiate more or less regularly from the margin of the cornea toward the equator of the globe, we may, at least, conclude that structures deeper than the conjunctiva are involved in the inflammatory process, and that the existence of iritis is probable.

Among the causes of iritis, syphilis deserves the most prominent place. Traumatism is another frequent cause, and not only when the iris itself is involved in the injury, but also when the cornea, lens, or ciliary body is wounded. Rheumatism and gout, diabetes, and the acute infectious diseases also deserve mention in this connection, and gonorrhea, though an infrequent cause, occasionally gives rise to it, the ocular inflammation having the same relation to the urethral disease that gonorrheal arthritis has. Iritis may also be a consequence of inflammation of other structures of the eye, as, for instance, abscess or perforating ulcer of the cornea.

There is also another cause of iritis to which I am disposed to attach importance, and which I believe to be an essential factor in the production of several apparently distinct varieties of the disease. I refer to an influence transmitted through sympathetic or "trophic" nerves, which is frequently reflex in character, and is probably dependent upon structural changes in gray nerve matter, either in the cerebral ganglia themselves, or in the ganglia connected with the fifth nerve, or in both. It is such an influence as this, I believe, that determines the development of sympathetic iritis, the iritis which is frequently found associated with herpes zoster ophthalmicus, that which occasionally follows malarial attacks, and probably also certain cases of 
serous iritis. In this category belong also those cases of iritis which rightfully, I think, have been ascribed to reflex dental and reflex uterine irritation, as well as certain intractable forms of irido-keratitis, that not infrequently are accompanied by anesthesia of the cor-

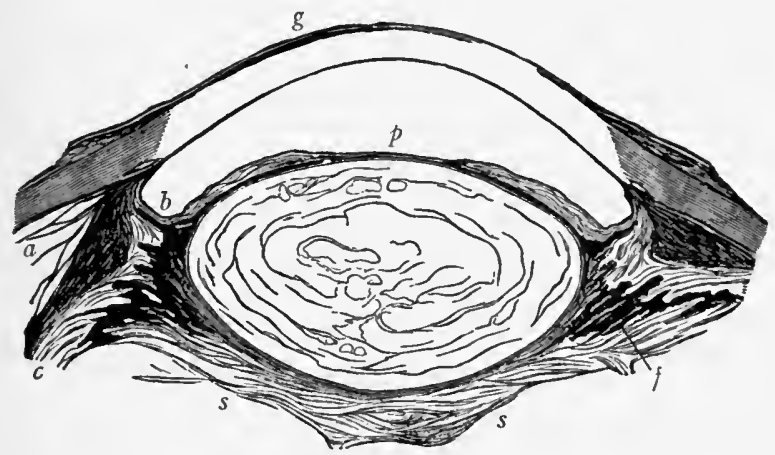

Fig. 104--Vertical section, showing total posterior synechia (Fuchs). The iris is adherent by its posterior surface to the anterior capsule of the lens and also to the anterior surface of the ciliary body. The posterior chamber consequently is obliterated and the anterior chamber deepened at its periphery, $b$; at this spot the iris is strongly retracted and at the same time is here the most thinned through atrophy. The exudate connecting the iris with the lens also stretches as a thin membrane, $p$, across the pupil. The exudate, $s$, springing from the ciliary body, envelops the posterior surface of the lens and by its shrinking draws the ciliary processes toward the center. As a result of this a separation of the ciliary body, $c$, from its bed has already taken place below, and in the intermediate space are seen the disjoined lamellx of the suprachoroil membrane, $a$. The pigment epithelium, $f$, of the ciliary processes has undergone proliferation. At the lower part of the cornea there is a zonular opacity, $g$. The lens is swollen and is opaque throughout; there is no hard undisintegrated nucleus (i.e., it is a soft cataract).

nea. Obstinacy and intractability are the common characteristics of these several varieties of iritis, and in the pathological changes which they exhibit, especially the tendency of the entire posterior surface of the iris to become glued to the lens capsule (Fig. I04), a condition seldom met with even in the worst cases, for 
example, of syphilitic iritis, there are also striking resemblances.*

The consequences of a severe attack of iritis which has been neglected or has been improperly treated are disastrous to the integrity of the eye in several ways. In the first place, especially in syphilitic iritis, other structures of the eye, for example, the ciliary body, choroid, retina, and lens, are liable to become involved in the inflammatory process, and may suffer irreparable damage (Fig. 105). Again, the pupil may be closed or obstructed by an organized membrane (occlusion), so that vision is reduced to mere perception of light; or the iris may become adherent to the anterior surface of the lens, at its pupillary margin only (exclusion), or throughout its whole extent (complete posterior synechia) (Fig. 104). In the two former conditions operative interference may accomplish great good; in the latter, whatever plan of treatment is adopted, the prognosis is much less favorable, and in time the deeper tunics are apt to suffer and the lens to lose its transparency. Sympathetic inflammation of the fellow-eye is another result of neglected iritis, which, though not of frequent occurrence, happens often enough to deserve mention.

Although there are so many causes of iritis, there are, strictly speaking, but three different kinds of iritis - plastic iritis, purulent iritis, and serous iritis (Descemetitis, uveitis). The first named variety, plastic iritis, characterized by the formation of an exudate, rich in

* The author realizes that to express such an opinion as this regarding the genesis of inflammation is to run counter to the teachings of modern pathology; but he is not convinced that the last word has yet been spoken upon this subject. (Compare foot-note on page 259.) 
cells, which tends to become organized, is by far the most comprehensive. It includes most cases of syphil-

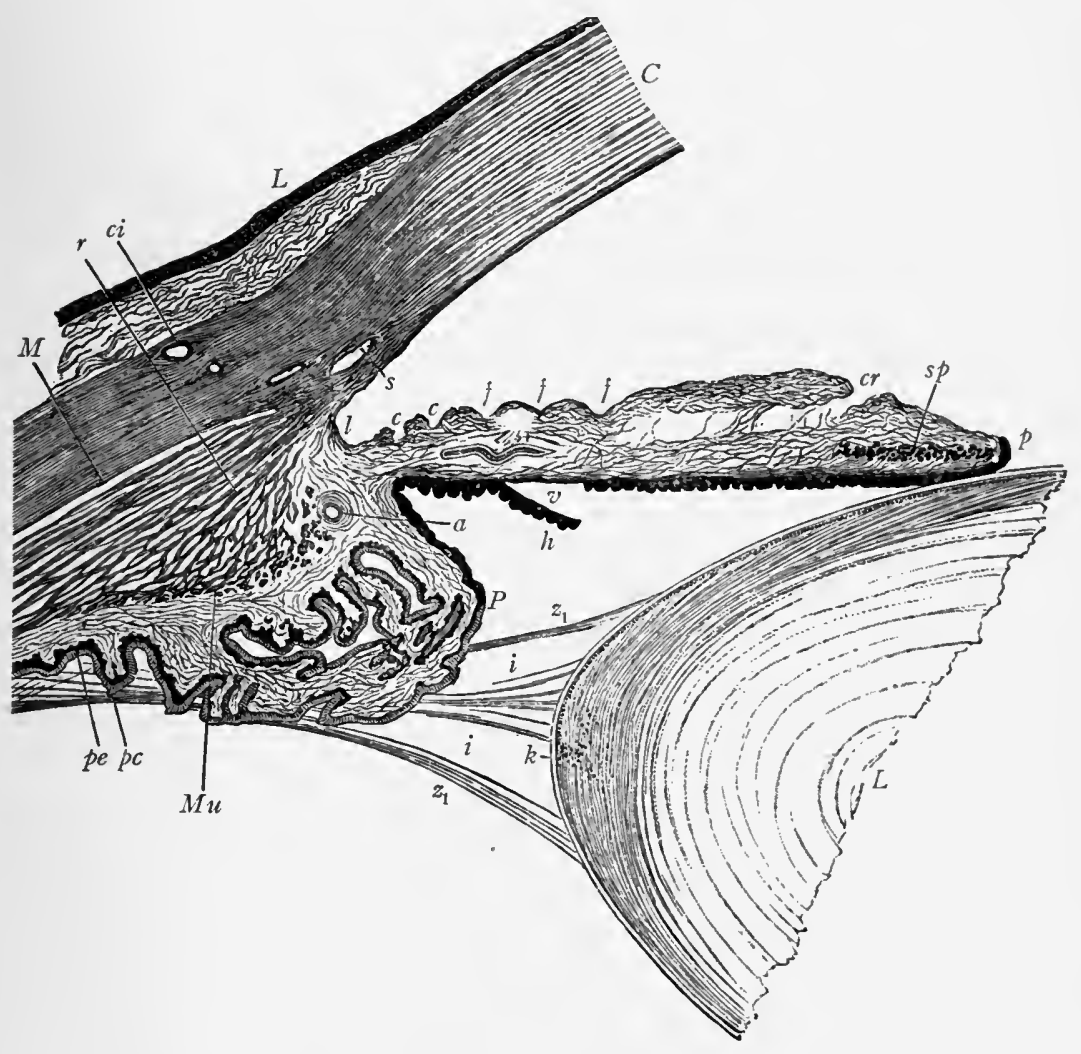

Fig. 105. - Meridional section through ciliary region, including the iris and part of the cornea and lens (Fuchs): $C$, Cornea; pe, $p c$, pigmented and non-pigmented cells of the pars ciliaris retina; $L$, lens; $M$, ciliary muscle; $r$, its radiating, $M u$, its circular fibers; $c i$, anterior ciliary artery; $s$, canal of Schlemm; $c, f$, anterior surface of iris; break at $c r ; s p$, sphincter pupillæ; $p$, edge of pupil; $P$, ciliary process; $h$, pigment lining iris, partly separated at $v ; a$, blood-vessel; $z_{1}, z_{1}$, fibers of suspensory ligament, enclosing spaces $i, i$; $k$, lens-capsule; $l$, ligamentum pectinatum.

itic, of rheumatic and gouty, of gonorrheal, and of sympathetic iritis. Many cases of traumatic iritis are 
also of this character, and so are most of those which have been spoken of as due to "trophic" nerve influence.

Purulent iritis is less common. It is usually the result of penetrating wounds of the eyeball, or of operations in which the globe is opened, and is almost always due to the presence of a pyogenic micro-organism. It may also follow severe suppurative inflammation of the cornea.

Serous iritis or, more correctly, uveitis, is a disease by no means of rare occurrence, yet it is one about the pathology of which we have much to learn. There is no doubt but that the iritis is usually only a part of an inflammatory process which involves the entire uveal coat. In some instances it seems to be dependent upon a rheumatic diathesis, and in others, as has already been intinated, upon a reflex or "trophic" nerve influence, while, of tener than not, it is impossible to assign a cause for it. The distinguishing characteristics of serous iritis (Plate VI, Fig. 3), which, fortunately, is rarely a binocular affection, are that the pupil is not contracted, as in other forms of inflammation of the iris; that posterior synechixe rarely occur; that there is a disposition to increased intraocular tension; that the vitreous humor is often diffusely clouded or contains numerous floating opacities; and, most typical of all, that there is commonly a deposit of minute opaque dots, of a yellowish-gray color, upon the inner surface of the cornea. These dots, which are due to the deposition of round cells and coagulated fibrin upon the corneal endothelium (Fig. I06), are usually arranged in a triangular shape, the base of the triangle being at the lower margin of the cornea, while its apex reaches up to, or may even extend beyond, the center of the cornea. 
Occasionally the affection exhibits a mixed type, and the characteristic dots upon the corneal endothelium, etc., are attended by a disposition to the formation of posterior synechix. It usually runs a protracted course, and responds far from satisfactorily to treatment. Unless the tension assumes a glaucomatous character, pain is not often complained of, and there is an absence of pronounced photophobia. Owing to the opacity of the vitreous humor and the condition of the cornea,

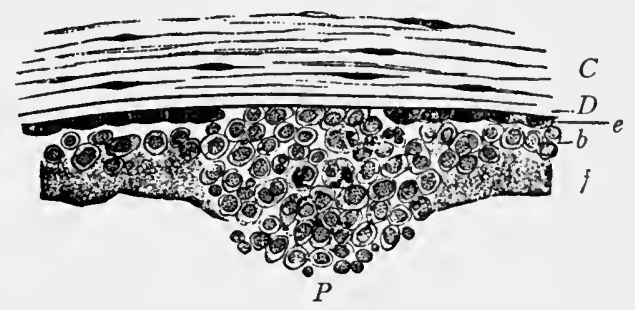

Fig. 106.-Section showing deposit of round cells and coagulated fibrin upon the inner surface of the cornea in uveitis. Magnified $140 \times I$ (Fuchs). The posterior surface of the cornea, $C$, is covered by Descemet's membrane, $D$, and the endothelium, $e$. The latter, which as a whole is of normal character, is wanting at the spot where the deposit, $P$, is situated. This deposit consists of an accumulation of cells with interspersed pigment granules which are partly free and partly inclosed in the round cells. In the place where no deposits are situated the posterior surface of the cornea is covered by a layer of exudation consisting of two strata, an anterior one, $b$, composed of round cells, and a posterior one, $f$, formed of coagulated fibrin.

vision during the height of the attack may be reduced to little better than light perception. Serious and permanent impairment of sight may result, if the increase of intraocular tension has been persistent and pronounced enough to injure the optic nerve, or if, as not infrequently happens, the retina has suffered in consequence of the general uveitis.

All varieties of plastic iritis (Plate VII, Fig. I), as has been stated, are characterized by a tendency to the for- 
mation of an organized exudate, but this tendency is much more marked in some than in others. It is especially so in sympathetic iritis, in the iritis of herpes zoster ophthalmicus, and, in fact, in all those forms of iritis which appear to be due to "trophic" nerve influence. In syphilitic and in rheumatic iritis this tendency usually manifests itself by the formation of adhesions between only the pupillary margin of the iris and the capsule of the lens (Fig. 107); but in sympathetic

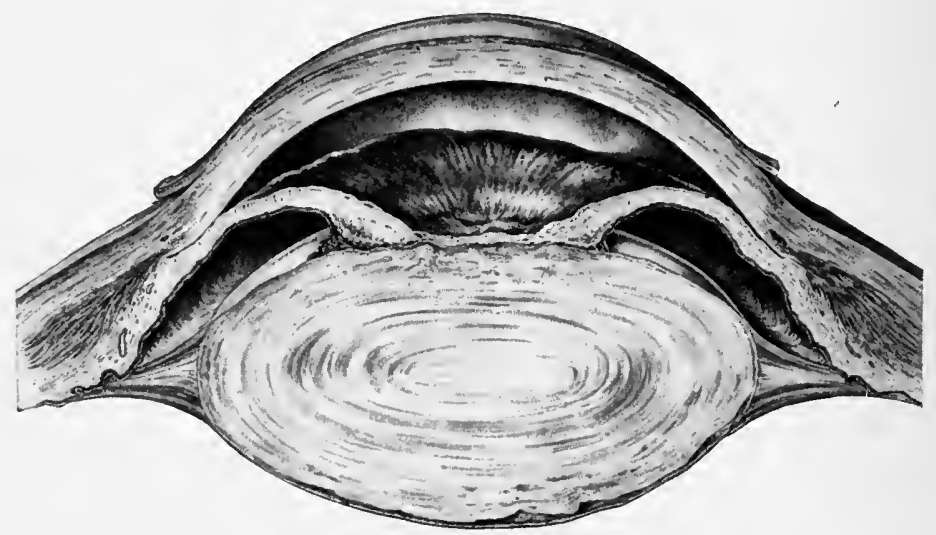

Fig. 107.-Annular posterior synechia, with occlusion of pupil and iris bombé (Deaver).

and other allied forms of iritis a felt-like exudation forms upon the posterior surface of the iris, causing it to adhere throughout its whole extent to the lens, and the pupil is commonly occluded by similar material (see Fig. 104). Under such circumstances, too, projecting portions of the anterior surface of the iris may become adherent (without ulceration) to the inner surface of the cornea, giving rise to anterior synechiæ.

Sypbilitic iritis, which is usually attended by severe pain, and by marked photophobia and lacrimation, 


\section{PLATE VII.}

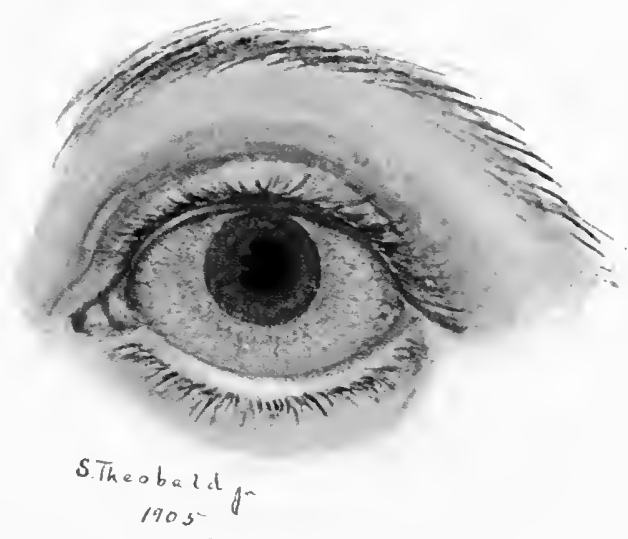

Fig. I.-Plastic Iritis. Pupil partly dilated by atropin, showing two posterior synechice.

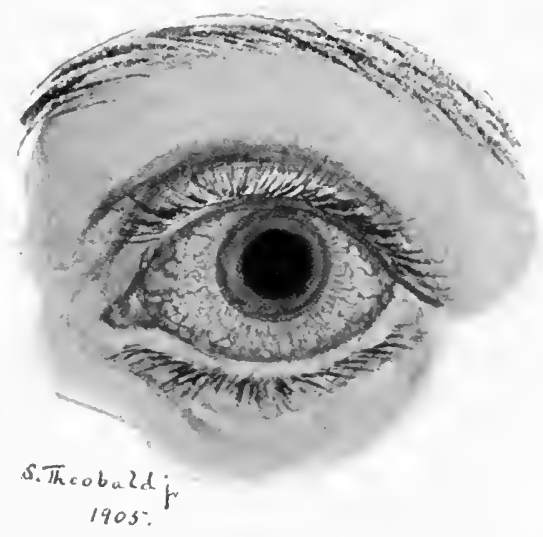

Fig. 2.-Inflamatory Glaucoma. 

commonly manifests itself during the secondary stage of the disease, in association with the macular eruption and the appearance of condylomatous growths. Less frequently it occurs in the tertiary stage. It is also met with in inherited syphilis, sometimes in infancy, sometimes even as a prenatal affection, but oftenest between the ages of seven and fifteen years, in connection with an outbreak of interstitial keratitis. About fifty per cent. of all cases of iritis are due to syphilis, inherited or acquired.

A pathognomonic, but by no means constant, feature of syphilitic iritis is the development upon the anterior surface of the iris of yellowish or reddish-brown nodules, which project forward into the anterior chamber, and sometimes even press against the cornea. Usually there are not more than one or two present; but they may be so numerous, and of such size, as to fill the anterior chamber. They occur more frequently in the iritis which develops during the secondary stage of the disease, and are then of the nature of condylomata (Fig. I08); those met with in the iritis of tertiary syphilis are gummatous in character. Hence the former variety of iritis is sometimes designated iritis condylomatosa, and the latter variety, iritis gummosa. They may undergo absorption, or may disappear through fatty or purulent degeneration. The inflammation of the iris tissue being more intense over the area which corresponds to their base, we find here a special tendency to the formation of adhesions to the lens capsule. All the varieties of iritis may be complicated by "hypopyon," though it is more common in the purulent and syphilitic types. It is due to the deposition from the aqueous humor of leukocytes or of round cells and fibrin, and, as a rule, undergoes absorption slowly. As the deposit may or 
may not consist of leukocytes the term, as commonly employed, is inexact.

Sympathetic Iritis.-Although exceptionally sympathetic ophthalmitis manifests itself as a neuroretinitis or choroido-retinitis, it very generally begins as an iritis or an irido-cyclitis, and, even in the rare instances in which the inflammation commences at the posterior pole of the eye, it soon extends to the iris and ciliary body unless promptly brought under control.

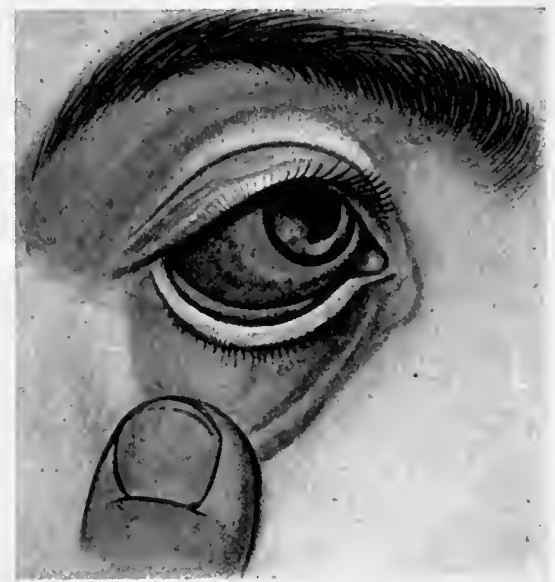

Fig. 108.- Syphilitic iritis, showing condyloma. Pupil dilated by atropin (Haab).

It is not inappropriate, therefore, to consider this affection in connection with other forms of iritis.

There are but few diseases of the eye in which the prognosis is more grave, and in which treatment is of less avail, than in sympathetic ophthalmitis. It is usually the result of a severe traumatic lesion, commonly of a penetrating wound, of the primarily affected or "exciting eye." Wounds involving the iris and ciliary body, and those complicated by the lodgment of a 
foreign body in the interior of the eye, are especially apt to give rise to it. It may also be induced by severe and protracted inflammation of the iris, ciliary body, and choroid of non-traumatic origin. It is interesting to note that a sympathetic inflammation leading to complete destruction of sight may be brought about by an injured or diseased eye which still retains a useful amount of vision. Another point of interest is that the disease has no definite period of "incubation," if we may use the expression, that the interval between the receipt of the injury or the occurrence of the inflammation in the exciting eye and the development of the sympathetic affection in the fellow-eye may vary from two or three weeks to very many years.

Usually, but not always, the outbreak of an attack of sympathetic inflammation is preceded by a period, which may be brief or very prolonged, of sympathetic irritation in the sympathizing eye. This condition, which is unattended by structural changes, and, therefore, is to be sharply differentiated from sympathetic ophthalmitis, is characterized by the existence of photophobia and lacrimation and by a "weakness" or irritability of the eye, which renders it incapable of near work, especially. Diminution of accommodative power and a tendency, upon slight provocation, to hyperemia of the conjunctival vessels are also frequently present. All these symptoms, constituting sympathetic irritation, it should be remarked, disappear promptly upon the removal of the exciting cause, that is to say, upon the enucleation of the primarily affected eye.

Unlike sympathetic irritation, sympathetic inflammation, when once established, though it may be favorably influenced, is seldom cut short by the removal of the exciting eye. On the contrary, though detected in its 
incipiency and combated by every known therapeutic means, it commonly leads, after a prolonged course attended by much suffering, to complete destruction of sight and ultimately to atrophy of the eyeball. The inflammation is usually of a plastic character, and when it involves the iris gives rise, as has been mentioned already, to the formation of a felt-like exudate, which occludes the pupil, and glues the entire posterior surface of the iris to the lens capsule (see Fig. 104). The choroid and retina are soon implicated, and in this way light perception is lost.

As to the manner in which the inflammation is lighted up in the sympathizing eye, and as to the rôle played by the exciting eye in the process, there is still much uncertainty and great difference of opinion. The soundness of the more prevalent view that the inflammation in the second eye is caused by micro-organisms which have migrated to it from the primarily affected eye, probably along the lymph-channels of the optic nerves, has certainly yet to be demonstrated; and, it may be observed, there are many features in the clinical history of the disease that militate against this view. On the other hand, there is, it seems to me, much evidence to support the older theory that sympathetic ophthalmitis is a neuropatbic affection; that the inflammatory changes in the sympathizing eye are the product of a reflex influence which is provoked by the intense and continued irritation of the ciliary nerves in the primarily affected eye, and that the peculiar characteristics of this inflammation-its intractability and its malignancy-are the result solely of this reflex influence, and not of especially virulent bacterial action. It is in accordance with this view that I have spoken of sympathetic iritis as belonging to the group of "trophic" 
nerve inflammations of the eye, along with herpes zoster ophthalmicus, reflex dental, and postmalarial iritis and keratitis, etc.

As I long ago pointed out, ${ }^{*}$ it is probable that the inflarnmatory manifestations in the sympathizing eye are preceded by, and are dependent upon, pathological changes in the ganglia connected with the fifth nerve and in the brain-centers which have to do with the metabolism of the eye. These changes, it seems probable, are not unlike those which occur in the gasserian ganglion and in the ganglia upon the posterior roots of the spinal nerves in herpes zoster. $\uparrow$ In this connection it is interesting to note the close resemblance which the iritis of berpes zoster opbtbalmicus bears to that which we meet with in sympathetic ophthalmitis. Like the latter, it is obstinate and intractable, and in each the iris shows the same tendency to become extensively adherent to the lens capsule-a tendency which, as has been stated, is commonly not manifested in the iritis of syphilis or of rheumatism.

Some authors describe a variety of inflammation of the iris which they call "spongy iritis." It is, however,

* In a paper upon the "Pathogeny of Sympathetic Ophthalmia," "Archives of Ophthalmology," Vol. XIII, No. I, I884.

$\dagger$ As a result of their painstaking investigation of the pathology of herpes zoster, Head and Campbell ("Brain,” Vol. XXIII, p. 353) assert that these changes consist of "an acute interstitial inflammation accompanied by necrosis of the ganglion cells." It is of interest to note, further, that they could find no evidence that bacteria play any part in either the ganglion or skin lesions, and that they regard the latter as being the result of "intense irritation of cells in the ganglion which normally subserve the function of pain," and not as due to "disturbance of special trophic nerves." If these conclusions are correct, their important bearing upon the etiology of sympathetic inflammation is obvious. (For a fuller consideration of this question, see a paper by the author upon "The Genesis of Sympathetic Ophthalmitis," published in the "Journal of the American Medical Association," Jan. 28, 1905.) 
only a type of the plastic variety, in which there occurs a low form of plastic exudation in the anterior chamber, that presents a cyst-like appearance, and might be mistaken for a dislocated lens. Such cases are commonly of rheumatic origin.

A cbronic form of plastic iritis is occasionally met with, in which the inflammatory symptoms are but slightly marked. It is often associated with a rheumatic or gouty diathesis, and shows a disposition to recurrence. Points of adhesion between the iris and lens are apt to take place before the true nature of the attack is discovered, as it develops insidiously, and is unattended by pain or other symptoms calculated to alarm the patient and induce him to seek medical advice.

Treatment.-In the treatment of iritis in general the indications are to control and overcome the inflammation as quickly as possible, and, by the use of a mydriatic, to keep the pupil widely dilated, so that adhesions shall not form between the posterior surface of the iris and the lens capsule. Nearly always constitutional as well as local measures are called for. The most important local remedy is atropin. Four grains to the ounce (about one per cent.) is the strength of the solution of atropin usually employed. In the different varieties of plastic and purulent iritis it must be used freely, the frequency of the applications being determined chiefly by the state of the pupil and the amount of ciliary neuralgia and photophobia. When there are recent pupillary adhesions, which we hope to break up (for we can usually accomplish this, unless the bands are firm and broad), an instillation every hour may be required, or even, for a short time, several instillations an hour may be permissible. Such frequent applications, however, can not be long continued without the 
constitutional effects of the drug becoming manifest, and, as cases of marked individual susceptibility to the action of belladonna are occasionally met with, due caution should be exercised in using atropin in this manner. Ordinarily, four to six applications a day are sufficient. In serous iritis atropin should not be used so frequently or in such strong solution, since in this affection the pupil yields readily to its influence, and moreover, owing to the tendency to increased intraocular tension which characterizes this disease, there is danger that its too liberal use may precipitate a glaucomatous condition.

Especially in plastic iritis, dionin, used in conjunction with atropin, is often beneficial, since it not only lessens the pain, and promotes the absorption of inflammatory products, but increases the mydriatic effect of the atropin. It may be used as often as three times a day in five per cent. solution.

Occasionally individuals are met with in whom atropin fails to produce a mydriatic effect, and others in whom it greatly irritates the conjunctiva, a few applications producing a conjunctivitis, which may be attended by an erythematous inflammation of the lids and cheek. Under such circumstances hyoscyamin hydrobromate or duboisin sulphate may be substituted for atropin. As these mydriatics, especially the latter, are more apt to produce constitutional effects when applied to the eye than atropin, greater caution is required in their use. Two grains to the ounce will usually be a strong enough solution of either of these to employ, and this should not be applied more than three or four times a day.

In many cases of iritis no other local treatment than the employment of a mydriatic is required; but, when 
the inflammation is of severe type, the application of three or four leeches to the temple may accomplish good, and, when there is severe pain, much relief is often experienced from the use of a lotion of opium (ext. opii, gr. x.-xv; aquæ, $ろ$ iv) or of belladonna (ext. belladonnæ, gr. xv; aquæ, 亏iv), which should be applied to the closed lids more or less constantly upon a pad of gauze or soft linen. The application in the same way, for half an hour at a time several times a day, of water as hot as can be borne is also a useful expedient under the same circumstances. In obstinate cases, more especially those of syphilitic origin, it is well to supplement the use of constitutional remedies by the application to the forehead and temples, two or three times a day, of mercurial ointment, to which extract of belladonna or extract of opium may be added in the proportion of $5 \mathrm{j}-\mathrm{ij}$ to $\overline{3} \mathrm{j}$.

Of constitutional remedies, the most valuable are mercury, potassium iodid, and the salicylates. If to this list are added quinin, which is especially useful in purulent iritis; opium, which seems not only to control the pain, but favorably to influence the inflammation; pilocarpin hydrochlorate, which is useful especially when there is increased intraocular tension; and some brisk purgative combination which, as a rule, should contain calomel, it will comprise all the drugs that are likely to be needed in treating any of the varieties of the disease. A supplemental list of less important but at times useful remedies would include arsenic, colchicum, lithia, iron and the Turkish bath.

In acute plastic iritis, whether of specific or nonspecific origin, sodium or lithium salicylate, given in liberal doses (gr. $\mathrm{x}$ to $\mathrm{xv}$ every two or three hours, according to the susceptibility of the patient), is, on 
the whole, the most promptly efficacious remedy that we have. In many cases, and especially in those of rheumatic origin, it not only relieves the pain very quickly, but hastens the resolution of the inflammation and promotes the absorption of effused material.

In syphilitic iritis, whether the disease be inherited or acquired, mercury in some form is usually demanded. It is also our chief reliance in sympathetic iritis, and is more useful than anything else-unless it be potassium iodid-in the iritis of herpes zoster ophthalmicus and in theother "trophic" nerve varieties. In the acute stage of syphilitic iritis it should be administered liberally, and in such shape as to impress the system promptly. Salivation is to be avoided, but in severe cases we must not stop far short of it. Small doses of calomel, frequently repeated (gr. $\frac{1}{4}$ every hour, or gr. ss. every three hours), supplemented, if necessary, by inunctions of mercurial ointment, afford the best means of accomplishing the desired result. There seem to be no contraindications to the administration of sodium salicylate and mercury at the same time, and I have obtained good results in this way. Opium may be given if the pain is severe, or if a purgative effect is produced by the mercury. In subacute cases, or when the symptoms are less urgent, biniodid of mercury, in doses varying from gr. $\frac{1}{24}$ to gr. $\frac{1}{12}$, may be given three times a day. This is a very efficacious and convenient method of administering mercury, and salivation is less apt to occur than when calomel is employed. It may be given in tablet triturates or pills, or preferabily in solution in water, a small quantity of potassium iodid being added to render the mercury soluble. When a prolonged course of mercury is required this, or the protoiodid, is decidedly the best form in which to administer it. 
Potassium iodid is valuable in rheumatic iritis, and it may also advantageously supplement the use of mercury in syphilitic iritis. In serous iritis it is the most efficacious remedy that we possess, but its good effects are not always manifest until it is given in liberal doses.

In sympathetic iritis the prognosis is grave, whatever treatment may be adopted. The most essential thing is that it should be commenced with the least possible delay. Although, as has been indicated, radically different views are held as to the etiology of sympathetic ophthalmitis, it is generally agreed that the chances of controlling the inflammation in the sympathizing eye are increased by the removal of the exciting eye. The condition of this eye, therefore, should be carefully determined, and if it is blind or so nearly blind that useful vision with it is impossible, although at the time it may be quiescent and apparently free from inflammation, it should be enucleated without a moment's unnecessary delay. On the other hand, if there is a reasonable probability that, with or without operation, a useful degree of vision may be retained in this eye, it is best not to sacrifice it; for it should be borne in mind that instances have occurred in which the sight of the sympathizing eye was entirely lost while serviceable vision was retained in the primarily affected eye. This question, whether or not to enucleate an eye which, though not itself sightless, has caused and is promoting the development of sympathetic inflammation in its fellow, is one of the most perplexing which fall to the lot of the ophthalmic surgeon to decide, and it is certainly one which no one without his especial training should attempt to decide.

Mercury given liberally, by the mouth and by inunction, so as to impress the system rapidly, and, it may 
be added, given persistently, is the remedy which has been chiefly relied upon to combat sympathetic inflammation, in whatever form it may manifest itself. At a later stage potassium iodid may be administered with benefit. Gifford, in several cases, has obtained surprisingly satisfactory results from the administration of heroic doses of sodium salicylate-" 150 to 200 grains in the course of the waking hours." $*$ His experience certainly seems to call for further experimentation in this direction.

In purulent iritis, which, as has been said, usually follows wounds of the eye or operations upon it, and is frequently accompanied by purulent infiltration of the cornea, the free administration of quinin sulphate offers the best prospect, though not a very promising one, of success. Pilocarpin hydrochlorate, which seems to be as efficacious when administered by the mouth as when introduced into the system by the hypodermic method, is sometimes useful in cases of serous iritis, in which the tension of the globe is high; and in any of the other varieties, if this condition obtains or if there is cloudiness of the vitreous humor, it may be administered with advantage. I have found it convenient to prescribe it in a solution of the strength of gr. $j$ to $5 j$. Ten drops of this solution, containing one-sixth of a grain of the salt, is the commencing dose, to be taken by the mouth, once a day. According to the effect produced, the dose is increased by adding each day two or three to the number of drops administered. In any severe attack of iritis an active cathartic may be given with advantage at the commencement of the treatment. A very efficacious one is the combination of two to five

* "Diseases of the Eye, Nose, Throat and Ear," Posey and Wright, p. 396. 
grains of calomel, two grains of scammony, and six of powdered rhubarb, already commended.

When the iritis is dependent upon a gouty diathesis, colchicum and the preparations of lithium are useful; and in the iritis which sometimes follows malarial attacks, and in that which accompanies ophthalmic shingles, arsenic, in the form of Fowler's solution, may be prescribed with benefit. The daily use of the Turkish bath is commended by Bull as a valuable remedy in arthritic iritis.

In the management of every case of iritis the question arises whether the patient should be confined to the house during the continuance of the attack. Undoubtedly, in acute cases, and especially when the inflammation is severe, this should be done if practicable. It is very rarely necessary, however, that he should be shut up in a dark room. With a shade and with dark glasses (London-smoke coquilles), he may safely be allowed the freedom of the house. This makes the treatment much less irksome to the patient, and does not in the least retard the cure. In subacute cases, and even in acute cases when there is but little pain or photophobia, the patient need not be confined to the house, unless, of course, the weather be unpropitious. Indeed, most patients with iritis are treated successfully as "outpatients," being seen by the medical attendant either at his office or at his hospital clinic.

Surgical interference is rarely required during the active stage of iritis. There are, however, some exceptions to this rule, as, for instance, in serous iritis, when the supervention of glaucomatous symptoms may demand the prompt performance of an iridectomy. To remedy the consequences of iritis, however, and to prevent recurrent attacks, operations upon the eye are 
frequently called for. When, after an attack of iritis, a few slender bands of adhesion between the margin of the pupil and the lens are left, probably no ill consequences will result therefrom, and for such a condition no operation is required. If, however, as happens not infrequently in neglected cases, the margin of the pupil is completely glued to the surface of the lens, an iridectomy should be performed without unnecessary delay, for soon the iris will be bulged forward by the accumulation of fluid behind it (see Fig. 107), and will undergo atrophy, while at the same time the deeper structures of the eye will suffer from the consequent disturbance of their nutrition. When, though not completely adherent, the margin of the pupil is attached to the lens by several broad bands, an iridectomy may be required, since recurrent attacks of inflammation are not infrequently induced in consequence of the irritation caused by the traction of these bands during the muscular movements of the iris.

When the pupil is closed, or is occluded by an organized exudate, an iridectomy is positively indicated, and, by yielding a clear artificial pupil, may restore almost normal vision to a nearly blind eye. If, however, the pupillary occlusion is attended, as it sometimes is after the more severe types of iritis, with adhesion of nearly the entire posterior surface of the iris to the lens capsule (see Fig. 104), the outcome of an iridectomy is apt to be much less satisfactory; for under such circumstances it is very difficult to obtain a clear pupil, since it frequently happens that the iris tissue is so friable that it can not be drawn out by the forceps, or the muscular coat only yields to the traction, while the pigment coat remains attached to the lens capsule. There is great danger, too, that the new pupil, if we are so fortunate 
as to secure one, will become occluded as a result of the inflammation arising from the performance of the iridectomy.

\section{DISEASES OF THE CILIARY BODY.}

Cyclitis.-Inflammation of the ciliary body, or cyclitis, though of common occurrence, is seldom met with as a disease per se. Usually it is but part of a more general inflammation of the uveal tract-of a choroiditis it may be, though oftener it occurs in association with iritis; indeed, it is present in most cases of severe iritis, especially those of syphilitic origin.

In cyclitis, as in iritis, the inflammation may be of plastic, or it may be of purulent or serous, type. Plastic cyclitis is commonly due to syphilis or to rheumatism; it also occurs, and in its worst form, in sympathetic ophthalmitis. Purulent cyclitis usually arises from penetrating wounds of the ciliary region or of the cornea and iris. It may occur also as a complication in operations, such as extraction of cataract, which involve opening of the eyeball. It is always a consequence of infection, and it leads commonly to a general suppurative inflammation of the eye-panophthalmitis,- - resulting in destruction of sight and ultimately in atrophy of the eyeball. Serous cyclitis is invariably but part of a general uveitis, which condition has been treated of already under the head of "serous iritis."

Intense pain, great photophobia, and, especially, exquisite sensitiveness of the ciliary region, together with marked pericorneal injection, are the characteristic signs of plastic and of purulent cyclitis, while decided impairment of vision is usual in all three varieties, owing to the cloudiness of the vitreous humor which is commonly present. 
Treatment.-The treatment of the different varieties of cyclitis, constitutional as well as local, is the same as in the corresponding types of iritis, mercury, the salicylates, and potassium iodid being indicated in the plastic and serous forms, and quinin in generous doses in the purulent form, while atropin in strong solution, dionin, and the lotion of opium are called for in the first and last named varieties.

In purulent panophthalmitis (see Fig. I30) prompt enucleation of the eye, which saves the patient from much intense suffering, is indicated. The risk of cerebral infection, which is supposed to attend the performance of the operation under such circumstances, is probably not appreciably greater than that to which the patient is exposed through retention of the suppurating eye.

Tumors of the ciliary body, sarcomatous growths more especially, are not so rare as tumors of the iris. Their early diagnosis, which is of the utmost importance, can be made only by means of the ophthalmoscope. When they are of malignant character, immediate enucleation of the eye is demanded.

Enucleation of the Eyeball.-One of the most definite advances in ophthalmic surgery in recent years is the method, now universally employed, of removing the eyeball.

The modern operation, known as "enucleation" of the eye, was devised by Bonnet, in I $84 \mathrm{I}$. The operation of "extirpation" of the eye, previously practised, a primitive and rude procedure, is aptly described by Fuchs as "cutting out the eyeball, together with the neighboring soft parts, in a not very different way from that in which a butcher is accustomed to do it."

The chief end in view, and a most important one, in 
the operation of "enucleation" is the preservation of all the soft parts contained in the orbit, especially the preservation of the conjunctiva and the muscles concerned in the movements of the globe, so that only the eyeball itself, freed from all its connections, is removed. This results in securing a relatively large cavity, lined by mucous membrane,- the conjunctiva,--well adapted to receive an artificial eye of the requisite size, and a movable "stump," made up of the preserved muscles, Tenon's capsule, and the fatty tissue of the
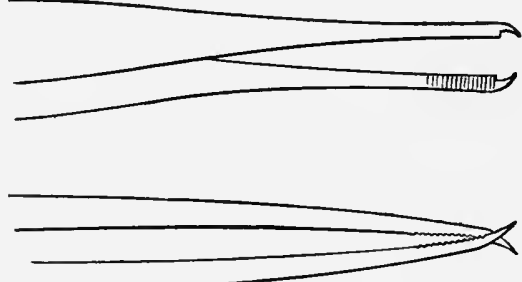

Fig. 109.-Bader's scleral fixation forceps. The long, sharp teeth bite into the sclera, and for this reason they are invaluable in the last step of enucleation of the eye-the division of the optic nerve.

orbit, which not only gives to the artificial eye a muchdesired prominence, but enables it, at least in considerable measure, to follow in a natural way the excursions. of the seeing eye.

The several steps of the operation, which, except when panophthalmitis is present or perforation of the globe has occurred, for example, from sloughing of the cornea, is a very simple surgical procedure, are well shown in the accompanying illustrations.

A general anesthetic and careful antiseptic precautions, of course, are demanded. The instruments required are a stop-speculum, a strabismus-hook, 
stout, blunt-pointed, scissors, curved on the flat, a pairpreferably two pairs - of ordinary fixation forceps, and

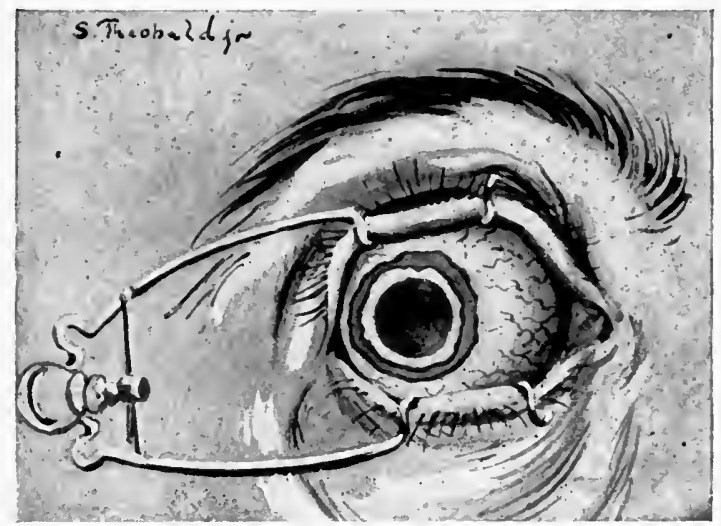

Fig. 110.-Enucleation of eye. The first step-the circumcorneal section of the conjunctiva-completed.

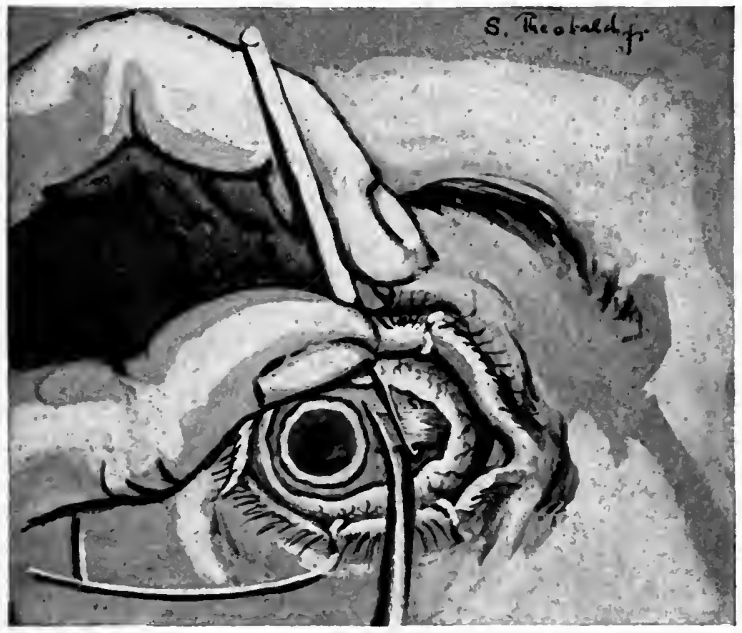

Fig. II1.-Enucleation of the eye. The second step-dissection of the recti muscles from their scleral attachment.

the scleral fixation forceps of Bader, shown in the cutFig. Iog. 
With the fixation forceps and the scissors the circumcorneal section of the conjunctiva (Fig. IIO) is easily made-the second pair of fixation forceps often being found useful to rotate the eye into a convenient position for seizing, with the other pair, the portion of the conjunctiva we wish to divide. This step completed, it is well, with the scissors, to separate the conjunctiva, in every direction, rather widely from the ball, so that the second step, the dissection of the recti

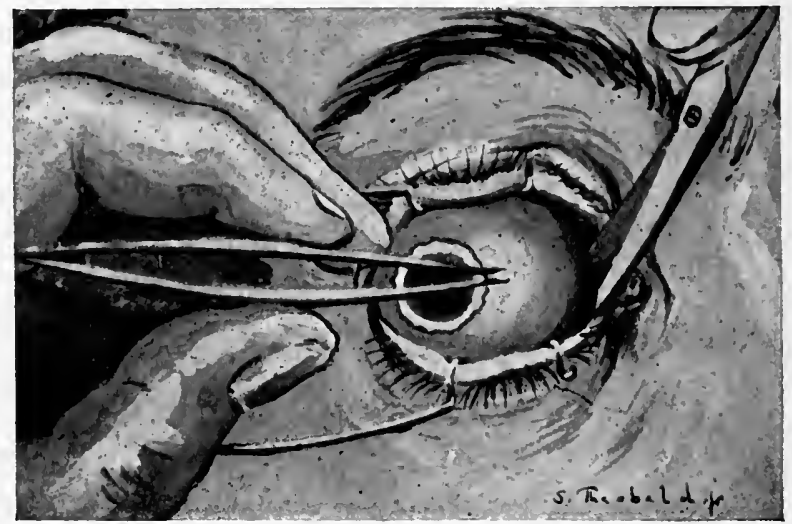

Fig. I I2.- Inucleation of the eye. Division of the optic nerve, the eyeball strongly rotated outward by means of Bader's scleral fixation forceps.

muscles from their scleral attachment (Fig. I I I), may be more readily accomplished.

The final step-the division of the optic nerve (Fig. I I2) - is not so easily executed, unless provision has been made for exerting traction upon the eyeball, so as to put the nerve somewhat upon the stretch. There are several ways of accomplishing this; but, to my mind, the most satisfactory is that shown in Fig. 112-the employment of the scleral fixation forceps of Bader. These forceps, it may be added, are especially helpful when it is desirable to sever the optic nerve at a con- 
siderable distance behind the scleral, as in enucleation for malignant intraocular growths or for sympathetic ophthalmitis.

Considerable hemorrhage follows the division of the optic nerve and the ciliary vessels that surround it, but, after the ball has been completely removed from the orbit by severing the attachments of the oblique muscles and any other adherent tissue, this is easily controlled by firm compression.

The after-treatment is very simple, and recovery from the operation, which is attended by little or no shock, astonishingly prompt. Sterile gauze "sponges" are applied over the lids-somewhat in the fashion of a graduated compress-and over these a tight bandage (Fig. IO). The next morning these compresses are removed, and a gauze pad, wet with a saturated solution of boracic acid, is applied in their stead, and is kept wet by repeated applications of the boracic solution.

As soon as the patient has recovered sufficiently from the effects of the anesthetic to wish to do so, he is permitted to sit up, and after three or four days a monocular eye-shade is substituted for the wet pad and bandage. A collyrium of alum and boracic acid (alum., gr. iij; acid. boracic., gr. xij; aquæe destil., $z_{j}$ ), to be dropped into the conjunctival sac three times a day, is now prescribed, and the patient, if he desires, may leave the hospital; and, at the expiration of a month from the date of the operation, he may begin to wear an artificial eye.

It is the practice of some surgeons, after the eye is removed, to bring together the edges of the conjunctival wound by means of a continuous suture, and others, more reprehensibly still, pack the orbit with sterile gauze. Both of these measures are as uncalled for as they are objectionable; and, in my opinion, the same 
274 PREVALENT DISEASES OF THE EYE.

criticism applies, because of the unnecessary traumatism involved, to the "finicky" procedures, supposed to increase the mobility of the "stump," of Suker, Schmidt, Priestley Smith, and others. 


\section{CHAPTER VIII.}

\section{GLAUCOMA.}

Of all the diseases of the eye there is none that it more important the general practitioner should be able to diagnosticate than glaucoma; for it is an affection that frequently runs so rapid a course that failure to recognize it, and to employ promptly the proper remedial measures, may result, in a few days, in irreparable blindness. It is not a disease, however, which, if it can be avoided, he should undertake to treat, since its proper management calls for the skill of the trained specialist. Still, as its onset is so often sudden, he is liable to be called upon at any time, especially in sections of the country remote from large centers of population, to recognize it, to point out its serious nature, and, at least, to suggest temporary measures of relief; and this much, unquestionably, he should be capable of doing.

As is well known, the essential feature of glaucoma is an increase in the intraocular tension-a hardening of the eyeball. This increase of tension may be slight and continuous or it may be excessive and intermittent in character. When it is pronounced, it is attended by certain characteristic symptoms which are not difficult of recognition. The severity of an attack of glaucoma and the amount of impairment of sight depend largely upon the degree of hardening of the eyeball. The impairment of vision is due to clouding of the media of the eye, to the pressure to which the optic nerve is subjected, and to interference with the blood- 
supply of the choroid and retina; the pain, which is often excessive, is largely the direct result of the increased tension.

There are two markedly different types of glaucomainflammatory glaucoma and simple glaucoma. These differ radically in their clinical features, in the treatment which they demand, and, probably, not less radically in their etiology. Besides these two varieties of "primary" glaucoma, certain inflammatory affections of the eye, and many traumatic lesions as well, may be complicated by an increase of intraocular tension, to which condition the name "secondary" glaucoma is applied.

Although secondary glaucoma may manifest itself at any period of life, primary glaucoma, of either variety, is extremely rare under thirty years of age, and is oftenest encountered in persons who have reached, or have passed, middle life. At the outset primary glaucoma is commonly monocular, but, sooner or later, both eyes are almost sure to become involved. In the rare instances in which increased intraocular tension is met with in the young there commonly occurs a gradual distention of the sclerotic coat and cornea, so that the whole eyeball becomes enlarged. Under such circumstances the depth of the anterior chamber is usually considerably increased, and the pupil and the iris itself are larger than normal. This condition, which is known as buphthalmos, is not infrequently of congenital origin (Fig. I I3). The enlargement of the ball finds its explanation in the fact that in the young the external tunic of the eye is less tough than in the adult, and, therefore, yields to the continued intraocular pressure.

Inflammatory Glaucoma (Glaucoma with Exacerbations).- It is this variety of glaucoma, with its pronounced inflammatory symptoms, that not infre- 
quently leads to rapid destruction of sight, though usually this is not the outcome of a first attack; for, as the subtitle given above indicates, the disease is commonly intermittent in character, though the periods of intermission are wholly irregular.

The symptoms, subjective and objective, of a wellmarked attack of inflammatory glaucoma are very characteristic. Severe pain, supraorbital and hemicranial as well as ocular, accompanied at times by nausea and vomiting; more or less complete anesthesia of the cornea;* pronounced imbairment of vision, and,

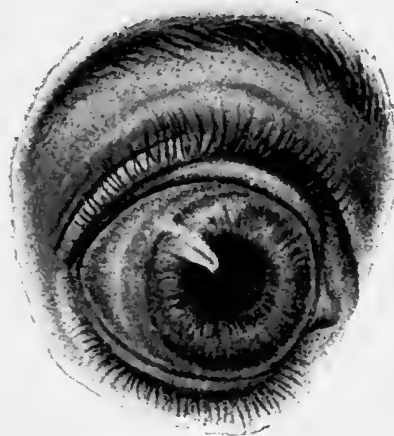

Fig. I13.-Buphthalmos (Haab).

when the sight is not too profoundly affected, the appearance of a halo, showing the rainbow colors, about the flame of a candle or a lighted match, are the chief subjective symptoms. The objective symptoms (Plate VII, Fig. 2) are marked pericorneal and general conjunctival injection; steaminess of the cornea, reminding one of the appearance produced by breathing upon a plate of cold glass; great enlargement of the pupil, which is often irregularly

* The sensibility of the cornea can be tested conveniently by touching it lightly with a slender spill of tissue or other thin paper. 
oval in shape, and shows little or no response to light; partial or complete obliteration of the anterior chamber, due to the lens and iris being pressed forward against the cornea; undue pupillary reflex, suggestive of opacity of the lens, and, above all, increased tension of the globe, easily detected by palpation through the upper lid while the eye is directed downward (see Fig. 4). The pain and injection of the ball are not characteristic, for they are not different from the pain and injection

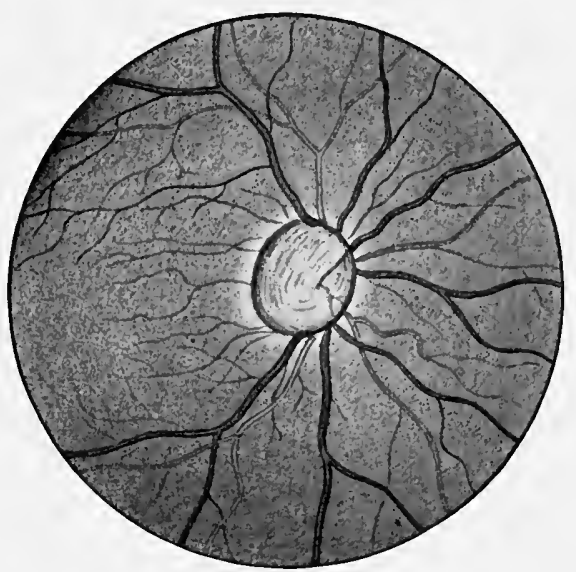

Fig. I 14.-Ophthalmoscopic appearance of the optic disc in advanced glaucoma (Jaeger).

encountered in iritis, in cyclitis, and in severe keratitis; but in these conditions we have a contracted, not a dilated, pupil, an anterior chamber of normal depth, no anesthesia of the cornea, and commonly no increase of intraocular tension.

Although during the premonitory stage of glaucoma and in the intervals between the exacerbations an ophthalmoscopic examination is usually required to establish the diagnosis (Fig. I I4), it is of little value 
during the height of an acute attack; for then the media are too cloudy to permit an inspection of the fundus of the eye, and, besides, the symptoms are so pathognomonic that the information which the ophthalmoscope affords under other circumstances is not missed.

Before the onset of such a severe attack as has been described, several, perhaps many, less marked exacerbations, in all probability, will have occurred, and the true character of these is not so easily recognized by the physician who is but little versed in the management of diseases of the eye. Among the earliest of the prodromal symptoms of inflammatory glaucoma is a rapid failure of the accommodative power of the eye, necessitating, at short intervals, an increase in the strength of the glasses worn in near vision. Then there occurs, in connection, perhaps, with an attack of indigestion or after prolonged use of the eyes, a transient obscuration of vision, attended by slight supraorbital pain and some pericorneal injection. If an examination of the eye is made at this time, a perceptible hardening of the ball will be detected, and a test with the candle flame will show the rainbow-colored halo. The ophthalmoscope would show, besides, a jerky pulse in the central retinal veins or, perhaps, a slight arterial pulse. After a night's rest the eye will have returned nearly or quite to its normal condition, and some weeks may elapse before a similar, but probably more pronounced, attack occurs. The recovery from this next exacerbation will be less prompt, and after several such attacks a perimetric test will reveal a perceptible contraction of the nasal half of the field of vision, and the ophthalmoscope will show a depression of the optic disc-the beginning of a glaucomatous "cup" (Fig. II5). After 
another interval of uncertain length, and again precipitated by some exciting cause, such as overstrain of the eyes, a spell of crying, etc., there will occur a wellmarked attack of glaucoma, and after this has subsided, if it does subside without radical treatment, vision will remain more or less seriously impaired.

Such is the usual history of a case of inflammatory glaucoma, until, in the course of time, a much more severe attack occurs, and sight is permanently lost. But even when this has happened the disease has

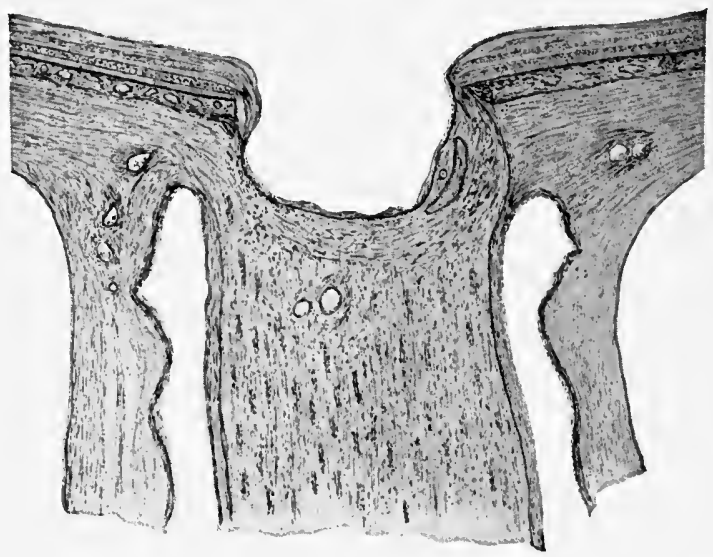

Fig. II5.-Section of optic nerve-head showing deep glaucomatous excavation (Lippincott).

not run its course, and much suffering is still in store for the unfortunate individual; for many acute exacerbations, caused by a temporary increase in the tension of the eye, and accompanied by intense pain, are liable to occur, until finally, the lens having already become cataractous, the eyeball undergoes partial atrophy, becomes abnormally soft, and remains comparatively quiescent. It is still, however, a source of danger, since in not a few instances such a blind and atrophied 
eye has been known to induce sympathetic ophthalmitis in the fellow-eye.

From the foregoing description it is evident that there is little excuse for mistaking inflammatory glaucoma for iritis, cyclitis, or keratitis, and still less for conjunctivitis. For confounding it with cataract, as the experience of every ophthalmic surgeon shows is done not very rarely, there is, of course, no excuse whatever, since cataract, unless complicated by other disease of the eye, is never attended by pain, injection of the ball, or other evidences of inflammation. To mistake it for a severe attack of facial neuralgia is less reprehensible; but such an error can be made only when one neglects to examine the eye, and so fails to note the steamy cornea, the enlarged pupil, the shallow anterior chamber, and the increased tension of the globe.

Let the general practitioner but once realize that glaucoma is to be suspected, and to be carefully searched for, whenever a painful inflammation of the eye attended by obscuration of sight is encountered in an individual who has reached, or has passed, middle life, and errors in diagnosis such as have been mentioned will become very rare indeed, and both his own reputation and the welfare of his patients will be great gainers thereby.

Much careful research has been undertaken with the view of throwing light upon the etiology of inflammatory glaucoma, but our knowledge upon the subject is as yet far from exact. In order to a clear comprehension of the matter, it must be borne in mind that there is a constant and very considerable flow of lymph through the chambers of the eye. This lymph-stream, which is necessary to maintain the non-vascular media of the eye-the vitreous humor, the crystalline lens, and the 
cornea-in a normal condition, is supplied in large part by the very vascular uveal coat, composed of the choroid, the ciliary body, and the iris. In the main the direction of the current is forward--from the vitreous chamber, through the circumlental space and through the pupil, to the anterior chamber, where it accumulates in considerable quantity as the aqueous humor (Fig. II6). Having performed its office the lymph must escape from the eye, and this is provided for by the existence of certain drainage channels, the chief one being at the periphery of the anterior chamber, where there are numerous "lymph-spaces" which connect with the canal of Schlemm. In addition, there are the

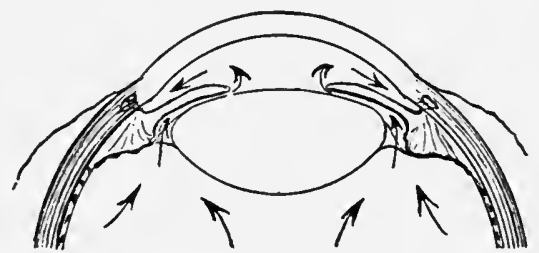

Fig. I $16 .-$ Direction of intraocular lymph-stream (Jackson).

lymphatic vessels which accompany the venæ vorticosæ in their passage through the sclera, and the lymphspaces which surround the optic nerve.

In order that the intraocular tension may be maintained at its normal standard, it is evident that an equilibrium must exist between the inflow and the outflow of this lymph-stream. If the inflow does not equal the outflow, the intraocular tension will be reduced, the eye will be abnormally soft; on the other hand, if the conditions are reversed the tension will be increased, the eye will be abnormally hard. A hardening of the eyeball, in other words, a glaucomatous condition, it will be seen, may be brought about in either of two ways 
- by an increase in the inflow of lymph beyond the normal capacity of the drainage apparatus, or by an obstruction or clogging of the outflow channels whereby the escape of lymph is impeded. It is probable that both of these conditions-an increase in the inflow and an obstruction of the outflow of lymph-are concerned in the causation of many cases of inflammatory glaucoma,

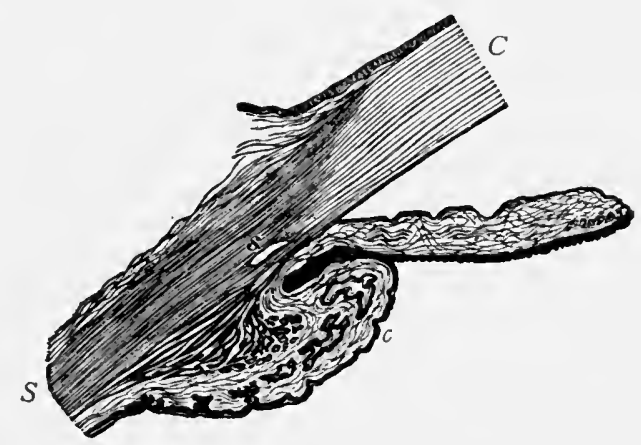

Fig. I17.-Section of iris and ciliary body in recent inflammatory glaucoma, showing obliteration of the filtration angle. Magnified $9 X_{I}$ (Fuchs). The ciliary process, $c$, is so greatly swollen that it pushes the root of the iris forward and presses it against the sclera, $S$, and the cornea, $C$. The sinus of the anterior chamber, which should lie somewhat behind Schlemm's canal, $s$, is thus closed. The ciliary muscle shows the pronounced development of the circular muscular fibers (Müller's portion), characteristic of the hypermetropic eye.

though the inclination at the present day is to regard the latter condition as the more potent factor.

It would be out of place to enter into a discussion of the many theories which have been advanced to explain the production of glaucoma. The view most widely accepted is that the hardening of the eyeball is due to obstruction of the lymph-spaces about the periphery of the anterior chamber, the so-called filtration angle (Figs. I 77 and I I8). Another view attaches more importance to compression of the lymphatic 
vessels which pass through the sclera with the venæ vorticosæ; another, to narrowing of the circumlental space, which has a normal width of but half a millimeter, whereby the flow of lymph from the vitreous chamber to the aqueous chamber is obstructed; while

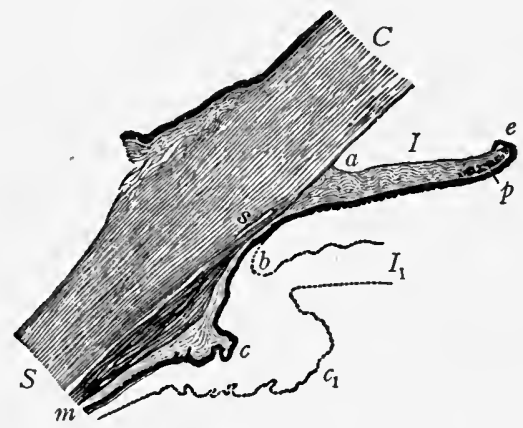

Fig. I18.-Section of iris and ciliary body in advanced inflammatory glaucoma, showing atrophy of the iris and adhesion of its periphery to the sclera and cornea, also pronounced atrophy of the ciliary body. Magnified $9 X_{I}$ (Fuchs). The dotted line gives the outline of the iris, $I_{1}$, and the ciliary body, $c_{1}$, in the normal condition. The root of the iris is adherent to the sclera, $S$, and the cornea, $C$, wherever it has been pressed against them by the ciliary body. The attachment of the iris is hence displaced forward and lies in front of Schlemm's canal, $s$. So, too, the sinus of the anterior chamber is displaced from $b$ to $a$. Wherever the iris has become adherent, it has been thinned through atrophy, so that in places- $b$, for example-it consists of scarcely anything more than the pigment layer. Even the free portion of the iris, $I$, appears, in consequence of its atrophy, narrower than the normal iris, $I_{1}$. Over the pupillary border, $e$, the retinal layer of pigment turns forward farther than usual, and the sphincter pupillæ, $p$, also shares to some extent in this eversion. The ciliary body, owing to its having become atrophic, has again separated from the iris, and in fact more so than in the normal condition, so that it is now removed from the iris by a broad interval. The atrophy affects both the ciliary muscle, $m$, and the ciliary process, $c$.

\section{still another maintains that under certain circumstances} there is an alteration in the consistency of the lymph -a serosity-which interferes with its escape through the drainage channels.

Those, of whom I am one, who consider that an in- 
crease in the lymph-stream, a hypersecretion of lymph, is not infrequently a factor in the production of glaucomatous tension, believe that this increase is often brought about, through the resulting hyperemia of the uveal coat, by the strain of accommodation due to errors of refraction. It is a well-known fact that hypermetropic eyes are especially disposed to develop glaucoma, though this has been explained upon the ground that in such eyes the circumlental space is narrower than in the emmetropic or myopic eye. The frequency with which astigmatism, and especially astigmatism "against the rule,"-a refractive error which gives rise to an exceptional amount of accommodative strain,-is found in association with glaucoma, attracted my attention many years ago, and was made the subject of a communication to the American Ophthalmological Society.* In this connection the fact, to which reference has been made, that attacks of glaucoma are frequently precipitated by prolonged use of the eyes in near workreading, writing, etc.-is significant.

Among the general conditions which are regarded as favoring the development of glaucoma may be mentioned, rheumatisn, gout, angiosclerosis, and the menopause. It should be mentioned also that the employment of a mydriatic in persons predisposed to glaucoma has been known frequently to precipitate an attack of the disease, the explanation being that when the pupil is widely dilated the iris is crowded into the periphery of the anterior chamber in such a manner as to obstruct the filtration angle. For this reason, the indiscriminate use of mydriatics in persons beyond middle life is regarded as reprehensible.

Writers commonly subdivide inflammatory glaucoma

*Transactions American Ophthalmological Soc., I 888. 
into three varieties-acute, subacute, and chronic. These subdivisions are more or less artificial, though they serve to emphasize the fact that in some instances the disease runs a rapid course, the exacerbations are of frequent occurrence and severe in character, and vision is soon destroyed; while in others the attacks occur at long intervals, and are comparatively mild, so that a much longer time elapses before sight is seriously impaired (Fig. II9). In what is known as "fulminating glaucoma" all the symptoms are greatly intensified, and sight may be destroyed in the brief period of

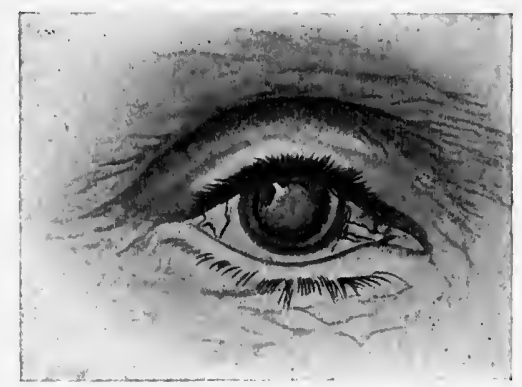

Fig. I I9.-Chronic inflammatory glaucoma, advanced stage (Ramsay).

forty-eight or even twenty-four hours. One of the most unpromising types of inflammatory glaucoma is that which is attended by recurrent intraocular hemorrhages-from the retinal vessels chiefly-and which, therefore, is known as "hemorrhagic glaucoma." "Malignant glaucoma" is a term chiefly employed by ophthalmic surgeons, it would seem, to characterize cases upon which they have operated unsuccessfully.

The impairment of vision in advanced glaucoma frequently exhibits a characteristic which is very striking. One of the early symptoms of the disease, as has been pointed out, is a narrowing of the nasal half of the 
visual field. In time this contraction of the field becomes general, and slowly progresses toward the point of fixation. As a result of this, in extreme cases, vision is lost everywhere except at the fovea. Under such circumstances the sight is much like that which one obtains in looking through a long, narrow tube, a gun-barrel, for example. Persons in this condition, though on the street they may seem to be quite blind, and have to be led about, often are able to distinguish comparatively small test-letters at twenty feet, when, after considerable difficulty, they have succeeded in "finding" them, and fixing their gaze directly upon them.

Treatment.-The sovereign remedy in the treatment of inflammatory glaucoma is the operation of iridectomy, and the sooner this is performed the more favorable is the prognosis. For this reason it is inadmissible for the general practitioner to lose valuable time in endeavoring to combat the disease by less efficient measures. On the contrary, it is incumbent upon him as soon as he has recognized its true character to refer the case, with the least possible delay, to the ophthalmic surgeon. To undertake the performance of the operation himself would be justifiable only if it were impracticable for him to do otherwise; for there are few operations in ophthalmic surgery which call more distinctly for especial skill and training than an iridectomy for glaucoma, particularly if it has to be done, as is often the case, during an acute exacerbation of the disease. The most favorable opportunity for the performance of the iridectomy is between the exacerbations; but, if the attack is a severe one, it is not permissible to defer the operation until the eye has become quiet, for before this happens serious and permanent damage may have been done to the sight. 
There are, however, certain measures which the physician under whose observation the case first comes should employ without delay, and which, possibly, may cut short the attack, or, if they do not accomplish this, may, at least, lessen its severity, and so place the eye in a more favorable condition for operation. In the first place, he should prescribe a solution of eserin for application to the eye. The good which eserin accomplishes in glaucoma is due to its mechanical effect upon the iris. As atropin tends to induce glaucoma by dilating the pupil, and thereby crowding the iris into the periphery of the anterior chamber, eserin tends to reduce increased tension by contracting the pupil, and drawing the iris away from the filtration angle. To obtain the desired effect, therefore, eserin must be used in such strength as to cause decided contraction of the pupil.

During an acute attack of glaucoma it is usually difficult, and not infrequently impossible, to induce the pupil to contract even by the most liberal use of eserin. Under such circumstances, therefore, it should be prescribed in strong solution-in a solution of the strength of four grains to the ounce (eserin sulphate, gr. ii; aquæ destil., $\tilde{3} \mathrm{ss}$ ) - and this should be applied from three to four times in twenty-four hours. After the subsidence of the attack, however, a much weaker solution-perhaps only half a grain, or even a quarter of a grain, to the ounce-suffices usually to maintain the pupil in a state of contraction; therefore such a weak solution, the weakest solution, in fact, that will produce the desired effect upon the pupil, should be employed under such circumstances, and this will probably not have to be used more than two or three times a day. Another useful local remedy is the lotion of opium, 
which is apt to be more grateful if applied warm. Dionin, in conjunction with eserin, has also proved useful in the acute exacerbations of inflammatory glaucoma, through its action as a lymphagogue and an analgesic.

The constitutional measures called for are the administration, without delay, of an energetic calomel purge (calomel, gr. iij-iv; pulv. scammonii virg., gr. ij; pulv. rad. rhei, gr. vj), and, when this has had its effect, the further administration, in liberal doses,- - ten grains every two hours - of sodium or lithium salicylate. Opium in some form may also be given to lessen the pain.

As has been stated, the prompt employment of these measures may control even a well-marked attack of glaucoma; but should so fortunate a result ensue it would not warrant unnecessary delay in resorting to operation, for the outcome of the next attack might be far different.

As to what may be promised from a well-executed and promptly performed iridectomy, it may be said that the result usually is extremely satisfactory, and this applies not only to the immediate but to the permanent result. If the operation is performed before serious damage has been done to the optic nerve and retina, the restoration of practicably normal vision may be anticipated, as well as complete relief from subsequent suffering. But even if so favorable an outcome is not to be expected, still the operation should be done with as little delay as possible; indeed, it is indicated for the relief of suffering, though there may be no hope whatever of restoring vision. Exceptionally, in the cases of so-called malignant glaucoma, iridectomy fails entirely to arrest the progress of the disease, and sight is destroyed in a very brief time. When this has happened, and the inflammation and pain per- 
sist, the eye should be enucleated, as this will put an end to all suffering. Fortunately cases of this character are extremely rare.

As inflammatory glaucoma is so invariable a binocular affection, every possible precaution should be taken when the disease has manifested itself in one eye to prevent its occurrence in the fellow-eye. These precautions should include, besides care in the use of the eyes, regulation of the bowels, and temperance in eating and drinking, the careful correction of any refractive error found to be present, and the use of eserin upon the appearance of the first premonitory symptoms of the disease.

Experience has convinced me that not only in dealing with the second eye but, if the case can be seen early in the prodromal stage, in controlling the development of the disease in the primarily affected eye, much is to be hoped for from the wearing of accurately adjusted glasses-of glasses which exactly correct any refractive or muscular anomaly that may be present, and thus do away with all strain in both distant and near vision.

The conviction forced upon me years ago, that complicated errors of refraction often play an important part in the causation of glaucoma, has grown stronger with more extended observation, and I venture the opinion that if in the incipient stage of every case of glaucoma refractive and muscular anomalies were carefully searched for, and as carefully corrected, there would be an appreciable diminution in the number of cases demanding operation. To those who have had considerable experience with refractive anomalies, and know how often accommodative strain gives rise not only to marked hyperemia but to a low grade of inflammation of the inner tunics of the eye, this statement will, 
perhaps, not seem extravagant. I would, therefore, strongly insist upon the importance of a thorough examination of the refraction in every case of incipient glaucoma, and especially upon its importance with reference to the uninvolved eye, when the disease has manifested itself in one eye only.

\section{Simple Glaucoma (Chronic Non-inflammatory} Glaucoma).- This disease, which is characterized by a slight and persistent increase of the intraocular tension, is attended by none of the signs of active inflammation observed in inflammatory glaucoma. There is no pain, no injection, no clouding of the media, if we except a very slight diminution in some instances of the corneal transparency, and little or no shallowing of the anterior chamber, and the pupil, though sluggish in its response to light, is but slightly enlarged. The increase of tension is often so inconsiderable as to be difficult of detection and, though it varies somewhat in degree from time to time, it is never very pronounced.

- The first symptom to direct the patient's attention to the condition of his eyes is failure of vision. This, which at first is scarcely perceptible, progresses slowly, until after the lapse of several years sight may be entirely lost. As in inflammatory glaucoma, there is early in the disease a contraction of the visual field, the nasal half of the field being first involved. Like inflammatory glaucoma, too, it is an affection of advanced life, being seldom observed in persons under forty-five or fifty years of age, and it almost invariably attacks both eyes. As to its etiology little is known. Gout, rheumatism, and angiosclerosis are looked upon as conditions predisposing to its development.

An ophthalmoscopic examination and a perimetric test of the field of vision are necessary to a diagnosis of 
simple glaucoma, and even with these aids it is not always easy to distinguish it from simple progressive atrophy of the optic nerve. In each of these conditions there is contraction of the field of vision and cupping of the optic disc; but in progressive atrophy of the nerve the contraction of the field does not begin in the nasal half, as in simple glaucoma, and the cupping of the disc is less marked in proportion to the loss of vision; there is, moreover, in simple glaucoma a halo-like ring about the disc-a ring of partial choroidal atrophywhich is not present in progressive atrophy of the nerve.

Treatment.-Whatever plan of treatment may be employed the prognosis is far from favorable. Iridectomy is by no means the effective remedy in simple glaucoma that it is in the inflammatory type of the disease. Indeed, its results are so uncertain that many experienced ophthalmic surgeons regard it as a measure to be resorted to only when all other remedies have been tried, and found of no avail. Failure to arrest the progress of the disease is not the only count that can be brought against it; for not rarely it is followed by a marked change for the worse in the state of the eye, the tension being decidedly increased, and a condition more like inflammatory glaucoma being precipitated, which may soon lead to complete destruction of sight. Still, as the operation in some instances does unquestionably control the disease, it is a warrantable procedure when other remedies have proved ineffectual, since, under such circumstances, it offers the only hope of preventing certain blindness.

Some excellent authorities, prominent among them being Dr. Charles Stedman Bull, of New York, it should be stated, look upon iridectomy in simple glaucoma with more favor, and believe that it should be 
performed as early in the disease as possible, as soon, indeed, as the diagnosis can be established with certainty: Sympathectomy, or excision of the superior ganglion of the cervical sympathetic nerve, is another operative procedure which has been recommended in simple glaucoma; but the claims made at first as to its utility have not been sustained by wider experience with the method.

The remedial measures, other than operative, are the use of eserin in such strength as to maintain the pupil in a state of moderate contraction-usually an eighth-of-a-grain-, or a quarter-of-a-grain-to-the-ounce solution, applied twice a day, will accomplish this; the correction of any error of refraction that may be present; moderation in the use of the eyes; regulation of the bowels, and attention to the condition of the system, with special reference to the possible dependence of the disease upon a rheumatic or gouty diathesis. Potassium iodid in moderate doses is especially worthy of trial.

Secondary Glaucoma.--Increased intraocular tension consequent upon an injury or upon pre-existent disease of the eye is denominated secondary glaucoma. The diseases and injuries most apt to lead to this condition are those which involve the cornea, iris, lens, or ciliary body. Among these may be mentioned, prominently, perforating ulcers and penetrating wounds of the cornea, complicated by the formation of anterior synechia; neglected iritis, leading to extensive adhesion of the iris to the lens capsule, and especially those cases in which there is complete adhesion of the pupillary margin to the capsule, since in these the flow of lymph from the vitreous to the aqueous chamber is prevented; penetrating wounds of the ciliary region, 
followed by prolapse of the ciliary body; and injuries causing dislocation of the lens, or rupture of its capsule and the consequent formation of a traumatic cataract. Intraocular growths, especially those of malignant type, such as sarcoma of the ciliary body or choroid coat, are also usually attended by increase of intraocular tension, and, as has already been stated, this may happen also in the course of serous iritis, or uveitis.

The symptoms and the consequences to sight, if the increase of tension is marked and is not soon controlled, are the same as in the inflammatory type of primary glaucoma.

Treatment. - This will vary with the conditions that have induced the glaucomatous tension. Iridectomy is indicated if there is anterior or posterior synechia; abscission, combined, perhaps, with iridectomy, if there is prolapse of the iris or ciliary body; extraction of the lens if there is a swollen traumatic cataract; and prompt enucleation of the eye if the existence of a malignant intraocular growth can be established. Sodium salicylate and in some instances mercury are very useful, and, especially when there is a traumatic cataract, potassium iodid, which promotes the absorption of the extruded lens substance. Eserin is seldom indicated, though in uveitis, if the pupil is much enlarged, it may be used with caution. If employed too freely in this condition it may aggravate the iritis, and cause the formation of posterior synechiæ. The lotion of opium, applied hot or cold as may be more grateful to the patient, is of ten of benefit. 


\section{DISEASES OF THE CRYSTALIINE LENS AND VITREOUS HUMOR.}

\section{DISEASES OF THE CRYSTALIINE LENS.}

Enclosed in its capsule, and held in place by its suspensory ligament, the zonule of Zinn, the crystalline lens lies in the hyaloid fossa, a saucer-like depression on the anterior surface of the vitreous humor. The capsule of the lens is a highly elastic, homogeneous membrane, capable of offering great resistance to chemical agents, heat (boiling water), and putrefactive influences. That part of it which covers the front of the lens, and which is known as the anterior capsule, is considerably thicker than the posterior half, which lies in contact with the vitreous humor. The central portion of the iris, the zone of contact varying in width with the size of the pupil, rests upon the anterior capsule. Being an epithelial structure, like the hair and nails, the lens continues to grow throughout life, and is considerably larger and heavier in old age than it is in youth. Its growth is provided for by the presence beneath the anterior capsule of a layer of cubical epithelial cells, which become elongated and converted into the sixsided prismatic fibers of which the lens is composed (Fig. 120).

In early life, up to about the thirtieth year, the whole lens is soft, the central and cortical portions being of the same consistency. After this period, through a process of sclerosis affecting the oldest fibers, which 
now constitute only the central portion of the lens, there is formed a relatively. hard nucleus. At first this central nucleus is small in proportion to the size of the whole lens, and is but slightly harder than the comparatively thick layer of newly formed lens fibers which surrounds it. With advancing age, however, it grows

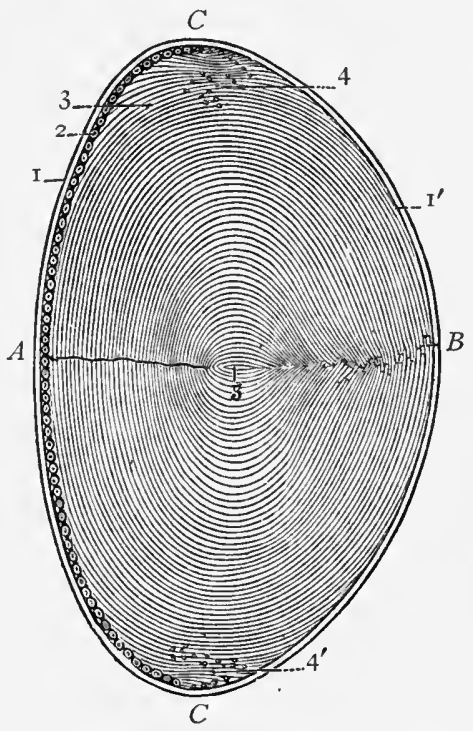

Fig. I 20.-Meridional section through human crystalline lens (Babuchin): $A$, Anterior, $B$, posterior surface; $C, C$, equatorial region; I, $\mathrm{I}^{\prime}$, anterior and posterior capsule; 2 , epithelium beneath anterior lens-capsule; 3 , lens substance composed of fibers; 4, transition zone where cells of anterior epithelium are converted into lens-fibers; 5, nucleus.

larger and increases in hardness, so that ultimately it constitutes the major part of the lens, the soft cortical layer which encloses it being now inconsiderable in thickness. Besides this change in its structure, which is attended by a diminution of its elasticity and an appreciable lessening of its transparency, the lens undergoes with advancing years an alteration in form, 
becoming flatter and less spherical than in childhood and youth.

These alterations in the structure and shape of the lens are of great significance. "Old-sight," or presbyopia, results from the loss of elasticity and flattening, while the presence or absence of a nucleus is of importance in connection with the development of cataract, since upon this hinges the character of the operative procedure which should be employed. The diminution of transparency is chiefly significant-for it does not appreciably lessen the acuity of vision-because in the aged it not infrequently leads to a mistaken diagnosis of cataract, with its attendant unpleasant consequences.

The lens, being without nutrient vessels, depends for its nutrition upon the lymph-stream which is supplied by the vascular uveal coat. To reach the lens the lymph must pass through the capsule, which it does chiefly at the equator, while it escapes through the anterior capsule. The maintenance of the lens in a normal state, it is evident, will depend upon the quality of the pabulum with which it is supplied. If through disease of the eye or constitutional disorder the intraocular lymph is materially altered in character, the lens, especially as to its transparency, is apt to suffer.

Cataract.-Although originally the name cataract, or cataracta, was employed to designate an opacity which, above all things, was held not to involve the crystalline lens, it is now applied only to opacities which are located in the lens. The origin of the name, which dates back to medieval times, is interesting:

The Greeks and Romans believed that the crystalline lens was the "seat of vision," and, as they knew that sight could be restored by the removal of a cataract, they were, perforce, driven to the conclusion that the 
opacity which they displaced from behind the pupil was not the lens, but an opaque substance which, cataract-like, had poured down over its anterior surface. Hence the Greek term bypocbyma and the Latin term, first used in medieval times, cataracta. Astonishing as it appears to us in the light of modern methods of research, this view was generally accepted up to the beginning of the eighteenth century, when Brisseau, a French surgeon, dissected the cataractous eye of a cadaver upon which he had previously performed the operation of depression, and discovered that the opacity which he had displaced from behind the pupil was, indeed, the crystalline lens. His discovery, which, of course, involved the abandonment of the view that the lens was essential to sight, was laid before the French Academy, but did not receive its endorsement until after three years of persistent opposition.

Cataract, though encountered most frequently in old age, occurs at all periods of life; indeed, as is well known, it is at times of congenital origin. Of the several classifications of cataract the one of greatest practical utility is that which is based upon the time of life at which the cataract develops, those which form after middle life being denominated senile or bard, and those which occur earlier than this juvenile or soft, cataracts, the hardness or softness of the cataract being dependent upon the presence or absence of a firm nucleus, the formation of which has already been described. Cataracts are also classified as general cataracts, those in which the opacity, sooner or later, involves the whole lens, and partial cataracts, in which, as a rule, this does not happen. Most cases of congenital and juvenile cataract and nearly all cases of senile cataract are "general" cataracts. Partial cataracts include zonular, or 
lamellar, cataract, anterior polar cataracts, and posterior polar cataracts. Again, cataracts may be classified with reference to their origin, as congenital cataracts, idiopatbic cataracts, complicated cataracts (those associated with or dependent upon other disease of the eye), traumatic cataracts, and cataracts due to constitutional disorder; and, still again, with reference to the stage of their development, as immature, mature, and bypermature cataracts; and, finally, after injuries of the lens or operations upon it we have secondary or capsular cataracts.

The significance of the first-mentioned classification is in its bearing upon the question of surgical treatment. Juvenile cataracts, since they have no hard nucleus, need not be removed from the eye, but may be broken up with a needle, and will, in time, undergo complete absorption. Senile cataracts, on the other hand, must be extracted, since their firm nucleus will not undergo solution in the fluids of the eye.

General Cataract.-As has been stated, most cases of congenital, of juvenile, and of senile cataract are included under this head; and, it may be added, traumatic cataracts also are nearly always of this character. General cataracts, whether juvenile or senile, and whether of idiopathic origin or due to constitutional cause or to traumatism, have certain features in common. They are all attended by marked and progressive impairment of sight; excepting those of traumatic origin, they are nearly always binocular, though they often do not develop in the two eyes concurrently; they are unattended by pain or other evidences of inflammation, unless complicated by injury or other disease of the eye; and, excepting again those due to traumatism, they are usually slow in forming, this being especially true of 
senile cataracts, which may be months or even years in reaching a state of maturity. The amount of impairment of vision which they cause depends upon the density of the opacity and upon its location in the lens. When they are fully formed vision is reduced to mere perception of light, though there are some exceptions to this rule in the case of senile cataracts; but, even when quite immature, vision may be little better than this, if the opacity is in the direct line of sight, that is, if it involves the nucleus of the lens or the central portion of the anterior or posterior cortex. On the other hand, the

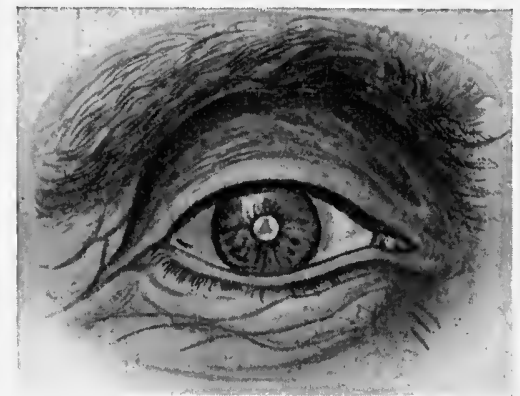

Fig. I2 I.-Senile cataract, mature (Haab).

existence of a considerable amount of opacity, if limited to the periphery of the lens, is not incompatible with normal sight, since this does not obstruct the entrance of light into the eye or interfere with the formation of a perfect image upon the retina.

Even in fully formed cataract (Fig. I2 I), it is important to remember, the pupil reacts to light almost if not quite as well as in the normal eye. When this is not the case, or when light perception is imperfect or absent, other and more grave disease of the eye is indicated.

As a rule, the cataracts which occur before middle 
life are whiter and more densely opaque, and, therefore, more conspicuous, than are those which develop in the aged. Indeed, in some cases of senile cataract, in which the nucleus of the lens is large and of amber color, the opacity is so inconspicuous that without the aid of the ophthalmoscope the true condition may be easily overlooked. Juvenile cataracts are also less constantly binocular than senile cataracts, and are commonly less slow in developing; and, because they are oftener due to other disease of the eye or to constitutional disorder, the prognosis in operating upon them is less uniformly favorable.

The presence of a comparatively large central nucleus, the characteristic feature of senile cataract, is the cause of this variety of cataract being less conspicuously white than are those which occur in early life. The nucleus itself is never white, and is seldom densely opaque. Occasionally it is colorless, but much oftener it is of a yellowish or amber tint, while at times it is almost black. As the layer of opaque cortical substance is not very thick, the color of the cataract partakes of that of its nucleus, so that we have in the aged yellowish, amber-colored, and so-called black cataracts, but rarely cataracts that are decidedly white.

In senile cataract the opacity usually manifests itself first in the cortical layers of the lens, and, as has been said, it commonly increases slowly, though in rare instances striking exceptions to this rule are encountered. While I have frequently seen cortical opacities remain stationary for years, I have, on the other hand, observed, in three instances, a senile cataract change from a state of incipiency to a state of maturity in one week's time. Doubtless, many old persons go to their graves without being aware that, for years, they have had incipient cat- 
aracts. This is not so remarkable, however, as the fact, of not very rare occurrence, that individuals may be blind in one eye from cataract for months, without being conscious that such is the case.

One of the earliest premonitory symptoms of senile cataract is the decline of presbyopia, the acquisition of so-called "second sight." When a person, who for years has been unable to read without presbyopic glasses, discovers that he can now put them aside and read without their assistance, he usually congratulates himself, and is congratulated by his friends, upon this restoration of youthful vision. The real significance of this change is that cataract is impending, and that in consequence of the degeneration of its fibers the lens is changing its shape, becoming more convex, and so giving rise to an acquired myopia, which enables small objects to be seen without the convex glasses that were previously required. It goes without saying that this improvement in vision is apt to be short-lived, and that it is hardly a matter for congratulation.

Our knowledge concerning the etiology of cataract is not as satisfactory as could be wished. We know, however, that the process which causes the lens to become opaque is a degenerative and not an inflammatory one. The lens fibers undergo degeneration, and in doing so lose their transparency. This degeneration, whether occurring in intrauterine life, in youth, or in old age, is commonly the result of faulty nutrition, which may be due to disease of the eye itself, to constitutional disorder, or to senile decay. Among the constitutional disorders which predispose to the development of cataract may be mentioned diabetes, inherited syphilis, rachitis, and angiosclerosis. A predisposition to cataract is also not infrequently inherited, an unusual 
number of cataracts being observed in successive generations of certain families.

The diseases of the eye most apt to lead to opacity of the lens are those which involve the uveal coat-the iris, ciliary body, and choroid. Cataract is also a usual consequence of unchecked inflammatory glaucoma. Lifelong accommodative strain, due to uncorrected refractive errors, through the congestion and inflammation which it induces in the inner tunics of the eye, is, I am persuaded, a far from uncommon cause of cataract. The frequency with which I have observed, especially in astigmatic eyes, incipient cataract associated with miliary choroido-retinitis, or with evidences of preexistent choroido-retinitis of this type, long since forced this conviction upon me.

Traumatic cataract is usually the result of injuries which involve the capsule of the lens, such as penetrating wounds of the cornea and lens, or of the cornea, iris, and lens. When a rent is made in the capsule, opacification of the entire lens commonly follows in a short time, in consequence of the action of the aqueous humor upon the lens substance. In rare instances, when the wound in the capsule is small it may close, and there may result only a circumscribed and stationary opacity, which, unless centrally located, may cause little or no impairment of vision. Usually when injured the lens swells considerably, and the pressure which it then exerts upon the iris and ciliary body may excite inflammation of these structures; secondary glaucoma may also be brought about in this way. Severe concussion of the eyeball, even when unattended by rupture of the lens capsule, is at times followed by the development of cataract.

Congenital cataract, which is almost invariably binoc- 
ular, is caused by disturbed nutrition or inflammation of the eye in intrauterine life. An inherited predisposition to this variety of cataract is especially common.

To the general practitioner the most important matter with reference to cataract is its diagnosis, the ability to recognize its existence, and to determine the stage of its development; for, unquestionably, the operative treatment of cataract should not be undertaken except by those who have had special training in this branch of surgery. The physician who is able to diagnosticate cataract in its early stages, and hence to give a correct prognosis as to the impending loss of sight, deserves, and will receive, no little credit; and if, further, he is qualified to decide as to its maturity-whether or not it has reached the stage when an operation should be performed-he is in a position to give to his patient advice of much value.

As a rule, when a cataract has progressed far enough to appreciably impair sight its recognition is not a matter of great difficulty, even to the non-specialist. This observation, as has been intimated, is especially true of cataract occurring in early life; but it is true also of senile cataract, if he will make use of the diagnostic aids which are within his reach.

To begin with, familiarity in the employment of "oblique illumination," which, as explained in the chapter upon "diagnosis," is not difficult of acquirement, will enable him to distinguish with certainty between lenticular and corneal opacities, between opacities situated behind and in front of the plane of the iris. It will enable him also to detect slight opacities in the lens, in its anterior portion especially, which might otherwise escape observation. Again, the use of an evanescent mydriatic (a one per cent. solution of hom- 


\section{PLATE VIII.}

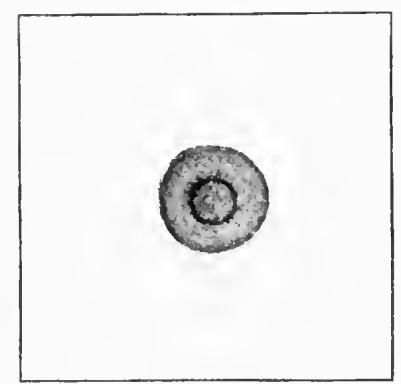

FIG. I.

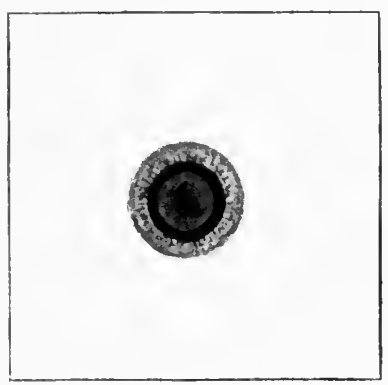

FIG. 3.

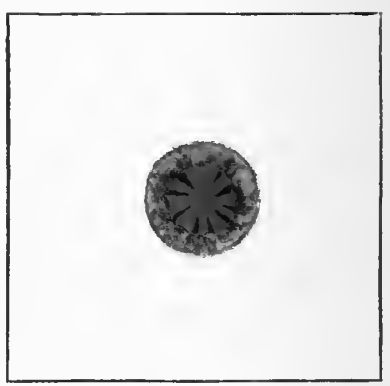

FIG. 2.

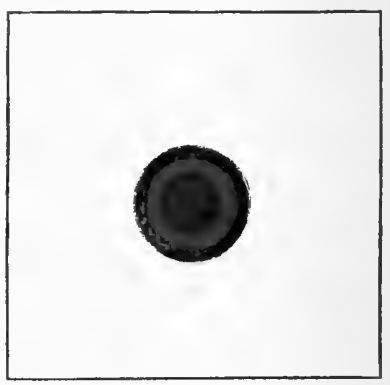

FIG. 4.

Fig. I.-Mature cataract, as seen by daylight or by oblique illumination (after Sichel).

Fig. 2.-Immature cataract, as seen by transmitted light (with ophthalmoscope or ear-mirror).

Fig. 3.-Zonular cataract, as seen, with pupil dilated, by oblique illumination (modified after Sichel).

Fig. 4.-Zonular cataract, as seen with ophthalmoscope or ear-mirror, (pupil dilated by atropin) (after Jaeger). 
atropin hydrobromate or a five per cent. solution of euphthalmin hydrochlorate), by exposing the lens more completely to inspection, will afford him further valuable assistance. With a widely dilated pupil, and the aid of oblique illumination, only opacities situated in the periphery of the lens or near its posterior pole are likely to escape detection, and the former, as has been stated, are not apt to disturb vision (Plate VIII, Fig. I).

The error into which the general practitioner, even with these aids, is most apt to fall, is in mistaking the apparent opacity of the senile lens for true cataract. For, with the pupil dilated, and the light focused upon the exposed lens, the yellowish color and the seeming opacity of the nucleus common in the aged are made especially conspicuous, so that the expert even may be inclined to believe that a cataract is present. Any doubts that he may entertain upon this point, however, are soon dispelled by the use of the ophthalmoscope, since by transmitted light the apparent opacity, made conspicuous by oblique illumination, disappears, and only such opacity as is real, as constitutes cataract, is seen. Real opacities observed in this way, the ophthalmoscopic mirror being held about twelve inches from the eye, no longer appear gray or yellowish, as they do by focused light, but, if the cataract is incomplete, are seen as blackish spokes or flocculi, or as a dark central area, against the red background of the eye (Plate VIII, Fig. 2).

This use of the ophthalmoscope does not require special training, so that those not skilled in ophthalmoscopy may make such an examination satisfactorily; and, if an ophthalmoscope be not at command, an ear or throat mirror will be found to answer almost as well, provided the light be placed at a 
greater distance than usual from the eye under examination, so that the illumination of the pupil shall not be too intense. In using such a mirror as a substitute for the ophthalmoscope, the observer must, of course, look through the opening in its center, otherwise the pupil will appear not red but black, and the lenticular opacities will not be seen. Ill-defined opacities limited to the periphery of the lens or situated at its posterior pole are comparatively difficult of detection even by transmitted light, and will hardly be recognized by those unfamiliar with the use of the ophthalmoscope.

The steamy appearance of the lens, exaggerated by the mistiness of the cornea and vitreous humor, observed in inflammatory glaucoma might be mistaken for cataract; but, as the inflammatory symptoms, the high tension, etc., point unmistakably to the true condition, an error of this character is inexcusable. With more warrant, the opaque exudate occluding the pupil which is at times observed as a sequel of iritis may lead to a mistaken diagnosis of cataract. Inspection by oblique illumination, however, would show that the opacity was upon and not beneath the capsule, and the application of a mydriatic, by the failure of the pupil to respond or by its irregular dilatation, would in all probability demonstrate the existence of posterior synechiæ.

The subjective symptoms of cataract, though not so pathognomonic as the objective signs, are nevertheless of diagnostic value. Mention has already been made of one of the most characteristic premonitory symptoms -the acquisition of "second sight." It should be remarked, however, that though this symptom indicates very clearly what is impending, it may, in exceptional instances, antedate by many months the development of such an amount of lenticular opacity as will seriously 
impair vision. The slowly progressive failure of sight, unattended by inflammatory symptoms, observed in cataract is not, in itself, characteristic, for we meet with this in other conditions, such, for example, as progressive atrophy of the optic nerve; but when it occurs without loss or diminution of pupillary reaction to light, the presumption is very strong that it is due to advancing lenticular opacity. Monocular diplopia or polyopia, most apt to be observed in regarding a bright light or a brilliantly illuminated object, such as the moon, is another symptom strongly suggestive of cataract, since it rarely occurs except in consequence of incomplete opacity of the lens, which causes the light in its passage toward the retina to be broken up into separate pencils. Better vision in subdued light, as after the setting of the sun, is another suggestive symptom, often mentioned by patients with incipient cataract, and which has its explanation in the increased size of the pupil under such circumstances. In line with this is the improvement in vision which often results in partially developed cataract from the application of a mydriatic.

Finally, it is to be stated, cataract should be suspected, and should be carefully searched for, whenever there is failure of sight, without other evident cause, in persons who have reached or who have passed middle age, in individuals known to be suffering with diabetes, and in infants or children with congenitally defective vision.

The determination of the maturity or "ripeness" of cataract is not so simple a matter as we were formerly taught to believe. Not very many years ago, a cataract was held to be mature if with the affected eye there was inability to count fingers, while it was regarded as imma- 
ture if this amount of vision was present. This "rule of thumb" is now known to be subject to so many exceptions that it can no longer be regarded as a trustworthy guide. Before speaking of these exceptions, it will be well to consider what is meant by a mature or ripe cataract.

A surgically mature cataract is one that is in a favorable condition for operation-one that may be easily and completely removed. In other words, it is a cataract in which all of the lens fibers have undergone degeneration, and in which, as one of the consequences of this change, the intimate connection that normally exists between the lens proper and its capsule has been lost. Such a cataract has been aptly compared to a ripe fruit, which may be readily removed from its rind. Now, it is a fact that there does not exist a constant relation between this condition and the degree of impairment of sight. That is to say, there are immature cataracts which reduce vision to mere light perception, while, on the other hand, there are mature cataracts, cataracts in a thoroughly satisfactory condition for operation, which impair sight much less markedly, not only ability to count fingers at several feet, but to distinguish large letters at this distance with no great difficulty, being retained. It is evident, therefore, that there are other factors, besides the amount of sight impairment, which must be taken into account in determining the surgical maturity of a cataract. The important point is to know how completely the lens has undergone degeneration.

If when inspected by oblique illumination portions of the lens are seen to be still transparent, the cataract is manifestly immature, the degeneration of the lens fibers is incomplete. The flocculent, glistening, mother- 
of-pearl appearance frequently seen in senile cataracts especially, and due to a lack of uniformity in the degeneration of the lens substance, is another evidence of immaturity, although when this condition is present vision is seldom better than light perception.

The cataracts which, notwithstanding their maturity, permit such a considerable degree of vision as has been described (ability to count fingers, etc.) are commonly observed in persons who are well advanced in yearsover sixty-five or seventy years of age-and in whom, therefore, the nucleus of the lens is relatively large and the overlying layer of cortical substance comparatively thin. The most striking examples of cataract of this character are those which exhibit a decidedly yellowish or amber color. It is the large size and the comparative clearness of the nucleus in such cataracts that explains the relatively good vision retained, and also the fact that not infrequently when the ophthalmoscope is used a sufficient amount of light reaches, and is reflected from, the fundus of the eye to give a reddish pupillary reflex, a thing which is never observed in the unripe, mother-ofpearl cataract or in even the far from mature juvenile cataract.

Almost without exception these amber-colored, seemingly unripe, cataracts prove to be in an ideal state for operation; that is, they are easily extruded from the capsule, and if any bits of the cortical substance are left behind they are dissolved speedily and soon disappear, because they have undergone previously such complete degeneration. In this respect they behave entirely unlike the clear lens substance of an immature cataract when left in situ, which increases in bulk as it becomes opaque, resists absorption for a considerable time, is apt to excite inflammation of the 
iris, and may in this way lead to the formation of a secondary cataract.

Treatment.-An important point to be impressed upon the general practitioner with regard to the treatment of cataract is that the only effectual method of dealing with the condition is by operation. One frequently hears of the claims put forth by charlatans that they can cure cataract, that they can dissipate lenticular opacities, by means other than operative; but, without exception, these claims, when subjected to investigation, have proved to be entirely without justification, to be, in fact, purely fraudulent.

In the incipient stage of cataract, when the opacity is confined to the periphery of the lens, if the ophthalmoscope affords evidence to warrant the belief that the lenticular changes are dependent upon a low grade of choroido-retinitis, it is proper to take measures to combat this latter condition; for, if this can be done successfully, there is ground for hope that the development of the cataract may be arrested or, at least, retarded. The most effectual means of doing this consist in the careful correction of any refractive errors that may be found to be present; in moderation in the use of the eyes; in inducing by suitable measures regular action of the bowels, and in the administration of small doses of biniodid of mercury (gr. $\frac{1}{40}$ to $\frac{1}{32}$ ) or of potassium iodid. If, however, the opacity has advanced so far as materially to impair sight, such measures will be without avail.

During the stage of immaturity, while the cataract perhaps is developing slowly, temporary but greatly appreciated improvement in vision may be obtained, in some instances, by keeping the pupil moderately dilated through the application of a mydriatic. The cases 
in which this is possible are those in which the opacity is limited to the central portion of the lens or, at least, is more dense there than it is in those parts of the lens that are commonly covered by the iris. A weak solution of atropin (gr. $\frac{1}{4}$ to $3 \mathrm{i}$ ) is best adapted to this purpose, and need not be applied, as a rule, oftener than once in three or four days. A single application will suffice to determine whether or not this will prove helpful. In using so weak a solution of atropin there is little danger, even in the aged, of causing an abnormal increase of intraocular tension; but the possibility of this should be borne in mind, and should any evidences of an induced glaucoma be observed, the mydriatic action of the atropin must be neutralized at once by the use of eserin.

It may be well to add that one should take care that the temporary improvement in the patient's vision caused by the mydriasis does not lead him to entertain false hopes that he is being cured. It is a common practice with quacks to employ a mydriatic in incipient cataract for this very purpose, and it is not difficult for them to persuade their dupes that an agent which, as the result of a single application, has made so marked an improvement in vision will in a short time effect a complete cure.

Another practical suggestion, having to do with the incipient stage of cataract, is as to the unwisdom of telling the average patient that a cataract is beginning to develop in his eye, which will in time lead to loss of sight. Months may elapse, in some instances even years, after the ophthalmoscope has revealed the presence of slight peripheral opacity of the lens before a considerable impairment of vision occurs, and during all this time the individual may be relieved of the dread of 
impending blindness, and the unhappiness to which this is sure to give rise, if the information gained by our inspection of the eye be kept from him. It is a case to which the proverb, "where ignorance is bliss," etc., is strikingly applicable. It is proper, however, for our own protection, if for no other reason, that some member of the patient's family should be made aware of the true condition. On the other hand, if the sight is already decidedly impaired it is best to tell the patient frankly what the trouble is, since we are then in a position to give him a very favorable prognosis, and so relieve his mind of much anxiety.

The question often arises as to the advisability of operating for cataract upon one eye, when the sight of the fellow-eye is as yet unimpaired. It may be stated, as a general truth, that the improvement in vision gained by doing this will not be very considerable; for the unaffected eye will still be used for all accurate seeing. The field of vision, however, will be widened, and there are certain other advantages to be gained which deserve consideration. In the first place, if the patient is still young, the improvement in personal appearance resulting from the removal of so serious a blemish as is caused by the presence of a monocular caratact is a matter not always to be ignored. Again, and this applies more particularly to cases in which there are incipient evidences of the formation of a cataract in the fellow-eye, it is a great comfort to the patient to feel that one eye has been operated upon successfully, and that he will have this eye to fall back upon when the sight of the other finally fails. Still another consideration is that cataracts in time, after having passed through the stage of maturity, tend to become overripe, or hypermature, in which condition, owing to the tough- 
ening of the capsule and the secondary degenerative changes in the lens, they are in a less favorable state for operation.

On the whole, taking into consideration the infrequency at the present day of unfavorable results in operations for cataract, it is best, I think, if there is evidence of beginning lenticular opacity in the relatively good eye, to operate upon the other eye, provided the cataract is fully mature. On the other hand, if it is immature, the operation should be deferred, at least until the sight of the better eye has become appreciably impaired. And here it may be remarked that when, as frequently happens, the lens opacity develops concurrently in the two eyes, so that the sight of each is much impaired, there is a disposition at the present day, more especially in dealing with persons who have reached the age of sixty-five or seventy, to operate upon the eye in which the opacity may be more advanced, without waiting for the cataract to become fully mature, the reason for this being that in the aged the nucleus of the lens is so large and the cortical substance so inconsiderable in amount that, even when it has not undergone complete degeneration, it is not apt to give rise to complications.

In persons under the age mentioned, if the sight of each eye is much affected, and the cataract in each is still immature, the procedure of Förster is indicated. This consists in the performance upon one eye of a preliminary iridectomy, accompanied by a bruising or "trituration" of the lens. The purpose of this procedure is to hasten the ripening of the cataract, so that its removal may be undertaken sooner than otherwise would be practicable. The desired result is not 
always secured; but not infrequently, within five or six weeks of the performance of the operation, the cataract will have reached a state of maturity which it might not have reached in many months had it been left to ripen in the usual way.

Although the operation of cataract extraction was known to the Romans of the period of the Empire, and was practised during the middle ages by the Arabians, it was not revived in Europe until the middle of the eighteenth century; and long after that, indeed as late as the middle of the last century, the real indications for its performance in preference to the more easily executed operations of couching or depression and of discission were but imperfectly understood. At the present day the manner in which a cataract shall be operated upon-whether it shall be "needled" and allowed to undergo solution in the eye or shall be extracted from the eye--is determined by its consistency, by its hardness or softness, that is to say, by whether it contains or does not contain a firm nucleus. Hence it is that all senile cataracts, indeed, all cataracts occurring in persons over thirty years of age, are extracted; while all juvenile cataracts are needled.

Formerly, in the operation of discission, as well as in that of couching, the cataract needle was introduced through the sclera, and the lens was attacked from behind. In the modern operation of discission, on the contrary, the needle is always introduced through the cornea, and the lens is attacked through the anterior capsule, in which a more or less extensive rent is made. The purpose of this is to expose the cataract to the action of the aqueous humor, which in time effects its solution and absorption. This process is a slow one, and commonly consumes several months, and not infrequently. 
more than one needling is required. It is, however, a much safer procedure, especially in infants and children, than the operation of extraction, and is less liable to accidental or other complications. At the time of the operation the pupil must be widely dilated by atropin, and this dilatation must be maintained until the absorption of the lens is complete. Under the influence of cocain the operation is painless; but in young children it is best usually to employ a general anesthetic, the primary anesthesia induced by chloroform being sufficient, as the needling requires but a few moments. Unless complications occur there is little or no after-suffering, and, except for a few days, the patient need be subjected to but slight restraint.

There are two methods of operating upon hard, or senile, cataracts in vogue at the present day-simple extraction and combined extraction, or extraction with iridectomy. Each has its advocates, though the latter procedure, because less liable to unpleasant complications, is probably more than holding its own. For myself, as the result of a considerable experience with both methods, I have come to prefer combined extraction, and for some years have operated by this method only.

With the eye thoroughly cocainized, the operation of cataract extraction, whichever procedure be employed, is seldom attended by pain that is at all intolerable, and frequently is entirely painless. The making of the corneal section is hardly ever painful, for the cornea is thoroughly anesthetized; but as the anesthesia of the iris is usually less complete, it is not uncommon for some pain to be experienced when it is drawn out and cut in the combined operation, or compressed and stretched in the extrusion of the lens in simple extrac- 
tion. Some discomfort in the eye, at times amounting to pain, is often felt during the twelve hours following the operation; but, if no complications occur, this usually constitutes the sum of the patient's suffering. For three days it is best that he should be kept in bed, with both eyes closed; but on the fourth he may sit up and have the use of the unoperated eye. At the end of a week the dressings are left.off the other eye, and usually at the end of two weeks he is able to leave the hospital, being provided with a pair of smoke-tinted glasses, to be worn until the eye is quite free from irritation and in a favorable condition for the adjustment of the "cataract glass," which, of course, is essential to clear vision, and which, thenceforth, he will wear continuously.

As to the chances of an operation for senile cataract being successful, it may be said that with the great help afforded by cocain, with the means at command for lessening the likelihood of post-operative accidents (Fig. 122), and with the careful antiseptic precautions employed at the present day the percentage of failures in the hands of experienced operators is extremely small-scarcely more than four per cent. In spite of every precaution, about two per cent. of the eyes operated upon are lost by infection, the infection in some instances doubtless being entogenous. The other two per cent. of failures is usually attributable to unruly behavior upon the part of the patient, either during or after the operation, to the existence of other disease of the eye apart from the cataract, or to some misadventure in the performance of the operation.

In one hundred consecutive cases of cataract extraction reported by the author a few years since* the opera* “American Journal of Ophthalmology," Dec., I899. 
tion was successful in ninety cases, the vision obtained varying from $\frac{20}{c c}$ to $\frac{20}{x \text { jii }}$, and partially successful, vision

- being less than $\frac{20}{c c}$, in six cases, while two eyes were lost from infection, and in two others no improvement in vision resulted, though recovery from the operation was smooth, owing to pre-existent disease of the retina.

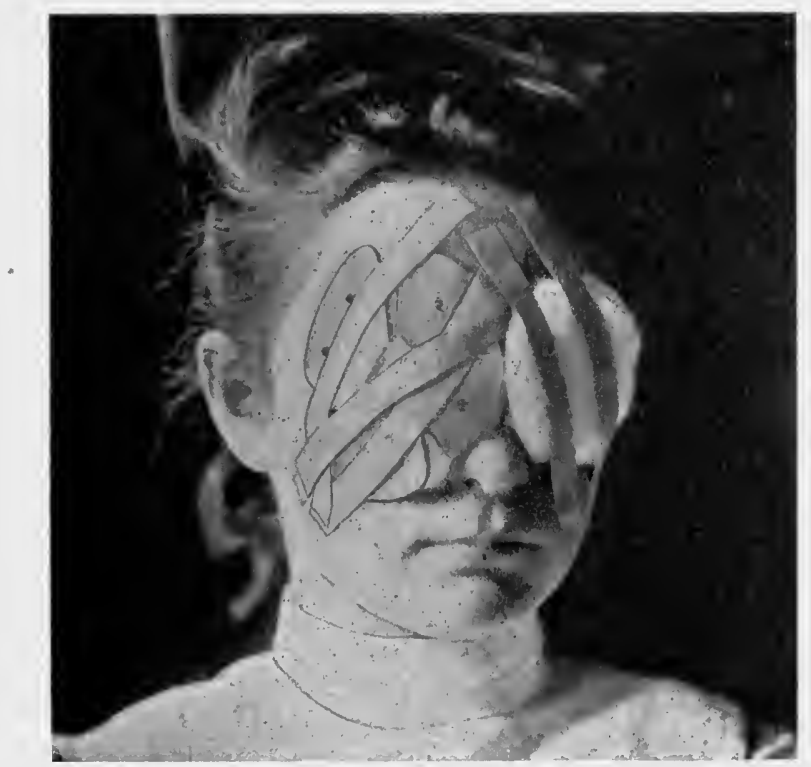

Fig. 122.-Dr. Murdoch's protective shield as applied after cataract extraction. The left eye is closed with a pad of gauze and absorbent cottona convenient dressing in less delicate operations.

In ninety-three operations performed since this report there have been four failures, two from suppurationexactly the same as in the first series-and two from other causes, a percentage of failures for the whole series of nearly two hundred cases of slightly less than 3. I I. Among the conditions which militate against the success of the operation of extraction of cataract may 
be mentioned the existence of diabetes. This should not be regarded as a contraindication to its performance, however, as in the great majority of such cases the operation proves successful. The presence of an arcus senilis was formerly regarded as influencing the prognosis unfavorably, but this is now known not to be the case. There is, moreover, little ground for the popular belief that advanced age lessens materially the chances of success of an operation for cataract. One often hears of persons who hesitate to submit to operation because they fear, or have been told, that they are too old to undergo it. My own experience is, and it agrees with that of other ophthalmic surgeons, that cataract operations upon octogenarians, if for their age they are in fairly good health, are as apt to be successful as are those performed upon persons who have not yet reached the biblical threescore years and ten.

Another popular misapprehension is as to the likelihood of a cataract returning after it has been removed. "If I have my eye operated upon, can I feel any assurance that the cataract will not return ?" is the way it is often put. Once removed, a cataract, strictly speaking, never returns; but capsular opacities, sometimes spoken of as secondary cataracts, occasionally develop even after the most successful operations, and may interfere with vision to such a degree as to require "needling." And in this circumstance, doubtless, is to be found the explanation of the misconception in question.

Not only do senile cataracts require to be extracted, but, under certain circumstances, soft, or juvenile, cataracts as well must be dealt with in this way. For example, a traumatic cataract occurring in a young person may become so swollen, and the anterior chamber so filled with opaque lens substance, as to cause much irrita- 
tion, and, perhaps, induce a glaucomatous condition; and a similar state of affairs may follow the operation of discission performed upon a soft cataract. Under such circumstances, the partial or complete removal of the cataract is called for, and this is accomplished by a linear extraction or by what is known as suction extraction. In the one case a linear incision, about 5 $\mathrm{mm}$. in length, is made in the cornea, a little in front of the plane of the iris, and the lens substance is coaxed out by carefully exerted pressure and counter-pressure. In the other, a similar incision is made, and the nozzle of a suction syringe, especially adapted to the purpose, is introduced into the anterior chamber, and the semifluid cataractous substance is cautiously sucked out. Successfully carried out, these procedures not only relieve the irritation and reduce the tension of the eye, but greatly hasten the restoration of vision, for which purpose alone they are sometimes employed.

It is well to remember, in dealing with congenital cataracts, that it is not safe to postpone operating too long after birth, since in the new-born permanent amblyopia is apt to result from nonexercise of the retina. In adults there is no risk of this sort.

In all operations for cataract a rule which I have invariably adhered to, and which I think should never be departed from, is to operate upon only one eye at a time. For, should the first operation not prove successful, the experience gained with this eye may be very helpful when we come to deal with the other eye. Again, an intercurrent infection may involve both eyes, or the failure of the operation upon one eye may lead to complications in the fellow-eye. In a word, as the homely proverb has it, we should never put all our eggs in one basket. 
Exceptionally, after excellent sight resulting from a cataract operation has been enjoyed for months, a gradual decline in vision may occur. This is due usually to a wrinkling of the posterior half of the lens capsule, and if the disturbance of vision is considerable a needle operation is called for; that is, a central rent should be torn in the capsule with a cataract needle or needleknife. This operation, under cocain anesthesia, is not painful, but it demands rigid antiseptic precautions; the outcome is commonly most satisfactory.

Partial Cataract.-Partial cataracts, which frequently are of congenital origin, differ from general cataracts, as has been said, in that they show little or no disposition to involve the whole lens, the opacity usually remaining circumscribed and stationary throughout life. There are several varieties of partial cataract, which differ radically as to their appearance, as to their etiology, and as to their effect upon vision. In two of these the opacity is limited to the anterior pole of the lens. These, therefore, are denominated anterior polar cataracts. There are also two varieties in which the opacity is confined to the posterior pole of the lens, and which are known as posterior polar cataracts. Finally, there is the variety known as zonular, or lamellar, cataract, in which there is, within the lens, a hollow, oblate sphere of opacity, which encloses, and is surrounded by, clear lens substance.

Anterior Polar Cataract.-Of the two varieties of anterior polar cataract the one less frequently encountered is due to the persistence of a portion of the embryonic pupillary membrane, which has adhered to the lens capsule and undergone calcification. A circumscribed, densely white opacity, occupying a limited portion of the pupillary area, and evidently lying upon, 
and not within, the capsule, is observed. Vision is not necessarily greatly disturbed.

The other variety of anterior polar cataract, known also as pyramidal cataract, occasionally develops during intrauterine life, but is commonly of postnatal origin, and has its starting-point in a central, perforating ulcer of the cornea, usually consequent upon ophthalmia neonatorum (Fig. I23). When a perforation of the cornea occurs, the aqueous humor escapes, the anterior chamber is obliterated, and the iris and lens are pressed forward so that they lie in contact with the cornea, where they remain until the anterior chamber is restored. When

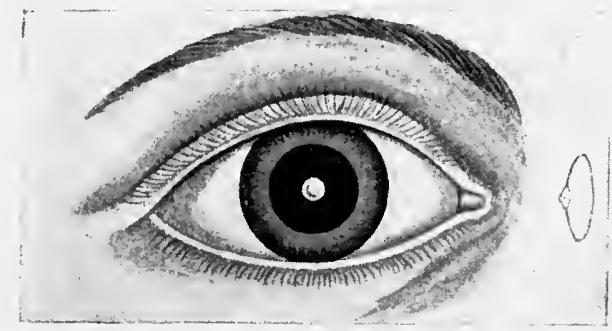

Fig. I23.-Anterior polar cataract (after Nettleship).

the perforation is so situated that the lens lies in direct contact with it, an irritation results which leads to proliferation of the subcapsular epithelial cells. The outcome of this is the formation, just beneath the anterior capsule, of a sort of fibrous tissue, which is white and opaque, and persists throughout life (Fig. I24). In addition to this, there is frequently a projecting mass of opaque material upon the external surface of the capsule, at a point corresponding with the intracapsular opacity. This consists of an organized exudate which remains adherent to the capsule when, in consequence of the re-accumulation of the aqueous humor, the lens is 
pushed away from the cornea, The pyramidal shape of this opacity is evidently the result of the traction to which it is subjected when the lens and cornea are thus forced apart. A perceptible corneal opacity usually marks the site where the ulcer perforated, and in some instances there may be seen a slender band of opaque tissue connecting this opacity with the apex of the epicapsular exudate. The degree of visual disturbance in pyramidal cataract depends largely upon the size of

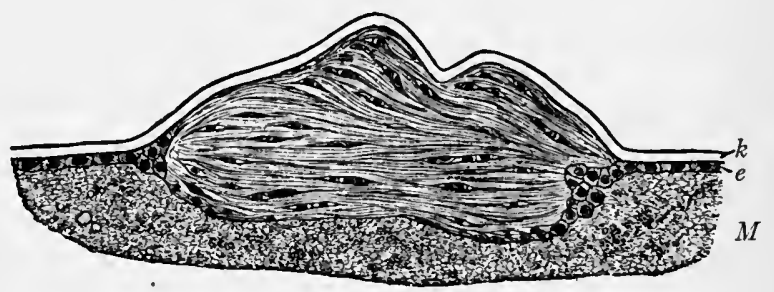

Fig. I 24.-Section of anterior polar cataract. Magnified $40 \times$ I (Fuchs). The capsular cataract forms a projection upon the anterior surface of the lens, covered by the capsule, $k$, which is unchanged and simply thrown into folds. The capsular epithelium, $e$, loses its regularity at the border of the cataract, its cells being increased in number and separated by the cataract from the capsule, so as to form for a short distance the posterior boundary of the cataract. The cataract consists of a fibrous tissue, with cells lying in the spindle-shaped gaps between the fibers. Succeeding the cataract posteriorly is liquor morgagni, $M$, which is coagulated into a pulverulent mass, separating the capsule from the cataractous layers of the lenticular cortex (which are not represented in the illustration).

the opacity relatively to that of the pupil, and upon its sharpness of definition. A dense opacity, if small and defined, is not incompatible with good vision. The corneal scar, though much less conspicuous, may produce far greater disturbance of sight.

Posterior Polar Cataract.-Opacities at the posterior pole of the lens, which are apt to cause greater impairment of vision than those at the anterior pole, can seldom be detected without the aid of the ophthalmoscope, 
though in some instances, with a widely dilated pupil, they may be fairly well seen by oblique illumination. Observed in this way, they are rather ill-defined and of a yellowish-gray color, whereas with the ophthalmoscope they appear black against the red background of the eye.

In the commoner form of posterior polar cataract the opacity, which may lie either just in front of the capsule or between it and the hyaloid fossa, is usually diffuse and ill-defined, sometimes exhibiting an imperfect star-

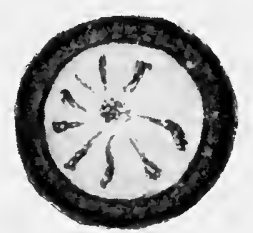

Fig. I 25.-Posterior polar cataract as seen by transmitted light (from a case of pigmentary degeneration of the retina) (Hopkins).

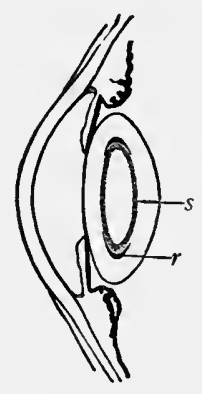

Fig. I 26.-Cross-section of zonular cataract. Schematic. Magnified $2 \times \mathbf{I}$ (Fuchs). The layers, $s$, lying between nucleus and cortex, are opaque, but the adjacent laver is so only in the equatorial region, $r$, indicating the presence of "riders."

shape (Fig. 125). This variety is commonly due to preexistent disease of the deeper tunics of the eye, which has interfered with the normal nutrition of the lens. Retinitis pigmentosa, diffuse choroido-retinitis, and myopia of high grade, attended by marked choroido-retinal changes, are the conditions most apt to give rise to it. The impairment of vision is apt to be considerable, and there is a greater probability than in any other form of partial cataract that the opacity may eventually involve the whole lens. 
The other, and rarer, form of posterior polar cataract is of congenital origin, and is due to the incomplete disappearance of the remains of the hyaloid artery, which in fetal life runs forward in the vitreous humor to the posterior pole of the lens. The opacity, which is upon, not within, the capsule, is small and defined, and seldom produces an appreciable disturbance of sight. Occasionally remnants of the hyaloid artery may be traced from the epicapsular opacity to the optic disc.

Zonular, or Lamellar, Cataract.-This is, perhaps, the most peculiar and interesting variety of partial cataract. The zone of opacity, which has been described (Fig. 126), varies considerably in size and also in thickness. Exceptionally there may be more than one opaque zone, the smaller zone being within the larger, and separated from it by a layer of transparent lens substance. Under such circumstances there is first a zone of clear lens next to the capsule, then an opaque zone, then another clear zone, and within this a second opaque zone enclosing a transparent nucleus.

This singular form of lens opacity, which nearly always affects both eyes, and a disposition to which is not infrequently inherited, develops either during the last months of fetal life or in early infancy, never in adult life. It is often found in association with inherited syphilis, rickets, or scrofula, and the majority of individuals in whom it occurs have suffered with infantile convulsions. Though its etiology is but imperfectly understood, it is probable that the explanation of its development is to be found in faulty nutrition, perhaps of intermittent degrees of intensity. Its frequent association with rachitic teeth is interesting, and helps to throw some light upon the way in which it is produced. 
There is commonly marked impairment of sight, though not so marked as in advanced general cataract. The amount of visual disturbance depends upon the thickness and density of the opaque zone, and there are cases in which this thickness and density are so slight as to interfere but little with vision. In most instances the opacity remains stationary, and retains its peculiarity, throughout life, though exceptionally the whole lens ultimately may become opaque.

The true character of zonular cataract seldom can be recognized until the pupil has been dilated by a mydriatic; for before this is done it presents much the appearance of an ordinary, immature cortical cataract. With the pupil widely dilated, however, the clear peripheral zone enclosing the smaller opaque zone may be made out easily by oblique illumination or with the ophthalmoscope or, as has been suggested, when an ophthalmoscope is not available, with the ordinary ear or throat mirror (Plate VIII, Figs. 3 and 4 ).

Treatment of Partial Cataract.-In anterior polar cataract, unless the vision is decidedly impaired, no treatment is indicated. If, however, the opacity is so considerable in extent as to occupy the greater part of the pupillary area, an iridectomy, made with the view of obtaining an artificial pupil opposite a clear portion of the lens, may be of decided benefit. The instillation of a mydriatic will indicate to what extent vision is likely to be improved by this procedure.

In posterior polar cataract an iridectomy is apt to be of little utility, and, as a rule, operative treatment is not indicated. When, however, the sight of both eyes is markedly impaired an endeavor to cause the whole lens to become opaque, by Förster's method or possibly by cautious needling, with a view to its subsequent re- 
moval, is justifiable. When the opacity is consequent upon choroido-retinitis, treatment of the latter condition is called for, and may be of some avail.

Zonular cataract, if it causes, as it usually does, marked impairment of sight, should be dealt with in one of two ways-either an artificial pupil should be made opposite the clear portion of the lens or discission should be performed. The former procedure is indicated when the cloudy zone is small and the clear zone relatively broad and free from the opaque spokes ("riders," as they have been called) which not infrequently are present, and when, moreover, dilatation of the pupil by a mydriatic is found to produce decided improvement in vision. Discission is called for when the opposite conditions exist, that is, when the opaque zone is wide, the clear zone narrow, when there are many and conspicuous "riders," and when inconsiderable improvement in sight results from mydriasis. The process of absorption of the lens through needling is a slow one in zonular cataract because so much of the lens is clear, and the breaking down of its fibers, which must precede their solution and absorption, takes a long time; but the ultimate result is apt to be more satisfactory than when an artificial pupil is made. After the lens has been absorbed a cataract glass must, of course, be worn.

Secondary, or Capsular, Cataract.-After operations upon the lens and after injuries involving the integrity of its capsule it may happen that some of the lens substance becomes imprisoned within the capsule in such a manner as to resist absorption. It may also happen that inflammatory exudates are deposited upon or within the torn capsule. In either case a more or less pronounced opacity, occupying the pupillary area and seriously interfering with vision, may result. Such opaci- 
ties are called secondary or capsular cataracts, and, if the disturbance of vision which they cause is considerable, must be dealt with by operative procedure.

Capsular cataracts are often so conspicuous that they may be detected at a glance; but they can be examined best, and their extent and character determined most satisfactorily, by oblique illumination, after the pupil has been widely dilated by a mydriatic.

Treatment.-This consists in making a rent in the opaque membrane-in its center, if possible-so that a clear area may be secured through which light can pass, unobstructed, to the retina. Exceptionally, it may be necessary to deal with these secondary opacities in a more radical way; but usually the desired result may be attained by a discission operation, that is, by introducing a cataract needle or needle-knife into the anterior chamber through the cornea, near its periphery, and cutting or tearing (for it is oftener a tear rather than a cut, the authorities to the contrary, notwithstanding) an opening in the opaque membrane. If the membrane is thick, it is best to divide it crucially; but when, as is often the case, it is cobweb-like in character, a linear incision suffices, as the elasticity of the capsule causes the rent to expand sufficiently for the end in view. The operation is done under cocain, with the strictest antiseptic precautions, and with the pupil dilated ad maximum.

Dislocation of the Crystalline Lens (Luxation of the Lens).-Dislocation of the crystalline lens, which occurs as a congenital as well as an acquired condition, may be complete or incomplete, and the displacement may be forward, into the anterior chamber, or backward, into the vitreous chamber. The lens is said to be incompletely dislocated when it remains in the hyaloid 
fossa, held measurably in place by remnants of the suspensory ligament or by the support of the vitreous humor. It is said to be completely dislocated when it has fallen back into the vitreous chamber or has passed through the pupil into the anterior chamber.

Congenital dislocation of the lens, which is commonly bilateral (Fig. 127), and to which there is not infrequently an inherited disposition, is usually incomplete, and is due to imperfect development or absence of the zonule of Zinn. Acquired dislocation is commonly the result of traumatism, though in certain conditions of the

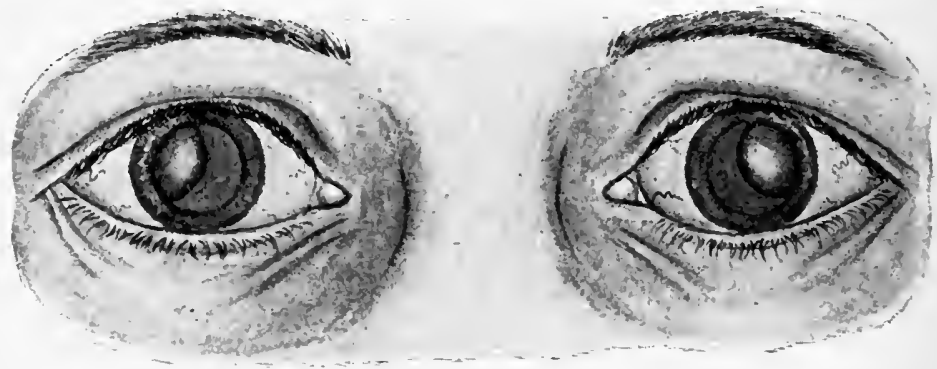

Fig. 127.-Congenital dislocation of the crystalline lenses, up and out (de Schweinitz).

eye-myopia of high grade, especially, or after chronic inflammation of the uveal coat-a very trivial accident, an inconsiderable blow upon the eye, for example, may suffice to bring it about. Dislocation of the lens beneath the conjunctiva (Fig. I28) is occasionally observed in severe injuries of the eye involving rupture of the sclera near the corneal border.

Displacement of the lens from behind the pupil, unless it happens to occur in a very myopic eye, necessarily causes marked impairment of vision. Moreover, by acting as a foreign body, the lens when loose in the vitreous chamber, and this is still more apt to happen 
when it is lodged in the anterior chamber, may cause great irritation, and excite severe inflammatory reaction.

The disturbance of vision from a partially dislocated lens, if the margin of the lens happens to be in line with the pupil, is especially annoying; for under such circumstances two images are formed upon the retina, a more distinct one by the rays of light which pass through the lens, and a less distinct one by those which reach the retina without passing through it, monocular diplopia being the result. A partially dislocated lens which lies behind the pupil in a tilted position-a con-

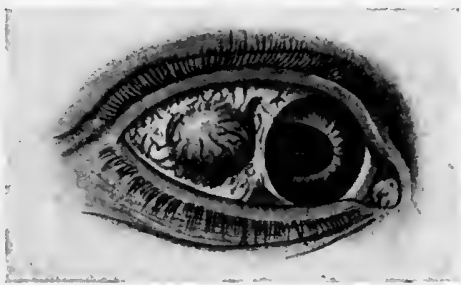

Fig. I 28. - Subconjunctival dislocation of lens (Haab).

dition not infrequently observed-also causes marked impairment of sight, since it necessarily produces a high degree of astigmatism.

A case of complete, probably congenital, dislocation of both lenses, exhibiting very unusual and interesting features, was observed, and an account of it published, by the author some years since*: In each eye of a lad, twelve years of age, the lens was completely dislocated into the vitreous chamber. In spite of this fact, and

* "Report of a case in which useful vision was maintained through a number of years by the aid of a totally dislocated lens." Trans. of the American Ophthalmological Society, I88I. With supplementary notes, as to the later history of the case, in the Transactions of the Society for I89I and 1893 . 
though he had never had compensating glasses, he had attended school, had been able to keep up with his classes, and had been much given to reading for pleasure.

In the course of my examination of the case it developed that his ability to read was due to his having acquired the knack of using one of the dislocated lenses. Without, of course, appreciating the significance of the maneuver, he was in the habit, whenever he wanted to see any small object, as in reading, of bending his head forward, with face to the ground. This brought the lens into position behind the pupil (as I satisfied myself by observation with the ophthalmoscope), and in this awkward fashion he was able to read with ease the finest print. He was given far and near glasses, which greatly improved his distant vision, and enabled him to read with his head in a natural position. Ten years subsequently, when he was twenty-two years of age, the capsule of the lens in the left eye ruptured. This was followed by considerable inflammatory reaction and decided impairment of vision. However, the lens gradually underwent absorption, though a fragment of the nucleus proved very obdurate, the inflammatory symptoms subsided, and vision regained its former standard. Six years after this the same thing happened to the right eye, and for a time there was the same decided inflammatory reaction, which was not without difficulty gotten under control.

Partial or complete dislocation of the lens backward is not always easy of detection. A completely dislocated lens-one that has fallen into the vitreous chamber - can be seen only with the help of the ophthalmoscope; a partially dislocated lens, after the pupil has been dilated by a mydriatic, may be satisfactorily inspected, 
and its position determined, by oblique illumination. A nearly constant symptom of backward dislocation of the lens is tremulousness of the iris-iridodonesis. This results from the iris having lost the support which the lens under normal conditions affords it. The existence of this symptom, therefore, should always raise a suspicion that the lens is more or less completely luxated.

When the lens lies in the anterior chamber (Fig. 129) the ease with which it may be detected by the non-expert depends largely upon whether it is transparent or catar-

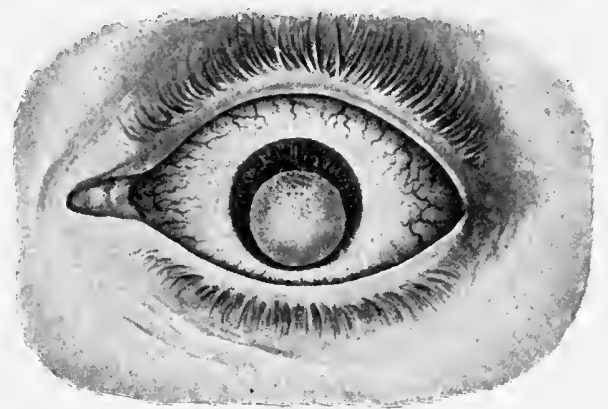

Fig. I 29.-Dislocation of lens into anterior chamber (Hansell and Sweet).

actous-for, it should be stated, dislocated lenses are very apt in time to become cataractous. An opaque lens in this situation should be detected at a glance, and the true state of affairs easily recognized. On the other hand, when the lens is clear it is not so easy as might be supposed to make a correct diagnosis. Oblique illumination would afford assistance, and a significant feature would be the unusual depth of the anterior chamber. In some instances a dislocated lens has a habit of gliding through the pupil, and being found now in front, and now behind, the iris.

Treatment.-When a dislocated lens lies in the an- 
terior chamber it is almost sure to give rise to much irritation, and to excite inflammation, it may be of a glaucomatous character. Its removal, therefore, is indicated. The operation is a delicate one, more so than an ordinary extraction of cataract, and should be undertaken only by one skilled in the performance of ophthalmic operations.

A partially dislocated lens may or may not require radical treatment. If it causes but little impairment of sight it should be left undisturbed, glasses being given if found to be of assistance; but if it is so placed as to interfere seriously with vision it may be extracted or, perhaps, needled, the latter procedure being indicated only in young subjects. The extraction of a displaced lens which has not left the hyaloid fossa, and is supported by a vitreous humor of normal consistency, is hardly more difficult than the ordinary removal of a cataract.

A lens completely dislocated into the vitreous chamber should not be disturbed, unless it is causing serious inflammatory reaction; for under such circumstances the vitreous humor is apt to be in a fluid or semifluid state, and an attempt to remove the lens is a hazardous procedure. It is possible, with the help of the ophthalmoscope, to needle a lens so situated, the needle being introduced through the sclera, and this procedure might be justifiable in a young person. Another possible procedure is to coax the lens by suitable manipulation through a dilated pupil into the anterior chamber, and, after imprisoning it there by the use of a strong myotic, to extract it through a corneal section

A lens dislocated beneath the conjunctiva may be extracted without difficulty. After the removal of a dislocated lens glasses, such as are prescribed after an operation for cataract, are, of course, necessary. 
DISEASES OF THE VITREOUS HUMOR.

Pathological changes in the vitreous humor seldom occur except as a result of traumatic lesions of the eye or in consequence of disease of the uveal coat or retina.

Purulent Panophthalmitis.- The vitreous humor affords an excellent medium for the growth of bacteria, and when a pyogenic organism finds lodgment

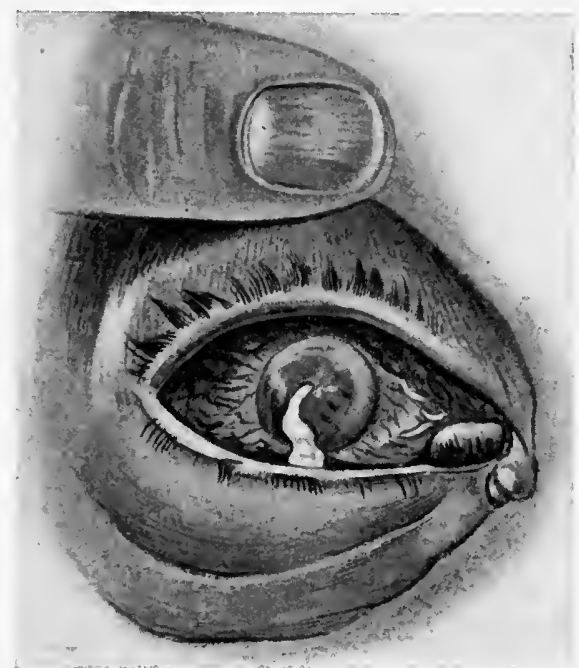

Fig. 130.-Panophthalmitis, from entrance of a piece of iron into the vitreous chamber (Haab).

there, as a result of a penetrating wound of the eye, operative or accidental, a destructive and usually uncontrollable suppurative panophthalmitis is apt to ensue (Fig. I30). Under such circumstances, in a very brief time, purulent infiltration of the entire vitreous body occurs; the uveal coat and cornea are soon involved; and after much suffering, attended by marked chemosis of the conjunctiva and great tumefaction of the lids, necrosis of the cornea or of the sclera at some point 
takes place. Then there is an escape of pus, with consequent reduction of the intraocular tension, 'and a measurable relief from pain is experienced. Complete loss of sight alwavs results, and ultimately atrophy of the eyeball.

Treatment.-So far as arrest of the suppurative process and preservation of sight are concerned, treatment is seldom of avail. Anodynes are indicated to control the pain, and hot fomentations - the lotion of opium, made stronger than is commonly necessary (ext. opii, gr. $x v$; aqux, $\overline{3}$ iv) -afford some relief. When it is evident that there is no hope of preserving sight, the eye should be enucleated without unnecessary delay. The danger of cerebral or systemic infection from the performance of the operation while the inflammation is still active seems to the author to have been much exaggerated, and the relief from suffering which it affords is almost instantaneous.

Fluidity of the Vitreous Humor (Synchysis). -This condition usually results from chronic inflammation of the uveal coat. It is, perhaps, oftenest met with in myopia of high grade attended by marked choroido-retinal changes. It is the outcome of malnutrition, and is frequently accompanied by floating opacities. The loss of consistency is seldom complete, though cases of this character are encountered. Fluidity of the vitreous body does not in itself cause inconvenience; but it probably predisposes to detachment of the retina, being usually attended by subnormal intraocular tension, and it may lead to complications in operations, such as extraction of cataract, or wounds which involve penetration of the coats of the eye. Treatment is ineffectual.

Opacities of the Vitreous Humor.-The vitreous 
humor may be diffusely clouded, or it may contain discrete opacities, varying greatly in size and number. Inflammation of the uveal coat, at all severe, is commonly attended by more or less marked loss of transparency of the vitreous body. At first the opacity is apt to be diffuse; but at a later stage, instead of a uniform cloudiness, we have ragged masses or shreds, resembling bits of cobweb, which float about freely, indicating, at least, partial synchysis. In time, and when the conditions are favorable, an opacity so dense as not only to preclude a view of the background of the eye with the ophthalmoscope, but to do away with all fundus reflex, and to reduce vision to mere light perception, may disappear completely, leaving no trace which the ophthalmoscope can discover. Discrete opacities, which seldom are stationary, but float about with considerable freedom, often cause much annoyance by obstructing the view especially of small objects through interference with the rays of light in their passage to the fundus of the eye, and by casting shadows upon the retina.

"Vitreous opacities" of considerable size can be detected easily with the ophthalmoscope, and their movements and position in the posterior chamber of the eye determined. Opacities too small to be seen in this way are not of moment, and yet such microscopic opacities, known as musca volitantes, which exist in all eyes, and under certain conditions, as when one looks toward a white wall or a light cloud, can always be perceived by the individual, give rise to much uncalledfor anxiety. There is a wide-spread popular belief, often difficult to combat, that the presence of "muscæe" is indicative of impending blindness-of the development of cataract or what not. It is undoubtedly true 
that they are more numerous and more conspicuous in eyes. made irritable by ref ractive or muscular anomalies, and to this extent they are significant; but the dread which they so often inspire is entirely unwarranted.

Hemorrhages into the vitreous humor which have undergone but partial absorption may give rise to large floating opacities, and opacities which are composed of an organized inflammatory exudate, accompanied by new-formed blood-vessels, and which shoot out into the vitreous humor from the retina, are observed in certain types of retinitis.

Treatment.-The treatment of opacities of the vitreous humor is the treatment of the condition or conditions upon which they depend. The major opacities, as has been stated, are usually caused by inflammation of the uveal coat, and this must be combated by the means described in the chapters in which diseases of the iris and ciliary body and of the choroid coat are discussed. The minor opacities (muscæ), when conspicuous, suggest, as has just been intimated, accommodative or muscular strain, and their existence should lead to a careful search for optical and muscular faults, and to an equally careful correction of these, by glasses or by operation, should they prove to be present. The lotion of opium, by lessening the irritability of the eyes, will also be found useful.

Hemorrhage into the vitreous humor occurs as a result of injuries of the eye, of disease of the choroid coat or retina, or in consequence of angiosclerosis or of alterations in the composition of the blood. It takes place usually from the vessels of the choroid, less often from those of the ciliary body or retina. It has been observed at times in association with frequent attacks of epistaxis, also, in recurrent form, in connec- 
tion with delayed menstruation. When the extravasation is considerable, vision may be reduced to light perception, and the ophthalmoscope may give only a black or reddish-black reflex. In some instances the blood is evenly diffused throughout the vitreous body, and then objects, which are seen indistinctly, have a reddish color, and the fundus of the eye is seen with the ophthalmoscope as through a red mist. Oftener it is in illdefined, opaque masses, which, according to their position, may or may not prevent a view of the optic disc and neighboring parts.

The absorption of the blood from the vitreous chamber is much more tedious than from the aqueous chamber, and is not so surely complete, floating opacities, as has been stated, being left in the vitreous humor, not infrequently, as the result of its incomplete disappearance. In cases of recurrent hemorrhage sight is apt to suffer serious and permanent injury; but usually after a single hemorrhage, when finally the extravasated blood has disappeared, vision regains its former standard.

Treatment.- Rest of the eyes and avoidance of active exercise, for a time at least, are indicated. Ergot is supposed to lessen the likelihood of a recurrence of the hemorrhage; but adrenalin, it would seem, should be still more efficacious. Habitual constipation, if present, should be corrected, aloin, in such doses as may be found necessary, being especially useful for this purpose. Potassium iodid should be given in five- to tengrain doses, as it unquestionably promotes the absorption of extravasated blood from the vitreous, as well as from the aqueous, chamber. Local remedies are of but little value; though, when the absorption of the hemorrhage is tardy, subconjunctival injections of salt solution are serviceable. 


\section{CHAPTER X.}

\section{DISEASES OF THE CHOROID COAT, RETINA, AND OPTIC NERVE.}

Although, without the help of the ophthalmoscope, it is impossible to diagnosticate with accuracy, and for this reason to treat intelligently, the several affections to be considered in this chapter, the physician who is not an ophthalmoscopist need not be wholly at a loss in dealing with these diseases of the deeper eye structures; for they are commonly attended by certain objective and subjective symptoms which are fairly pathognomonic, and which when taken into account permit, at least, of approximate accuracy in diagnosis.

As a rule, it may be stated, no external signs of inflammation are observable in choroiditis, in retinitis, or in optic neuritis. Nor are these affections commonly accompanied by ocular pain, or by photophobia, or lacrimation. Obscuration, more or less marked, of both distant and near vision is the most constant and conspicuous subjective symptom, while enlargement of the pupil and sluggishness in its response to light are the chief objective symptoms. Exceptionally, as in intraocular neuritis, or choked disc, and in diseases affecting chiefly the periphery of the choroid or retina, very good central vision may exist in spite of the fact that pronounced changes observable with the ophthalmoscope are present; and in inflammation of the choroid, if the ciliary body be involved, there will almost surely be present pericorneal injection, pain, photophobia, sensi- 
tiveness of the eyeball to pressure, etc. But these are the exceptions which lend force to the rule that has been enunciated, and, speaking broadly, it may be said that disease of the optic nerve or retina, or of the choroid and retina (for the retina seldom escapes involvement when there is considerable inflammation of the choroid), is to be suspected when the pupil is enlarged and responds imperfectly to light, and when, glaucoma having been excluded, distant as well as near vision is impaired, and is growing progressively worse.

It should be borne in mind, moreover, in endeavoring to reach a diagnosis, that the affections under consideration, though encountered in infancy and childhood, are more common in middle and advanced life; that they are usually binocular; that they are generally due to some constitutional disorder, such as syphilis, nephritis, diabetes, angiosclerosis, etc.; that they may occur during the course of the exanthematous fevers or as a complication in pregnancy; and as to optic neuritis that it is very commonly the result of coarse intracranial disease. However, it goes without saying that in these grave affections the physician should not rest content with a supposititious diagnosis, but that he should call to his aid a competent ophthalmoscopist, so that all uncertainty as to what is the true condition may be dispelled.

The treatment of these deeper diseases of the eye, local measures being of but little value, is mainly constitutional, and should be directed to the underlying systemic disorder upon which they depend. The prognosis, so far as sight is concerned, depends largely upon whether this underlying malady is or is not remediable. As a rule, those affections that are of syphilitic origin are the most amenable to treatment; while, as might be 
supposed, the prognosis is least favorable in those due to diabetes, to nephritis, to degenerative changes in the vessel walls, and to non-luetic intracranial disease.

\section{DISEASES OF THE CHOROID COAT.}

Choroiditis, or inflammation of the choroid coat, occurs as a purulent, as a serous, and as a plastic process. Purulent choroiditis is but another name for purulent panophthalmitis, which has been considered in the preceeding chapter, while serous choroiditis, which is synonymous with serous uveitis, has been treated of in connection with diseases of the iris.

Plastic Choroiditis.-This is the more common variety of choroidal inflammation, and is oftenest due to syphilis, inherited or acquired. It is also frequently present in progressive myopia, and is not rarely a result of traumatism.

Syphilitic inflammation of the choroid occurs usually in association with iritis and cyclitis, but well-marked cases of choroiditis are encountered in which neither the iris nor the ciliary body is involved; while in some instances, reversing the usual order, the inflammation begins in the choroid and extends from there to the ciliary body and iris. It occurs oftenest in the secondary stage of the disease, but may manifest itself at a later period. In every pronounced case of luetic choroiditis the retina is invariably implicated, and, if the macular region is involved in one of the areas of more intense inflammation which are characteristic of the disease, decided and permanent impairment of vision usually results. On the other hand, if this region is not seriously involved, there may be but little permanent damage to sight, though for a time it may be reduced to mere 
light perception, owing largely to opacity of the vitreous humor.

Loss of transparency of the vitreous humor is a usual feature of syphilitic choroiditis. At first the opacity is diffuse, and often so dense as to render a view of the background of the eye with the ophthalmoscope impossible. At a later stage, instead of this uniform clouding, we have ragged, cobweb-like opacities, which float more or less freely in the relatively clear vitreous

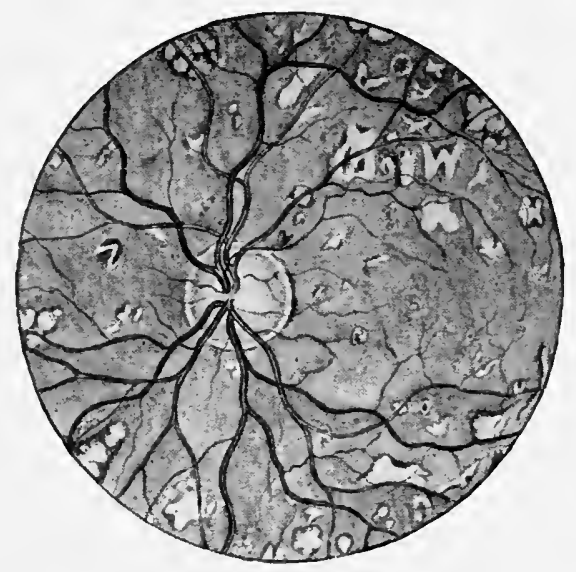

Fig. 131.-Fundus changes consequent upon severe (probably syphilitic) choroiditis, as seen with the ophthalmoscope (de Wecker).

body, and impair sight to a greater or less extent according to their position with reference to the visual axis. Ultimately these floating opacities usually disappear, and with their disappearance excellent vision may be regained, provided the retina, its central portion more especially, has not suffered serious damage (Fig. I3I).

The disease runs a tedious course, a course of months rather than weeks, and is apt to attack both eyes, though energetic treatment not infrequently prevents 
involvement of the second eye. Its existence should be suspected whenever, especially during the secondary stage of syphilis, marked impairment of vision, unattended by pain or injection of the eye, occurs. The presence of pain and circumcorneal injection indicates, as has been pointed out, that the iris and ciliary body are involved in the inflammatory process.

In the subjects of inherited syphilis plastic choroiditis occurs most frequently in association with interstitial keratitis, and under such circumstances iritis also is often present. It may make its appearance at any period of life, even as a prenatal affection; but, like interstitial keratitis, it is encountered oftenest between the ages of six and fifteen or sixteen years. The prognosis is unfavorable; but much may be accomplished by promptly instituted treatment. Opacity of the lens is a not uncommon consequence of unchecked syphilitic choroiditis.

The choroiditis of myopia of high grade is less intense than the syphilitic type; but as it occurs at the posterior pole of the eye and frequently involves the macular region, and as the retina is always implicated, the consequences to vision are often disastrous (Fig. I32). Floating vitreous opacities, large enough to cause considerable annoyance, are usually present, and detachment of the retina, and the development of cataract from disturbance of the nutrition of the lens, are complications to be apprehended.

Plastic choroiditis of traumatic origin, with which iritis and cyclitis are often associated, arises most frequently from penetrating wounds of the eye complicated by hernia of some portion of the uveal coat. The lodgment of a foreign body in the eye is especially apt to give rise to it. When such injuries are attended by the 
entrance of a pyogenic micro-organism into the interior of the eye a purulent panophthalmitis is apt to supervene; but when this is not the case a less intense inflammation, of plastic character, usually results. The prognosis in these cases is distinctly unfavorable, and enucleation of the eye is often demanded to prevent sympathetic implication of the fellow-eye.

Miliary Choroido-retinitis.-As a result of accom-

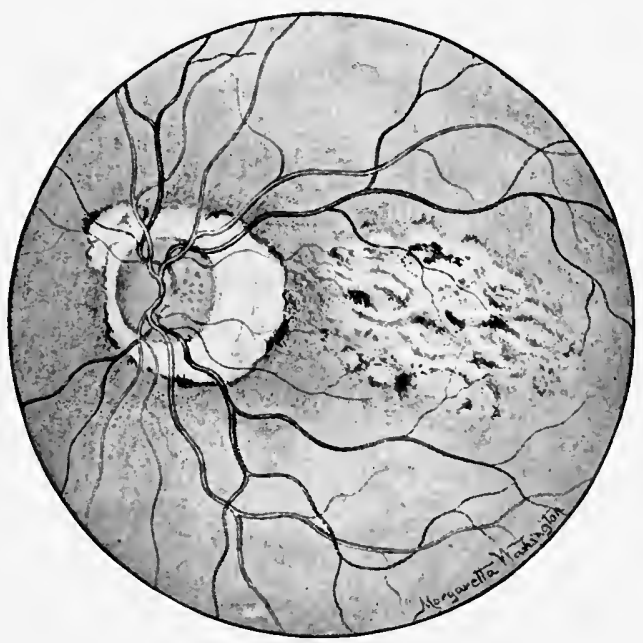

Fig. 132.-Eye-ground in progressive myopia. Large posterior staphyloma surrounding the nerve-head. Macular region occupied by an area of semi-atrophic choroido-retinitis (de Schweinitz).

modative strain, especially in astigmatic eyes which without glasses or with improperly adjusted glasses are much taxed in reading, writing, etc., a low grade of choroiditis, or rather choroido-retinitis, is of very common occurrence; and because of the frequency of its occurrence, and the serious consequences, near and remote, to which it gives rise, this affection deserves fuller consideration than is commonly accorded it in text- 
books upon eye diseases. In treating of the etiology of glaucoma and of cataract the significance of this condition has already been emphasized.

Unlike the severer types of choroiditis which have been described, this affection, in its early stages at all events, is not attended by marked impairment of sight. Instead, we have irritability of the eyes, photophobia, easily provoked lacrimation and conjunctival hyperemia, and, not infrequently, blepharitis marginalis and frontal headache-in a word, we have, in intensified form, the complex of symptoms which constitutes asthenopia. At first the ophthalmoscope shows a markedly hyperemic disc and, especially in the neighborhood of the disc and the macula, an undue retinal reflex, indicative of edema of the retina. After a time this retinal edema disappears, and, instead, we find, and again most marked in the region of the macula and between it and the disc, miliary changes in the choroido-retinal pigment-an appearance as though fine black pepper had been dusted over the background of the eye. (See Frontispiece.) In after years, when possibly incipient cataracts or the premonitory symptoms of glaucoma are manifesting themselves, there is observed a general thinning of the pigment of the choroid and retina, showing too plainly the choroidal vessels, with the islands of pigment between them--the so-called "patchy" choroid with which every ophthalmoscopist is familiar.

The frequency with which this state of the choroid, indicative, as I believe it is, of a precedent "miliary" choroido-retinitis, is found in cataractous and in glaucomatous eyes must have attracted the attention of every one who has carefully studied such eyes. To my mind it is clear that the connection between these conditions is not merely accidental. On the contrary, I 
am persuaded that a definite causative relation exists between the strain of accommodation, the miliary choroido-retinitis to which it gives rise, and the later occurrence of cataract or glaucoma, as the case may be. Other factors doubtless play a part, especially with reference to the supervention of glaucoma, but the long-continued accommodative strain, not infrequently, is the dominant one.

How many of these serious maladies of advanced life might be prevented by the early recognition and careful correction of the errors of refraction with which they are so often found associated it is difficult to say. - But I venture to predict that when the medical profession and the laity have learned how important a rôle these errors play in the causation of ocular diseases, and, furthermore, that their determination and correction,- so far from being a trivial matter to be left to the tyro who, calling himself an "examining optician," undertakes to "fit glasses,"--demand the best efforts of the physician trained in this department of medicine, there will be a very material reduction in the number of eyes requiring operation for glaucoma or for cataract, and probably, too, of eyes in which sight is irretrievably impaired through pathological changes in the retina or its bloodvessels.

Treatment.-In choroiditis of syphilitic origin local remedies are of but little value; but one should be on the lookout constantly for the supervention of iritis, and immediately upon the appearance of symptoms indicative of its occurrence, such as pericorneal injection, pain, photophobia, and lacrimation, should employ atropin freely. The constitutional remedies chiefly to be relied upon are mercury and potassium iodid, and these to be effectual must be given persistently and in liberal doses. 
The biniodid of mercury in doses of a sixteenth of a grain, or the protoiodid in one-fourth of a grain doses, should be given three times a day, and with either of these potassium iodid may often be combined advantageously. In addition, inunctions of mercurial ointment should be employed in severe or intractable cases. Heroic doses of potassium iodid not infrequently prove valuable. As a rule, in the cases due to inherited lues less energetic treatment is demanded, though here, too, it must be persisted in. One should not be disappointed if the response to the remedies employed is tardy, for this is what must be expected.

In the choroiditis of high myopia it is of the first importance that the eyes should be taxed but little in near vision, and that carefully adjusted glasses should be prescribed-glasses which, as a rule, leave a part of the myopia uncorrected, but which take into account any astigmatism or muscular fault that may be present. The long-continued use of the lotion of opium and boracic acid is also of undoubted value. It should be applied at bedtime on gauze or linen pads, and the pads, kept in place by a light bandage, should be allowed to remain in position until morning. It may seem that such a remedy, applied in this way, could hardly be efficacious; but it unquestionably lessens the irritability of the eyes, and probably reduces the hyperemia of the deeper ocular tunics. We know that belladonna used in a similar manner finds its way into the circulation of the eye, and produces its characteristic effect, and there is no reason why opium should not do the same.

In non-suppurative traumatic choroiditis sodium salicylate, in generous doses, is the most useful remedy that we possess. Mercury also is at times serviceable. Atropin and the lotion of opium and boracic 
acid are indicated when, as is often the case, pain and photophobia are present.

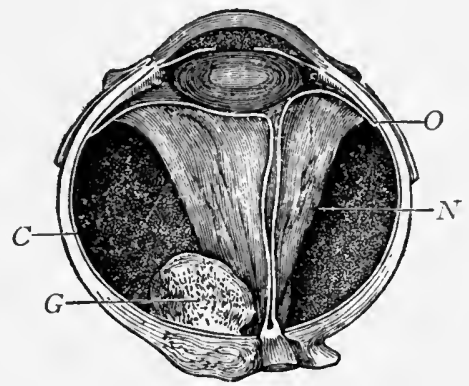

Fig. I33.- Sarcoma of the choroid with complete detachment of the retina (Leber). The tumor, $G$, rises from the choroid, $C$, which cverywhere lies in contact with the sclera. The retina, $N$, on the contrary, is detached entirely from its bed under the form of a folded funnel. It retains its connection only with the papilla behind, and with the choroid along the ora serrata, $O$, in front.

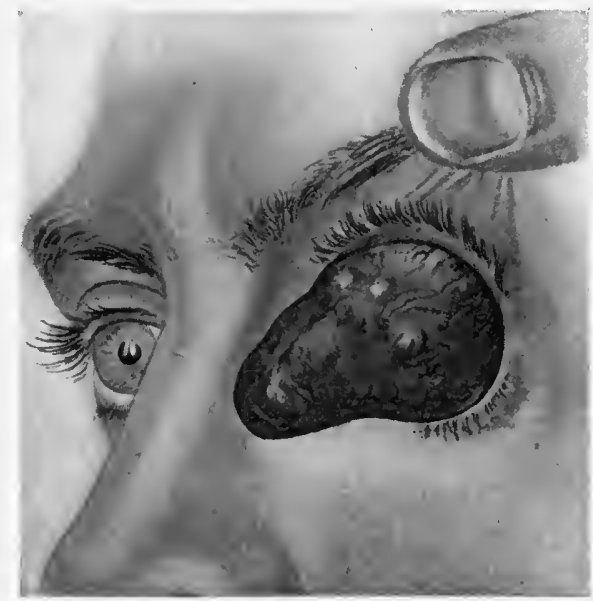

Fig. 134.-Sarcoma of the choroid, advanced stage (Haab).

As might be supposed, in dealing with miliary choroido-retinitis the most important indication is the careful correction of the refractive error or muscular fault 
which has brought it about. Exceptionally, prolonged rest of the eyes is called for; but usually the trouble itself and the symptoms which characterize it disappear promptly when proper glasses are prescribed. The lotion of opium, just spoken of, is very useful in this condition also, and the patient's general condition, especially the digestive apparatus and the state of the bowels, should be looked after.

$\mathcal{T}$ umors of the choroid are of infrequent occurrence, and are commonly of malignant type, sarcoma of the choroid being oftenest encountered (Figs. I33 and 134). As soon as their malignant nature is recognized, immediate enucleation of the eye is demanded; but even when this measure is resorted to at an early stage of their development there is no assurance that the disease will not manifest itself elsewhere.

Congenital anomalies of the choroid are comparatively rare, and need not engage our attention.

\section{DISEASES OF THE RETINA.}

RETINITIS.

Retinitis, or inflammation of the retina, occurs as a primary and as a secondary affection. When mild in type, and attended only by hyperemia and edema, it is known as serous retinitis; when more severe, and accompanied by hemorrhage and round-cell infiltration, it is denominated parenchymatous retinitis. Inflammation of the retina is not attended, as might be supposed, by photophobia, nor is it accompanied by pain. Impairment of vision is the chief subjective symptom; enlargement and sluggishness of the pupil are almost the only objective symptoms, apart from the evidence afforded by the ophthalmoscope. When the inflammation involves the region of the macula the disturbance 
of sight is pronounced; when it is limited to the outlying portions of the retina there is commonly only circumscription of the visual field, which may cause but little annoyance. Parenchymatous retinitis is usually bilateral, and commonly runs a tedious course, and, if unchecked, leads to atrophy of the nervous elements of the retina, with attendant hypertrophy of the supporting tissue, and obliteration of the blood-vessels.

The usual causes of primary retinitis are syphilis, nephritis, diabetes, splenic leucocythemia, pernicious anemia, gout, and angiosclerosis. It may also result from embolism or thrombosis of the central artery of the retina, or one of its chief branches, and from thrombosis of the central vein, while exceptionally it is produced by some direct exciting cause, such as undue exposure of the eye to the direct rays of the sun or to the electric arc light. Prolonged accommodative strain is another cause, though this commonly gives rise to a choroidoretinitis, of which we have already spoken.

Secondary retinitis is usually consequent upon inflammation of the uveal coat or of the optic nerve. It may occur also as a result of serious injury of other eye structures.

Retinitis Albuminurica (Nephritic Retinitis).Retinitis occurs in all forms of kidney disease attended by albuminuria, but especially in chronic interstitial nephritis. It is met with also in the albuminuria of pregnancy and in that of scarlatina. It is not infrequently a comparatively early symptom of nephritis, and is almost always bilateral. It is said that a majority of the patients suffering with kidney disease die within two years of the appearance of the retinal lesion, and that to live for five years is rare. As it commonly involves the region of the macula, it is usually 
attended by marked impairment of vision. The occurrence of a hemorrhage in this region may cause a sudden and pronounced impairment of sight. The severity of the eye lesion, it should be remarked, does not bear a constant relation to the severity of the kidney affection.

The retinal changes consist of degeneration of the walls of the blood-vessels, round-cell infiltration, numerous hemorrhages, and rapidly occurring fatty degener-

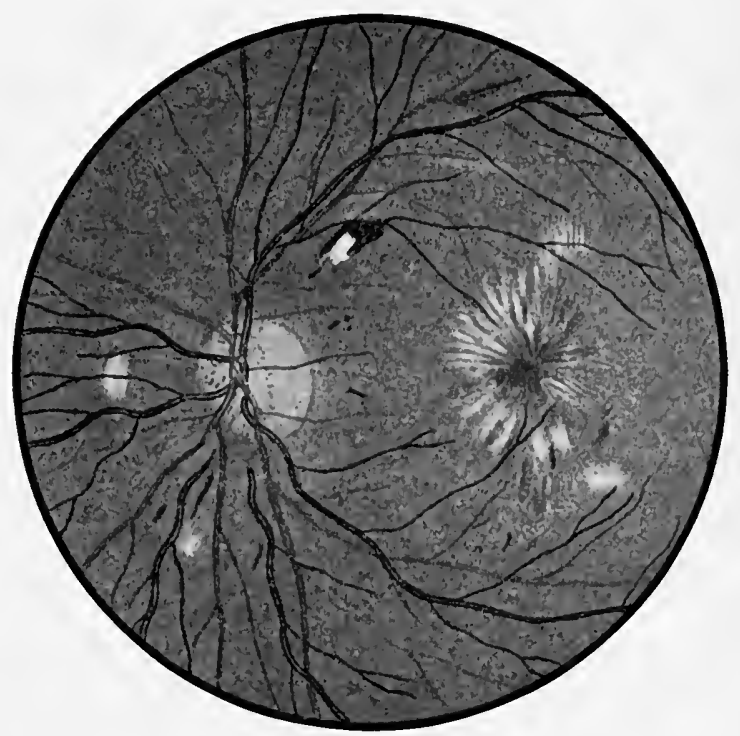

Fig. I35.-Albuminuric retinitis (Haab).

ation of the infiltrate and of the retina itself. The ophthalmoscopic picture presented by these changes is very characteristic (Fig. 135), and for this reason it often happens that an examination of the eyes reveals the true nature of the patient's malady before other suggestive symptoms have manifested themselves. The discovery of retinitis should always lead to a careful analysis of the urine. 
A sudden and pronounced impairment of vision, due to uremia, may occur in any of the varieties of albuminuria, but especially in the albuminuria of pregnancy and in that due to the eruptive fevers (uremic amblyopia). With improvement in the patient's general condition vision commonly returns to its former standard.

In the albuminuria of pregnancy and of scarlatina the prognosis as to vision is favorable; but, as might be supposed, the outlook is grave when this condition is due to organic disease of the kidneys. Even under such circumstances, however, I have occasionally seen the retinal lesion improve decidedly, and seemingly as a result of the treatment instituted. Fortunately, sight is seldom completely lost, for death usually occurs before this happens.

Treatment.-So far as the eyes themselves are concerned the treatment consists solely in care in their use,not necessarily absolute avoidance of reading, writing, etc., but very moderate indulgence in such occupations-and in the careful adjustment of glasses to correct any refractive or muscular fault that may be present. This latter measure is of much importance, and, by relieving the eyes of all strain, may do more than anything else to preserve the sight. Apart from these local measures, treatment is to be directed to the nephritis and to the patient's general condition. Tincture of iron, in liberal doses, is at times distinctly beneficial. Basham's mixture is also useful, and so, in some instances, is potassium iodid, administered in small doses.

Diabetic Retinitis.- This occurs as a late manifestation of the disease, and is always bilateral. It is not infrequently complicated by cataract and occasionally by plastic iritis or glaucoma. The fundus changes are not wholly unlike those observed in neph- 
ritic retinitis, but are less sure to involve the region of the macula. Hemorrhages into the retina are common, and extravasations of blood into the vitreous humor occur. As in other forms of retinitis, the extent to which sight is impaired depends in large measure upon the involvement or non-involvement of the macula in the inflammatory process. The prognosis, of course, is unfavorable, and depends upon whether or not the patient's general condition can be improved. An ophthalmoscopic examination is demanded whenever failure of sight occurs during the progress of a case of diabetes. It may reveal either retinitis or incipient cataract.

Treatment.-Besides moderation in the use of the eyes and the prescribing of suitable glasses, treatment consists chiefly in regulating the diet and habits of life of the patient.

Leucocythemic Retinitis.-This seldom occurs except in the splenic variety of the disease. The retinal changes consist of emigration of leucocytes and numerous hemorrhages. As seen with the ophthalmoscope, the light color of the background of the eye, with the distended and rose-red veins, the contracted and orangeyellow arteries, and the pale optic disc form a striking picture. Both eyes are involved, and the ocular lesion commonly increases with the progress of the general disease. The treatment is that of the systemic condition.

Retinitis of Pernicious Anemia.-This affection closely resembles leucocythemic retinitis, and the ophthalmoscopic changes are of much the same. character. One or both eyes may be involved, and after temporary improvement relapses frequently occur. The prognosis as to vision is distinctly unfavorable.

Treatment, as recommended by Osler, consists of rest 
in bed, with a light nutritious diet, massage, and increasing doses of arsenic.

Syphilitic Retinitis.-Syphilitic inflammation of the retina, without accompanying involvement of the choroid, is rare, but is described by most authors, and is said to occur in the inherited as well as in the acquired form of the disease. Exudations, especially along the retinal vessels, and vitreous opacities make their appearance, while hemorrhages are rare, and there is an absence of the pigment changes seen in syphilitic choroido-retinitis. One or both eyes may be involved, and the affection may develop within a few months of the initial lesion or at a much later period. The process is usually a chronic one, and not infrequently results in marked impairment of sight. If treatment is promptly instituted, however, the prognosis is not unfavorable.

Treatment.-Energetic antisyphilitic treatment is indicated. The biniodid of mercury, with or without potassium iodid, is especially useful, and in individuals showing an insusceptibility to mercury, its administration may be supplemented by inunctions of blue ointment.

Retinitis from Exposure of the Eyes to Intense Light.-Retinitis resulting from undue exposure of the eyes to the direct rays of the sunoftenest brought about by observing an eclipse of the sun without proper protection of the eyes-and to intense electric light, as in electric welding, usually manifests itself in the region of the macula, where at first edema, and later miliary pigment changes, are observable with the ophthalmoscope. The visual disturbance is characterized at the outset by a persistent after-image, and this is followed by a decided, usually small, central 
scotoma, which may be attended by subjective sensations of light. From the action of the electric light, in addition to the retinitis, considerable ciliary irritation and conjunctivitis sometimes result, an effect, it would seem, of the ultra-violet rays, comparable to that which is produced by the too-prolonged action of the " $\mathrm{X}$ " rays or by the emanations of radium.

The symptoms commonly disappear slowly, and the eyes ultimately resume their normal state; but exceptionally the outcome is not so favorable, and a more or less pronounced impairment of central vision remains.

Treatment consists in complete rest of the eyes, and in their protection from bright light by smoke-tinted glasses. The lotion of opium and boracic acid is indicated, and the occasional administration of a purgative may do good.

Retinitis. Pigmentosa (Pigmentary Degeneration of the Retina).-This singular and interesting affection, which invariably involves both eyes, makes its appearance in childhood, when it is not congenital, and progresses slowly with advancing age. Little is known as to its etiology except that, not infrequently, inheritance seems to be an important factor, and that it is met with sufficiently often in the offspring of consanguineous marriages to make the fact noteworthy. Probably the latter circumstance explains its frequent association with congenital anomalies, such as harelip, mental deficiency, deaf-mutism, etc. From five to ten per cent. of congenital deafmutes, it is said, are afflicted with this disease. Instances in which cases have occurred in several generations of the same family are not uncommon. For some reason, as yet not understood, females are less susceptible to the disease than males. 
Inherited syphilis is usually given as one of the causes of retinitis pigmentosa. My own experience is not in accord with this view, and I am disposed to believe it is the result of confounding syphilitic disseminated choroido-retinitis with true retinitis pigmentosa, which it sometimes closely resembles.

One of the earliest symptoms of the disease, and the most characteristic, is night-blindness; progressive, concentric contraction of the visual field is another prominent symptom. Ultimately there is marked failure of central vision, though complete blindness is rare, or, at all events, does not come on until quite late in life. Nystagmus is not infrequently present, especially in cases of congenital origin.

The failure of vision is due to a slowly progressive atrophy of the retina and optic nerve, attended by marked narrowing of the retinal vessels, and by the peculiar deposition of pigment from which the disease derives its name. The deposits of pigment, which often lie along the course of the vessels, are stellate in form, and resemble bone corpuscles as seen with a microscope of low power. At first these deposits are confined to the periphery of the fundus, and are detected, perhaps, with some difficulty with the ophthalmoscope; they slowly advance, however, toward the optic nerve and macula, and ultimately are scattered over the whole background of the eye. The ophthalmoscopic picture presented is a very characteristic one, and should not be mistaken for any other condition (Fig. I36).

The decline of vision, as has been said, is very gradual, but the prognosis as to the final outcome is most unfavorable, for treatment is of little or no avail. In every case of defective night vision, especially if of con- 
siderable duration and progressive in character, the existence of retinitis pigmentosa should be suspected, and a careful ophthalmoscopic examination, directed particularly to the periphery of the eye-grounds, should be made. One of the later complications of the disease, not infrequently observed, is the development of posterior polar cataract, which, of course, further markedly impairs vision.

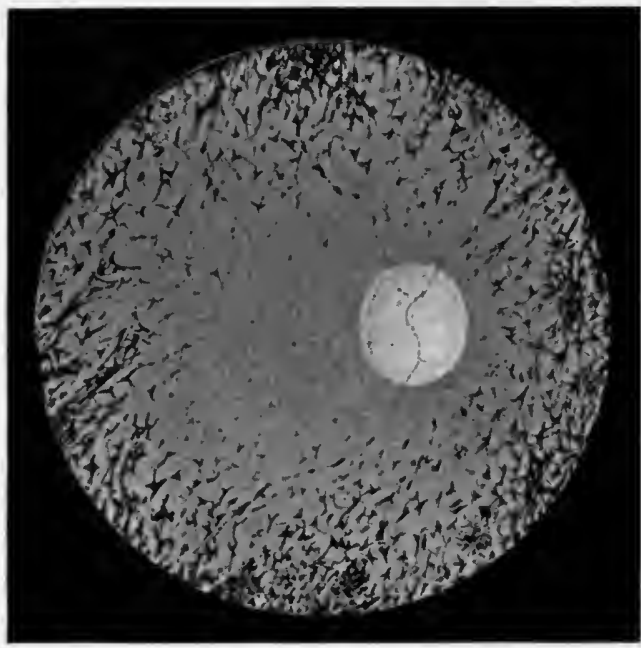

Fig. 136.-Advanced stage of retinitis pigmentosa. The optic nerve shows pronounced atrophy, and the retinal vessels have nearly disappeared (Jaeger).

Treatment.-As pronounced refractive errors are often associated with retinitis pigmentosa, they should be carefully searched for, and as carefully corrected by glasses if found to be present. Moderation in the use of the eyes should be enjoined and the avoidance of occupations which involve much reading, writing, sewing, and the like. Strychnin given from time to time, in moderate doses and for considerable periods, 
seems in some cases to be of benefit; galvanism also is recommended, and potassium iodid may be tried when other measures have failed.

\section{Embolism of the Central Artery of the Retina.} -The lodgment of an embolus in the central artery of the retina, which is usually a consequence of valvular disease of the heart or of aneurism, causes sudden and complete loss of sight of the affected eye. In rare instances the embolus becomes dislodged or breaks down, and vision is restored; but such improvement in sight as occurs occasionally from the establishment of a collateral blood-supply almost always proves to be but temporary. When the obstruction takes place in a branch of the artery central vision may remain unimpaired, and there may result only a more or less marked contraction of the visual field.

Pronounced edema of the retina develops within a few hours of the lodgment of the embolus, and this is accompanied by marked diminution in the size of the retinal arteries. Hemorrhages, especially in the macular region, may occur. Ultimately atrophy of the retina and optic nerve ensues.

The picture revealed by the ophthalmoscope is very characteristic and striking (Fig. I37). The edematous condition of the retina gives to the general eye-ground a whitish appearance; but as the edema does not involve the macula the red color of the choroid shows plainly at this point. The contrast in color is pronounced, and the condition is spoken of as "the cherry-red spot at the macula."

It is probable that a not inconsiderable number of the cases which in the past have been diagnosticated as "embolism" of the central retinal artery, but in which none of the cardiac or vascular conditions apt 
to give rise to the formation of an embolus was present, were really cases of rapidly forming thrombotic obstruction of the artery.

Treatment.-This has for its object the possible dislodgment of the embolus, so that it may find its way into one of the subdivisions of the artery, where the ill con-

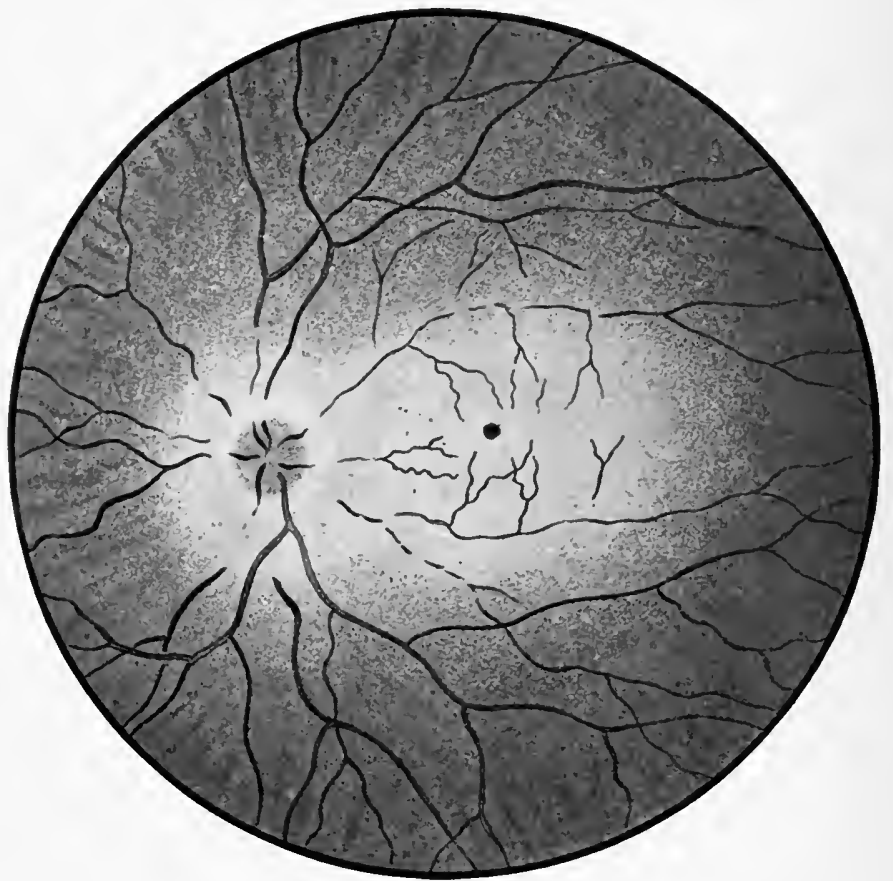

Fig. I37.-Embolism-or thrombosis-of the central artery of the retina (Haab).

sequences of its presence will be of less moment. With this end in view the eye should be rather energetically massaged, and nitrite of amyl should be administered by inhalation for the purpose of causing transient dilatation of the artery. These measures have in some instances proved effectual. 
Thrombosis of the central artery of the retina commonly results from degenerative changes in the arterial coats or from alterations in the composition of the blood. The symptoms which attend it are similar to those observed in embolism, except that the final loss of sight is often preceded by transient obscurations of vision and by attacks of giddiness, faintness, and headache. The ophthalmoscopic picture is essentially the same. (See Fig. I37.)

It seems probable that the cases of sudden loss of sight following severe hemorrhage, especially hemorrhage from the stomach, are due in most instances to thrombotic obstruction of the retinal artery, exceptionally, perhaps, to similar obstruction of the retinal vein.*

The treatment is the same as that employed in embolism.

Thrombosis of the central retinal vein occurs usually in elderly persons who are afflicted with organic disease of the heart or angiosclerosis; it may result also from alterations in the state of the blood, such as occur from excessive hemorrhage, or in consequence of orbital disease or disease of the cavernous sinus. Facial erysipelas, through extension to the orbit, may give rise to it. One or both eyes may be involved.

Loss of sight is not so sudden as in embolism of the artery, and the prognosis is somewhat less hopeless, though blindness from atrophy of the retina and optic nerve is the usual outcome. In some instances a glau-

* In the report of "A case of atrophy of the optic nerve following hemorrhage from the stomach, with a consideration of the causes of post-hemorrhagic blindness," published in the "Am. Journal of Ophthalmology" for May, 1899, and in the "Johns Hopkins Hospital Bulletin" of the same date, this view was advocated by the author, and evidence set forth in support of it. 
comatous condition supervenes, and gives rise to much suffering.

The ophthalmoscope shows edema of the retina with, perhaps, more definite areas of exudation, enormous distention and great tortuosity of the retinal veins, and numerous flame-shaped hemorrhages scattered over the entire fundus (Fig. I 38). "Hemorrhagic retinitis"

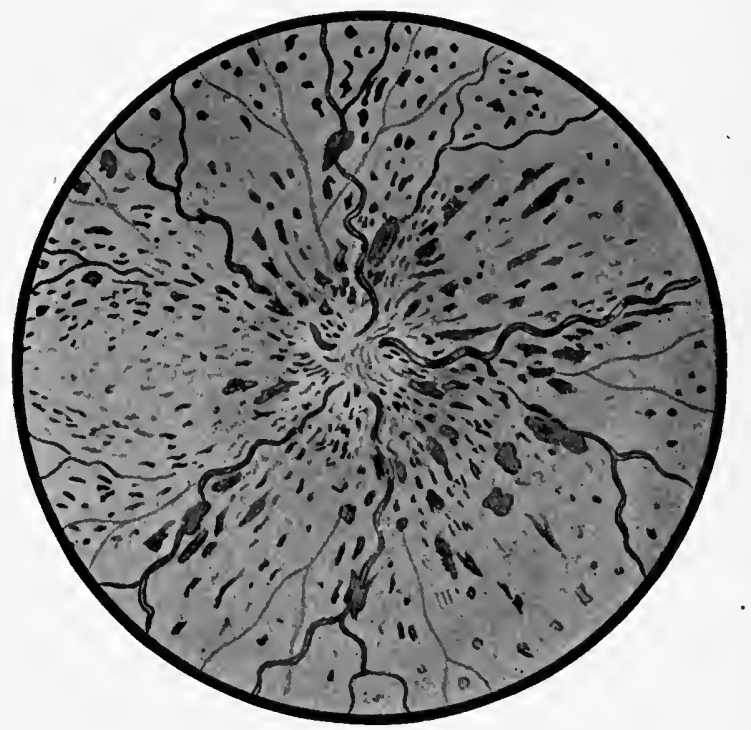

Fig. 138 . - Thrombosis of the central retinal vein (Haab).

was the name formerly given to this condition, before its essential nature was understood.

Treatment is of but little avail. An energetic cathartic, followed by potassium iodid, may possibly accomplish some good.

Detachment of the Retina.-In view of the fact that the retina is attached only about the optic nerve margin and at the ora serrata, and that elsewhere it is kept in apposition with the choroid simply through the 
support afforded by the vitreous humor, it is little matter for surprise that it should at times become "detached," or separated from the other coats of the eye. Indeed, the wonder is that this misadventure does not happen more frequently than it does; and I can not but feel that some of the elaborate and far-fetched theories which have been advanced to explain its occurrence are as uncalled for as they are unsatisfying. For example, one of these theories, which has been accepted by many, holds that bands which have formed in the vitreous humor, by contraction, drag upon the retina with such force as finally to tear a rent in it, and that through this rent fluid from the vitreous chamber passes, and causes the detachment. Why the retina does not yield directly to the traction, and what causes it, since it is not adherent to the choroid, to resist the drag upon it to the point of being torn, the proposers of the theory do not explain; nor do they tell us why the subretinal fluid might not be supplied by the vascular uveal coat quite as well as by the non-vascular vitreous body.

As a matter of fact, detachment of the retina, when it is not due to a serious traumatism, usually occurs in eyes which have been the seat of long-standing disease, especially disease of the uveal coat, myopia of high grade being the condition in which it of tenest takes place. It is also a common result of the development of intraocular tumors. That in these several conditions a predisposition exists to the occurrence of hemorrhagic or serous effusion from the very vascular choroid coat goes without saying, and it is doubtless in this way that detachment of the retina usually is brought about, the immediate cause of such an effusion being, very often, a violent spell of coughing or vomiting, a fall, a blow upon the head, or an unusual strain, as in lifting a heavy 
weight. In intraocular growths there is also, not infrequently, a mechanical elevation of the retina by the growth itself, and in high myopia the staphylomatous yielding of the sclera at the posterior pole of the eye is an additional factor tending to promote separation of the retina from the outer tunics.

The detachment may begin at any point, and may be partial or complete. When it commences in the upper part of the retina it usually extends downward, owing to the gravity of the subretinal fluid, and as this takes place the upper and first detached portion may resume its normal position with reference to the choroid, a striking illustration, it would seem, of the important rôle which the effusion plays in the process. Strictly speaking, the retina never becomes completely detached; for, though everywhere else separated, it always remains attached at the optic nerve entrance and at the ora serrata, presenting under these circumstances an appearance suggestive of a nearly closed umbrella (see Fig. 133).

Besides causing marked impairment of vision, the degree of impairment depending largely upon its location and extent, detachment of the retina is usually attended by subjective light sensations and by a "flickering" and confusion of sight that make an eye in which this condition is present a source of much greater annoyance to its unfortunate possessor than if it were quite blind. There are two ways in which detachment of the retina may impair vision. In the first place, the separated portion of the retina is itself incapable of useful vision; in the next place, it may hang or float in front of a part of the retina which is, perhaps, in a nearly or quite normal condition, the macular region, for example, and cut off the light from it. If the macular region is 
involved in the detachment or is covered by it in the manner just described, the sight of the eye will be of but little value. On the other hand, if the detachment is confined to the periphery of the retina, good central vision may be present, and there may be only a limitation of the visual field. A late complication, more apt to occur in myopic eyes, is the development of cataract.

When the symptoms described make their appearance, as they usually do, suddenly, and especially in an eye known to be decidedly myopic, retinal detachment should be suspected, and an ophthalmoscopic examination should be made. This view would be strengthened if the tension of the eye was found to be below normal; for this is usually the case in uncomplicated detachment of the retina. On the other hand, if increased tension of the globe is found in association with retinal detachment the presence of an intraocular growth is to be feared.

The prognosis in detachment of the retina is most unpromising. In rare instances a spontaneous rẹcovery takes place, and, almost as rarely, a recovery occurs seemingly as a result of treatment. Usually the detachment increases, and vision goes from bad to worse. Unless some inflammatory complication ensues pain is not experienced. The affection is commoner in males than in females, probably because they are more liable to such accidents as may bring it about, and occurs much oftener in advanced life than in youth.

Treatment.-This consists, at the outset, in rigid confinement to bed and in the administration of increasing doses of pilocarpin, so that marked salivation and sweating may be induced. Later potassium iodid may be substituted for the pilocarpin. Avoidance of constipation is indicated. Subconjunctival injections of 
sterile salt solution are worthy of trial, though the results obtained, as in all other methods of treatment, are usually disappointing. The injections should be repeated once in two or three days, the strength of the solution being increased gradually from two per cent. to five per cent. and the quantity injected from 15 to 25 minims. If improvement is manifested, the treatment should be persisted in for several weeks.

Numerous operative procedures have been suggested for the cure of this condition, when less radical measures have proved unavailing. The most rational of these, and the one as apt to prove. efficacious as any, consists in the drawing off of the subretinal fluid by means of an incision, made subconjunctivally, through the sclera at a point corresponding with the detachment. With careful antiseptic precautions this procedure is attended with little risk. Rest in a recumbent posture for some days after the operation should be enjoined. It is claimed, by Stillson, that more permanent results are obtained from perforating the sclera at one or two points beneath the detachment by means of the galvanocautery. I have had no experience with this method, but it impresses me as being a rather severe procedure.

Glioma of the Retina.-Although this very malignant intraocular tumor is certainly, and it may be added fortunately, not one of the commoner diseases of the eye, it has seemed to me best to treat of it, for the reason that the early recognition of its existence is of the first importance, and before such cases are brought to the attention of the specialist they are very apt to fall under the observation of the general practitioner. It is true that, in its early stages, a positive diagnosis can not be made without the aid of the ophthalmoscope; nevertheless a very shrewd guess as to what the trouble is 
may be hazarded as a result simply of a careful daylight inspection of the eye, aided by the employment of a mydriatic.

Glioma of the retina is essentially a disease of childhood, and generally makes its appearance during the first three years of life; indeed, it is not infrequently of congenital origin. In about one case in four both eyes are involved, and it occurs with equal frequency in males and in females. It has its starting-point commonly in the inner granular layer of the retina, and is of rapid growth. It shows a strong disposition to invade the optic nerve, and in time causes a rupture of

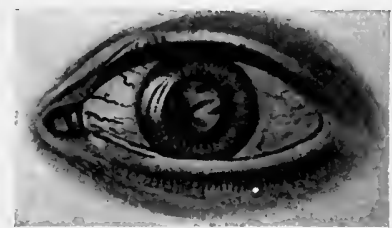

Fig. I39.-Glioma of the retina, early stage (Haab).

the eyeball, usually either in the neighborhood of the optic nerve or through the cornea.

In its early stages it is attended neither by pain nor by external signs of inflammation. The first evidences of its existence are a somewhat enlarged and sluggish pupil and a peculiar yellowish or grayish reflex from the pupil, with decided impairment of the sight of the affected eye, though the determination of this point is of ten difficult, because of the early age at which it commonly develops. This striking appearance of the pupil led the earlier authors to designate the condition "amaurotic cat's eye" (Fig. 139).

As the tumor increases in size there is usually an elevation of the intraocular tension, and when this is at 
all pronounced pain manifests itself. At this period several enlarged and tortuous subconjunctival vessels often make their appearance. Before long the shape of the eye undergoes alteration, the iris becomes muddy, the cornea cloudy, and there is great intensification of the pain; and presently rupture of the eyeball occurs. If this takes place posteriorly pronounced proptosis quickly develops; if anteriorly, a fungoid mass sprouts out rapidly from the place of rupture (Fig. I 40), and increases in size so fast that in a comparatively short time it may be as large, or nearly as large, as the child's head, and we

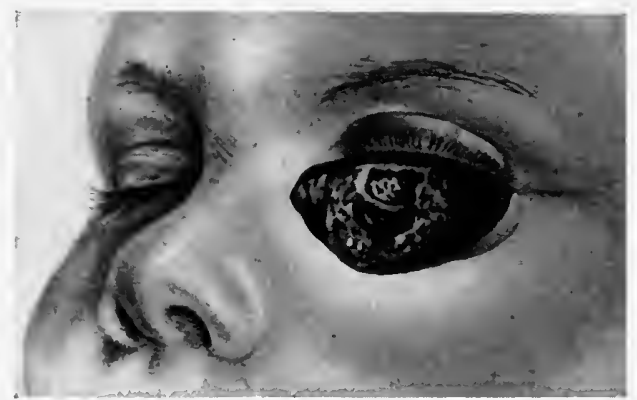

Fig. 140.-Glioma of the retina, more advanced stage (Haab).

have the condition which formerly was known as "fungus bematodes oculi." In the meantime, by extension along the optic nerve, the disease, perhaps, has reached the brain; or, less frequently, by metastasis, has invaded the liver or other distant organs, and death, not any too soon, comes to put an end to the sufferings of the wretched little patient. There are few tumors which are as malignant as glioma of the retina, and there are not many diseases in which the prognosis is so wholly unpromising.

Treatment consists in enucleation of the eye at the earliest moment possible after the establishment of the 
diagnosis, care being taken to divide the optic nerve as far behind the eyeball as practicable. If this is done before the growth has invaded the orbit or has extended to the optic nerve, there will, in all probability, be no local return of the disease, and, in very exceptional instances, no development of it elsewhere. Usually, however, the child dies within two or three years, with symptoms which indicate that the growth has recurred in the brain.

If the tumor has invaded the orbit not only the eye, but the whole contents of the orbit should be removed, although even when this is done in the most radical manner a local recurrence of the growth is extremely probable.

\section{DISEASES OF THE OPTIC NERVE.}

\section{OPTIC NEURITIS.}

Two principal varieties of inflammation of the optic nerve are recognized-a usually more intense form, which tends to involve the nerve throughout its whole course, is commonly consequent upon intracranial disease, is nearly always bilateral, and is attended by marked inflammatory changes in the papilla; and a less intense form, which is limited to the orbital portion of the nerve, has no relation to intracranial disease, is usually monolateral, and is accompanied by relatively slight intraocular changes. The last-named variety, known as retrobulbar, or orbital, neuritis, is a type of peripheral neuritis, and occurs as an acute and as a chronic affection. The first-mentioned variety, now commonly known as intraocular neuritis, papillitis, or choked disc, was formerly subdivided into descending neuritis and intraocular neuritis or cboked disc, and, though definite pathologico-anatomical evidence to 
support this classification is lacking, from a clinical standpoint there seem to be good reasons for adhering to it.

According to this view, a "descending" optic neuritis is an inflammation of the optic nerve in which the inflammatory process, as the name indicates, progresses along the optic nerve from the brain to the eye; while a "choked disc," or "intraocular neuritis," is one in

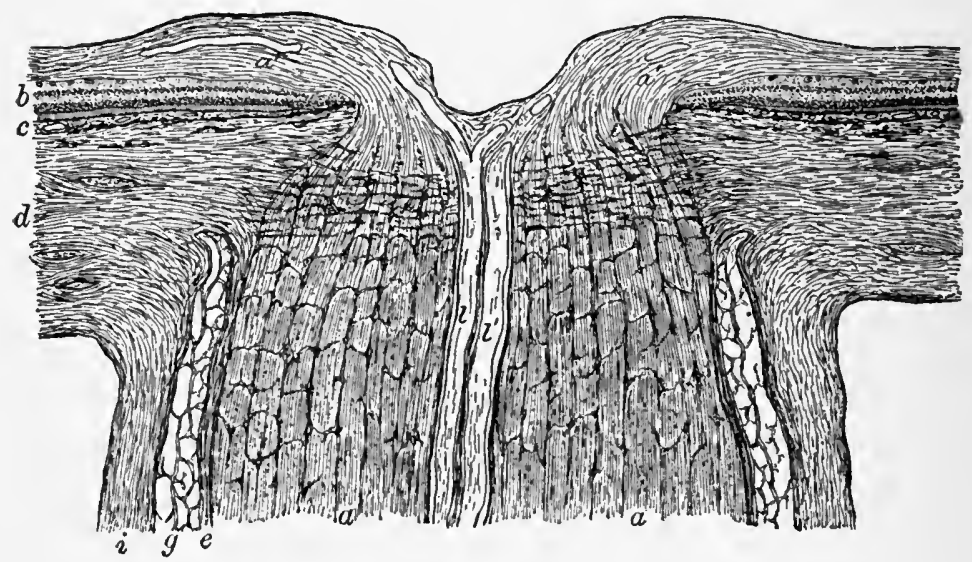

Fig. I41.-Longitudinal section of the optic nerve-head (Piersol): $a, a$, bundles of optic nerve fibers, which spread out over retina at $a^{\prime}, a^{\prime} ; b$, layers of retina; $c$, choroid; $d$, sclera, continued across optic nerve as the lamina cribrosa; $e, g, i$, respectively the pial, arachnoid, and dural sheaths of optic nerve, enclosing subdural and subarachnoidal lymph-spaces; $l, l^{\prime}$, retinal blood-vessels cut longitudinally.

which the inflammation, believed to be caused by obstruction of the blood- or lymph-currents of the optic nerve, or by the presence of toxins or other pathological products in the lymph-channels of the nerve (Fig. I4I), begins at or in the neighborhood of the papilla, and tends to extend thence toward the brain. Both of these types of optic neuritis are characterized by vascular congestion and swelling of the papilla and by engorgement and 
tortuosity of the central retinal veins; but this swelling and the engorgement of the retinal veins are much more marked in choked disc than in descending neuritis, and for this reason it is usually possible, with the ophthalmoscope, to differentiate the two forms (Fig. 142). In addition to the engorgement of the veins and the swelling of

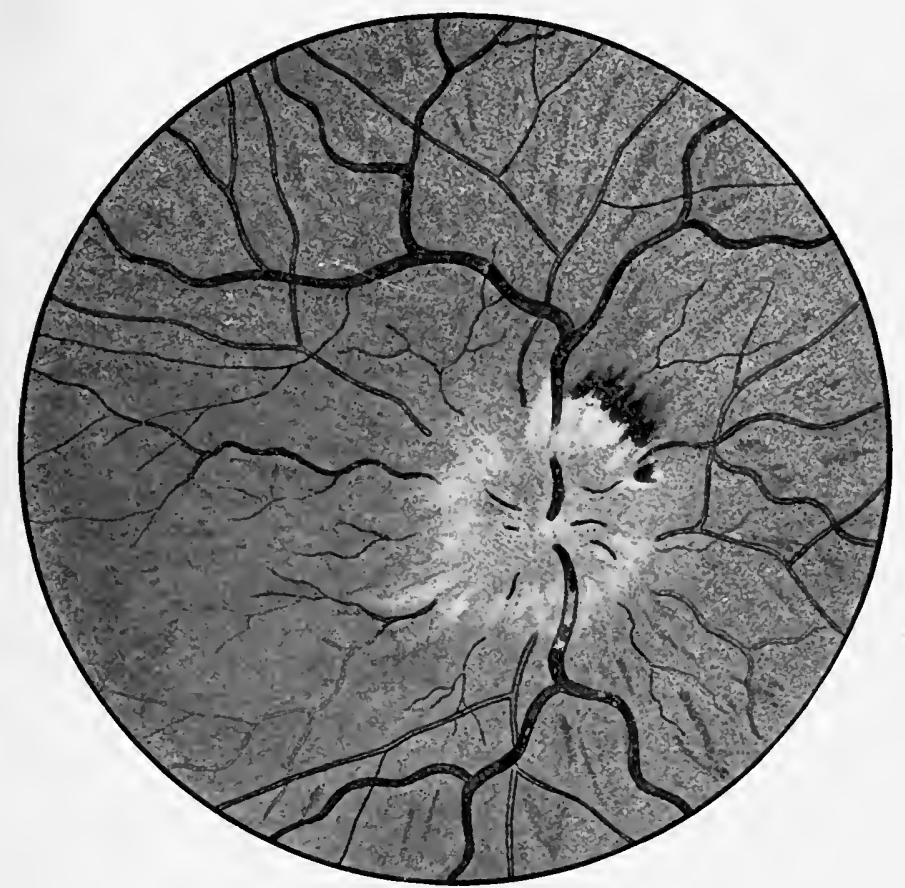

Fig. 142.-Choked disc, or papillitis (Haab).

the papilla, which latter is due to edema and inflammatory exudation as well as to hyperemia, and which, extending somewhat into the retina, obliterates the usually well-defined margin of the disc, both types are commonly attended by hemorrhages upon and around the papilla, and not infrequently by inflammatory changes in the neighboring portions of the retina. When these 
changes in the retina are pronounced the condition is called neuro-retinitis.

Choked Disc (Papillitis). - In this type of intraocu-

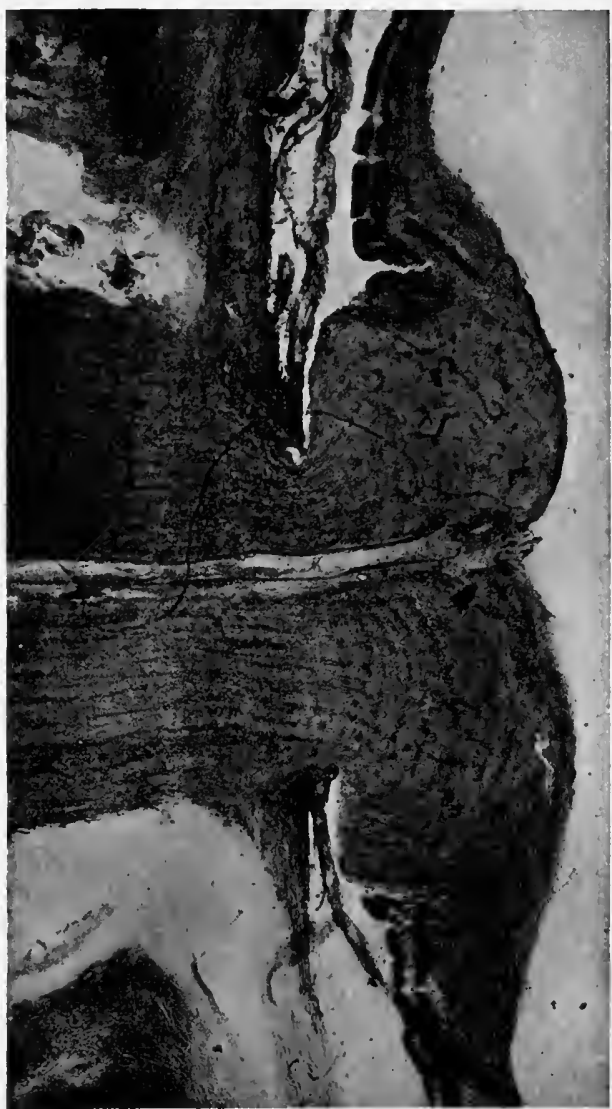

Fig. 143.-From a photo-micrograph by Dr. James Wallace of a section of a choked disc prepared by Dr. William Thomson.

lar neuritis, the supposed etiology of which has been referred to, and which, as has been said, is characterized by great swelling of the papilla and great engorgement of the retinal veins (Fig. I43), sight is often, and it may 
be for a long time, insignificantly impaired, the impairment being very much less than the pronounced fundus changes would lead one to expect. Not infrequently an enlargement of the normal "blind spot" is the only visual disturbance observed, until finally atrophy of the nerve-fibers ensues, when, if this is complete, sight is entirely lost. Owing to this circumstance and the further fact that, except perhaps for a slight dilatation and sluggishness of the pupil, the eye exhibits no external evidences of the serious malady with which it is afflicted, it often happens that the true condition is first discovered not by the oculist but by the physician concerned with affections of the brain and nervous system, whose advice is sought for the relief of headache or other symptoms pointing to intracranial disease. The process is a chronic one, often lasting for months, and, as has been intimated, it usually ends in atrophy of the nerve and loss of sight.

The commonest cause of choked disc is intracranial tumor, though almost any coarse disease of the brain may give rise to it. The character of the tumor, whether malignant or benign or of syphilitic origin, and its size and location seem to have but little influence in determining the result, so far as the involvement of the optic nerve is concerned. Optic neuritis, and usually of the choked disc type, is present in from eighty to ninety per cent. of intracranial new-growths. It is obvious, therefore, that its existence, while it throws no light upon the location of the growth, is of great diagnostic value in this class of cases, and that it should be sought for ophthalmoscopically whenever there is reason to suspect the presence of a brain tumor.

Other conditions which may give rise to choked disc are meningitis, acute and chronic,-in children tuber- 
culous meningitis especially, - abscess and softening of the brain, thrombosis of the sinuses, hydrocephalus, traumatic lesions of the head, the acute infectious diseases,' such as scarlet fever, smallpox, measles, diphtheria, influenza, pneumonia, and typhoid fever; disturbances of nutrition, such as occur in nephritis, diabetes, disorders of menstruation, pregnancy, and the puerperal state; syphilis, either directly or through the development of gummata, meningitis or periostitis; and, finally, affections of the orbit-new-growths, periostitis, etc. When arising from pathological changes in the orbit choked disc is usually monolateral, but under other circumstances it is almost always bilateral.

Descending optic neuritis, characterized, as has been said, by less marked swelling of the papilla, by decidedly less engorgement and tortuosity of the retinal veins, and, perhaps, by greater disposition to involvement of the retina, is distinctly a disease of intracranial origin. Its most common cause, probably, is basilar meningitis, especially that of tuberculous origin, which is usually accompanied by inflammation of the contiguous brain substance, though it may be produced by a tumor situated at the base of the brain, and which has given rise to a localized cerebritis. It commonly runs a less protracted course than the choked disc type of optic neuritis, and vision is sooner impaired.

As to the ultimate outcome of the two conditions -choked disc and descending optic neuritis-the prognosis is much the same. When either, as is so often the case, is dependent upon incurable intracranial disease, or upon such irremediable affections as chronic nephritis, diabetes, and the like, it is thoroughly bad; but, on the other hand, when due to affections that are themselves amenable to treatment, such as syphilis, 
the acute infectious diseases, rheumatism, etc., it is more' hopeful, and under such circumstances much may be accomplished by energetic and promptly employed remedial measures.

Treatment.-This, of course, depends in large measure upon the condition that has given rise to the neuritis. If it be consequent upon intracranial disease every effort should be made to combat this, and in exceptional instances radical operative procedures - the removal of a cerebral tumor, for example-may be demanded. Mercury, in liberal doses, and potassium iodid, heroically administered, are the constitutional remedies chiefly to be relied upon, not only because they may possibly exert a favorable influence upon the intracranial affection, but because of their direct effect upon the neuritis. They should be employed the more persistently whenever there is reason to believe that syphilis may be a factor, direct or indirect, in the causation of the neuritis; for under such circumstances much may be hoped for from their influence. Pilocarpin, so administered as to produce marked salivation and sweating, is another remedy which at times proves useful, while sodium salicylate in large doses may be beneficial, especially, but not solely, in cases of rheumatic origin.

The urine should be examined in every case of optic neuritis, as this may afford important information as to its etiology and as to the line of treatment to be employed. The bowels should be regulated, any tendency to constipation being carefully controlled, and from time to time an energetic purgative may be given with good effect. If the neuritis be dependent upon orbital disease this should receive appropriate treatment, operative or otherwise. From the application of remedies to the 
eye itself little benefit is likely to result; it should, however, be protected from undue exposure to bright light, and should be given as complete rest as possible. Prompt response to treatment is hardly to be expected, even in the more favorable cases.

Retrobulbar Neuritis (Orbital Neuritis).-Two types of retrobulbar neuritis are recognized, an acute and a chronic form. They resemble each other chiefly in that in each the inflammation affects the orbital portion of the nerve, and that in each the papillomacular fibers-those fibers which supply the macular region-are the ones first attacked. In each, too, if the process is not arrested, all of the nerve-bundles in time are involved, and ultimately undergo atrophy.

Acute Retrobulbar Neuritis.-There are a variety of causes which seem to be capable of giving rise to this affection The most common are syphilis, rheumatism, gout, the exanthemata, influenza, sudden suppression of the menstrual flow, chilling of the surface of the body from exposure to wet and cold, and the ingestion of considerable quantities of methylic or wood alcohol. It may also be secondary to other orbital disease. It is usually unilateral, and the symptoms which indicate its existence are rapid decline of central vision, moderate dilatation and sluggishness of the pupil in response to light, and not infrequently retrobulbar pain when the eye is turned in different directions or is pressed toward the apex of the orbit.

As the nerve fibers which supply that portion of the retina lying between the optic disc and the macula are those primarily affected, the visual disturbance manifests itself at first in the form of a paracentral scotomaa circumscribed area of impaired vision near to, and perhaps involving, the center of the visual field. At the 
outset this impairment of vision may be slight, amounting only to inability to distinguish colors, but it may increase rapidly, so that within this area objects can no longer be distinguished; and, if the inflammation extends from the papillo-macular fibers to the rest of the nerve, a general disturbance of vision will ensue, which may eventuate in total loss of sight.

The disease runs a much less protracted course than do the intraocular forms of neuritis, and the prognosis, as a rule, is less grave; still, the outcome is always uncertain, and permanent impairment of vision, especially a persistent central scotoma, may result.

Treatment.-Potassium iodid, alone or in combination with biniodid of mercury, sodium salicylate, especially when the affection is of rheumatic origin, and, at a later stage, strychnin, are the remedies of greatest value. Local treatment, except perhaps the abstraction of blood by leeches, or the application of a blister to the forehead or temple, is of little efficacy. Complete rest of the eyes and their protection from bright light should, of course, be enjoined.

Chronic Retrobulbar Neuritis (Toxic Amblyopia).-This disease, as the subtitle indicates, is caused by certain toxic agents, which, acting usually for a considerable time upon the system, eventually induce a chronic, peripheral, interstitial inflammation of the optic nerve. As in the acute form of the disease, the papillo-macular fibers are the first to suffer. (See Plate IX.) The affection is commonly bilateral, and its outcome, unless the inflammatory process can be arrested, is blindness from atrophy of the optic nerves.

The first complaint of the patient is of a "fogginess" of vision, as though he were seeing objects through a smoky atmosphere. A common accompanying symp- 
tom is recurrent frontal headache. A characteristic early symptom, to be determined by a perimetric examination, is a nearly central color scotoma-an area of red-green blindness extending from the fixation point to the normal blind spot. Any degree of impairment of central vision may be present, a visual acuteness of one-fourth to one-tenth normal being not unusual at the time medical advice is sought. The fact that the failure of sight is very gradual probably explains this singular circumstance.

Although in exceptional instances the disease under consideration is produced by such agents as nitrobenzol (used in the manufacture of certain explosives), bisulphid of carbon (employed in vulcanizing rubber), arsenic, lead, iodoform, etc., it is usually caused by tobacco or alcohol or, as is more of ten the case, by the combined action of the two. For this reason it is encountered much more frequently in males than in females. The habitual smoker and the habitual dramdrinker are the ones in whom it is most likely to develop. In some instances it has been observed in persons whose employment compelled them to inhale air laden with tobacco dust, as in tobacco manufacturing establishments. It is rarely met with in persons under thirty-five years of age.

Although the intraocular signs of chronic retrobulbar neuritis are not very marked or characteristic, the ophthalmoscope is helpful in arriving at a diagnosis. In the early, the inflammatory, stage of the disease there is usually marked hyperemia of the optic disc. Later on, when the stage of atrophy is reached, the disc becomes pale and perceptibly cupped, and the lamina cribrosa is seen with too great distinctness, these changes commonly being more marked in the lower 


\section{PLATE IX.}
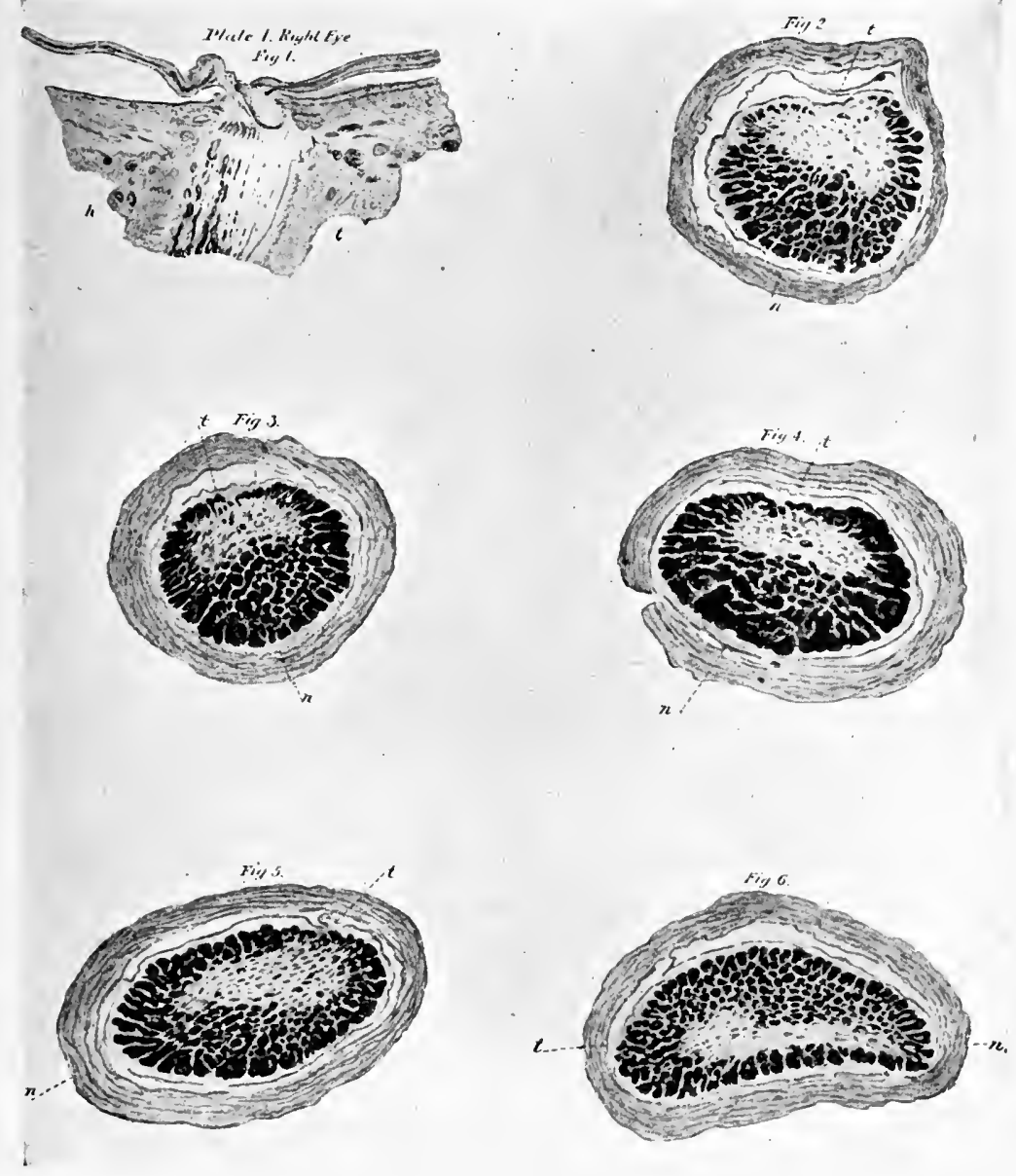

Sfctions of the Right Optic Nerve of Dr. G. E. De Schwfinitz's Case of Tobacco Aublyopia, Silowing Degfineration of tile Paplllomacular Bundle; Weigert's Stain.

Fig. I.-Longitudinal section of the posterior half of the right bulbus and five millimeters of the optic nerve.

Figs. 2 and 3.-Transverse sections of the optic nerve, eight and thirteen millimeters, respectively, behind the globe.

Figs. 4 and 5.- Transverse sections of the optic nerve in the region of the optic foramen.

Fig. 6.- Transverse section of the nerve in the intracranial region. 

temporal quadrant, which corresponds with the situation of the papillo-macular fibers.

The prognosis, especially in the cases which are due to tobacco and alcohol, is not unfavorable, even when vision is greatly impaired, if the atrophic process has not already progressed too far.

Treatment. - The most important feature of the treatment is the absolute withdrawal of the toxic agent which has provoked the neuritis. In the case of smokers and dram-drinkers total abstinence from the use of tobacco and alcohol must be insisted upon. In addition to this, and especially during the inflammatory stage, indicated by the hyperemic papilla, moderate doses $\left(\frac{1}{32}\right.$ to $\frac{1}{24}$ of a grain) of biniodid of mercury should be prescribed, and in combination with this strychnin should be given in ascending doses. There is no excuse, in my judgment, for administering the strychnin hypodermically, as some authorities advise. Beginning with a thirtieth of a grain, given three times a day, and directly after meals, the dose may be gradually increased, as tolerance is established, until finally it reaches five or six times this amount. It has not been my experience, in a single instance, to meet with the cumulative effect which some contend is liable to occur from the prolonged exhibition of this drug.

Quinin Blindness.-As a result of the administration of heroic doses of quinin, and in susceptible individuals of doses which could not be so regarded, pronounced impairment of vision, at times complete loss of sight, occasionally is produced. Investigation has shown that the visual disturbance, which is usually accompanied by deafness and marked tinnitus, is due to excessive spasm of the vessels of the retina and optic nerve. The resulting ischemia, if it persists for a con- 
siderable time, may give rise to degeneration of the ganglion cells and nerve-fibers of the retina and ultimately to an ascending atrophy of the optic nerve, as Ward Holden has pointed out. Fortunately, the outcome of these cases is usually not so serious, the blindness often proving to be transient, and normal vision being regained, it may be after a few hours, or, perhaps, not until the lapse of some days. While the blindness persists the pupils are widely dilated and non-responsive to light. Not infrequently the loss of sight is preceded by headache, unsteadiness of gait, and, in some instances, by visual hallucinations.

Treatment consists in inhalations of nitrite of amyl and in the administration of nitroglycerin, to be followed by ascending doses of strychnin. The giving of quinin should, of course, be stopped at once.

Atrophy of the Optic Nerve.-Two definite types of optic nerve atrophy are recognized-a primary, noninflammatory atrophy, which is usually consequent upon spinal disease, and an atrophy consecutive to optic neuritis or retinitis. With the ophthalmoscope it is usually possible to distinguish these two forms, for although in each the optic disc is pale, or conspicuously white if the atrophy is advanced, there are present in the post-neuritic type certain definite signs of the precedent inflammation which are wanting in the primary type. Clinically the two forms differ in that while the prognosis is grave in each, it is more entirely hopeless in primary than it is in consecutive atrophy. The progress of the former also is slower, and in it both eyes are almost always involved, whereas the latter is not infrequently unilateral.

Primary, simple, or non-inflammatory atrophy of the optic nerve is oftenest encountered in tabes 
dorsalis; it is not uncommon also in the general paralysis of the insane, and it may be due to syphilis, venereal excesses, diabetes, or the toxic action of certain drugs. There is also a hereditary form, which occurs almost exclusively in males, and there is still another form which is produced by compression of the nerveas by the growth of a tumor, periosteal thickening and the like-either within the cranium or within the orbit. As has been said, the affection is almost always bilateral, the cases in which only one eye is involved being usually those which are consequent upon orbital disease.

Among the earliest symptoms are inability to distinguish small objects - as, for example, the letters of a printed page - by subdued light, diminution or loss of color perception, and, as shown by the perimeter, concentric, or perhaps quite irregular, contraction of the visual fields. Slowly the decline of vision progresses, until ultimately, it may be not until after many months, sight is completely lost. The failure of vision is accompanied by expansion, exceptionally, in certain spinal affections, by contraction, of the pupil, and by its sluggish response, or complete lack of response, to light stimulation. Especially in cases of tabetic origin, the Argyll-Robertson symptom is observed-failure of the pupil to contract to light, but its prompt contraction when accommodation and convergence are called into play.

The ophthalmoscopic signs of simple atrophy - and in no case of suspected optic nerve atrophy should an ophthalmoscopic examination be omitted-are very striking. The papilla, at first only slightly pale, grows progressively whiter as the nerve-fibers atrophy and the fine blood-vessels, which give to the normal papilla its pinkish color, disappear. At the same time its outlines 
remain sharply defined, its surface becomes more or less markedly depressed, and the lamina cribrosa is seen with too great distinctness, and not over a limited area only, as when a deep physiological cup is present, but over the whole, or a considerable part, of the disc (Fig. 144). Accompanying these alterations in the nerve-head there is commonly some diminution in the caliber of the retinal arteries; but, apart from this, and

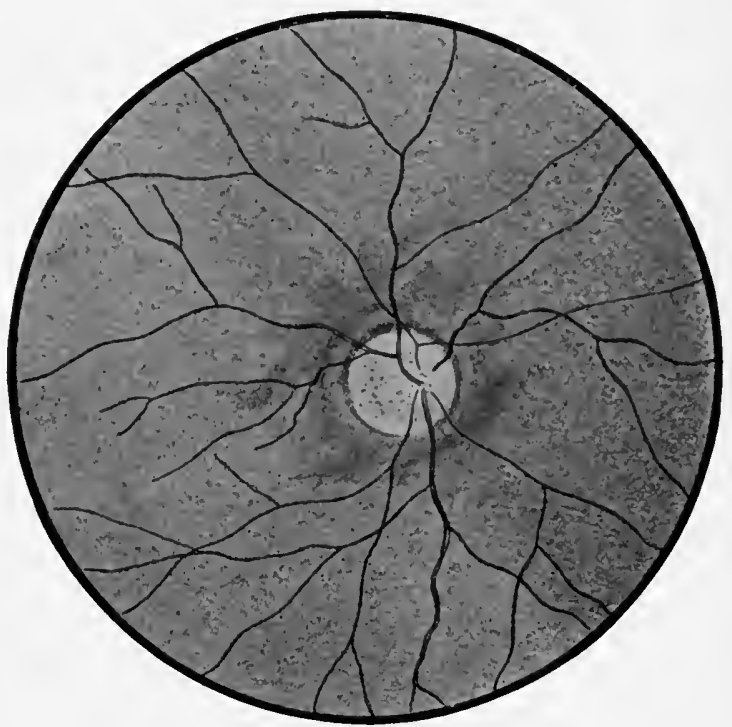

Fig. I44.-Primary atrophy of the optic nerve (Haab).

in striking contrast to what is observed in post-neuritic or post-retinitic atrophy, there are no other fundus changes-no thinning or heaping up of the retinal or choroidal pigment. In tabes, atrophy of the optic nerve is often an early symptom, making its appearance before the occurrence of the ataxia. It should, therefore, always be sought for whenever there is a suspicion of the existence of this affection. 
Treatment is of but little avail, as may be inferred from what has been said as to the irremediableness of the affection. Strychnin, in increasing doses, should be given a thorough trial, and if there is a history of lues potassium iodid should be prescribed in full doses. Galvanism and massage, which some commend, are of little or no value.

Consecutive Atrophy of the Optic Nerve (Inflammatory Atrophy).-This type of degeneration of the nerve may follow any one of the several forms of optic neuritis, or may be secondary to uncontrolled retinitis; it may also be consequent upon embolism or thrombosis of the central artery of the retina or thrombosis of the central vein. It is especially prone to occur after the so-called intraocular forms of neuritis, and is less common after retrobulbar neuritis.

The subjective symptoms are much the same as in primary atrophy, though the failure of sight is usually more rapid, and the affection is less constantly bilateral. The pupil is dilated and responds sluggishly, or not at all, to light. Apart from this, the eye exhibits no external signs of disease.

The diagnosis is to be definitely established only by an ophthalmoscopic examination. The fundus changes differ appreciably from those seen in simple atrophy. The papilla is white, but its margins are ill-defined, and, owing to the presence of newly formed connective tissue resulting from the precedent inflammation, it lacks the translucency observed in primary atrophy, and for this reason the lamina cribrosa is not seen. The indistinctness of the disc-margin is in part due to this same cause and in part to pigment changes in the adjacent retina. The retinal vessels upon and in the neighborhood of the papilla are frequently bordered 
by white streaks, due to thickening of their lymphsheaths. The arteries are diminished in size, the veins for a considerable time often distended and tortuous. When the atrophy is consequent upon retinitis, the pigment and other changes usually left by this condition are found disseminated over the eye-ground, and the retinal vessels, veins as well as arteries, are commonly much diminished in caliber.

The prognosis in consecutive atrophy depends in large measure upon the stage which the atrophic process has reached at the time the case comes under observation and upon the nature of the affection primarily responsible for it. If the inflammatory process is still active, and the nerve-fibers are suffering from the compression of exudates the absorption of which it may be possible to bring about, much may be hoped for from energetic treatment. On the other hand, if the case is not seen until a considerably later period, when the inflammation is a thing of the past, and the degeneration of the nerve is advanced, the outlook is wholly unpromising. It is most unpromising also when the primary affection of the nerve has been caused by serious intracranial disease or by such intractable maladies as chronic nephritis, diabetes, etc., or has followed degeneration or severe inflammation of the retina.

Treatment.-This must depend largely upon the conditions existing at the time the case is seen. Potassium iodid and mercury are the remedies to be relied upon to promote the absorption of exudates, and relieve the nerve fibers from compression, and therefore are of greatest value when given at an early stage of the affection. In addition, strychnin, in increasing doses, is indicated, at this stage as well as at a later period, when it is, in fact, our only reliance. 
Hemianopsia (Hemiopia).--Hemianopsia or halfblindness, a serious disturbance of vision, sudden in onset, involving both eyes, and of most unfavorable prognosis, finds its explanation in the semi-decussation of the optic nerves. When the nerve-fibers of each optic tract reach the chiasm, it will be remembered, they separate into two bundles - an outer bundle which proceeds to the eye of the same side, and an inner bundle which crosses in the chiasm and proceeds to the opposite eye. It will be remembered also that the non-decussating fibers supply the outer or temporal half of the retina of the eye to which they go, and that the decussating fibers supply the inner or nasal half. As a consequence of this, it is evident that the fibers coming from the right side of the brain, and forming the right optic tract, supply the right half of the retina of each eye, that is to say, the temporal half of the retina of the right eye and the nasal half of that of the left eye; while the conditions are reversed as to the fibers coming from the left side of the brain. From this it is further evident that a lesion, for example, of the right optic tract, or the visual centers of the right half of the brain with which it is in relation, will destroy the perceptive power of the right half of each retina, causing left-sided blindness or loss of the left half of the visual field of each eye; that a lesion which affects the decussating fibers only will cause blindness of theinner or nasal half of each retina, with loss of the outer or temporal visual field of each eye, and that one which involves only the non-decussating fibers will affect the outer half of each retina and result in blindness of both nasal fields. A further possibility is that the nervefibers supplying either the upper or the lower half of each retina may be destroyed, and, as a consequence, that 
the lower or upper half, as the case may be, of the visual field of each eye may be lost.

All of these forms of hemianopsia-which a study of the accompanying illustration (Fig. I45) will make more

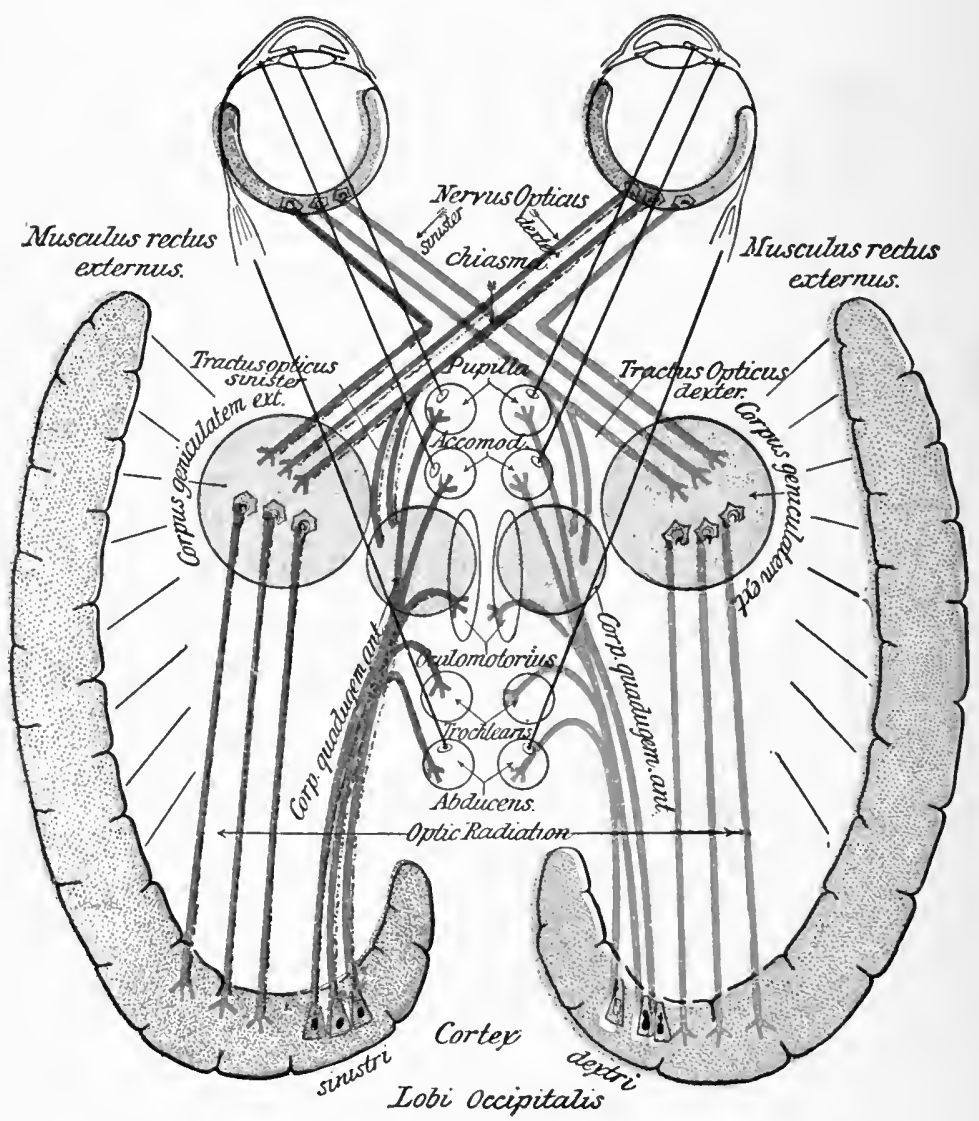

Fig. 145.-Scheme of the optic tracts (after von Monakow).

easy of comprehension-are met with; but the two lastdescribed, known respectively as binasal bemianopsia and borizontal bemianopsia, for obvious reasons-because a lesion which shall affect the non-decussating 
fibers at each extremity of the chiasm, without involving the decussating fibers lying between them, or one which shall destroy only the fibers lying close to the upper or lower surface of the chiasm, permitting the others to escape, must necessarily be of rare occurrence-are very uncommon. On the other hand, the two first-mentioned forms - that in which the right, or it may be the left, half of the visual field of each eye is lost, known as bomonymous lateral hemianopsia, and that in which both temporal fields are lost, known as bitemporal bemianopsia,--are more frequently encountered, and demand fuller consideration.

Homonymous Lateral Hemianopsia.-This variety of hemianopsia, in which there is loss of the right or left half of each visual field, is the one oftenest met with. It may be caused by a lesion of either cortical visual center or by one which produces a break in the visual tract anywhere between this center and the optic chiasm, that is, in the optic radiations, in the primary visual ganglia, or in the optic tract itself. Among the conditions apt to produce such a lesion may be mentioned hemorrhage, embolism, softening, abscess, intracranial syphilis, and the pressure of a tumor.

The visual disturbance, which may be preceded by headache and accompanied by giddiness and nausea, is commonly sudden in onset, and is at times attended by other evidences of serious brain injury, such as hemiplegia, aphasia, etc. The loss of vision in the affected half of the field is usually complete; but fortunately the macular region nearly always escapes serious involvement, and good central vision is retained. This is due to the fact-doubtless a result of the action of the law of the survival of the fittest - that this most essential and most highly specialized portion of the retina is 
supplied by fibers from both sides of the brain. Because of the one-sided character of the blindness, the patient frequently imagines that one eye only is affected, and is much surprised when made aware of the true condition. At first he experiences much inconvenience from the curtailment of his field of vision, but in time he becomes less conscious of the defect. As might be supposed, the affection is more common in advanced life, when the walls of the blood-vessels are undergoing degenerative changes. For some time after the onset of hemianopsia the ophthalmoscope shows no alteration in the papilla, but later evidences of partial atrophy may make their appearance.

Attacks of transient bemianopsia, lasting usually for but a few moments, and of very different import from the serious affection under consideration, are not uncommon. They are doubtless due to some temporary disturbance in the circulation of the visual centers, and, in my experience, are oftenest brought on by eye-strain from refractive or muscular faults.

Bitemporal Hemianopsia.-This form of half-vision, of much less frequent occurrence than the one just described, is produced by a lesion involving the central portion of the chiasm, and injuring the decussating fibers only. It may be the result of hemorrhage, aneurism, syphilis, of a tumor, or of a fracture of the base of the skull. It also occurs in acromegaly as a consequence of enlargement of the pituitary body. The disturbance of vision is very annoying because of the great circumscription of the lateral fields.

In bitemporal as well as in binasal hemianopsia the prognosis is somewhat more favorable than in the homonymous form. Homonymous hemianopsia has little or no localizing value, as is evident from what has been 
said as to the various conditions which may give rise to it; but the bitemporal and binasal forms have, since they indicate that the lesion is in the neighborhood of the chiasm. The importance in all cases of cerebral disease of measuring the visual fields, to determine whether or not hemianopsia is present, is manifest.

The treatment of the several varieties of hemianopsia is usually without avail. The prognosis, as a rule, is more promising in the cases of syphilitic origin. Potassium iodid and mercury are the remedial agents from which most is to be hoped. 


\section{CHAPTER XI.}

\section{ANOMALIES OF REFRACTION AND ACCOMMO- DATION.}

\section{ANOMALIES OF REFRACTION.}

It is not the purpose of this chapter to encourage the general practitioner to undertake the determination and correction of the refractive anomalies of the eye, or to teach him how to do this. On the contrary, one of its purposes, at least, is to impress upon him, what so many physicians fail to realize, that there is no branch of ophthalmic practice which more imperatively demands the skill and training of the specialist than does this matter of the measurement and correction of the refractive errors and muscular faults of the eyes. There are, indeed, few misconceptions which call for more vigorous combating than the belief, so common among medical practitioners who have paid but little attention to diseases of the eyes, that the adjustment of glasses is a trivial matter which may be undertaken by any physician who may have provided himself with test-cards and a trial case, or may be safely left to the hap-hazard methods of the nearest vender of spectacles, or to the jeweler's clerk who, after a few weeks of instruction in an "optical college," announces himself as an "examining," or an "ophthalmic," optician. How much harm the community suffers from the pretentious ignorance of such tyros it would be difficult to estimate. Let the general practitioner but bear in mind that the vender of spectacles-the optician-has a knowledge of the eye 
and its diseases about equivalent to that which the vender of drugs - the apothecary-has of general medicine, and he will not be so apt to fall into the error which I have tried to emphasize. Not only the welfare of the eye, it should be borne in mind, but in many instances the general well-being of the individual hinges upon the accuracy with which these optical and muscular faults are corrected.

How important a rôle errors of refraction play in the causation of many serious diseases of the eye few who have not made a special study of the subject realize. Frequent reference has already been made to this fact, and in treating of the etiology of cataract, of glaucoma, of choroido-retinitis, of chronic conjunctivitis, and of blepharitis marginalis much stress, it will be recalled, was laid upon the important influence exerted by accommodative strain in the production of these diseases. From my own observation, I am well satisfied that even such affections as albuminuric and diabetic retinitis, syphilitic choroido-retinitis, and the inflammatory processes of the eye dependent upon a rheumatic diathesis are, at least, unfavorably influenced, if not in some instances actually precipitated, by the strain caused by uncorrected refractive and muscular anomalies.* That not only strabismus but many less obvious disturbances in the ocular muscle-balance are largely dependent upon errors of refraction is, of course, well established.

As to the importance of the rôle played by eye-strain in the production of other disorders than those of the eye

* This statement may seem extravagant; but it will not be denied that in such disorders it is the tissues the resisting powers of which are poorest that are most apt to suffer. And, unquestionably, this is the condition of the internal tunics of an eye which, as a result of long-continued accommodative strain, are already in a state bordering upon inflammation. 
itself there can be no question; though it must be admitted that, as to this, there has been much exaggeration. The claim, seriously put forward, that sterility, menstrual disorders, habitual constipation, epilepsy, etc., are frequently due to refractive errors, and may be cured by their correction, is, of course, absurd; but, on the other hand, there can be no doubt that such errors

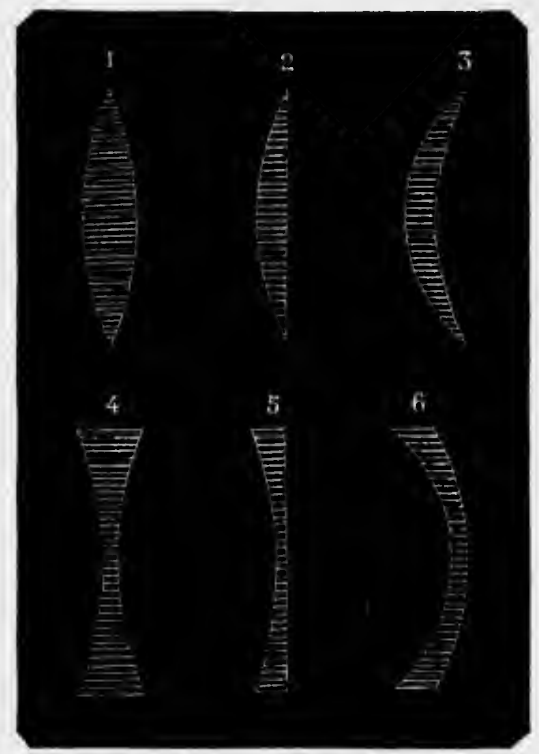

Fig. 146.-Types of spherical lenses prescribed in ametropia and presbyopia: I, Biconvex lens; 2, plano-convex lens; 3 , concavo-convex lens, convergent meniscus; 4, biconcave lens; 5 , plano-concave lens; 6 , convexoconcave lens, divergent meniscus (de Schweinitz).

are a common cause of headache, of neurasthenia, of vertigo, of insomnia, of somnolency, and of disturbance of mental concentration; that they are a less frequent cause of nausea, indigestion, tinnitus aurium, and chorea, especially of the facial muscles; and that, exceptionally, they exert a by no means unimportant influence upon epilepsy. 
Before describing the refractive errors, it will not be amiss to speak of some of the prevalent misconceptions regarding these errors and concerning the indications for glasses and their influence upon sight (Fig. I46).

In the first place, it is a mistake to suppose that errors of refraction-which are really faults in the conformation of the eye-may be "outgrown," or may be gotten rid of by any therapeutic procedure, such as prolonged rest, systematic exercise of the eyes, massage, etc., and, as a corollary of this, that the wearing of glasses should be regarded as a dernier ressort.

In the next place, it is as great a mistake to imagine that glasses are injurious to the eyes, that they "weaken the sight," as the common expression is, and that if their use is begun in early life a time may come when it will not be possible to find lenses that will afford the needed help. If glasses really are needed, the eyes soon learn to accept the help which they give, and, of necessity, become dependent upon this help; but so far from injuring the eyes or "weakening the sight," the effect, of course, is exactly the reverse. Furthermore, though glasses of considerable strength be prescribed, as is often necessary, for children not yet in their "teens," there need be no fear, though they live to be octogenarians, that the required lenses can not be easily supplied. In a word, the popular prejudice against the wearing of glasses, and especially against their use by young persons, is entirely without warrant. Unquestionably, from a cosmetic point of view objection may be urged against their use; but this, of necessity, must give way to considerations of greater weight.

Again, it should be remembered that glasses are not given solely with a view to making vision more acute. As a matter of fact, in many instances, especially in 
early life, there may be urgent need for them, and yet normal acuteness of vision, for both near and distant objects, may exist. Under such circumstances, and this applies, of course, to many cases in which, besides, they do render vision more acute, they are demanded for the purpose of relieving the strain which the optical error imposes upon the accommodative apparatus. Indeed, the very fact that young eyes, in spite of the existence of marked refractive errors, often do see sharply is evidence of the great tension under which they perform their office. It may be well to add that though glasses unquestionably tend to prevent the increase of certain refractive errors-myopia especially-it is not to be expected that they will cure the optical defect, for which they are prescribed. For this reason, the hope can seldom be held out that after a time the eyes will not need their assistance. In exceptional instances this may happen, as, for example, in low grades of hypermetropia or astigmatism, when the asthenopic symptoms have manifested themselves for the first time after an illness which has lowered the general tone of the system; but such instances, it is well to remember, are distinctly exceptional:

Finally, it should be stated that not every eye needing the help of glasses makes a direct appeal for their aid. Not infrequently, when the remote consequences of eyestrain are pronounced, there may be little or no complaint of the eyes themselves. Therefore one should not be too ready to conclude that persistent headache, obscure neurasthenic symptoms, etc., can not be due to eye-strain, because there is little or no asthenopia. In illustration of this, a case may be mentioned which came under my observation not long since. A young woman, who was unconscious of anything being amiss 
with her eyes, frequently awakened in the middle of the night with attacks of pronounced vertigo. Her physician, not being able to discover a cause for these attacks or a means of relieving them, thought of the possibility of their being dependent upon eye-strain, and sent her to me for advice. A moderate amount of astigmatism was discovered, and correcting glasses were prescribed, with some misgivings as to the result; but after they had been worn a very short time the vertiginous attacks ceased.

Emmetropia.-In order to a proper understanding of the refractive errors of the eye one should have a clear conception of what constitutes an optically normal, or,

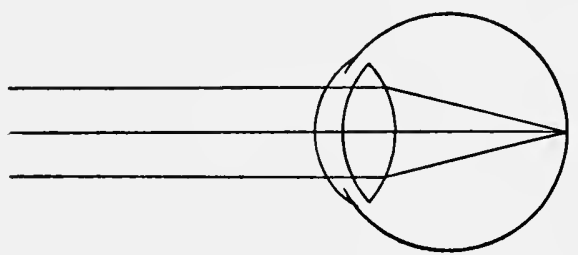

Fig. I47.-Diagram of emmetropic eye.

as it is called, an emmetropic eye. An eye is said to be emmetropic when it is so constructed, when the focal length of its system of lenses with reference to the distance between the cornea and the retina is such, that, with the accommodative apparatus at rest, parallel rays of light, those coming from distant objects, are brought to a focus exactly upon the retina (Fig. I47). In such an eye, it will be seen, accommodative effort is required only when near objects, the light from which reaches the eye in divergent pencils, are regarded, since sharp images of distant objects are projected upon the retina without action upon the part of the ciliary muscle. An emmetropic eye, it should be added, is not necessarily a 
sharp-seeing eye; indeed, it may be blind, for example, from atrophy of the optic nerve; but, however this may be, it is at all events one that is normal from the optical point of view.

Ametropia.-This term, which is opposed to emmetropia, denotes a departure from the normal in the optical construction of the eye. If from any fault in the conformation of the eye sharp images of distant objects are not formed upon the retina, or are so formed only by an effort of accommodation, such an eye is ametropic. The term is a general one, and under it are included all the optical errors, all the refractive faults, of the eye. If, for example, the lens system has an abnormally short focal length, while the eyeball is of normal dimensions, or if the focal length of the lens system is normal, while the eyeball is elongated in its antero-posterior diameter, that form of ametropia which is known as myopia, or short-sightedness, results. If, on the contrary, the focal length of the lens system is longer than it should be, without a corresponding elongation of the eyeball, or if the eye is preternaturally flat, without a compensating increase of its refractive power, there will result the type of ametropia known as hypermetropia, or far-sightedness. If, again, there is a lack of symmetry in the curvature of the cornea or the lens, or if the latter sets obliquely with reference to the visual axis, so that the rays of light entering the eye are not focused accurately at any point, we shall have still another form of ametropia, which we call astigmatism.

Each of these refractive faults, which are of common occurrence, and which in certain important respects differ radically in their essential nature, in their mode of origin, and in their clinical course, has its characteristic shortcomings, which will be considered presently. 
Hypermetropia (Far-sightedness). - This type of refractive error, which very generally is of congenital origin, is the most prevalent of all the optical defects of the eye. Indeed, it is so prevalent that it is a question whether the normal eye is not one that is slightly hypermetropic - that is, when its accommodation is paralyzed by a cycloplegic-rather than exactly emmetropic. It goes without saying, therefore, that the very low grades of hypermetropia are, as a rule, of little moment, and seldom require correction.

The fault of the hypermetropic eye is that it is incapable of focusing upon the retina even parallel rays of light, without an effort of accommodation. The outcome of this is that so long as the eyes are in use the ciliary muscle is never at rest. But this is not the whole task imposed upon the hypermetropic eye. When it is employed in near vision, its accommodative apparatus must do double duty; its ciliary muscle must do what is required of the ciliary muscle of the emmetropic eye plus the effort which it necessarily puts forth to secure clear distant vision. Whether, under such circumstances, the muscle must perform only considerably more work than is required of it under normal conditions, or twice or three times as much, depends upon the grade of the hypermetropia. Indeed, if the refractive fault is excessive, and the same is true with lower degrees of hypermetropia when the power of accommodation has been partly lost through advancing age, it may prove unequal to the task imposed upon it, and as a result there will be indistinctness of vision for both far and near objects. Such a condition is known as absolute or non-facultative bypermetropia, while that in which clear vision is obtained through the action of the ciliary muscle is denominated facultative bypermetropia. 
It is this ever-present strain upon the accommodative apparatus, greatly increased when the eyes are employed in near vision, that gives rise to the asthenopia, headache, blepharitis marginalis, and the intermittent indistinctness of vision which are so frequently observed in hypermetropia of considerable degree. And it is by relieving this strain, by doing for the eyes what they strive to do for themselves at such cost, that glasses afford in this condition the complete relief they invariably do.

But there is still another difficulty with which the hypermetrope has to contend. In emmetropia there is a constant harmony between the effort of accommodation and the effort of convergence of the visual axes, between the stimulus to action sent by the brain to the ciliary muscles and to the internal recti muscles. In distant vision both sets of muscles are completely relaxed; in near vision both act in unison, a convergence for twelve inches, as in reading, for example, being accompanied by a corresponding accommodative effort. In hypermetropia this harmony is radically disturbedaccommodation is always and necessarily in excess of convergence. In regarding distant objects, if clear vision is to be had, the ciliary muscles, as has been explained, must act, while, if binocular vision is to be maintained, the internal recti muscle must remain quiescent. In near vision the difficulty is the same, the ciliary muscles must put forth a much greater effort than is required of the interni. In a word, the effort to annul the normal relation between accommodation and convergence, to secure sharp vision and the same time to maintain binocular fixation, is one of the hypermetrope's most trying tasks.

It is matter for little surprise, then, that the hy- 
permetrope should elect, not infrequently, to rid himself of this task by a sacrifice of binocular vision; that, in order to restore the harmony between accommodation and convergence, he should permit one eye to squint. In his disposition to do this is to be found the explanation of the occurrence of convergent strabismus in hypermetropia. As, however, many hypermetropes do not squint, it is manifest that there are other factors which influence the development of this fault. Among those tending to favor its occurrence may be mentioned insufficiency of the external recti muscles and subnormal accommodative power; while it is manifest that insufficiency of the internal recti muscles and a power of accommodation above the normal must have the contrary effect. Still, beyond question, by far the most potent factor in the causation of non-paralytic convergent squint, as Donders taught us, is hypermetropia, and for the reasons just set forth.

It has been stated already that hypermetropia is nearly always a congenital anomaly, and that it consists either in an abnormal flatness of the eyeball or in a lack of refractive power in the lens system of the eye. The first-mentioned type, in which the antero-posterior axis of the eye is shorter than it should be, is the more common form, and is known as axial bypermetropia (Fig. 148). The type in which there is a lack of power in the refractive media, the cornea or lens, or both, being less convex than normal, is known as curvature bypermetropia, and is not only less common but is less uniformly congenital in origin, since it may be acquired through flattening of the cornea consequent upon inflammation, especially inflammation attended by loss of tissue.

Congenital hypermetropia, it should be clearly understood, is not a pathological condition; it is simply a 
fault, often an inherited fault, in the conformation of the eye. It is also a fault which does not increase in degree; indeed, in early life, it of ten diminishes appreciably, but if it be considerable in amount its disappearance is not to be looked for.

The ill consequences of hypermetropia, if the defect is of high grade, manifest themselves very early in life, as soon, indeed, as the child begins to use the eyes in regarding small objects, as in looking at picture books or in learning to read. There is usually no complaint of the eyes, but it is seen that the child has difficulty in dis-

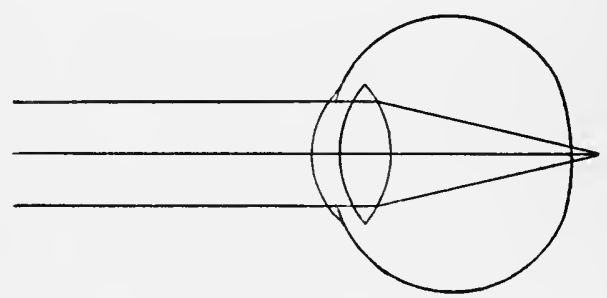

Fig. 148.-Diagram of axial hypermetropia.

tinguishing small letters, and it is at this time that the squint, if it is to occur, is apt to develop.

If the hypermetropia be non-facultative, if it be of so high a degree that sharp vision can not be maintained even by the greatest effort which the accommodation can put forth, the condition frequently simulates, and is mistaken for, myopia of high grade. The child holds the printed page very close to the eyes, since in this way a considerably larger retinal image is obtained, which more than offsets the increased blurring. If the defect be less pronounced, it may give rise to no inconvenience until the child is old enough to spend more time in study, when the appearance of asthenopia, headache, and, not improbably blepharitis, may be looked for. 
If of still lower grade, its existence may be unsuspected until, with the failure of accommodation consequent upon increasing years, it manifests itself through uncomfortable and defective near vision and through the need, at an exceptionally early age, of presbyopic glasses.

Treatment.-It is evident, from what has already been said, that the whole treatment of hypermetropia is comprised in the careful adjustment of glasses. With suitable glasses the hypermetropic eye is relieved of all strain, and is capable of doing the work which the nor-

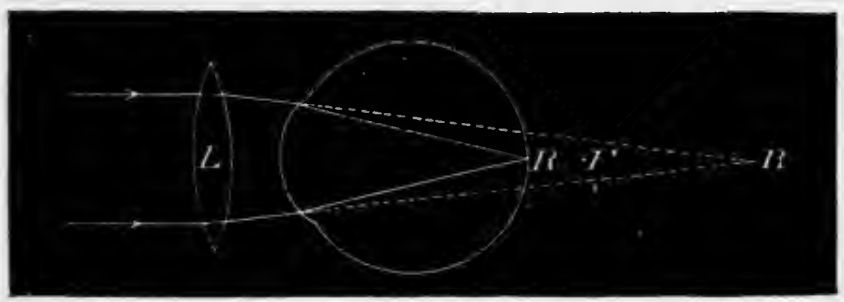

Fig. I49.-Correction of hypermetropia by a convex glass. The lens $L$ gives to parallel rays a convergence toward the point $R^{\prime}$; they will consequently be united upon the retina $R$ without an effort of accommodation (de Schweinitz).

mal eye does without assistance (Fig. I49). There need be no fear of ill consequences, therefore, in permitting the young hypermetrope, with his refractive error corrected, to pursue his studies as others do, or to adopt any vocation in life that may be thought desirable.

Whether he should be required to wear the glasses constantly, in far as well as in near vision, will depend largely upon the degree of the refractive fault, but in no small measure, also, upon his power of accommodation and upon the relative strength of the external and inter- 
nal recti muscles. Theoretically, it would appear that the glasses should be worn constantly; but, practically, if the fault is not too pronounced, complete relief is often obtained by their use in near vision only. It follows that in determining the course to be pursued the muscle balance for both far and near vision should be tested with the same care as the refractive error. The same considerations should influence one also in deciding upon the strength of the glasses to be prescribed in any particular case. If the accommodation is active, and if the external recti muscles are relatively strong as compared with the internal recti, a very considerable part of the hypermetropia may be left uncorrected; while, on the other hand, if the power of accommodation is poor and the external recti muscles relatively weak, glasses which give full or nearly full correction may be called for to insure complete relief.

The practice followed, especially in this country, by many distinguished oculists of prescribing glasses which correct the total refractive error as revealed by a cycloplegic, without regard to the muscle balance, or, in other words, without reference to the ability of the eyes to help themselves, is, in my judgment, unwarrantable, since it not only fails to take account of the fact that a low degree of hypermetropia is hardly to be regarded as an abnormal condition, but, by giving the eyes more help than is called for, it disturbs too radically the established relation between accommodation and convergence, and thus substitutes for a difficulty of one sort a difficulty of another sort, which is nearly as intolerable. Moreover, it is objectionable because it renders the eyes more absolutely helpless, more completely dependent upon glasses, than is necessary.

The older practice of correcting only that part of 
the hypermetropia which is manifest, ${ }^{*}$ that is to say, the part which can be made evident without the employment of a cycloplegic, provided this affords a normal muscle-balance in both distant and near vision, I can not but regard as a much more rational procedure. Not to be misunderstood, it may be well to add that it is to the considerably greater amount of hypermetropia that can be rendered manifest by painstaking binocular testing, and not to the less amount discoverable when the eyes are tested separately, that reference is had in this statement. Before resort to the binocular test the manifest fault of each eye should, of course, be carefully determined.

With the gradual failure of accommodation that comes with increasing age the ill consequences of uncorrected hypermetropia become more and more pronounced. Thus it happens that a hypermetropia which has caused no inconvenience in childhood may become very troublesome with the passing of youth; for it must be remembered that the power of accommodation begins to decline very early in life, and that even a child ten or fifteen years of age has already lost a part of the ability to focus the eyes which it possessed when it was several years younger. From this it follows, other things being equal, that the need for glasses increases with the age of the hypermetrope, and that as he grows older he will not only require a fuller correction, but will more surely need to wear glasses in far as well as in near vision. This increasing dependence upon glasses need give rise to no anxiety for the future, however; for, with suitable lenses, his eyes will continue to

* The manifest hypermetropia is indicated by the strongest convex glass with which sharp distant vision can be maintained, without, of course, a cycloplegic being employed. 
serve him as well as though they had from the first been free from refractive fault. If, however, his error of refraction has not been recognized, or if, having been advised to wear glasses, as not infrequently happens, he has disregarded the advice, it will be matter for little surprise if the years of strain to which his eyes have been subjected should sooner or later give rise to serious consequences, such as choroido-retinitis or, possibly, glaucoma or cataract.

From what has been said as to the manner in which convergent squint arises in hypermetropia, it is evident that we have in glasses a very efficient means of preventing this deformity, and, in some instances, even of correcting it after it has become established. Indeed, it is safe to say that it might be prevented in almost every instance, if the refractive error could be corrected soon enough. The practical difficulty in the way of doing this is the very early age-when the wearing of glasses is almost out of the question-at which the squint commonly develops. Still, it is surprising how little difficulty is experienced in inducing a child that is decidedly hypermetropic, though it may be only three or four years old, to wear glasses; for the relief they afford is so pronounced that even so young a child soon appreciates it, and, instead of objecting to them, actually prefers to have them on.

As to the possibility of correcting a convergent squint by glasses alone, without resort to operation, it may be said that this can be done always, if the case is seen before the habit has become firmly fixed, that is to say, during the stage when the squint is inconstant, is as yet periodic in character; and it is to be remembered that all cases of non-paralytic squint pass through such a stage, unless, indeed, as sometimes happens, the fault continues in- 
definitely to be periodic. As it falls to the lot of the general practitioner much oftener than to the specialist to see these cases during the formative stage of the squint, the responsibility which devolves upon him under the circumstances, and the great value of the advice which he is in a position to give, can not be too strongly emphasized.

Before dismissing the subject of the treatment of hypermetropia it remains only to observe that, more often than not, hypermetropia is complicated by the coexistence of astigmatism, and that in view of this and of the fact, to which reference has already been made, that a clear comprehension of the muscular anomalies of the eyes and of the influence which glasses exert upon them is essential to the proper correction of this refractive fault, it is evident that the treatment of hypermetropia is a matter to be undertaken only by the physician who has had especial training and experience.

Myopia (Short-sightedness, Near-sightedness). -This defect is much less common than hypermetropia, from which it differs in several essential respects. In the first place, unlike hypermetropia, it is nearly always an acquired, seldom a congenital, fault. In the next place, it is a fault which is commonly progressive; and, in the third place, it is usually accompanied by, indeed is dependent upon, a pathological condition of the eye. As is hypermetropia, inheritance often plays in myopia an important rôle; but it is not the defect itself that is transmitted from one generation to another, but rather a predisposition to its development.

The essential fault in myopia is that rays of light entering the eye from distant objects are brought to a focus not upon the retina, as they should be, but in front of it. This results either from the antero-posterior axis of the 
eye being abnormally long or from an excess of refractive power in the lens system of the eye. The first-mentioned type is known as axial myopia (Fig. I50); the second, as curvature myopia. Axial myopia is the more common type and is very generally due to a yielding of the eyeball at its posterior pole, to the development, as it is called, of a posterior staphyloma. The sclerotic coat in the region of the macula and optic nerve entrance is incapable of resisting the intraocular pressure and, being unsupported here, as it is laterally, by the recti muscles, becomes distended and thinned. The choroid and retira also participate in the staphylomatous process, and in myopia of high grade usually exhibit, ophthalmo-

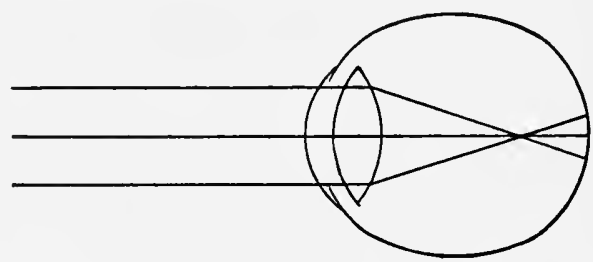

Fig. I50.-Diagram of axial myopia.

scopically, marked signs of inflammation and degeneration. (See Fig. 132.) It is this insufficiency of the sclera, this disposition to the development of posterior staphyloma, that is inherited, and it is this that accounts for the family tendency to myopia often observed.

Much less frequently axial myopia is the result of a gradual enlargement of the eyeball, of a general yielding of the sclera and cornea, such as occurs in the glaucoma of youth.

In curvature myopia, which, like axial myopia, is commonly an acquired condition, the cornea is usually the seat of the fault. As a result of inflammation or injury or in consequence of some inherent insufficiency the 
cornea becomes abnormally convex. In the condition known as conical cornea (see Fig. I02) we have one of the most striking examples of curvature myopia, which is often excessive in amount. Another type of curvature myopia is produced by an increase in the convexity of the lens, such as is frequently observed in the incipient stage of cataract, or by the lens, from any cause, assuming a position farther from the retina than it normally occupies.

Axial myopia, being the type of near-sightedness usually encountered, calls for fuller consideration.

While the inherited disposition to the development of posterior staphyloma unquestionably plays an important rôle in the causation of axial myopia, and in my opinion deserves greater consideration than is usually accorded it by recent authors, there are other etiological factors which are hardly less significant. Among the most important of these, unquestionably, are uncorrected astigmatism and unrecognized anomalies of the ocular muscles.

In their descriptions of the causes of myopia the use of the eyes in near vision-as in reading, writing, sewing and the like-is usually given more prominence by writers, it seems to me, than it deserves; for if this, of itself, were sufficient to produce near-sightedness, the fault would be far more common than it is. The normal eye, it is safe to say, is seldom harmed by, is rarely incapable of doing without injury, the work of this character which is demanded of it. The eyes that really suffer-unless indeed the work required of them is beyond reason, and is rendered exceptionally trying by the unfavorable conditions as to light, position, unhygienic surroundings, etc., under which it must be performed-are the ones that start with some inherent de- 
fect; the ones that are astigmatic or anisometropic, or in which there is a faulty muscle-balance, or, finally, in which there exists an inherited predisposition to myopia. Unquestionably, myopia is much less apt to develop in such eyes if they are called upon to do but little near work, and to this extent the use of the eyes in near vision deserves to be regarded as a factor in the causation of near-sightedness, but not as a factor of prime importance.

The causative relation between the astigmatism or the muscle fault and the myopia is easily traced. The strain induced by these anomalies leads to congestion of the inner tunics of the eye and in time to a low grade of choroido-retinitis, and this, in turn, to a disturbance in the nutrition of the sclera and to the development of posterior staphyloma. In no other country are the refractive and muscular faults of the eyes so diligently searched for, and so carefully corrected, as in the United States, and the outcome of this, as Dr. Risley, of Philadelphia, has pointed out, is that myopia of high grade is by no means as common among us at the present day as it was thirty or forty years ago. On the other hand, its prevalence has rather increased than diminished during this period in Germany, where there is a disposition to deride, and to regard as "finicky," the tendency of the American ophthalmologists to prescribe glasses for the lower grades of these defects.

It is not improbable that, as a result of inheritance, there exists among the Germans an exceptional predisposition to myopia, which doubtless, in no small measure, explains the wide prevalence of this fault among them; but I venture the prediction that they will meet with but little success in overcoming this predisposition until they have learned to emulate, rather than 
deride, the example which has been set them by the ophthalmologists on this side of the water.

It is during the early years of life, especially before the age of twenty-five, that the disposition of myopia to increase is chiefly observed. After that age, if the defect is not excessive, it is apt to become stationary. It is of the greatest importance, therefore, that during these early years every effort should be made to overcome this tendency to progression; for while a moderate degree of myopia is only an inconvenience, a high degree is a matter of serious moment, which may even eventuate in loss of sight. Choroido-retinitis, involving especially the macular region, is a common accompaniment of high myopia, and among the complications to be feared are intraocular hemorrhage, detachment of the retina, and the development of cataract. Very near-sighted eyes also are prone to suffer serious consequences from slight injuries, which would do no harm to the normal organ.

The myope has to contend with another difficulty comparable to, but exactly the opposite of, that which besets the hypermetrope. The latter, as has been explained, with his fault uncorrected, must always put forth an effort of accommodation in excess of that of convergence. The myope, on the other hand, with bis fault uncorrected, must converge more strongly than he accommodates-must call upon the internal recti muscles to work in excess of the ciliary muscles. Indeed, if his near-sightedness is of considerable degree, because of the proximity of his far point, he must converge more strongly even than the emmetrope, and at the same time must do his best to suppress entirely all accommodative effort. To the myope this derangement of the normal relation between accommodation and convergence is as 
trying as the derangement of an opposite character is to the hypermetrope, and when it becomes intolerable he, too, abandons the effort to maintain binocular fixation, and seeks to restore the parallelism between these two functions by permitting one eye to squint outward, just as the hypermetrope accomplishes the same result by allowing one eye to squint inward. If he does not find relief in this manner he is apt to suffer, in much the same way that the hypermetrope does, with asthenopia, headaches, etc., while, of greater moment still, the growth of the myopia is promoted by the continual strain.

While this disturbance of the normal relation between accommodation and convergence is doubtless the chief cause of the occurrence of divergent squint in high myopia, there are other factors that favor its development, especially the considerably greater length of the eyeball, which, for mechanical reasons, renders the converging of the eyes in near vision more difficult. It is also one of the chief causes of the asthenopia frequently observed in myopia of moderate degree, and it is in considerable measure through its elimination that the growth of near-sightedness is so favorably influenced by properly adjusted glasses. To rid themselves of the discomfort to which it gives rise, many myopes, whose fault has not been corrected by glasses, acquire the habit of reading with one eye, closing the other or covering it with the hand.

In some instances myopia is a result of acute systemic disease, especially of the exanthematous fevers. A softening of the sclera, a diminution of its resisting power, occurs, and this leads to the development of posterior staphyloma. Without having sufficient warrant for the belief, it has seemed to me probable that 
the extreme degrees of myopia not infrequently encountered among the negroes in the South commonly originate in this way.

The most characteristic symptom of myopia is indistinctness of distant vision. There is a prevalent belief that the existence of myopia incapacitates one from reading at the usual distance; but this, of course, is erroneous, since it is only of the higher grades of the defect that it is true. Besides poor distant vision, the myope is apt to complain of muscæ volitantes and, as has been said, of asthenopia. To enable him to see distant objects better, he frequently acquires the habit of keeping the eyes half closed, of nipping the lids, and it is from "this habit that the name "myopia" is derived. However, none of these symptoms is pathognomonic, for they all are met with in other refractive errors, notably in astigmatism of marked degree.

The ophthalmoscope affords a certain and the readiest means of making a positive diagnosis. It enables one also to determine with approximate accuracy the amount of the defect, and, besides, throws much light upon the probable prognosis; for, even when the myopia is of considerable degree, if there are no gross pathological changes in the fundus of the eye, the outlook for the future is not unpromising. Other things being equal, it may be said that the earlier in life myopia develops, and the higher the grade already reached in childhood, the more unfavorable is the prognosis, and for the reason already referred to-that it is in adolescence that the defect is chiefly progressive.

Treatment.-If special training, experience, and painstaking effort are required for the proper treatment of hypermetropia, they are the more urgently demanded in the treatment of myopia; for the defect is not only, in 
itself, more difficult to measure and correct, and as often complicated by the presence of astigmatism and faulty muscle-balance, but its existence implies an unsound condition of the eye, so that not only the comfort of the individual, but, in many instances, the preservation of sight is at stake. The question whether the defect shall increase to the danger-point, or shall be arrested before the deeper structures of the eye have suffered irreparable damage, hinges, in large measure, upon the skill exercised in its correction by glasses (Fig. I 5 I).

If the astigmatism so often found in association with myopia is ignored or inaccurately corrected,

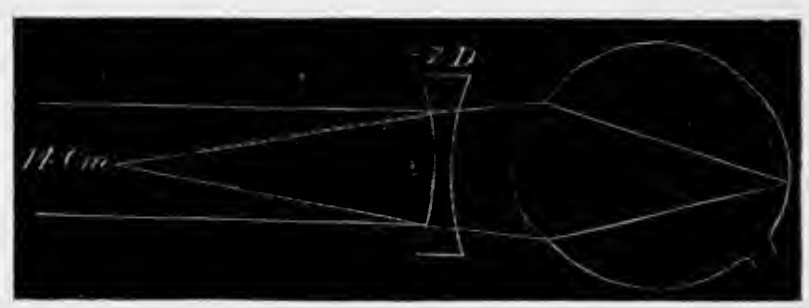

Fig. I5 I.-Manner in which a concave lens causes parallel rays to diverge as from the far point of a myopic eye (de Schweinitz).

or if the muscle-balance in far and in near vision and the accommodative power of the eyes are not taken into account, the glasses prescribed are more apt to be productive of harm than of good. On the other hand, if in their selection regard is had for each of these points they can hardly fail to exert a distinctly beneficial influence. It is not too much to expect that they will relieve the asthenopic symptoms, and enable the eyes to be used in near vision, at least in moderation, without the risk of increasing the myopia. It is true that, exceptionally, there are instances-as when near-sightedness of high grade is already present in 
childhood-in which, even with the help of glasses, the near use of the eyes is not to be permitted; but such cases are comparatively rare, and, as a rule, if the glasses afford the help which they should, we shall run but little risk in permitting the eyes to be used in near work, provided we see to it that they are not taxed immoderately, that the printed page or other object to be regarded is well lighted and kept at the proper distance, and that a stooping posture of the head is avoided.

There is a wide-spread belief that in myopia glasses are needed for distant vision only, and that because fine print can be read without their assistance they may be dispensed with in near vision. Seldom is this belief well founded. Indeed, very generally it is far more important for the welfare of the eyes that they should be worn in near, than that they should be used in distant, vision. In distant vision they are a convenience; in near vision they are a therapeutic agent of great value. If the myopia is of moderate degree, is equal in the two eyes, is uncomplicated by astigmatism, and if, in addition, as seldom happens, the muscle-balance at the reading distance is what it should be, glasses for near vision may be dispensed with; but, as a matter of fact, such conditions seldom obtain. It is often a difficult matter to persuade the myope to wear reading glasses, and a contention is apt to arise when this point is broached; but in the end he seldom fails to realize the help which they afford. Exceptionally, the contention takes a different form-a willingness is shown to wear near glasses, while objection is made to their use in distant vision. It is not always so necessary to combat this view, and the point may be yielded, provided the eyes are free from muscular fault and are not decidedly astigmatic.

Whether the same glasses may be worn in both far 
and near vision will depend upon the degree of the myopia, upon the age and accommodative power of the individual, and upon the relative strength of the external and internal recti muscles. In youth, if the myopia does not exceed two or three diopters, and there is normal power of accommodation, it is usually good practice to prescribe glasses which give satisfactory distant vision, and permit these to be worn for all purposes. Later in life, when the eyes have lost part of their accommodative power, this is impracticable, and weaker glasses must be provided for near vision. Again, in myopia of high grade it is usually best not to correct the entire fault, and, under such circumstances, the same glasses may often be worn in both far and near vision.

Whenever a considerable amount of myopia is present in childhood the eyes should be examined from time to time, in order to learn whether the fault is increasing, and if this is found to be the case greater moderation in the use of the eyes in near vision should be insisted upon.

Apart from the systematic wearing of carefully adjusted glasses, and the exercise of such precautions as have been set forth in the near use of the eyes, the progress of myopia is favorably influenced by measures which improve the general health. In youth, especially, reading for pleasure should be discountenanced, and participation in outdoor occupations and amusements should be encouraged. Rough sports, however, such as football, boxing, and the like, should be avoided. The digestive apparatus should be kept in good condition, and constipation of the bowels should be avoided. When the eyes are irritable and the nearsightedness inclined to increase, I have found decided benefit result from the long-continued use of the opium and boracic acid lotion (ext. opii, gr. x; acid. boracic., 
gr. xl; aq. destil., そiv) which I have had occasion to commend so often. It should be used systematically during the sleeping hours, not too heavy pads of gauze or soft linen, wet with the lotion, being laid over the eyes, and kept in place by a light bandage.

Operative procedures are seldom called for, though, exceptionally, when there is pronounced insufficiency of the internal recti muscles a guarded tenotomy of one or both of the opponent muscles may be indicated. Removal of the crystalline lens for the correction of myopia of very high grade, a practice which of late years has come into vogue, is a procedure of doubtful advantage, and one attended by considerable risk, and the cases in which it is justified are, in my judgment, extremely rare.

Astigmatism.- Next to hypermetropia astigmatism is the commonest of the refractive anomalies. An astigmatic eye, as the name indicates ( $\dot{a}$ arirka, "without a point"), is one that is incapable of bringing the rays of light which enter it to a focal point. There are two types of astigmatism-regular astigmatism, usually of congenital origin, and irregular astigmatism, which is frequently an acquired fault. Irregular astigmatism has been described already in treating of "Opacities of the Cornea" and does not call for further consideration.

Regular astigmatism, of ten transmitted from one generation to another, is commonly due to asymmetry in the curvature of the cornea, to the different meridians of the cornea varying in their convexity; less frequently, to asymmetry in the curvature of the crystalline lens, or to the lens occupying an oblique position with reference to the visual axis. The result of each of these faults is that the rays of light passing through the different meridians of the dioptric media of the eye are un- 
equally refracted, those which pass through the most convex meridian, or through the meridian to which the obliquity. of the lens corresponds, being most quickly focused, those through the less convex meridians less quickly, and those through the least convex meridian, or through the meridian corresponding to the axis about which the lens is rotated, least quickly. The outcome of this is an imperfect, a blurred, retinal image, which is very annoying, and which the eye, at the expense of much effort, endeavors to make more distinct. The amount of blurring depends upon the measure of success which attends this effort, as well as upon the degree of the astigmatism.

In some eyes a large part of the static astigmatism is corrected, is rendered "latent," through the action of the ciliary muscle, which, contracting asymmetrically, produces a compensatory lenticular astigmatism or, perhaps, a tilt of the lens, which serves the same purpose. It is the ever-present strain involved in this effort that is the chief cause of the asthenopia, the headaches, etc., so commonly associated with astigmatism. A fact confirmatory of this is that when the astigmatism is of very high grade there is usually little complaint of asthenopia, only of imperfect vision, because, under such circumstances, the eye soon learns that by no effort which it is capable of making can clear vision be secured, and so abandons the task and accepts the inevitable.

Many astigmatic persons also learn to improve their vision, as myopes do, by nipping the lids, whereby they not only secure the advantage of a narrow, slit-shaped pupil, but, through the pressure exerted by the lids, actually alter to their advantage the curvature of the cornea. In order to obtain a larger retinal image they 
also frequently hold the printed page abnormally close to the eyes. For these reasons, and because their distant vision is manifestly indistinct, they are often supposed to be myopic. Unlike that of the myope, however, their vision, if the defect is considerable in degree, is imperfect at all distances. They are more sure, too, to be asthenopic, and, as has been pointed out, they are liable to many grave lesions of the eye in consequence of the ever-present strain to which their accommodative apparatus is subjected.

The simplest form of astigmatism is that in which the eye is emmetropic in one meridian and hypermetropic or myopic in the opposite meridian. This type of the defect, simple astigmatism, as it is called, is, however, the exception, and it is usually with compound astigmatism that we have to deal, that is, with astigmatism in which there is hypermetropia or myopia, as the case may be, in one meridian and a greater amount of the same defect in the opposite meridian. More rarely mixed astigmatism is encountered-there is hypermetropia in one meridian and in the opposite meridian myopia.

In corneal astigmatism the meridian of shortest focus is apt to be approximately vertical, the meridian of longest focus approximately horizontal. In lenticular astigmatism, on the other hand, it is usual to find the conditions reversed. The first type, being the more common, is known as astigmatism with, or according to, the rule; the last, as astigmatism against the rule. A low grade of astigmatism "with the rule," a quarter of a diopter, for example, usually gives rise to little or no inconvenience, indeed, is so common as hardly to be regarded as an abnormality. On the contrary, astigmatism "against the rule," generally located, as has been 
said, in the lens, deserves to be regarded as a wider departure from the normal, and, though it be ever so slight in degree, is apt to cause trouble. Even the lowest grades of astigmatism against the rule, therefore, should be corrected-the more so because the discrepancy between the manifest and the total defect is apt to be greater than is the case in astigmatism with the rule.

The fact, of frequent observation, that astigmatic glasses after having been worn for some months have to be increased in strength, finds its explanation in this latency of a part of the error. Latent hypermetropia usually can be made evident by a few instillations of a cycloplegic; but this is not true of latent astigmatism. Only as a result of the correction of the manifest defect does the induced, the compensatory, lenticular astigmatism slowly disappear, and the static error become fully manifest. Thus it happens that an eye which is made comfortable for half a year or more by a cylindrical glass of a certain strength may eventually call for one twice as strong. In exceptional instances, especially in progressive myopia, there may be an actual increase of astigmatism; but commonly it remains unchanged, and there is only an apparent increase due to the defect becoming more and more manifest.

There is a popular impression that an astigmatic glass once right is always right; but, like many such impressions, this one is without warrant. Indeed, not only may an increase in the strength of the glass be called for, but also a change in its position; for the astigmatism may undergo what is known as orientation-a change may occur in the direction of the principal meridians, ${ }^{*}$ doubtless another re-

* The meridians of greatest and of least convexity are called the "principal meridians," and are at right angles to each other. 
sult of the effort which the eye puts forth to correct its own defect.

Although regular astigmatism is usually a congenital fault and frequently, as has been said, an inherited one, it may also be acquired. Acquired astigmatism is often of traumatic origin. Any wound of the eye which produces a permanent change in the curvature of the cornea may give rise to it. A familiar example of this is the astigmatism against the rule which very generally follows the operation of cataract extraction. Severe inflammation of the cornea may also produce it, more especially a perforating corneal ulcer. It is more inconstant than congenital astigmatism, being liable to vary in degree as the asymmetry of the cornea increases or diminishes, and because of this tendency a readjustment of the glasses prescribed for its correction is more often necessary.

Because the common belief is to the contrary, it is well to emphasize the fact that the existence of a degree of astigmatism capable of giving rise to pronounced asthenopic symptoms is not incompatible with normal acuteness of vision. Especially is this true, in my experience, of astigmatism against the rule, of which the eye seems more intolerant, and with which, so far as sharpness of vision is concerned, it seems to cope more successfully. Often have I met with cases of this defect in which, without glasses, vision was fully up to the normal standard, and yet in which the prescribing of weak cylinders has afforded immediate relief from headaches, asthenopia, etc.

The detection of the lower grades of astigmatism is a matter which often taxes the skill of the specialist; but when the error is marked in degree it is not difficult to prove its existence. The easiest way to do this is by 
means of a' stenopaic disc, or, if this is not available, by means of an improvised stenopaic apparatus which any one can make by cutting a narrow slit in a visiting-card. When such a contrivance is held in front of, and close to, an eye the vision of which is defective from any refractive error, an appreciable improvement in vision, especially in distant vision, will result. If the defective vision is due to a symmetrical error, for example, to myopia, the improvement will be the same in whatever direction the slit is turned. If, however, it is due to astigmatism, vision will be much sharper when the slit is held in a certain easily found position, and much less sharp when it is held in the opposite direction. The test is a rough one; but, if the difference in vision is considerable in the two positions, it is conclusive, and warrants a positive diagnosis of astigmatism. It also indicates the direction of the principal meridians, since the greatest improvement in sight is obtained when the slit is held at a right angle to the faulty meridian, or, if both are at fault, to the one which is most so. However, as this test is conclusive only when the astigmatism is considerable in degree, it should be borne in mind that a negative result does not exclude the possible existence of a significant amount of the defect.

Since astigmatism is so prevalent an error, and is so often a chief factor in the causation not only of a host of ocular maladies but of many obscure disturbances of the nervous system, such as headache, neurasthenia, nausea, nervous dyspepsia, vertigo, insomnia, somnolency, incapacity for mental concentration, chorea, etc., its significance, its etiologic importance, can not be too strongly impressed upon the general practitioner. "Remember the eyes" is a dictum which, if taken to heart, will often stand him in good stead, and enable 
him to solve many a diagnostic riddle which otherwise might prove insolvable.

Treatment.-There is but one way to treat astigmatism, and that is, with painstaking care, to correct it by means of glasses; for, being simply a defect in the conformation of the eye and not a pathological condition, there are no means by which it can be eliminated or even lessened in amount. Theoretically, the glasses prescribed for its correction should be worn constantly; but, practically, this is not always necessary, for when the defect is of low grade, and is approximately with the rule, complete relief is not infrequently obtained by their systematic use in near vision only. In astigmatism against the rule, even if the error be slight in degree, it seldom happens that relief is secured unless the glasses are worn constantly. This is usually the case also when there is a considerable difference in the amount of the astigmatism in the two eyes, or when it is with the rule in one eye and against the rule in the other. As oftener than not other refractive faults are found associated with astigmatism, the question whether the glasses shall be worn only in near vision or constantly may depend upon the nature of the associated error.

It is by means of cylindrical lenses that we are enabled to correct astigmatism. If the astigmatism is "simple" a plano-cylindrical lens suffices for its correction-a planoconcave cylinder (Fig. 152) if the astigmatism is myopic, a plano-convex cylinder (Fig. I 53) if it is hypermetropic. If the astigmatism is "compound" or "mixed," a sphero-cylindrical lens is usually necessary, that is, a lens one surface of which is spherical-concave or convex, as the case may be-and the other cylindrical. In the direction of its axis a plano-cylinder has only the refractive value of a plate of glass with parallel surfaces, while in 
the direction perpendicular to its axis its refractive power is greatest. Hence the effect of a cylindrical lens upon vision depends upon the position in which the axis is placed. It is necessary, therefore, in prescribing glasses for the correction of astigmatism to determine with accuracy not only the required strength of the cylinder, but also the exact position in which it is to be worn. The question whether a plano-cylinder or a spherocylinder is required has also to be determined, and, if the latter is called for, what shall be the strength of the spherical surface. And here, again, it is of the utmost

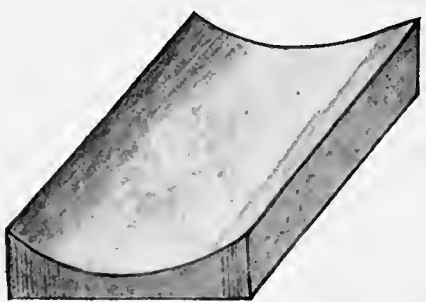

Fig. 152.-Plano-concave cylinder.

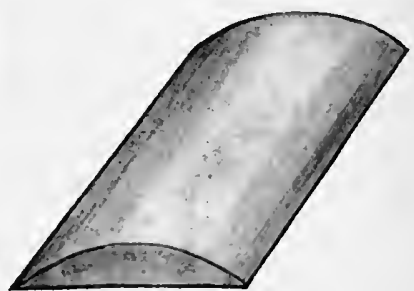

Fig. 153.-Plano-convex cylinder.

importance that the muscle-balance of the eyes should be taken into account.

The correction of astigmatism by means of cylindrical lenses has a twofold effect-vision is greatly improved, in most instances brought up to the normal standard, and, even more important than this, the previously existing accommodative strain is relieved. The latter effect may well be called the more important, since it is through this that relief is obtained from the many ill consequences, local and remote, to which uncorrected astigmatism usually gives rise.

It is well to bear in mind that the relief which cylindrical lenses afford is not appreciated, in many instances, until they have been worn for a time-long enough for 
the eyes to learn to adjust themselves to the new conditions, and to accept the help which they give. Indeed, when they are first put on a transient aggravation of the asthenopic symptoms is experienced not infrequently. In children this seldom happens, but in persons more advanced in age it is often pronounced. It is well, therefore, that the patient should be warned beforehand what to expect, otherwise the glasses may be petulantly thrown aside under the impression that they "do not suit," and much undeserved opprobrium heaped upon the one who has prescribed them.

How long the glasses first given will continue to afford the needed relief is a matter of much uncertainty. Exceptionally, especially in astigmatism against the rule, a change may be called for within a few months, while in other instances they may be worn with comfort for many years.

In youth, so far, at least, as the astigmatism is concerned, the same glasses may be worn for all purposes, for near as well as for distant vision; but after the presbyopic age has been reached a lens especially adapted for near vision becomes necessary-one that, in addition to correcting the astigmatism, will afford the needed magnifying power. As a rule, this can be done most conveniently by means of a bifocal lens, by adding to the distance glass a "lenticular" of the required strength.

There are several methods by which the existence of astigmatism can be ascertained and its direction and degree determined. The ophthalmoscope affords the readiest means of doing this, and this method has the great advantage that it does not necessitate the use of a cycloplegic; but even in the hands of the most expert the information which it gives is only approximately exact, and must be confirmed by other means. The 
ophthalmometer, which some hold in high esteem, is not to be relied upon, since it gives only the astigmatism of the anterior surface of the cornea, and tells us nothing of asymmetry either of the posterior corneal surface or of the lens. Skiascopy, the shadow test, or, as it is sometimes called, retinoscopy, affords much more exact results, and is the most trustworthy of the objective methods of measuring astigmatism as well as other refractive errors, but its employment necessitates the use of a cycloplegic.

Of all the methods of measuring astigmatism the most reliable-the court of last resort, as it has been called-is the subjective method, the test with glasses, test-types, and the astigmatic dial. Even here contradictory results and inconsistencies are not uncommon, and it often happens that in endeavoring to reach a definite conclusion the skill and patience of the most experienced are sorely tried. The beginner usually derives much help from the use of a cycloplegic, and in the opinion of many this is essential to the attainment of accurate results. With this view, however, I am not in accord; for my experience is that, in many instances, the dilatation of the pupil attendant upon the employment of a cycloplegic introduces as many $x$ quantities as are eliminated through the suppression of the power of accommodation. The advantage gained from the paralysis of the ciliary muscle is unquestionably great; but this is nearly, if not quite, offset by the disadvantage of having to make the visual tests with a widely dilated pupil. The problem would be different did we possess a cycloplegic which was not a mydriatic - a pupil dilator-as well, or had Nature been less niggardly, and made the whole of the cornea and the whole of the crystalline lens as optically perfect as she 
has the visual zone of each; but such a cycloplegic has yet to be found, and, as the ophthalmoscope and skiascopy have shown us, Nature, as yet, has busied herself but little in perfecting those outlying portions of the cornea and lens which, under usual conditions, take no part in the formation of retinal images. ${ }^{*}$ To show how great may be the lack of agreement between the results of tests made with and without a cycloplegic, I may mention that in a few instances I have found the total hypermetropia to be less than the manifest, and in a few others have seen the direction of an astigmatism exactly reversed through the induction of mydriasis. Still, I would not be understood as being opposed in general to the use of a cycloplegic as an aid in the determination of refractive errors; for I recognize the fact that, in not a few instances, a dilated pupil is a lesser evil than an irritable ciliary muscle, which is changing its tension from moment to moment.

There is a popular impression that after glasses prescribed for the correction of astigmatism have been worn for a time, and have given the needed relief, they may be put aside without detriment. It is hardly necessary to say that this is rarely the case. In low grades of astigmatism with the rule, especially if the work required of the eyes becomes less exacting, this sometimes happens; but, as a general truth, it may be said that cylindrical glasses once needed are always needed. "Until you get to heaven," is the way I sometimes put it in answer to the frequently propounded ques-

* It is interesting to note that this is especially true of the eye of the negro. I am not aware that attention has been called to this fact; but from my own observation I have no hesitation in stating it as a fact. Especially does one find frequently in the eye of the negro marked examples of "symmetrical aberration," as defined by Jackson. 
tion, "How long shall I have to wear these horrid glasses?"

Anisometropia is the rather awkward term employed to designate a difference between the refractive state of the two eyes. When of moderate degree this condition often gives rise to asthenopia, because of the unequal accommodative effort which it necessitates; when pronounced, it tends to promote the development of strabismus. Exceptionally, it proves a blessing in disguise, one eye being used with satisfaction in distant vision, the other with equal satisfaction in near vision. This is the case especially when one eye happens to be moderately myopic, the other nearly emmetropic; for under such circumstances the emmetropic eye does good service in distant vision, while the myopia of the other eye serves the purpose of a convex lens, and obviates the necessity of presbyopic glasses. As a change in the refraction of either eye is liable to occur, the difference between the two may vary, the anisometropia becoming, in the course of time, greater or less, as the case may be.

Treatment.-As a rule, it is practicable and best to equalize the focus of the eyes by giving to each the lens which its refractive fault calls for. In most instances, by making the sight and the accommodative effort of the two equal, this results in the establishment of comfortable binocular vision. However, it is not always feasible to do this. In the first place, the difference between the required glasses may be so great as to render impracticable fusion of the retinal images, which under such circumstances would differ considerably in size. In the next place, when the eyes have been long divorced, so to speak, pronounced muscular faults. are apt to develop. These give rise to no inconvenience 
as long as the eyes make no effort to work together, but are liable to cause much annoyance when, through the action of glasses, binocular vision is reestablished. Such muscular faults frequently disappear as a result of the new relations established between the eyes; but this is not always the case.

The difference in strength which it is practicable to make between the glasses prescribed for the correction of anisometropia varies considerably in different individuals. In some instances a difference of 4 or 5 diopters proves acceptable, while in others a difference appreciably less than this may give rise to annoyance. In the very high grade of anisometropia which exists when one eye has been operated upon for caratact, and the other eye still retains good sight, it is seldom possible, by any arrangement of glasses, to secure comfortable binocular vision. Under such circumstances it is best, therefore, to give a glass to the sharper-seeing eye only. If this happens to be the one that has been operated upon for cataract, it may be necessary, in order to establish the habit of using this eye, to "exclude" the other eye for a time by placing an opaque disc or a ground glass before it.

\section{ANOMALIES OF ACCOMMODATION.}

A sharp distinction is to be drawn between the anomalies of accommodation and the anomalies of refraction. The latter, as has been explained, are the expression of certain faults in the conformation of the eye; the former have to do with the capacity of the eye to alter its focus, to adjust itself to the sharp-seeing of objects at varying distances. To a comprehension of the anomalies of accommodation a clear conception of the normal accommodation of the eye is essential. 
According to the commonly accepted theory of the accommodation of the eye, propounded by Helmholtz, the crystalline lens, when unrestrained, has an inherent tendency to become more convex. When the eye is fixed upon distant objects this tendency is held in check through the traction exerted upon the lens capsule by the zonule of Zinn. The tension of the zonule is controlled by the action of the ciliary muscle. When this muscle is at rest the tension of the zonule is at the max-

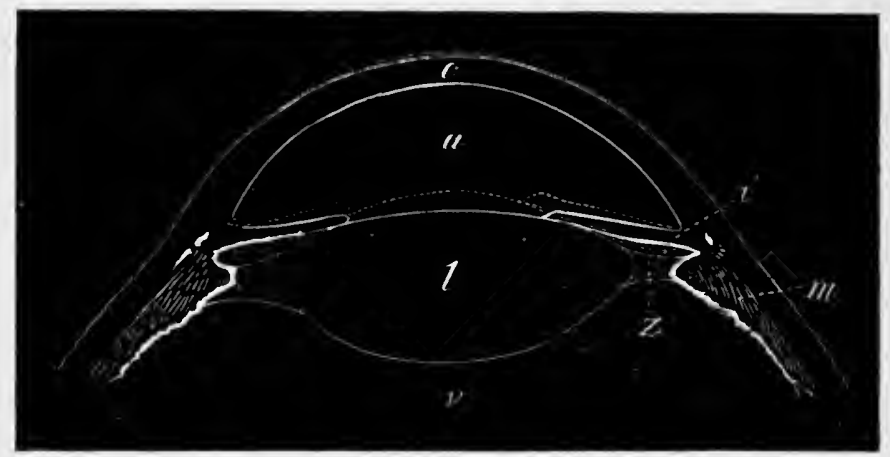

Fig. 1 54.-Changes in the conformation of the lens during accommodation. The solid white outline of the lens, $l$, shows its form when the zonule, or suspensory ligament, is tense. The dotted line shows the increased curvature of the anterior surface during accommodation, and its advancement into the anterior chamber, $a . \quad z$ is the suspensory ligament; $m$, the ciliary muscle; and $i$, the iris (Landolt).

imum; when it contracts the tension is lessened. The adjustment of the eye for the sharp-seeing of near objects is brought about, then, by the contraction of the ciliary muscle, which relaxes the zonule and permits the lens to become more convex (Fig. I54). The exact degree of relaxation of the zonule necessary to produce the required change in the focus of the eye is learned through experience, and varies in different individuals, being markedly influenced by age. 
From this description it is evident that the ability of the eye to accommodate itself for different distances may be impaired in any one of several ways: The lens may lose, to a greater or less degree, its elasticity, its tendency upon relaxation of the zonule to become more convex. It may, as a congenital fault, possess this tendency to a subnormal degree. The ciliary muscle may lose its power, may become paralyzed, or it may become spasmodically contracted, or it may be, congenitally, weak and inefficient. All of these conditions are encountered, and each of them produces a definite disturbance in the accommodative power of the eye.

The first-mentioned condition-the loss of elasticity of the lens-occurs in every eye as a result of advancing age, and, when it has reached a certain degree, constitutes the fault known as presbyopia or, popularly, as old-sightedness. The second and last conditions-congenital inelasticity of the lens, and congenital inefficiency of the ciliary muscle-are of not infrequent occurrence, and, existing separately or conjointly, give rise to the anomaly which I have called "subnormal accommodative power." The third condition-paralysis of the ciliary muscle-may occur suddenly at any time of life, and is oftenest due to syphilis or to diphtheria. The fourth condition-spasm of the ciliary muscle-is met with occasionally as a complication in astigmatism and other refractive errors, but is of less frequent occurrence than some authorities would have us believe.

Presbyopia (Old-sightedness).-As a result of its growth and the sclerosis of its older central fibers, which in time come to form the hard nucleus characteristic of the senile lens (see Chap. IX), the crystalline lens gradually loses its elasticity, its capacity to become 
more convex upon relaxation of the zonule. This diminution of elasticity begins to manifest itself, as has been mentioned already, very early in life, even in childhood; but usually does not become sufficiently marked to cause inconvenience until about the forty-fifth year, when it interferes with the sharp-seeing of near objects, as in reading, sewing, and the like. Its gradual development also lessens the ability of the eye to cope with refractive faults, and so, if these are present, may lead, as, for example, in hypermetropia, to indistinctness of distant as well as of near vision.

With the advent of presbyopia the complaint is often heard that the light, especially the light which one has been in the habit of reading or sewing by at night, is not as good as it formerly was, or that the newspaper is not as well printed as it used to be. The threading of a needle becomes a difficult task, and the printed page or the sewing is held inconveniently far away from the eyes. If these hints are acted upon, and the needed glasses procured, satisfactory and comfortable near vision is obtained at once; but if the individual, as often happens, continues to struggle along without their help, hoping to avoid, or at least postpone, this confession of advancing years, the strain upon the eyes soon begins to tell, and asthenopic symptoms, or headaches, perhaps, manifest themselves.

In rare instances the emmetropic eye retains its ability to see near objects distinctly considerably beyond the usual presbyopic age; but, nearly always, when this ability is present after the forty-seventh or forty-eighth year, it will be found that one or both eyes are myopic, and only exceptionally are such persons not helped by properly adjusted glasses, for only exceptionally does it happen that the myopia is just of the requisite degree 
to neutralize the presbyopia. The failure of near vision before the forty-fifth year commonly indicates the existence of hypermetropia or, perhaps, of hypermetropic astigmatism.

Treatment.-There is but one way to deal with presbyopia, and that is by giving the needed glasses. There is no warrant for the claim, put forth by unprincipled quacks, that it can be "cured," or even that its development can be postponed, by such procedures as massage, the use of "eye-cups," and the like.

There is a common belief that in the selection of glasses for "old-sight" the services of the oculist may be dispensed with; that they, at all events, can be "fitted" by any one who "carries a stock" of spectacles, or can be chosen with safety by the individual himself. The fallaciousness of this view can not be too emphatically insisted upon. In prescribing glasses for presbyopia one often has to take into account unsuspected refractive faults, such as astigmatism or anisometropia. The muscle-balance also has to be considered, and the influence which the glasses that seem to be indicated exert upon it. The neglect of these points, which fall definitely within the province of the medical specialist, gives rise to much unnecessary discomfort, and not infrequently to the more serious consequences apt to follow long-continued eye-strain.

It should be borne in mind that presbyopia is a progressive condition, and that the glasses prescribed for its correction must be increased in strength from time to time. Usually, if they have been accurately adjusted, they afford the needed help for about two years; but there are many exceptions to this rule, in some instances a change being called for sooner than this, and in others not so soon. After the seventieth year it is not often 
necessary to increase further the strength of the glasses, for by this time the eye has lost entirely its power of accommodation. In exceptional instances, with the acquisition of so-called "second sight," which, as has been explained, is usually a premonitory symptom of developing cataract, the glasses previously worn have to be considerably weakened, or may even be put aside altogether.

In presbyopia, if glasses are required for distant vision as well as for near, it is commonly best to prescribe

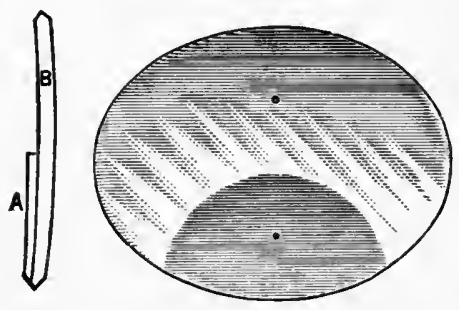

Fig. 155.-Cemented bifocal lens. $B$, Correction for distant vision; $A$, "lenticular," added for near vision.
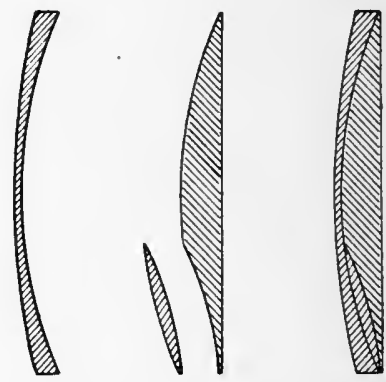

Fig. 156.- "Invisible" bifocal lens. The "lenticular," which is countersunk, is made of glass having a very high index of refraction.

bifocal lenses (Figs. 155 and 156), as this does away with the necessity for two pairs of glasses and the inconvenience of having to change frequently from one to the other. At first such lenses often prove annoying; but in a short time the eyes become accustomed to them, and they afford much comfort.

Paralysis of the Ciliary Muscle.-The, most common causes of this anomaly, as has been stated, are diphtheria and syphilis. Other conditions which may give rise to it are affections of the central nervous system-tabes dorsalis, especially-influenza, diabetes, 
ptomaine poisoning, and contusion of the eyeball. It is a prominent symptom, too, in poisoning by belladonna (I have observed it in one instance in a susceptible individual from the application of a belladonna plaster); and it is to be remembered that it is sometimes due to the accidental and unconscious application of atropin to the eye, as in an instance which came under my observation recently where a physician rubbed one of his eyes with his finger after handling a hypodermic tablet of atropin and morphin.

Not infrequently paralysis of the ciliary muscle is accompanied by paralysis of the sphincter pupillæ and consequent mydriasis. This is more apt to be the case when the paralysis is of syphilitic origin or when it occurs in the course of tabes. It happens less often in post-diphtheritic paralysis. In cases due to acquired syphilis it is not uncommon to find not only the sphincter pupillæ implicated, but all the extraocular muscles supplied by the third nerve. In post-diphtheritic cycloplegia both eyes are usually involved, while cases dependent upon syphilis are commonly unilateral.

The prominent symptom is impairment of sight, which usually manifests itself suddenly. In emmetropic eyes only near vision is affected; but both far and near vision are impaired in eyes that are hypermetropic, because such eyes require an effort of accommodation to see distinctly even distant objects. If the paralysis is complete, ability to read ordinary print is lost. The diagnosis is established by finding that a convex glass of ten or twelve inches focus enables fine print to be read with ease.

The prognosis, as a rule, is favorable, especially in the post-diphtheritic cases, which commonly recover within a few weeks. The most unfavorable cases are 
those which are dependent upon disease of the central nervous system.

Treatment.-This necessarily depends upon the primary cause of the affection. In post-diphtheritic cases and in cases following influenza tonics containing iron and quinin, and especially strychnin, are indicated. In cases of luetic origin potassium iodid, in generous doses, and strychnin are the most useful remedies. Local remedies are of but little value, though eserin, in weak solution, is usually commended.

Spasm of the Ciliary Muscle (Spasm of Accommodation).- The most typical spasm of the ciliary muscle is that which is produced by the action of eserin upon the eye. The contraction of the muscle causes a marked lessening of the tension of the zonule of $\mathrm{Zinn}$, which, in persons who have not yet reached the presbyopic age, is attended by an exceptional increase in the convexity of the crystalline lens. This results in the production of a transient myopia, the degree depending upon the age of the individual and the strength of the eserin solution employed.

Exceptionally, something equivalent to this occurs in ametropic eyes which, without suitable glasses, have been strained by much near work. It is more prone to occur in astigmatic eyes, particularly in astigmatism against the rule, or when the astignatism is complicated by insufficiency of the internal recti muscles. It is usually attended by marked asthenopic symptoms, and not infrequently the existence of a low grade of choroidoretinitis is revealed by the ophthalmoscope. It necessarily masks the true refractive condition, causing hypermetropic astigmatism and even considerable degrees of hypermetropia to simulate myopia, and exaggerating any real myopia that may be present. 
Treatment.-The muscle-balance in far and near vision having been determined, a cycloplegic (atropin or hyoscyamin) should be prescribed. In most instances this will quickly overcome the ciliary spasm, though exceptionally its use may have to be continued for some days before this is accomplished. As soon as this has been brought about, a careful measurement of the refractive condition of the eyes should be made, and glasses, usually for constant wear, should be ordered. If the choroido-retinitis is marked in degree, a period of abstinence from near use of the eyes should be insisted upon, and a lotion of opium and boracic acid, to be employed until all symptoms of irritation have subsided, should be prescribed.

Subnormal Accommodative Power.-In a paper published in the "Transactions of the American Ophthalmological Society," in I89I, I described a condition which I believed to be a not infrequent cause of asthenopia in young persons, and for which I proposed the name "Subnormal accommodative power." Although the detection and correction of this defect can hardly be said to fall within the province of the general practitioner, I may, perhaps, be excused for going somewhat into detail in describing it.

The characteristic symptoms of this condition, as set forth in my paper, are quite different from those of presbyopia. There is no complaint of indistinctness of near vision, but of asthenopia and not infrequently of headache. The underlying cause in most instances was assumed to be a congenital insufficiency of the ciliary muscle. A congenital rigidity, or lack of elasticity, of the crystalline lens would account for the condition as satisfactorily. The anomaly may exist independently of any other fault of the eye, or may compli- 
cate other errors, refractive or muscular. The early development of presbyopia occasionally observed in emmetropic individuals is one of its manifestations.

Its existence is not to be determined by the tests employed in presbyopia,--for the finest print can be read with facility and, for a short while, at least, at as near a point as the age of the individual would lead one to expect,--but is demonstrated by a lack of accordance between the lateral muscle-balance in far and in near vision, as shown by the vertical diplopia test of von Graefe.

This test, in eyes that are in every way normal, shows an orthophoric muscle-balance in distant vision, but at the reading distance shows an exophoria of from $3^{\circ}$ to $5^{\circ}$. Indeed, whatever the muscle-balance in distant vision may be, unless certain modifying conditions to be described presently exist, the test will show in near vision a difference, in the sense of exophoria, of $3^{\circ}$ to $5^{\circ}$. For example, if an esophoria at $20^{\prime}$ of $5^{\circ}$ is shown, orthophoria or, at most, an esophoria of $\mathrm{I}^{\circ}$ or $2^{\circ}$ should be found at $13^{\prime \prime}$. If, on the other hand, an exophoria of $5^{\circ}$ is shown in distant vision, $8^{\circ}$ to $10^{\circ}$ of exophoria may be predicted in near vision.

A difference between the far and near muscle-balance, in the sense of exophoria, appreciably greater than $5^{\circ}$ indicates that the convergence effort is not supported, as it is under normal conditions, by a corresponding accommodative effort. This is what happens in uncorrected myopia, and it explains the excess of exophoria at the reading distance characteristic of this defect. On the other hand, a difference appreciably less than $3^{\circ}$ indicates that an unusual accommodative effort is being put forth, an effort in excess of the convergence effort which normally should accompany it. 
A typical example of this condition is shown when a cycloplegic has been used, and the eyes have not fully recovered from its influence. If under such circumstances, even when the accommodation has recovered sufficiently to enable fine print to be read, a test of the near muscle-balance be made by von Graefe's method, a result will be obtained very different from that shown by the same test before the cycloplegic was used. Instead of an exophoria of $3^{\circ}$ or $4^{\circ}$ or $5^{\circ}$, the test will show no exophoria at all, or, at all events, several degrees less than was found previously. The meaning of this is that the still somewhat enfeebled ciliary muscle requires excessive stimulation to enable it to perform the work required of it, and that, because of the intimate relation which exists between the accommodative effort and the convergence effort, this is necessarily accompanied by a corresponding stimulation of the conjointly acting interni.

No better illustration than this could be offered of what occurs in the condition for which I have suggested the name subnormal accommodative power. In the one case we have a transient enfeeblement of the ciliary muscle; in the other, a ciliary muscle congenitally weak, or, what amounts to the same thing, an inelastic crystalline lens, to cope with which demands inordinate action upon the part of the normal ciliary muscle; but, so far as the muscle-balance test in near vision is concerned, the result is the same in each.

Asthenopic symptoms, then, manifest themselves in subnormal accommodative power, as they usually do when the normal parallelism between the two functions is disturbed, because the accommodative effort is in excess of the convergence effort.

The rule for the detection of subnormal accommoda- 
tive power, deducible from what has gone before, is this: Ascertain, by the vertical diplopia test, the musclebalance in far and in near vision. If the latter does not show a difference, in the sense of exophoria, of at least $2^{\circ}$, the existence of subnormal accommodative power is indicated. It is well to make the test both with and without correction of any refractive fault that may be present; but the result - the difference between the far and near muscle-balance--is usually the same under both conditions. In applying the test one should be careful to exclude the possible influence, such as has been described, of a cycloplegic. As to this, it may be well to state, my experience shows that for, at least, eight days after the discontinuance of a two-grain solution of hyoscyamin hydrobromate the result of the test is almost sure to be misleading.

Treatment.-It is manifest that we have in convex glasses the means of getting rid of the unpleasant consequences of subnormal accommodative power. In uncomplicated cases, that is to say, in cases in which no refractive or other muscular fault exists, they will be required for near vision only. Under such circumstances, the strength of the glass needed is easily determined by following this simple rule: Ascertain by trial the weakest convex spherical glass that will give, at $13^{\prime \prime}$, the minimum amount of normal exophoria $\left(2^{\circ}\right.$ to $\left.3^{\circ}\right)$, and prescribe this for systematic use in near vision. Should the strength of this glass be so considerable as to bring the binocular far point inconveniently close to the eyes, reduce it, and add esophoric prisms of such strength as will give the required exophoria.

In complicated cases the correction needed for distant vision should be determined by the usual tests, and then, with this correction, the test for subnormal accommoda- 
tive power should be employed in the manner just described, convex glasses or, possibly, sphero-prisms, being added until the required exophoria at the reading distance is obtained. This, of course, involves the necessity, in such cases, for two pairs of glasses, one for far, the other for near, vision, unless, as a matter of convenience, bifocal lenses are preferred.

As set forth in a recent paper,* published in the "Transactions of the American Ophthalmological Society" for 1904, and in the "Johns Hopkins Hospital Bulletin" for January, 1905, the results which I have obtained in this condition of subnormal accommodative power by following the rules just given-prescribing at times for young asthenopic emmetropes convex glasses of considerable strength for near vision, and for young hypermetropes stronger glasses for near than for distant vision, and occasionally combining with these esophoric prisms-have been so eminently satisfactory, that the practice has become as much a matter of course with me as the correction of astigmatism or of hypermetropia itself.

* "The Importance of Testing the Ocular Muscle-balance for Near, as well as for Distant Vision." 


\section{CHAPTER XII.}

\section{MUSCULAR ANOMAIIES OF THE EYES.}

The muscular anomalies of the eyes are divisible into two major groups-manifest muscular anomalies, and latent muscular anomalies. The first group comprises the several varieties of squint-convergent, divergent, and vertical; the second group, the different varieties of heterophoria, or insufficiency of the ocular musclesesophoria, exophoria, and hyperphoria. The essential difference between the two is that in the one case there is a sacrifice of binocular vision, while in the other binocular vision is maintained, but only at the cost of a constant struggle against an ever-present tendency to squint.

Again, the muscular anomalies of the eyes may be divided, with reference to their etiology, into those of paralytic origin, those of congenital origin, and those dependent upon defects in the conformation of the eye - upon refractive errors.

The anomalies of paralytic origin commonly belong to the first major group, though, as improvement in the palsy occurs, the squint may disappear, and they may pass over into the second group, to remain there for a shorter or longer time or, it may be, permanently. The anomalies of congenital origin, for the most part, belong to the second group, though, exceptionally, they may give rise to actual squint. The anomalies due to errors of refraction constitute a very considerable part of both major groups. 
The manifest muscular anomalies-the actual squints - are not, in themselves, provocative of eye-strain, of asthenopia, but are of moment chiefly because of the deformity which attends them, and because the squinting eye usually becomes rapidly amblyopic. When they are of paralytic origin, and, therefore, develop suddenly, they are also commonly attended by very annoying diplopia and by vertigo. On the other hand, the latent anomalies, while they have no cosmetic significance, are of moment because of the marked asthenopic symptoms to which they give rise. The asthenopia is the expression of the constant effort required to maintain binocular vision, in the presence of a disposition to squint; the establishment of the squint means the abandonment of this effort, and frequently results in the disappearance of the asthenopia.

Squints provoked by errors of refraction are lateral squints, and, according to the nature of the refractive fault, the misdirected eye may turn in or out, the squint may be convergent or divergent. Convergent squints, as has been explained in the preceding chapter, are usually associated with, and in most instances are dependent upon, hypermetropia, and commonly develop in early childhood. Divergent squints are oftenest associated with myopia, and may develop at any period of life.

Squints due solely to muscular faults of congenital origin, as has been said, are rare; but when such muscular faults happen to be associated with refractive errors they may, and often do, play an important part in the production of squint.

Paralytic squints may occur at any age, and, depending upon the muscle involved, the squinting eye may turn in or out, upward or downward. To a proper 
comprehension of squints of this character familiarity with the nervous supply of the extrinsic muscles of the eye is essential.

\section{MANIFEST MUSCULAR ANOMALIES.}

Paralytic Squint.-It will be recalled that no less than three of the cranial nerves are distributed to the extrinsic ocular muscles (Fig. 157). The abducens, or

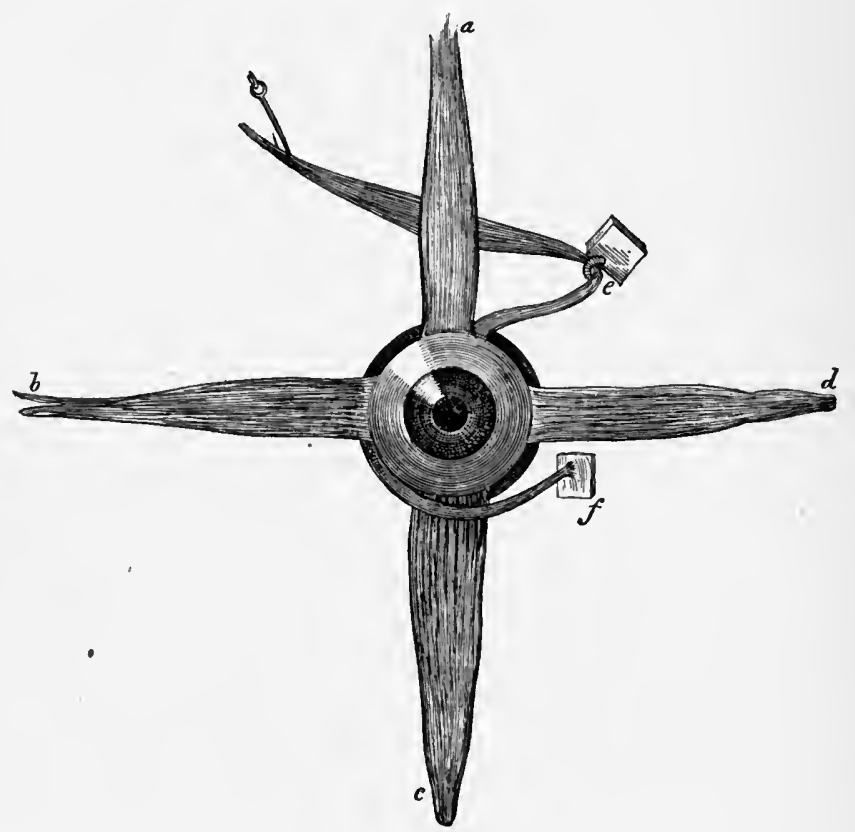

Fig. 157.-The ocular muscles-the recti muscles, $a, b, c, d$, separated from their attachments at the apex of the orbit, the bony attachment of the inferior oblique muscle $(j)$, and the trochlea of the superior oblique $(e)$, diagrammatically represented (Nunneley).

sixth nerve, supplies the external rectus; the trochlear, or fourth nerve, the superior oblique; and the oculomotor, or third nerve, all the other external muscles of the eyeball, as well as the levator of the upper lid, the ciliary mus- 
cle, and the sphincter pupillæ. From this it follows that but a single muscle is affected in paralysis of the fourth or sixth nerve, but that many are involved, or may be involved, in paralysis of the third nerve. In complete paralysis of any one of these three nerves a squint develops, and, as has been said, is attended by very annoying diplopia. If the paralysis is incomplete, the squint and the diplopia may manifest themselves only when an effort is made to turn the eyes in the direction of the affected muscle. Under any circumstances the diplopia disappears when either eye is excluded from vision. The sudden occurrence of diplopia, which can be gotten rid of by the exclusion of one eye, is an almost certain indication of paralysis of some one of the extrinsic eye muscles. The squint which attends the paralysis results, as a matter of course, from the unrestrained action of the opponent muscle. Paralytic squints, as a rule, can be distinguished from concomitant squints (those due to refractive errors) by the fact that the squint increases in degree when the eyes are turned toward the faulty muscle. An inability to rotate the affected eye in the direction of this muscle is also usually evident; though, in incomplete paralysis, this is not always demonstrable.

Paralysis of the ocular muscles (Fig. 1 58) arises from a variety of causes, and may be central or peripheral in its origin. Acquired syphilis is one of the commonest. Among other causes may be mentioned disease of the central nervous system, locomotor ataxia, for example, rheumatism, influenza, diphtheria and other acute affections, diabetes, renal and vascular disease, "cold," traumatism,tumors, or other coarse pathological changes, within the cranial cavity, and similar pathological pro- 
cesses involving especially the apex of the orbit, through which the nerves pass in their course to the eye.

In paralyses of intracranial origin, the lesion may be cortical, or it may involve the association centers, the nerve nuclei, the fibers which connect these centers, or, finally, the nerve-trunks in their course along the base of the brain. Although the lesion may develop as a primary affection, it is much more frequently the re-

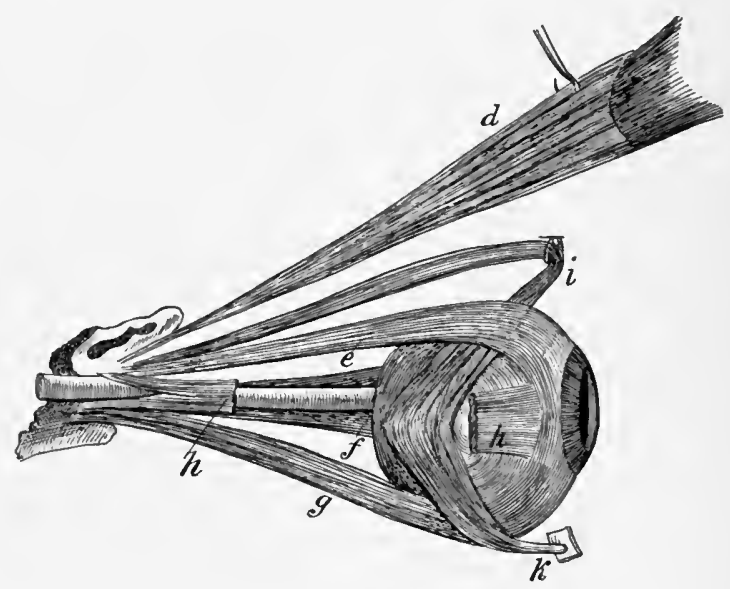

Fig. 158.-Lateral view of the ocular muscles (Nunneley). The external rectus $(h)$ is divided, so as to show the attachments of the oblique muscles $(i, k)$ to the eyeball. The attachments of the four recti muscles $(e, f, g, h)$, the superior oblique $(i)$, and the levator palpebra $(d)$ at the apex of the orbit are also shown.

sult of pathological processes in neighboring structures, which involve secondarily, by compression or otherwise, the nerves or their nuclei. In orbital paralyses, the nerve-lesion may be primary, the result of exposure to cold, for example, or it may be secondary to other disease, such as periostitis or gumma at the apex of the orbit.

Paralysis of the External Rectus Muscle (Paralysis of the Sixth Nerve).-This is the commonest of the ocular 
palsies. The lesion is usually orbital-an inflammation of the nerve itself or of its sheath-and is commonly the result of exposure to cold. If the paralysis is complete, there will be an easily recognized inward squint of the affected eye (Fig. 159), which will become more pronounced if an effort is made to rotate the eyes in the direction of the paralyzed muscle. Slight pain in the region of the orbit or one-sided headache may be complained of, but the chief complaint will be of diplopia, which is commonly attended by vertigo and not infrequently by nausea. If the paralysis is incomplete, the
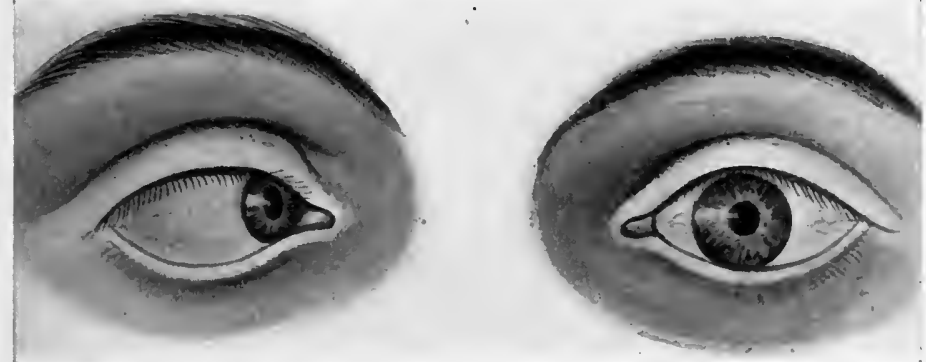

Fig. 159.-Convergent strabismus (Dalrymple).

squint will be evident, and the diplopia will manifest itself, only when the eyes are turned toward the affected muscle. Closure of either eye will cause the diplopia to disappear. The affection is nearly always unilateral, and the prognosis is distinctly favorable.

Treatment.-The most efficacious remedy is potassium iodid in moderate-five- to ten-grain-doses. In conjunction with this strychnin may be given. The application of a blister to the temple has also seemed to me to be beneficial. In order to get rid of the annoyance caused by the diplopia, the affected eye should be excluded from vision by means of a patch or a ground glass. 
Paralysis of the Superior Oblique Muscle (Paralysis of the Fourth Nerve).-This is much less common than paralysis of the external rectus, and is not so easily diagnosticated. Although it may arise from any of the causes mentioned as capable of producing ocular palsies, in its etiology and pathology it usually resembles the affection just described, and the prognosis is equally favorable.

As the action of the superior oblique, besides rotating the eye about its sagittal axis so that the upper extremity of the vertical meridian is inclined inward, is to turn the eye downward and outward, the diplopia and squint which attend paralysis of this muscle are more marked, or may occur only, on looking down As this is true also of paralysis of the inferior rectus, the differentiation of the two conditions is to be made only by carefully taking into account the character of the diplopia, whether the images are "crossed" or "homonymous," and whether the "false image" tilts toward or away from the median line; but as such tests do not fall within the province of the general practitioner, a detailed description of them may be omitted, especially as the treatment of the two conditions is essentially the same.

Treatment. - The therapeutic measures recommended in paralysis of the external rectus are indicated.

Paralysis of the Oculomotorius or $\mathcal{T}$ hird Nerve. -Next to paralysis of the sixth nerve, this is the commonest of the ocular paralyses. It is oftenest dependent upon acquired syphilis, is occasionally bilateral, and is not infrequently accompanied by palsy of other motor nerves of the eye. When all the branches of the nerve are involved a striking and very characteristic picture is exhibited. From loss of power of the levator palpebræ 
the upper lid droops, and cannot be elevated (ptosis) (see Fig. 36). The pupil is semidilated and near vision greatly impaired, because the sphincter pupillæ and the muscle of accommodation are paralyzed; and, as all the extrinsic eye-muscles, except the external rectus and the superior oblique, are affected, there is a downward and outward squint, with inability to turn the eye upward, inward, or directly downward. Diplopia is not complained of because the drooping lid excludes the affected eye from participation in vision; it becomes manifest, however, when the lid is held up. If the fourth and sixth nerves are also involved there will be inability to move the eye in any direction, and; especially in this condition, known as opbthalmoplegia totalis, there will be marked exophthalmos, since the eyeball is deprived of the restraining influence of all of the recti muscles.

Not infrequently only the extrinsic muscles supplied by the third nerve are affected, and the intrinsic muscles, the sphincter pupillæ and the ciliary muscle, escape. This type of paralysis, known as opbthalmoplegia externa, is necessarily of nuclear origin, and finds its explanation in the fact that the nuclei for the sphincter pupillæ and the ciliary muscle lie appreciably in front of those for the extrinsic muscles. For the same reason it may happen that the extrinsic muscles escape, while only the intrinsic muscles are involved. This constitutes the condition known as opbthalmoplegia interna, and is characterized by mydriasis and loss of power of accommodation. Diphtheria is oftenest responsible for this type of ocular paralysis, which has been considered in the preceding chapter, in treating of "anomalies of accommodation." Isolated paralysis of other muscles supplied by the third nerve is observed, but more rarely. 
It may result from orbital disease, or may be congenital in origin. Congenital ptosis, from paralysis or imperfect development of the levator palpebræ superioris (see Fig. 35), is a familiar example. It is usually bilateral, and is at times accompanied by congenital paralysis of the superior recti.

The prognosis in paralysis of the third nerve, especially when the affection is of luetic origin, is favorable, provided the requisite therapeutic measures are employed without delay.

Treatment.-Mercury and potassium iodid are, of course, the chief reliance in cases due to syphilis, and the latter is useful in other types of the disease. Strychnin may be given in combination with either of these agents, and some authorities have faith in the efficacy of electricity.

Operative procedures for the correction of paralytic squints and acquired ptosis are not to be resorted to hastily. Indeed, they should be employed only after other measures have been tried thoroughly, and have proved of no àvail.

Conjugate, or Associated, Ocular Paralyses.This interesting form of ocular palsy results from lesions which involve the centers for the associated movements of the eyes-the convergence center, for example, which presides over the conjoint action of the internal recti muscles, or the center which controls the conjoint action of the internal rectus of one eye and the external rectus of the other, and has to do with the lateral movements of the eyes. A lesion of the first-named center will annul the associated action of the internal recti, though these same muscles will act normally in rotating the eyes to the right or left. On the other hand, a lesion of the center for 
the lateral movements of the eyes will prevent their being turned toward the affected side, though the convergence movement remains intact. This latter condition usually leads to a more or less marked deflection of the eyes toward the unaffected side-conjugate deviation of the eyes. Conjugate paralyses are usually the result of destructive lesions of the brain, particularly cerebral hemorrhage.

The prognosis depends upon the seriousness of the cerebral lesion, to which the treatment is to be directed.

Nystagmus.- This affection, in which there is a rapid, oscillatory movement of the eyes, usually lateral, but more rarely vertical or rotary, is commonly of congenital origin. It is met with also as an acquired condition, and then usually develops in infancy or early childhood. An interesting form of acquired nystagmus occurs in miners, as a result of the abnormal conditions to which their eyes are subjected.

Congenital nystagmus, and this is true also of the nystagmus acquired in early childhood, is commonly associated with other congenital ocular defects, such as zonular cataract, coloboma of the choroid, corneal opacities, albinism, refractive errors of high grade, etc. Like the conjugate paralyses just described, this affection also is dependent upon an abnormal condition of the centers which preside over the associated movements of the eyes.

Although from a cosmetic point of view nystagmus is of moment, it seems, of itself, except, perhaps, in the case of miner's nystagmus, to give rise to no subjective inconvenience. It may be added in parenthesis, however, that it is the bete noire of the ophthalmoscopist and of the "refractionist," so called.

Treatment.-Little can be done for the amelioration 
of congenital or early acquired nystagmus, except to correct carefully any refractive fault that may be present. Miner's nystagmus may disappear in time as a - result of a change of occupation.

Concomitant Squint (Strabismus Concomitans). -A concomitant squint, as opposed to a paralytic squint, is one in which the squint remains constant in degree in whatever direction the eyes may be turned; that is, the squinting eye always follows the movements of the fixing eye. In paralytic squint, as has been explained, this is not the case. The squinting eye does not follow in all directions the movements of the fixing eye, and therefore the squint varies in amount, increasing when the eyes are turned in the direction of the paralyzed muscle, and diminishing or, perhaps, disappearing when they are turned in the opposite direction.

The misdirected eye in concomitant squint may turn in or out, upward or downward, or it may squint both vertically and laterally. The squint may be constant, or it may be inconstant, or periodic, as it is termed. It may affect always the same eye, or it may be alternating - may shift from one eye to the other. It is never bilateral, since one or the other eye must necessarily be directed toward the object regarded. Diplopia is rarely complained of in concomitant squint, because, in the first place, the fault usually develops in early childhood, and, in the next place, in the young the habit of mentally suppressing the image formed in the squinting eye is very quickly acquired.

Reference has already been made to the causes of concomitant squint. Enumerated in the order of their importance, they are: anomalies of refraction, congenital muscular defects (insufficiencies), acquired mus- 
cular defects. A marked difference in the visual acuteness of the two eyes also tends to promote the development of squint, and so does a pronounced difference in their refraction.

Apart from the deformity which attends it, the most serious consequence of concomitant squint is the amblyopia of the squinting eye to which it gives rise. It is true, there are those who contend that the amblyopia nearly always found in the squinting eye (in non-alternating strabismus) is a cause, rather than a consequence, of the squint; but, to my mind, the evidence to the contrary is so strong as to be practically conclusive.*

It is a mistake to designate, as most writers do, the amblyopia of a squinting eye as "amblyopia exanopsia," for it is essentially different in its origin from this condition. As an example of true amblyopia exanopsia, or, in other words, of amblyopia from non-use of an eye, may be cited the impairment of vision which results from permitting a monocular, congenital cataract to remain too long unoperated upon. Under such circumstances, the cataractous eye becomes amblyopic simply because its retina and optic nerve are not exercised as they should be. In concomitant squint something very different from this occurs. In order to get rid of the diplopia, which at the outset necessarily manifests itself each time that the as-yet-not-fully-established squint recurs, an active mental effort is made to suppress the image formed upon the retina of the squinting eye; and in the young this effort proves so successful that in a comparatively short time the diplopia disap-

* The author's views upon this point, and the grounds upon which they are based, are set forth in a paper "The Amblyopia of Squinting Eyes: Is it a Determining Cause or a Consequence of the Squint ?" published in the "Medical News" of September 4, I886, and in the "Trans. of the American Ophthalmological Society" for I 886. 
pears, and concurrently with this the sight of the squinting eyes becomes markedly impaired.

As pointed out in the paper to which reference has been made, the regional character of the amblyopia found in squinting eyes is very significant, and affords strong evidence in support of the view that it is an acquired, and not a congenital, condition, and that it is produced in the manner just described.

If we ask, what must be the chief sources of annoyance to an individual who has just begun to squint, we must conclude that he has two especial difficulties to contend with-one, the doubling of every object upon which he fixes his attention, in consequence of the "false" position of the retinal image of this object in the squinting eye; the other, the confusion of vision which must result from the images of different objects falling upon the macular region of the two eyes.

To get rid of these annoyances he has a twofold task to accomplish: To eliminate the diplopia, he must induce that part of the retina of the squinting eye that habitually receives the false image of the object he is regarding with the other eye not to take cognizance of this image; to prevent the image of some object which he is not regarding from being (mentally) superposed and fused with the object which he is regarding, he must ignore all images formed upon the macula of the squinting eye. The successful accomplishment of this task will result in the production of an amblyopia in the squinting eye regional in charactermost marked (I) in the neighborhood of the macula and (2) in that portion of the retina which receives the false image of the object regarded by the properly directed eye. Now, as a matter of fact, the amblyopia found in squinting eyes does exhibit just these characteristics, 
and this circumstance seems to afford, as I have said, almost conclusive proof that the defect is not a congenital one, but is a product of the squint.*

This question of the origin of the amblyopia of squinting eyes is one not merely of theoretical interest, but of practical importance; for, if it can be established that the amblyopia is a consequence of the squint, the advisability of early operation would hardly be open to doubt.

It is a fortunate circumstance when a squint shows a disposition to alternate, or when, as sometimes happens, the eye which squints in distant vision is the fixing eye in near vision, and vice versa; for, under such circumstances, both eyes usually retain good vision. A marked difference in the refraction of the two eyes also has a tendency to lessen the likelihood of the squinting eye becoming amblyopic, because the retinal image in this eye, being ill-defined, causes less annoyance, and for this reason is not so energetically "suppressed."

Concomitant squint is always alternating in the sense that if the fixing eye is covered the squint shifts to this eye, and the other eye, for the moment, becomes the fixing eye. It is true the fixation with this eye is often uncertain, and may be eccentric, because of its regional amblyopia. The "secondary" squint thus induced

* It is an interesting fact, and one having an important bearing upon the question under consideration, that when a squint, which for years has been unattended by diplopia, is over-corrected by too free tenotomizing - so that, for example, a convergent squint is converted into a divergent one-a persistent and annoying diplopia not infrequently manifests itself. The explanation, of course, is that owing to the change in the direction of the squint the false image now falls upon an entirely different part of the retina, a part which has not learned to ignore images formed upon it, and, therefore, is not amblyopic. The diplopia which it is usually possible to produce in a strabismic individual by causing the false image of a candle flame, by means of a prism, to fall upon an unusual part of the retina is to be explained in the same way, and is equally significant. 
in the usually properly directed eye commonly equals in degree the "primary" squint; but, especially in convergent strabismus, it is not infrequently greater in amount, because the ciliary muscle of the squinting eye is not exercised as habitually as that of the fixing eye, and must, therefore, put forth a greater effort in order to obtain a sharply defined retinal picture. The result is the same when the refractive error is considerably greater in the squinting eye. On the other hand, when, as is seldom the case, the refractive error in this eye is considerably less than in the fixing eye the secondary squint may be less than the primary.

The detection of a pronounced squint is not a difficult matter; but, if one relies solely upon "appearances," it is easy to fall into error. For not only is it often impossible to recognize in this way slight degrees of squint, but there is a simulation of convergent squint in myopia of high grade, and of divergent squint in hypermetropia of like character, "calculated" to deceive even the elect.

The "cover-test" affords, except when the fault is very slight in degree, a trustworthy means of determining the existence or non-existence of squint, and it is not difficult of application. The patient, with both eyes open, is directed to gaze fixedly upon a candle-flame ten to twenty feet away. The supposedly fixing eye is then covered quickly with a small screen, while the other eye is watched closely to see if it moves, in order to "fix" the flame. If it has been squinting previously it is obvious that it must make a "movement of correction," as it is termed, in order to look directly at the candleflame. In very low degrees of squint this correcting movement is so slight that it may be difficult to detect; but, except under such circumstances, it is easily observed, and when observed the existence of a squint is 
proved. The direction of the movement will show the character of the squint-whether it is convergent, divergent, or vertical.

Convergent Concomitant Squint or Strabismus.This is the commonest variety of concomitant squint (see Fig. I 59). It nearly always develops in early childhood, when the eyes are beginning to be used in regarding near objects, and, except in rare instances, it is found in association with hypermetropia or hypermetropic astigmatism. Its etiology, and the rôle which hypermetropia plays in its production, have been considered in the preceding chapter (pages 396 and 397), in treating of the ill consequences of that refractive fault.

Donders, who, so far as the anomalies of refraction are concerned, brought order out of chaos, was the first to recognize the intimate dependence of concomitant convergent squint upon hypermetropia. $\mathrm{He}$, of course, realized that hypermetropia is a far more common condition than convergent squint, and he explained this fact-why some hypermetropes squint and so many do not - in a thoroughly satisfactory manner.* The desire for binocular vision and the abhorrence of diplopia, he tells us, suffice to prevent the occurrence of squint in the great majority of hypermetropes. Weakness of the internal recti muscles and, he might have added, exceptionally energetic accommodative power, tend to the same end. Among conditions conducing to its occurrence he mentions congenital weakness of the external recti muscles, congenital or acquired difference in the visual acuteness or the refractive condition of the two eyes, and the exceptionally large value of the angle alpha (the angle formed by the

* "The Anomalies of Accommodation and Refraction of the Eye," pp. 294 et seq. 
visual line and the axis of the cornea) in hypermetropia. .And, again, he might have added, inefficient, or subnormal, accommodative power.

It is evident why weakness of the internal recti muscles, and why exceptional power of accommodation, in association with hypermetropia, should render the occurrence of convergent squint less probable; and why insufficiency of the external recti muscles and subnormal accommodative power should favor its development. And since the advantages of, and the desire to maintain, binocular vision, as well as the annoyances arising from diplopia, are greatly lessened by the existence of a marked difference in the acuteness of vision or the refraction of the two eyes, it is not difficult to comprehend why each of these conditions should have a like effect. As to the influence exerted by a large angle alpha, it must be admitted that it is not so obvious.

What has been said as to "periodic" and "alternating" squint applies especially to the variety of squint under consideration. There can be little doubt that most cases of concomitant convergent squint, at the outset, are periodic, and the cases which remain so permanently are nearly always of this type, and this is true also of the majority of cases of alternating squint.

Although so frequently found in association with hypermetropia, concomitant convergent squint occurs, exceptionally, in myopia of high grade. In these seemingly anomalous cases there is, in the first place, doubtless a congenital lack of balance between the external and internal recti muscles-the former being insufficient and the latter possessing unusual strength. Then, it commonly happens that this type of squint is encountered in myopes who have used their eyes a great deal in near vision, without correcting glasses, holding 
the printed page, for example, very close to the eyes, and reading with forced convergence. The effect of this habitual overaction of the internal recti, and the attendant stretching of the externi, is to exaggerate the preponderance of the former, until finally there comes a time when, in regarding distant objects, the visual axes can not be brought into proper relation, and a convergent squint, which of ten continues to manifest itself only in distant vision, is established.

Treatment.-In view of the disfigurement which attends convergent squint, and the serious impairment of vision which nearly always results in the deviating eye, there seem to be the best of reasons why the defect should be corrected, with as little delay as possible; and, it may be added, there are few cases in which this can not be done, provided the remedial measures indicated are employed as promptly as they should be.

These measures consist in the adjustment of glasses for the correction of the usually present refractive fault, and in operative procedures upon the ocular muscles. And here it may be well to state, parenthetically, that, in view of the interdependence of the two conditions, no one who is incapable of accurately determining and correcting refractive anomalies is justified in operating for concomitant squint.

Only in exceptional instances can concomitant convergent squint be dealt with satisfactorily, without the aid of glasses. Whether it can be corrected by glasses alone, without operation, depends largely upon the character and duration of the squint, as well as upon the degree of the refractive error and the visual acuteness of the deviating eye. As a general truth, it may be said that when a squint has passed the periodic stage, and has become firmly established, 
it is seldom possible to correct it without operation. The cases likely to prove exceptions to this rule are those in which hypermetropia of high grade exists, with comparatively good central vision in the deviating eye, such as is usually found in alternating squint. On the other hand, when the squint is still in the formative stage, is still periodic in character, it is always possible to correct it by glasses alone; although, even under such circumstances, an operation may be indicated to improve the muscle-balance and relieve asthenopic symptoms In every case of squint, whether an operation is to be performed or not, a cycloplegic should be employed, and the refractive condition and visual acuteness of each eye should be carefully determined. If astigmatism is present it, as well as the general refractive fault, must be corrected.

The ideal result aimed at in the treatment of squint is the re-establishment of binocular vision. Whether this can be accomplished or not depends, in great measure, upon the character and degree of the amblyopia in the deviating eye: In some instances, too, we have to contend with a positive disinclination to fuse images formed in the two eyes upon identical retinal points. As a result of operation the muscle-balance may be practically normal, and yet the squinting eye may show no inclination whatever to "fix" the object which the properly directed eye is regarding. The condition is a discouraging one, and can seldom be overcome. Systematic exercise of the vision of the eye which is at fault, the other being carefully excluded, offers the only hope of accomplishing this. If, on the other hand, the sight of the deviating eye is not greatly impaired, and an inclination to binocular fixation exists, the ideal result spoken of can in most instances be attained. In securing this 
result we are greatly aided often by combining esophoric prisms with the lenses which correct the refractive error.

As to the operative procedure best adapted to the correction of convergent squint there is not a unanimity of opinion. There are those who prefer the operation of advancement, though the great majority of ophthalmic surgeons prefer tenotomy. My own decided preference is for tenotomy, and I resort to advancement only exceptionally, and usually to increase the effect of a previously performed tenotomy. The advantages of tenotomy are that it is a simpler procedure, involving considerably less traumatism and much less pain than advancement; that its effect can be more exactly gauged; that, if necessary, it can be repeated more readily; and that it accomplishes the end in view, at least, as satisfactorily.

Of the several methods of performing tenotomy the simplest-the operation of Arlt-it seems to me, is decidedly the best, since it is the easiest to execute and the least painful, and its effect can be graduated with much precision. For its performance there are required a speculum * (Fig. I60), a pair of straight, slightly blunt-pointed scissors (Fig. I6I), a strabismushook (Fig. I62), and delicate straight forceps, with teeth -two on one blade and one on the other-that project but slightly (Fig. 163). If the conjunctival wound is to be closed by a stitch, a curved needle and a suitable

* This speculum, contrived by the late Dr. Russell Murdoch, of Baltimore, should be used far more generally than it is. Especially for delicate operations, such as extraction of cataract, it is, in my opinion, decidedly the best speculum that we have. Its advantages are that it is self-locking, that it exerts a minimum degree of pressure upon the eye, that it can be easily and quickly removed from between the lids, and that it affords an exceptionally unobstructed field to the operator. 
needle-holder (Fig. I64) should also be provided. The steps of the operation are as follows: The speculum

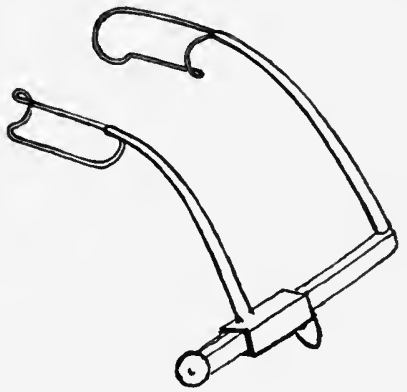

Fig. I60.-Murdoch's self-locking speculum (about two-thirds actual size).

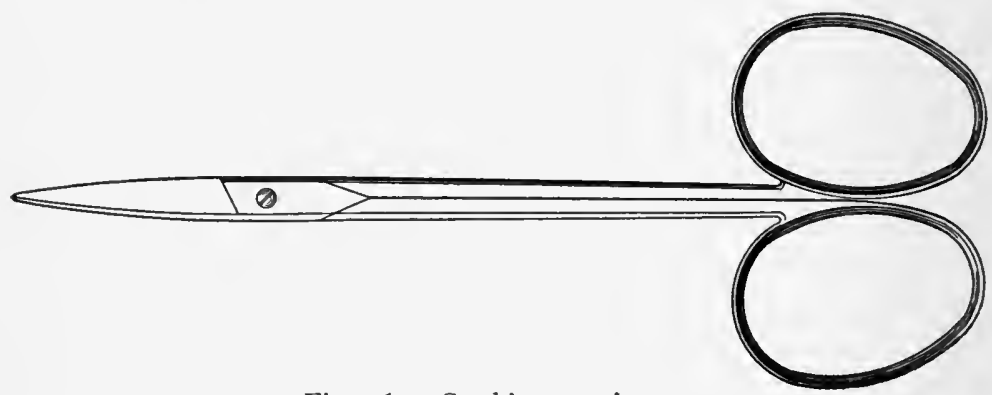

Fig. I6I.-Strabismus scissors.

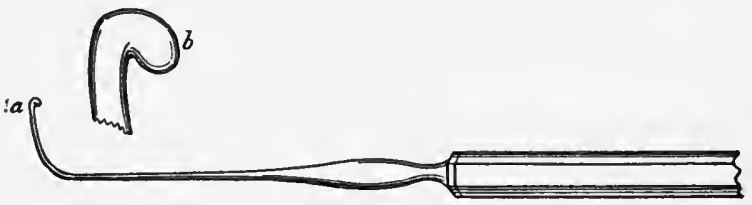

Fig. 162.-Author's crochet-pointed strabismus hook: $a$, Actual size of hook; $b$, enlarged view of crochet point.

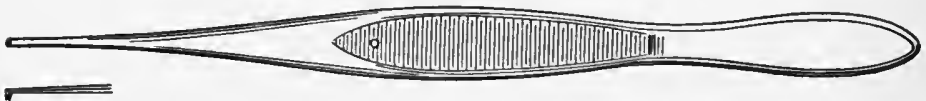

Fig. 163. - Strabismus forceps.

having been introduced, the conjunctiva just over the insertion of the tendon to be divided is seized with the 
forceps, care being exercised not to include in their grasp the underlying fascia, and with the scissors a vertical incision, somewhat less than $\mathrm{I} \mathrm{cm}$. in length, is made in the slightly elevated conjunctiva (Fig. 165). The points of the scissors are next introduced through this opening, and the conjunctiva is rather widely separated from the underlying fascia. This step is especially important in operating upon the rectus internus, because, if it is donethoroughly,--the scissor-points being carried well toward the inner canthus - the unsightly sinking of the caruncle

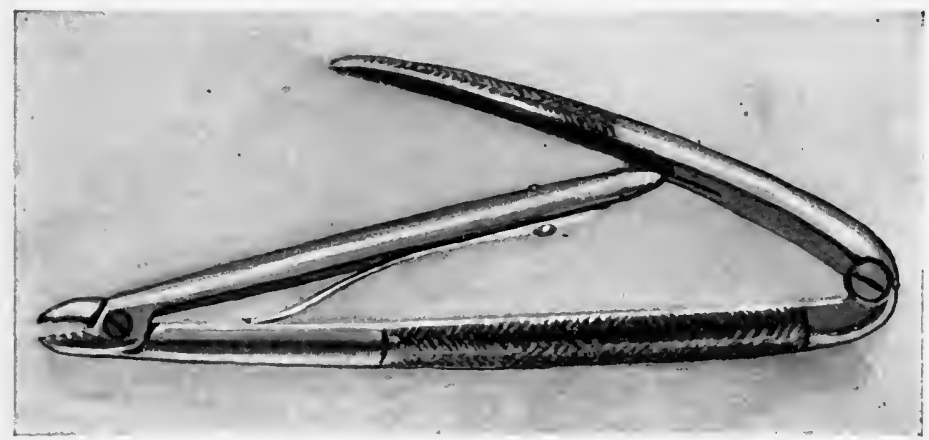

Fig. 164.-Author's needle-holder.

so often observed in awkwardly executed tenotomies is entirely obviated. The exposed tendon is now seized just back of its attachment, is somewhat elevated, and is separated from the sclera by a few snips of the scissors (Fig. I66). If the tenotomy is intended to produce only a moderate effect, the operation, except for the closure of the conjunctival wound, may stop at this point; but if, as is usually the case in operations for squint, a more decided effect is desired, the incision, previously limited to the tendon, must be extended, both upward and downward, through Tenon's capsule. And here, for the first 
time, to facilitate this last step of the operation, it becomes necessary to use the strabismus-hook. And this is one of the chief advantages of Arlt's operation, for the manipulations with the hook are more apt to cause

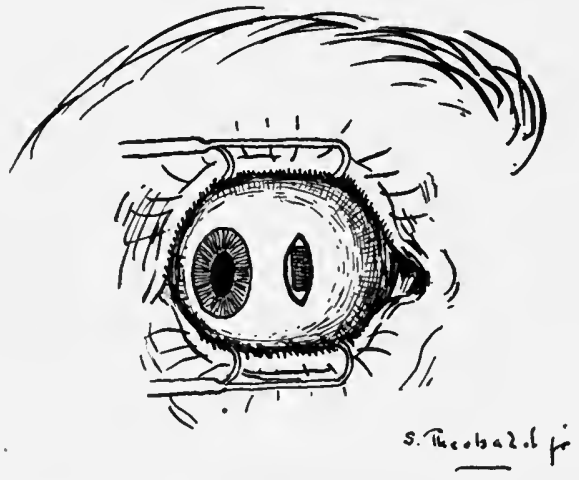

Fig. I 65.-Tenotomy of the internal rectus of the right eye by Arlt's method. The first step of the operation-the conjunctival incision-completed, exposing the tendon.

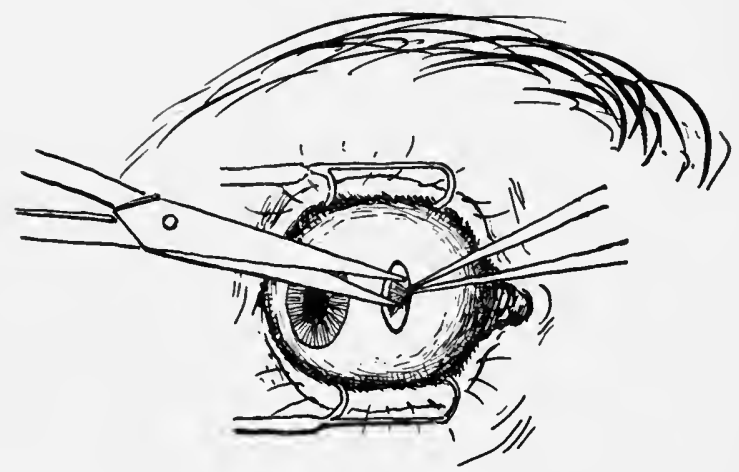

Fig. I66.-Dissection of the tendon from its scleral attachment. (The operator is standing behind the patient.)

pain than the actual cutting with the scissors, and therefore it is desirable that it should be employed as little as possible.

The conjunctival wound is now brought together by 
means of a single stitch of fine black silk (Fig. 167)except in young children, because with them the removal of the stitch is usually attended with considerable difficulty -and a light compress bandage is applied (see Fig. Io). On the following day the bandage is dispensed with, and after three days the stitch is removed.

With the eye thoroughly under the influence of cocain, and with delicacy of manipulation, this operation in most instances is practically painless. In children, simply because it is impossible otherwise to control

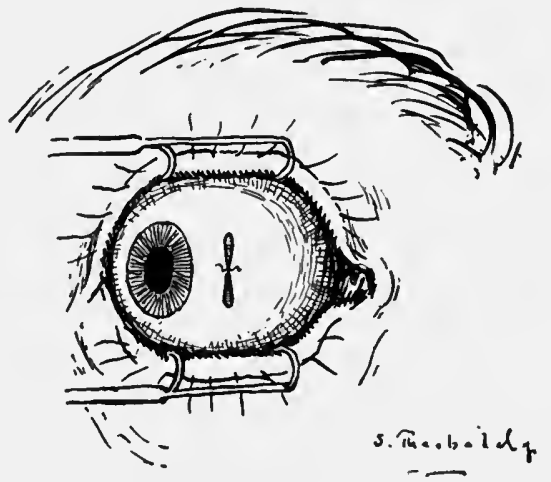

Fig. I67.-The operation completed. Conjunctival wound closed by a single black-silk suture.

their movements, general anesthesia is required; but with adults this is never necessary. Alternating with the applications of cocain, it is advantageous to make a few instillations of a I : I 000 adrenalin solution, as this lessens, and sometimes entirely obviates, hemorrhage, besides increasing the anesthetic action of the cocain. Since in skilful hands, and with proper antiseptic precautions, the operation may be said also to be practically without risk-infection being almost unheard of-there seems to be no good reason why it should 
not be resorted to whenever there is a clear indication for its performance.

Should the cutting of a single muscle leave, as it often does, a residual squint, either of two courses may be adopted. The internal rectus of the opposite eye may be tenotomized somewhat less freely, or an endeavor may be made to overcome the residual defect by correcting the usually present refractive error and combining esophoric prisms with the required lenses. The latter course is to be preferred, if the residual squint is slight and the refractive error of high degree; the former, if there is still a decided squint left, and especially if there is not a considerable amount of hypermetropia to be corrected.

In former times, when operations for squint were done in clumsy fashion,- - the muscle being divided at a considerable distance behind its point of attachment to the sclera,-and the refractive state of the eyes was ignored, it frequently happened that the eye operated upon "went the wrong way," as it was expressed, a scarcely less unsightly divergent squint being substituted for the previously existing convergent squint. In consequence of this the operation fell into well-deserved disrepute, which to the present day it has not entirely outgrown. It is scarcely necessary to point out that the modern operation is a wholly different procedure, and that in skilful hands it is attended by no such risk.

It only remains to add that the earlier a convergent squint can be corrected the better; since, other things being equal, the longer it is allowed to exist the greater will be the amblyopia of the squinting eye. We are unquestionably handicapped in operating at so early an age that the help afforded by glasses can not be availed 
of; but, notwithstanding this fact, I believe more is lost than is gained by postponing operation.

Divergent Concomitant Squint or Strabismus. -In the preceding chapter it has been pointed out that myopia plays almost as important a rôle in the causation of divergent squint (Fig. I68) as hypermetropia does in that of convergent squint, and an explanation of how this occurs has been given. The importance of this influence is shown by the fact that myopia exists in about two-thirds of all cases of divergent squint. Other factors which conduce to the development of this variety of strabismus are congenital or acquired insufficiency

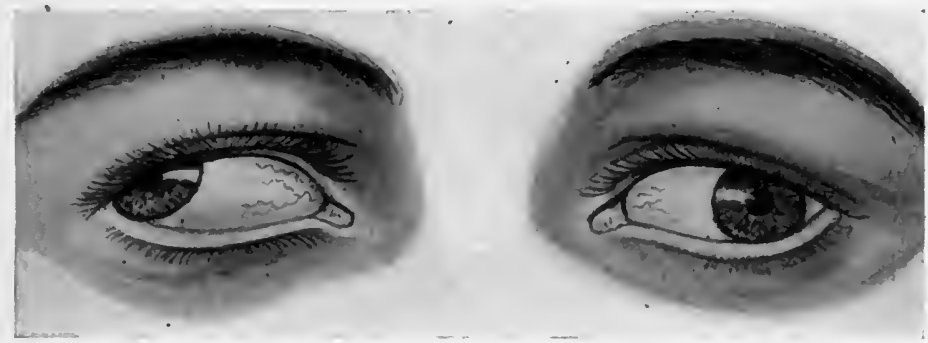

Fig. I68.-Divergent strabismus (Dalrymple).

of the internal recti muscles and marked difference in the visual acuteness of the two eyes.

When, from any cause, the sight of one eye is decidedly impaired, so that the advantages of, and the disposition to maintain, binocular vision are in large measure lost, the defective eye is prone to squint in one direction or another. It will squint inward, if the external recti muscles are relatively weak, or if the better eye is decidedly hypermetropic. On the other hand, if there is insufficiency of the internal recti or the better eye is myopic, a divergent squint is very apt to occur. Unlike convergent squint, divergent squint, which is 
less common, usually develops in adults, and is observed only exceptionally in children.

In myopia of considerable degree, because of the nearness of the far-point of distinct vision and the altered relation of accommodation and convergence, the difficulty of maintaining binocular fixation is greatest in near vision, as in reading, writing, etc. For this reason it often happens that the squint manifests itself at first only when near objects are regarded. Indeed, it is not uncommon for it to continue to occur only under such circumstances, binocular fixation being maintained in distant vision. At the outset the squint is usually periodic, and only after a considerable time becomes constant. It is seldom alternating, except in the sense that one eye may be used in distant, the other in near, vision. In divergent concomitant squint, probably because the true and false images are usually so far apart, there is but little complaint of diplopia, and for this reason, and because the defect seldom develops in childhood, the regional amblyopia characteristic of the convergently squinting eye is rarely observed.

Treatment.-Only exceptionally is it possible to correct a divergent squint without operation. The cases in which this may be accomplished are usually those in which a marked difference exists between the refractive condition of the two eyes, or in which the squint is associated with myopia of considerable degree, and occurs only in near vision. In the condition first mentioned the blurred image formed upon the macula of the more ametropic eye proves a source of annoyance, and the squint may be the expression of a desire to get rid of this annoyance rather than a result of the existence of decided muscular imbalance. If, therefore, by means of glasses the vision of each eye can be brought up to about the 
same standard, the squint not infrequently will disappear, and the eyes thereafter will work in harmony.* In the cases associated with myopia of considerable degree, in which the squint occurs only in near vision, glasses that correct a part of the near-sightedness, by removing the far-point of distinct vision to a comfortable distance from the eyes, and by restoring more nearly the normal relation between accommodation and convergence, will render binocular fixation very much easier, and may cause the disappearance of the squint.

Such cases, however, as has been said, are exceptional, and, as a rule, the surgeon may congratulate himself if, even by the help of an operation, he is able to correct a divergent squint so exactly as to re-establish comfortable binocular vision. In high degrees of myopia it is not always best to attempt this, for the progress of the myopia is often favorably influenced by the abandonment of binocular fixation and the convergence tension which it implies.

Divergent squint can not so surely be corrected by tenotomy alone as can convergent squint. Not infrequently tenotomy of the external rectus must be supplemented by advancement of the internal rectus. However, free tenotomy of both external recti muscles, not, as a rule, performed at the same time, though this may be warrantable, often yields a very satisfactory result, even in cases in which the squint is pronounced. The procedure of Arlt, already described, it should be stated, is as well adapted to the correction of divergent, as it is to that of convergent, squint. In securing the effect desired glasses afford valuable assistance, especially in myopic cases and in cases in which there is ani-

* In the "Johns Hopkins Hospital Bulletin" for April, r 89o, Vol. I, No. 4, the author has reported several cases of this character. 
sometropia. With the lenses called for by the refractive fault exophoric prisms may often be combined with advantage.

When the sight of the squinting eye is very defective, a lessening of the deformity is the most that can be hoped for from operation, since under such circumstances the restoration of binocular vision is not to be expected. Even for this purpose, however, a tenotomy may be justifiable, since the cosmetic effect is often very gratifying.

Vertical concomitant squint is rare, and is usually the result of a precedent paralysis of one of the oblique muscles, or of one of the superior or inferior recti muscles. It is not uncommon, however, in both convergent and divergent squint, to find a considerable amount of vertical deviation associated with the lateral fault.

Treatment.-Vertical squint can seldom be corrected without operation, and is more difficult to deal with than lateral squint, because, in the first place, glasses do not afford us the same help in securing the desired result, and, in the next place, we can depend upon the eyes, themselves, for comparatively little assistance, since they are capable of overcoming but a slight residuum of vertical squint.

\section{LATENT MUSCULAR ANOMALIES.}

Under this head are included, as has been explained, all the varieties of heterophoria, or, in other words, all the muscular anomalies of the eyes, in which, despite a tendency to squint, binocular vision is maintained.

Although heterophoric conditions, because less common, are not so frequent a cause of asthenopia as are errors of refraction, they are quite as capable of pro- 
ducing the manifold symptoms, local and remote, which we have learned to attribute to eye-strain. Among the ocular disturbances to which they give rise may be mentioned pain, intermittent blurring of vision,- - attended at times by diplopia,-conjunctival hyperemia, and blepharitis marginalis; among the more remote, headache, vertigo, nausea, neurasthenia, insomnia, and indigestion. When associated with ametropia, they may greatly aggravate the ill consequences of the refractive fault; but, to their credit be it said, they may exert exactly the contrary effect, as, for example, when a not too considerable exophoria is associated with hypermetropia, or a not too pronounced esophoria with myopia.

Of the several varieties of heterophoria-esophoria, exophoria, and hyperphoria-the last named, in which there is a tendency to vertical squint, is the one most sure to give rise to unpleasant consequences, since the eyes are less capable of coping with it successfully. However, both esophoria and exophoria often cause marked asthenopia, headache, etc., and the latter condition, when associated with myopia, exerts a further baneful influence, since it tends to promote the increase of the refractive fault.

It has been stated that a very considerable part of the latent muscular anomalies of the eyes are due to errors of refraction. It is equally true that a not inconsiderable part are wholly independent of refractive errors. Those which belong to the first-mentioned class may properly be termed apparent, those which belong to the latter class actual, muscular anomalies.

There are extremists who contend that refractive errors are responsible for all latent muscular anomalies, not of paralytic origin. There are other extremists who 
hold exactly the opposite view, who belittle the influence which errors of refraction exert upon muscular faults, and who do not hesitate to tenotomize, let us say, the internal rectus for an apparent esophoria, without having determined, with even approximate accuracy, the refractive condition of the eyes. The truth lies between these extremes. It seems, indeed, inexplicable that any one who has had experience in ophthalmic practice, and whose powers of observation are not below mediocrity, should, on the one hand, deny the reality of muscular insufficiencies, or, on the other, fail to recognize the important rôle which errors of refraction play in the causation of heterophoria.

While it is true that refractive errors, when present, markedly influence real muscular faults, there are muscular faults, as has been said, which are in no sense dependent upon ametropia, and which are just as real as ametropia itself. Such faults, it may be admitted, are not very often met with in emmetropic eyes; but this circumstance finds its explanation in the comparative rarity of emmetropia.

It is not a difficult matter to determine whether an observed muscular anomaly is actual or apparent. The first step, of course, is to ascertain the true refractive condition of the eyes, and to do this it is usually necessary to employ a cycloplegic. If the test fails to reveal the existence of ametropia, or if it shows an error of refraction incapable of producing the muscular anomaly, the reality of the latter is demonstrated. If, again, it reveals a refractive error which might be expected to cause the muscle-fault, this error must be corrected by glasses, and the test for muscular imbalance repeated. Should these tests now show a practically orthophoric condition, we may conclude that the muscular fault is 
not real, that it is wholly the result of the ametropia. Should they, on the other hand, still show an appreciable amount of heterophoria, we are warranted in regarding this residual fault, at least, as being real. It should be added that the muscle-balance tests just mentioned should be made not only while the eyes are under the influence of the cycloplegic, but after they have recovered fully from its effect.

From what has been said as to the significance of the latent muscular anomalies of the eyes, and as to the ill consequences to which they give rise, it is evident that their determination and correction are of prime importance; that, in fact, they demand attention as imperatively as do the faults of refraction.

As the determination of heterophoria does not fall within the province of the general practitioner, I have not thought it necessary to describe the muscle-balance tests to which reference has been made. I may say, however, in this con-

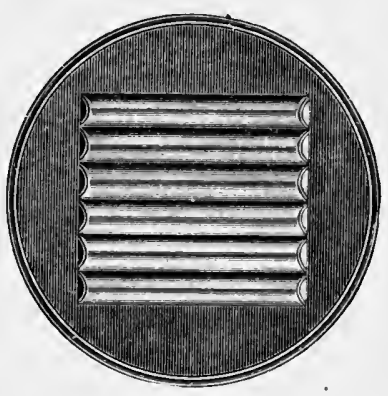

Fig. 169.-Multiple Maddox rod. nection, that I consider the simplest contrivances for measuring the muscle-balance the best, and that I have found no occasion to employ for this purpose cumbersome apparatus, such, for example, as the phorometer of Stevens. My chief reliance, in the determination of hyperphoria, is upon the multiple Maddox rod (Fig. I69) and, in the measurement of esophoria and exophoria, upon the modified vertical diplopia test of von Graefe; and in applying these tests I make use of prisms taken from my trial case. The muscle-balance for near vision, I consider, should be 
determined with as much care as for distance, and in doing this, especially in searching for hyperphoria, I have found the "pin-hole" light of Schild,* used in conjunction with the Maddox rod, of great assistance (Figs. I 70 and 171 ).

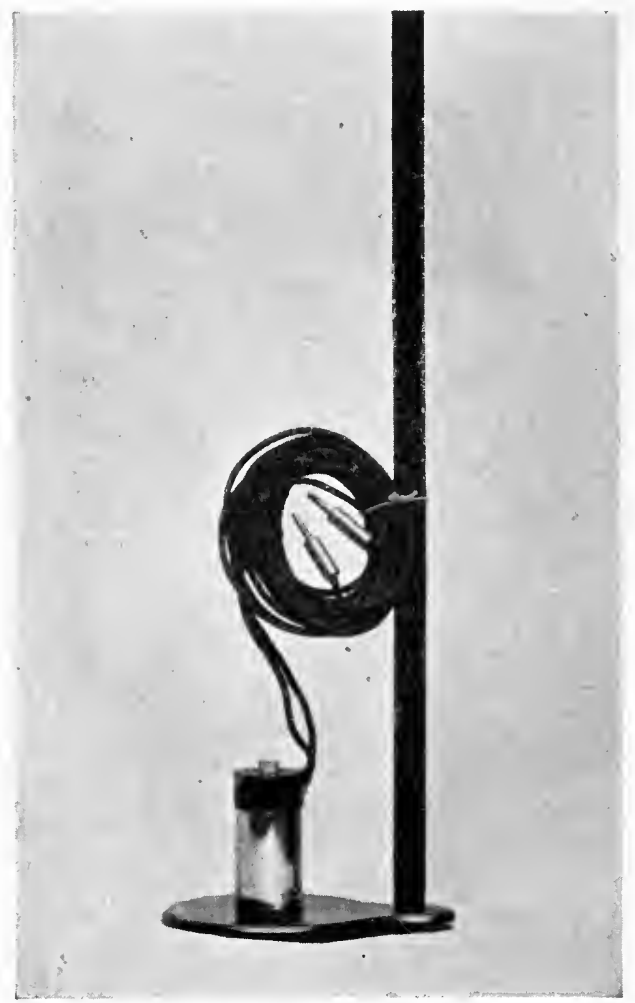

Fig. I70.-Schild's pin-hole electric light.

The correction of heterophoria is to be accomplished by glasses or by operative procedure. If the musclefault is unreal, if it is dependent wholly upon an error of refraction, it will disappear with the correction of the

* Described in "The Ophthalmic Record" for June, 1904, and made by Chas. A. Euker \& Co., 312 N. Howard St., Baltimore. 
ametropia. If it is real, prismatic glasses must be prescribed, or an operation performed-the former, if the fault is not marked; the latter, without hesitation, if it is pronounced. Although some ophthalmic surgeons are skeptical as to the advisability of operating for latent muscular anomalies, my own experience is that in suitable cases much can be accomplished by operation; and here, as in squint, my preference is for tenotomy of the stronger, rather than for advancement of the

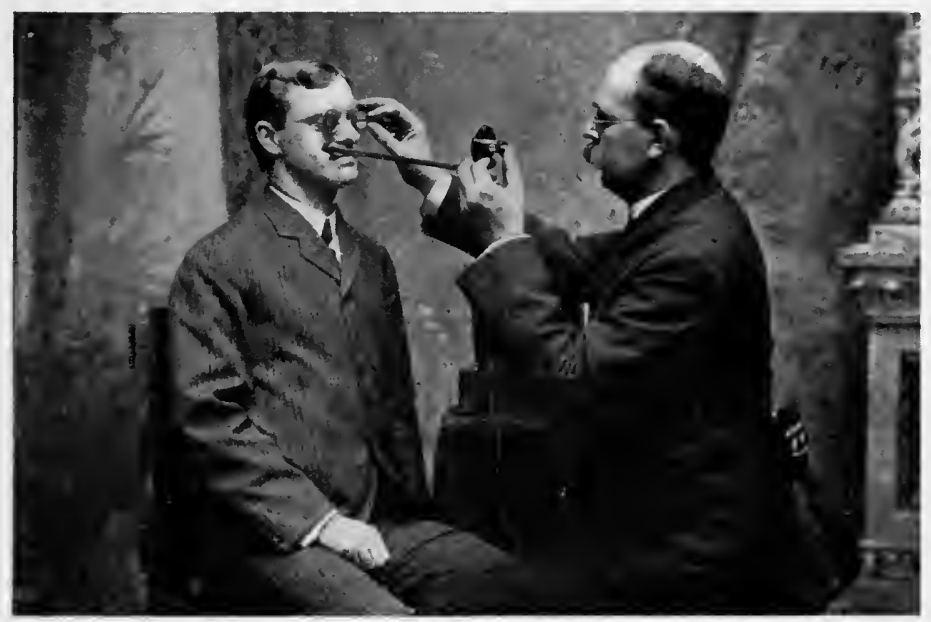

Fig. I 7r.-Schild's pin-hole light as employed in the Maddox-rod test, at the reading distance.

weaker, muscle, though exceptionally I deviate from this rule, especially in cases of marked exophoria.

Exophoria (Insufficiency of the Internal Recti Muscles).- Since myopia, as has been pointed out, plays so important a part in the causation of both apparent and actual insufficiency of the internal recti muscles, a careful test of the refraction should be made in every case of exophoria. If the test reveals a considerable amount of myopia, and especially if the exophoria is 
chiefly noticeable in near vision, the muscle-fault will often prove to be wholly, or in great part, unreal. If, on the other hand, it reveals emmetropia, and all the more if it shows hypermetropia or hypermetropic astigmatism, the reality of the muscular insufficiency is proved.

Treatment.-If the exophoria is associated with myopia, and is chiefly manifest in near vision, the partial or complete correction of the refractive error by glasses - the strength of the glasses to be determined not only by the degree of the myopia, but by the age and accommodative power of the individual and the amount of the muscular defect-will usually eliminate the exophoria, or reduce it to a facultative degree. If this is not the case, exophoric prisms must be combined with the glasses selected for near vision. If the muscular fault is present in both distant and near vision, and, as usually happens under such circumstances, is not eliminated by the correction of the myopia, prisms for constant use must be prescribed, or, if the fault is of such a degree as to warrant it, a guarded tenotomy of the external rectus or an advancement of the internal rectus must be made.

Exophoria occurring independently of myopia is susceptible of correction only by prisms or by operation. If it is not pronounced, or if it is present, as sometimes happens, only in near, or possibly only in distant, vision, the former method is indicated. If it is pronounced, and present in both far and near vision, a tenotomy of the external rectus of one or both eyes-a considerable interval, however, being allowed to elapse between the two operations - should be performed, and will commonly prove markedly beneficial. In extreme cases, as has been intimated, advancement of one or both of the internal recti muscles may be combined with tenotomy of the externi. 
Esophoria (Insufficiency of the External Recti Muscles).-In the causation of esophoria hypermetropia and hypermetropic astigmatism play even a more important rôle than myopia does in that of exophoria, and, since these faults are so prevalent, their existence should be suspected and sought for in every case in which there is apparent weakness of the external recti muscles.

The connection between hypermetropia and esophoria is the same as that between hypermetropia and convergent squint. In hypermetropia, as has been explained, the normal parallelism between the effort of accommodation and the effort of convergence is destroyed-accommodation is always in excess of convergence. Under such circumstances there is an everpresent tendency to restore the parallelism between these two functions. Convergent squint results when this tendency is more powerful than the desire to maintain binocular vision; esophoria, when it is less powerful, when the desire for binocular vision prevails. The abandonment of the effort to maintain binocular vision means, usually, the disappearance of the previously experienced asthenopia; the maintenance of binocular vision, with the attendant esophoria, means its persistence. The complete disappearance of the esophoria, as a result of the correction of the refractive error, proves that the external recti muscles were only seemingly at fault; its incomplete disappearance shows an actual insufficiency of these muscles. The existence of a considerable amount of hypermetropia, without esophoria, indicates one of three things-exceptionally energetic accommodative power, unusually strong external recti muscles, or actual insufficiency of the internal recti.

It must not be supposed, however, that esophoria is 
always dependent upon hypermetropia. On the contrary, marked examples of this fault are met with in emmetropes and in individuals who are but slightly hypermetropic, and other cases are encountered in which the full correction of the hypermetropia eliminates but a fraction of the muscle-fault. These are the cases in which there is actual insufficiency of the external recti muscles. Whether, under such circumstances, these muscles are imperfectly developed, or are attached to the eyeball in such fashion as to lessen their efficiency, or whether they are only relatively weak as compared with their opponents, the internal recti, or whether, finally, the fault is in their nervous supply, it is seldom possible to determine; but there can be little doubt that every case of real insufficiency of the external recti muscles finds its explanation in the existence of one, or, it may be, more than one, of these conditions.

The existence of esophoria and its degree are to be determined by the same tests that have been mentioned as best adapted to the measurement of exophoria, and here, too, the muscle-balance for near vision should be ascertained with as much care as for distance. It goes without saying that in every case of esophoria a careful test of the refraction should be made, and the effect upon the muscle-balance of the correction of any refractive anomaly that may be found noted; for only in this way can we know whether we are dealing with an actual, or merely an apparent, muscular fault.

Treatment.-From what has just been said, it is evident that the first step in the treatment of esophoria consists in the determination of the refractive condition of the eyes, and to this end a cycloplegic is usually necessary. If hypermetropia or astigmatism is discovered, this must be corrected, and the influence which this 
correction exerts upon the muscle-balance ascertained, the muscle-tests being made not only while the eyes are under the influence of the cycloplegic, but after they have recovered fully from its effect. If a marked error of refraction is found to be present-a hypermetropia, let us say, of 2 D. or 3 D., and especially if this is complicated by astigmatism - it is commonly best to be satisfied, for the time being, with a fairly full correction of this defect, even though this may leave a considerable residuum of esophoria; for, with the disappearance of the asthenopia and the tension of accommodation, it is not unusual to find a decided improvement in the muscle-balance. If, however, in spite of such correction, the asthenopia persists, and the residual esophoria does not diminish, one of two things must be done-either prisms must be combined with the previously prescribed lenses, or a tenotomy must be performed.

No hard and fast rule can be laid down as to when one and when the other of these measures should be resorted to; but, in general, it may be said that when more than $6^{\circ}$ of esophoria in distant vision, and its equivalent, $2^{\circ}$ or $3^{\circ}$, in near vision, remain after the correction of the refractive error, or when the asthenopic symptoms persist notwithstanding the correction of $4^{\circ}$ of esophoria by prisms, a tenotomy is indicated. This same rule as to the choice between prisms or a tenotomy, it may be added, holds good when no, or only a trivial, error of refraction exists.

It should be borne in mind, however, that the ocular muscle-balance is often markedly influenced by the general condition of the individual, and that a pronounced change for the better in this respect may follow temporary rest of the eyes, and a building up of the system by suitable tonics. One should hesitate, 
therefore, to resort to operation if there are reasonable grounds for supposing that the symptoms are but the expression of a run-down state of the system, of a general lack of muscular tone. When, as not infrequently happens, the muscle-balance is at fault in distant vision only, or, it may be, in near vision only, a tenotomy is contraindicated, since it must necessarily do as much harm as good, substituting for one sort of heterophoria another sort, quite as apt to give rise to annoying symptoms.

As to the advisability of operating at all for latent muscular anomalies there is still, as has already been mentioned, considerable diversity of opinion. My own convictions upon this point are very definite. Conservatism is to be commended, and an operation is not to be thought of until the influence of the refraction upon the muscle-balance has been carefully studied. When, however, this source of error has been eliminated, and there are clear indications, as I view them, for a tenotomy, I operate with the fullest confidence that marked benefit will result. When, some years ago, I first began to tenotomize non-squinting eyes, I confess I did so with some trepidation; but abundant experience has convinced me that few surgical procedures yield more gratifying results than a clearly indicated tenotomy for the relief of muscular asthenopia.

Perhaps, I should make it plain that by "tenotomy" I mean a real division of the tendon. There is, in my opinion, no room for the so-called graduated or partial tenotomies. If the muscle-fault is so trivial that it can be relieved by such a procedure, it is too trivial to require operation at all; it can be more satisfactorily dealt with by means of glasses. On the other hand, if it is sufficiently pronounced to demand operation, it will 
certainly not be relieved by a procedure which is little better than a pretence. A "guarded tenotomy," that is to say, one in which the tendon is completely divided, but the section is not extended to Tenon's capsule, is frequently indicated; but an operation which accomplishes less than this had better be left undone.

It has been a matter of surprise to me how much tendon-cutting is not only permissible, but is demanded, in certain cases of lateral heterophoria. Not very infrequently in marked esophoria a free tenotomy of both internal recti muscles, and in pronounced exophoria an equally free division of both external recti muscles, will hardly suffice to restore a normal musclebalance, although a squint has never manifested itself. It is noteworthy that an actual squint is often corrected by less free tenotomizing than is sometimes demanded in these latent muscular faults.

Hyperphoria.-This condition, in which there is a suppressed inclination to vertical squint, is one of the most annoying of the latent muscular faults. From exophoria and esophoria it differs essentially in that it is but little influenced by the refractive condition of the eyes-anisometropia, in which the eyes are of unequal focus, being the only ametropic condition which seems to promote its development. More constantly, therefore, than the lateral forms of heterophoria it is distinctly a muscular fault. Not infrequently, it would seem, it is the result of an incompletely-recovered-from paralysis of one of the muscles which have to do with the vertical movements of the eyes. In other cases it is due to some congenital or acquired insufficiency of one of these same muscles.

Hyperphoria is capable of producing any and all of the distressing symptoms, local and remote, which have 
been described as arising from eye-strain, and, although it is not a common defect, it should be looked for in every case of asthenopia. Exceptionally it is present only in near, or it may be only in distant, vision; it should be sought for, therefore, in both. The best test which has been devised for its detection and measurement is the multiple rod of Maddox, supplemented in the tests for near vision, as has already been mentioned, by the pin-hole light of Schild.

Treatment.-Hyperphoria may be corrected by prisms or by operation. If the defect is pronounced, a guarded tenotomy should be performed. If it is not pronounced, it is much better to correct it by means of prisms. Experience has taught me that it is more difficult to predicate the effect of a tenotomy upon a superior or an inferior rectus muscle than of one upon either of the lateral recti. I am, therefore, little disposed to operate, as some surgeons do, for the correction of the lower degrees of vertical heterophoria. Such cases, that is to say, cases in which there are not more than $3^{\circ}$ or $4^{\circ}$ of hyperphoria, can almost always be dealt with satisfactorily by means of vertical prisms, either alone or in combination with such other correction as may be demanded. And even in the higher grades the tenotomy should be distinctly "guarded"; for it is better to accomplish too little-and later, perhaps, operate upon the other eye-than to do too much.

In operations upon the lateral muscles glasses usually afford us much assistance, and, besides, these muscles are more fully under the control of the will, so that a slight over-correction or under-correction is not a matter of moment, since the eyes come to our assistance, so to speak, and help us greatly in securing the result desired. In operations upon the vertical muscles this is not the 
case, and a slight over-correction will sometimes give quite as much discomfort as the original defect.

Again, it is not always possible to secure a result which is equally satisfactory in both far and near vision. If the superior rectus of the upward-tending eye be divided, just the effect desired in near vision may be obtained, but when the eyes are directed to distant objects there may be trouble. On the other hand, if the inferior rectus of the opposite eye be severed, all may be well in distant vision, but not in near vision. In a word, more caution is requisite, and less assurance as to the outcome can be felt, in operating for the correction of vertical, than for the correction of lateral, heterophoria.* At the same time, there are, unquestionably, cases in which an operation is clearly demanded, and in which relief can be obtained in no other way.

* In a paper, "Are tenotomies for hyperphoria necessarily more uncertain in their results than those for esophoria and exophoria," published in the "Trans. of the American Ophthalmological Society" for I903, and in the "Maryland Medical Journal," Jan., I904, the author's views upon this point have been more fully set forth, and illustrative cases reported. 


\section{CHAPTER XIII.}

\section{INJURIES OF THE EYE AND ITS APPENDAGES.}

Injuries of the eyelids, of moment, are not of common occurrence, and, therefore, the subject does not demand extended consideration. A chief aim in dealing with traumatic lesions of the lids is to prevent subsequent deformity, especially malposition of the

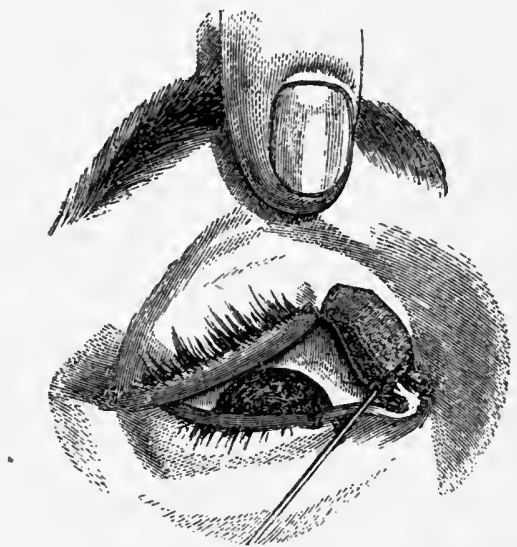

Fig. I 72.-Great deformity of the lid from a knife wound-the deformity being in large measure due to lack of early surgical attention (Lawson).

lid-margins and of the lacrimal puncta, since this is not only attended by considerable facial disfigurement, but is apt to give rise to troublesome epiphora (Fig. I 72). The occurrence of anchyloblepharon (adhesion of the lid-margins) (Fig. I 73) and of symblepharon (adhesion of the lid to the eyeball) is also to be carefully guarded against. As the tissues of the lids are very vascular, 480 
INJURIES OF THE EYE AND APPENDAGES. 48 I sloughing is not apt to occur, and stitches may be used freely to secure accurate apposition.

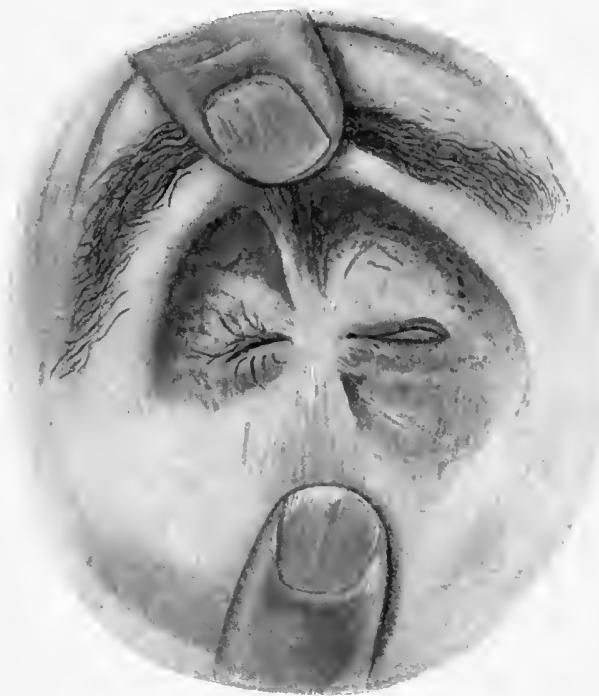

Fig. 173.-Symblepharon and anchyloblepharon following burn by molten iron (Haab).

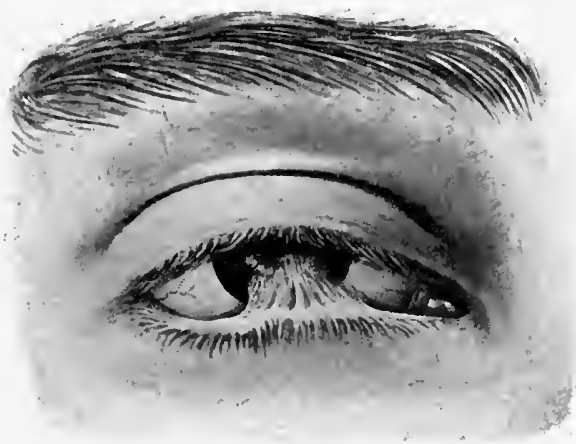

Fig. 174.-Symblepharon following lime burn (Hansell and Sweet).

Burns of the external surface of the lids are often difficult to deal with, and, if severe, are prone to produce ectropion. Burns of their conjunctival surface from 
caustic agents, molten metal, etc., tend to the production of entropion and symblepharon (Fig. I74).

Treatment.-Incised wounds of the lids (Fig. 175), after having been thoroughly cleansed with a I : 5000 sublimate solution, should be neatly closed by a liberal use of stitches (fine black silk), and dressed with silverfoil and collodion. Lacerated wounds commonly do better with a wet dressing - a gauze pad, wet with a saturated solution of boracic acid, and kept in place with a light bandage. The presence of any foreign substance in the wound should be carefully searched for. Loss of a considerable part of the external integument of the lid

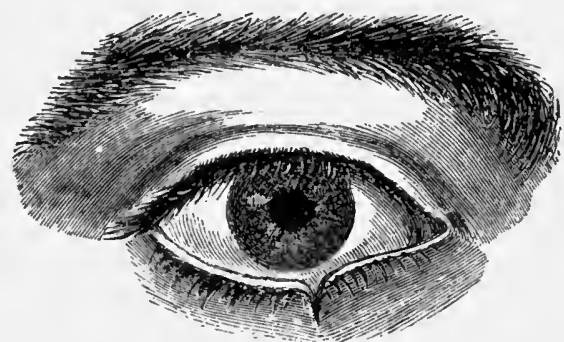

Fig. I 75.-Incised wound of the palpebral margin (Lawson).

must be replaced by skin grafts, the thin grafts of Thiersch being best adapted for this purpose. Slitting of the canaliculus, the lower one especially, is at times indicated for the relief of epiphora consequent upon wounds of the lids.

Burns of the external surface of the lids should be anointed with borated vaselin, to which cocain may be added, or they may be covered with absorbent gauze wet with a solution of sodium bicarbonate. Carbolized oil and lime-water is also a useful application. ' In burns of the palpebral or bulbar conjunctiva a solution of atropin (alkaloid) in castor-oil (gr. iv- $\_j$ ) is useful. 
Gunpowder burns are especially annoying from the disfigurement which they cause. Efforts to remove mechanically the disintegrated powder-grains are usually unsuccessful. If, however, these efforts are supplemented by the application of hydrogen peroxid to the stained tissue better results are obtained. The application is conveniently made by means of a sharp toothpick, armed with a little absorbent cotton.

Destruction of the external integument of the lids resulting from burns may call for Thiersch grafts, which are best applied after the burned surface has become covered with granulations. When the burn has involved the lid-margins or the palpebral and bulbar conjunctiva, the occurrence of anchyloblepharon and symblepharon should be guarded against by repeated separation of the opposed raw surfaces and the liberal application of vaselin.

Injuries of the Bulbar Conjunctiva.-Considerable wounds of the bulbar conjunctiva, without involvement of the deeper tunics of the eye, are rare. Occasionally, however, the conjunctiva may be so lacerated as to require the careful insertion of stitches, a procedure fraught with little or no risk.

Severe burns of the conjunctiva, from " concentrated lye," lime, strong acids, and molten metal (Fig. I 76), are more common, and, as they often involve the cornea, and are apt, as has been pointed out, to cause symblepharon, they are of serious concern.

Treatment.--In burns of the conjunctiva, if the fornix has escaped injury, it is usually possible, through the measures described in treating of injuries of the lids, to prevent the occurrence of symblepharon; but when this, as well as the tarsal and bulbar conjunctiva, is involved, our efforts in this direction are almost sure to prove unavailing. 
When the burn has been caused by a caustic substance, such as lye, lime, or a strong acid, and the case is seen promptly, the eye should be bathed freely with a chemically neutralizing agent, such as diluted vinegar, if the burn has been produced by lime or lye, or a solution of sodium bicarbonate, if it is the result of an acid. Any remnants of the caustic substance should, of course, be carefully removed. The subsequent treatment con-

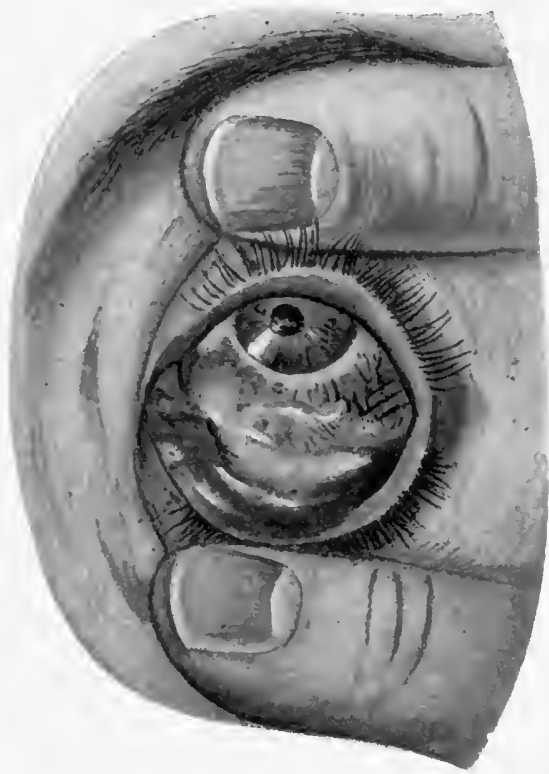

Fig. i 76.-Recent burn of the palpebral and ocular conjunctiva by a piece of hot iron (Haab).

sists in douching the eye with a fifteen-grain solution of boracic acid, in the instillation of a four-grain solution of atropin (alkaloid) in castor-oil, and in efforts to prevent, as has been explained, the formation of adhesions between the lids and the eyeball.

In incised or lacerated wounds of the conjunctiva, after the parts, if they are not disposed to fall together, 
have been united by stitches, a collyrium of boracic acid should be prescribed, and, if there is much discomfort and tumefaction, a lotion of opium and boracic acid, to be applied over the lids on absorbent gauze.

Superficial Injuries of the Cornea.-Burns of the cornea are of serious concern because, when at all severe, they are apt to leave a persistent opacity, which, if central, will greatly impair vision (Fig. I7 7 ). One of the most unfortunate cases of this kind that $I$ have encountered resulted from dropping aqua ammoniæ into the eye in mistake for a collyrium. A severe and obstinate keratitis followed, and an opacity was left which markedly and permanently impaired the sight.

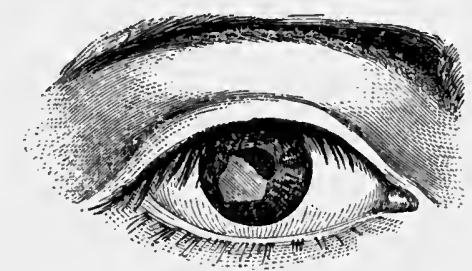

Fig. 177.-Leucoma resulting from a lime burn (Lawson).

Non-penetrating wounds of the cornea are seldom attended by untoward results, unless they happen to become infected, or involve the visual zone and give rise to persistent opacity. Abrasions, which simply disturb the corneal epithelium, though they may cause acute suffering for a short time, soon heal, as the epithelium is quickly regenerated.

Treatment.-If there is not much discomfort, and the wound is not infected, no other treatment than the instillation of a ten-grain solution of boracic acid is called for. If there is considerable irritation, photophoria, and lacrimation, atropin (one to four grains to the 
ounce) should be added to the boracic acid solution. A one- to two-grain solution of the local anesthetic holocain hydrochlorate is also a useful application, as it affords prompt relief from pain, is measurably antiseptic, and does not disturb the corneal epithelium and thereby favor infection, as does cocain, which is much oftener employed in like circumstances.

If it is evident from the presence of pus in the wound and in the neighboring corneal tissue that infection has occurred, the wound should be cleaned, and carefully cauterized with pure carbolic acid. The cleaning and the application of the acid can be done effectually and conveniently by means of a sharp, wooden toothpick, about the tip of which a very little absorbent cotton has been tightly wound. The acid should be used in minute quantity, and should not be permitted to come in contact with the uninjured corneal surface. Afterward the eye should be flushed with a boracic acid or normal salt solution. Further treatment should consist in dropping freely into the eye, as often as once in three hours, freshly prepared, undiluted chlorin water, which is one of the most efficient, and at the same time one of the best borne, ocular antiseptics. In addition, atropin or holocain should be prescribed, and, if there is much pain, an opium lotion. Should the wound after twentyfour hours still present an unhealthy appearance, it should be cleaned, and the carbolic acid again applied. In burns of the cornea the solution of atropin in castoroil, already mentioned, is an excellent application.

The Superficial Lodgment of Foreign Bodies in the Eye.-This is one of the commonest accidents to which the eye is subject, and, though seldom followed by serious consequences, it often gives rise to much suffering, which can be immediately relieved by the 
exercise of a little dexterity upon the part of the physician whose help may be sought. On the other hand, the display of a considerable measure of sinisterity on his part (if the word is permissible) is not apt to be soon forgotten by the unfortunate patient.

Mechanics, particularly those engaged in metalgrinding and polishing, stone-cutting, and similar occupations, are especially subject to this accident, and so are persons who are much exposed to wind and dust and to flying cinders.

Almost without exception, foreign bodies which do not penetrate the globe find lodgment-if they find it at " all, for many are washed out by the tears or removed by the individual's own efforts-in one of two places: either they attach themselves to the cornea, or they adhere to the inner surface of the upper lid, that is to say, to the tarsal conjunctiva (Fig. 178), for they rarely find their way into the superior retro-

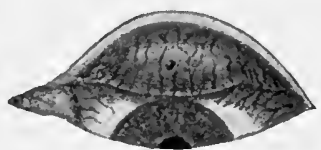

Fig. I 78.-Foreign body adherent to inner surface of upper lid. Lid everted to show a usual point of lodgment (Jackson). tarsal fold. In the exceptional instances in which a foreign body adheres to the bulbar conjunctiva, it will be found that it has hit the eye with considerable force, or was hot at the moment of impact, and has partly or completely penetrated this membrane.

The cornea is the usual place of lodgment for foreign bodies which are driven, so to speak, into the eye, and for this reason it is there they are commonly discovered in the case of metal-grinders, stone-cutters, etc. Indeed, it is not unusual to find the cornex of persons engaged in such occupations studded over with innumerable little nebulous opacities, each marking the point of impact of a bit of steel or stone, or a particle of 
emery. Cinders, and such like substances, which simply "fly into the eye," have no such definite predilections, and attach themselves as often to the superior tarsal conjunctiva as to the cornea.

The canaliculus is one of the unusual places in which foreign bodies are found, and yet they sometimes lodge there, especially detached eyelashes, which commonly enter but for part of their length, the projecting portion causing much discomfort by coming in contact with the sensitive bulbar conjunctiva.

There is a class of foreign bodies, met with from time to time, that seem to deserve especial mention, because their true character is apt to be overlooked by the inexperienced, and because, when once they have

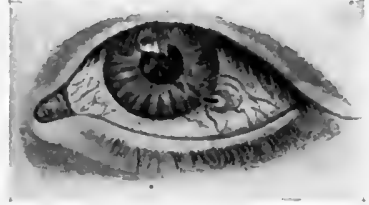

Fig. I 79.- "Chaff-particle" attached to the corneal limbus (Haab). attached themselves to the eye, they have a habit of remaining attached for an almost interminable period. I have preserved quite an interesting collection of these intractable little intruders, which consist of a portion of the outer shell of some tiny seedweed or grass seed (Fig. 179). They have several peculiarities, which explain their unusual behavior: They are concavo-convex in shape, they are semitransparent, and they are capable of resisting for a very long time the solvent action of the secretions of the eye. Their shape makes them adhere firmly to the eye,-they are almost always found upon the cornea,-their indestructibility renders them very nearly everlasting, and their transparency causes their true character to be easily overlooked. In my collection there is one of these shells which remained attached to the cornea for twelve months, and there are 
several which maintained their hold for from two to five months; and I may add, in support of what I have said as to the possibility of a mistaken diagnosis being made in these cases, that one of my earlier patients, with this sort of foreign body, narrowly escaped an energetic course of mercury at the hands of his family physician.

The suffering caused by the presence of a foreign body in the eye varies greatly in different individuals; but usually it is severe, and most intense when the body is sharp or rough, and is attached to the tarsal conjunctiva in such a position as to cause it, from the movements of the eye and lids, to scrape the surface of the cornea. Under such circumstances the upper part of the cornea is sometimes found extensively denuded of its epithelium.

In reaching a diagnosis as to the presence of a foreign body in the eye, it is well to bear in mind, in the first place, that a foreign body frequently enters the eye without the knowledge of the individual or any suspicion on his part that such an accident has occurred, and, in the next place, that patients often insist that a foreign body is present, when such is not the case. Whatever the patient's convictions, it is always best, in every case in which the symptoms even remotely suggest the propriety of so doing, to search carefully for the possible presence of some irritating foreign substance. The cornea should first be inspected,-and here "oblique illumination" (see page 19) will often prove of invaluable assistance-and then the upper lid should be everted, as described on page 22, and the tarsal conjunctiva carefully scanned. If a foreign body is not found in either of these places, and is not, as may possibly happen, lying loose upon the bulbar conjunctiva or in the lower retrotarsal fold, it may safely be concluded that none is present. 
Treatment.-In general, whether a foreign body be lodged upon the cornea or beneath the upper lid, it can be most easily removed-simply wiped from its point of attachment-by the help of a sharp, wooden toothpick, having a little absorbent cotton wrapped, moplike, over its point (Fig. 180). This will not suffice, however, if the foreign body has been driven forcibly

Fig. 180.-Toothpick armed with cotton for removal of foreign bodies.

into the eye, and is embedded in the corneal tissue. Under such circumstances an old-fashioned couching needle, or a similar needle such as is made nowadays expressly for this purpose (Fig. I8I), should be employed, as the foreign body must be picked out of its bed. To prevent possible infection the needle should, of course, be previously sterilized by immersion

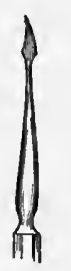

Fig. I8I. - Needle for removal of foreign bodies embedded in the cornea. in boiling water.

In removing a foreign body from the cornea it is always best to employ cocain, which tends to loosen its hold, besides rendering the operation painless and, therefore, much easier of performance. This is not necessary, however, when the body is attached to the inner surface of the lid, as then its removal is a painless procedure.

After the removal of a scale of iron or steel, or a bit of emery, from the cornea it often happens that a brownish stain is left, which conveys the impression that the removal has not been complete. Further efforts, at the moment, to detach this are apt to prove abortive, and considerable traumatism may result if these efforts are 

PLATE X.

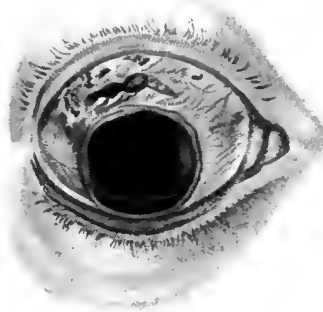

Rupture of the Sclera, with Hemorrhage into the Anterior Chamber (After Sichel). 
persisted in. It is better to wait for twenty-four or forty-eight hours, when it will be found that the seeming remnant of the foreign body, which is, in fact, only stained cornea tissue, has become loosened, and can be easily removed.

If keratitis has resulted from the irritation produced by a foreign body, or if infection of the wound has occurred, the measures prescribed for the treatment of simple or infected wounds of the cornea, as may be indicated, should be promptly employed.

The extraction of a foreign body which is deeply embedded in the cornea, and which may easily be displaced into the anterior chamber, is a delicate procedure, which had best be left to expert hands.

Contusions of the eye are of common occurrence, but fortunately, owing to the protection afforded by the bony orbital margin and the elastic cushion of fat which occupies the depth of the orbit and permits of considerable recession of the eyeball, serious injury seldom results, only, indeed, when the blow is exceptionally severe or is of unusual character. But for these provisions of nature, many eyes only "blacked" by blows would, doubtless, be irreparably damaged.

Blows from objects of such shape or size as not to be arrested by the orbital margin are the ones which are apt to be disastrous. Rupture of the eyeball (see Plate X), dislocation of the lens, laceration of the iris (Fig. I 82), iridodialysis (Fig. I83), detachment or other injury of the retina and choroid coat, and extensive intraocular hemorrhage, are among the consequences of such injuries. On the other hand, even severe blows from larger objects, such as the fist, are seldom followed by anything more serious than swelling and ecchymosis of the lids and extravasation of blood 
beneath the conjunctiva and into the loose cellular tissue of the orbit. When we consider the delicate structure of the eye it is indeed surprising to what rough usage it may be subjected without serious injury. However, it is only sound eyes that exhibit such immunity. Myopic eyes, as has been stated already, are

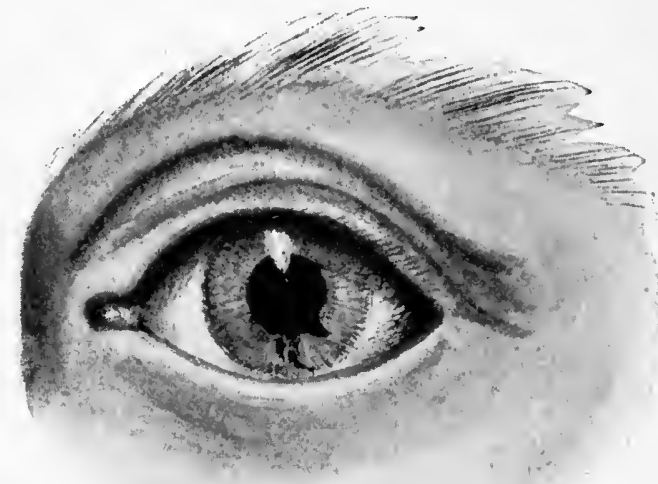

Fig. I 82.- Radiating and pupillary rupture of the iris (Hansell and Sweet).

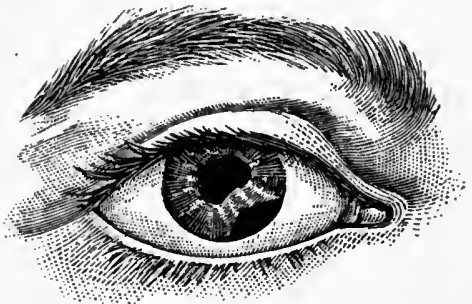

Fig. 183.--Iridodialysis caused by the explosion of a firecracker (Lawson).

especially liable to be seriously damaged by comparatively trivial traumatisms, and so are those which have suffered from previous attacks of iritis, choroiditis, etc.

Opacity of the lens, traumatic cataract, is one of the not uncommon results of severe contusions of the eye. And this may happen without rupture of the lens cap- 
sule, simply from the concussion to which the eye is subjected. Mydriasis and loss of the power of accommodation from paralysis of the sphincter pupillæ and the ciliary muscle, usually transient, also occur. Hemorrhage into the anterior chamber, and marked exophthalmos from extravasation of blood into the cellular tissue of the orbit, are observed, and rarely such injury of the muscles or motor nerves of the eye as may lead to the production of squint. Injury of the lacrimal sac and nasal duct from blows is not unusual, and may result in occlusion of the duct and persistent and annoying epiphora.

Among the commoner causes of severe contusions of the eye may be mentioned blows from flying chips in chopping and splitting wood, from stones thrown by accident or design, from pebbles or bullets shot from air-guns, from nails awkwardly struck, from limbs of trees, and from corks driven with violence from champagne or soda-water bottles. In one instance I saw an eye that was irreparably damaged from having been struck by a "ball" from a Roman candle, which had been foolishly aimed at a party of boys.

Treatment.--This will depend, of course, upon the nature of the injury, which may be so severe as to demand immediate enucleation of the damaged eye, or so trivial as to call only for the application of ice-cloths and a few days' rest from work. The lotion of opium and boracic acid is a most useful application, and atropin is often indicated, especially when the iris is injured. Sodium salicylate, in liberal doses, has a marked influence in controlling supervening inflammation, particularly of the deeper structures of the eye.

In severe contusions of the eye the danger of sympathetic ophthalmitis should not be lost sight of, the cases 
in which this is most to be feared being those in which rupture of the tunics has occurred, with subsequent partial atrophy of the ball. Enucleation of the injured eye is indicated under such circumstances, and should not be unnecessarily deferred.

Penetrating wounds of the eye are always of serious concern, not only because of the immediate damage involved and the inflammation liable to supervene, but because of the danger of infection of the wound, which, once established, is very apt to end in destructive panophthalmitis.

Penetrating wounds of the cornea often involve the iris and not infrequently the lens, and when this is the case prolapse of the iris and traumatic cataract usually result. Wounding of the lens constitutes a grave complication, not only because of the ultimate effect upon vision, but because the swelling of the lens, which usually quickly supervenes, adds materially to the risk of serious inflammation of the iris and ciliary body. It may also give rise to a glaucomatous condition of the eye, resulting, if not soon controlled, in permanent damage to the optic nerve and retina. Incised wounds of the cornea which do not involve the iris or lens usually do well, though they not infrequently give rise to displacement of the pupil and to the formation of an anterior synechia. If central, they are apt to impair vision markedly, because of the resulting opacity and disturbance of the corneal curvature.

Wounds of the sclera and ciliary body are of grave import, and are often complicated by hernia of the ciliary body or iris (Fig. 184) and prolapse of the vitreous humor. Infection is especially apt to occur under such circumstances, since the vitreous. humor furnishes an admirable culture medium for the growth of pyogenic bacteria. 
Penetrating wounds of the sclera, back of the ciliary region, though they usually involve the choroid and retina, are commonly not so serious in their consequences as are those of the ciliary body. If, however, infection occurs they result disastrously, and, even without this happening, if they are extensive and lacerated, they usually lead to loss of sight and ultimately to atrophy of the eyeball (Fig. I 85 ).

Treatment.-In view of the attendant dangers, and the serious complications liable to occur, the treatment of penetrating wounds of the eye should be consigned, if

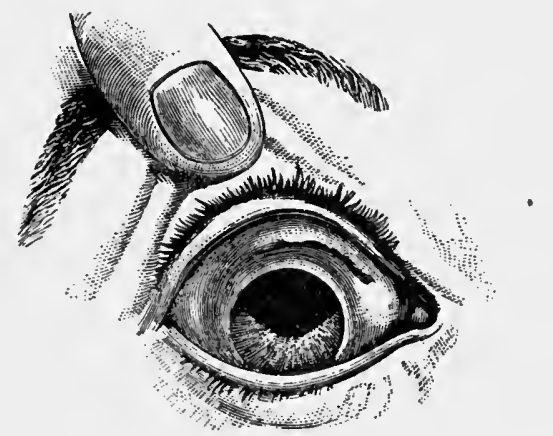

Fig. 184.-Rupture of the sclera with incarceration of the iris from a blow (Lawson).

practicable, to the hands of the specialist. For, in the first place, the question whether it is worth while to attempt to save the eye, whether immediate enucleation is advisable or not, of ten presents itself. Then, if there is a prospect of saving the eye, careful antiseptic precautions are called for, and, very probably, the abscission or replacement of prolapsed portions of the iris or ciliary body, or possibly the extraction of a wounded or dislocated lens.

As a "first aid" in such cases, the lids and the eye itself should be gently bathed with a I: 8000 sublimate 
solution, and a gauze pad, wet with the same solution or a sterilized lotion of opium and boracic acid, should be applied over the closed lids, and kept in place by a

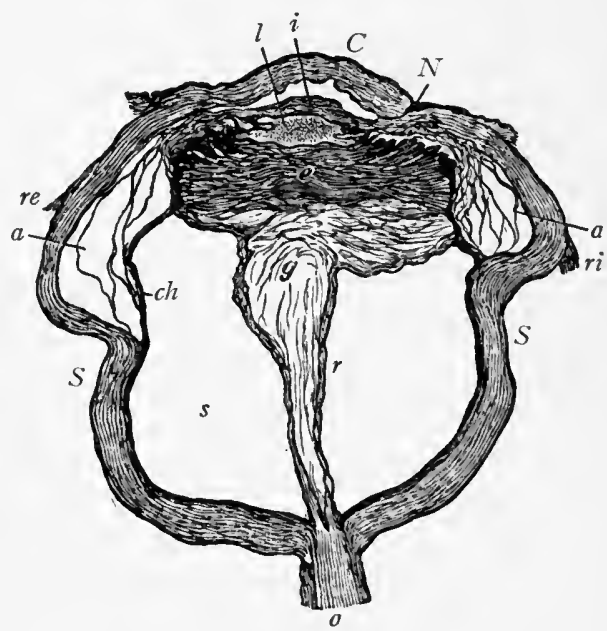

Fig. 185.-Atrophy of the eyeball, the result of severe iridocyclitis caused by a penetrating wound (Fuchs, in part after Wedl-Bock). The umbrellalike (the usual form of) detachment of the retina is well shown. The eye is smaller and of irregular shape, chiefly from the wrinkling of the sclera, $S$, behind the points of attachment of the ocular muscles, the rectus internus, $r i$, and the rectus externus, re. The cornea, $C$, is diminished in size, flattened, and wrinkled especially on its posterior surface. At its inner border it bears the depressed cicatrix, $N$, which was produced by the injury. The anterior chamber is shallow; the iris, $i$, is thickened and forms an unbroken surface, because the pupil is closed by exudate. Behind the iris lies the shrunken lens, $l$, and behind this is the great hull of cyclitic membrane, $c$, the shrinking of which is the cause of the atrophy of the eyeball. By reason of this shrinking, the ciliary processes, the pigment layer of which has markedly proliferated, are drawn in toward the center, and, together with the adjacent choroid, $c h$, are detached from the sclera; between the two structures are seen the disjoined lamellæ of the suprachoroid membrane, $a$. The retina, $r$, is detached and folded in the form of a funnel, which incloses the remains of the degenerated vitreous, $g$. The subretinal space, $s$, is filled with a fluid rich in albumin. The optic nerve, $o$, is thinner than usual and atrophic.

light bandage. And here, if practicable, as I have said, the general practitioner's care of the case should cease. 
If, in spite of antiseptic precautions, definite signs of infection of the eye exhibit themselves, enucleation is commonly indicated, and, if promptly performed, will save much unnecessary suffering. As has already been pointed out, the danger of cerebral or general infection is not appreciably greater from operating under such circumstances than from permitting the panophthalmitis to run its tedious course. There is also the risk of sympathetic ophthalmitis from extensive wounds of the cornea and iris, or of the sclera and ciliary body, and this is another reason why, if there is no prospect of useful vision being preserved, enucleation should be resorted to without unnecessary delay.

Wounds of the Eye Complicated by the Lodgment of Foreign Bodies within the Ball.-The dangers attendant upon penetrating wounds of the eye are greatly increased by the lodgment of a foreign body within the ball. In the first place, the risk of infection is much greater (see Fig. I30), and, even if this does not occur, the irritation caused by the continued presence of the foreign body is almost certain to set up an insidious inflammation of the deeper structures of the eye, which ends in loss of sight. Again, there is greater probability of sympathetic ophthalmitis supervening. Indeed, this dreaded complication is more often due to the presence of a foreign body in the primarily affected eye than to any other cause.

Much depends, it is true, upon the nature of the foreign body, and upon its position within the ball. Foreign bodies which, though sterile, undergo chemical changes, such as bits of iron or steel or copper, are most apt to give rise to disastrous consequences. On the other hand, spicules of glass or small fragments of stone are not so surely destructive of sight, since they may 
become encysted, and in this way be rendered innocuous. A striking case of this character has come under my observation, in which a small piece of glass, after entering the anterior chamber, fell through the pupil, and lodged in the ciliary processes. Sharp inflammatory reaction followed, but soon subsided, and evidently resulted in the glass becoming encysted; and to this day, the accident having occurred more than thirty years ago, no ill effects have been experienced, the injured eye being as free from irritation, and as capable of performing its daily task, as its fellow.

As to the influence of location, a foreign body lodged within the lens capsule, although almost sure to cause the development of cataract, may not provoke inflammatory complications, and this, in less measure, is true of one suspended in the vitreous humor. As opposed to this, a foreign body embedded in the iris, ciliary body, or choroid is more certain to excite destructive inflammation, and is also more apt to cause sympathetic implication of the fellow-eye. Wounds of the eye from bird-shot are especially dangerous, partly from the character of the wound, and partly from the nature of the foreign body.

In order to penetrate the tunics of the eye a foreign body must have considerable weight relatively to its size, and must be driven with great force. This is especially true of those that impinge upon the very tough sclera, which is less frequently penetrated than the cornea. Foreign bodies which pass through the sclera commonly fall into the vitreous chamber, and in time come to rest upon the retina, at the bottom of the eyeball. Those which enter through the cornea exceptionally fall into the anterior chamber, but more often lodge in the iris or pass through it into the vitreous 
body, frequently wounding the lens in their course, and giving rise to a traumatic cataract (Fig. 186). Those which lie loose in the anterior chamber usually excite iritis, and eventually become encysted in the narrow space between the lower margin of the iris and the cornea.

Treatment.-Before the days of the electro-magnet and skiagraphy, the lodgment of a foreign body within the eyeball usually meant loss of sight and, sooner or later, enucleation of the eye. Nowadays, many such eyes are saved, and often with useful vision. By means

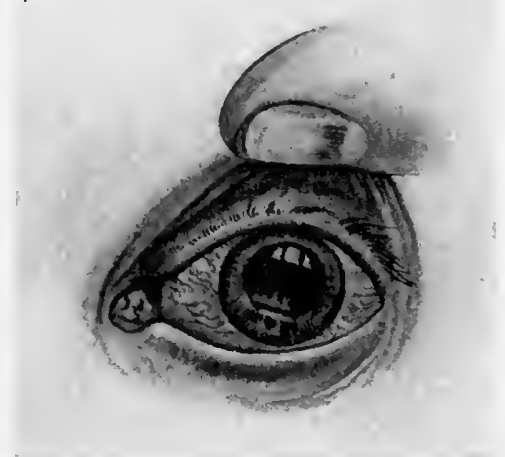

Fig. 186.-Spicule of iron in the vitreous (extracted); laceration of the iris, traumatic cataract, and turbidity of the vitreous (Haab).

of skiagraphy the exact location of nearly all foreign bodies can be determined, and this, it is evident, greatly facilitates their removal (Figs. 187, 188, 189). By the help of the magnet bits of iron and steel (and they constitute a very large proportion of the foreign bodies which enter the eye), in most instances, can be extracted, and, not infrequently, so as to leave a serviceable eye. For this purpose the powerful electro-magnet of Sweet is the instrument $I$ have found most effective (Fig. 190). 
By means of oblique illumination foreign bodies lying in the anterior chamber or superficially in the lens, or that are partially embedded in the iris, can usually be detected without difficulty, unless, as sometimes happens, they are hidden by hemorrhage; but only exceptionally can those that have passed into the vitreous chamber be discovered, for in most instances the media

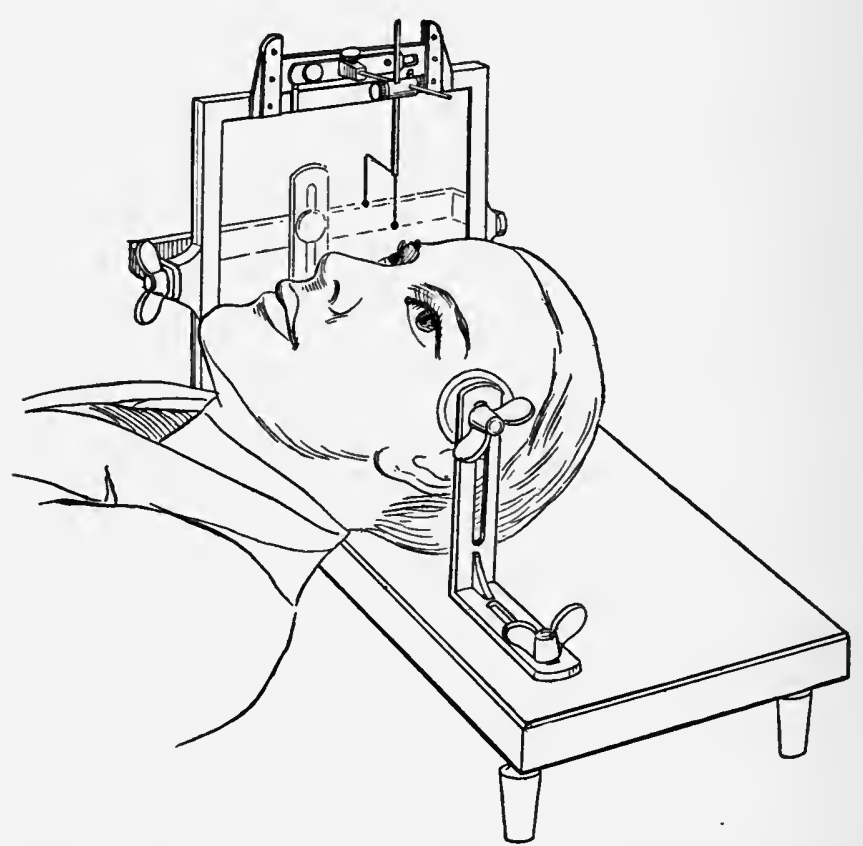

Fig. 187.--Sweet's apparatus for localizing foreign bodies in the eye with the Röntgen rays.

are too cloudy to permit of a satisfactory ophthalmoscopic examination. And it is here that skiagraphy proves invaluable. When this test is not available the "pain reaction" test may be employed, provided the foreign body is magnetic. This consists in approaching a strong magnet very close to the eye, or bringing it 
INJURIES OF THE EYE AND APPENDAGES. 5OI

in actual contact with it. The pull which it exerts upon fragments of iron or steel, especially if they are of considerable size, usually causes decided pain. A negative result does not definitely exclude the presence of a magnetizable foreign body, but, at least, it renders it improbable.

In every case in which there is reason to fear that a foreign body has entered the eye the tests described-

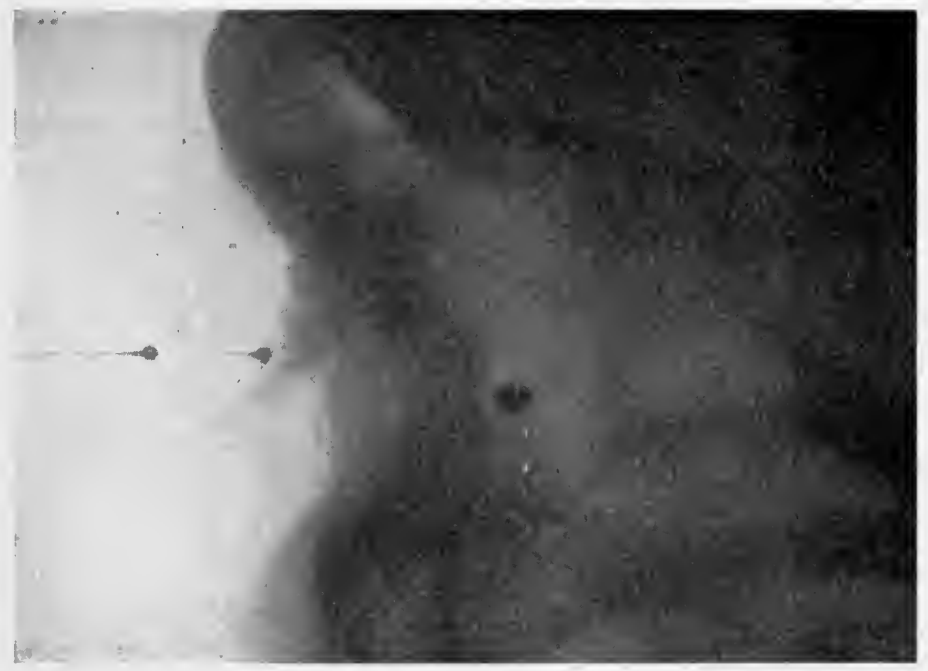

Fig. 188.-Radiograph showing foreign body (Sweet).

especially the skiagraphic test-should be employed with as little loss of time as possible; for the sooner it is discovered and removed the greater, other things being equal, is the likelihood of saving the eye. In the meantime, until the case can be placed in the hands of a specialist, antiseptic measures, such as have been mentioned in connection with the treatment of penetrating wounds of the eye, should be employed, a sterilized fourgrain solution of atropin (Fig. I9I) should be instilled 
every three or four hours, and the eye should be closed with a pad of gauze, wet with a $\mathrm{I}: 8000$ sublimate solution, or a sterilized lotion of opium and boracic acid, and a light bandage.

Non-magnetic foreign bodies should be located, if possible, by means of oblique illumination, the ophthalmoscope, or skiagraphy, and removed, through a suitably placed incision, with toothless forceps, a traction hook, or a small curet.

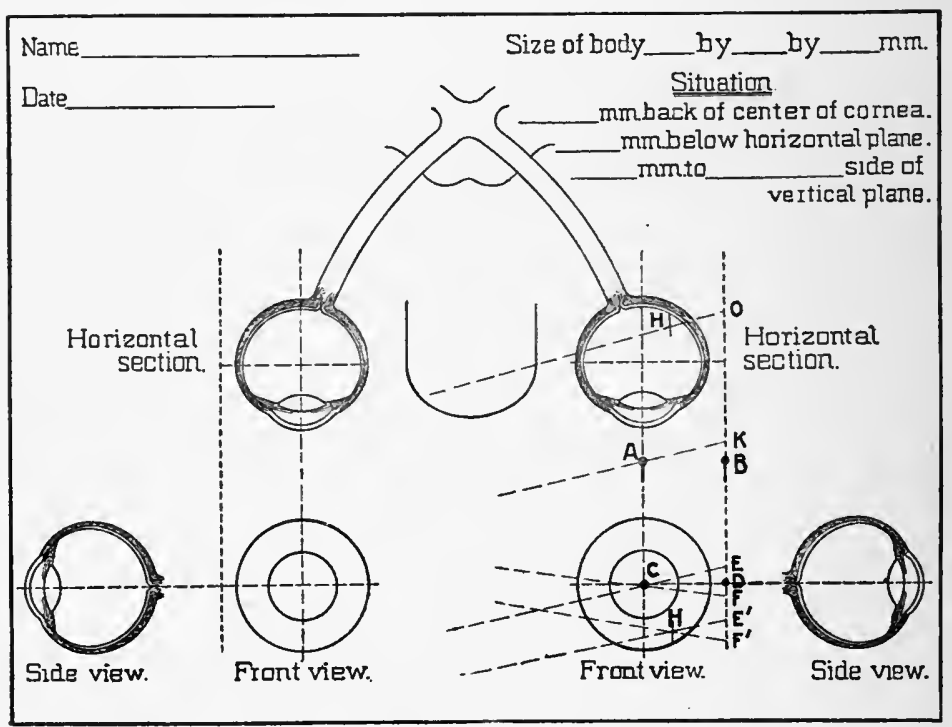

Fig. I89.--Reduced drawing of Dr. Sweet's localizing chart, illustrating method of plotting position of foreign body in the eyeball.

The removal of foreign bodies from the anterior chamber is not so easily accomplished as might appear at first sight. If magnetic they may, perhaps, be drawn out with a strong magnet through the wound of entrance or through an incision made for the purpose. If they can not be removed in this way, they must be extracted with toothless forceps; but as the aqueous 
humor escapes, and the anterior chamber is obliterated, as soon as an incision is made in the cornea, this is often a difficult procedure. Indeed, when the foreign body is embedded in the iris it is frequently necessary to remove the portion of the iris to which it is attached--that is to say, to perform an iridectomy.

In an unual case which came under my observation some years since, as the result of a blow from a chip of wood an eyelash was driven through the cornea into the anterior chamber, and lay, across the pupil, upon

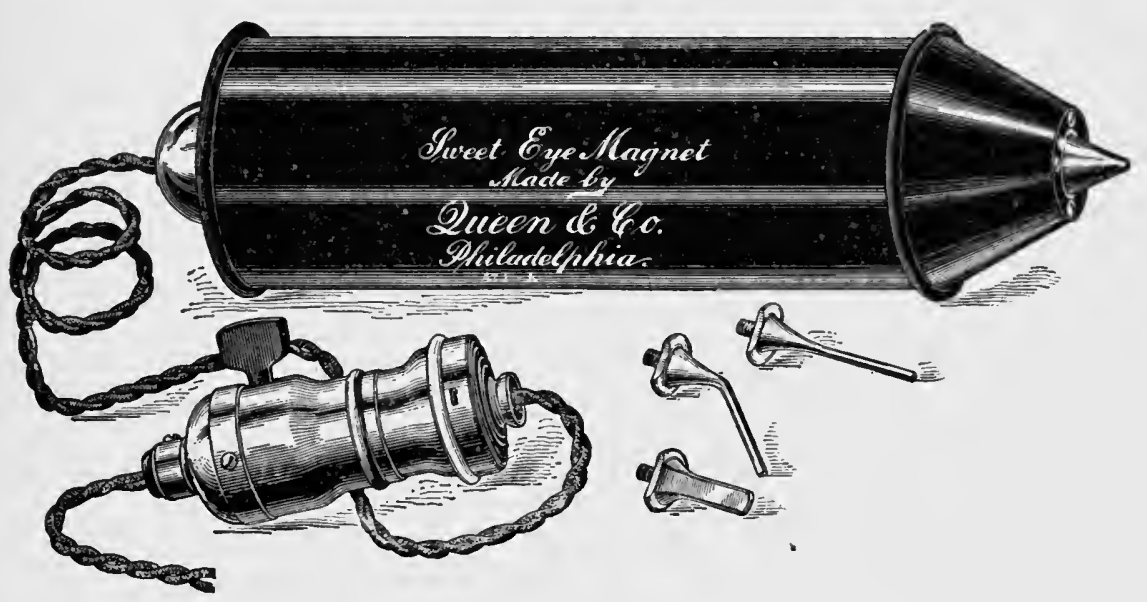

Fig. I9o.-Sweet's electro-magnet.

the surface of the iris. With considerable difficulty it was removed through a peripheral corneal incision, without injury to the iris, and, though a slight anterior synechia was left, useful vision was preserved.

Exceptionally, and particularly in gunshot wounds, a foreign body may pass through the posterior, as well as the anterior, coats of the eye, and lodge in the orbit, and in other exceptional instances it may lodge in the orbit without wounding the eyeball. Its removal, 
under such circumstances, is difficult of accomplishment, and may not be necessary, unless it proves a

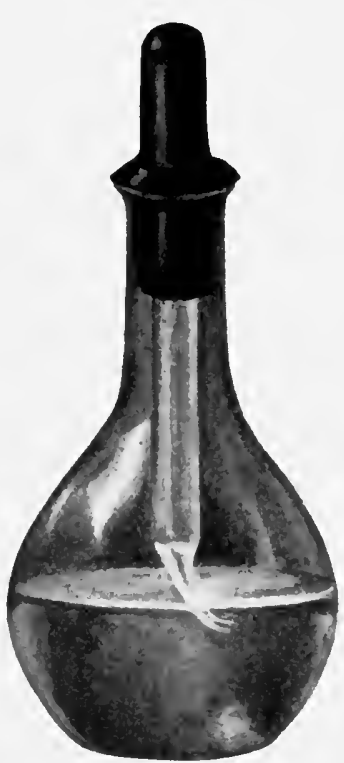

Fig. 191.-Florence flask for sterilizing collyria (about two-thirds actual size). See foot-note.* source of irritation.

Wounds of the eyeball caused by penetrating foreign bodies are sometimes so extensive or of such a character that enucleation is clearly called for, the mere removal of the foreign body offering no hope of preserving sight. This is especially true of lacerated wounds of the cornea and ciliary region, such as are produced by jagged pieces of iron or steel, struck from the edge of a hammer or chisel, flying against the eye with great force. In such cases removal of the eye is the only procedure to be thought of; and it can not be done too quickly, for delay means not only much needless suffering, but the added risk of sympathetic ophthalmitis.

* This flask, fitted with a "Barnes eye-dropper," I greatly prefer to the Stroschein flask commonly used, for it is not only less expensive, but much less fragile. The mouth of the flask is plugged with cotton, in the usual manner, when the solution is being boiled. The eyedropper is boiled separately, and, when the solution has cooled, is fitted quickly into the flask. The neck of the I-ounce flasks, as they are found on the market, is too large to fit the Barnes eye-dropper; but Messrs. Whitall, Tatum \& Co., of Philadelphia, who furnish them, will "draw it in" to the size desired. 


\section{APPENDIX.}

The following formulæ, for the most part in general use, are indicated, and will be found efficacious, in the conditions mentioned.

\section{COLLYRIA.}

To be applied by means of an eye-dropper, preferably one with a bent tip. Except when solutions of atropin, hyoscyamin, and other such poisonous drugs are prescribed, they may be instilled freely, and it is not necessary to direct exactly how many drops should be used.

Acid. boracic. gr. $x$

Aquæ destil. 3 .

A few drops three or four times a day.

Hyperemia of the conjunctiva, mild conjunctivitis, inflammation of the conjunctiva following injuries, operations, the intrusion of foreign bodies, etc., and for the relief of smarting and burning of the eyes.

Acid. boracic...................gr. x

Aquæ camphor.................. $5 \mathrm{ij}$

Aquæ destil.................... $3 \mathrm{vj}$.

A few drops three or four times a day.

Hyperemia of the conjunctiva, and for the relief of smarting and burning of the eyes.

Sodii chlorid..................... gr. iij-v

Aquæ destil. ..................... $3 \mathrm{j}$.

Indicated in the same conditions, and to be used in the same manner, as the foregoing. 
Acid. boracic.

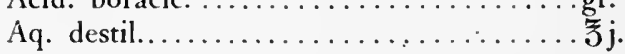

For cleansing the eye and for instillation every hour or half hour in purulent ophthalmia, and for less frequent instillation, in connection with other remedies, in ulcerative and suppurative keratitis.

Zinci sulphat.................. ss

Acid. boracic. ...................

Aquæ destil...............

A few drops three times a day.

Catarrhal and follicular conjunctivitis.

Hydrarg. bichlorid.................gr. $\frac{1}{16}$

Sodii chlorid.................... . iij-v

Aqux destil. ..................

A few drops three times a day.

Vernal catarrh, follicular conjunctivitis, and blennorrhea of the lacrimal sac. In the last-mentioned condition, to be dropped into the inner corner of the eye, after pressing out the contents of the sac.

Alum. gr. iij

Acid. boracic.

Aquæ destil. 号 j.

A few drops morning and night.

For habitual application to the conjunctival sac when an artificial eye is being worn.

Sol. Protargol. $40 \%$

For daily application, with cotton mop or eye-dropper, in purulent ophthalmia, and every second day in acute stage of trachomatous conjunctivitis.

Sol. Argyrol...................... $40 \%$ to $50 \%$

Indications the same. May be applied as often as three times a day in purulent ophthalmia. 
Sol. Protargol.................... 10\%

For application twice daily in purulent ophthalmia, in addition to the daily application of the stronger solution, and for use, two or three times a day, in trachomatous conjunctivitis.

Sol. Argyrol...................... $20 \%$

Indications the same. May be used more freely.

Sol. Argyrol.................... $5 \%$ or

Protargol........................

Drop into the inner corner of the eye three times a day, after pressing out the contents of the lacrimal sac.

Blennorrhea of the lacrimal sac.

Aqua chlorinii (freshly prepared).

To be dropped into the eye, if practicable upon the cornea, every three hours.

Infected corneal ulcer, whether of idiopathic or traumatic origin.

Holocain hydrochlorat................gr. j

Acid. boracic...................... $x$

Aquæ destil....................

A few drops every three hours.

Ulcer of cornea.

Atropiæ sulphat....................gr. j

Acid. boracic...................... $x$

Aquæ destil.................... $\mathrm{z} \mathrm{j}$.

One or two drops three times a day.

Phlyctenular conjunctivitis and keratitis and other mild forms of keratitis. 
Atropix sulphat..................... gr. ij

Acid. boracic....................gr. $x$

Aquæ destil.....................

In like conditions, when photophobia and lacrimation are more marked.

Atropiæ sulphat................... gr. iv

Acid. boracic...................gr. $\mathrm{x}$

Aquæ destil....................

One or two drops every three hours.

Iritis, cyclitis, severe keratitis, or sclero-keratitis, and, three times daily, in episcleritis, sclero-conjunctivitis, and interstitial keratitis.

Atropiæ sulphat...................gr. $\frac{1}{4}$

Acid. boracic...................... gr. $\mathrm{x}$

Aquæ destil.....................

One or two drops every third day.

To improve the vision in incipient cataract, when the opacity of the lens is chiefly central, by maintaining semi-dilation of the pupil.

Atropix (alk.).................... gr. iv

Ol. ricini.................

One or two drops three or four times a day. Burns of the cornea and conjunctiva.

Eserin sulphat. ................. gr. ij-iv

Acid. boracic..................... gr. $x$

Aquæ destil.................... $\mathrm{z} \mathrm{j}$.

One or two drops three or four times a day. Inflammatory glaucoma.

Eserin sulphat. gr. $\frac{1}{8}-\frac{1}{4}$

Acid. boracic...................... gr. $x$

Aquæ destil.....................

One or two drops morning and night.

Glaucoma simplex, chronic glaucoma, and in inflammatory glaucoma between exacerbations. 
Pilocarpin hydrochlorat..............gr. iv-viij

Acid. boracic................... gr. $x$

Aquæ destil....................

One or two drops three or four times a day.

Inflammatory glaucoma, when eserin is not well borne.

Pilocarpin hydrochlorat..............gr. j-ij

Acid. boracic.

gr. $x$

Aquæ destil . .

One or two drops two or three times a day.

As a substitute for the weaker solution of eserin, in glaucoma simplex, chronic glaucoma, and in inflammatory glaucoma between exacerbations.

Sol. Dionin. ................. $5 \%$

One or two drops two or three times a day.

Iritis and keratitis, in conjunction with atropin, inflammatory glaucoma, in conjunction with eserin, and to promote the absorption of recent corneal opacities, of inflammatory exudates in the anterior chamber, and of extruded cortical lens substance, after cataract operations or wounds of the lens.

Hyoscyamin hydrobromat. ............ gr. ij

Aquæ destil....................

One or two drops every three or four hours.

Iritis, keratitis, etc., as a substitute for atropin, when atropin is not well borne. Also as a cycloplegic in determining refractive errors. When used for this purpose, two applications of a single drop, an hour or two apart, should be made the evening before, and three applications, at similar intervals, during the forenoon of, the day on which the examination is to be made. 
Homatropiæ hydrobromat............... ij

Aquæ destil................... $3 \mathrm{ij}$.

An evanescent and but slightly toxic cycloplegic, used in measuring refractive errors. Three applications at half-hour intervals the evening before, and seven or eight applications the morning of, the examination, the last three twenty minutes apart, the others at intervals of an hour.

Homatropiæ hydrobromat..............gr. ij

Aqux destil....................

One or two applications.

An evanescent mydriatic. To facilitate ophthalmoscopic examinations, to permit inspection of the lens in suspected cataract, and to determine the presence of iritis.

Euphthalmin hydrochlorat............gr. vj

Aqua desil................ $3 \mathrm{ij}$.

A transient mydriatic, to be used in the same way, and for the same purposes, as the foregoing.

Cocain hydrochlorat................. xx $(4 \%)$

Acid. boracic................... xv

Aquæ destil.................

Three to five applications, at intervals of two or three minutes.

To induce anesthesia. In all important operations should be sterilized by boiling before being used. Distinctly objectionable, and not to be employed, as a therapeutic agent. 
Holocain hydrochlorat................. gr. x $(2 \%)$

Aquæ destil.................

A local anesthetic, to be applied in the same manner as cocain, and to be used as a substitute for it when it is desirable to avoid the mydriasis and the disturbance of the corneal epithelium which cocain produces. Is in itself an antiseptic.

Sol. Hydrarg. bichlorid............ I : 8000 .

For sterilizing the eye preparatory to operations. Several hours before the time fixed for the operation the lids should be cleansed with soap and water, the conjunctival sac flushed with this solution, and a gauze pad wet with it bound over the lids. The flushing of the conjunctiva should be repeated three times, at intervals of about an hour, before the operation is begun.

LOTIONS.

To be applied on absorbent gauze, or soft linen, pads over the closed lids.

\section{Saturated Solution of Boracic Acid.}

Acid. boracic....................gr. Ixxij

Aquæ destil....................

Useful in wounds of the lids and eyeball, after enucleation of the eye, in lid abscess, etc. By covering the pad with oiled-silk or oiled-muslin a cleanly and excellent poultice is provided. 


\section{Lotion of Opium and Boracic Acid.}

(Frequently commended in the text.)

Ext. opii..................... $\mathrm{x}$

Acid. boracic. .................

Aq. destil. ..................

Valuable in any painful condition of the eye, especially in traumatic lesions, in keratitis, iritis, glaucoma, acute inflammation of the lacrimal sac, lid abscess, panophthalmitis, cellulitis of the orbit, etc. Also useful in asthenopia, in miliary choroido-retinitis, dependent upon strain of accommodation, and in the choroidoretinitis of high myopia. May be given a poultice-like action by covering the pad on which it is applied with oiled-silk or oiled-muslin. May be applied hot if found more soothing.

Lotion of Belladonna.

Ext. belladonnæ................... xv

Aquæ destil..................

Iritis, cyclitis, keratitis, etc.

\section{Sublimate Solution.}

Hydrarg. bichlorid...................gr. j

Aquæ destil..................... Oj.

Wounds of the lids and eyeball. The lids should first be bathed, and the conjunctival sac flushed, with this same solution.

\section{Hot Water.}

Interstitial keratitis, iritis, and glaucoma. Should be applied as hot as can be borne without complaint.

\section{Iced Water.}

Pads of gauze should be kept lying upon a block of 
ice, and a fresh pad should be applied to the eye at short intervals.

Purulent ophthalmia in the adult.

\section{OINTMENTS. "Vaselin Cerate."}

Ceræ flav...................... I part Vaselin........................ parts.

To be melted together, and stirred while cooling.

A useful base, of proper consistency and with little tendency to become rancid, for ointments to be applied to the lids.

Hydrarg. ox. flav..................gr. viij

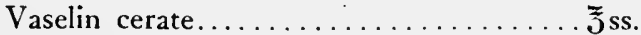

To be applied to the lids at bedtime, after removing any crusts that may be present by bathing with warm water.

Blepharitis marginalis and eczema of the lids.

Hydrarg. ox. flav................... iv. iv

Vaselin......................

A piece the size of a rice-grain to be applied to the eye once a day, preferably in the morning. The application can be conveniently made with a flat wooden toothpick or, by untrained hands, with a small camel's-hair brush.

Phlyctenular conjunctivitis and keratitis, vernal catarrh, chronic trachoma with pannus.

Zinci oxid.

Acid. boracic.................. $\bar{a} \bar{a} \bar{Z} s s$

Vaselin cerate...................

To be applied morning and night. 
Eczema of the lids.

Acid. salicylic. gr. iv-viij

Vaselin cerate

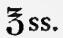

To be applied morning and night.

Eczema of the lids, and, exceptionally, in blepharitis marginalis, when the yellow oxid of mercury ointment fails to cure.

Ext. belladonnæ................ $5 \mathrm{j}$

Ung. hydrarg................

To be rubbed upon the forehead and temples three times a day.

Syphilitic iritis and irido-cyclitis, sympathetic ophthalmitis.

AGENTS TO BE APPLIED, WITH EXACTNESS, TO THE EYE OR LIDS.

Acid. carbolic.

For application to infected corneal ulcers or wounds, and to abort styes.

A very small quantity of the acid should be applied directly to the ulcer (the eye being under the influence of cocain) by means of a sharp-pointed, wooden toothpick, armed with a few fibers of absorbent cotton. After the application the eye should be flushed with a boracic acid or normal salt solution. In the treatment of styes, the point of the toothpick, after having been dipped into the acid, should be insinuated as far as possible into the infected follicle, which can generally be recognized upon the lid-margin.

Tinct. iodin. 
To be applied in the same careful way as carbolic acid.

Infected corneal ulcers.

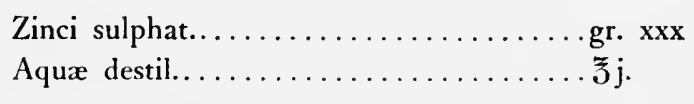

Should be applied with the finger-tip, every half-hour, to the external surface of the lid, over the sensitive area, care being exercised not to let the solution enter the eye. - To abort styes.

Pointed crayon of silver nitrate.

To be applied lightly to the lid-margin after removal of crusts.

Blepharitis marginalis.

Copper sulphate crystal.

For application to the conjunctival surface of the everted lids, every second or third day.

Chronic trachomatous conjunctivitis.

Veratriæ Oleat.................... $10 \%$

A little to be rubbed upon the forehead and temples once a day, preferably in the morning. Care should be exercised to prevent its getting into the eye, as this causes severe and persistent irritation.

Asthenopia and frontal headache due to accommodative or muscular strain. To lessen the irritability of the ciliary muscle preparatory to testing errors of refraction. 


\section{CONSTITUTIONAL REMEDIES.}

Elix. ferri et quiniæ et strychniæ phosphat. (Wyeth's.*)

A teaspoonful three times a day for adults; for children two-thirds to one-third of a teaspoonful, according to age. When less than a teaspoonful is prescribed, it should be diluted to the required degree with elix. simplex, so that the quantity to be given shall be one teaspoonful.

Phlyctenular conjunctivitis and keratitis, eczema of the lids, and eczematous blepharitis marginalis.

Syr. ferri et quiniæ et strychnix phosphat. (Sharp and Dohme's.*)

Dose and indications for administration the same as the foregoing. May be diluted with simple syrup.

Quiniæ sulphat. in three-grain capsules.

One every three or four hours.

Ulcer and abscess of the cornea, herpetic keratitis, purulent iritis, and threatening panophthalmitis.

Ext. nucis vom.................. gr. $x$

Quiniæ sulphat................gr. xl-lxxx

Ferri carbonat. (Vallet's).............. gr. lxxx.

Ft. capsules xl.

One three times a day.

A well-known tonic combination, often found useful in the treatment of eye diseases.

Tinct. nucis vom.................. $\mathrm{z} j$

Tinct. cinchonæ compos.............

Two teaspoonfuls three times a day.

* These particular preparations are mentioned because they contain a considerably larger proportion of iron and quinin than most of the preparations which are sold under the same name. 
Another excellent tonic combination, especially useful in asthenopia following exhausting diseases.

Syr. ferri iodid.

Eight to twenty drops, in a wineglassful of water, three times a day.

Phlyctenular conjunctivitis and keratitis, accompanied by definite signs of struma.

Potassii iodid. (saturated solution).

In increasing doses.

Rheumatic and syphilitic inflammations of the eye, especially those occurring in the tertiary stage of syphilis, uveitis, sclero-keratitis, scleritis, paralysis of the ocular muscles, and to promote the absorption of blood in the vitreous or anterior chamber, and of cortical lens substance after cataract operations or injuries of the lens.

Hydrarg. biniodid. gr. $\mathrm{j}-\mathrm{ij}$

Potassii iodid. gr. $x$

Aqua. כुiv.

A teaspoonful three times a day, after meals.

Syphilitic affections of the iris, ciliary body, choroid, retina, and optic nerve; especially those occurring during the secondary stage of the disease; also in glaucoma, retrobulbar neuritis, sympathetic ophthalmitis, etc. When prompt action is demanded, its administration may be supplemented by inunctions of mercurial ointment, and when thought desirable the proportion of potassium iodid may be increased, so that the dose prescribed shall contain five to ten grains.

Hydrarg. biniodid. gr. j

Potassii iodid.

Syr. ferri iodid. 予ss

Aqux 予ijss. 
A teaspoonful, in a wineglassful of water, three times a day, after meals.

Interstitial keratitis and other chronic syphilitic affections.

Hydrarg. bichlorid. gr. j

Tinct. ferri chlorid. ................

Aquæ..........................

A teaspoonful, in a wineglassful of water, three times a day, after meals.

A useful substitute for the foregoing, especially when the iodides are not well borne.

Potassii iodid. ..................... lxiv-xcvj

Syr. ferri iodid. ................

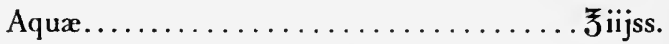

A teaspoonful, in a wineglassful of water, three times a day, after meals.

Interstitial keratitis.

Tablet triturates of calomel (gr. $\left.\frac{1-1}{1-\frac{1}{2}}\right)$.

One every two hours, guarded by opium if found necessary, and supplemented by inunctions of mercurial ointment.

When prompt mercurialization is called for, as in severe syphilitic inflammation of the iris, choroid, retina, optic nerve, etc., and in sympathetic ophthalmitis.

Sodii salicylat. (In solution or in capsules.)

Ten grains every two or three hours.

Iritis, cyclitis, scleritis, sclero-conjunctivitis, especially when they are dependent upon a rheumatic diathesis, acute glaucoma, inflammation of the uveal coat due to traumatism, whether accidental or operative, and in much larger doses, pushed to the point of toleration, in sympathetic ophthalmitis. 
Strychniæ sulphat. (In solution or in tablet triturates.)

In increasing doses, to be given directly after meals. Incipient atrophy of the optic nerve or retina, chronic retrobulbar neuritis, paralysis of the ocular muscles.

Pilocarpin hydrochlorat...............gr. iv

Aquæ ...............................

- Ten drops once a day, the dose on each succeeding day to be increased by two or three drops, according to the effect produced.

Choroido-retinitis, uveitis, glaucoma, detachment of the retina.

Sodii pyrophosphat................ $\mathbf{j} \mathrm{j}$

Aquæ..........................

A tablespoonful, in water, every two or three hours. Acute inflammation of the lacrimal sac, cellulitis of the orbit, abscess of the lid.

Natural lithia water.

To be drunk freely.

Gouty affections of the eye-scleritis, iritis, retinitis, etc.

\section{"Compound Calomel Powder."}

(Frequently mentioned in the text.)

Hydrarg. chlorid. mitis ............. gr. ij-iv

Pulv. scammonii................. gr. ij

Pulv. rad. rhei................ gr. vj.

Ft. capsules ij (or may be given in powder form).

The two capsules, or the whole powder, to be given at bedtime. The dose may often be repeated with advantage after forty-eight hours. 
As a first measure in the treatment of phlyctenular kerato-conjunctivitis, especially when there is attendant eczema of the lids, face, etc., also in acute glaucoma, iritis, and whenever an energetic purgative is called for.

Tablet triturates of aloin (gr. $\left.\frac{1}{10} \frac{1}{4}\right)$.

One to be taken at hedtime.

Habitual constipation. 


\section{INDEX.}

NotE.-The bold-face type indicates the pages on which the subjects are especially treated of (or considered).

Abducens, paralysis of. See $\mathrm{Pa}$ ralysis of external rectus muscle.

Ablatio retinæ. See Retina, detachment of.

Abscess and ulcer of cornea, 207 of lacrimal sac. See Dacryocystitis.

of orbit, 108

prelacrimal, 137

Accommodation, 426

abnormal power of, $4_{28}$

anomalies of, 425

different ways in which may be impaired, 427

disturbance of normal relationship between convergence and, 396

in hypermetropia, 396, 397, 400 asthenopia caused by, 396 convergent squint caused by, 397

in myopia, 407,408

asthenopia caused by, 408

divergent squint caused by, 408

in subnormal accommodative power, 434,435 asthenopia caused by, 435

how remedied, 436

failure of, from advancing age, 427 Helmholtz's theory of, 426

mechanism of, 426

normal relationship between convergence and, 396

how disturbed in hypermetropia, 396

in induced cycloplegia, 435

in myopia, 407,408

in subnormal accommodative power, 434, 435

paralysis of, 427 . See also $\mathrm{Pa}$ ralysis of ciliary muscle.

symptoms which indicate, 32 progressive decline of, 427,428
Accommodation, rapid failure of, an early symptom of glaucoma, 279 spasm of, 427. See also Spasm of ciliary muscle.

subnormal power of, 427. See also Subnormal accommodative power.

Accommodative power, subnormal. See Subnormal accommodative poner.

Acromegaly in etiology of hemianopsia, 386

Actual cautery in corneal ulcers, 214 Adrenalin in operation for pterygium, 101

in recurrent intraocular hemorrhage, 337

in treatment of strictures of nasal duct, 143

Advancement of muscle in convergent squint, 457

in divergent squint, 465

in exophoria, $47^{2}$

in heterophoria, $47 \mathrm{I}$

After-cataract. See Cataract, capsular.

Age, influence of, in development of convergent squint, 402, 439, 448

in diseases of choroid and retina, 339

in glaucoma, 276

in interstitial keratitis, 223

in myopia, 407,4 I 2

upon cataract, 298, 299, 30 I, $313,314,318$

upon hypermetropia, 398 , 4 ㄷ upon the accommodation, 426, $427,428,429$

upon the crystalline lens, 295,296

Agents for exact application to eye or lids, 514, 515

Albuminuric retinitis. See Retinitis, albuminuric. 
Alcohol, methylic, in retrobulbar neuritis, 374

Alcoholism in hyperemia of conjunctiva, 15.3

in retrobulbar neuritis, $37^{6}$

Aloin in habitual constipation, 520 in recurrent hordeola, 69

in retinal hemorrhage, 337

Alternating strabismus. See Squint, alternating.

Alum and boracic acid, formula for collyrium of, 506

after enucleation of eye, 273 , 506

in blennorrhea of lacrimal sac, 148

crystal in trachoma, I 8I

"Amaurotic cat's eye." See Retina, glioma of.

A mblyopia exanopsia, 319, 449 misuse of term, 449

of squinting eye, how induced, 449 toxic. See Optic neuritis, retrobulbar, chronic.

uremic, $35 \mathrm{I}$

Ametropia, 394

definition of, 394

varieties of, 394

Ammonium chlorid in treatment of stricture of nasal duct, I 49

Ammon's, von, operation for shortening lid, 93

Anagnostakis-Hotz operation for entropion, 86

Anatomy of cornea, 204

of crystalline lens, 295,296

of eyelids, 68

of iris and ciliary body, $25 \mathrm{I}$

of lacrimal apparatus, I 18 , I 2 I, I 26,137

pathological, of glaucoma, ${ }_{2} 8_{3}$, 284

of interstitial keratitis, 224, 225

of stenosis of nasal duct, 125 , $137,13^{8}$

of trachomatous conjunctivitis, $175,176,177,178,180$

of ulcerative keratitis, 205,206 , 207, 209, 2 I0

Anchyloblepharon, 480,483

Anemia, acute, thrombosis of retinal artery in, 359

pernicious, retinitis in, 352

Anesthesia from cocain, 51, 5 10 and adrenalin, IOI, I 43

from holocain, 5 I I

general, in enucleation of eye, 270 in tenotomy in children, $46 \mathrm{I}$

of cornea, how determined, 35
Anesthesia of cornea, in glaucoma, 277

in neuropathic keratitis, 217

Angiosclerosis, 291, 302

Angle alpha in hypermetropia, 453 , 454

Anisometropia, 424

a factor in causation of strabismus, 424

consequences of, 424

definition of, 424

difference in size of pupils due to, 25

exceptionally a blessing in disguise, 424

treatment of, 424,425

Anomalies, muscular. See Muscular anomalies of the eyes.

of accommodation, 425

of refraction, $\mathbf{3 8 8}$

and accommodation, 388

etiological importance of, 38.9 , 390

general observations upon significance of, $3^{88}$

harm which results from unskilful correction of, 388 , $3^{89}$

prevalent misconceptions regarding, 390, 391, $39^{2}$

special skill and training required in correction of, 388

Anterior chamber, examination of, by oblique illumination, 37,500

eyelash lodged in, 503

filtration angle of, 282

alteration of, in glaucoma, 283,284

inspection of, 35

obliteration of, in glaucoma, 278

paracentesis of, 2 I 5

pus in, 211,215

removal of foreign bodies from, 503

polar cataract, $\mathbf{3 2 0}$

staphyloma. See Staphyloma of cornea.

synechia. See Synechia, anterior.

Antisepsis in wounds of eye, 495,5 or preparatory to operations upon eye, 327,46 r, 5 I I

Antiseptic agents, 50, 5I, 5 II, 5 I 2

Antitoxin, diphtheria, 6r

Apothecaries, a reprehensible habit of, 43

Appendix, 505

Application of carbolic acid to eye. See Carbolic acid, application of. of collyria, 4I, 42, 43, 44 
Application of ointments to eye and lids, 45

Aqueous humor, inspection of, 35,36 lessened transparency of, in iritis, 247

Arcus senilis, 235 pathology of, 235

Argyll Robertson symptom, 27, 379

Argyria conjunctivæe, 193 etiology of, 193 signs of, 193 treatment of no avail in, 193

Argyrol, 51, 157 in dacryocystitis, 137 in purulent conjunctivitis, ${ }^{6} 6_{4}$

Argyrosis. See Argyria conjunctiva. Arlt's operation for ectropion, 95 tenotomy, 457,465

Arsenic in herpes zoster ophthalmicus, 266

in neuropathic keratitis, 222

in retinitis of pernicious anemia, 353

Artificial eye, 241, 273, 506 pupil, 238, 267, 325, 326

Associated ocular paralyses, 446

Asthenopia, symptoms characteristic of, 32

Astigmatism, $4 I_{3}$

according to the rule, $4^{15}$ usually located in cornea, 4 I 5 acquired, 4I 7

advantages and disadvantages of a cycloplegic in determination of, 422,423

a factor in the causation of cataract, 303,345

of glaucoma, 285,345

of miliary choroido-retinitis, 343

of myopia, 406

after cataract extraction, 4I 7

against the rule, 415

special significance of, 285 , 4 I $5,416,4$ I 7

usually located in lens, $4 \mathrm{I} 5$

apparent increase of, how explained, $4 \mathrm{I} 6$

a significant amount of, not incompatible with normal acuteness of vision, $4 \mathrm{I} 7$

asthenopia, how caused by, 4I4

compound, $4 \mathrm{I} 5$

consequences of, 285, 303, 343, $345,406,4^{1} 8$

corneal, 4 I 5

correction of, by cylindrical glasses, 419,420

definition of term, 4I 3
Astigmatism, increase of, $4 \mathrm{I} 6$

irregular, 237, $4^{13}$

latent, $4 \mathrm{I} 4,4 \mathrm{I} 6$

explanation of, $4 \mathrm{I} 4$

how rendered manifest, $4 \mathrm{I} 6$

lenticular, $4 \mathrm{I} 5$

manifest, $4 \mathrm{I} 6$

may be acquired, 417

may be due to asymmetry or ob-

liquity of crystalline lens, $4 \mathrm{I} 3$

measurement of, $42 \mathrm{I}, 422$

by skiascopy, 422

with lenses, test-type, and astigmatic dial, 422

with ophthalmometer, 422

with ophthalmoscope, $42 \mathrm{I}$

mixed, 4I5

nature of visual disturbance in, 4I 3,4 I 4

of ten inherited, $4 \mathrm{I} 3$

often mistaken for myopia, 415

one of the commonest rerractive

anomalies, $4 \mathrm{I} 3$

orientation of, $4 \mathrm{I} 6$

principal meridians in, 416

regular, $4 \mathrm{I} 3$

seat of, 4I3

simple, 4 I 5

method of detecting pronounced degrees of, 417,418

static, $4^{\mathrm{I}} 4$

symptoms of, 4I 4,4 I 5

the cornea the usual seat of, $4 \mathrm{I} 3$

the ophthalmometer an untrust-

worthy means of measuring, 422

treatment of, 4 I 9

usually a congenital fault, $4 \mathrm{I} 3$

varieties of, 4I3,415

Astringents and antiseptics, indications for employment of, 40

Atresia of canaliculi, 130

of lacrimal puncta, 126

Atrophy of conjunctiva, 176

of eyeball from glaucoma, 280

from penetrating wounds of globe, 495,496

from purulent panophthalmitis, 334

of lacrimal gland, I 24

of optic nerve, 378

inflammatory, 38r

non-inflammatory, 378

of retina, in retinitis pigmentosa, 355

Atropin, 46

and boracic acid collyria, 507, 508 follicular conjunctivitis from pro-

longed use of, $4^{8}$

glaucoma induced by, 47,288 
Atropin, idiosyncrasies displayed towards, 48,26 I

indications for employment of, 40, 46

persistent effect of, 47

to be used with caution in serous iritis, $26 \mathrm{I}$

weak solutions of, in incipient cataract, 3 I I

Author's crochet-pointed strabismus hook, $45^{8}$

doctrine of subnormal accommodative power, 433

eye-bandage, 55

lacrimal probe for use by patients, 149

probes, I 4 I

measurements of nasal ducts, I4I needle-holder, 459

series of cataract extractions, 316 supplementary lacrimal probe, I 45 theory of the etiology of post-hemorrhagic blindness, 359

of the genesis of pterygium, 198 views on the amblyopia of squinting eyes, 449, 450, $45^{1}$

on the genesis of sympathetic ophthalmitis, 258,259

Auto-infection, 208

BAciltus, Klebs-Löffler, I 70, 2 II Weeks, 156

Bader's scleral fixation forceps, 270 , 272

Bandage, Author's eye, 55,56

Bandages, eye, indications for use of, $5^{6}$

Basham's mixture, $35 \mathrm{I}$

Belladonna and mercury, ointment of, 5 I 4 lotion of, 512

Bichlorid of mercury. See Mercury, bichlorid.

Bifocal lenses, $42 \mathrm{I}, 430$ cemented, $43^{\circ}$ invisible, 430

Blennorrhea of lacrimal sac, I 32

Blepharitis marginalis, $63,389,467$ etiology of, 64,65 .

treatment of, 65,66

Blepharo-adenitis, $6_{3}$

"Blind spot," enlargement of, in choked disc, $37 \mathrm{I}$

Blindness, monocular, often present without knowledge of patient, 33

Blisters in acquired ptosis, Ioo in paralysis of facial nerve, 107 of sixth nerve, 443
Blisters in retrobulbar neuritis, 375

Bonnet, operation of enucleation of eye devised by, 269

Boracic acid, after removal of pterygium, 200

conditions in which, is useful, $5 \mathrm{I}$

formulæ for collyria of, 504

in asthenopia, $5 \mathrm{I}$

in corneal ulcers, 2 I 2

in diphtheritic conjunctivitis, 172

in hyperemia of conjunctiva, 153

in keratomalacia, 216

in membranous conjunctivitis, I 69

in mild catarrhal conjunctivitis, in ${ }^{51}$ phlyctenular conjunctivitis, 190

in superficial injuries of cornea, 485

ointment of, I 57

saturated solution of, 5 I I conditions in which, is useful, 5 II

in purulent conjunctivitis, 163

in wounds of the lids, 482

use of, as a poultice, $53,5 \mathrm{II}$

Bowman's membrane, 204

never regenerated, 203

operation for fistula of lacrimal gland, I 2 I

for ptosis, IO2

of division of canaliculus, I 40

probes, inadequate size of, 140

Bull, C. S., 266, 292

Buller's shield, I 66

Buphthalmos, 276

characterized by general enlargement of eyeball, 276

etiology of, 276

Burns of conjunctiva, $481,482,483$ entropion from, 482

symblepharon from, 482

treatment of, $482,483,484$

of cornea, 485

consequences of, 485

leucoma from, 485

treatment of, 485,486

of lids, $48 \mathrm{I}$

ectropion from, $48 \mathrm{I}$

from gunpowder, 483

treatment of, 482,483

Thiersch grafts in, 483

CAlomel purge, value of, in inflammatory affections of the eye, $6 \mathbf{r}$ 
Canal of Schlemm, 282

Canaliculi, atresia of, $\mathbf{r} 30$ etiology of, I 30 treatment of, I 30

Canaliculus, division of lower, 128 of upper, I 30

foreign bodies in, 131,448

knife, Weber's, I 29 modified, I 29

polypus in, I3 I

Capsular cataract. See Cataract, capsular.

Carbolic acid, application of, to corneal ulcers, 51, 2 I 3,2 I 4, 2 I 5 , 5 I 4

in treatment of hordeolum, 70 , $5 \mathrm{I} 4$

Caries of orbit, I 11

treatment of, I I 3

hydrochloric acid in, I 13

resulting from stricture of nasal duct, ${ }_{4} 8$

Castor oil in burns of the eye, 54 solution of atropin in, indications for use of, 54

\section{Cataract, 297}

accommodative strain in causation of, $3 \circ 3,344,3^{89}$

a mber-colored, characteristics of, 301,309

anterior polar, 320. See also Cataract, pyramidal.

black, 3 OI

Brisseau's discovery of true nature of, 298

capsular, 326

diagnosis of, 327

etiology of, 326

treatment of, 327

couching of, 314

discission of, as formerly practised, 3 I 4

as now performed, 314

encountered oftenest in the aged, 298

erroneous ideas long prevalent as to nature of, 297

extraction of, 3I 4,3 I 5

combined, 3 I 5

linear, 319

prognosis in, 316

simple, 315

suction, 319

extractions, author's series of, 3 I 6

Förster's operation in immature, $3^{1} 3$

general, 299

acquisition of "second-sight" a premonitory symptom of, 302
Cataract, general, amblyopia consequent upon, 3 I9

capsular opacity after extraction of, 3 I 8

conditions which point to probable existence of, 307

constitutional disorders which predispose to development of, 302

determination of maturity of, $307,308,309$

diagnosis of, 304,305

errors in, 35, 305, 306

help afforded by a mydriatic in, $304,3 \circ 5$

by oblique illumination in, 304,306

by ophthalmoscope in, 305

nature of visual impairment helpful in, 300,307

subjective symptoms to be considered in, 306, 307

differences in rapidity of development of, $30 \mathrm{I}$

due to degeneration of lens fibers, 302

etiology of, $302,303,304$

normal response of pupil to light in, 300

ocular diseases which predispose to, $3 \circ 3$

predisposition to, often inherited, 302

prenatal development of, 303, 304

traumatic lesions which tend to production of, $3 \circ 3$

treatment of, $310-320$

atropin in, 310

only operative, of avail, 3 ro post-operative, 3 I 6

prophylactic, 310

when immature, $3 \div 3$

when only one eye is involved, 3I 2, 3I 3

variations in color of, 301

varieties of, 299

in detachment of retina, 363

in high myopia, 342

origin of name, 297,298

partial, 320

definition of, $32 \mathrm{C}$

etiology of, $320,321,322,323,324$

varieties of, 320

posterior polar, 322, $35^{6}$

etiology of, 323,324

treatment of, 325

varieties of, 323,324 
Cataract, pyramidal, $32 \mathrm{r}, 322$ etiology of, 321,322 vision in, 322 ripeness of, $3 \circ 7$ secondary. See Cataract, capsular.

traumatic, 294, 299, 303, 318, 492, $494,498,499$

varieties of, 298,299

zonular, 324

diagnosis of, 325

etiology of, 324

impairment of vision in, 325 treatment of, 326

Catarrhal conjunctivitis. See Conjunctivitis, catarrhal.

Cathartic, energetic, value of, in inflammations of the eye, 61

Caustic potash, treatment of entropion of lower lid with, 82

Cautery, actual, in corneal ulcers, 2 I 4

Cellulitis of orbit, 108

romplications in, 109

diagnosis of, 109

etiology of, 108

symptoms of, 108

treatment of, Io9

Central artery of retina, embolism of. See Embolism of central artery of retina.

thrombosis of. See Thrombosis of central artery of retina. retinal vein, thrombosis of. See Thrombosis of central retinal rein.

Chalazion, 73

clinical history of, 73,74

diagnosis of, 73

etiology of, 73

operation for, 75

treatment of, 74

Childhood, glaucoma in, 276

Children, examination of eyes of, 57

Chlorin water in corneal ulcers, 212 , 221,507

Choked disc, $37^{\circ}$ commonly caused by intracranial new-growths, $37 \mathrm{I}$

consequences of, $37 \mathrm{r}$

etiology of, $3^{67}, 3^{68,} 371,372$

ophthalmoscopic picture of, 369 , 370

pathology of, $368,3^{60}$

prolonged course of, $37 \mathrm{I}$

sight often insignificantly impaired in early stages of, $37 \mathrm{r}$ symptoms of, $37 \mathrm{I}$

treatment of, 373

usually bilateral, 372
Chorea, 390, 418

Choroid coat, diseases of, 340

retina, and optic nerve, diseases of, 338

etiology of, 339

impairment of vision in, $33^{8}$

prognosis in, 339,340

recognition of existence of, without the aid of the ophthalmoscope, 338,339

symptoms indicative of, 338,339

treatment of, to be directed to the underlying systemic cause, 339

sarcoma of, 348

tumors of, 348

commonly of malignant type, 348

treatment of, 348

Choroiditis, 340

of high myopia, $34^{2}$

cataract a consequence of, $34^{2}$

characteristics of, 342

detachment of retina in, 342

impairment of vision in, 342

treatment of, 346

plastic, 340

etiology of, 340

of traumatic origin, 342

etiology of, 342

serious import of, 343

sympathetic ophthalmitis

from, 343

treatment of, 346

varieties of, 340

purulent, 340. See also Panophthalmitis, purulent.

serous, 340. See also Iritis, serous.

syphilitic, 340

impairment of vision in, 340

iritis frequently associated with, $34^{\circ}$

loss of transparency of vitreous humor in, $34^{\circ}$

occurs in inherited syphilis, 340 pathological changes in, 340, 341 symptoms of, 342

treatment of, 345

Choroido-retinitis, miliary, 343

a common consequence of eyestrain, 343

a factor in causation of cataract and glaucoma, $285,3 \circ 3,344$, 345 
Choroido-retinitis, miliary, deserving of more consideration than is commonly accorded it, 343 etiology of, 343 ophthalmoscopic picture of, 344 "patchy choroid" a result of, 344

pathological changes in, 344

symptoms of, 344

treatment of, 347,348

Ciliary body, diseases of, 268

hernia of, 494

tumors of, 269

muscle, insufficiency of, in subnormal accommodative power, 433,435

paralysis of, 430

spasm of, 432

Circumlental space, in glaucoma, 284

Clonic spasm of lids from reflex dental irritation and from phimosis, 223

Cocain hydrochlorate, formula for solution of, 5 Io

in treatment of stricture of nasal duct, 143

valuable as an anesthetic, but not as a remedial agent per se, $5 \mathrm{I}$

Colchicum, 244, 262

"Cold" as a factor in causation of catarrhal conjunctivitis, I 56 of dacryoadenitis, I 19

of dacryocystitis, I 33

of neuropathic keratitis, 2 I9

of paralysis of sixth nerve, $4+3$ of retrobulbar neuritis, 374

Collyria, 40-44, 46-52

application of $41,42,43,44$

contamination of, by apothecaries, 43

formulæ for, 505-5I I

precautions necessary in using poisonous, 43

rose-water objectionable in, 44 sterilization of, $50 \mathrm{I}$

"Compound calomel powder," formula for, 519

in acute dacryoadenitis, 120

in acute dacryocystitis, 136

in blepharitis, 66

in glaucoma, 280

in phlyctenular conjunctivitis, 192

in recurrent hordeola, $7 \mathrm{~J}$

Concomitant squint, 448 . See also Squint, concomitant.

Condylomatous iritis, 255
Conical cornea, 241

galvano-cautery in, 242

marked impairment of vision in, $24 \mathrm{~J}$

myopia of high grade, a consequence of, 24 I

symmetrical aberration in, $24 \mathrm{I}$ treatment of, 24 I

Conjugate ocular paralyses, 446

etiology of, 446,447

pathology of, 446,447

symptoms of, 446,447

treatment of, 447

varieties of, 446

Conjunctiva, diseases of, I $5 \mathbf{I}, 467$

hyperemia of, I $\mathbf{5 2}$

in juries of, $48 I, 482,483$

Conjunctivitis, I 54

catarrhaJ, I 54

acute, I 55

bacteriology of, I 56

diagnosis of, I 55

etiology of, 156

nearly always binocular, I 55

prognosis in, 156

treatment of, I 57

chronic, 157

etiology of, I 57

treatment of, 157

croupous, 168

character of membrane in, I 60

etiology of, I 68

symptoms of, I 69

treatment of, I 60

diagnostic signs and symptoms of, I 5

diphtheritic, $\mathbf{1 6 9}$

atypical forms of, I 7 I

bacteriology of, I 7o, $7^{2}$

character of membrane in, I 70

consequences of, 17 I

entropion from, $I 7$

infection, modes of, in, $17 \mathrm{I}$

symptoms of, 170

treatment of, 172

follicular, I73

pathology of, 173

symptoms of, I 73

treatment of, I 73

gonorrheal, 158. 'See also Conjunctivitis, purulent.

lymphatica. See Conjunctizitis, phlyc'enular.

phlyctenular, I 86

associated pathological conditions in, 192

catarrhal type of, 187

consequences of, 187

diagnosis of, I 87 
Conjunctivitis, phlyctenular, etiology of, 188 pathology of, 186 symptoms of, 186 treatment of, 189 purulent, $\mathbf{I}_{5} \mathbf{8}$

bacteriology of, 158,162

Buller's shield in, I 65

consequences of, 160

etiology of, 159

prognosis in, 160

prophylaxis in, 167

symptoms of, 158

treatment of, $I_{6} 1$

toxic, 48 ; 50,192

etiology of, 193

symptoms of, 193

treatment of, 193

trachomatous, I 74

consequences of, 176

entropion from, I 77

etiology of, 174,178

implication of cornea in, 174,177 pannus in, development of, 177 pathology of, $174,1_{7} 6$

symptoms of, I 74

treatment of, 179

mechanical, I8 I

varieties of, 154

vernal, 183

bulbar type of, $18_{3}$

palpebral type of, 184

pathology of, 184

significance of name, 183

symptoms of, 185

treatment of, 185

varieties of, i 83

Consecutive atrophy of optic nerve, $38 \mathrm{I}$

Constipation, habitual, aloin useful in, 520

Constitutional remedies useful in diseases of the eye, 39 formulæ for, $5^{16-520}$

Contusions of eye, 491 observations upon, $57-62$

commoner causes of severe, 493 eyeball, how protected against, $49 \mathrm{I}$

injuries that may result from, $49 \mathrm{I}, 492,493$

myopic eyes most apt to suffer from, 407,492

rupture of eyeball from, $49 \mathrm{I}$

sympathetic ophthalmitis from, 493

traumatic cataract from, 492

treatment of, 493,494

why so seldom disastrous, 49 I
Convergence center, lesions of, in associated ocular paralyses, 446

Convergent concomitant squint, 453

Copper citrate in trachomatous conjunctivitis, 182

sulphate in trachomatous conjunctivitis, $180,181,515$

Cornea, abscess and ulcer of, 207

bacteriology of, 210

carbolic acid in treatment of, 2 I 3

clinical history of, 207, 209

diagnosis of, 208

etiology of, 208, 2 I 0

malignancy of, factors which determine, 2 10

pathology of, 207,210

symptoms of, 208

treatment of, 2 I I, 221

varieties of, 2 I I

and sclera, diseases of, 202

anatomy of, 204

anesthesia of, in glaucoma, 277

in neuropathic keratitis, 217 , 218,220

method of determining, 35, 277

conical. See Conical cornea.

diseases of, 202

dots on, in uveitis, 252

eczema of, I 86

inflammation of. See Keratitis.

leucoma of, 236

lodgment of foreign bodies on, 487 , 488

loss of transparency of, in glaucoma, 277

macula of, 236

massage of, 23 I

nebula of, 236

opacities of, 235

leucomatous, 236

macular, 236

nebular, 236

opacity of, senile, 235

penetrating wounds of, 494. See also Wounds of eye, penetrating.

anterior synechia from, 494

impairment of vision from, 494

sensibility of, how determined, 277 staphyloma of. See Staphyloma of cornea.

superficial injuries of, $\mathbf{4 8 5}$

tattooing of, 238

tumors of, 242

ulcer of, 207

dendritic, 21 I, 220

herpetic, 2 I I, 220 
Cornea, ulcer of, hypopyon, 2 I I

"infected," 2 10

marginal, or ring, 2 I I

mycotic, 211

neuropathic, 217,218

phlyctenular, 205

post-malarial, 220

progressive, 209

regressive, 209

serpent, 2 I I

simple, 2 10

Credé's prophylactic measures, 167

Croupous conjunctivitis, $\mathbf{6} 68$

Crystalline lens, anatomy and physiology of, 295

an epithelial structure, 295

changes in, from advancing age, 296

characteristics of, in early life, 295

development of nucleus of, 296

diseases of, 295

dislocation of. See Dislocation of crystalline lens.

growth of, 295

nutrition of, 297

transparency of, impaired by malnut rition, 297

Curet, chalazion, 75

use of, in corneal abscess, 2 I 5

in corneal ulcers, $2 \mathbf{I}_{4}$

in periostitis of orbit, 113

Curettage, $\mathrm{II}_{3}, 2 \mathrm{I} 4$

Cyclitis, 268

etiology of, 268

impairment of vision in, 268

plastic, 268

oftenest due to syphilis, 268

sympathetic ophthalmitis worst type of, 268

treatment of, 269

purulent, 268

infection in, 268

treatment of, 269

serous, 268 . See also Iritis, serous.

symptoms of, 268

treatment of, 269

usually associated with iritis, 268

Cycloplegia, post-diphtheritic, 43 I

Cyst, hydatid, of orbit, II 4

sebaceous, of lid. See Milium.

of orbit, II 4

tarsal. See Chalazion.

watery, of lid. See Hydrocystoma.

\section{DACRYOADENITIS, I I 9}

acute, IIO

diagnosis of, I 19
Dacryoadenitis, acute, etiology of, I 19

mistaken for cellulitis of orbit, I 19

symptoms of, I I9

treatment of, I 20

chronic, I I9

symptoms of, I 20

treatment of, 120

Dacryocystitis, I 3 I

acute, I 33

chronic, 132

etiology of, r $3 \mathbf{r}$

in new-born, 150

prognosis favorable in, I 50

treatment of, 150

symptoms of, 132

treatment of, 136

usually dependent upon stricture of nasal duct, I 3 I

Dacryoliths, $\mathbf{I} 2$

in canaliculi, $\mathbf{I} \mathbf{3}$

treatment of, $\mathbf{I} 3 \mathrm{I}$

Dacryops, I 2 I

etiology of, I 2 I

Graefe's operation for, 122

treatment of, I 2 I

Daylight, inspection of eye by, 34

"Dead" teeth, 222

Dendritic keratitis, 220

Dental irritation, reflex, keratitis and paralysis of accommodation from, 222, 223

Descending optic neuritis, 372

Detachment of retina, 360 . See also Retina, detacliment of.

Determination of acuteness of vision, 27

of corneal sensibility, 35,277

of intraocular tension, 23

of visual field, 29

Diabetes, 248, 302, 3 $8,339,349$, $372,379,3^{82}$

Diabetic retinitis. See Retinitis, diabetic.

Diagnosis, helpful questions to be asked in endea voring to reach a, 3 I

importance of an early, in eye diseases, $4^{\circ}$

Diagnostic significance of symptoms olsserved in ocular inflammations, 3 I

Diagnosticating diseases of the eye, difficulties with which the general practitioner has to contend in, 17

Dionin, $\mathbf{5}^{2}$

formula for collyrium of, 509

in glaucoma, 52, 289 
Dionin in iritis, 52, 261

in keratitis, 52, 213

in opacities of the cornea, 52

Diphtheria, I69, 1 70, I 71, 430, 445 antitoxin, 6r, 172

Diphtheritic conjunctivitis, r69

Diplococcus of Sattler, I 74

Diplopia, crossed, 444

homonymous, 444

in concomitant squint, 448,449 , $450,451,453,454,464$

in heterophoria, 467

in paralytic squint, $439,441,443$, 444,445

monocular, in incipient cataract, 307

of sudden onset, significance of, 33

Discharge from eye, character and amount of, diagnostic value of, 36

Discission of cataract, 3r4. See also Cataract, general, treatment of.

Diseases of the eye, value of constitutional remedies in, 38

Dislocation of crystalline lens, $\mathbf{3 2 7}$ acquired, 328

beneath the conjunctiva, 328 congenital, 328

consequences of, 328,332

diagnosis of, $33^{\circ}, 33^{1}$

effect upon vision of, 328,329

etiology of, 328

in high myopia, 328

interesting case of, 320

into the anterior chamber, 327,33 I

iridodonesis a symptom of, 331

traumatic, $328,33^{2}$

treatment of, $331,33^{2}$

varieties of, 327,328

of lacrimal gland, 122

Donders on the etiology of convergent squint, 397,453

Duboisin, 48

Dyspepsia, induced by eye-strain, 418

\section{Ectropion, 88, 48 I}

cicatricial, 89

epiphora a common symptom of, 88

etiology of, 88

from chronic conjunctivitis, 9 I from facial paralysis, 88

often induced by lacrimal disease, 89
Ectropion, organic, 89

Arlt's operation for, 95

commonly of traumatic origin, 89

etiology of, 89

operative treatment of, 95

employment of Thiersch and

senile, 88 Wolfe grafts in, 97

treatment of, $91, \mathrm{Q}_{2}$

spasmodic, 88

comparable to paraphimosis, 89

etiology of, 88

Snellen's operation for, 92

treatment of, 92

varieties of, 88

Eczema conjunctivæ, 186

of eyelids, $7 \mathrm{I}$

etiology of, 7 I

treatment of, $7 \mathrm{r}, 72$

Edema of conjunctiva induced by dionin, 52

of lids in purulent conjunctivitis, 163

of retina in miliary choroido-retinitis, 344

Electrolysis in treatment of lacrimal stricture, 149

Electro-magnet, Sweet's, 499

Embolism of central artery of retina, 357

consequences of, 357

etiology of, 357

ophthalmoscopic picture of, 357

thrombotic obstruction mis.

taken for, 357

treatment of, $35^{8}$

Emmetropia, 393

definition of, 393

vision not necessarily acute in, 393

Entogenous infection of eye after cataract extraction, 3I 6

Entropion, 8o

from diphtheritic conjunctivitis, 171

from trachomatous conjunctivitis, 177,182

from trauma, 482

of lower lid, 82

treatment of, 82

with caustic potash, 82

of upper lid, operation for, 84

organic, $8 \mathrm{I}$

etiology of, $8 \mathrm{r}$

Green's operation for, 86

Hotz's operation for, 86

treatment of, $8 \mathrm{I}$

senile, 8 I 
Entropion, spasmodic, $8 \mathrm{r}$

etiology of, 81

treatment of, 82

varieties of, 80

Enucleation of eyeball, 269, 294

after-treatment of, 273

Bader's scleral forceps in, 270 , 272

control of hemorrhage in, 273

general anesthesia required in, $27^{\circ}$

instruments needed in, 270

objectionable practices in con-

nection with, 273

steps of operation of, 272

when panophthalmitis is present, 269, 334

Epilation forceps, 183

Epiphora a characteristic symptom of disease of the lacrimal drainage apparatus, 126

of long standing, significance of, 32

Episcleritis, 244

diagnosis of, 244

etiology of, 244

pathology of, 244

symptoms of, 244

treatment of, 245

Epithelioma of lid, 79

Ergot, 337

Erysipelas, facial, a cause of thrombosis of central retinal vein, 359

dacryocystitis mistaken for, I 33

Eserin, 49

and boracic acid collyrium, formula for, 508

follicular conjunctivitis from prolonged use of, $5^{\circ}$

in ulcerative keratitis, 213

precautions necessary in use of, 49

production of iritis from injudicious use of, 49

rules for employment of, in glaucoma, 49

value of, in glaucoma, 288, 293

Esophoria, 473

detection and measurement of, 469 , 470,474

etiology of, 396, 473, 475

state of refraction to be determined in every case of, 474

symptoms of, 466,467

treatment of, $470,471,474$

by glasses, 474,475

by operation, $475,476,477$

Euphthalmin hydrochlorate, 47

formula for collyrium of, 510

in diagnosis of cataract, 305

of iritis, 247
Eversion of lacrimal puncta, $\mathbf{2} \mathbf{2 8}$

of lower lid, 22

of upper lid, 22

Examination of eyes of unruly children, 57

Exanthematous fevers, $139,192,208$, 408

Excision of chalazion, absurdity of attempting, 75

of lacrimal gland, 124, 149

sac, 149

of orbital growths, I 6

"Exciting eye," 256, 257

Exophoria, 47I

detection and measurement of, 469 , 470

etiology of, $407,408,438,467,468$, $47 \mathrm{I}$

symptoms of, 466,467

treatment of, $470,471,472,476$, 477

by glasses, $47^{2}$

by operation, $47^{2}$

Exophthalmos from hypertrophy of lacrimal gland, 123

from ophthalmoplegia totalis, 445

from tumors of lacrimal gland, 125

of orbit, I 14

in cellulitis of orbit, 108, 109

in periostitis of orbit, I I I

External recti muscles, insufficiency of. See Esophoria.

rectus muscle, paralysis of. See Paralysis of external rectus muscle.

Extraction of cataract. See Cataract, general, treatment of.

Eye, ametropic, 394

artificial, 273

circulation of lymph in, 28I

emmetropic, 393

exciting, 256,257

infection of, 316, 317, 319, 494, 495,497

injuries of, 480

inspection of, by daylight, 34

by oblique illumination, 36

speculum, 457

squinting, amblyopia of, 449

sympathizing, 257

Eyeball, contusions of, 49I

foreign bodies in. See Wounds of eye complicated by lodgment of foreign bodies within ball.

gunshot wounds of, 498

penetrating wounds of, 494

protrusion of. See Exophthalmos. 
Eye-dropper, right, and wrong, way to hold, 42

suggestions regarding use of, $4 \mathrm{I}$, 42

transference of infectious material by, $4 \mathrm{I}$

Eyelid, epithelioma of, 79

eversion of, 22

malignant tumors of, 79

sarcoma of, 80

warts upon, 78

Eyelids, anatomy of, 68

diseases of, 63

injuries of, 480

Eye-strain a factor in causation of blepharitis, $64,3^{89}$

of cataract, $3 \circ 3,344,389$

of choroido-retinitis, $343,3^{80}$

of chronic conjunctivitis, 157 , 389

of glaucoma, $285,290,344$, $3^{89}$

of headache, 390

of indigestion, 390

of insomnia, 390

of nausea, 390

of neurasthenia, 390

of somnolency, 390

of styes, 60

of tinnitus aurium, 390

of vertigo, 390

constitutional disorders due to, 389

etiological importance of, 389

ocular maladies caused by, 389 , 390

symptoms indicative of, 32

FACIAL erysipelas, I 33, 359

nerve, paralysis of. See Paralysis of facial nerve.

Far-sightedness. See Hypermetropia.

Fellow-eye in diphtheritic conjunctivitis, 173

in glaucoma, 290

in purulent conjunctivitis, 165 , 166

in sympathetic ophthalmitis, 257

Fevers, exanthematous, I 39, I 92 , 208,408

Field of vision, contraction of, in detachment of retina, $3^{6} 3$

in embolism of retinal artery, 357

in glaucoma, $279,287,29 \mathrm{I}$

- in primary atrophy of optic nerve, 379
Field of vision, contraction of, in retinitis pigmentosa, 355

determination of, 29,30

in hemianopsia, $383,384,385$, 386

simple method of measuring, $29^{\circ}$

Filtration angle of anterior chamber, 283

obstruction of, in glaucoma, $28_{3}$, 284

Fistula, lacrimal, 134, I 48

of lacrimal gland, 120

Fixation, binocular, disinclination to, observed in some cases of squint, 456

the ideal result aimed at in operating for squint, $45^{5}$

Fixing eye, determination of, in squint, $45^{2}$

Florence flask for sterilizing collyria, 504

Fluidity of the vitreous humor, 334

Follicular conjunctivitis, I73

induced by atropin, 48

induced by eserin, 50

Foreign bodies in anterior chamber, $5 \circ 3$

in iris, 500

in lens, 498

in orbit, 504

in vitreous chamber, 500

superficial lodgment of, in eye, 486

means of detecting 489

misconceptions of patient in regard to, 489

occupations which predispose to, 487

symptoms which point to, 32,480

treatment of, $490,49 \mathrm{r}$

within the eyeball. See Wounds

of eye complicated by lodgment of foreign bodies within ball.

Formulæ for agents for exact application to eye and lids, 514,5 I 5 for collyria, 505, 506, 507, 508, 500, $5 \mathrm{IO}, 5 \mathrm{II}$

for constitutional remedies useful in eye diseases, $516,5^{17}, 5_{18}$, 519,520

for lotions, 5 I I , 5 I 2

for ointments, $5^{1} 3,5^{1} 4$

Förster's operation, 31 3, 325

Fourth nerve, paralysis of. See Paralysis of superior oblique muscle.

Fowler's solution in herpes zoster ophthalmicus, 266 
Fuchs on identity of herpes cornex febrilis and keratitis dendritica, 22 I

on pathology of pinguecula, 195 of pterygium, 197 of vernal conjunctivitis, 184

Fulminating glaucoma, 286

Fungous growth in dendritic keratitis, 2 I I

Fungus hematodes oculi. See Retina, glioma of.

Galvanism in acquired ptosis, 100 in retinitis pigmentosa, 357

of little value in primary atrophy of optic nerve, $3^{8} \mathrm{I}$

Galvano-cautery, 149,215

Gasserian ganglion, 208

Gifford's use of sodium salicylate in sympathetic ophthalmitis, 265

Gland, lacrimal. See Lacrimal g!and.

Glands of eyelid, 68

Glasses, action of, in ametropia, 392

bifocal, $421,43^{\circ}$

in presbyopia, 429,430

in subnormal accommodative power, $43^{6}$

cylindrical, 4 I 9

do not "weaken sight," $39 \mathrm{I}$

effect of, in astigmatism, 419,420 in hypermetropia, 396, 399, 400, 401,402

in myopia, 406, 408, 410

not every eye needing their help makes a direct appeal for, 392

not given solely with a view to making vision more acute, $39 \mathrm{r}$

not to be regarded as a dernier ressort, $39 \mathrm{I}$

popular misconceptions regarding, 390, 391, 392

smoke-tinted, 55

sphero-cylindrical, 419, 420

Glaucoma, 274

fulminating, 286

hemorrhagic, 286

inflammatory, 276

clinical history of, 279,280

constitutional disorders predisposing to the development of, 285

diagnosis of $277,278,279,28 \mathrm{I}$ duty of general practitioners in management of, 287

etiology of, $281,282,283,285$

intermittent character of, 279
Glaucoma, inflammatory, narrowing of the visual field in, 286

ophthalmoscopic picture of, 278

sympathetic ophthalmitis caused by, 280

symptoms of, objective, 277 , 278

subjective, 277

treatment of, 287,289

constitutional remedies in, 289

correction of refractive errors in, 290

dionin in, 289

eserin in, 288

iridectomy the sovereign remedy in, 287,289

of fellow-eye in, 290

varieties of, 286

malignant, 286, 289

secondary, 293

definition of, 293

etiology of, 293, 294

symptoms of, 294

treatment of, 294

simple, 29r

diagnosis of, 291, 292

etiology of, 291

symptom of, 29 I

treatment of, 292

iridectomy in, 292

non-operative, 293

sympathectomy in, 293

Glioma of retina, 364

Gonococcus, 158,162

Gonorrhea a cause of dacryoadenitis, ii 9

Gonorrheal conjunctivitis. See Conjunctivitis, purulent.

iritis, 248

ophthalmia. See Conjunctivitis, purulent.

Gout in etiology of dacryoadenitis, . I 9

of eczema of lids, $7 \mathrm{I}$

of glaucoma, $285,291,293$

of iritis, $248,251,266$

of retinitis, 349

of retrobulbar neuritis, 374

of scleritis, 242

Gouty affections of eye, lithia water

in, 5 I 9

Graduated tenotomies, no room for, 476

Graefe's, von, operation for dacryops, 122

vertical diplopia test, 434,469

Grandmont's, de, operation for ptosis, 102 
Granular conjunctivitis. See Conjunctivitis, trachomatous.

lids. See Conjunctivitis, trachomatous.

Green's operation for entropion, 86

"Ground-glass" cornea, 223

Gruening's operation for ptosis, I02

Gummata in lacrimal sac and nasal duct, 139

Gummatous iritis, 255

Gunshot-wounds of eye, 498

HALSTED's use of silver foil as a surgical dressing, 96

Head and Campbell's investigation of pathology of herpes zoster, 259

Headache in ametropia, $32,390,398$ in astigmatism, 418

in choked disc, $37 \mathrm{I}$

in latent muscular anomalies, 43I, 467

in toxic amblyopia, 375

Helmholtz's theory of accommodation, 426

Hemianopsia, 383

binasal, 384

bitemporal, 385

etiology of, 383,386

localizing value of, 387

prognosis in, 386

symptoms of, 383

treatment of, 387

etiology of, 383,385

explanation of, 383

homonymous lateral, $\mathbf{3 8 5}$

central vision retained in, 385

etiology of, 385

pathology of, 385

symptoms of, 385,386

transient type of, 386

treatment of, 387

without localizing value 386

horizontal, 384

symptoms of, $38_{3}$

varieties of, 383,384

Hemiopia. See Hemianopsia.

Hemorrhage into vitreous humor, 336

subconjunctival I93

Hemorrhagic glaucoma, 286

Hernia of ciliary body, 494

of iris, 494

of lacrimal gland. See Lacrimal gland, dislocation of.

Herpes corneæ febrilis, 220

zoster ophthalmicus, 218, 259

etiology of, 2 I 8

impairment of vision from, 2 I 8
Herpes zoster ophthalmicus, iris of ten involved in, 218 symptoms of, 218 treatment of, 22 I

Heterophoria, 466, 467, 469, 470, 477. See also Muscular anomalies of the eyes, latent.

Holden, Ward, 378

Holocain, action of, in promoting healing of corneal ulcers, 213

formulx for collyria of, 507,5 I I in corneal ulcers, $181,212,213$

indications for use of, 507

in episcleritis, 245

in keratomalacia, 216

in neuropathic keratitis, $22 \mathrm{I}$

Homatropin hydrobromate, 47

formula for collyrium of, 5 IO

in diagnosis of cataract, 304, 305 of iritis, 247

Hordeolum, 67

etiology of, 67,69

symptoms of, 67

treatment of, $69,70,7 \mathrm{I}$

abortive, 69

Hot water, $5^{12}$

in interstitial keratitis, 229

in iritis, 262

in purulent conjunctivitis, $\mathrm{I}_{3}$

Hotz's operation for entropion, 86

Hutchinson, Jonathan, 223

teeth, 227

Hyoscyamin hydrobromate, 48, 436 formula for collyrium of, 509

Hyperemia, diagnostic significance of the different types of bulbar, 35

of conjunctiva, 152

acute, 153

chronic, I 53

etiology of, I 53

treatment of, I 53

Hypermetropia, 394

absolute, 395

axial, 397

commonly of congenital origin, 395

consequences of, $396,398,399$, 401,402

correction of convergent squint in, 402

curvature, 397

definition of, 395,397

disturbed relation of accommodation and convergence in, 396 , 402

factors which influence development of squint in, 397

how acquired, 397

influence of glasses in, 396, 399, 402 
Hypermetropia, manifest, $40 \mathrm{I}$ most prevalent type of ametropia, 395

non-facultative, 395,398

not a pathological condition, 397 often complicated by astigmatism, 403

often inherited, $39^{8}$

presbyopia influenced by, $40 \mathrm{I}$

relation of convergent squint to, 397,402

rules for prescription of glasses in, $399,400,401,402$

tension of accommodation in, 395 , 396

treatment of, 399, 400, 401, 402, 403

varieties of, $395,397,401$

Hyperopia. See Hypermetropia.

Hyperphoria, 477

definition of, 477

detection and measurement of, $47^{8}$ etiology of, 477

ill consequences of, 477,478

treatment of, 478,479

Hypertrophy of lacrimal gland, 123

Hypochyma, 298

Hypopyon in iritis, 255

ulcer of cornea, 2 II

\section{ICE-CLOTHS, $4 \mathrm{I}, \mathrm{I}_{54}, 5^{\mathrm{I}} 2$}

in purulent conjunctivitis, ${ }^{6} 6_{3}$

Iced water, 4I, 5 I 2

Illumination, oblique. See Oblique illumination.

Imbalance, muscular, 468

Incised wounds of cornea, 494 of lids, 482

Incubation period, indefinite, in sympathetic ophthalmitis, 257 in purulent conjunctivitis, 159

Indigestion, 279

induced by eye-strain, 390, 467

Infection of eye, after cataract extraction, 316, 317, 319

from penetrating wounds, 494 , 495,497

Injuries of bulbar conjunctiva, 483 consequences of, 483 from caustic agents, 483 symblepharon from, 483 treatment of, $483,484,485$

of cornea, superficial, 485 consequences of, 485

from caustic agents, 485

treatment of, 485,486

when wound is infected, 486

of eye and its appendages, 480
Injuries of eyelids, 480

anchyloblepharon from, 480 , 482,483

ectropion from, 48 I

from gunpowder, 483

malposition of the lacrimal puncta from, 480

symblepharon from, 480,482

treatment of, 482,483

Thiersch grafts in, 483

Insomnia induced by eye-strain, 390 , 418,467

Inspection of eye by daylight, 34

Insufficiency of external recti muscles, 473. See also Esophoria. of internal recti muscles, $47 \mathrm{I}$. See also Exophoria.

Internal recti muscles, insufficiency of. See Exophoria.

Interstitial keratitis. See Keratitis, interstitial.

Intraocular lymph-stream, 282

tension, determination of, 23 how noted, 24

Inunctions of mercurial ointment, $5^{8}$

Inversion of lacrimal puncta, 128 of lid, 80

Iodin, tincture of, application of, to corneal ulcers, $5^{1}$

ointment, 124

Iridectomy, $161,238,240,241,266$, 267

extraction of cataract with, 315

in gla ucoma, 49, 287, 289, 292, 294

in partial cataract, 325

in zonular cataract, 326

preliminary to extraction of cataract, 3 I 3

Iridocyclitis, 256,268

Iridodialysis, $49 \mathrm{I}$

I ridodonesis, 331

Iris and ciliary body, diseases of, $\mathbf{2 4} 6$

diseases of, 246

hernia of, 494

wounds of, $494,495,498$

Iritis, 246

a common cause of blindness, 246

character of vascular injection in, 247

consequences of neglected, 250 , 267

diagnosis of, 246

diagnostic value of mydriatic in, 247

etiology of, 248

excited by injudicious use of eserin, 49

general management of patient in, 266 
Iritis, gonorrheal, 248 .

hypopyon in, 255

involvement of deeper eye structures in, $25^{\circ}$

iridectomy in, 266, 267

myotics contraindicated in, $5 \circ$

neuropathic. See Iritis, trophic nerve.

of herpes zoster ophthalmicus, 218 , 259

oftenest dependent upon syphilis, 255

plastic, 250, 253

characteristics of, 250

chronic, 260

etiology of, 25 I

iridectomy in, 267

treatment of, $260,26 \mathrm{I}$

types of posterior synechia in, 254

varieties of, $25 \mathrm{I}$

purulent, 252

etiology of, 252

treatment of, 260,265

serious consequences of failure to recognize, 246

serous (uveitis), $\mathbf{2 5 2}$

characteristics of, $25^{2}$

disposition to increased intraocular tension in, 252

etiology of, $25^{2}$

marked impairment of vision in, 253

treatment of, $264,265,266$

spongy, 259

sympathetic, 256

bacteria probably play no part in etiology of, $25^{8}$

character of posterior synechia in, $25^{8}$

clinical history of, 257,258

consequences of, $25^{8}$

etiology of, $256,258,259$

"incubation period" indefinite in, 257

malignancy of, 258

ophthalmitis from neglected, $25^{\circ}$

probably neuropathic in origin, $25^{8}$

removal of exciting eye in, 264

resemblance of, to iritis of herpes zoster ophthalmicus, 259

symptoms of, 258

treatment of, 260,264

mercury in, 264

sodium salicylate in, 265

symptoms of, 246,247
Iritis, syphilitic, 254

condylomatosa, 255

diagnosis of, 255

gummosa, 255

met with in inherited syphilis, 255

occurs as a prenatal affection, 255

occurs oftenest in secondary stage of disease, 253

symptoms of, 254

treatment of, $260,262,263,264$

treatment of, 260

"trophic" nerve, $\mathbf{2 4 8}$

character of posterior synechia in, 249, 254, 259

characteristics of, 249

etiology of, 248,259

formation of anterior synechia in, 254

herpes zoster ophthalmicus a variety of, 259

sympathetic ophthalmitis a variety of, 259

varieties of, 248,259

varieties of, 250

Iron chlorid in interstitial keratitis, 230

in retinitis albuminurica, 35 I

iodid, 5 I 7

in blepharitis of strumous origin, 67

in interstitial keratitis, 230

in periostitis of orbit, I I 2

in phlyctenular conjunctivitis, I9I

in scleritis, 245

in strumous affections of the eye, 60

Irritation, sympathetic, $\mathbf{2 5 7}$

JAEGER's test types, 28

Japanese stove, 4I

Jequirity, 183

Juvenile cataract, 298

\section{Keratitis, 202}

bacteriology of, 204

consequences of, 203

dendritic, 220

etiology of, 203

from reflex dental irritation, 222 herpetic, 220

impairment of vision from, 203

interstitial, 223

a disease of childhood, 223 
Keratitis, interstitial, always dependent upon inherited syphilis, 223

chronicity a marked feature of, 225

diagnostic signs of, 224

etiology of, 223

ground-glass appearance of cornea in, 223

Hutchinson teeth often associated with, 227

iritis in conjunction with, 226

marked impairment of vision in, 226

pathology of, 224

recurrent attacks of, not rare, 226 symptoms of, 223

treatment of, 227

neuropathic, 2 I 6

anesthesia of cornea in, 217

disturbance of metabolism of cornea a prime factor in causation of, 217

etiology of, 217,222

gasserian ganglion in, 2 I 6

milder forms of, 2 I 8

anesthesia of cornea in, 219 clinical characteristics of, 219

etiology of, 2 I 9

malarial fever a factor in causation of, 220

ophthalmic ganglion in, 219

symptoms of, 2 I 9

treatment of, $22 \mathrm{I}$

usually unilateral, 220

varieties of. 218

ophthalmic ganglion in, 217

treatment of, 221

varieties of, 216

pannitic, 23 I

canthotomy in, 233

diagnosis of, 233

etiology of, 231

treatment of, 233

phlyctenular, 205

etiology of, 1 88

impairment of vision from, 205

symptoms of, 205

treatment of, 189,206

post-malarial, 220

suppurative, 204

bacteriology of, 204

etiology of, 204

factors which determine tracta-

bility or intractability of, 204 symptoms of, 202

"trophic nerve." See Keratitis, neuropathic.
Keratitis, ulcerative, 207

varieties of, 207

Keratoconus. See Conical cornea.

Keratomalacia, 2 I 5

etiology of, 215

malignancy of, 216

night-blinḋness a

symptom of, 2 I 6

premonitory

symptoms of, 2 I 6

treatment of, 216

Kerato-scleritis, 242

Klebs-Löffler bacillus, I 70, I 7 I , 2 I I

Knapp's operation for anterior staphyloma, 241

roller-forceps, 118

LACRIMAL apparatus, diseases of, I I 8 drainage apparatus, 125

duct. See Nasal duct.

fistula, I 34, 148

etiology of, 134

treatment of, 148

gland, anatomy of, 121

atrophy of, 124

diseases of, I I 9

dislocation of, 122

excision of, 149

fistula of, 120

hypertrophy of, $\mathbf{I} 23$

exophthalmos from, 123

treatment of, 124

Velpeau's operation for, I 24

inflammation of. See Dacryoadenitis.

tumors of, 125

symptoms of, 125

treatment of, 125

varieties of, 125

probe, author's, for use by patient, I 49

supplementary, 145

introduction of, 144

probes, author's, I 4 I

puncta, atresia of, 126

etiology of, 127

treatment of, 127

eversion of, 128

inversion of, 128

malpositions of, 128

treatment of, r 28

sac, abscess of. See Dacryocystitis.

blennorrhea of, 132 etiology of, 132

symptoms of, 132

destruction of, 149

excision of, 149

gummata of, I39 
Lacrimal sac, inflammation of, 132. See also Dacryocystitis.

primary inflammation of, I $3 \mathbf{I}$ stricture. See Stricture of nasal duct.

Lamellar cataract. See Cataract, zonular.

Latent muscular anomalies, 466

Lateral illumination. See Oblique illumination.

Lead acetate, opacities of cornea from, 237

Leber, 222

Leeches in acute dacryoadenitis, I 20

Lens, crystalline. See Crystalline lens.

dislocation of. See Dislocation of crystalline lens.

foreign bodies in, 498

"trituration" of, $3^{\mathbf{I}} 3$

wounds of, $303,494,498$

Lenses, bifocal, $43^{\circ}$

cylindrical, 419

spherical, 390

types of, $39^{\circ}$

Leucocythemic retinitis, 352

Leucoma, i 87,236

adherens, 236

with anterior synechia, 236

Leucomata, 236

Light not the reprehensible thing it was once supposed to be, 54 perception, definition of, 28 protection of inflamed eyes from undue exposure to, 54

Lithia water, 5 I 9

Local remedies useful in diseases of the eye, 39

formulæ for, $5^{\circ} 5^{-5} 5^{15}$ observations upon, $39-57$

Locomotor ataxia, 44I

Lotion of belladonna, formula for, 512

in iritis, 262

"of opium and boracic acid," formula for, 5 I 2

in cellulitis of orbit, 109

in corneal ulcers, 213

in dacryocystitis, 136

in glaucoma, 288, 294

in iritis, 262

in miliary choroido-retinitis, 348,433

in myopia, 346, $4 \mathrm{I} 2$

in panophthalmitis, 334

in scleritis, 245

in traumatic lesions of eye,

"Lotions," formulæ for, 511,512
Lower lid, ectropion of, induced by lacrimal disease, 89 operation for entropion of, 82

Luxation of lens. See Dislocation of crystalline lens.

Lymph-spaces of eye, 282,283

Lymph-s.ream of eye, $281,282,283$ increase of, in glaucoma, 284

Macula lutea, 344

Maculæ of cornea, 236

Macular region, 407

Maddox-rod, $469,470,478$

Magnet, Sweet's, 499

Malarial fever, keratitis induced by, 220

“Malignant" glaucoma, 286, 289

tumors of choroid, 348

of ciliary body, 269

of lacrimal gland, 125

of lid, 79

of orbit, I I 4

of retina, 364

Maturity of cataract, 308, 309

Meibomian glands, 68

Membranous conjunctivitis, 168

Meningitis basilar, 372

in causation of optic neuritis, $37 \mathrm{I}$, 372

tuberculous, 371

Mercurial inunctions, $263,264,353$

Mercury, administration of, in eye diseases, 58

bichlorid, $50,5 \mathrm{I}$

addition of sodium chlorid to collyria of, $5^{\mathrm{I}}$

as nasal spray in treatment of stricture of nasal duct, 148

formula for collyrium of, with sodii chlorid, 506

formulæ for solutions of, $5 \mathrm{II}$, 512

in blennorrhea of lacrimal sac, I 37

in croupous conjunctivitis, 169

in diphtheritic conjunctivitis, 172

in follicular conjunctivitis, I 73 in penetrating wounds of eyeball, 495

in stricture of nasal duct, 148 internally, with tincture iron, in interstitial keratitis, 230 , 5 I 8 in vernal conjunctivitis, 185 in wounds of eyelids, 482

contraindicated in suppuration and ulceration of cornea, $5^{8}$, 212 
Mercury in acquired ptosis, 100 in choroiditis, 345

in choroido-retinitis, 310

in chronic dacryoadenitis, I 20 in cyclitis, 269

indications for administration of, $5^{17}, 5^{18}$

in diseases of the eye, 57

in herpes zoster ophthalmicus, 263

in inierstitial keratitis, $229,23 \circ$

in iritis, 262, 263

in optic neuritis, 373

in paralysis of third nerve, 446

in periostitis of orbit, 112

in recrobulbar optic neuritis, 375

in secondary glaucoma, 294

in sympathetic ophthalmitis, 264

in syphilitic orbital growths, I 17 retinitis, 353

tarsitis, 80

yellow oxid, formulx for ointments of, $5^{\mathrm{I} 3}$

in blepharitis, 54,65

in chalazion, 74

in corneal opacities, 238

ulcers, $2 \mathrm{I} 2$

in eczema of lids, $7 \mathrm{I}, 19^{\circ}$

in hordeolum, 69, 70

in phlyctenular conjunctivitis, 189

in vernal conjunctivitis, 185

of no value in interstitial keratitis, 229

Metallic foreign bodies, 497, 502, 504

Miliary choroido-retinitis, $3 \circ 3,343$

Milium, 77 treatment of, 77

Moist heat, method of applying, to eye, 4 I

Monocular blindness, individuals not rarely unaware of existence of, 33

Mucocele, I32

Mumps, I I 9

Murdoch's eye-speculum, $457,45^{8}$ protective shield, $3 \mathbf{I} 7$

Muscæ volitantes, 335

more numerous and conspicuous in ametropic eyes, $33^{6}$

popular misconceptions as to significance of, 335

present in all eyes, 335

Muscle-balance, determination of, 469

Maddox-rod in, 460

Schild's pin-hole light in, 470

in correction of hypermetropia, $40 \mathrm{I}$

of myopia, 410
Muscle-balance in subnormal accommodative power, $434,435,436$

Muscular anomalies of the eyes, 438

actual, 467,468

apparent, 467,468

dependent upon refractive errors, 438

etiology of, 438

latent, $438,439,466$

asthenopia, how caused by, 439,467

consequences of, 439,466 , 467

contradictory views regarding etiology of, 467,468

correction of, $470,+7 \mathrm{I}$

by glasses, 470,471

by operation, $470,47 \mathrm{~L}$, 476,477

determination of, 460,470

etiology of, 467,468

heterophoria a synonym of, 466

influence of refractive errors upon, $46 \%, 468$

multiple Maddox-rod in measurement of, 460

ocular disturbances from, 467

Schild's pin-hole light in measurement of, 470,478

treatment of, $470,47 \mathrm{I}$

varieties of, 467,468

whether apparent or actual, how determined, 468

manifest, $438,439,440$

comprise all the varieties of squint, 438 .

not in themselves provocative of asthenopia, 439

oftenest due to refractive errors, 438,439

only exceptionally due solely to muscular faults, $438,+39$

the deformity and rapidly developing amblyopia of misdirected eye most serious consequences of, 439

treatment of. See Squint, treatment of.

when of paralytic origin, develop suddenly, 439

of congenital origin, 438

of paralytic origin, $43^{8}$

varieties of, 438,466

imbalance, 468

Mycotic ulcer of cornea, 2 I I 
Mydriasis, accidentally induced, 431 conditions which give rise to, 26 disadvantages of, in measuring refractive errors, 422

from application of belladonna plaster, $43 \mathrm{I}$

from reflex dental irritation, 223

in ophthalmoplegia interna, 445

in paralysis of accommodation, $43 \mathrm{I}$

of oculomotorius, 445

Mydriatic, value of transient, in searching for pathological changes in eye, 37

Mydriatics, indiscriminate use of, reprehensible in advanced life, 285

Myopia, 403

a pathological condition, 403

apparent, from spasm of ciliary muscle, 432

asthenopia in, 408, 409

astigmatism a potent factor in causation of, $405,406,410$

axial, 404, 405

cataract in, 407

caused by conical cornea, 241, 405

choroido-retinitis in, 407

curvature, 404

definition of, 403,404

detachment of retina in, 407

development of divergent squint in, 407,408

of posterior staphyloma in, 404

diagnosis of, 409

disturbed relation of accommodation and convergence in, 407

etiology of, $403,404,405,406$, 408

exceptionally a consequence of systemic disease, 408

exophoria in, 407,408

factors which influence selection of glasses in, 4 IO, 41 I, 412

glasses a therapeutic agent of great value in, $4 \mathrm{I} I$

influence of age upon progress of, 407,409

influence of glasses in, 4IO, 4 I I, 412

muscæ volitantes common in, 409

not excluded by ability to read at usual distance, 33

pathology of, $404,405,406,407$

predisposition to, often inherited, 403,405

removal of crystalline lens in high, 4 I 3

symptoms of, 409

tenotomy in, 4 I 3
Myopia, treatment of, 409, 4 io, 4 I I, 412,413

usually an acquired fault, $4 \circ 3$

Myopic eyes liable to injury from trivial traumatisms, 407, 492

glasses, 410

Myosis, conditions which may give rise to, 26

in spinal disease, 379

Myotics, action of, in glaucoma, 48

rules for employment of, 49

NASAL disease, etiological importance of, in causation of lacrimal stricture, 1 35, 138, 139

duct, a natomy of, 137

author's measurements of, I 41 stricture of, 137

Nausea induced by eye-strain, 390, 418,467

Near-sightedness. See Myopia.

Nebulæ of cornea, 236

Necrosis of cornea, ro9, I I 5, I 60, 2 I 5 of orbital walls, I I I

Needle for removal of foreign bodies, 490

operation for capsular cataract, 327

for juvenile cataract, 3 I 4

Nephritis in causation of optic neuritis, 372

of retinitis, 349

Neurasthenia induced by eye-strain, $390,418,467$

Neuritis, optic. See Optic neuritis.

Neuroepithelioma. See Retina, glioma of.

Neuropathic iritis. See Iritis, "trophic" nerve.

keratitis, 2 I 6 milder forms of, 2 I 8

origin of sympathetic ophthalmitis, $25^{8}$

Neuro-retinitis, 370

Night-blindness in keratomalacia, 216

in retinitis pigmentosa, 355

Nitrite of amyl in embolism of central retinal artery, $35^{8}$ in quinin blindness, 378

Nitroglycerin in quinin blindness, 378

Non-magnetic foreign bodies, 502

Normal salt-solution, 5I, $5 \circ 5$

Notched and pegged teeth, 227

Noyes, H. D., and E. Williams, first to use large lacrimal probes, I4O 
Nystagmus, 447

acquired, 447

commonly of congenital origin, 447

etiology of, 447

miner's, 447,448

treatment of, 447,448

Oblique illumination, 19, 36, 304, 500

information afforded by, 36

Ocular muscles, 440, 442

anatomy of, 440

associated movements of, 446 paralyses of, 446

insufficiency of. See Muscular anomalies, latent.

latent anomalies of, 446 manifest anomalies of, 440 nerves distributed to, $44^{\circ}$ operations upon, $457,45^{8}$ paralysis of 441,442 . See also Squint, paralytic.

paralyses, conjugate. See Conjugate ocular paralyses.

Oculomotorius, paralysis of. See Paralysis of oculomotorius.

Ointments, application of, to eye and to lids, 45

formulæ for, $5^{\mathrm{I}} 3,5^{\mathrm{I}} 4$

indications for use of, 53

"Old-sight," 297

Old-sightedness. See Presbyopia.

Opacities of cornea, 235

dionin in treatment of, 238

etiology of, 235,236

from lead acetate, 237

how distinguished from lenticu. lar opacities, 238

impairment of vision from, 236 iridectomy for, 238

tattooing of, 239

treatment of, 238

varieties of, 236

of lens. See Cataract.

of vitreous humor, 334

secondary capsular, 326

Ophthalmia, granular. See Conjunctivitis, trachomatous.

neonatorum, I 59, 161, I6 63

purulent. Sec Conjunctivitis, purulent.

sympathetic. See Iritis, sympathetic.

Ophthalmic ganglion, 208

Ophthalmitis, sympathetic, ${ }_{25} 6$. See also Iritis, sympathetic.
Ophthalmoplegia externa, 445 necessarily of nuclear origin: 445

interna, 445. See also Paralysis of ciliary muscle.

oftenest dependent upon diphtheria, 445

totalis, 445

characteristic picture of, 444,445 exophthalmos a symptom of, 445

Ophthalmoscope, diagnosis of glioma of retina without aid of, $3^{64}$

ear or throat mirror as substitute for, 305

in diagnosis of cataract, 305

in hands of general practitioner rarely trustworthy aid to diagnosis, 19

Opium and morphin in diseases of eye, 60

as a local remedy in diseases of the eye, 53

lotion of, formula for, $5 \mathrm{I} 2$ mode of application of, 53

Optic nerve, atrophy of, $\mathbf{3 7 8}$ consecutive, 38 I

diagnosis of, 38 I

etiology of, $3^{8}$ I

ophthalmoscopic picture of, $3^{81}, 3^{82}$

prognosis in, ${ }_{3} \mathrm{~S}_{2}$

symptoms of, 381

treatment of, $3^{32}$

primary, 378

Argyll-Robertson symptom in, 379

behavior of pupil in, 379

etiology of, 378

hereditary form of, 379

often an early symptom of tabes, 380

ophthalmoscopic picture of, $379,3^{80}$

symptoms of, 379

treatment of, $3 \mathrm{~S}_{\mathrm{I}}$

unfavorable prognosis in, $379,3^{S_{1}}$

varieties of, $37^{8}$

diseases of, 367

inflammation of. See Optic neuritis.

neuritis, 367

descending, $37^{2}$

etiology of, $37^{2}$

ophthalmoscopic picture of, $368,369,372$

treatment of, 373

etiology of, 367,368

intraocular. See Choked disc. 
Optic neuritis, ophthalmoscopic picture of, 369

orbital. See Optic neuritis, retrobulbar.

pathology of, $367,368,369$

retrobulbar, $\mathbf{3 7 4}$

acute, 374

etiology of, 374

impairment of vision in, 374

pathology of, 374

prognosis in, 375

symptoms of, 374

treatment of, 375

chronic, 375

etiology of, 375,376

more common in males, 375

ophthalmoscopic changes in, 376

pathology of, 375

prognosis in, 377

symptoms of, 375,376

treatment of, 377

varieties of, 367,368

Orbicularis muscle, anatomy of, 92

Orbit, abscess of, 108

benign tumors of, I 14

cellulitis of, 108

diseases of, 107

exenteration of, I 6

malignant tumors of, I I 4

sarcoma of, I I 5

tumors of, II 3

Orbital optic neuritis. See Optic neuritis, retrobulbar.

walls, periostitis of, I I I

Orthophoria, 434

Orthophoric condition, 468

muscle-balance, 434

"Pain-Reaction" test, 500

Palsy. See Paralysis.

Panas's operation for ptosis, 104

Pannitic keratitis, 23I

Pannus, $174,177,182,231$

diagnosis of, 233

etiology of, $23^{1}$

treatment of, 233

Panophthalmitis, purulent, 240, 268, 269,333

consequences of, 334

course of, 333

etiology of, 333

symptoms of, 333,334

treatment of, 334

Papillitis. See Choked disc.
Paracentesis of anterior chamber, 2 I 5

Paralyses, conjugate ocular. See Conjugate ocular paralyses.

Paralysis of accommodation. See Paralysis of ciliary muscle.

and mydriasis from reflex dental irritation, 223

of ciliary muscle, 430

etiology of, 430, $43 \mathrm{I}$

from application of belladonna plaster, $43 \mathrm{I}$

from reflex dental irritation, 223

of ten accompanied by mydriasis, $43 \mathrm{I}$

prognosis usually favorable in, 431

symptoms of, 43 I

treatment of, $43^{2}$

vision, how impaired in, $43 \mathrm{I}$

of external rectus muscle, $\mathbf{4 4 2}$

commonest of ocular palsies, 442

etiology of, 443

pathology of, 443

prognosis favorable in, 443

symptoms of, 443

treatment of, 443

usually of orbital origin, 443

of facial nerve, ro5

epiphora from, I05

etiology of, Io6

keratitis in, 105

symptoms of, 105

treatment of, 106

of fourth nerve, 444

of oculomotorius, 444

etiology of, $444,445,446$

one of the commonest ocular palsies, 444

prognosis in, 446

ptosis a usual symptom of, 445

symptoms of, 445

treatment of, 446

varieties of, 445

of sixth nerve. See Paralysis of

external rectus muscle.

of superior oblique muscle, 444

diagnosis of, 444

etiology of, $4+4$

symptoms of, 444

treatment of, 444

of third nerve, 444

Paralytic squint, 440. See also Squint, paralytic.

Parenchymatous keratitis. See Keratitis, interstitial. 
Penetrating wounds of eye, 494. See also Wounds of eye, penetrating.

Pepsin, essence of, administered in conjunction with potassium iodid and the salicylates, to prevent gastric irritation, 59

Pericorneal injection, significance of, 35

Perimeter, standard, 30

Periostitis of orbital walls, I I I consequences of, I I I etiology of, I I I symptoms of, I I I treatment of, II 2

Permanganate of potash in diphtheritic conjunctivitis, 172

Pernicious anemia, retinitis in, $35^{2}$

Persistent pupillary membrane, 320

Phimosis, spasm of orbicularis palpebrarum from, 223

Phlyctenular conjunctivitis. See Conjunctivitis, phlyctenular.

keratitis. See Keratitis, phlyctenular.

Phosphates of iron, quinin and strychnin, 516

in blepharitis marginalis, 67

in phlyctenular conjunctivitis, I9I

in recurrent hordeola, $7 \mathrm{I}$

value of, in diseases of the eye, 60

Photophobia, not a symptom of retinitis or neuritis, 32

Phthisis bulbi, 496

Physiological salt solution, 5 I

Pigmentary degeneration of the retina, 354. See also Retinitis pigmentosa.

Pilocarpin hydrochlorate, formula for collyrium of, 509

in detachment of retina, $3^{6} 3$

in iritis, 262

in optic neuritis, 373

in serous iritis, 265

internal administration of, 6I, 265 , 510

Pinguecula, 194

diagnosis of, 195

etiology of, 195

origin of name, I95

pathology of, 195

treatment of, 195

"Pink eye," I 56

Pituitary body, enlargement of, 386

Plastic cyclitis. See Cyclitis, plastic. iritis. See Iritis, plastic.
Pneumococcus in catarrhal conjunctivitis, 156

in suppurative keratitis, 204, 2 I I

Polyopia, monocular, in incipient cataract, 307

Polypus in canaliculus, I 3 I

Posterior polar cataract, 322 staphyloma, 404, 406, 408 synechia. See Synechia, posterior.

Post-hemorrhagic blindness, etiology of, 359

Post-malarial keratitis, 220

Post-neuritic atrophy of optic nerve. See Atrophy of optic nerve, consecutive.

Potassium iodid, 59, 5 I 7

in acquired ptosis, 100

in choroiditis, 345,346

in chronic dacryoadenitis, 120

in cyclitis, 269

in detachment of retina, 363

in diseases of the eye, 59

in episcleritis, 245

in facial paralysis, 107

in glaucoma simplex, 293

in hemorrhage into vitreous chamber, 337

in hypertrophy of lacrimal gland, 124

in interstitial keratitis, 229,230

in iritis, $26_{2}, 26_{3}, 26_{4}, 26_{5}$

in neuropathic keratitis, 222

in optic neuritis, 373

in paralysis of ciliary muscle, 432

of external rectus, 443

of third nerve, 446

in periostitis of orbit, I I 2

in retinitis albuminurica, $35 \mathrm{I}$

in retrobulbar optic neuritis, 375

in scleritis, 244

in secondary glaucoma, 294

in syphilitic orbital growths, I I 7 retinitis, 353

Prelacrimal abscess, I $\mathbf{3 7}$

Presbyopia, 297, 427

advent of, ${ }_{42} 8$

a progressive condition, 429

asthenopia from neglect of, 428

decline of, in incipient stage of cataract, $302,43^{\circ}$

explanation of, 427,428

how influenced by ametropia, 399 , 428,429

late development of, 428

symptoms of, 32,428

treatment of, $429,43^{\circ}$

bifocal lenses in, 430 
Presbyopia, treatment of, fallacy of the popular belief that little skill is required in selection of glasses in, 429

glasses the only remedy in, 429

Primary atrophy of optic nerve, $\mathbf{3 7 8}$

Prismatic glasses, $47 \mathrm{I}$

Prisms from trial case in measurement of heterophoria, 460

in correction of squint, 462

in esophoria, 475

in exophoria, 422

in hyperphoria, 478

in $\mathrm{subnormal}$ accommodative power, 437

Protargol, 5I, 157, 169

in dacryocystitis, I 37

in purulent conjunctivitis, I 64 , I68

in trachomatous conjunctivitis, I 80,181

Pseudo-pt rygium, 197

Pterygium, 195

description of, 195

etiology of, 197

genesis of, author's theory of, 198 impairment of vision from, 196

operation for removal of, 199

treatment of, 198

Ptomaine poisoning, $43 \mathrm{I}$

Ptosis, 98

- acquired, 98

etiology of, 98

symptoms of, 98

treatment of, 100

Bowman's operation for, 102

congenital, 98

characteristic facial expression, in, 99

etiology of, 98

treatment of, 99, 101, 102, 103, I04, 105

vicarious action of occipitofrontalis in, 98

de Grandmont's operation for, 102 Gruening's operation for, I02, 103

Panas's operation for, 104,105

varieties of, 98

Pulsation of retinal vessels, 279

Puncta lacrimalia. See Lacrimal puncta.

Pupil, 24

Argyll Robertson, 27

consensual reflex action of, 25

direct reflex action of, 24

enlargement of, in glaucoma, 277 , 291

occlusion of, in iritis, $249,250,254$, $25^{8}$

Pupillary reactions, 24
Pupils, associated action of, 25 normal variations in size and activity of, 25

size of, influenced by refractive state of eyes, 25

size and reactions of, conditions which influence, 25

unequal size of, in anisometropia, 25

Purulent choroiditis, 340. See also Panophthalmitis, purulent.

conjunctivitis. See Conjunctivitis, purulent.

cyclitis. See Cyclitis, purulent.

iritis. See Iritis, purulent.

panophthalmitis. See Panophthalmitis, purulent.

Pyramidal cataract, $32 \mathrm{I}$

Pyrophospate of sodium. See Sodium pyrophospate.

QUESTIONS helpful in reaching a diagnosis, $3 \mathrm{I}$

Quinin blindness, 377

pathology of, 377,378

symptoms of, 377,378

treatment of, 378

in neuropathic keratitis, 221

in purulent cyclitis, 269

iritis, 262, 265

in suppurative keratitis, 206, 212 value of, in abscess and ulcer of cornea, 60

RANDOLPH, .R. L., 186

Rays of light, parallel, 393

course of, in emmetropic eye, 393
in hypermetropic eye, 395 ,
398
in myopic eye, 403,404

Reading distance, importance of testing muscle-balance for, 469

Reconstruction of lid margin (Hotz) in entropion, 87

Reflex dental irritation in keratitis, 222

paralysis of ciliary muscle from, 223

spasm of orbicularis palpebrarum from, 223

Refraction, anomalies of, 388

Refractive and muscular anomalies, symptoms which suggest existence of, $3^{2}$ 
Remedies, constitutional, useful in diseases of the eye, 39 formulæ for, $516-520$

observations upon, 57-62

local, useful in diseases of the eye, 39

formula for, 505-5 I 5

obscrvation upon, $39^{-57}$

Retina, detachment of, 360, $49 \mathrm{I}$

cataract a late complication in, 363

diagnosis of, 363

etiology of, $36 \mathbf{r}$

far-fetched theories of genesis of, $3^{6} \mathrm{I}$

in high myopia, $34^{2}, 36 \mathrm{I}$

in intraocular growths, 362

subconjunctival injection of salt solution in, 363

symptoms of, 362,363

treatment of, $36_{3}, 36_{4}$

unfavorable prognosis in, $3^{6} 3$

diseases of, $34^{8}$

glioma of, 364

a disease of childhood, 365

clinical course of, $365,366,367$

diagnosis of, 364,365

malignancy of, 366

symptoms of, 365,366

treatment of, 366,367

unfavorable prognosis in, 367

pigmentary degeneration of, 354

Retinal artery, embolism of, 357 pulsation in, 279

thrombosis of, 359

Retinitis, 348

albuminuric, 349

etiology of, 349

impairment of vision in, 349 , $35^{\circ}$

in pregnancy, $349,35 \mathrm{I}$

in scarlatina, $349,35^{\mathrm{I}}$

ophthalmoscopic picture of, $35^{\circ}$

pathology of, $35^{\circ}$

prognosis in, $35 \mathrm{I}$

treatment of, $35 \mathrm{I}$

uremic amblyopia in, 35 I

diabetic, $35 \mathrm{I}$

cataract in association with, 35 I

fundus changes in, $35^{\mathrm{I}}$

impairment of vision in, $35^{2}$

iritis and glaucoma in associa-

tion with, $35 \mathrm{I}$

prognosis in, $35^{2}$

treatment of, $35^{2}$

from exposure of the eyes to intense light, 353

conjunctivitis in association with, 354
Retinitis from exposure of the eyes to intense light, fundus changes in, 353

how produced, 353

prognosis in, 354

symptoms of, 353

treatment of, 354

hemorrhagic. See Thrombosis of central retinal vein.

leucocythemic, $35^{2}$

ophthalmoscopic picture of, $35^{2}$

pathology of, $35^{2}$

treatment of, $35^{2}$

of pernicious anemia, $35^{2}$

fundus changes in, $35^{2}$

prognosis in, $35^{2}$

treatment of, $35^{2}, 353$

parenchymatous, 348,349

pigmentosa, 354

clinical history of, 354,355 , $35^{6}$

congenital anomalies frequently associated with, $35+$

development of posterior polar cataract in, $35^{6}$

etiology of, 354

in association with deaf-mutism, 354

night-blindness a characteristic symptom of, 355

nystagmus not infrequently present in, 355

ophthalmoscopic picture of, 355

pathology of, 355

progressive contraction of visual field in, 355

slow progress of, 354,355

symptoms of, 355

treatment of, 356

unfavorable prognosis in, 355

primary, 349

secondary, 349

symptoms of, $34^{8}$

syphilitic, 353

fundus changes in, 353

prognosis in, 353

treatment of, 353

varietics of, 348

Retino-choroiditis. Sce Choroidoretinitis.

Retinoscopy, 422

Retrobulbar neuritis, 374

Rheumatic diathesis, 244,389

Rheumatism, I I9, 242, 248,268 , 291, 374

"Ring ulcer" of cornea, 2 I $r$

"Ripeness" of cataract, determination of, 307 
Risley, S. D., on the lessened prevalence of high myopia in the United States, 406

Roller-forceps, Knapp's, I 81 , I 82 in vernal conjunctivitis, 186

Röntgen-ray apparatus, Sweet's, 500

Röntgen-rays in detection of foreign bodies in eyeball, 499, 500, 50r, $5^{\circ} 3$

Salicylate of sodium. See Sodium salicylate.

Salicylates, the, in inflammatory conditions of eye, 59

Salicylic acid, ointment of, $5^{\text {I } 4}$ in blepharitis, 66 eczema of lids, 7 I

vernal catarrh, I 85

Salt solution, subconjunctival injections of, in detachment of retina, 363,364

Sarcoma of choroid, 347,348

of ciliary body, 269

of lacrimal gland, 125

of lid and orbit, 80

of orbit, I1 15

Sattler's diplococcus, 174

Schlemm's canal, 282

Sclera and ciliary body, wounds of, 494,495

diseases of, 242

Scleritis, 242

acute, 242

chronic, 243

diagnosis of, 243

etiology of, 242

symptoms of, 243

treatment of, 243

usually of rheumatic or gouty

origin, 242

varieties of, 242

Sclero-conjunctivitis, 242

Sclero-keratitis, 242

Sclerosis of lens fibers, 295

Sclerotitis. See Scleritis.

Scopolamin, 48

Scotoma, central, 353,376

color, 376

paracentral, 374

Scrofulous conjunctivitis, I86, I 88 . See also Conjunctivitis, phlyctenular.

ophthalmia, 188. See also Conjunctivitis, phlyctenular.

"Second sight" a premonitory symptom of cataract, 302,306

Secondary cataract. See Cataract, capsular.
Seed-shells lodged on cornea, 488 easily overlooked, 488 peculiar behavior of, 488

Senile, 236

cataract, 298, 299, 300, 301, 302

changes in crystalline lens, 295, 296,297

decay, 302

Senilis, arcus, 235

Senility, 208

Serous cyclitis. See Iritis, serous. iritis. See Iritis, serous.

Serpent ulcer of cornea, 2 I I

Shadow test, 222

Shortening lid, von Ammon's operation for, 93

Short-sightedness. See Myopia.

Sichel's cataract knife, I 45

Silver foil as a dressing in lid operations, 96

as a surgical dressing, suggested by Halsted, 96

nitrate in blepharitis marginalis, 66,515

in purulent conjunctivitis, 164 , I 68

in severer types of conjunctivitis, $5 \mathbf{I}$

in trachomatous conjunctivitis, I $80,18 \mathrm{I}$

Simple atrophy of optic nerve, 378 glaucoma. See Glaucoma, simple.

Simulated blindness, 33

Sixth nerve, paralysis of. See Paralysis of external rectus muscle.

Skiagraphy in detection of foreign bodies in eyeball, 499, 500, 50r, $5 \circ 3$

Skiascopy, 422

Skin-grafting, 88, 94, 95, 96, 97 silver-foil as dressing in, 96,98

Smith's, Nathan R., knife for dividing strictures of the nasal duct, $\mathbf{I}_{\mathbf{4}} 2$

Snellen's operation for spasmodic ectropion, 92

test-types, 27

Sodium chlorid, formula for collyrium of, $5 \circ 5$

subconjunctival injection of, $23^{8}, 337,3^{6} 3,3^{6} 4$

with bichlorid of mercury, $5 \mathrm{I}$, 506

pyrophosphate in cellulitis of orbit, I 10

in dacryoadenitis, 120

in dacryocystitis, 136

value of, in suppurative processes of the lids, lacrimal sac, and orbit, 62 
Sodium salicylate, $59,5^{18}$

in choroiditis, 346

in cyclitis, 269

in episcleritis, 245

in glaucoma, 289

in iritis, 262,263

in optic neuritis, 373

in periostitis of orbital walls, III

in retrobulbar optic neuritis, 375

in scleritis, 244

in secondary glaucoma, 294

in severe contusions of eye, 493

in sympathetic ophthalmitis, 265

in traumatic choroiditis, 346

Solar retinitis, 353

Somnolency induced by eye-strain, 390,418

Spasm of accommodation, 432

of ciliary muscle, 432

etiology of, 432

from eserin, $43^{2}$

symptoms of, 432

transient myopia produced by, 432

treatment of, 433

true refractive condition of eyes masked by, 432

uncorrected astigmatism a

Spectacles, 420 common cause of, $43^{2}$

bifocal, 430

Sphincter pupillæ, paralysis of, 26 , $4.3 \mathrm{I}, 445$,

undue contraction of, 26,379

"Spongy" iritis, 259

Spring catarrh. See Conjunctivitis, vernal.

Squint, concomitant, $\mathbf{4 4 8}$

alternating, $448,45 \mathrm{I}, 554$

amblyopia of misdirected eye in, 449

how induced, 449, 450, $45^{\mathrm{I}}$

not an example of "amblyopia exanopsia," 449

origin of, not merely of theoretical interest, $45^{\mathrm{I}}$

regional character of, $45^{\circ}$ significance of, 450

characteristics of, 448

consequences of, 449

constant, 448

convergent, 453

etiology of, 396, 397, 453, 454

Donders' dicta regarding, 397,453
Squint, concomitant, convergent, hypermetropia most potent factor in causation of, 397 in myopia, 454

oftenest develops in early childhood, 453

treatment of, $402,403,455,456$ by glasses alone, 402,455 , $45^{6}$

by operation, $455,457,45^{8}$, $459,460,461,462$

definition of, $44^{8}$

detection of, $45^{2}$

cover test in, $45^{2}$

divergent, $46_{3}$

development of, in myopia, $+64$

etiology of, $407,408,463$

may develop at any time of life, $46_{3}, 46_{4}$

myopia most potent factor in causation of, 463,464

seldom alternating, 464

treatment of, 464

by glasses alone, 464,465

by operation, 465,466

etiology of, $438,439,448$

periodic, 448,454

primary, $45^{2}$

secondary, $45 \mathrm{I}, 45^{2}$

varieties of, 448

detection of, $45^{2}$

paralytic, 440

diagnosis of, $44^{\mathrm{I}}$

diplopia a characteristic symptom of, $44^{\mathrm{I}}$

etiology of, $4+1,4+2$

external rectus of tenest involved in, 442

pathology of, 44I, 442

symptoms of, 44I

primary, $45^{2}$

secondary, $45^{1}, 45^{2}$

vertical, 466

etiology of, 466

glasses of little assistance in correction of, 466

treatment of, 466

Squints, 439

characteristic features of, 439

due solely to muscular faults, rare,

439

etiology of, 439

of paralytic origin occur at any time of life, 439

Staphylococcus aureus in keratitis, 204, 210

in phlyctenular conjunctivitis, I 89 
Staphyloma, anterior. See Staphyloma of cornea.

of cornea, I6I, 239

anatomy of, 239

etiology of, 239

impairment of vision in, 239

iridectomy for, 241

Knapp's operation for, 241

partial, 239

total, 239

treatment of, 240

posterior, in myopia, 404, 406, 408

Sterilization of co'lyria, 501

of instruments by brief boiling, I 44

Stilling, I 42

Stillson, 364

Strabismus. See Squint.

Streptococcus in suppurative keratitis, 204, 2 I I

Stricture of canaliculus, 130 of nasal duct, 137

division of, practised by Nathan R. Smith, in 1846 , 142

etiology of, 1 $37,138,139$

location of, 139

Nathan R. Smith's knife for dividing, 142

often consequent upon nasal disease, 138

often multiple, I-39, I 40

transient, in new-born, I 50

treatment of, $140-15^{\circ}$ employment of large probes in, 143,146

varieties of, 140

Strumous diathesis, 19 I

Strychnin, 519

in acquired ptosis, 100

in diseases of the eye, 60

in facial paralysis, 107

in neuropathic keratitis, 222

in paralysis of ciliary muscle, 432 of sixth nerve, 443

of third nerve, 446

in primary atrophy of optic nerve, $3^{81}$

in quinin blindness, 378

in retinitis pigmentosa, $35^{6}$

in retrobulbair optic neuritis, 375 , 377

in secondary atrophy of optic nerve, $3^{82}$

preferably administered by the mouth, 60

Stye. See Hordeolum.

Subconjunctival hemorrhage, I93 diagnosis of, I 94
Subconjunctival hemorrhage, etiology of, 194

treatment of, 194

injection of salt solution, 238,337 , $36_{3}, 364$

Subnormal accommodative power, 433

a not infrequent cause of asthenopia, 433

asthenopia, how produced in, 435

described by author in r89r, 433

early development of presbyopia a manifestation of, 434

how detected, 434, 435, 436 may exist independently of other faults, or may complicate other errors, refractive or muscular, 433

of transient character, after use of cycloplegic, 435

rule for correction of, 436 for detection of, 435

symptoms of, $433,434,435$

treatment of, 436,437

when complicated by refractive or other muscular faults, 436

satisfactory results of, 437

underlying causes of, 433,435

"Sugar of lead," 237

Sulphate of copper. See Copper sulphate.

Sulphonal, 60

Superficial lodgment of foreign bodies in eye, 486

Superior oblique muscle, paralysis of. See Paralysis of superior oblique muscle.

Suspensory ligament of lens, 295, 328

Sweet's electro-magnet, 499, 503

localizing chart, 502

Röntgen-ray apparatus, 500

Symblepharon, 480, 481, 482, 483

Sympathectomy, 293

Sympathetic iritis. See Iritis, sympathetic.

irritation, 257

prompt disappearance of, upon removal of exciting eye, 257

symptoms of, 257

ophthalmitis, 250, 256, 28I, 493, 497, 504. See also Iritis, sympathetic.

etiology of, $256,258,259$

Sympathizing eye, $257,258,259$

Synchysis, 334 
Synechia, anterior, 37, I61, 206, 236, $254,293,294,494,504$

posterior, $21,26,37,247,249,250$, $252,254,255,258,259,260$, $267,293,294$

Syphilis a common cause of retinitis, 349

acquired ptosis oftenest due to, 98

a factor in causation of lacrimal stricture, I 39 , I 49

cataract in inherited, 302

characteristic physiognomy of inherited, 227,228

commonest cause of iritis, 248, 255 disease of deeper tunics of eve often dependent upon, 339

facial paralysis from, 106

importance of rôle played by, in etiology of diseases of the eye, 57

in acute retrobulbar neuritis, 374

in choked disc, $37^{2}$

in dacryoadenitis, I 19

in descending optic neuritis, 372

in hemianopsia, 385,386

in paralysis of ciliary muscle, 430 of ocular muscles, $44 \mathrm{I}$

in primary atrophy of optic nerve, 379

in retinitis, 353

interstitial keratitis due to inherited, 223

iritis in inherited, 226, 255

notched and pegged teeth in inherited, 227

observations upon treatment of, $5^{8}$

paralysis of third nerve oftenest due to, 444

periostitis of orbit oftenest due to, I I I

plastic choroiditis oftenest due to, 340,342

cyclitis from, 268

tarsitis from, 79

tumors of orbit due to, II4, II 5 , I 7

zonular cataract in inherited, 324

Syphilitic choroiditis. See Choroiditis, syphilitic.

iritis. See Iritis, syphilitic.

keratitis. See Keratitis, interstitial.

retinitis, 353

TABEs dorsalis, $37^{8}$

Tarsal cartilages, 85

cyst. See Chalazion.
Tarsitis, 79

treatment of, 80

usually consequent upon acquired syphilis, 79

Tattooing of cornea, 238

Teeth, "dead," 222

Hutchinson, 227

Tenon's capsule, division of, in "free" tenotomies, 459

non-division of, in "guarded" tenotomies, 477

Tenotomies, graduated, little better than a pretence, 477

Tenotomy, 455, 457, 458, 459, 460, $461,462,465$

adrenalin helpful in operation of, 461

Arlt's method of performing, 457, $4^{6} 5$

awkward methods of performing, formerly in vogue, 462

best method of performing, 457

description of operation of, 457

early resort to, indicated in convergent squint, 462

for correction of convergent squint, 457

how sinking of caruncle may be prevented in, 459

why preferable to advancement, 457

of divergent squint, 465

of esophoria, 475,476

of exophoria, $47^{2}$

of hyperphoria, 478,479

had best be "guarded," 478

indications for, $47^{8}$

little assistance afforded by glasses in, 478

uncertainties which attend, 478,479

of latent muscular anomalies,

$471,476,477$

of vertical squint, 466

"guarded," 4 I $3,477,478$

how effect of, may be modified, $459,+77$

in children, ${ }_{4} 6 \mathrm{I}$

infection almost unheard of in operation of, 46 I

instruments required in performing, $45^{8}$

operation of, practically free from risk, ${ }_{4} 6 \mathrm{I}$

Tension, intraocular, 23,282

increase of, collyria indicated in, 508,500

in gliucoma, $274,276,278$, $280,282,291$ 
Tension, intraocular, increase of, in glioma of retina, $36_{5}$ in intraocular growths, 363 in serous iritis, $252,26 \mathrm{I}$ method of determining and noting, 23,24

subnormal, 282

in detachment of retina, 363

Theobald's lacrimal probes, I4I. See also Author's.

Therapeutic agents called for in the treatment of discases of the eye, 39

Thiersch-grafts in correction of ectropion, 94, 97 of entropion, 88

silver-foil as covering for, 96

Third nerve, paralysis of. See

Paralysis of oculomotorius.

Thrombosis of central artery of retina, $359,3^{8} \mathrm{I}$

etiology of, 359

post-hemorrhagic blindness probably due to, 359

symptoms of, 359

treatment of, 359

retinal vein, $359,3^{81}$

consequences of, 359

etiology of, 359

ophthalmoscopic picture of, 360

treatment of, 360

Tinnitus aurium from eye-strain, 390

Tobacco a factor in causation of retrobulbar neuritis, 376

Toxic amblyopia. See Optic neuritis, retrobulbar, chronic.

conjunctivitis, 192

Trachoma. See Conjunctivitis, trachomatous.

usual cause of organic entropion, $8 \mathrm{r}$

Transient hemianopsia, 386

Treatment of diseases of the eye, general observation upon, 38-62

Trional, administration of, after operations upon the eye, 60

"Trituration" of lens, $3^{1} 3$

Trochlear nerve, paralysis of. See Paralysis of superior oblique muscle.

"Trophic nerve" keratitis. See Keratitis, neuropathic.

Tuberculosis of lacrimal gland, i i 9 of nose, 139

Tuberculous periostitis of orbit, I I I

Tumors of choroid, 348

of ciliary body, 269
Tumors of orbit, I 13

diagnosis of, 115

etiology of, II 3

treatment of, I 6

varieties of, I I 4

of retina, 364

Turkish bath in iritis, 262, 266

Typhoid fever, 208

UlCER of cornea, 207. See also Cornea, ulcer of.

Uremic amblyopia, 35 I

Urine, importance of testing, in optic neuritis, 373 in retinitis, $35^{\circ}$

Uveitis, 252

VASCULAR injection of eyeball, significance of different types of,

"Vaseline cerate," formula for, 46 , $5^{1} 3$

Velpeau's operation for removal of lacrimal gland, 124

Venæ vorticosæ, 282

Veratria oleate, formula for, $5_{15}$ indications for, 515

Vernal conjunctivitis, 183

Vertical diplopia test of von Graefe, 469 squint, 466

Vertigo, in ametropia, 390 in astigmatism, 418 in heterophoria, 467

in paralytic squint, 439,443

Visual acuity, determination of, 27 for near objects, 28 when sight is greatly impaired, 28

Vitreous humor, diseases of, $\mathbf{3 3 3}$

fluidity of, 334

consequences of, 334

etiology of, 334

oftenest met with in myopia, 334

hemorrhage into, 336

absorption of, 337

effect upon vision of, 337

etiology of, $33^{6}$

ophthalmoscopic appearances of, 337

origin of, $33^{6}$

serious consequences of, when

recurrent, 337

treatment of, 337

opacities of, 334

diagnosis of, 335 
Vitreous humor, opacities of, disappearance of, 335

disturbance of vision from, 335

etiology of, 335,336

of little moment when microscopic, 335

treatment of, 336

varieties of, 335

WARTS upon lid margin, 78

treatment of, 78

Weber's canaliculus knife, 129

Weeks' bacillus, 156

Weeks on bacteriology of phlyctenular con junctivitis, 180

Williams, E., and H. D. Noyes, first to use large lacrimal probes, I 40

Wolfe-grafts in operations for ectropion, 94

Wounds. See also Injuries.

of eye complicated by lodgment of foreign bodies within the ball, 497

cataract caused by, 498

consequences of, 497 , $498,499,5 \circ 4$

danger of infection in, 497

interesting cases of, $498,5 \circ 3$

sympathetic ophthalmitis from, $497,498,504$

treatment of, 499 antiseptic precautions in, 501 electro-magnet in, 499, 500, 503 enucleation of eye not inf requently demanded in, 504

pain-reaction test in, 500

skiagraphy in, 499, 500,501

when foreign body is non-magnetic,

penetrating, 494
Wounds of eye, penetrating, always of serious concern, 494, 495

antiseptic precautions demanded in, 495, 496

complicated by involvement of lens, 494

consequences of, 494

danger of infection occurring in, 494, 495

enucleation of injured eye in, 495, 497

"first aid" in, 495, 496

hernia of iris and ciliary body from, 494

involving the choroid and retina, 495

the cornea, iris, and lens, 494,497

the sclera and ciliary body, 494,497

sympathetic

from, 497

traumatic cataract a not uncommon result of, 495

treatment of, $495,496,497$ of eyelids, 480

Wyeth's elixir of phosphates of iron, quinin, and strychnin, 60

Xerophthalmia, i 76

$\mathrm{X}$-rays. See Röntgen-rays.

YeLlow oxid of mercury. See Mercury, yellow oxid.

Zinc oxid and boracic acid, ointment of, 7 I

formula for, $5^{13}$

sulphate, 50

contraindicated in phlyctenular conjunctivitis, i 89

formula for colly rium of, 506

in catarrhal conjunctivitis, 50 , 157

in follicular conjunctivitis, 173

in hordeolum, 60,515

in membranous conjunctivitis, 169

in vernal conjunctivitis, 185

Zonular cataract, 324

Zonule of Zinn, 205, 328 



\section{SAUNDERS' BOOKS}

\section{on \\ Skin, Genito-Urinary \\ Diseases, Chemistry, and Eye, Ear, Nose, and Throat}

\section{W. B. SAUNDERS COMPANY 925 WALNUT STREET \\ PHILADELPHIA}

9, HENRIETTA STREET, COVENT GARDEN, LONDON

\section{MECHANICAL EXCELLENCE}

NOT alone for their literary excellence have the Saunders publications become a standard on both sides of the Atlantic: their mechanical perfection is as universally commended as is their scientific superiority. The most painstaking attention is bestowed upon all the details that enter into the mechanical production of a book, and medical journals, both at home and abroad, in reviewing the Saunders publications, seldom fail to speak of this distinguishing feature. The attainment of this perfection is due to the fact that the firm has its own Art Department, in which photographs and drawings of a very high order of merit are produced. This department is of decided value to authors, in enabling them to procure the services of artists specially'skilled in the various methods of illustrating medical publications.

A Complete Catalogue of our Publications will be Sent upon Request 


\section{Stelwagon's \\ Diseases of the Skin}

A Treatise on Diseases of the Skin. For Advanced Students and Practitioners. By Henry W. Stelwagon, M. D., Ph.D., Professor of Dermatology in the Jefferson Medical College, and Clinical Professor of Dermatology in the Woman's Medical College, Philadelphia. Handsome octavo volume of I I 35 pages, with 258 text-cuts and 32 fullpage colored lithographic and half-tone plates. Cloth, \$6.0o net; Sheep or Half Morocco, $\$ 7.00$ net.

\section{JUST ISSUED-NEW (4th) EDITION, REVISED FOUR LARGE EDITIONS IN THREE YEARS}

The demand for four editions of this work in a period of three years, and the many gratifying review notices indicate beyond a doubt the practical character of the book. In preparing the work the predominant aim kept in view was to supply the physician with a treatise written on plain and practical lines, giving abundant helpful case illustrations. In this edition, although some fifty new illustrations have been added, the size of the work has not been increased, many old illustrations having been eliminated and the text, wherever possible, made more concise.

\section{PERSONAL AND PRESS OPINIONS}

John T. Bowen, M.D.,

Assistint Professor of Dermatology, Hirvard University Medical School, Boston.

"It gives me great pleasure to endorse Dr. Stelwagon's book. The clearness of description is a marked feature. It is also very cárefully compiled. It is one of the best text-books yet published and a credit to American dermatology."

George T. Elliot, M. D.,

Professor of Dermatology, Cornell University.

"It is a book that I recommend to my class at Cornell, because for conservative judgment, for accurate observation, and for a thorough appreciation of the essential position of dermatology, I think it holds first place."

\section{Boston Medical and Surgical Journal}

"We can cordially recommend Dr. Stelwagon's book to the profession as the best textbook on dermatology, for the advanced student and general practitioner, that has been brought strictly up to date. . . The photographic illustrations are numerous, and wany of them are of great excellence." 


\title{
DeSchweinitz's Diseases of the Eye
}

\author{
Just Issued-The New (5th) Edition, Enlarged
}

Diseases of the Eye: A Handbook of Ophthalmic Practice. By G. E. DeSchweinitz, M.D., Professor of Ophthalmology in the University of Pennsylvania, Philadelphia, etc. Handsome octavo of 894 pages, 3 I 3 text-illustrations, and 6 chromo-lithographic plates. Cloth, $\$ 5.00$ net; Sheep or Half Morocco, $\$ 6.00$ net.

\section{WITH 313 TEXT-ILLUSTRATIONS AND 6 COLORED PLATES}

For this new edition the text has been very thoroughly revised, and the work enlarged by the addition of new matter to the extent of some one hundred pages. There have been added, amongst other subjects, chapters on the following : X-Ray Treatment of Epithelioma, Xeroderma Pigmentosum ; Purulent Conjunctivitis of Young Girls; Jequiritol and Jequiritol Serum; X-ray Treatment of Trachoma ; Infected Marginal Ulcer; Keratitis Punctata Syphilitica ; Uveitis and Its Varieties ; Eye-ground Lesions of Hereditary Syphilis; Macular Atrophy of the Retina; IVorth's Amblyoscope ; Stovain, Alypin; Motais' Operation for Ptosis : KuhntMüller's Operation for Ectropion; Haab's Method for Foreign Bodies: and Sweet's X-Ray Method of Localizing Foreign Bodies. Other chapters have been rewritten. The excellence of the illustrative feature has been maintained.

\section{PERSONAL AND PRESS OPINIONS}

\section{Samuel Theobald, M.D.,}

Clinical Profe'ssor of Ophthalmology, Johns Hopkins University, Baltimore.

"It is a work that I have held in high esteem, and is one of the two or three books upon the eye which I have been in the habit of recommending to my students in the Johns Hopkins Medical School."

\section{W. Franklin Coleman, M. D.,}

Professor of Diseases of the Eye, Postgraduate Medical School, Chicago.

"I am very much pleased with deSchweinitz's work and will recommend it to the members of my class as a most reliable, complete, and up to date text-book."

\section{British Medical Journal}

"A clearly written, comprehensive manual. One which we can commend to students as a reliable text-book, written with an evident knowledge of the wants of those entering upon the study of this special branch of medical science." 
GET

THE BEST

American

THE NEW

STANDARD

\section{Illustrated Dictionary}

Just Issued-New (4th) Edition

The American Illustrated Medical Dictionary. A new and complete cictionary of the terms used in Medicine, Surgery, Dentistry, Pharmacy, Chemistry, and kindred branches; with over IOO new and elaborate tables and many handsome illustrations. By W. A. Newman Dorland, M. D., Editor of "The American Pocket Medical Dictionary." Large octavo, nearly 840 pages, bound in full flexible leather. Price, $\$+. j 0$ net; with thumb index, $\$ 5.00$ net.

\section{WITH 2000 NEW TERMS}

In this edition the book has been subjected to a thorough revision. The author has also added upward of two thousand important new terms that have appeared in medical literature during the past few months.

Howard A. Kelly, M. D.,

Professor of Ginctologr. Johns Hopkins University, Baltimore.

"Dr. Dorland's Dictionary is admira'le. It is so well gotten up and of such convenient size. No errors have been found in my use of it."

\section{Theobald's \\ Prevalent Diseases of the Eye}

Prevalent Diseases of the Eye. By Samuel Theobald, M. D., Clinical Professor of Ophthalmology and Otology, Johns Hopkins University. Octavo of 550 pages, with 200 text-cuts and several colored plates.

\section{JUST READY-FOR THE PRACTITIONER}

With few exceptions all the works on diseases of the eye, although written ostensibly for the general practitioner, are in reality adapted only to the specialist ; but Dr. Theobald in his book has described very clearly and in detail only those conditions, the diagnosis and treatment of which come within the province of the general practitioner. The therapeutic suggestions are concise, unequivocal, and specific, in every case only one course of definite treatment being given. Over 200 text-illustrations and several colored plates greatly aid in presenting the subject in a lucid and practical way. It is the one work on the Eye written peremptorily for the general practitioner. 


\section{American Text-Book of Eye, Ear, Nose, and Throat}

American Text-Book of Diseases of the Eye, Ear, Nose, and Throat. Edited by G. E. DE Schweinitz, M. D., Professor of Ophthalmology in the University of Pennsylvania; and B. Alexander Randall, M. D., Clinical Professor of Diseases of the Ear in the University of Pennsylvania. Imperial octavo, I25 I pages, with 766 illustrations, 59 of them in colors. Cloth, \$7.00 net; Sheep or Half Morocco, $\$ 8.00$ net.

This work is essentially a text-book on the one hand, and, on the other, a volume of reference to which the practitioner may turn and find a series of articles written by representative authorities on the subjects portrayed by them. Therefore, the practical side of the question has been brought into prominence. Particular emphasis has been laid on the most approved methods of treatment.

\section{American Journal of the Medical Sciences}

"The different articles are complete, forceful, and, if one may be permitted to use the term, 'snappy.' in decided contrast to some of the labored but not more learned descriptions which have appeared in the larger systems of ophthalmology.'

\section{Hyde and Montgomery's Syphilis and Venereal}

Syphilis and the Venereal Diseases. By JAnes Nevins Hyde, M. D., Professor of Skin, Genito-Urinary, and Venereal Diseases, and Frank H. Montgomery, M. D., Associate Professor of Skin, GenitoUrinary, and Venereal Diseases in Rush Medical College, in Affiliation with the University of Chicago, Chicago. Octavo volume of 594 pages, profusely illustrated. Cloth, \$4.00 net.

\section{SECOND EDITION, REVISED AND GREATLY ENLARGED}

In this edition every page has received careful revision; many subjects, notably that on Gonorrhea, have been practically rewritten, and much new material has been added. A number of new cuts have also been introduced, besides a series of beautiful colored lithographic plates.

\section{American Journal of Cutaneous and Genito-Urinary Diseases}

"It is a plain, practical, and up-to-date manual containing just the kind of information that physicians need to cope successfully with a troublesome class of diseases." 


\section{Brühl, Politzer, and Smith's Otology}

Atlas and Epitome of Otology. By Gustav BrüHL, M. D., of Berlin, with the collaboration of Professor Dr. A. Politzer, of Vienna. Edited, with additions, by S. MacCuen Smith, M.D., Professor of Otology in the Jefferson Medical College, Philadelphia. With 244 colored figures on 39 lithographic plates, 99 text illustrations, and 292 pages of text. Cloth, $\$ 3.00$ net. In Saunders' HandAtlas Serie's.

\section{INCLUDING ANATOMY AND PHYSIOLOGY}

The work is both didactic and clinical in its teaching. A special feature is the very complete exposition of the minute anatomy of the ear, a working knowledge of which is so essential to an intelligent conception of the science of otology. The association of Professor Politzer and the use of so many valuable specimens from his notably rich collection especially enhance the value of the treatise. The work contains everything of importance in the elementary study of otology.

Clarence J. Blake, M. D.,

Professor of Otology in Harvard University Medical School, Boston.

"The most complete work of its kind as yet published, and one commending itself to both the student and the teacher in the character and scope of its illustrations."

\section{Haab and deSchweinitz's Operative Ophthalmology}

Atlas and Epitome of Operative Ophthalmology. By Dr. O. HАAв, of Zurich. Edited, with additions, by G. E. DESchwernitz, M. D., Professor of Ophthalmology in the University of Pennsylvania. With 30 colored lithographic plates, I 54 text-cuts, and 375 pages of text. In Saunders' Hand-Atlas Series. Cloth, $\$ 3.50$ net.

\section{RECENTLY ISSUED}

Dr. Haab's Atlas of Operative Ophthalmology will be found as beautiful and as practical as his two former atlases. The work represents the author's thirty years' experience in eye work. The various operative interventions are described with all the precision and clearness that such an experience brings. Recognizing the fact that mere verbal descriptions are frequently insufficient to give a clear. idea of operative procedures, Dr. Haab has taken particular care to illustrate plainly the different parts of the operations.

Johns Hopkins Hospital Burletin

"The descr ptions of the various operations are so clear and full that the volume can well hold p'ace with more pretentious text-books." 


\section{Haab and DeSchweinitz's External Diseases of the Eye}

Atlas and Epitome of External Diseases of the Eye. By Dr. O. HAAB, of Zürich. Edited, with additions, by G. E. DESchweInITZ, M. D., Professor of Ophthalmology, University of Pennsylvania. With 98 colored illustrations on 48 lithographic plates and 232 pages on text. Cloth, \$3.00 net. In Saunders' Hand-Atlas Series.

\section{SECOND REVISEDD EDITION-RECENTLY ISSUED}

Conditions attending diseases of the external eye, which are often so complicated, have probably never been more clearly and comprehensively expounded than in the forelying work, in which the pictorial most happily supplements the verbal description. The price of the book is remarkably low.

The Medical Record, New York

"The work is excellently suited to the student of ophthalmology and to the practising physician. It cannot fail to attain a well-deserved popularity."

\section{Haab and DeSchweinitz's Ophthalmoscopy}

Atlas and Epitome of Ophthalmoscopy and Ophthalmoscopic Diagnosis. By Dr. O. HAAB, of Zürich. From the Third Reaised and Enlarged Gcman Edition. Edited, with additions, by G. E. DESchweinitz, M. D., Professor of Ophthalmology, University of Pennsylvania. With 152 colored lithographic illustrations and 85 pages of text. Cloth, \$3.00 net. In Saundcrs' Hand-Atlas Scrics.

The great value of Prof. Haab's Atlas of Ophthalmoscopy and Ophthalmoscopic Diagnosis has been fully established and entirely justified an English translation. Not only is the student made acquainted with carefully prepared ophthalmoscopic drawings done into well-executed lithographs of the most important fundus changes, but, in many instances, plates of the microscopic lesions are added. The whole furnishes a manual of the greatest possible service.

\section{The Lancet, London}

"We recommend it as a work that should be in the ophthalmic wards or in the library of every hospital into which ophthalmic cases are received." 


\section{Barton and Wells' Medical Thesaurus}

A NEW WORK-RECENTLY ISSUED

A Thesaurus of Medical Words and Phrases. By Wilfred M. Barton, A. M., Assistant to Professor of Materia Medica and Therapeutics, and Lecturer on Pharmacy, Georgetown University, Washington, D. C. ; and Walter A. Wells, M. D., Demonstrator of Laryngology, Georgetown University, Washington, D. C. Handsome I $2 \mathrm{mo}$ of 534 pages. Flexible leather, $\$ 2.50$ net; with thumb index, $\$ 3.00$ net.

\section{THE ONLY MEDICAL THESAURUS EVER PUBLISHED}

This work is unique in that it is the only Medical Thesaurus ever published. Instead of supplying the meaning to given words, as an ordinary dictionary does, it reverses the process, and when the meaning or idea is in the mind it endeavors to supply the fitting term or phrase to express that idea. This Thesaurus will be of service to all persons who are called upon to state or explain any subject in the technical language of medicine.

\section{Boston Medical and Surgical Journal} readers."

"We can easily see the value of such a book, and can certainly recommend it to our

\section{Saxe's Urinalysis}

Examination of the Urine. By G. A. De Santos Saxe, M. D., Pathologist to Columbus Hospital, New York City. I mo of 39I pages, fully illustrated. Flexible leather, \$I.5O net.

\section{RECENTLY ISSUED}

This work is intended as an aid in diagnosis, by interpreting the clinical significance of the chemic and microscopic urinary findings.

\section{Francis Carter Wood, M. D.}

Adjunct Professor of Clinical Pathology, Columbia University.

"It seems to me to be one of the best of the smaller works on this subject; it is indeed, better than a good many of the larger ones." 


\section{Gradle's \\ Nose, Pharynx, and Ear}

Diseases of the Nose, Pharynx, and Ear. By Henry Gradle, M. D., Professor of Ophthalmology and Otology, Northwestern University Medical School, Chicago. Handsome octavo of 547 pages, illustrated, including two full-page plates in colors. Cloth, \$3.50 net.

\section{INCLUDING TOPOGRAPHIC ANATOMY}

This volume presents diseases of the Nose, Pharynx, and Ear as the author has seen them during an experience of nearly twenty-five years. In it are answered in detail those questions regarding the course and outcome of diseases which cause the less experienced observer the most anxiety in an individual case. Topographic anatomy has been accorded liberal space.

\section{Pennsylvania Medical Journal}

"This is the most practical volume on the nose, pharynx, and ear that has appeared recently. . . . It is exactly what the less experienced observer needs, as it avoids the confusion incident to a categorical statement of everybody's opinion."

\section{Kyle's \\ Diseases of Nose and Throat}

Diseases of the Nose and Throat. By D. Braden Kyle, M. D., Professor of Laryngology in the Jefferson Medical College, Philadelphia; Consulting Laryngologist, Rhinologist, and Otologist, St. Agnes' Hospital. Octavo, 669 pages; over I 84 illustrations, and 26 lithographic plates in colors. Cloth, $\$ 4.00$ net.

\section{RECENTLY ISSUED-THIRD REVISED EDITION}

Three large editions of this excellent work fully testify to its practical value. In this edition the author has revised the text thoroughly, bringing it absolutely down to date. With the practical purpose of the book in mind, extended consideration has been given to treatment, each disease being considered in full, and definite courses being laid down to meet special conditions and symptoms.

\section{Dudley S. Reynolds, M. D.,}

Formerly Professor of Ophthalmology and Otology, Hospital College of Medicine, Lowisville.

"It is an important addition to the text-books now in use, and is better adapted to the uses of the student than any other work with which I am familiar. 1 shall be pleased to commend Dr. Kyle's work as the best text-book." 


\section{Grünwald and Grayson's Diseases of the Larynx}

Atlas and Epitome of Diseases of the Larynx. By Dr. L. GRÜNWALD, of Munich. Edited, with additions, by Charles P. Grayson, M. D., Clinical Professor of Laryngology and Rhinology, University of Pennsylvania. With 107 colored figures on 44 plates, 25 text-cuts, and 103 pages of text. Cloth, \$2.50 net. In Saunders' Hand-Atlas Series.

\section{British Medical Journal}

"Excels everything we have hitherto seen in the way of colored illustrations of diseases of the larynx. . . . Not only valuable for the teaching of laryngology, it will prove of the greatest help to those who are perfecting themselves by private study."

\section{American Text-Book of Genito-Urinary, Syphilis, Skin}

American Text=book of Genito=Urinary Diseases, Syphilis, and Diseases of the Skin. Edited by L. Bolton Bangs, M. D., late Prof. of Genito-Urinary Surgery, University and Bellevue Hospital Medical College, New York; and W. A. Hardaway, M. D., Professor of Diseases of the Skin, Missouri Medical Collegre. Imperial octavo, I 229 pages, with 300 engravings, 20 colored plates. Cloth, \$7.00 net.

\section{Journal of the American Medical Association}

"This voluminous work is thoroughly up-to-date, and the chapters on genito-urinary diseases are especially valuable. The illustrations are fine and are mostly original. The section on dermatology is concise and in every way admirable."

\section{Senn's \\ Genito-Urinary Tuberculosis}

Tuberculosis of the Genito-Urinary Organs, Male and Female. By N. Senn, M. D., Ph. D., LL.D., Professor of Surgery in Rush Medical College. Octavo of 3 I 7 pages, illustrated, Cloth, $\$ 3.00$ net.

\section{British Medical Journal}

"The book will well repay perusal. It is the final word, as our knowledge stands, upon the diseases of which it treats, and will add to the reputation of its distinguished author." 


\section{Mracek and Stelwagon's Diseases of the Skin}

Atlas and Epitome of Diseases of the Skin. By Prof. Dr. Franz Mracek, of Vienna. Edited, with additions, by Henry IV. Stelwagon, M. D., Professor of Dermatology in the Jefferson Medical College, Philadelphia. With 77 colored plates, 50 half-tone illustrations. and 280 pages of text. In Saunders' Hand-Atlas Series. Clo., \$4.00 net.

\section{RECENTLY ISSUED-NEW (2nd) EDITION}

This volume, the outcome of years of scientific and artistic work, contains, together with colored plates of unusual beauty, numerous illustrations in black, and a text comprehending the entire field of dermatology. The illustrations are all original and prepared from actual cases in Mracek's clinic, and the execution of the plates is superior to that of any, even the most expensive, dermatologic atlas hitherto published.

\section{American Journal of the Medical Sciences}

"The advantages which we see in this book and which recommend it to our minds are: First, its handiness; secondly, the plates, which are excellent as regards drawing, color, and the diagnostic points which they bring out."

\section{Mracek and Bang's' Syphilis and Venereal}

Atlas and Epitome of Syphilis and the Venereal Diseases. By Prof. Dr. Franz Mracek, of Vienna. Edited, with additions, by L. Bolton Baxgs, M. D., late Prof, of Genito-Uninary Surgery, University and Bellevue Hospital Medical College, New York. With 7 I colored plates and 122 pages of text. Cloth, \$3.50 net. In Saunders' Hand-Atlas Series.

\section{CONTAINING 71 COLORED Plates}

According to the unanimous opinion of numerous authorities, to whom the original illustrations of this book were presented, they surpass in beauty anything of the kind that has been produced in this field, not only in Germany, but throughout the literature of the world.

\section{Robert L. Dickinson, M. D.,}

Art Editor of "The American Tcxt-Book of Obstctrics."

" The book that appeals instantly to me for the strikingly successful, valuable, and graphic character of its illustrations is the 'Atlas of Syphilis and the Venereal Diseases.' I know of nothing in this country that can compare with it." 


\section{Holland's Medical Chemistry and Toxicology}

A Text-Book of Medical Chemistry and Toxicology. By JAMES W. Holland, M.D., Professor of Medical Chemistry and Toxicology, and Dean, Jefferson Medical College, Philadelphia. Octavo of 592 pages, fully illustrated. Cloth, $\$ 3.00$ net.

\section{RECENTLY ISSUED}

Dr. Holland's work is an entirely new one, and is based on his thirty-five years' practical experience in teaching chemistry and medicine. Recognizing that to understand physiologic chemistry, students must first be informed upon points not referred to in most medical text-books, the author has included in his work the latest views of equilibrium of equations, mass action, cryoscopy, osmotic pressure, d:ssociation of salts into ions, effects of ionization upon electric conductivity, and the relationship between purin bodies, uric acid, and urea. More space is given to toxicology than in any other text-book on chemistry. American Medicine

"Its statements are clear and terse; its illustrations well chosen; its development logical, systematic, and comparatively easy to follow. . . : We heartily commend the work."

\section{Grünwald and Newcomb's Mouth, Pharynx, and Nose}

Atlas and Epitome of Diseases of the Mouth, Pharynx, and Nose. By DR. L. Grünwald, of Munich. From the Second Revised and Enlarged German Edition. Edited, with additions, by James E. Newconb, M. D., Instructor in Laryngology, Cornell University Medical School. With 102 illustrations on 42 colored lithographic plates, 41 text-cuts, and 219 pages of text. Cloth, \$3.00 net. In Saunders' Hand-Atlas Series.

\section{INCLUDING ANATOMY AND PHYSIOLOGY}

In designing this atlas the needs of both student and practitioner were kept constantly in mind, and as far as possible typical cases of the various diseases were selected. The illustrations are described in the text in exactly the same way as a practised examiner would demonstrate the objective findings to his class. The illustrations themselves are numerous and exceedingly well executed. The editor has incorporated his own valuable experience, and has also included extensive notes on the use of the active principle of the suprarenal bodies.

\section{American Medicine}

"Its conciseness withnut sacrifice of clearness and thoroughness, as well as the excellence of text and illustrations, are commendable." 


\section{Jackson on the Eye}

A Manual of the Diagnosis and Treatment of Diseases of the Eye. By Edward Jackson, A. M., M. D., Emeritus Professor of Diseases of the Eye in the Philadelphia Polyclinic. I 2 mo volume of 535 pages, with 178 beautiful illustrations, mostly from drawings by the author. Cloth, \$2.50 net.

The Medical Record, New York

"It is truly an admirable work. . . . Written in a clear, concise manner, it bears evidence of the author's comprehensive grasp of the subject. 'The term 'multum in parvo' is an appropriate one to apply to this work. It will prove of value to all who are interested in this branch of medicine."

\section{Grant on the Face, Mouth, and Jaws}

A Text-Book of the Surgical Principles and Surgical Diseases of the Face, Mouth, and Jaws. For Dental Students. By H. Horace Grant, A. M., M. D., Professor of Surgery and of Clinical Surgery, Hospital College of Medicine, Louisville. Octavo of 23I pages, with 68 illustrations. Cloth, \$2.50 net.

\section{Annals of Surgery}

"The book is well illustrated, the text is clear, and on the whole it serves well for the purpose for which it is intended."

\section{Friedrich and Curtis' Nose, Larynx, and Ear}

Rhinology, Laryngology, and Otology, and Their Significance in General Medicine. By Dr. E. P. FrIEDrich, of Leipzig. Edited by H. Holbrook Curtis, M.D., Consulting Surgeon to the New York Nose and Throat Hospital. Octavo volume of 350 pages. Cloth. $\$ 2.50$ net.

\section{Boston Medical and Surgical Journal}

"This task he has performed admirably, and has given both to the general practitioner and to the specialist a book for collateral reference which is modern, clear, and complete." 


\section{Ogden on the Urine}

Clinical Examination of Urine and Urinary Diagnosis. A Clinical Guide for the Use of Practitioners and Students of Medicine and Surgery. By J. Bergen Ogden, M. D., Late Instructor in Chemistry, Harvard University Medical School; Formerly Assistant in Clinical Pathology, Boston City Hospital. Octavo, 418 pages, 54 illustrations, and a number of colored plates. Cloth, $\$ 3.00$ net.

\section{SECOND REVISED EDITION-RECENTLY ISSUED}

In this edition the work has been brought absolutely down to the present day. Important changes have been made in connection with the determination of Urea, Uric Acid, and Total Nitrogen ; and the subjects of Cryoscopy and Beta-Oxybutyric Acid have been given a place. Special attention has been paid to diagnosis by the character of the urine, the diagnosis of diseases of the kidneys and urinary passages; an enumeration of the prominent clinical symptoms of each disease; and the peculiarities of the urine in certain general diseases.

\section{The Lancet, London}

"We consider this manual to have been well compiled; and the author's own experience, so clearly stated, renders the volume a useful one both for study and reference."

\section{Vecki's Sexual Impotence}

The Pathology and Treatment of Sexual Impotence. By VICTOR G. Vecki, M. D. From the Second Revised and Enlarged German Edition. I $2 \mathrm{mo}$ volume of 329 pages. Cloth, $\$ 2.00$ net.

\section{THIRD EDITION, REVISED AND ENLARGED}

The subject of impotence has but seldom been treated in this country in the truly scientific spirit that its pre-eminent importance deserves, and this volume will come to many as a revelation of the possibilities of therapeutics in this important field. The reading part of the English-speaking medical profession has passed judgment on this monograph. The whole subject of sexual impotence and its treatment is discussed by the author in an exhaustive and thoroughly scientific manner. In this edition the book has been thoroughly revised, and new matter has been added, especially to the portion dealing with treatment.

\section{Johns Hopkins Hospital Bulletin}

"A scientific treatise upon an important and much neglected subject. . . . The treatment of impotence in general and of sexuai neurasthenia is discriminating and judicious." 
American Pocket Dictionary

Fourth Edition, Revised Recently Issued

The American Pocket Meidcal Dictionary. Edited by W. A. Newman Dorianid, M. D., Assistant Obstetrician to the Hospital of the University of Pennsylvania. Containing the pronunciation and definition of the principal words used in medicine and kindred sciences. Flexible leather, with gold edges, \$1.00 net; with thumb index, \$1.25 net.

James W: Holland, M. D..

Professor of Medical Chemistry and Toxicology, and Dean, Jefferson Medical College. Philadelphia,

"I am struck at once with admiration at the compact size and attractive exterior. I can recommend it to our stidents without reserve.'

\section{Stelwagon's Essentials of Skin}

Sixth Revised Edition Recently Issued

Essentials of Diseases of the Skin. By Henry W. Stelwagon, M. D., PH.D., Professor of Dermatology in the Jefferson Medical College, Philadelphia. Post-octavo of 276 pages, with 72 text-illustrations and 8 plates. Cloth, \$I.oo net. In Saunders' Question-Compend Series.

\section{The Medical News}

"In line with our present knowledge of diseases of the skin. . . Continues to maintain the high standard of excellence for which these question compends have been noted,"

\section{Wolff's Medical Chemistry}

Sixth Edition, Revised Recently Issued

Essentials of Medical Chemistry, Organic and Inorganic. Containing also Questions on Medical Physics, Chemical Physiology, Analytical Processes, Urinalysis, and Toxicology. By LAwRENCE WolfF, M. D., Late Demonstrator of Chemistry, Jefferson Medical College. Revised by Smith Ely Jelliffe, M. D., Ph.D., Professor of Pharmacognosy, College of Pharmacy of the City of New York. Post-octavo of 222 pages. Cloth, \$1.00 net. In Saunders' Question-Coripend Series.

\section{New York Medical Journal}

"The author's careful and well-studied selection of the necessary requirements of the student has enabled him to furnish a valuable aid to the student."

\section{Martin's Minor Surgery, Bandaging, and the Venereal Diseases \\ Second Edition, Revised}

Essentials of Minor Surgery, Bandaging, and Venereal Diseases. By Edward Martin, A. M., M. D., Professor of Clinical Surgery, University of Pennsylvania, etc. Post-octavo, 166 pages, with 78 illustrations. Cloth, \$1.00 net. In Saunders' Question-Compend Series.

\section{The Medical News}

" The best condensation of the subjects of which it treats yet placed before the pro feselion." 


\section{Wolf's Examination of Urine}

A Laboratory Handbook of Physiologic Chemistry and Urine-examination. By Charles G. L. Wolf, M. D., Instructor in Physiologic Chemistry, Cornell University Medical College, New York. I 2 mo volume of 204 pages, fully illustrated. Cloth, $\$ 1.25$ net. British Medical Journal

"The methods of examining the urine are very fully described, and there are at the end of the book some extensive tables drawn up to assist in urinary diagnosis."

\section{Jackson's Essentials of Eye}

Third Revised Edition

Fssentials of Refraction and of Diseases of the Eye. By Edward Jackson, A. M., M. D., Emeritus Professor of Diseases of the Fye, Philadelphia Polyclinic. Post-octavo of 26 I pages, 82 illustrations. Cloth, \$I.OO net. In Saunders' Question-Compend Series. Johns Hopkins Hospital Bulletin

"The entire ground is covered, and the points that most need careful elucidation are made clear and easy."

\section{Gleason's Nose and Throat}

Third Edition, Revised

Essentials of Diseases of the Nose and Throat. By E. B. Gleason, S. B., M. D., Clinical Professor of Otology, MedicoChirurgical College, Philadelphia, etc. Post-octavo, 241 pages, I 2 illustrations. Cloth, \$I.OO net. In Saunders' Question Compends.

\section{The Lancet, London}

"The careful description which is given of the various procedures would be sufficient to enable most people of average intelligence and of slight anatomical knowledge to make a very good attempt at laryngoscopy."

\section{Gleason's Diseases of the Ear}

Third Edition, Revised

Essentials of Diseases of the Ear. By E. B. Gleason, S. B., M. D., Clinical Professor of Otology, Medico-Chirurgical College, Phila., etc. Post-octavo volume of 2 I 4 pages, with I 4 illustrations. Cloth, \$I.OO net. In Saunder' Question-Compend Series.

\section{Bristol Medico-Chirurgical Journal}

"We know of no other small work on ear diseases to compare with this, either in freshness of style or completeness of information."

\section{Wilcox on Genito-Urinary and Venereal Diseases $\begin{gathered}\text { Just } \\ \text { Issued }\end{gathered}$}

Essentials of Genito-Urinary and Venereal. Diseases. By Starling S. Wilcox, M. D., Professor of Genito-Urinary Diseases and Syphilology, Starling Medical College, Columbus, Ohio. I 2 mo of 3 I 3 pages, illustrated. Cloth, \$I.OO net. Saunders' Compends.

\section{Stevenson's Photoscopy}

Just Ready

Photoscopy. (Skiascopy or Retinoscopy) By Mark D. StevEnson, M. D., Ophthalmic Surgeon to the Akron City Hospital. I 2 mo of 200 pages, illustrated.

Dr. Stevenson's work fully and clearly explains the use of this objective test and elucidates the reasons of the various phenomena observed. The illustrations have been drawn with special attention to their practical usefulness. 






\section{.}





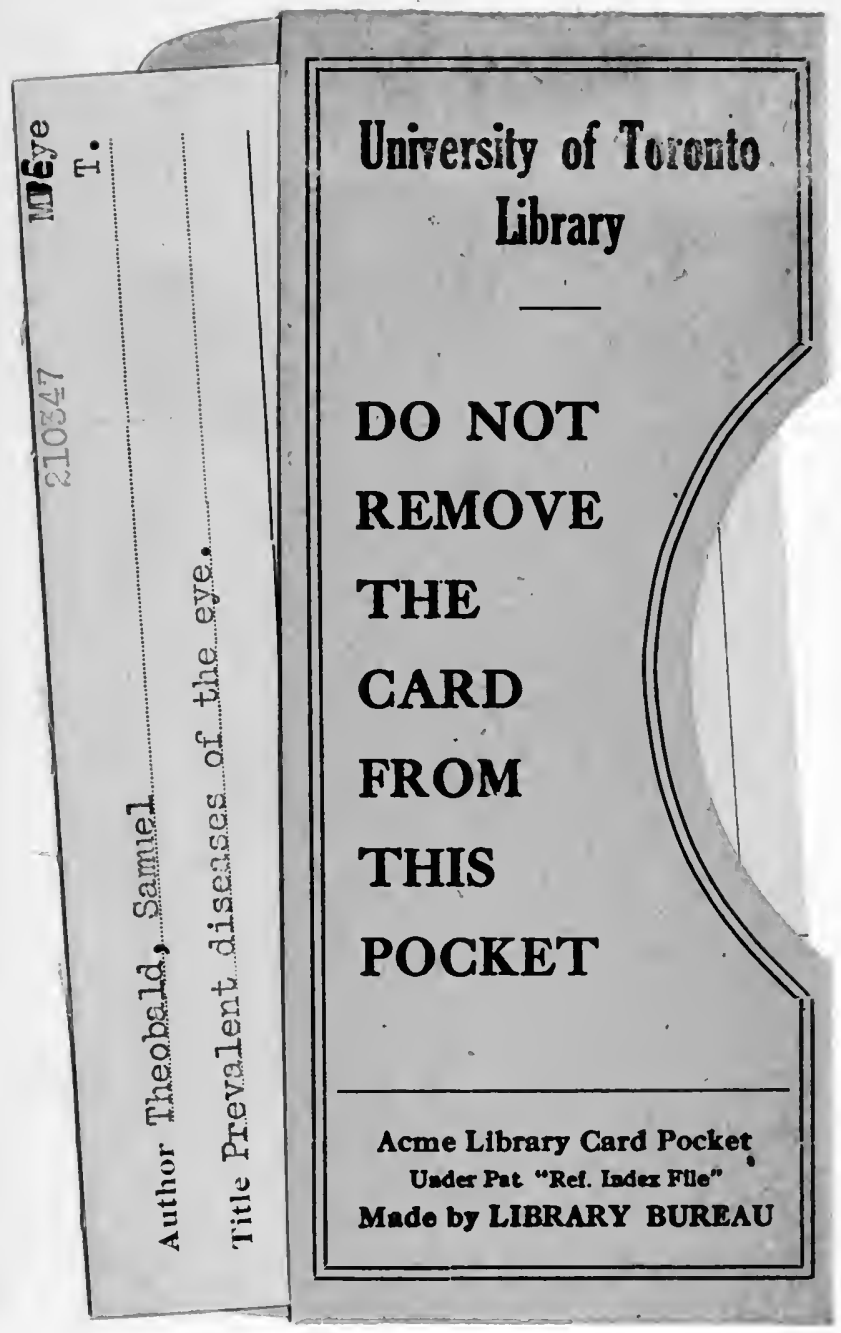


funlites

n- $4 y_{0}$

ㅂ. 